\title{
Computational Analysis of Single Drops and Sprays for Spray Cooling Applications
}

Murat Dinc

Follow this and additional works at: https://researchrepository.wvu.edu/etd

\section{Recommended Citation}

Dinc, Murat, "Computational Analysis of Single Drops and Sprays for Spray Cooling Applications" (2015). Graduate Theses, Dissertations, and Problem Reports. 5497.

https://researchrepository.wvu.edu/etd/5497

This Dissertation is protected by copyright and/or related rights. It has been brought to you by the The Research Repository @ WVU with permission from the rights-holder(s). You are free to use this Dissertation in any way that is permitted by the copyright and related rights legislation that applies to your use. For other uses you must obtain permission from the rights-holder(s) directly, unless additional rights are indicated by a Creative Commons license in the record and/ or on the work itself. This Dissertation has been accepted for inclusion in WVU Graduate Theses, Dissertations, and Problem Reports collection by an authorized administrator of The Research Repository @ WVU.

For more information, please contact researchrepository@mail.wvu.edu. 


\title{
Computational Analysis of Single Drops and Sprays for Spray Cooling Applications
}

\author{
Murat Dinc \\ Dissertation submitted \\ to the Benjamin M. Statler College of Engineering and Mineral Resources \\ at West Virginia University
}

in partial fulfillment of the requirements for the degree of

Doctor of Philosophy in

Civil Engineering

\author{
Donald D. Gray, Ph.D., Chairman \\ Leslie Hopkinson, Ph.D. \\ Wade W. Huebsch, Ph.D. \\ John M. Kuhlman, Ph.D. \\ Lian-Shin Lin, Ph.D.
}

Department of Civil and Environmental Engineering

\author{
Morgantown, West Virginia \\ 2015
}

Keywords: Single Drop Splash; Spray; Spray Cooling; Fluid Dynamics; Computational Fluid Dynamics; Heat Transfer 


\begin{abstract}
Computational Analysis of Single Drops and Sprays for Spray Cooling Applications
\end{abstract}

\title{
Murat Dinc
}

Spray cooling is a key technology in the thermal management of the next generation electronic, aircraft and spacecraft systems. There have been relatively fewer computational studies of spray cooling because simulating all the detailed physics and dynamics of a spray consisting of millions of drops per second is computationally very expensive.

In this study, computational approaches have been used to analyze single drops and sprays for spray cooling applications. The commercially available Computational Fluid Dynamics (CFD) code ANSYS Fluent (versions 14, 14.5, 15) has been used to perform single drop and spray simulations on two desktop workstations and the High Performance Computing (HPC) cluster at West Virginia University (WVU).

Single drop impingement on wet surfaces has been studied using the 2D axisymmetric Volume of Fluid (VOF) model in ANSYS Fluent 14 and 14.5. The free surface shape and hydrodynamics of single drops after they impact on wet surfaces have been validated with the experiments performed by members of the WVU Spray Cooling team in the Mechanical and Aerospace Engineering Department (MAE) at WVU. Initial film thickness, initial drop diameter, initial drop shape and gravity effects have been investigated for water at room temperature. It has been concluded that gravity has significant effects on the drop and film dynamics while drop shape does not have any significant effects.

The 2D axisymmetric Discrete Phase Model (DPM) with the wall film submodel in ANSYS Fluent 14 has been used to perform simulations of spray impact on flat surfaces. The effects of the nozzle-to-surface distance, spray half angle, spray coolant, spray mass flow rate and gravity on spray variables (e.g. average drop diameters, drop velocities, etc.) have been analyzed for a full cone spray based on the Spraying System 1/8 G nozzle operating at $40 \mathrm{psi}$ which has been used in the spray experiments performed by members of the WVU Spray Cooling team.

Full cone 40 psi water spray cooling simulations with phase change have been performed in 3D coordinates using the DPM, Eulerian Wall Film (EWF) and the Species Transport Model (STM) in ANSYS Fluent 15. The free surface shape and hydrodynamics of the film have been analyzed. The film thickness results have been compared with experiments. The effects of the surface temperature, spray temperature and air temperature on the film characteristics (e.g. film thickness, film velocity magnitude) and heat transfer (e.g. surface heat flux) have been studied. It has been concluded that air temperature does not have a significant effect on the film characteristics and heat transfer whereas spray temperature has significant effects. Increasing the spray temperature $50 \mathrm{~K}$ (from $300 \mathrm{~K}$ to $350 \mathrm{~K}$ ) causes a $62 \%$ decrease in the surface heat flux.

Full cone 40 psi water spray cooling simulations have been also performed in 2D axisymmetric coordinates using the Eulerian Multiphase (EM) model in ANSYS Fluent. The computed average surface heat flux value was $8 \%$ different compared to the 3D DPM-EWFSTM model. However, there has been a large discrepancy in the film characteristics between these two models and also between the EM model and experiments. In conclusion, the 3D DPMEWF-STM model is the preferred method in order to analyze spray cooling at the present time. 


\section{TABLE OF CONTENTS}

ABSTRACT

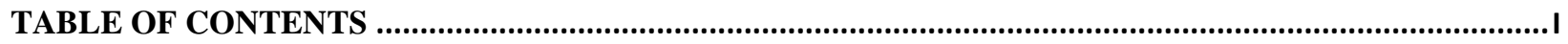

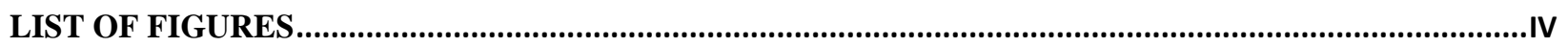

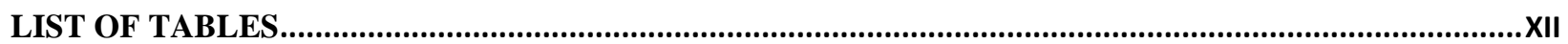

ACKNOWLEDGMENTS ...................................................................................................................................

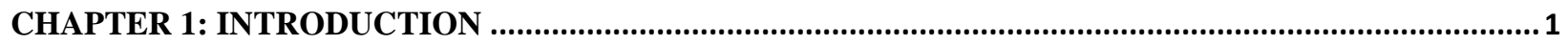

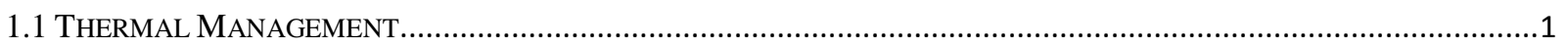

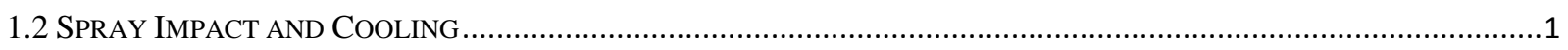

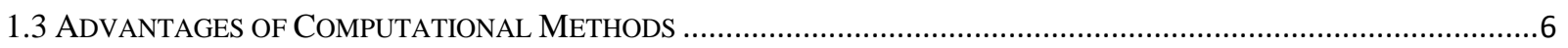

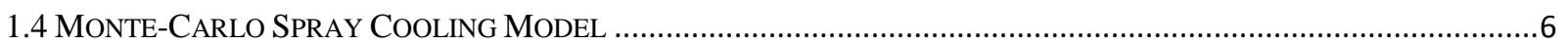

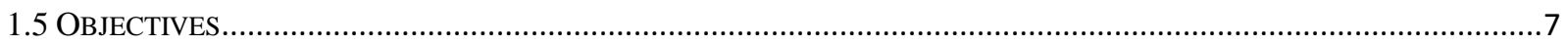

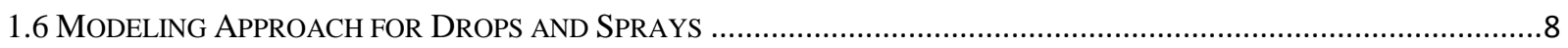

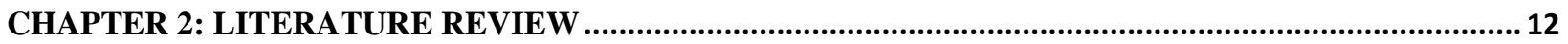

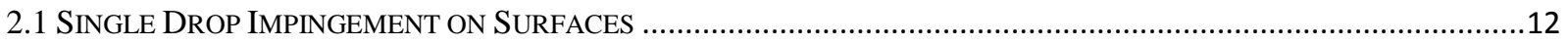

2.2 SPRAY IMPINGEMENT ON SURFACES AND SPRAY COOLING .................................................................

CHAPTER 3: SIMULATIONS OF SINGLE DROP IMPACT ON LIQUID LAYERS ................................. 23

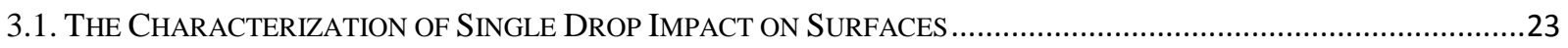

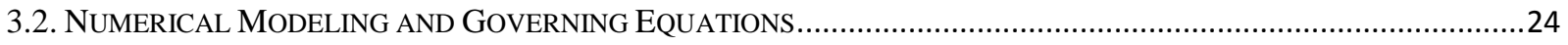

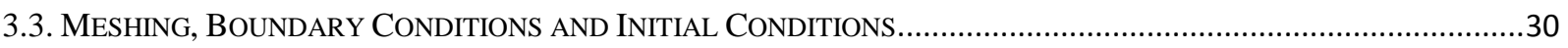

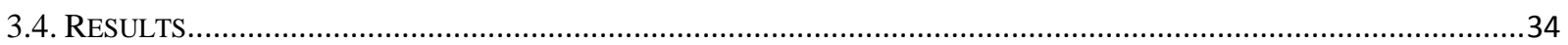

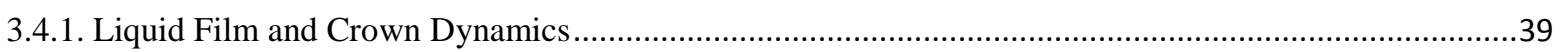

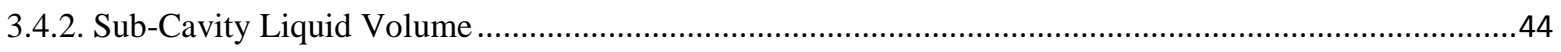

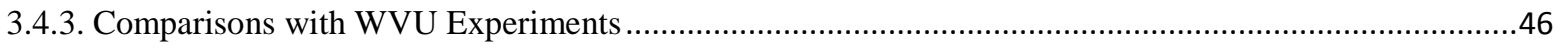

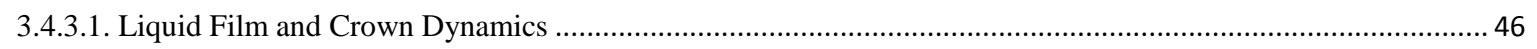

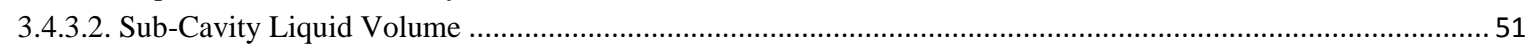

3.5. EFFECTS OF VARYING GRAVITY ON SINGLE DROP IMPACT CHARACTERISTICS...........................................55

3.6. EFFECTS OF DROP SHAPE ON SINGLE DROP IMPACT CHARACTERISTICS .....................................................62

CHAPTER 4: SPRAY IMPACT SIMULATIONS USING THE DISCRETE PHASE MODEL (DPM)......... 68

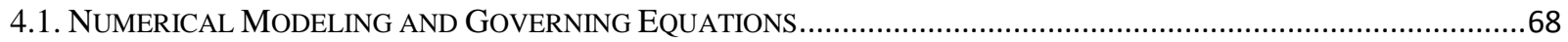

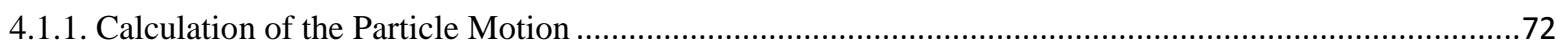

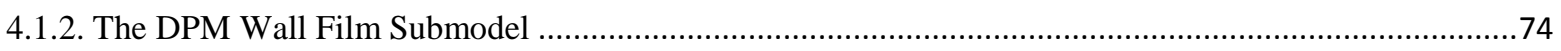

4.2. Preliminary Simulations of Sprays Impacting Plane Surfaces Using the Discrete Phase Model

(DPM)

4.2.1. Results of the Preliminary Full 3D Hollow Cone Spray Simulations .....................................................77

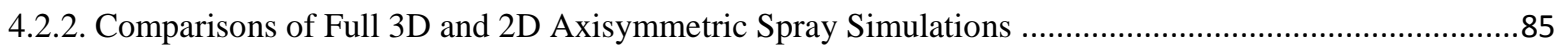

4.3. Simulations of Full Cone 2D Axisymmetric Sprays Using the Discrete Phase Model (DPM) ........90

4.3.1. Effects of Varying Nozzle-to-Surface Distance $(\mathrm{h})$..........................................................................92

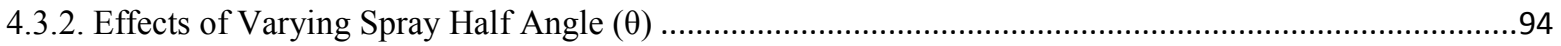




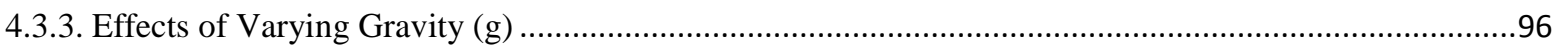

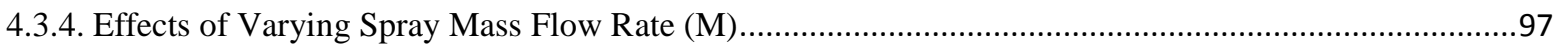

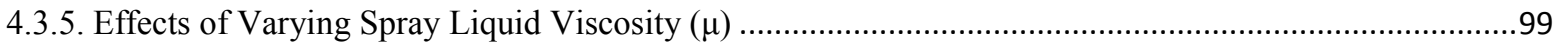

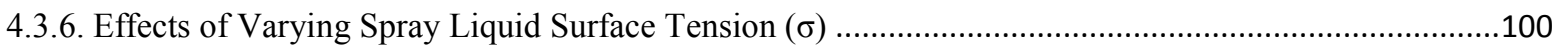

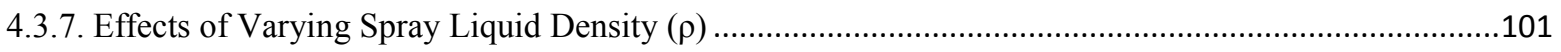

4.4. SUMMARY OF THE 2D AXISYMMETRIC FULl CONE SPRAY SiMULATIONS ...................................................103

4.5. Limitations OF THE 2D AXISYMMETRIC MODEL FOR THE CALCULATIONS OF SPRAY-WALl INTERACTIONS105

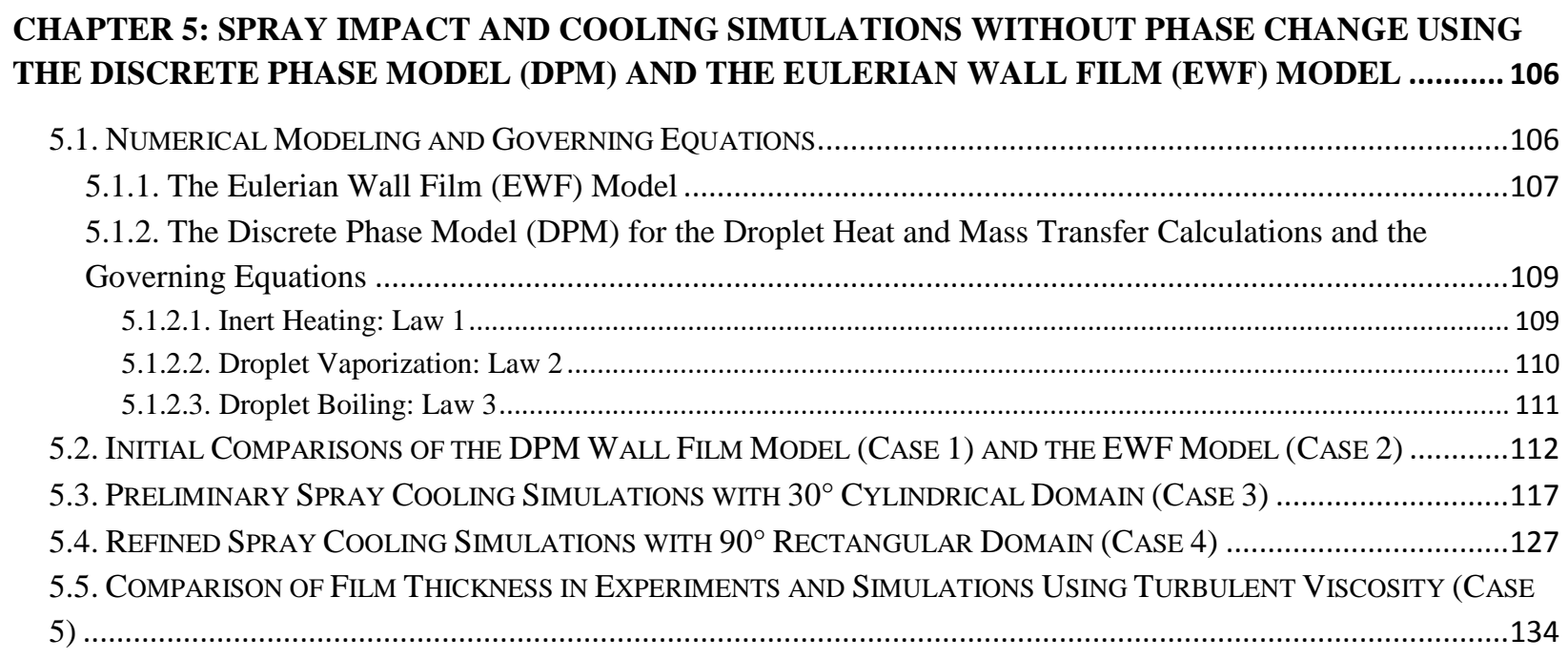

CHAPTER 6: SPRAY IMPACT AND COOLING SIMULATIONS WITH PHASE CHANGE USING THE DISCRETE PHASE MODEL (DPM), THE EULERIAN WALL FILM MODEL (EWF) AND THE SPECIES TRANSPORT MODEL (STM). 148

6.1. LiMitations OF THE EWF FOR HEAT TRANSFER CALCULATIONS IN FLUENT 14.5 .......................................148

6.2. THE SPECIES TRANSPORT MODEL (STM) AND THE CONSERVATION EQUATIONS FOR SPECIES........................152

6.3. Spray CoOling Simulations with Phase Change using the Discrete PhaSe Model, the Eulerian Wall Film Model with Turbulent Viscosity (M) AND THERmal Conductivity (K) AND THE SPeCies

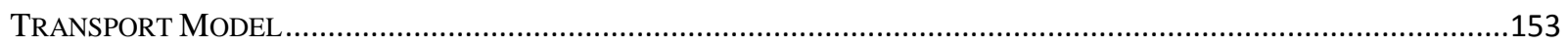

6.3.1. Spray Cooling Simulations with Phase Change: Case I...............................................................153

6.3.2. Spray Cooling Simulations with Phase Change: Case II .................................................................163

6.3.3. Spray Cooling Simulations with Phase Change: Case III ...................................................................170

CHAPTER 7: SPRAY IMPACT AND COOLING SIMULATIONS USING THE EULERIAN

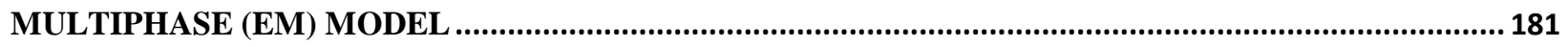

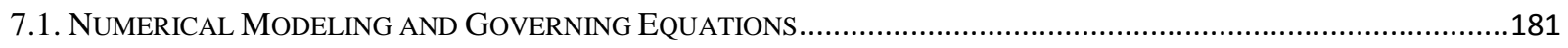

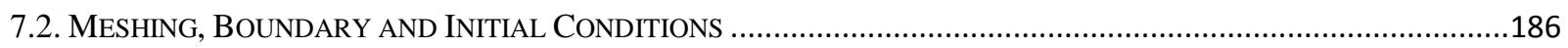

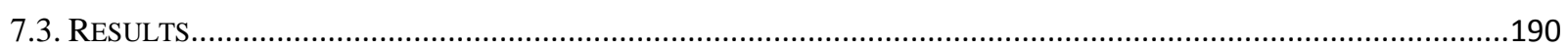

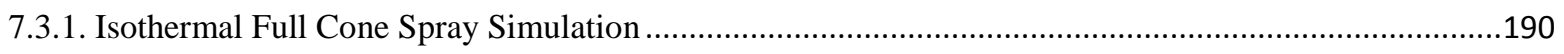

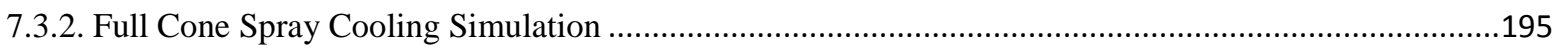

CHAPTER 8: CONCLUSIONS AND FUTURE WORK ……....................................................................... 200

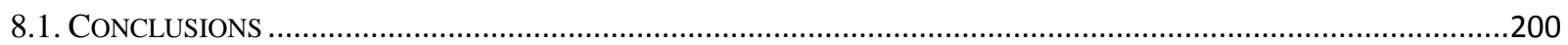

8.1.1. Simulations of Single Drop Impact on Wetted Surfaces .....................................................................200

8.1.2. Simulations of Sprays Impacting on Dry Surfaces at Isothermal Conditions .......................................201 


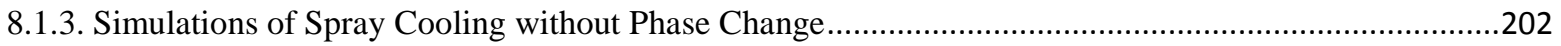

8.1.4. Simulations of Spray Cooling with Phase Change ..........................................................................202

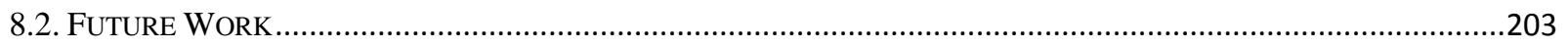

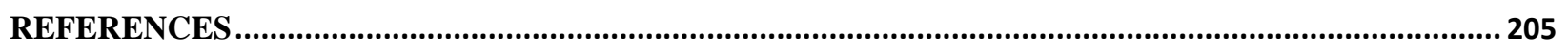

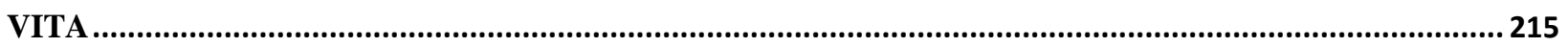




\section{LIST OF FIGURES}

Figure 1.1 Spray impact mechanisms. (Left image was obtained from the experimental part of the project at WVU and it shows a full cone spray formed from a 40 psi Spraying Systems 1/8-G FullJet nozzle which is impacting on a circular disk, right image is a cartoon that represents the liquid film formation on the impact surface)......3

Figure 1.2 Spray cooling mechanisms. (Silk et al., 2008).....

Figure 1.3 Spray schematic that shows some of the spray variables and two spray regions. (1. Region: Spray drop region, 2. Region: The impact region where drops interact with liquid film and bubbles which are formed from nucleation sites over the heated surface)......

Figure 1.4 The outline of the CFD approaches that are used in this study. ...........................................................11

Figure 2.1 Initial and boundary conditions of the 3D two phase simulation model of Sarkar and Selvam (2009). ....17

Figure 2.2 Temperature and heat flux distributions on the $\mathrm{x}-\mathrm{y}$ plane and over the surface for the 3D two phase model of Sarkar and Selvam, (2009). (Note: Comments in the boxes have been added later by present author.) ........17

Figure 2.3 Comparison of heat flux (Sarkar and Selvam, 2009). ....................................................................18

Figure 2.4 Left image shows heat flux corrected for sensible heating and wall superheat, and right image shows the average CLL length for the center eight heaters as a function of the wall superheat (Horacek et al., 2005). ( $T_{w}$

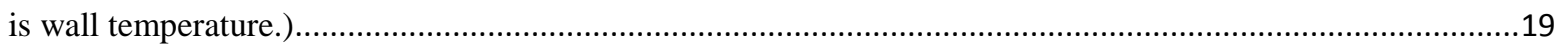

Figure 2.5 Simulation results for varying size bubbles (open circle) and impinging droplets (black dot) with a

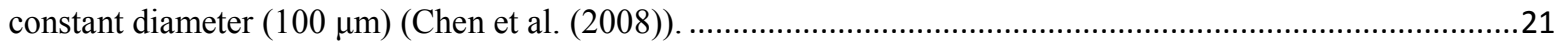

Figure 3.1 Typical single drop impact on a wet, flat surface: first impact at $t_{l}=t_{0}$ (top image), maximum crown height at $t_{2}=t_{\max }$ (middle image) and crown collapse as liquid refills crater at $t_{3}>t_{\max }$ (bottom image). (Note

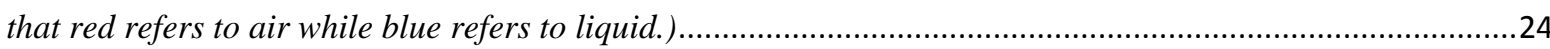

Figure 3.2 Solution procedure in the Explicit VOF model (ANSYS Fluent User's Guide, 2011)...........................27

Figure 3.3 Solver options and the VOF Scheme in Fluent (ANSYS Fluent User's Guide, 2011)..........................28

Figure 3.4 Comparison of two different interface calculation schemes: a) Simple Line Interface Calculation (SLIC) b) Piecewise Linear Interface Calculation (PLIC) (Rider and Kothe, 1998). ("Reconstructed interfaces (shaded regions) for a circle (continuous line) using the SLIC and PLIC methods. The piecewise constant approximation in SLIC forces the reconstruction to align with selected mesh logical coordinates, whereas the piecewise linear approximation in PLIC allows the reconstruction to align naturally with the interface.

Numbers in the cells denote volume fractions. ", directly taken from Rider and Kothe, 1998) ........................28

Figure 3.5 Calculation of gradients using Least Squares Cell Based Method (ANSYS Fluent User's Guide, 2011)..29 Figure 3.6 The PISO Algorithm and Solution Convergence Criteria (Versteeg, 2007). ..........................................30

Figure 3.7 Schematic of a single drop above a thin liquid film before the impact. (Note: only half of the domain is shown; blue represents the liquid while white represents the surrounding gas.)

Figure 3.8 2D Axisymmetric boundary conditions, computational domain $(0.05 \mathrm{~m} \mathrm{x} 0.05 \mathrm{~m})$ reflected across the axis.

Figure 3.9 Level-4 adaptive mesh refinement at the interface of liquid and air at $3 \mathrm{~ms}$ for Case $2(h / D=0.113)$

(Kuhlman et al., 2014). (Note: Blue refers to liquid drop, green refers to liquid layer and red refers to air)....33

Figure 3.10 Non-uniform and non-adaptive triangular mesh results for Case 5: We $=2009, D=4.2 \mathrm{~mm}$ and $h / D=$

0.5. (Note: Blue refers to air and red refers to liquid)

Figure 3.11 Comparison of results for Case 5 for $4.2 \mathrm{~mm}$ diameter droplet impacting onto a liquid layer with a thickness of $2.1 \mathrm{~mm}: R e=1168, W e=2009, F r=631$, and $h / D=0.5$. (Experimental images from Wang and Chen (2000) in the left column, numerical results from Asadi and Passandideh-Fard (2009) in the middle column, and numerical results of the current study in the right column with blue representing water and red representing air.)

Figure 3.12 Comparison of the upper crown diameter for Case 6 with $4.2 \mathrm{~mm}$ diameter droplet impacting on a thin liquid film with a dimensionless thickness of 0.67, We $=668$ (a) Experimental results (Cossali et al., 2004) and 
simulation results of Asadi and Passandideh-Fard (2009). (Image taken from Asadi and Passandideh-Fard (2009).) (b) The present simulation results. (Note: Lines are extended from experimental results of Cossali et al. (2004) in order to compare with current simulation results.)

Figure 3.13 2D axisymmetric simulation results: (a) late splash for Case 1 with $Y=3.5$ (b) crater collapse without late splash for Case 2 with $Y=0.398$ (c) late splash for Case 3 with $Y=4.7$. (Note: red is air, green is film liquid and blue is drop liquid. Only half of the solution domain is shown for clarity.) ....................................38

Figure 3.14 Three dimensional model liquid volume fraction results for Case 1 with respect to the dimensionless time $(t /(D / U))$. (Note: only quarter of the domain was simulated) (Hillen et al. (2012)) ...................................39

Figure 3.15 Case 1 comparisons of the 2D axisymmetric Level-4 and 3D Level-3 results of dimensionless centerline film thickness $\left(h_{0} / D\right)$ versus dimensionless time $(\tau)$ (Hillen et al. $\left.(2012)\right)$.....................................................40

Figure 3.16 Case 1 comparisons of dimensionless bottom crown radius $\left(R_{B} / D\right)$ versus dimensionless time ( $\tau$ ): (a) 2D axisymmetric Level-4 and (b) 3D Level-3 results with computational results of Nikolopoulos et al. (2007). (Hillen et al. (2012))

Figure 3.17 Comparison of Case 1 with $h / D=0.116$, Case 2 with $h / D=0.614$, and Case 3 with $h / D=0.5$ based on liquid (drop and film) on surface. (Note: dashed lines refer to drop liquid while solid lines refer to film liquid.)

Figure 3.18 Case 1 centerline drop and film liquid thickness $\left(h_{0}\right)$ during the initial stage. ......................................42

Figure 3.19 Case 2 centerline drop and film liquid thickness $\left(h_{0}\right)$ during the initial stage. ......................................43

Figure 3.20 Case 3 centerline drop and film liquid thickness $\left(h_{0}\right)$ during the initial stage. ....................................43

Figure 3.21 Drop liquid and layer liquid percentages (\%) below the cavity along the cavity radius (mm) for Case 1.

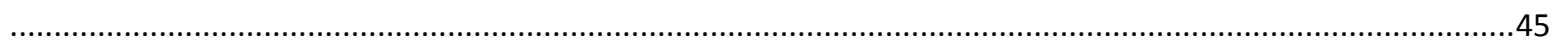

Figure 3.22 Drop liquid and layer liquid percentages (\%) below the cavity along the cavity radius (mm) for Case 2 .

Figure 3.23 Drop liquid and layer liquid percentage (\%) below the cavity along the cavity radius ( $\mathrm{mm}$ ) for Case 3.46

Figure 3.24 Case 1 comparisons of the high-speed video images (top) and the CFD simulations (bottom) for times $t$ $\approx 0,3.08,12.9$, and $28.6 \mathrm{~ms}$. (Hillen et al. (2012)) (Red refers to the air phase and blue refers to liquid phase (drop and layer). $)$.

Figure 3.25 Case 2 comparisons of the high-speed video images (top) and the CFD simulations (bottom) for times $t$ $\approx 0,3.67,8.92$, and $17.8 \mathrm{~ms}$. (Hillen et al. (2012)) (Red refers to the air phase and blue refers to liquid phase (drop and layer).).

Figure 3.26 Case 3 comparisons of the high-speed video images (top) and the CFD simulations (bottom) for times $t$ $\approx 0,4.42,33.2$, and $69.1 \mathrm{~ms}$. (Hillen et al. (2012)) (Red refers to the air phase and blue refers to liquid phase (drop and layer).).

Figure 3.27 Case 1 centerline film thickness comparisons between the average experimental results and simulation results. (Hillen et al. (2012)) .

Figure 3.28 Case 2 centerline film thickness comparisons between the average experimental results and simulation results. (Hillen et al. (2012))

Figure 3.29 Case 3 centerline film thickness comparisons between the average experimental results and simulation results. (Hillen et al. (2012)) .

Figure 3.30 Comparison of the dimensionless bottom crown diameter experimental and simulation results with respect to the dimensionless time for Case 1. (Hillen et al. (2012)) ................................................................50

Figure 3.31 Comparison of the dimensionless bottom crown diameter experimental and simulation results with respect to the dimensionless time for Case 2. (Hillen et al. (2012)) ................................................................50

Figure 3.32 Comparison of the dimensionless bottom crown diameter experimental and simulation results with respect to the dimensionless time for Case 3. (Hillen et al. (2012)) ..........................................................51

Figure 3.33 Comparison of film thickness variation with time and radius for experiments (top) and CFD (bottom) for Case 1 (Kuhlman et al., 2014) 
Figure 3.34 Comparison of film thickness variation with time and radius for experiments (top) and CFD (bottom) for

Case 2 (Kuhlman et al., 2014)...

Figure 3.35 Comparison of film thickness variation with time and radius for experiments (top) and CFD (bottom) for

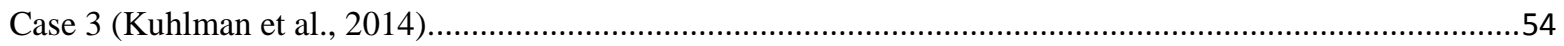

Figure 3.36 The effect of $F r$ on the time at which the crater started to refill......................................................57

Figure 3.37 The effect of $\mathrm{Fr}$ on the time at which the Worthington jet reached the undisturbed film thickness. .......58

Figure 3.38 Centerline liquid film thickness, $h_{0}$, as a function of time for Solar, Jupiter and Earth gravity. .............58

Figure 3.39 Centerline liquid film thickness, $h_{0}$, as a function of time for Earth, Mars and Vesta gravity. ...............59

Figure 3.40 Case 1: single drop impact onto a wet surface in Solar gravity. (Note: blue is water and white is air.

"ms" refers to milliseconds. Each image is cropped in order to zoom in on the impact and crater region.)......59

Figure 3.41 Case 4: single drop impact onto a wet surface in Mars gravity. (Note: blue is water and white is air.

"ms" refers to milliseconds.) ...................................................................................................................6

Figure 3.42 Case 10: single drop impact onto a wet surface in "negative Earth gravity". The ceiling is at the bottom of this figure. (Note: blue is water and white is air. "ms" refers to milliseconds.) .........................................61

Figure 3.43 Case 10: liquid crown falling off the surface for upward moving drop hitting the downward facing water film in Earth gravity ("Negative Earth Gravity"). The ceiling is at the top of this figure. (Note: original domain size is shown. Blue is water and white is air. "ms" refers to milliseconds.). .61

Figure 3.44 Single drop impact onto a wet surface in Earth gravity for: (a) Cylindrical drop with $D=4.48 \mathrm{~mm}, L=$ $2.987 \mathrm{~mm}$ (equal volume cylinder) (b) Case 3 spherical drop with $D=4.48 \mathrm{~mm} . \ldots \ldots \ldots \ldots \ldots \ldots \ldots \ldots \ldots \ldots \ldots \ldots \ldots \ldots \ldots . . .64$

Figure 3.45 Variation of the centerline liquid layer thickness, $h_{0}$, with time for the Case 3 sphere and the cylindrical

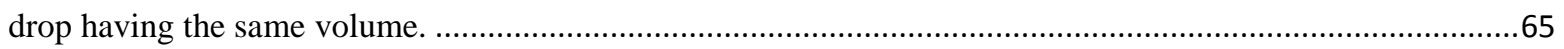

Figure 3.46 Equal altitude cylindrical drop impact onto a wet surface in Earth gravity with $D=4.48 \mathrm{~mm}, L=4.48$

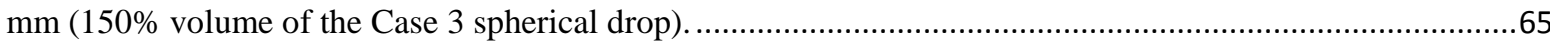

Figure 3.47 Half altitude cylindrical drop impact onto a wet surface in Earth gravity with $D=4.48 \mathrm{~mm}, L=2.24$ $\mathrm{mm}$ (75\% volume of the Case 3 spherical drop).

Figure 3.48 Comparison of bubble for (a) Equal altitude cylinder at $t=3.6 \mathrm{~ms}$, (b) Half altitude cylinder at $t=3.7$

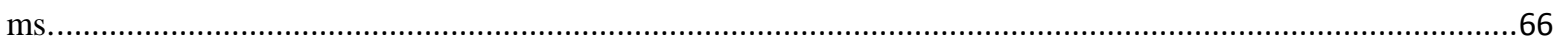

Figure 4.1 A flow regime map for a spray with 100 micron droplets.................................................................70

Figure 4.2 A high speed video image that shows a full cone spray formation for a nozzle (Kreitzer and Kuhlman, 2010).

Figure 4.3 Interaction mechanisms during impact with a boundary in the DPM wall film submodel in ANSYS Fluent

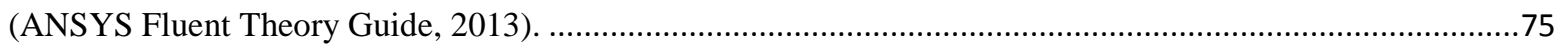

Figure 4.4 3D computational domain, domain specifications, boundary conditions and spray half angle $(\theta)$...........77 Figure 4.5 Spray injection, spray impact and liquid film formation on the surface for Case $1\left(h=40 \mathrm{~mm}, \theta=10^{\circ}\right)$. Particles are colored by the velocity magnitude-dark blue refers to lower velocity range $(0-3 \mathrm{~m} / \mathrm{s})$ and red

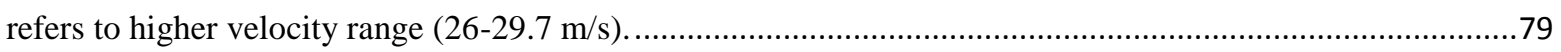

Figure 4.6 Spray injection, spray impact and liquid film formation on the surface for Case $2\left(h=35 \mathrm{~mm}, \theta=18^{\circ}\right) .80$ Figure 4.7 Spray drop diameter distribution $(\mathrm{m})$ for Case $1\left(h=40 \mathrm{~mm}, \theta=10^{\circ}\right)$ at $5 \mathrm{~ms}$. (Note that red refers to

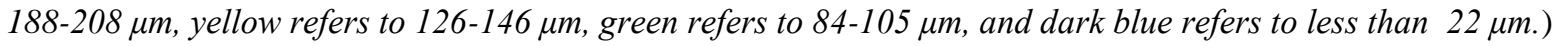

Figure 4.8 Spray drop diameter distribution $(\mathrm{m})$ for Case $2\left(h=35 \mathrm{~mm}, \theta=18^{\circ}\right)$ at $5 \mathrm{~ms}$. (Note that red refers to 301-334 $\mu$ m, yellow refers to 201-234 $\mu \mathrm{m}$, green refers to 134-168 $\mu \mathrm{m}$, and dark blue refers to less $35 \mu \mathrm{m}$.)...81

Figure 4.9 The Sauter mean drop diameter $\left(d_{32}\right)$ on the left and the arithmetic mean drop diameter $\left(d_{10}\right)$ on the right with respect to time (ms) for Case $1\left(h=40 \mathrm{~mm}, \theta=10^{\circ}\right)$ and Case $2\left(h=35 \mathrm{~mm}, \theta=18^{\circ}\right)$. (Note that initial impact time is close to $1.5 \mathrm{~ms}$ and Case 2 drops hit the wall earlier than Case 1).

Figure 4.10 Variation of the total liquid film mass, $m_{s}(\mathrm{mg})$, on the surface with respect to time $(\mathrm{ms})$ for Case $1(h=$ $\left.40 \mathrm{~mm}, \theta=10^{\circ}\right)$ and Case $2\left(h=35 \mathrm{~mm}, \theta=18^{\circ}\right)$. (Note that $t=0 \mathrm{~ms}$ is the time when spray is injected, $t \sim$ 
$1.5 \mathrm{~ms}$ is the time when spray drops start to impinge and accumulate on the surface, and the simulation end time is $7.5 \mathrm{~ms}$.)

Figure 4.11 Contours of the liquid film height at the impact surface for Case 1 at $5 \mathrm{~ms}$. (Note that red refers to 2.04$2.27 \mu \mathrm{m}$, yellow refers to 1.36-1.59 $\mu \mathrm{m}$, green refers to 0.91-1.13 $\mu \mathrm{m}$, and dark blue refers to less than 0.27 $\mu m$.).

Figure 4.12 Contours of the liquid film height at the impact surface for Case 2 at $5 \mathrm{~ms}$. (Note that red refers to 1.46$1.62 \mu \mathrm{m}$, yellow refers to 0.97-1.13 $\mu \mathrm{m}$, green refers to 0.65-0.81 $\mu \mathrm{m}$, and dark blue refers to less than 0.16 $\mu m$.)........

Figure 4.13 Variation of the spray impact efficiency, $\eta(\%)=M_{s} / M$, with respect to time (ms). (Note that $t=0 \mathrm{~ms}$ is the time when spray is injected, and $t \sim 1.5 \mathrm{~ms}$ is the time when spray drops start to impinge and accumulate on the surface.)

Figure 4.14 Comparison of the average drop diameters (a) $d_{32}$ (b) $d_{10}$ for Case $1\left(h=40 \mathrm{~mm}, \theta=10^{\circ}\right) \ldots \ldots \ldots \ldots \ldots \ldots . . .86$

Figure 4.15 Comparison of Case $1\left(h=40 \mathrm{~mm}, \theta=10^{\circ}\right)$ (a) the liquid film mass $\left(m_{s}\right)$ and (b) the spray impact efficiency, $\eta(\%)$.

Figure 4.16 Comparison of full 3D (on the left column, perspective view) and 2D axisymmetric (on the right column, vertical plane view) spray droplet velocity distribution for Case $1\left(h=40 \mathrm{~mm}, \theta=10^{\circ}\right)$ from $t=0$ $2.5 \mathrm{~ms}$. (Particles are colored by the velocity magnitude-dark blue refers to lower velocity range and red refers to higher velocity range $(26-29.7 \mathrm{~m} / \mathrm{s}$.)......

Figure 4.17 Comparison of full 3D (on the left column, perspective view) and 2D axisymmetric (on the right column, vertical plane view) spray droplet velocity distribution for Case $1\left(h=40 \mathrm{~mm}, \theta=10^{\circ}\right)$ from $t=3.5$ $6 \mathrm{~ms}$. (Particles are colored by the velocity magnitude-dark blue refers to lower velocity range and red refers to higher velocity range $(26-29.7 \mathrm{~m} / \mathrm{s})$.)......

Figure 4.18 Boundary conditions and computational cells for the 2D axisymmetric full cone spray cases...............91 Figure 4.19 (a) The Sauter mean drop diameter $\left(d_{32}\right)$ (b) the arithmetic mean drop diameter $\left(d_{10}\right)$ for Case A $(h=$ $25.4 \mathrm{~mm})$, Case B $(h=31.75 \mathrm{~mm})$ and Case $\mathrm{C}(h=38.1 \mathrm{~mm})$ with $\theta=26.5^{\circ}$

Figure 4.20 (a) The liquid film mass accumulation $\left(m_{s}\right)$ (b) the spray impact efficiency $(\eta)$ for Case $\mathrm{A}(h=25.4$ $\mathrm{mm})$, Case B $(h=31.75 \mathrm{~mm})$ and Case $\mathrm{C}(h=38.1 \mathrm{~mm})$ with $\theta=26.5^{\circ}$.

Figure 4.21 Spray drop velocity and diameter distributions for (a) Case A $(h=25.4 \mathrm{~mm})$ and (b) Case C $(h=38.1$ $\mathrm{mm}$ ) with same spray half angle, $\theta=26.5^{\circ}$. (Note: the results are shown at the equal effective time which is $t_{\text {eff }}$ $=t-t_{\text {impact }}=6.5 \mathrm{~ms}$ for both cases. $\mathrm{g}$ refers to gravity. Left part of images: Red refers to faster (also fastest) droplets $(18-20 \mathrm{~m} / \mathrm{s})$ while dark blue refers to slower (also slowest) droplets $(0-2 \mathrm{~m} / \mathrm{s})$. Right part of images: Green refers to bigger droplets (0.11-0.155 mm) while dark blue refers to smaller droplets $(0.002-0.026 \mathrm{~mm})$.)

Figure 4.22 (a) The Sauter mean drop diameter $\left(d_{32}\right)$ (b) the arithmetic mean drop diameter $\left(d_{10}\right)$ for Case $\mathrm{C}(\theta=$

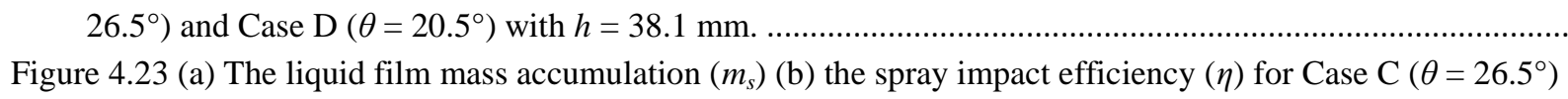
and Case $\mathrm{D}\left(\theta=20.5^{\circ}\right)$ with $h=38.1 \mathrm{~mm}$.

Figure 4.24 The droplet velocity and diameter distributions for (a) Case $\mathrm{C}\left(\theta=26.5^{\circ}\right)$ (b) Case $\mathrm{D}\left(\theta=20.5^{\circ}\right)$ with same nozzle-to-surface distance, $h=38.1 \mathrm{~mm}$. (Note: the results are shown at the equal effective time which is $t_{\text {eff }}=t-t_{\text {impact }}=6.5 \mathrm{~ms}$ for both cases. Left part of images: Red refers to faster (also fastest) droplets (18-20 $\mathrm{m} / \mathrm{s}$ ) while dark blue refers to slower (also slowest) droplets $(0-2 \mathrm{~m} / \mathrm{s})$ Right part of images: Green refers to bigger droplets (0.11-0.155 mm) while dark blue refers to smaller droplets $(0.002-0.026 \mathrm{~mm})$.)...................96

Figure 4.25 (a) The Sauter mean drop diameter $\left(d_{32}\right)$ (b) the arithmetic mean drop diameter $\left(d_{10}\right)$ for Case $\mathrm{C}(g=-$ $\left.9.81 \mathrm{~m} / \mathrm{s}^{2}\right)$, Case $\mathrm{E}\left(g=-275 \mathrm{~m} / \mathrm{s}^{2}\right)$ and Case $\mathrm{F}\left(g=+9.81 \mathrm{~m} / \mathrm{s}^{2}\right)$. (Note that negative sign means gravity acting toward the surface.)

Figure 4.26 (a) The liquid film mass accumulation $\left(m_{s}\right)$ (b) the spray impact efficiency $(\eta)$ for Case $\mathrm{C}(g=-9.81$ $\left.\mathrm{m} / \mathrm{s}^{2}\right)$, Case $\mathrm{E}\left(g=-275 \mathrm{~m} / \mathrm{s}^{2}\right)$ and Case $\mathrm{F}\left(g=+9.81 \mathrm{~m} / \mathrm{s}^{2}\right)$. 
Figure 4.27 (a) The Sauter mean drop diameter $\left(d_{32}\right)$ (b) the arithmetic mean drop diameter $\left(d_{10}\right)$ for Case $\mathrm{C}(M=$

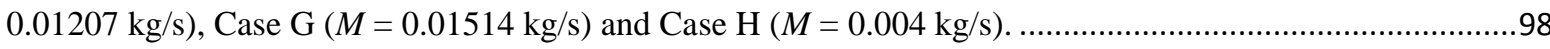

Figure 4.28 (a) The liquid film mass accumulation $\left(m_{s}\right)$ (b) the spray impact efficiency $(\eta)$ for Case $\mathrm{C}(M=0.01207$

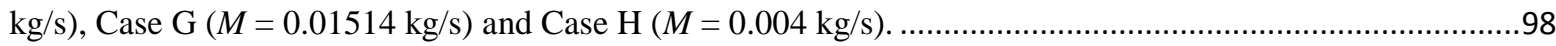

Figure 4.29 (a) The Sauter mean drop diameter $\left(d_{32}\right)$ (b) the arithmetic mean drop diameter $\left(d_{10}\right)$ for Case $\mathrm{C}$ (water: $\mu$ $=0.001 \mathrm{~kg} / \mathrm{m} . \mathrm{s})$ and Case I (HypV: $\mu=0.005 \mathrm{~kg} / \mathrm{m} . \mathrm{s})$.

Figure 4.30 (a) The liquid film mass accumulation $\left(m_{s}\right)$ (b) the spray impact efficiency $(\eta)$ for Case $\mathrm{C}$ (water: $\mu=$ $0.001 \mathrm{~kg} / \mathrm{m} . \mathrm{s}$ ) and Case I (HypV: $\mu=0.005 \mathrm{~kg} / \mathrm{m} . \mathrm{s})$ 100

Figure 4.31 (a) The Sauter mean drop diameter $\left(d_{32}\right)$ (b) the arithmetic mean drop diameter $\left(d_{10}\right)$ for Case C (water: $\sigma$ $=0.072 \mathrm{~N} / \mathrm{m})$ and Case J (HypST: $\sigma=0.01 \mathrm{~N} / \mathrm{m})$. 101

Figure 4.32 (a) The liquid film mass accumulation $\left(m_{s}\right)$ (b) the spray impact efficiency $(\eta)$ for Case C (water: $\sigma=$ $0.072 \mathrm{~N} / \mathrm{m}$ ) and Case J (HypST: $\sigma=0.01 \mathrm{~N} / \mathrm{m})$. 101

Figure 4.33 (a) The Sauter mean drop diameter $\left(d_{32}\right)\left(\right.$ b) the arithmetic mean drop diameter $\left(d_{10}\right)$ for Case C (water: $\rho$ $\left.=998 \mathrm{~kg} / \mathrm{m}^{3}\right)$ and Case K (HypD: $\left.\rho=3000 \mathrm{~kg} / \mathrm{m}^{3}\right)$. .102

Figure 4.34 (a) The liquid film mass accumulation $\left(m_{s}\right)$ (b) the spray impact efficiency $(\eta)$ for Case $\mathrm{C}$ (water: $\rho=$ $998 \mathrm{~kg} / \mathrm{m}^{3}$ ) and Case K (HypD: $\left.\rho=3000 \mathrm{~kg} / \mathrm{m}^{3}\right)$.

Figure 4.35 Effects of basic spray parameters on total number of drops $(N)$. 104

Figure 5.1 Computational domain and boundary conditions for the EWF model and DPM wall film submodel comparisons. (Note that surface below the impact surface is also assigned a wall boundary condition) .........113

Figure 5.2 Comparisons of the liquid film velocity magnitude for the DPM wall film model (Case 1) in the left column and the EWF model (Case 2) in the right column at a) $3.75 \mathrm{~ms}$, b) $17.75 \mathrm{~ms}$, c) $30.75 \mathrm{~ms}$ simulation times. (Red refers to the maximum velocity and blue refers to the minimum velocity, but the color scales differ between the two models and between times for the same model. Note that the initial spray impact time on the surface is $1.75 \mathrm{~ms}$. All images show the top view of the impact surface.).

Figure 5.3 Comparisons of the liquid film thickness for the DPM wall film model (Case 1) in the left column and the EWF model (Case 2) in the right column at a) $3.75 \mathrm{~ms}$, b) $17.75 \mathrm{~ms}$, c) $30.75 \mathrm{~ms}$ simulation times. (Red refers to the maximum thickness and blue refers to the minimum thickness, but the color scales differ between the two models and between times for the same model. Note that the initial spray impact time on the surface is 1.75 ms. All images show the top view of the impact surface.)

Figure 5.4 Computational domain and boundary conditions for the preliminary spray cooling simulations using the DPM and EWF models, Case 3. The viewpoint is above the impact surface looking toward the axis of symmetry.

Figure 5.5 Case 3 drop velocity magnitude at $11.75 \mathrm{~ms}$. (Red refers to the maximum velocity and blue refers to the minimum velocity. Initial spray impact time is $1.75 \mathrm{~ms}$.)

Figure 5.6 Case 3 flooded contours of the liquid film thickness at a) $11.75 \mathrm{~ms}$, b) $51.75 \mathrm{~ms}$. The color scale is different in a) and b). (All images show the top view of the impact surface.)

Figure 5.7 Case 3 film thickness variation with time at the symmetry surface parallel to the $x$-axis......................122

Figure 5.8 Case 3 flooded contours of the liquid film velocity magnitude at a) $11.75 \mathrm{~ms}$, b) $51.75 \mathrm{~ms}$. The color scales are nearly identical in parts a) and b). (All images show the top view of the impact surface.)..... .123

Figure 5.9 Case 3 flooded contours of the mid depth film temperature at a) $11.75 \mathrm{~ms}$, b) $51.75 \mathrm{~ms}$. The color scales differ slightly in a) and b). (All images show the top view of the impact surface.).

Figure 5.10 Case 3 mid depth film temperature variation with time along the symmetry surface parallel to the $x$-axis.

Figure 5.11 Case 3 flooded contours of the surface heat flux at a) $11.75 \mathrm{~ms}$, b) $51.75 \mathrm{~ms}$. The color scales differ in a) and b). (All images show the top view of the impact surface.).

Figure 5.12 Case 4 computational domain and boundary conditions for the spray and spray cooling simulations using the DPM and EWF models. The viewpoint is above the impact surface looking toward the axis of 
symmetry. (Note that the missing two boundary conditions on the outer vertical surfaces are pressure outlet.)

Figure 5.13 Case 4 flooded contours of the liquid film velocity magnitude at a) $5.975 \mathrm{~ms}$, b) $51.725 \mathrm{~ms}$. The color scale differs in a) and b). (All images show the top view of the impact surface.) .130

Figure 5.14 Case 4 flooded contours of the liquid film thickness at a) $5.975 \mathrm{~ms}$, b) $51.725 \mathrm{~ms}$. The color scale differs in a) and b). (All images show the top view of the impact surface.). 131

Figure 5.15 Case 4 flooded contours of the mid depth film temperature at a) $5.975 \mathrm{~ms}$, b) $51.725 \mathrm{~ms}$. The color scale is the same in a) and b). (All images show the top view of the impact surface.) 132

Figure 5.16 Case 4 flooded contours of the surface heat flux at a) $5.975 \mathrm{~ms}$, b) $51.725 \mathrm{~ms}$. The color scale differs in a) and b). (All images show the top view of the impact surface.).

Figure 5.17 Case 5 updated computational domain and boundary conditions for the spray and spray cooling simulations using the DPM and EWF models. The viewpoint is above the impact surface looking toward the axis of symmetry. (Note that not all of the boundary conditions (BC) are shown. Pressure outlet BCs at side surfaces are not included in this view.)....

Figure 5.18 Computed liquid film thickness at $70 \mathrm{~ms}$ for laminar (CFD_Laminar) and constant turbulent viscosity $\left(\mu_{e}=5 \mu\right)\left(\mathrm{CFD}_{-}\right.$Turbulent) assumptions compared with experimental data (EXP) for 40 psi water spray case.

Figure 5.19 Case 5 flooded contours of the liquid velocity magnitude at a) $50 \mathrm{~ms}$, b) $100 \mathrm{~ms}$. The color scale is not the same in a) and b). Red refers to the maximum velocity, blue refers to the minimum velocity. (All images show the top view of the impact surface.).

Figure 5.20 Case 5 flooded contours of the liquid film thickness at a) $50 \mathrm{~ms}$, b) $100 \mathrm{~ms}$. The color scale is the same for both times. Blue refers to 150 micron, red refers to 1500 micron (1.5 mm). (All images show the top view of the impact surface.).

Figure 5.21 Case 5 flooded contours of the mid depth liquid film temperature at a) $50 \mathrm{~ms}$, b) $100 \mathrm{~ms}$. The color scale is almost the same in a) and b). (All images show the top view of the impact surface.).....

Figure 5.22 Case 5 flooded contours of the surface heat flux at a) $50 \mathrm{~ms}$, b) $100 \mathrm{~ms}$. The color scale differs in a) and b). The color scale is almost the same in a) and b). All images show the top view of the impact surface. Blue refers to $0 \mathrm{~W} / \mathrm{m}^{2}$ and red refers to $1.9 \mathrm{MW} / \mathrm{m}^{2}$.

Figure 5.23 Case 5 film thickness along the $x$-axis at various times for $300 \mathrm{~K}$ spray impacting on liquid film with 150 micron initial height and $323 \mathrm{~K}$ initial film temperature. (Impact surface temperature is $372 \mathrm{~K}$.

"Isothermal Experiment" shows the experimental film thickness measurements for the same spray case using 40 psi full cone nozzle without heat transfer)

Figure 5.24 Case 5 mid depth liquid film temperature along the $\mathrm{x}$-axis for various times for $300 \mathrm{~K}$ spray impacting on liquid film with 150 micron initial height and $323 \mathrm{~K}$ initial temperature. (Impact surface temperature is 372 K.)

Figure 5.25 Case 5 average heat flux variation on the surface (22 $\mathrm{mm} \times 22 \mathrm{~mm})$. 147

Figure 6.1 Flooded contours of film thickness at $90 \mathrm{~ms}$ simulation time for a 40 psi full cone spray a) The ANSYS Fluent 15 EWF model without surface tension b) The ANSYS Fluent 14.5 EWF model with surface tension.

(The color scales are the same in $(a)$ and $(b)$. All images show the top view of the impact surface.).

Figure 6.2 Flooded contours of film velocity magnitude at $90 \mathrm{~ms}$ simulation time for 40 psi full cone spray a) The ANSYS Fluent 15 EWF model without surface tension b) The ANSYS Fluent 14.5 EWF model with surface tension. (The color scales are very close in $(a)$ and $(b)$. All images show the top view of the impact surface.)

Figure 6.3 Case I: a) 40 psi full cone water spray drops at $80 \mathrm{~ms}$ colored by droplet velocity magnitude b) Perspective view of flooded contours of the corresponding film thickness at $80 \mathrm{~ms}$ simulation time. (a) Red refers to the maximum droplet velocity range: $14.9-15 \mathrm{~m} / \mathrm{s}$; blue refers to the minimum droplet velocity range: 
12.5-12.61 m/s. b) red refers to the maximum film thickness: $724-762 \mu \mathrm{m}$; blue refers to the minimum film thickness: $0-38 \mu \mathrm{m}$.)......

Figure 6.4 Flooded contours of film velocity magnitude for 40 psi full cone water spray, Case I a) 50 ms simulation time b) $85 \mathrm{~ms}$ simulation time. (The color scale is the same for both times. Red refers to the maximum velocity range: $0.593-0.624 \mathrm{~m} / \mathrm{s}$; blue refers to the minimum velocity range: $0-0.031 \mathrm{~m} / \mathrm{s}$. The black arc indicates the edge of the spray. All images show the top view of the impact surface.).

Figure 6.5 Flooded contours of film thickness for 40 psi full cone water spray, Case I a) 50 ms simulation time b) 85 ms simulation time. (The color scale is the same for both times. Blue refers to 150 microns, red refers to 778 microns. The black arc indicates the edge of the spray. All images show the top view of the impact surface.)

Figure 6.6 Flooded contours of mid depth film temperature for 40 psi full cone water spray, Case I a) $50 \mathrm{~ms}$ simulation time b) $85 \mathrm{~ms}$ simulation time. (The color scale is almost the same in a) and b). Blue refers to 300 $K$, red refers to $366 \mathrm{~K}$. The black arc indicates the edge of the spray. All images show the top view of the impact surface.)

Figure 6.7 Flooded contours of surface heat flux for 40 psi full cone water spray, Case I a) 50 ms simulation time b) $85 \mathrm{~ms}$ simulation time. (The color scale is almost the same in a) and b). Blue refers to $0 \mathrm{~W} / \mathrm{m}^{2}$ and red refers to $2.07 \mathrm{MW} / \mathrm{m}^{2}$. The black arc indicates the edge of the spray. All images show the top view of the impact surface.)

Figure 6.8 Flooded contours of water vapor mass fraction for 40 psi full cone water spray, Case I a) $50 \mathrm{~ms}$ simulation time b) $85 \mathrm{~ms}$ simulation time. (The color scale is almost the same in a) and b). Blue refers to 0 and red refers to 0.116. The black arc indicates the edge of the spray. All images show the top view of the impact surface.).

Figure 6.9 Flooded contours of film velocity magnitude for 40 psi full cone water spray, Case II a) 50 ms simulation time b) $85 \mathrm{~ms}$ simulation time. (The color scale is the same for both times. Red refers to the maximum velocity range: $0.594-0.625 \mathrm{~m} / \mathrm{s}$; blue refers to the minimum velocity range: $0-0.031 \mathrm{~m} / \mathrm{s}$. The black arc indicates the edge of the spray. All images show the top view of the impact surface.)......

Figure 6.10 Flooded contours of film thickness for 40 psi full cone water spray, Case II a) 50 ms simulation time b) $85 \mathrm{~ms}$ simulation time. (The color scale is the same for both times. Blue refers to 150 microns, red refers to 778 microns. The black arc indicates the edge of the spray. All images show the top view of the impact surface.).

Figure 6.11 Flooded contours of mid depth film temperature for 40 psi full cone water spray, Case II a) $50 \mathrm{~ms}$ simulation time b) $85 \mathrm{~ms}$ simulation time. (The color scale is almost the same in a) and b). Blue refers to 300 $K$, red refers to $368 \mathrm{~K}$. The black arc indicates the edge of the spray. All images show the top view of the impact surface.)

Figure 6.12 Flooded contours of surface heat flux for 40 psi full cone water spray, Case II a) $50 \mathrm{~ms}$ simulation time b) $85 \mathrm{~ms}$ simulation time. (The color scale is different in $a$ ) and $b$ ). Blue refers to $0 \mathrm{~W} / \mathrm{m}^{2}$ and red refers to $0.0083 \mathrm{MW} / \mathrm{m}^{2}$ in a) and $0.737 \mathrm{MW} / \mathrm{m}^{2}$ in b). The black arc indicates the edge of the spray. All images show the top view of the impact surface.). 168

Figure 6.13 Flooded contours of water vapor mass fraction for 40 psi full cone water spray, Case II a) $50 \mathrm{~ms}$ simulation time b) $85 \mathrm{~ms}$ simulation time. (The color scale is different in a) and b). Blue refers to 0 and red refers to 0.23 in a) and 0.205 in b). The black arc indicates the edge of the spray. All images show the top view of the impact surface.).

Figure 6.14 Flooded contours of film velocity magnitude for 40 psi full cone water spray, Case III a) $50 \mathrm{~ms}$ simulation time b) $85 \mathrm{~ms}$ simulation time. (The color scale is the same for both times. Red refers to the maximum velocity range: $0.594-0.625 \mathrm{~m} / \mathrm{s}$; blue refers to the minimum velocity range: $0-0.032 \mathrm{~m} / \mathrm{s}$. The black arc indicates the edge of the spray. All images show the top view of the impact surface.).

Figure 6.15 Flooded contours of film thickness for 40 psi full cone water spray, Case III a) 50 ms simulation time b) $85 \mathrm{~ms}$ simulation time. (The color scale is almost the same for both times. Blue refers to 150 micron, red refers 
to 778 micron. The black arc indicates the edge of the spray. All images show the top view of the impact surface)

Figure 6.16 Flooded contours of mid depth film temperature for 40 psi full cone water spray, Case III a) $50 \mathrm{~ms}$ simulation time b) $85 \mathrm{~ms}$ simulation time. (The color scale is the same in a) and b). Blue refers to $300 \mathrm{~K}$, red refers to $368 \mathrm{~K}$. The black arc indicates the edge of the spray. All images show the top view of the impact surface.)

Figure 6.17 Flooded contours of surface heat flux for 40 psi full cone water spray, Case III a) 50 ms simulation time b) $85 \mathrm{~ms}$ simulation time. (The color scale is different in a) and b). Blue refers to $0 \mathrm{~W} / \mathrm{m}^{2}$ and red refers to $2.07 \mathrm{MW} / \mathrm{m}^{2}$ in a) and $2.08 \mathrm{MW} / \mathrm{m}^{2}$ in $\mathrm{b}$ ). The black arc indicates the edge of the spray. All images show the top view of the impact surface.).....

Figure 6.18 Flooded contours of water vapor mass fraction for 40 psi full cone water spray, Case III a) $50 \mathrm{~ms}$ simulation time b) $85 \mathrm{~ms}$ simulation time. (The color scale is same in a) and b). Blue refers to 0 and red refers to 0.116. The black arc indicates the edge of the spray. All images show the top view of the impact surface.)

Figure 6.19 Liquid film thickness (a) and liquid film velocity magnitude vs. radius for Cases I, II and III at $100 \mathrm{~ms}$.

Figure 6.20 Variation of the mid depth liquid film temperature (a) and water vapor mass fraction (b) over the impact radius for Cases I, II and III at $100 \mathrm{~ms}$.

Figure 7.12D axisymmetric computational domain with the boundary conditions.

Figure 7.2 Location of the velocity inlet boundary condition cells (left) and flooded contours of liquid volume fraction (right). (Red shows the maximum liquid volume fraction (0.845\%) and blue shows zero. Note that the whole domain is not shown.).

Figure 7.3 Computational cells for the whole domain (top) and for the near wall region (below). 189

Figure 7.4 Flooded contours of liquid volume fraction at $100 \mathrm{~ms}$ simulation time near the impact surface (a) isosurface values for 0.00845-1 (0.845\%-100\%) (Red is 1.0 and blue is 0.845.) and (b) iso-surface values for 0.5$1(50 \%-100 \%)$ (Red is 1 and blue is 0.5. Note that the whole domain is not shown. Areas in a and $b$ are the same. Horizontal distance is 320 micron and vertical distance is 200 micron.)

Figure 7.5 Contours of liquid volume fraction near the impact surface at (a) $30 \mathrm{~ms}$ (b) $50 \mathrm{~ms}$ (c) $70 \mathrm{~ms}$ (d) $100 \mathrm{~ms}$ simulation time. (Red is 1.0 and blue is 0 . Note that the whole domain is not shown. Areas in $a, b, c$ and $d$ are the same. Horizontal distance is 450 micron and vertical distance is 250 micron. Each cell is 12.5 micron in this figure.).

Figure 7.6 Liquid film thickness time average and Root Mean Square (RMS) thickness values vs. radius over the time interval from $30 \mathrm{~ms}-110 \mathrm{~ms}$

Figure 7.7 Relative thickness fluctuations in the liquid film (Relative thickness fluctuations $=100\left(h_{R M S} / h_{\text {mean }}\right)$ ). 194 Figure 7.8 Liquid film thickness with respect to simulation time at different radial locations..............................195

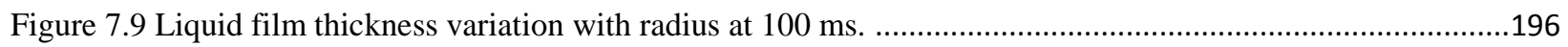

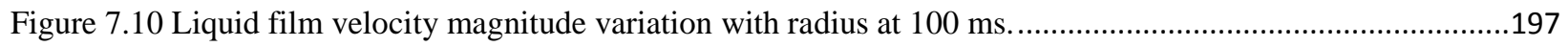

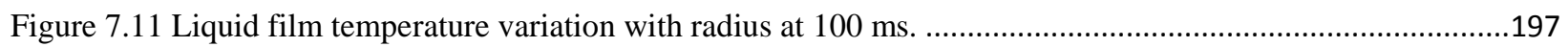

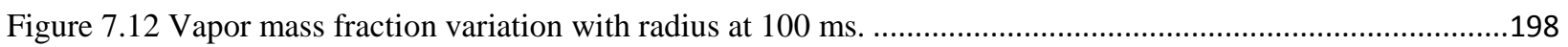




\section{LIST OF TABLES}

Table 1.1 Basic parameters that can affect spray impact and cooling.

Table 3.1 Simulations performed and corresponding dimensionless numbers. (Note: Water was used as drop and film liquid for all cases except Case 5 and Case 6 where water-70\% glycerol was used.)

Table 3.2 The average of the percentages (\%) of drop liquid and layer liquid in the whole sub-cavity for the entire cavity lifetime.

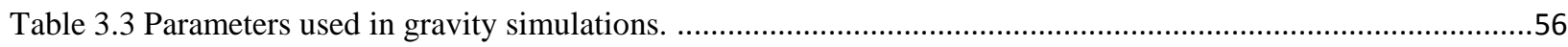

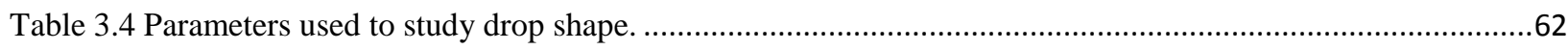

Table 3.5 The effect of drop shape on the time at which the crater started to refill..............................................67

Table 3.6 The effect of drop shape on the time at which the Worthington jet reached the undisturbed layer thickness.

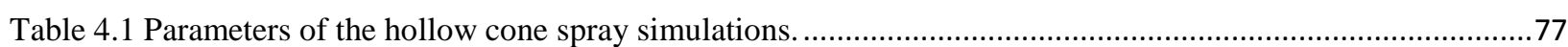

Table 4.2 General spray characteristics at $t=5 \mathrm{~ms}$ for the hollow cone spray cases. ..........................................78

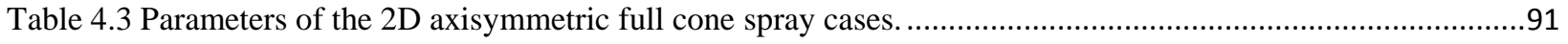

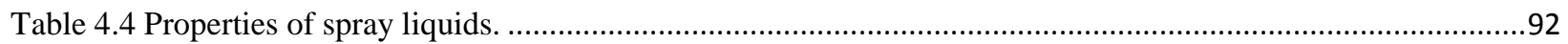

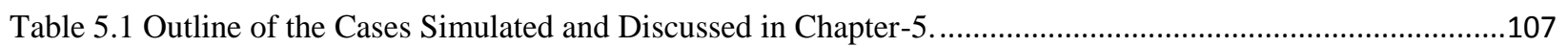

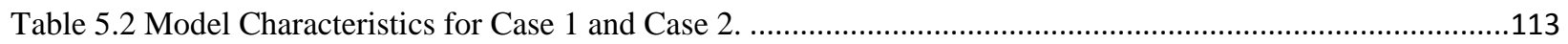

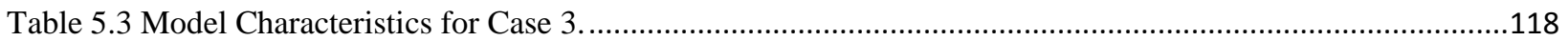

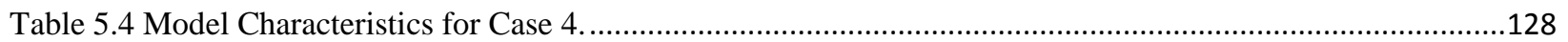

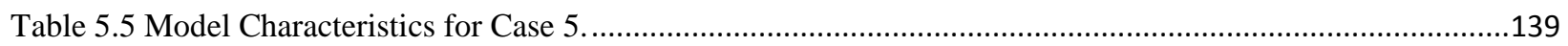

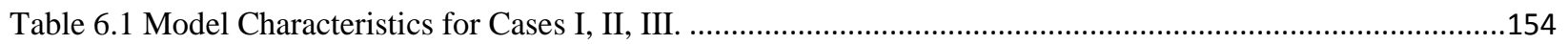

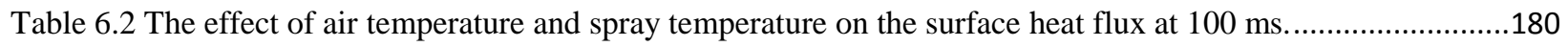

Table 7.1 Model parameters for Eulerian Multiphase (EM) Simulations..............................................................191

Table 7.2 Computed wall heat flux values for phase change simulations. ...............................................................198 


\section{ACKNOWLEDGMENTS}

I would like to thank my advisor Dr. Donald D. Gray for his great encouragement, supervising and everlasting support during my studies in Morgantown, WV. I also want to thank Dr. John M. Kuhlman for his great support and for all the helpful discussions that we made at our research meetings and also for being my committee member.

I would also like to thank Dr. Leslie Hopkinson, Dr. Lian-Shin Lin and Dr. Wade W. Huebsch for being my committee members and for their time and thoughts in reviewing my dissertation.

I would like to express my deep appreciation to NASA for their financial support throughout the entire project under Contract Number NNX10AN04A. I would like to thank Dr. Eric Silk of NASA Goddard Spaceflight Center and Dr. Kirk Yerkes of the Air Force Research Laboratory for their valuable suggestions and contributions on the research project.

I would like to thank my fellow students Nicholas Hillen, Steven Taylor and Krishna Medam for all the helpful discussions that we made at our research meetings and also for being good friends.

Last but not least, I would like to thank my mom and dad, Deniz and Kemal, and my fiancée, Rémah, for their great support, motivation and encouragement throughout my doctoral studies. 


\section{CHAPTER 1: INTRODUCTION}

\subsection{Thermal Management}

With the latest developments and advances in technology and science, the size of electronic devices is decreasing causing the power consumption/volume ratio to increase dramatically. Overheating can cause damage or even failure of an electronic device during operation. Some high level electronics such as Insulated Gate Bipolar Transistors (IGBT's) and Laser-Diode Arrays (LDA's) can dissipate more than $1500 \mathrm{~W} / \mathrm{cm}^{2}$ (Sehmbey et al., 1995). There is a certain need to effectively remove heat from these devices.

It has been shown by many researchers that spray cooling has the greatest potential for removing heat from surfaces (Silk et al., 2008). It has many practical applications in the field of thermal management for the current and future high level computers, electronic devices and space based systems (e.g. space shuttles, high performance computational devices, lidar systems, etc.).

Spray cooling has some important advantages over the other high heat flux cooling methods (micro-channel cooling, pool boiling, liquid jet impingement, etc.). Spray cooling is characterized by uniform and high heat removal, low liquid velocity (e.g. compared to the liquid jet cooling method) and small fluid inventory. Another advantage of spray cooling over the other methods is the requirement of low flow rate for a given heat flux. Spray cooling can offer the most significant heat removal from surfaces due to phase change and other important heat transfer mechanisms (e.g. transient heat conduction, convection, bubble formation from the nucleation sites over the heated surface, etc.) that occur. The highest rates of heat transfer from microelectronic components and other high energy density devices have been achieved using spray cooling. Yang et al. (1996) reported achieving a heat flux up to $1000 \mathrm{~W} / \mathrm{cm}^{2}$ for spray cooling on flat surfaces. Pais et al. (1992) also reported that up to $1200 \mathrm{~W} / \mathrm{cm}^{2}$ was obtained using water as a coolant liquid.

\subsection{Spray Impact and Cooling}

Even though spray cooling is the most effective cooling method, a universal empirical model which includes the basics of spray cooling has not been well defined yet because all of the heat transfer mechanisms and the importance of these mechanisms on spray cooling have not been 
fully understood. In order to understand spray cooling and its heat transfer mechanisms, one should also understand in detail the spray impact characteristics on a surface and the effects of some of the basic parameters on both spray impact and spray cooling (Table 1.1). Spraying system, spray liquid (coolant), and impact surface and ambient conditions are three major sets of these basic parameters. These three major groups consist of several parameters, some of which are given in Table 1.1. The effects of each of these three major groups on spray impact and cooling should be well understood in order to develop a universal empirical model for spray cooling.

Table 1.1 Basic parameters that can affect spray impact and cooling.

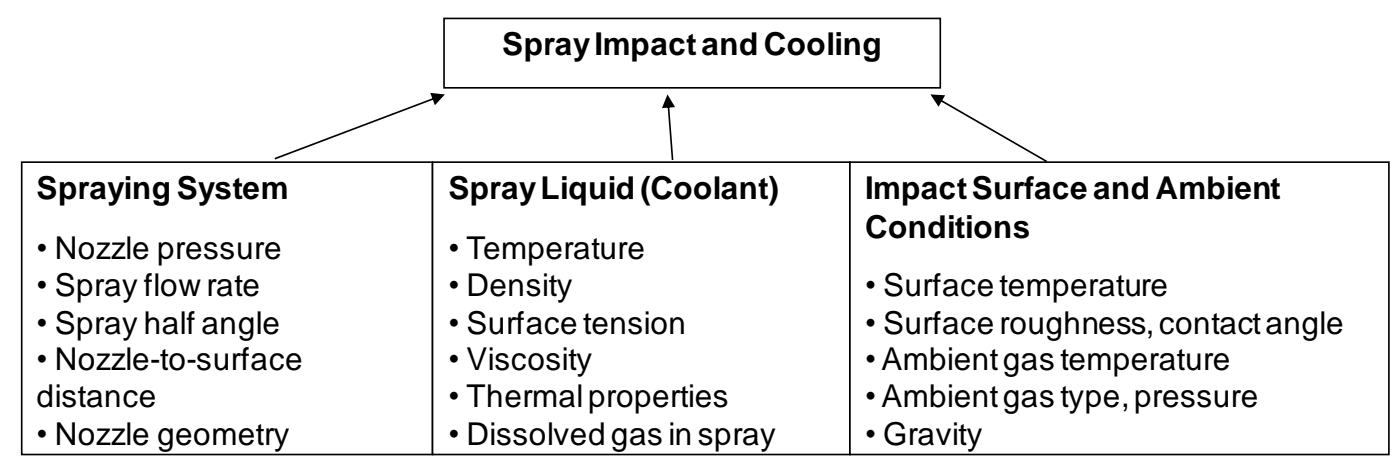

Sprays can include millions of drop impingements per second on a surface which is generally referred as the impact (heated) surface. After a spray impacts a dry surface, a liquid film is formed on that initially dry surface with the film thickness ranging between a few microns to hundreds of microns (Fig. 1.1). The extreme complexity of the flow created by the impact of millions of droplets each second is very challenging (Fig. 1.1 and Fig. 1.2), and this impact can cause different heat transfer mechanisms to occur (e.g. convective heat transfer, transient heat conduction between liquid drops and impact (heated) surface, free surface evaporation of liquid film, generation of vapor bubbles from nucleation sites on the surface, etc.). The understanding of these heat transfer mechanisms and which are the most dominant is not complete yet, although several studies have been performed both experimentally and computationally. 


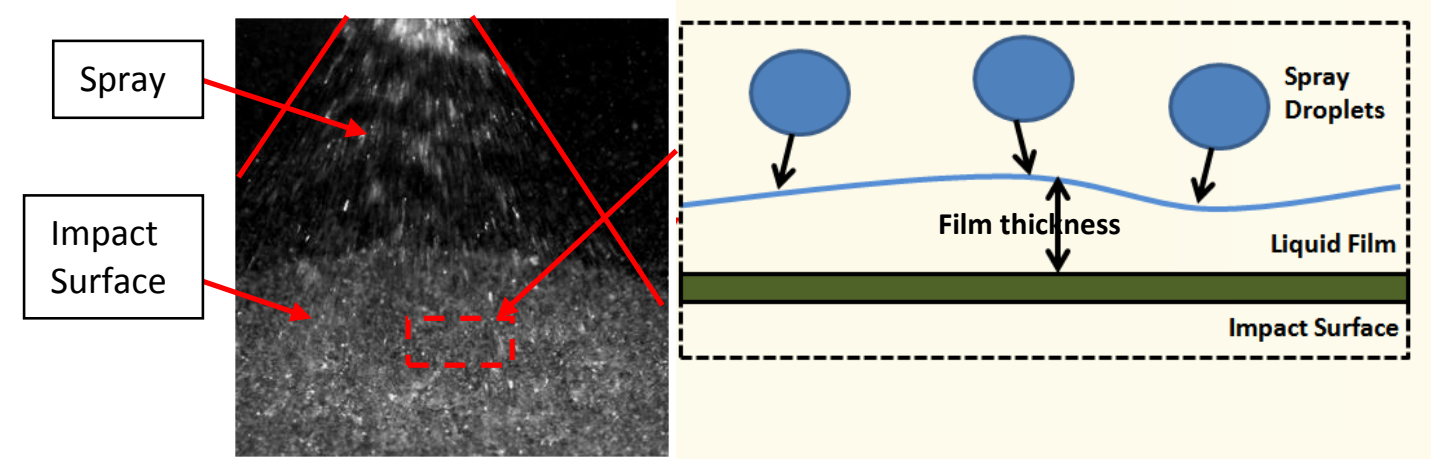

Figure 1.1 Spray impact mechanisms. (Left image was obtained from the experimental part of the project at WVU and it shows a full cone spray formed from a 40 psi Spraying Systems 1/8-G FullJet nozzle which is impacting on a circular disk, right image is a cartoon that represents the liquid film formation on the impact surface)

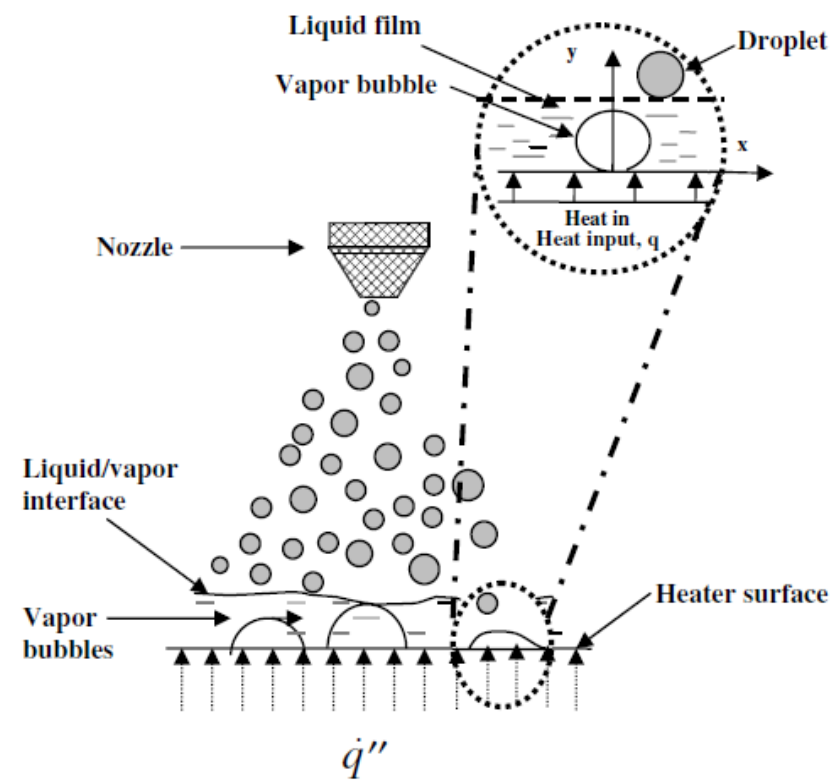

Figure 1.2 Spray cooling mechanisms. (Silk et al., 2008)

Figure 1.3 shows a spray schematic which includes some of the spray variables considered in the dimensional analysis of heat flux $(q)$ in spray cooling. 

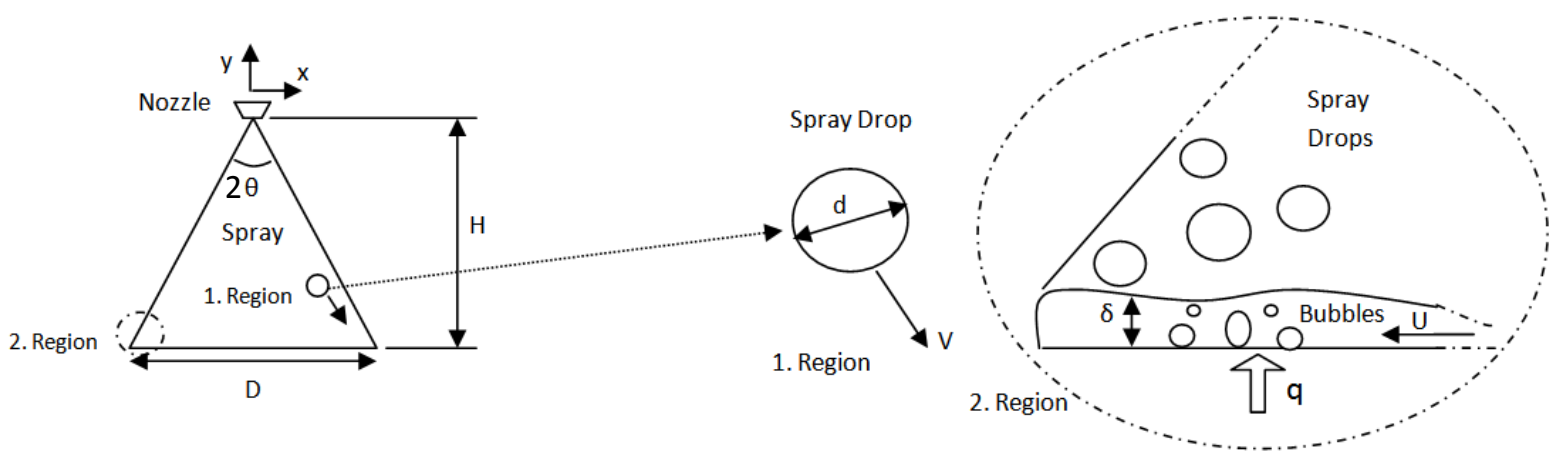

Figure 1.3 Spray schematic that shows some of the spray variables and two spray regions. ( 1 . Region: Spray drop region, 2. Region: The impact region where drops interact with liquid film and bubbles which are formed from nucleation sites over the heated surface)

The independently controllable variables that are generally considered to affect the heat flux in spray cooling are

- Nozzle type

- Nozzle gage pressure: $P$

- Nozzle-to-impact surface distance: $H$

- Radial position: $r$

- Liquid spray temperature: $T_{\text {liq }}$

- Liquid density: $\rho$

- Liquid surface tension: $\sigma$

- Liquid kinematic viscosity: $v$

- Liquid thermal conductivity: $k$

- Liquid specific heat: $c_{p}$

- Liquid enthalpy of vaporization: $h_{f g}$

- Surface temperature: $T_{\text {sur }}$

- Gravity: $g$

- Ambient gas temperature: $T_{a m b}$

- Ambient gas pressure which determines the liquid saturation temperature: $T_{\text {sat }}$

The selection of the nozzle type, gage pressure, and distance from the surface $H$ determines the spray flowrate $Q$, the spray angle $2 \theta$, the Sauter mean drop diameter $d_{32}$, the drop velocity distribution $V$, and the spray radius at the surface $R$. The choice of the coolant and its temperature $T_{l i q}$, together with the ambient gas pressure determine the fluid properties $\rho, \sigma, v, k$, $c_{p}, h_{f g}$ and $T_{s a t}$. The surface temperature is obviously important for heat transfer. The ambient 
gas temperature may also be important, but the other properties of the gas have been found to have negligible effects on the flow and heat transfer. Gravity $g$ may also affect the flow and heat transfer. Based on these considerations, a typical dependent variable such as the local heat flux $q$ at radius $r$ at steady state is given by this dimensional expression:

$$
q=f\left(T_{\text {liq }}, T_{\text {sur }}, T_{\text {sat }}, T_{a m b}, P, r, R, k, c_{p}, v, \rho, \sigma, g, \theta, h_{f g}\right)
$$

The subcooling is defined as

$$
\Delta T_{\text {sub }}=T_{\text {sat }}-T_{\text {liq }}
$$

The superheat is defined as

$$
\Delta T_{\text {super }}=T_{\text {sur }}-T_{\text {sat }}
$$

The spray radius $R$ at the impact surface is given by $R=H \tan (\theta)$.

Therefore the dimensional expression can be written as

$$
q=f\left(\Delta T_{\text {sub }}, \Delta T_{\text {super }}, T_{\text {sat }}, T_{a m b}, P, r, R, k, c_{p}, v, \rho, \sigma, g, \theta, h_{f g}\right)
$$

Based on dimensionless analysis, the following dimensionless relationship can be derived.

$$
\frac{q R}{k \Delta T_{\text {super }}}=f\left(\frac{\Delta T_{\text {sub }}}{\Delta T_{\text {super }}}, \frac{T_{\text {amb }}}{\Delta T_{\text {super }}}, \frac{T_{\text {sat }}}{\Delta T_{\text {super }}}, \frac{P v}{k \Delta T_{\text {super }}}, \frac{R}{r}, \frac{C_{p} \Delta T_{\text {super }} R^{2}}{v^{2}}, \frac{\rho v^{3}}{k \Delta T_{\text {super }} R^{2}}, \frac{\sigma v}{k \Delta T_{\text {super }} R}, \frac{g R^{3}}{v^{2}}, \theta, \frac{h_{f g} R^{2}}{v^{2}}\right)
$$

Some of the parameters can be rearranged and may be written as

$$
N u=f\left(\frac{\Delta T_{\text {sub }}}{\Delta T_{\text {super }}}, \frac{T_{\text {amb }}}{\Delta T_{\text {super }}}, \frac{T_{\text {sat }}}{\Delta T_{\text {super }}}, \frac{P v}{k \Delta T_{\text {super }}}, \frac{R}{r}, M o, J a, P r, B o, \frac{\rho v^{3}}{k \Delta T_{\text {super }} R^{2}}, \theta\right)
$$

where $N u$ is the Nusselt number which is the ratio of convective to conduction heat transfer $(N u$ $=h R / k), M o$ is the Morton number which is used to characterize the shapes of bubbles or drops 
$\left(M o=g \rho^{3} v^{4} / \sigma^{3}\right), J a$ is the Jakob number which is the ratio of sensible to latent energy $(\mathrm{Ja}=$ $c_{p} \Delta T_{\text {super }} / h_{f g}$ ), $\operatorname{Pr}$ is the Prandtl number which is the ratio of the momentum and thermal diffusivities $\left(\operatorname{Pr}=c_{p} v \rho / k\right), B o$ is the Bond number which is the ratio of gravitational and surface tension forces $\left(B o=\rho g R^{2} / \sigma\right)$.

This dimensionless form of heat flux shows the complexity of two-phase spray cooling since heat flux depends on many variables as shown equation 1.6.

\subsection{Advantages of Computational Methods}

Although laboratory experiments are essential to understand spray cooling, there are several advantages of the computational approaches. One of the advantages of computational studies over experiments is the ability to visualize important features such as the liquid vapor interface or the temperature and velocity distribution in the very thin liquid film over the heater surface (Sarkar, 2008). Experimental methods can be more time consuming and can be very expensive to modify. For instance, in order to investigate gravity effects, experiments should vary gravity. Also, it is difficult to vary several different parameters independently at the same time, for example the coolant properties such as thermal conductivity, latent heat of vaporization and specific heat. This is not possible using experimental methods since once the coolant is changed all the physical and thermal properties of the coolant are also changed. However, using the computational approaches one can study the effects of each individual property of the coolant keeping the others constant.

\subsection{Monte-Carlo Spray Cooling Model}

Even though traditional computational fluid dynamics can be preferable compared to experiments, there are also some disadvantages in computational methods. For example, there should be very small computational elements where small spray drops, bubbles and liquid films occur in a spray cooling simulation. This need for very small computational cells increases the total computational time and also the storage in order to keep all data and results saved. This decreases efficiency, especially when the number of simulations needed is large. Many of the simulations presented in this dissertation have required run times of several days on state of the art workstations and clusters. 
A first principles simulation of a spray of millions of drops is not practical without using supercomputers. Therefore, there is a need for an accurate predictive model that is physically accurate yet computationally effective. Kuhlman and Kreitzer (2010) at West Virginia University (WVU) have developed a Monte Carlo model to satisfy this need. The Monte Carlo method is a statistical approach to track individual droplets, and it utilizes randomly generated numbers to make calculations that result in final averaged predictions. This Monte Carlo model is a flexible computer model that consists of some correlations and assumptions obtained from literature regarding single drops, sprays and spray cooling. Some of these correlations include the drop diameter and velocity distribution and radial number flux for a specific spray nozzle, drop splashing mechanisms and the number of drop impacts resulting in a splash, the penetration depth of impinging drops, the crown growth and the impact crater size and lifetime (Kreitzer and Kuhlman, 2010). More details about this model can be found in Kreitzer's dissertation (Kreitzer, 2010).

\subsection{Objectives}

Although the initial results of the Monte Carlo model have been promising, it relies on correlations that at present are based on insufficient data or unconfirmed assumptions. For instance, the Monte Carlo model results showed significant disagreement in the calculation of a surface heat flux versus the temperature difference between the heater surface and spray liquid (Kreitzer, 2010). This disagreement was explained by Kreitzer and Kuhlman (2010) as due to a correlation that was needed for the calculation of the number of nucleation sites in the boiling region on the heater surface. Kreitzer and Kuhlman (2010) also pointed out that the Monte Carlo model should include the effects of different nozzles, different spray mass flow rates, different geometries and different fluids on the calculation of spray cooling.

The main objective of the present work was to obtain computational models that could be used to derive additional necessary correlations for the Monte Carlo spray cooling model that has been previously developed by Kreitzer and Kuhlman (2010). These computational studies are described in two different sections: Chapter 3 includes simulations of a single drop impact on initially undisturbed liquid films, and Chapters 4-7 include simulations of a spray impact on flat unheated and heated surfaces. 
The minimum liquid film thickness created in the cavity formed by a droplet impacting a preexisting liquid film is important because it helps to understand the details of spray impact and cooling mechanisms on a heated surface. A closely related question is the volume of liquid under the impact crater (the sub-cavity liquid volume). The relative percentages of impinging drop liquid and pre-existing film liquid in a sub-cavity volume are also important to understand the effects of the impinging spray drops on liquid film dynamics and heat transfer. These characteristics can help to accurately predict the onset of boiling and the time when dry-out of the thin liquid film could happen. It is believed that this sub-cavity liquid volume dry-out could contribute to the onset of Critical Heat Flux (CHF) (Kuhlman et al., 2007). Thus, simulations of a single drop impact on surfaces have been performed to obtain this information.

The effects of primary spray variables (drop diameter, velocity, number of total drops, etc.) and spray-wall variables (liquid film mass, liquid film height, average total liquid film velocity, etc.) have been studied in order to investigate the effects of nozzle-to-surface distance, nozzle spray geometry (e.g., spray half angle), spray coolant properties (e.g., different coolants) and gravity (e.g., microgravity, hypergravity) on sprays. Spray simulations have been performed to calculate the spray variables and spray-wall interactions in both isothermal and heat transfer conditions. Surface temperature variation on the surface and in the liquid film, evaporation of liquid film and generation of vapor bubbles from nucleation sites on the heater surface are some of the important phenomena that need to be considered in spray cooling simulations. Several of these parameters have been studied in this dissertation.

For these purposes, the commercially available Computational Fluid Dynamics (CFD) code ANSYS Fluent (versions 14, 14.5, 15) was used to model single drop and spray simulations on two desktop workstations each having Quad Core Xeon Processors and 24 GB of memory. Additional simulations were performed using the High Performance Computing (HPC) cluster at West Virginia University using 6 cores and 48 GB memory. More details about the models that are used for single drop and spray simulations are explained in the next section and also in the later chapters of the dissertation.

\subsection{Modeling Approach for Drops and Sprays}

The Volume of Fluid (VOF) method is an interface tracking method used for the calculation of the interfaces between two or more immiscible fluids. Single drop simulations have been studied 
using the Volume of Fluid (VOF) model in ANSYS Fluent. A 2D axisymmetric domain was generated to perform the single drop simulations which are presented in Chapter 3. Different color schemes were used for film liquid, drop liquid and air phases to differentiate each phase before, during, and after the impact (e.g., during crater formation). The adaptive mesh refinement method was utilized in order to reduce the total computational time. The effects of initial liquid film thickness, initial drop diameter and velocity, gravity, and initial drop shape on cavity formation and sub-cavity liquid parameters (thickness, radius, volume, etc.) were investigated for water.

The Discrete Phase Model (DPM) in ANSYS Fluent is a Lagrangian-Eulerian based multiphase model, and it has been used for the calculation of dispersed phase flows presented in Chapter 4. In the DPM method, a large number of particle trajectories can be calculated in the Lagrangian formulation while the continuous phase is calculated by using the standard Eulerian conservation equations. The DPM has built-in submodels to calculate basic spray characteristics e.g. droplet atomization, two-way coupling between liquid and gas phases and spray and impact surface interactions via the DPM wall film model. Hollow cone spray simulations were performed using both full 3D and 2D axisymmetric (2D-Axi) computational domains using the DPM to investigate the predicted effects of nozzle-to-surface distance and spray half angle at isothermal conditions. Results for 2D axisymmetric and 3D simulations were compared for the primary spray variables (drop diameter, velocity, number of total drops, etc.) and spray-wall interactions (liquid film mass, liquid film height, average total liquid film velocity, etc.). It was concluded that 2D axisymmetric spray models were sufficient to capture all the necessary details of the spray variables and spray-wall interactions. Therefore, the 2D axisymmetric DPM model was chosen to perform spray impact and cooling simulations using full cone sprays. A full cone spray (based on the Spraying System 1/8 G nozzle which was used in spray experiments at WVU) impingement onto a non-heated surface was simulated to analyze the effects of the some of the parameters shown in Table 1.1. Nozzle-to-surface distance, nozzle spray geometry (e.g. spray half angle), and spray coolant properties were analyzed using the 2D axisymmetric DPM model in ANSYS Fluent. The details of these studies are explained in Chapter 4.

However, there are some shortcomings in both the VOF interface tracking method and the DPM dispersed multiphase model. Even though an adaptive mesh refinement method has been implemented in VOF, the computational time was long even for single drop impingement 
simulations (4-5 days for a single case from the initial impact until the liquid crown collapse). Since the VOF model requires the resolution of all involved length scales, if more drops were added, the total computational time would increase dramatically making simulation of large numbers of cases studies almost impossible. The DPM model can be successfully used to simulate spray atomization and spray wall interactions with heat transfer. However, the DPM model as implemented in ANSYS Fluent does not include boiling of the film even though evaporative phase change is included for the spray drops. In addition, even though the DPM wall film model gave good results for the film thickness distribution over the impact surface at early simulation times, later simulation times showed unrealistic film thickness results at and near the axis for 2D-axisymmetric simulations. Therefore, other modeling approaches were studied to calculate the spray-wall interactions.

The Eulerian Wall Film (EWF) model in ANSYS Fluent has been used with the DPM to perform spray and spray wall-film calculations in 3D coordinates as presented in Chapter 5. Spray cooling without phase change was also studied using the EWF and DPM. Film thickness results were compared with the experimental measurements. In order to calculate phase change in the film and drops, the Species Transport Model (STM) was needed to couple with the DPM and EWF. Simulations of spray cooling with phase change were successfully performed using the DPM-EWF-STM models. The details of spray cooling simulations with phase change are explained in Chapter 6.

Finally, the Eulerian Multiphase (EM) model has also been used to perform spray cooling simulations in 2D axisymmetric coordinates with boiling modeled using the Rensselaer Polytechnic Institute (RPI) boiling model of Kurul and Podowski (1991) as summarized in Chapter 7. The purpose of using the EM model was to investigate which modeling approach could be more accurate with the least amount of computational time for the simulations of spray cooling with phase change. However, this approach gave unrealistic results. In addition, a simulation of spray cooling with phase change using the EM took about 5 times longer than the DPM-EWF-STM model. Figure 1.4 shows the outline of the computational approaches that are used for the modeling of single drops, sprays and spray cooling. 


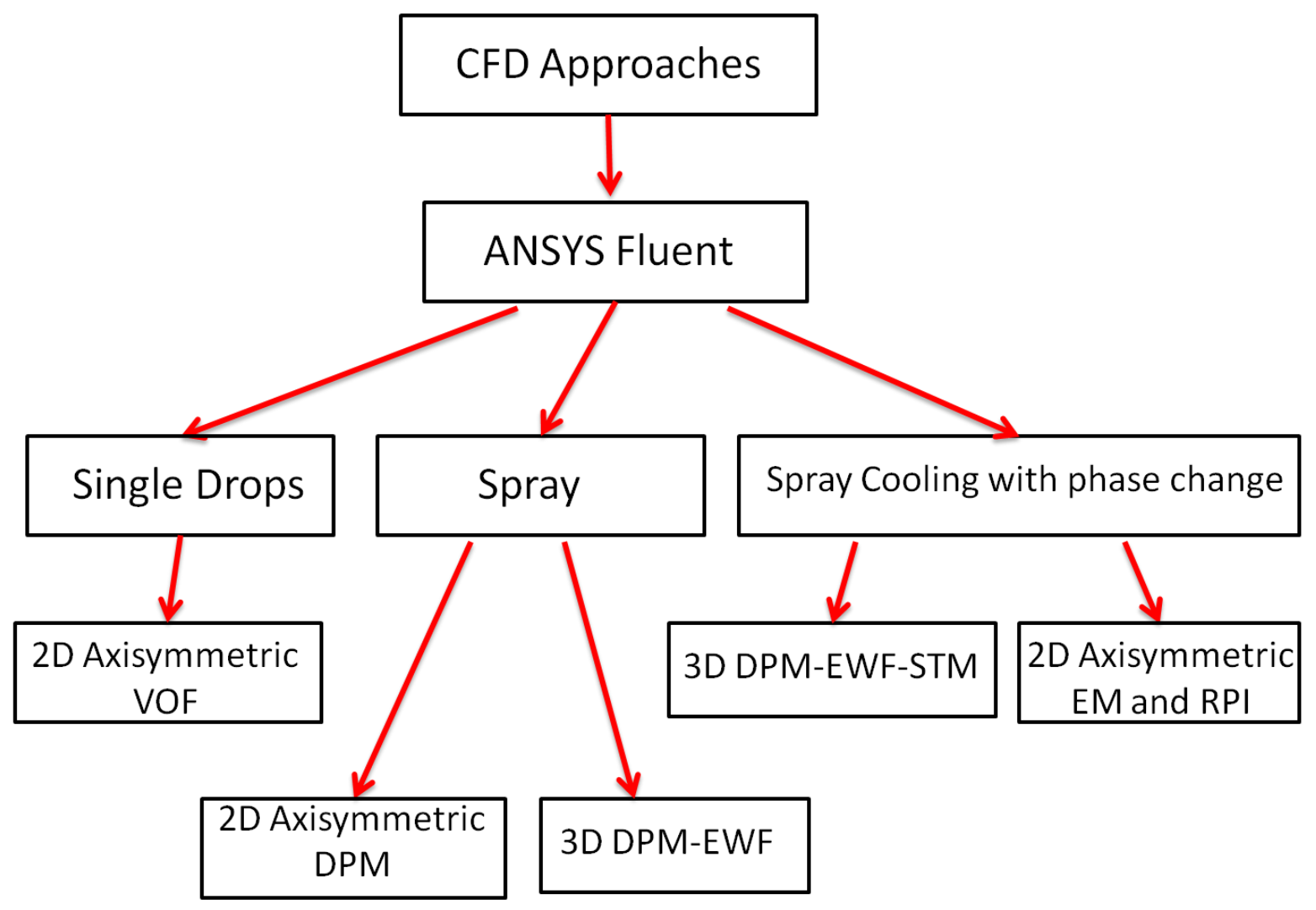

Figure 1.4 The outline of the CFD approaches that are used in this study. 


\section{CHAPTER 2: LITERATURE REVIEW}

In this chapter, a brief literature review about the previous experimental and computational studies related to single drop impingement on surfaces and spray cooling is presented.

There are important dimensionless numbers that are used in single drop and spray/spray cooling studies. For instance, the interaction between a drop and a surface for the unheated case may involve inertial, viscous, surface tension, and gravitational forces. A sufficient set of dimensionless numbers to describe the interaction of these forces is the Reynolds number ( $R e=$

$\rho U D / \mu)$, the Weber number $\left(W e=\rho U^{2} D / \sigma\right)$, and the Froude number $\left(F r=U^{2} / g D\right)$, where $g$ is the acceleration due to gravity, $\rho$ is the liquid density, $U$ is the drop velocity, $D$ is the drop diameter, $\mu$ is the liquid dynamic viscosity and $\sigma$ is the liquid surface tension. The Reynolds number is proportional to the ratio of inertial forces to viscous forces, the Weber number is proportional to the ratio of inertial to surface tension forces, and the Froude number is proportional to the ratio of inertial to gravity forces. Many authors prefer to use the Ohnesorge number $\left(\mathrm{Oh}=\mathrm{We} / \mathrm{Re}^{2}\right)$ in place of $R e$. The Ohnesorge number is proportional to the ratio of the viscous forces to the square root of the product of the inertial and surface tension forces. Another key dimensionless number is the relative film thickness $\left(h^{\prime}=h / D\right)$ where $h$ is the initial liquid film thickness.

\subsection{Single Drop Impingement on Surfaces}

Among the many theoretical, numerical, and experimental studies of the impact of a single drop on wet and dry surfaces, a few recent papers have been selected for comparison to the results in the next chapter. These selected papers will be briefly explained.

Asadi and Passandideh-Fard (2009) numerically studied drop impingement on a thin liquid film for a range of Weber (We) and Ohnesorge $(\mathrm{Oh})$ numbers. They employed a finite volume code with a Volume of Fluid (VOF) model based on Young's (1984) algorithm. They investigated the liquid corona (liquid crown) height and crater characteristics. They reported that their results agreed well with the experiments in the literature.

Yokoi et al. (2009) experimentally and numerically analyzed a single drop impact on a smooth, flat, dry surface. They utilized a coupled VOF-level set method in which the VOF method was used to calculate the interface motion and the level set method accounted for surface 
tension and wettability. Yokoi et al. (2009) used a special version of the VOF-level set method called the tangent of hyperbola for interface capturing/weighed line interface calculation (THINC/WLIC) method for interface capturing. They developed a dynamic contact angle model to calculate more accurately the dynamics of drop impact on a dry surface during the spreading and retracting stages, and they concluded that only the dynamic contact angle model (with the angle parameters obtained from their experiments) gave results that matched closely with the experiments.

Peduto et al. (2011) numerically studied a drop impact on deep and shallow liquid films using a VOF model with dynamic mesh adaption. They reported that both axisymmetric and three dimensional simulations demonstrated excellent agreement with each of their experiments.

Wang and Chen (2000) developed a novel experimental method in order to perform a single drop impingement on a very thin liquid film $\left(h^{\prime}<0.1\right)$. The critical Weber number $\left(W e_{c r}\right)$ is used to define when splashing would occur after a drop impact on a wetted surface. Above this value, splashing is expected. They measured the critical Weber number for splashing $\left(W e_{c r}\right)$ and concluded that if the film is relatively thin, $W e_{c r}$ does not depend on film thickness. They also concluded that $W e_{c r}$ for $h^{\prime}<0.1$ depends on $O h$ and the characteristics of the surface under the liquid film.

\subsection{Spray Impingement on Surfaces and Spray Cooling}

In Chapters 4-7 spray simulations are performed using the Discrete Phase Model (DPM), the Eulerian Wall Film (EWF) Model and the Eulerian Multiphase (EM) Model in ANSYS Fluent. In this section, some of the important experimental and computational studies related to spray cooling are described.

The DPM has been used by many authors to model particles in a variety of flow conditions including the calculation of rain drop trajectories around a car windshield (Ghani et al., 2011) and snow flake trajectories around snowplow trucks (Dinc, 2011). The DPM has also been used to model spray particle trajectories, particle diameter distributions, and heat transfer (Guechi et al., 2012; Gant, 2006; Tao et al., 2009; Yigit et al., 2011; Issa and Yao, 2005; Alkhedhair et al., 2013).

Guechi et al. (2012) used the Eulerian Multiphase (EM) model in ANSYS Fluent for their computational spray cooling studies in addition to the DPM. They also experimentally 
investigated sprays with the nozzle water flow rate between $109 \mathrm{ml} / \mathrm{min}$ and $130 \mathrm{ml} / \mathrm{min}$ at a supply pressure of 3 bar which generated droplets with a 214 micron Sauter mean diameter. They used the wall film submodel in the DPM to calculate the spray-wall interaction when using the DPM for spray cooling simulations. They concluded that the Eulerian Multiphase model in ANSYS Fluent gives heat transfer coefficient and surface temperature results which agree more closely with the experiments then does the DPM wall film submodel. They also concluded that the Realizable k-epsilon turbulence model was more accurate for spray cooling compared to the Standard k-epsilon and RNG k-epsilon turbulence models in ANSYS Fluent.

Gant (2006) used the EM and the DPM for modeling of 3D water spray barriers. They compared spray envelope radius and air velocity vectors with the experiments of St-Georges and Buchlin (1994). They concluded that both models gave good agreement with the experiments but the DPM gave slightly better results than the EM.

Tao et al. (2009) used the DPM with the wall film submodel in ANSYS Fluent for 3D spray and spray cooling using water. They used the Standard k-omega turbulence model for air flow calculations. They analyzed droplet Sauter mean diameter, droplet collision speed, thickness of liquid film and impact surface temperature. They compared Sauter mean droplet diameter results with the experiments in the literature (Estes and Mudawar, 1995) and concluded that their simulation results agreed with the experiments within $13.8 \%$.

Yigit et al. (2011) experimentally and numerically studied water mist impact on a heated surface in regimes ranging from nucleate to film boiling. They used the 2D axisymmetric DPM in ANSYS Fluent for water mist calculations and the k-epsilon turbulence model for air flow calculations. They concluded that their numerical surface heat transfer coefficient results agree well with their experiments within around $13 \%$.

Issa and Yao (2005) numerically studied single droplet stream and full spray-wall interactions and spray cooling using the 2D axisymmetric DPM in ANSYS Fluent for surface temperatures ranging from nucleate boiling to film boiling. They used the standard k-epsilon turbulence model for the air flow calculations. They compared their single droplet stream surface heat flux results at different ambient pressures with experiments in the literature and concluded that their numerical results agree well with the experiments.

Alkhedhair et al. (2013) numerically studied steady state 3D air cooling with water sprays using the DPM in ANSYS Fluent to enhance the performance of Natural Draft Dry Cooling 
Towers (NDDCT). They used the Standard k-epsilon turbulence model in order to include the turbulence effects in simulations. They studied the effects of droplet diameter and air velocity on the cooling performance (droplet evaporation and transport). They concluded that the spray cooling efficiency was higher at lower air velocities.

Pautsch and Shedd (2006) conducted experiments using FC-72 coolant to measure the liquid film thickness at a low flow rate using a single nozzle, and at a high flow rate using a fournozzle array. They mentioned that there were several different heat transfer mechanisms in the liquid film associated with spray cooling. These heat transfer mechanisms were heat conduction, heat convection, bubble nucleation and secondary nucleation caused by gas bubbles entrained by impacting droplets. They explained that each of these mechanisms contributed to the total heat transfer in spray cooling, but it was not understood which was dominant.

Yang et al. (1996) developed a Nusselt number correlation as a function of the liquid film thickness for spray cooling in the nucleate boiling regime. They concluded that the secondary nucleation sites increase once the spray flow rate increases. They also concluded that the liquid film thickness depends on the liquid properties and the spray volumetric flow rate.

Galvan et al. (2011) experimentally investigated the liquid film thickness and heat transfer in spray cooling. R134a refrigerant was sprayed using a full cone nozzle. They used a high speed camera with a long distance microscope to measure the film thickness over the square heated surface. They explained that the spray efficiency $\left(\eta=100\left(C H F /\left(\rho Q h_{f g}+\rho Q c_{p} \Delta T_{\text {sub }}\right)\right)\right.$ where CHF is the Critical Heat Flux) increases as the Weber number decreases. They concluded that a spray with a lower droplet density is more efficient for the same flow rate. They also concluded that the highest average Nusselt number is obtained with the highest Weber number. They measured the film thickness in the zone outside the spray cone (impact area) over the square heater. They found that the film closer to the spray cone was thicker, and the average film thickness increased as the Weber number increased. As the heat flux increased, the local film thickness also increased due to the increase in the amount of vapor generated inside the film in the nucleate boiling regime. One of their most important results was that three different zones could be defined in the nucleate boiling regime of the boiling curve as a function of the heat flux. The Nusselt number and the dimensionless film thickness varied in a similar manner as the normalized heat flux. 
Estes and Mudawar (1995) performed experiments to investigate nucleate boiling and Critical Heat Flux (CHF) using full cone sprays. Spray nozzle, volumetric flux, sub-cooling and spray coolant were varied in their experiments. They explained that the CHF increased with higher flow rate and sub-cooling. Higher CHF was obtained with smaller drops. They also found that the Sauter mean diameter was highly dependent on the orifice diameter and the Weber and Reynolds numbers based on the orifice flow conditions for full cone sprays.

Chen et al. (2004) conducted experiments to investigate the effects of droplet velocity, droplet diameter and droplet flux on the heat transfer coefficient and CHF using water as a coolant liquid. They found that droplet velocity was the most effective parameter to obtain a higher heat transfer coefficient and CHF. After droplet velocity, droplet flux was the next most effective parameter while droplet diameter was the least important parameter.

Horacek et al. (2004) performed experiments using FC-72 coolant to investigate the effects of nozzle stand off distance (the distance from nozzle to impact surface) on the heat transfer distribution under one and two spray nozzles. They explained that more uniform heat flux was observed across the heater surface by increasing the nozzle-to-surface distance. They reported that there is an optimum value for the nozzle-to-surface distance in order to achieve a uniform heat flux distribution.

Sarkar and Selvam (2009) investigated single phase and two phase heat transfer mechanisms relevant to spray cooling using detailed 3D numerical simulations of single drop impacts at different wall superheat conditions. Two cases were considered in their analysis: 1) a droplet impacting on the thin liquid film without a vapor bubble for a single phase heat transfer model, and 2) a droplet impacting on the thin liquid film with a vapor bubble growing in the thin liquid film on a hot surface for a two phase heat transfer model. Figure 2.1 shows the initial and boundary conditions of their 3D simulation. The locations where the fluid immediately adjacent to the heater surface was cooled significantly is shown in Figure 2.2. The maximum heat flux was obtained more accurately with two phase heat transfer compared to single phase heat transfer (Sarkar and Selvam, 2009). The maximum heat flux was larger for the two phase simulation at $40{ }^{\circ} \mathrm{C}$ wall superheat compared to the single phase simulation at $40{ }^{\circ} \mathrm{C}$ wall superheat, and the two phase simulation gave closer results to the experiments (Fig. 2.3). The maximum heat flux was obtained after the bubble collapsed due to the droplet impact. This created a way for the colder liquid to spread over the dry and hot surface area formerly occupied 
by the bubble, causing rapid transient conduction for a very short time (Sarkar and Selvam, 2009). This transient heat conduction was reported to be the dominant heat transfer mechanism in spray cooling by the authors.

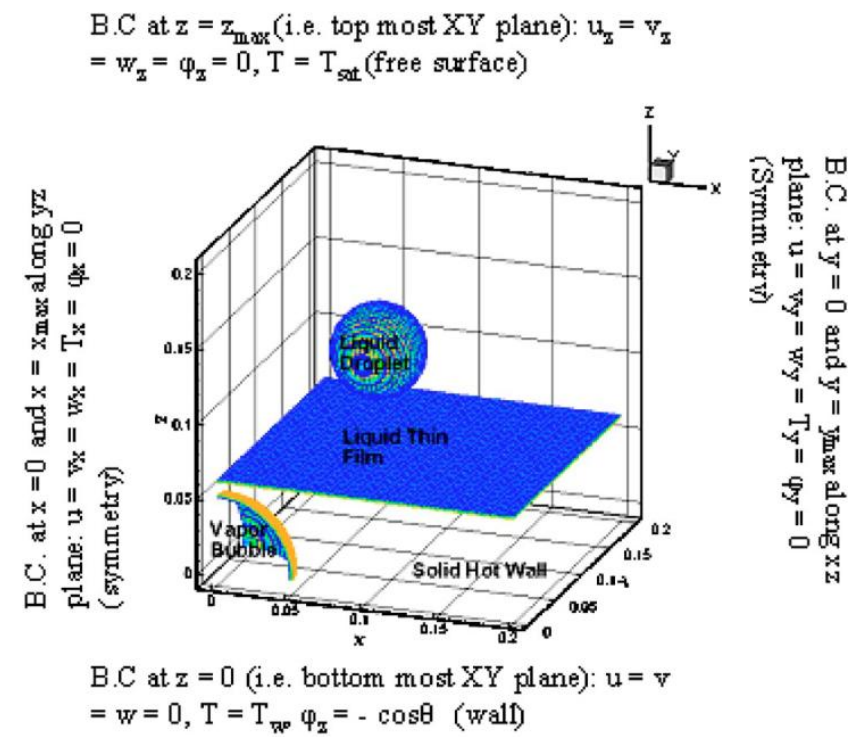

Figure 2.1 Initial and boundary conditions of the 3D two phase simulation model of Sarkar and Selvam (2009).
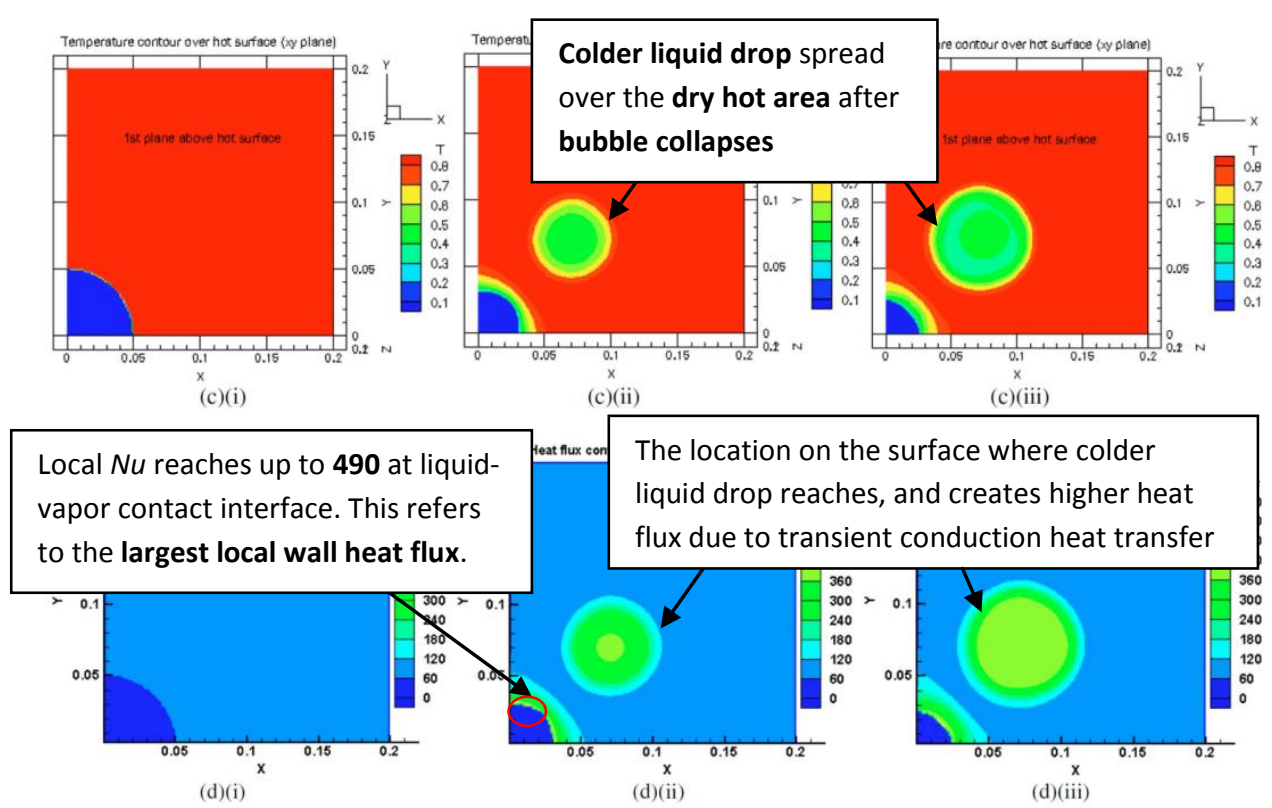

Figure 2.2 Temperature and heat flux distributions on the $\mathrm{x}-\mathrm{y}$ plane and over the surface for the 3D two phase model of Sarkar and Selvam, (2009). (Note: Comments in the boxes have been added later by present author.) 


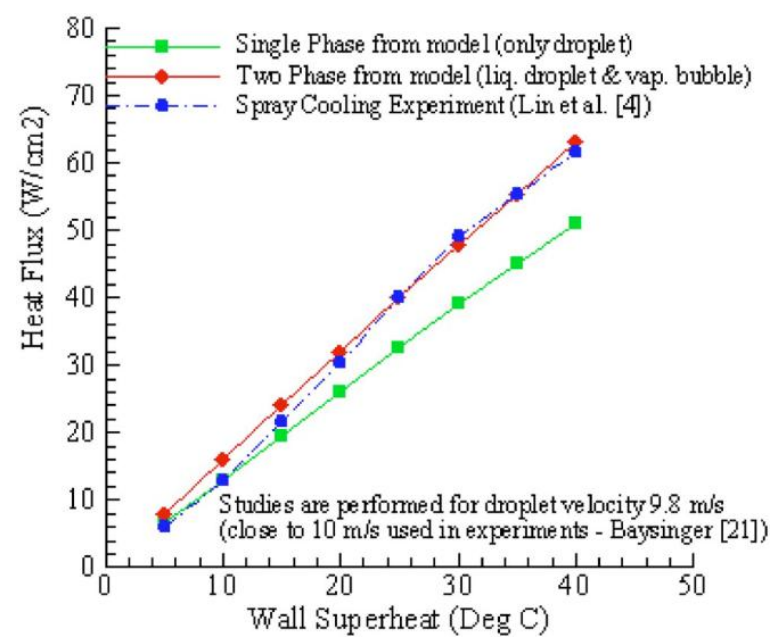

Figure 2.3 Comparison of heat flux (Sarkar and Selvam, 2009).

Yoshida et al. (2001) experimentally investigated the effects of gravity on spray cooling heat transfer using FC-72 as a coolant. The gravity was varied from $0.01 g$ to $1.8 g$ in flight experiments. They concluded that heated surface orientation and gravity had no effect on CHF under low flow rate conditions. Reduced gravity significantly affected the spray cooling heat transfer at higher flow rates. The CHF was found to be influenced by both gravity and heater surface orientation. They also observed significantly lower heat transfer in the transient boiling regime in the low gravity environment. They reported that spray cooling of a downward facing surface had a significantly lower heat flux than an upward facing surface. This was attributed to splashed droplets re-impacting on the upward facing surface.

Yerkes et al. (2006) experimentally studied the effects of reduced gravity on single phase spray heat transfer using air-saturated FC-72. They showed that $N u$ decreased with an increase in $\left(\mathrm{Fr}^{1 / 2} \mathrm{Ga}\right)^{1 / 2}\left(\mathrm{Fr}\right.$ : Froude Number, $\mathrm{Ga}$ : Galileo Number $\left.=g L^{3} / v^{2}\right)$ at constant We number. In other words, the heat transfer increased as gravity decreased.

Lin and Ponnappan (2003) experimentally studied the effects of dissolved gas on spray cooling heat transfer. They used four different liquid coolants (FC-72, FC-87, water and methanol). Two major conclusions were obtained for air as a dissolved gas in FC-72 coolant: the spray cooling curves moved to higher wall temperatures and the CHF increased. They explained that when air was sprayed along with the liquid, the droplet diameter decreased and droplet velocity increased which caused a thinner liquid film and improved heat transfer. They also mentioned that the dissolved air increased the surface evaporation in spray cooling. 
Horacek et al. (2005) also studied the effects of the amount of dissolved gas on heat transfer using air as the dissolved gas and FC-72 as coolant. Their results confirmed the Lin and Ponnappan (2003) results. Horacek et al. (2005) stated that CHF increased with dissolved air; but it occurred at similar wall superheats. Horacek et al. (2005) defined Contact Line Length (CLL) to explain the liquid-vapor interface that occurs during the heat transfer between the impinging liquid drops and the superheated surface. CLL was used for the statistical quantification of the images obtained by directing light from a light source to the liquid-vapor interface on the heated surface. When the light reached the liquid-vapor interface, it was transmitted into the liquid and reflected at a sloped interface. Figure 2.4 shows the variation of CLL with respect to the wall superheat. The general results related to CLL and its importance according to Horacek et al. (2005)'s analyses are that:

- CLL increases with the wall superheat.

- CLL has the maximum value at the superheat where the critical heat flux (CHF) occurs (at around $30{ }^{\circ} \mathrm{C}$ wall superheat). Then, CLL decreases as the surface dries out (Figure 2.4 , right image).

- As shown in Figure 2.4, the curve shapes of the heat flux profiles (left image) and CLL (right image) show very similar behavior. The authors concluded that CLL has a very significant correlation with the heat transfer.

- The authors also concluded that the similarity between heat flux and CLL curves shows that the phase change heat transfer is directly related to the CLL.
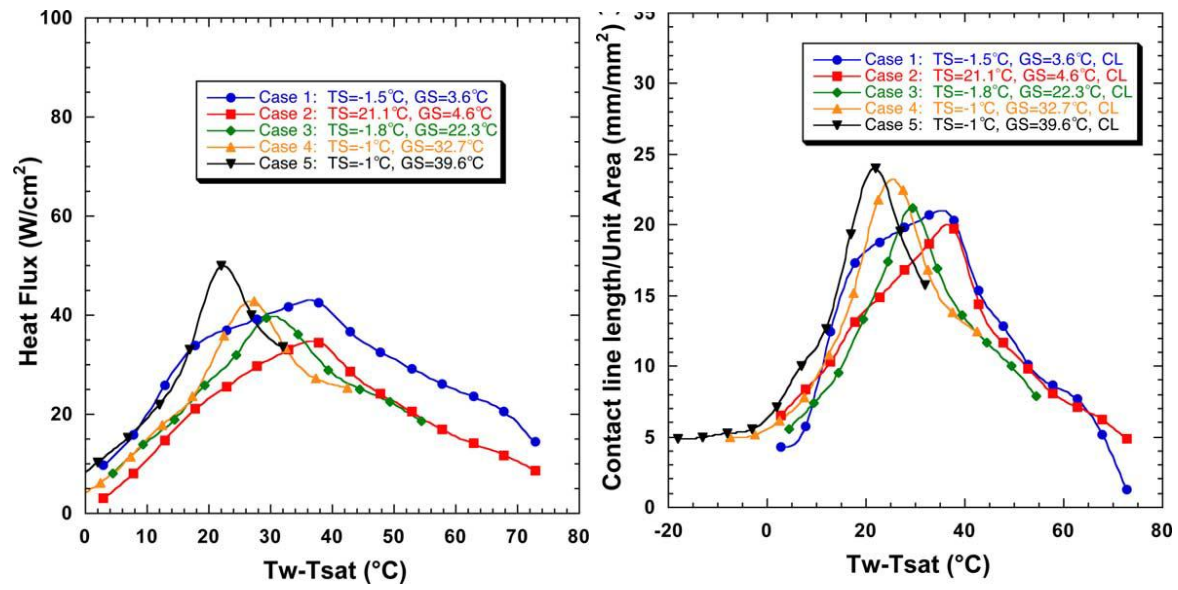

Figure 2.4 Left image shows heat flux corrected for sensible heating and wall superheat, and right image shows the average CLL length for the center eight heaters as a function of the wall superheat (Horacek et al., 2005). ( $T_{w}$ is wall temperature.) 
Some experimental studies have been performed to investigate the effects of surface characteristics on spray cooling heat transfer. Kim et al. (2004) and Hsieh and Yao (2006) studied the effects of micro-structured surfaces. It was observed that the heat transfer from the micro-structured surface was 50\% higher than the uncoated normal surface. Silk et al. (2004) studied the effect of surface geometry on spray cooling heat transfer using a $2 \times 2$ hollow cone nozzle array with FC-72 coolant and straight fin, cubic fin and pyramidal fin coated copper surfaces. They reported that the pyramidal surface had higher heat transfer compared to cubic and straight fin surfaces which gave similar heat transfer results. They also concluded that all structured surfaces were much more effective than flat surfaces.

Rini et al. (2002) experimentally investigated the bubble growth rates and bubble site density. They reported that the bubble site density increased as the droplet number flux increased. They found that the bubble lifetimes during spray cooling were more than an order of magnitude smaller than during pool boiling.

Chen et al. (2008) investigated the dynamic effects of impinging droplets and bubbles on enhancing nucleate boiling heat transfer using computational approaches. These dynamic effects included: bubble merging, bubble puncturing by impinging droplets, bubble size distribution, bubble diameter at puncture, and secondary nucleation. Their simulation model included submodels to account for the bubble growth from the heated surface and for secondary nucleation, bubble merging, bubble-droplet interaction, and distribution of secondary nuclei. They mentioned that these submodels were developed based on the previous experimental spray cooling studies and related pool boiling results. Their simulation results are shown in Figure 2.5 for impinging droplets and secondary nuclei. In this figure, droplets with constant $100 \mu \mathrm{m}$ diameter are represented as black dots, and bubbles with varying diameter are represented as open circles (Chen et al., 2008). 


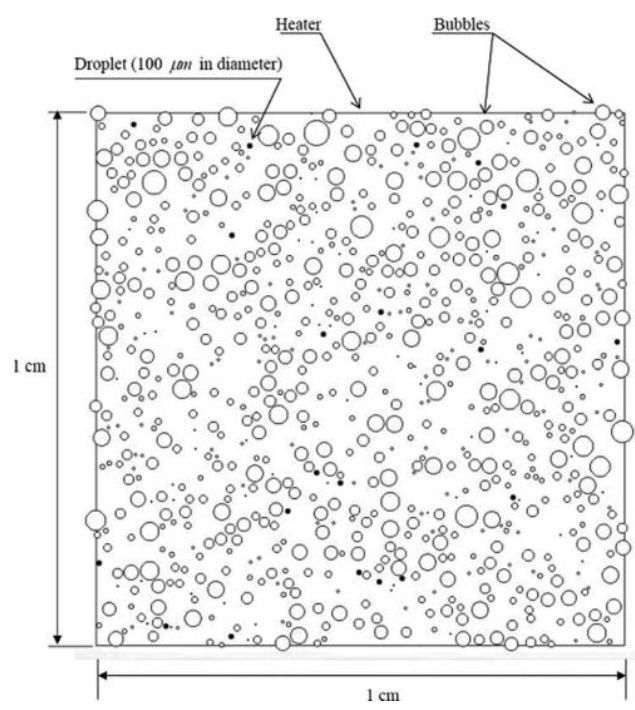

Figure 2.5 Simulation results for varying size bubbles (open circle) and impinging droplets (black dot) with a constant diameter $(100 \mu \mathrm{m})($ Chen et al. (2008)).

The major conclusions from the simulations of Chen et al. (2008) are:

- Bubble density was not affected by the number of surface nuclei because of the large values of droplet flux.

- Bubble merging frequency increased with droplet flux since more secondary nuclei were captured on the liquid surface.

- For the purpose of enhancing nucleate heat transfer, increasing the number of secondary nuclei was not as effective as increasing the bubble puncturing frequency by the impinging droplets.

- Increasing the droplet flux caused an increase in bubble density and a decrease in mean bubble diameter. Thus increasing droplet flux improves nucleate heat transfer.

- Furthermore, the authors concluded that to increase the heat transfer efficiency of spray cooling, there should be a higher bubble density with more small bubbles.

Olsen and Cloete (2009) computationally studied the hydrodynamics of gas stirred ladles to investigate the behavior of the liquid in the ladle, the gas above the liquid, and the bubbles in the liquid at higher gas flow rates using the coupled Volume of Fluid (VOF) and Discrete Phase Model (DPM). The Lagrangian approach (the DPM) was used to track bubbles and the Eulerian approach (the VOF model) was used to calculate the liquid and gas phase above the liquid and 
the interface between them. They explained that the Lagrangian bubbles are connected to the Eulerian phase with a two-way coupling using interchange terms, e.g. drag force in the momentum equations. No coupling was done in the continuity equation because they considered that the presence of a DPM particle (a bubble) in a volume cell did not affect the continuous phase of that cell due to low void fraction. Their numerically coupled DPM and VOF model with the k-epsilon turbulence model was validated with the experimental results for liquid velocity profiles at different heights above the gas release point.

Arienti et al. (2011) computationally studied a spray hitting a surface to calculate the wall liquid film formation and breakup using coupled VOF and DPM methods using ANSYS Fluent version 6.3 to refine the Atomization Model Interfaced with Surface Tracking (AtoMIST) model. This coupled model included some submodels such as secondary atomization, liquid column breakup, splashing and film breakup models. A film formation model based on the refined level set grid method of Herrman (2008) was also included. They concluded that their computational results agreed well with the phase Doppler particle analyzer data and nonintrusive film thickness point measurements of Shedd et al. (2009). 


\section{CHAPTER 3: SIMULATIONS OF SINGLE DROP IMPACT ON LIQUID LAYERS}

In this chapter, the modeling of single drops using ANSYS Fluent software is discussed, and the analysis of different single drop simulations is explained. The effects of varying gravity and drop shape on the characteristics of liquid film dynamics are analyzed. In this chapter and in the other chapters, the variables are defined separately. Thus, the symbols in this chapter are often not the same as the symbols shown in the other chapters. In addition, cases are generally numbered independently in different sections. This is because the studies which will be presented in this chapter are primarily based on several publications in which the author of this dissertation (Dinc) was a co-author, namely Hillen, Kuhlman, Dinc, Gray (2012), Dinc and Gray (2012, 2013) and Kuhlman, Hillen, Dinc, Gray (2014).

\subsection{The Characterization of Single Drop Impact on Surfaces}

Figure 3.1 shows the typical flow when a drop with an initial diameter $(D)$ and initial velocity $(U)$ impacts normally onto a wet surface with a liquid layer thickness $(h)$. The impact drives the liquid outward from the point of impact forming a crater or cavity whose leading edge takes the form of a crown. This may be accompanied by the ejection of secondary drops known as prompt splashing (Yarin, 2006). The crown grows in height as it spreads until it reaches a maximum crown height $\left(H_{\max }\right)$ at time $t_{\max }$. The crown may become unstable to the formation of jets which in turn may pinch off to form drops (late splashing). In the final stage the crown collapses and the crater refills, with the possible formation of a Worthington jet at the center due to the converging inflow. Drops may also pinch off from the Worthington jet. Generally, inputs are $D$, $U, h$, type of liquid (density, viscosity, surface tension), and gravity, and the outputs are crown characteristics (height $(H)$, radius, shape) and crater characteristics (the volume of sub-cavity liquid, the cavity radius $\left(R_{B}\right)$ vs. time, etc.). 

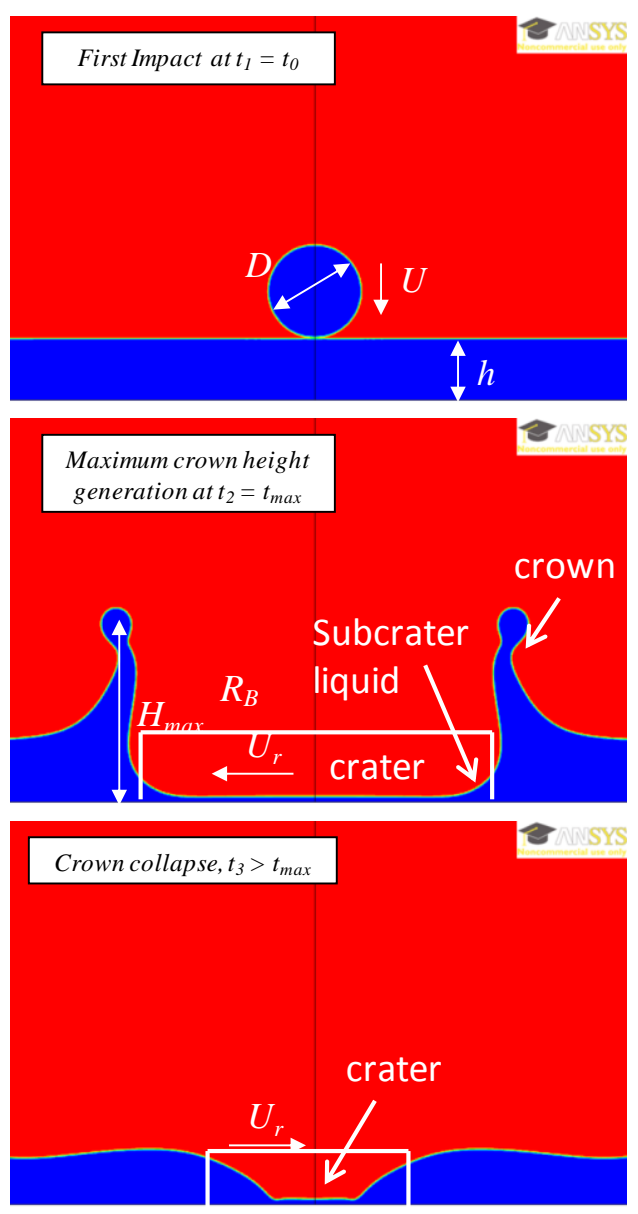

Figure 3.1 Typical single drop impact on a wet, flat surface: first impact at $t_{1}=t_{0}$ (top image), maximum crown height at $t_{2}=t_{\max }$ (middle image) and crown collapse as liquid refills crater at $t_{3}$ $>t_{\max }$ (bottom image). (Note that red refers to air while blue refers to liquid.)

\subsection{Numerical Modeling and Governing Equations}

The Navier-Stokes and continuity equations were solved in 2D axisymmetric coordinates using the finite volume method for unsteady, incompressible, isothermal and laminar flow. In this flow, it is essential to be able to determine the location of the free surface of the liquid. This was accomplished by using the explicit Volume of Fluid (VOF) method (Hirt and Nichols, 1981).

In the VOF, each fluid was treated as incompressible, and volume weighted fluid properties were used in cells which contained both air and water. Surface tension was incorporated into the Navier-Stokes equation by using the Continuum Surface Force (CSF) model, which accounts for the curvature of the interface (Brackbill et al., 1992). Velocity and pressure coupling was achieved with the Pressure Implicit with Splitting of Operators (PISO) 
algorithm (Issa, 1986). The sharpness of the interface was enhanced by use of the georeconstruct (Piecewise Linear Interface Calculation, PLIC) scheme (Rider and Kothe, 1998). Discretization of the advective terms in the momentum equations used second order upwinding. Solution convergence was assured by monitoring the mass imbalance at some locations in the domain and also by checking the residual values of variables such as the velocity far from the impact region.

For incompressible flow, the continuity equation in axisymmetric cylindrical coordinates ( $x$-axial, $r$-radial coordinate) can be written as

$$
\nabla \cdot \underline{U}=\frac{\partial\left(U_{x}\right)}{\partial x}+\frac{\partial\left(U_{r}\right)}{\partial r}+\frac{U_{r}}{r}=0
$$

The Navier-Stokes equations are

$$
\rho\left(\frac{\partial(\underline{U})}{\partial t}+\underline{U} \cdot \nabla \underline{U}\right)=-\nabla P+\mu \nabla^{2} \underline{U}+\underline{F}_{S}+\rho \underline{g}
$$

where $\underline{U}$ is the total velocity vector, $\rho$ is the density, $\mu$ is the dynamic viscosity, $g$ is the gravity vector, $P$ is the pressure, and $\underline{F}_{s}$ is the body force caused by the surface tension $(\sigma)$ between two phases. This is determined using the Continuum Surface Force (CSF) model (Brackbill et al., 1992) shown in equation 3.3.

$$
\underline{F_{S}}=\sigma \frac{\rho k \nabla f_{i}}{\left(\frac{1}{2}\right)\left(\rho_{L}+\rho_{G}\right)}
$$

where $f_{i}$ is the volume fraction of the fluids in a computational cell for $i=1$ and 2 where 1 refers to the drop phase and 2 refers to the layer phase. The VOF multiphase model was implemented such that the drop liquid and the film liquid could be distinguished, even though they are physically identical. For each liquid an advective transport equation was solved for an indicator function defined as the fraction of a computational grid cell occupied by that liquid. If the sum of these liquid indicator functions was less than 1 , the cell contained an air-water interface at which 
surface tension acted. The volume fractions of the drop and film phases, $f_{1}$ and $f_{2}$, are calculated separately from the advection equation (e.g. $i=1$ for drop phase, $i=2$ for film phase)

$$
\frac{\partial\left(f_{i}\right)}{\partial t}+\underline{U} \cdot \nabla f_{i}=0
$$

From equation 3.4, volume fraction of liquid phases (drop and film) is calculated at the interface and then gas volume fraction is obtained from

$$
\sum_{i=1}^{3} f_{i}=f_{1}+f_{2}+f_{3}=f_{\text {drop }}+f_{\text {layer }}+f_{\text {air }}=1
$$

where $i$ represents phases in domain. $f_{i}$ can take on the value of between 0 and 1 .

If $f_{1}=0$ and $f_{2}=0$ the cell is empty of the liquid (100\% gas).

If $f_{1}=1$ the cell is full of drop liquid phase (100\% drop).

If $f_{2}=1$ the cell is full of film liquid phase (100\% film).

If $0<f_{3}<1$ the cell contains gas and liquid. For instance if $f_{1}=0.4$ and $f_{2}=0.3,40 \%$ of the cell is drop liquid, $30 \%$ is film liquid, and $30 \%$ is air.

Mixture properties are obtained using the equations 3.6 and 3.7 based on the volume fraction, $f_{i}$. The surface curvature of the interface, $k$ and the surface normal vector, $\widehat{n_{l}}$ are calculated using equations 3.8 and 3.9.

$$
\begin{aligned}
& \rho=\sum_{i=1}^{3} f_{i} \rho_{i} \\
& \mu=\sum_{i=1}^{3} f_{i} \mu_{i} \\
& k=-\nabla \cdot \widehat{n_{\imath}} \\
& \widehat{n_{\iota}}=\frac{\nabla f_{i}}{\left|\nabla f_{i}\right|}
\end{aligned}
$$


Where $k$ is the curvature of the interface; $\rho_{L}$ is the liquid density, $\rho_{G}$ is the gas density; $\mu_{L}$ is the liquid dynamic viscosity, $\mu_{G}$ is the gas dynamic viscosity and $f_{i}$ is the volume fraction, $i$ is the number of phases (drop, film, air).

Discretization of the advective terms in the momentum equations was obtained via a second order upwind scheme. The discretized equations were solved via the explicit method in ANSYS Fluent 14. The explicit method solves the volume fraction in sub time-steps which are determined by the Courant Number (Fig. 3.2). The maximum number of iterations per time step and the relaxation factor were chosen to ensure solution convergence. The pressure based solver was used since it is suggested for VOF simulations (Fig. 3.3, ANSYS Fluent User's Guide, 2011). The Piecewise Linear Interface Calculation (PLIC) interface calculation scheme (Rider and Kothe, 1998) was used because this method gives a sharper interface compared to other interface calculation schemes such as SLIC. These schemes are illustrated in Fig. 3.4.
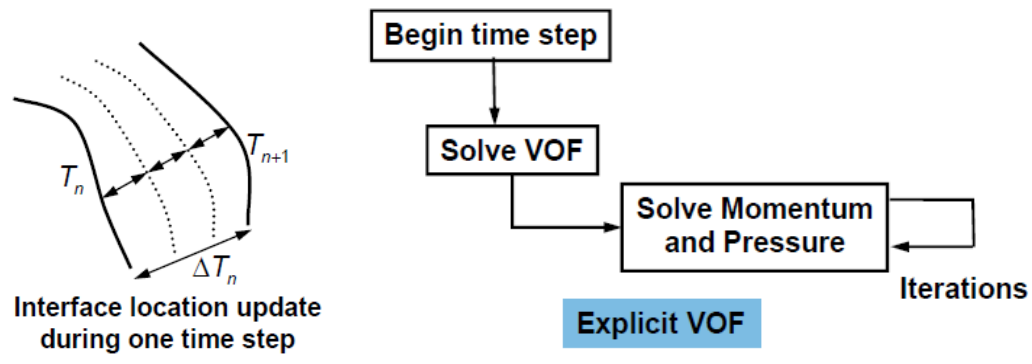

Figure 3.2 Solution procedure in the Explicit VOF model (ANSYS Fluent User's Guide, 2011). 

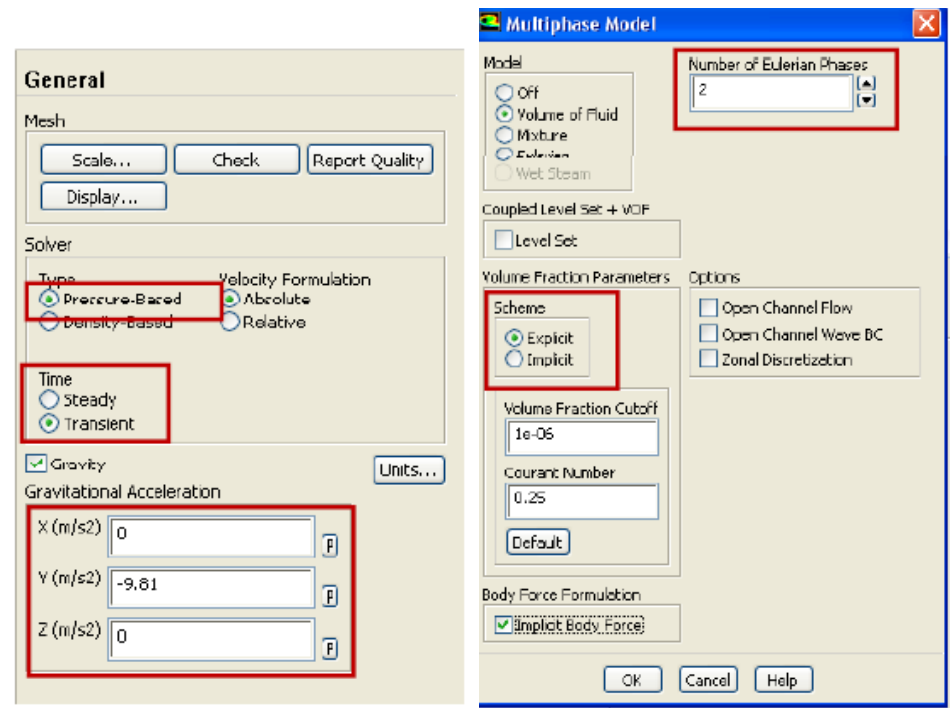

Figure 3.3 Solver options and the VOF Scheme in Fluent (ANSYS Fluent User's Guide, 2011).
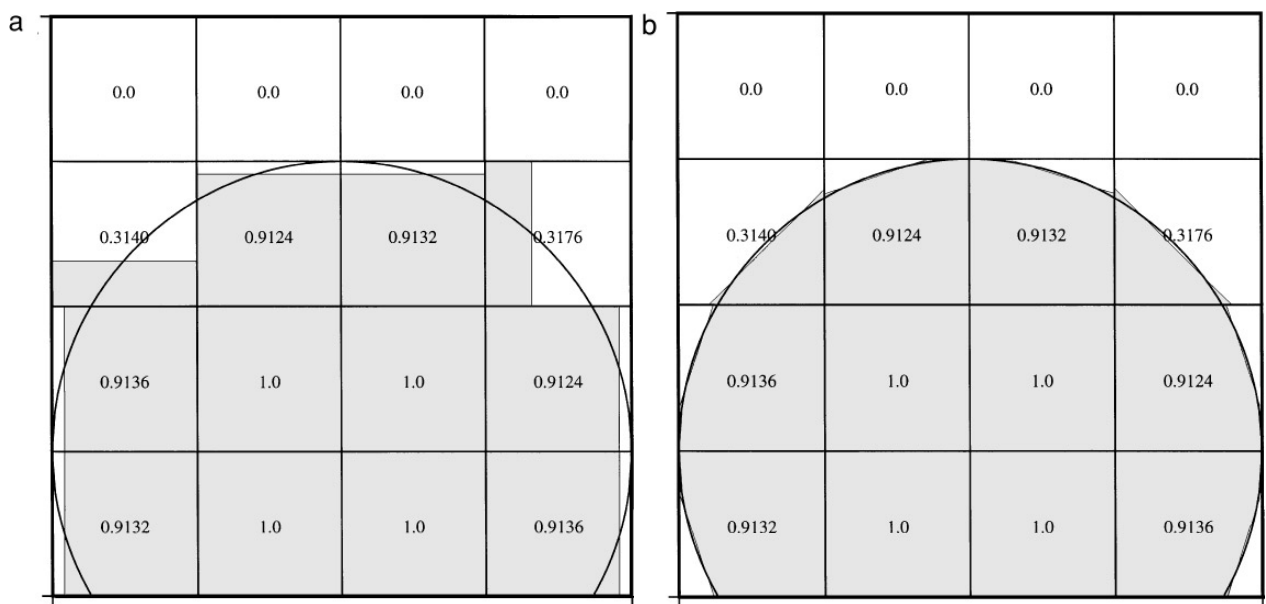

Figure 3.4 Comparison of two different interface calculation schemes: a) Simple Line Interface Calculation (SLIC) b) Piecewise Linear Interface Calculation (PLIC) (Rider and Kothe, 1998). ("Reconstructed interfaces (shaded regions) for a circle (continuous line) using the SLIC and PLIC methods. The piecewise constant approximation in SLIC forces the reconstruction to align with selected mesh logical coordinates, whereas the piecewise linear approximation in PLIC allows the reconstruction to align naturally with the interface. Numbers in the cells denote volume fractions. ", directly taken from Rider and Kothe, 1998)

Gradients were needed in order to determine the values of a scalar at the cell faces and for computing secondary diffusion terms and velocity derivatives. The gradients of transport terms were calculated using the Least Squares Cell Based method in ANSYS Fluent (Fig. 3.5). In 
this method the solution is assumed to change linearly between cell centroids. The equation given in the Figure 3.5 shows the change in values between cell $c 0$ and cell $c i$ along the vector $r_{i}$ from the centroid of cell $c 0$ to cell $c i$ (ANSYS Fluent User's Guide, 2011).

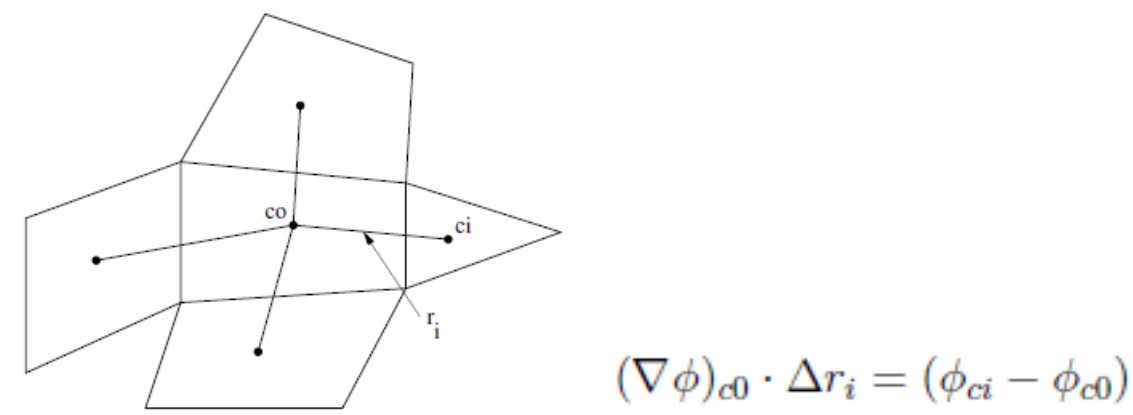

Figure 3.5 Calculation of gradients using Least Squares Cell Based Method (ANSYS Fluent User's Guide, 2011).

The Pressure-Implicit with Splitting of Operators (PISO) pressure-velocity coupling scheme is used for the coupling between pressure and velocity to solve the unsteady NavierStokes Equations. In this method, calculations are repeated until the momentum balance is satisfied after pressure correction equation is solved (Fig. 3.6). The PISO algorithm executes two additional corrections in order to improve the efficiency of the calculations compared to the other basic algorithms. 


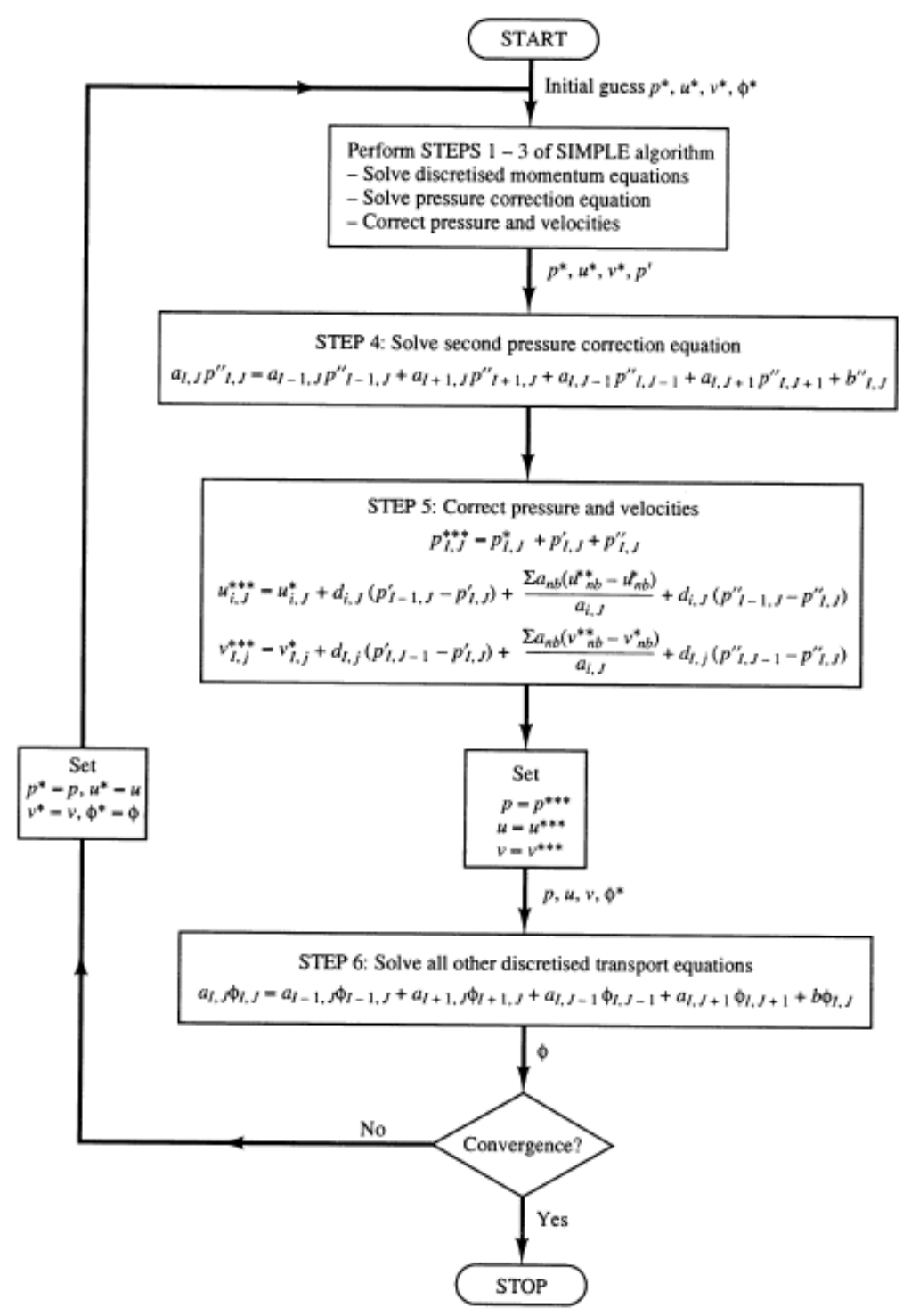

Figure 3.6 The PISO Algorithm and Solution Convergence Criteria (Versteeg, 2007).

\subsection{Meshing, Boundary Conditions and Initial Conditions}

The initial conditions for the axisymmetric simulations are shown in Figure 3.7. At the start of the simulations, the drop centroid was $1.5 \mathrm{D}$ above the free surface of the initial liquid film, but the simulation time was set to zero when the drop first makes contact with the film surface in order to be consistent with the experiments. The initial drop diameter, $D$; the liquid film thickness, $h$; and the drop velocity, $U$ were defined as initial conditions. The velocity of the droplet at $t_{0}$ (at the first impact time) was greater than the velocity at the start of the simulation 
because of the acceleration due to gravity, but the initial velocity $U$ has been used to calculate the dimensionless parameters.

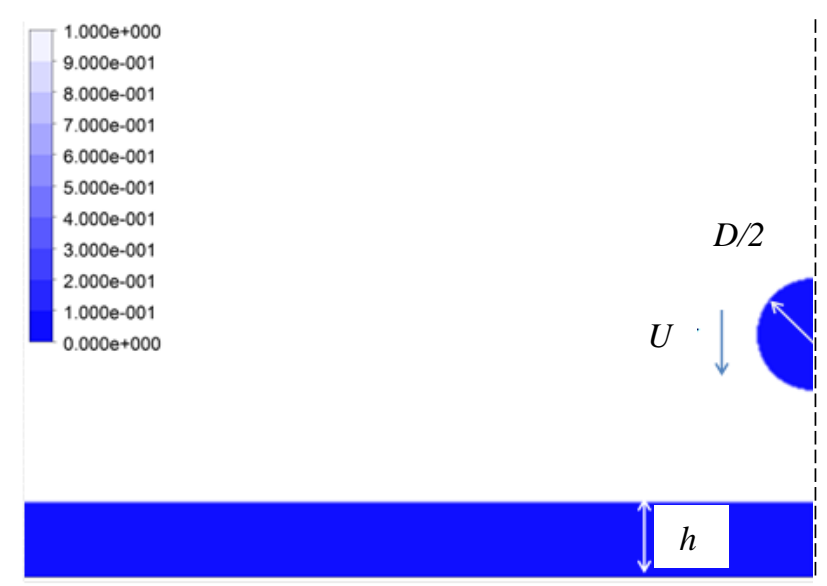

Figure 3.7 Schematic of a single drop above a thin liquid film before the impact. (Note: only half of the domain is shown; blue represents the liquid while white represents the surrounding gas.)

The boundary conditions are shown in Fig. 3.8. The impermeable no-slip wall boundary condition was applied on the bottom boundary, and the axis was a symmetry boundary. Pressure outlet boundary conditions were defined on the top boundary. A wall boundary condition was implemented at the maximum radius. However, no disturbance was observed due to the corona reaching the confining wall in these simulations.

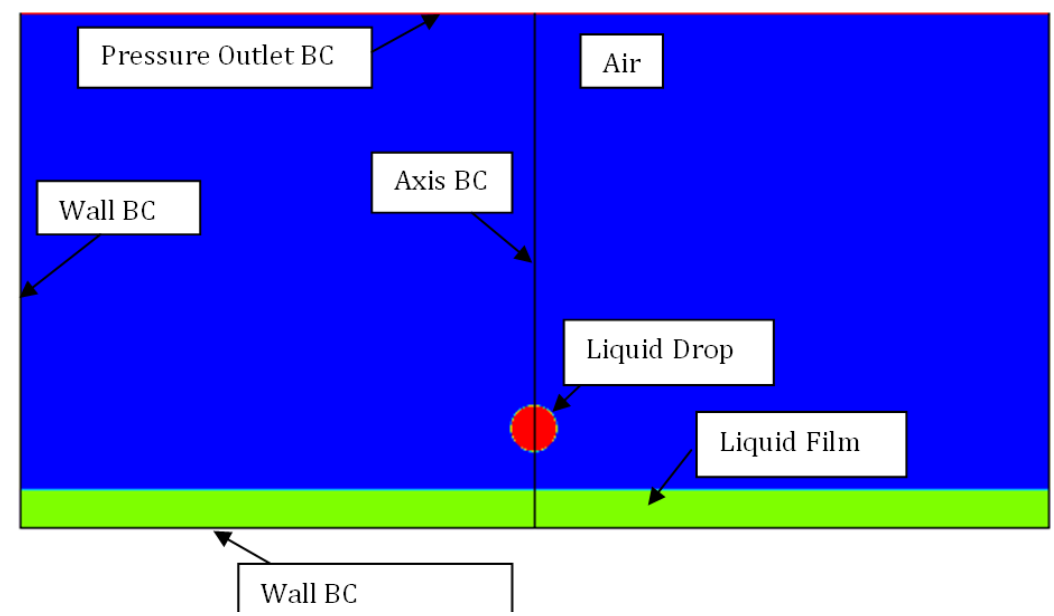

Figure 3.8 2D Axisymmetric boundary conditions, computational domain (0.05 m x $0.05 \mathrm{~m})$ reflected across the axis. 
The importance of using optimum size computational cells, especially at the interface of the liquid and air, was demonstrated by comparing simulations having minimum mesh sizes of $D / 80, D / 168, D / 336$, and $D / 1344$ where $D$ is the initial drop diameter for non-uniform (triangular) and uniform (rectangular) cells. The implementation of grid refinement especially on the critical zones, allowed the calculation of correct transport values (velocity, pressure, etc.) as well as liquid volume fraction.

The minimum mesh size of $D / 80$ has been commonly used in the literature. The smaller the mesh size was, the more accurate the results were at the interface (e.g. very small air bubbles at the interface of a drop and a thin film were resolved with the smallest mesh elements $(D / 1344)$ at the initial impact). However, decreasing the mesh size also increased the computational time required and did not always make significant changes in the liquid crown and cavity characteristics. Fine quadrilateral and adaptive refined meshes (using Level-4 adaption, with D/5 far from the impact region and $D / 80$ in the impact region) were implemented in order to increase the accuracy of the free-surface flow calculations while maintaining reasonable computational times. For the present domain, a uniform grid of square D/80 cells would contain 325,000 cells. By using adaptive mesh refinement of Level-4 with the largest cells measuring $D / 5$ and the smallest cells measuring D/80, only 25,000 cells were needed. The Level-4 dynamic mesh adaption can be seen in Fig. 3.9. Mesh size D/80 occurs at the interface of each phase (film-gas, film-drop, drop-gas interfaces), and the mesh gets larger and reaches a maximum size of $D / 5$ away from the interfaces. 


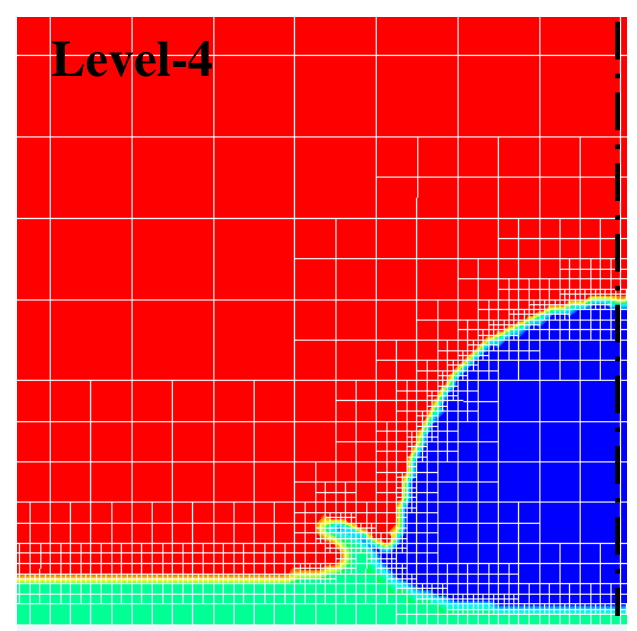

Figure 3.9 Level-4 adaptive mesh refinement at the interface of liquid and air at $3 \mathrm{~ms}$ for Case 2 ( $h / D=0.113)$ (Kuhlman et al., 2014). (Note: Blue refers to liquid drop, green refers to liquid layer and red refers to air)

Under certain conditions, a drop impact creates a crown which forms a series of jets which breakup into a series of discrete droplets. This is the phenomenon known as secondary (late) splashing. Cossali et al. (1997) developed a correlation for secondary splashing based on their experiments. They found that the occurrence of secondary splashing depends on the value of the dimensionless parameter $Y$, given as

$$
Y=\frac{W e O h^{-0.4}}{2100+5880\left(\frac{h}{D}\right)^{1.44}}
$$

Late splashing was found to occur for $Y>1$ while for $Y<1$ deposition without splashing and jet break-up was observed (Cossali et al., 1997). The range of validity of this criterion is 0.1 $<h / D<1$ and $O h>0.007$ and also $h / D<0.2$ for $O h=0.0022$ (for water).

Table 3.1 gives the dimensionless parameters used in the simulations that were carried out to establish confidence in the computational method. 
Table 3.1 Simulations performed and corresponding dimensionless numbers. (Note: Water was used as drop and film liquid for all cases except Case 5 and Case 6 where water-70\% glycerol was used.)

\begin{tabular}{|c|c|c|c|c|c|c|c|c|c|c|c|}
\hline Case & $\begin{array}{c}\text { 2D axi } \\
\text { or 3D }\end{array}$ & $\begin{array}{c}\boldsymbol{D} \\
(\boldsymbol{m m})\end{array}$ & $\begin{array}{c}\boldsymbol{h} \\
(\boldsymbol{m m})\end{array}$ & $\begin{array}{c}\boldsymbol{U} \\
(\boldsymbol{m} / \mathbf{s})\end{array}$ & $\boldsymbol{R e}$ & $\boldsymbol{W e}$ & $\boldsymbol{O h}$ & $\boldsymbol{F r}$ & $\boldsymbol{h} / \boldsymbol{D}$ & $\boldsymbol{Y}$ & $\begin{array}{c}\text { Late } \\
\text { Splash? }\end{array}$ \\
\hline 1 & $2 \& 3$ & 6.25 & 0.725 & 2.631 & 17460 & 597 & 0.0014 & 113 & 0.116 & 3.5 & yes \\
\hline 2 & 2 & 3 & 2 & 2 & 6370 & 166 & 0.0020 & 135 & 0.614 & 0.398 & no \\
\hline 3 & 2 & 6 & 3 & 4.2 & 26754 & 1460 & 0.0014 & 300 & 0.5 & 4.7 & yes \\
\hline 4 & 2 & 4.2 & 2.1 & 5.098 & 21304 & 1496 & 0.0018 & 631 & 0.5 & 4.52 & yes \\
\hline 5 & 2 & 4.2 & 2.1 & 5.098 & 1168 & 2009 & 0.0384 & 631 & 0.5 & 1.73 & yes \\
\hline 6 & 2 & 4.2 & 2.814 & 2.94 & 673 & 668 & 0.0384 & 210 & 0.67 & 0.67 & no \\
\hline 7 & 2 & 4 & 8 & 4 & 16987 & 883 & 0.0017 & 408 & 2 & 0.62 & no \\
\hline 8 & 2 & 4 & 4 & 4 & 16987 & 883 & 0.0017 & 408 & 1 & 1.403 & yes \\
\hline 9 & 2 & 4 & 2 & 6 & 25480 & 1988 & 0.0017 & 917 & 0.5 & 5.9 & yes \\
\hline 10 & 2 & 4 & 2 & 4 & 16987 & 883 & 0.0017 & 408 & 0.5 & 2.62 & yes \\
\hline 11 & 2 & 4 & 2 & 2 & 8494 & 221 & 0.0017 & 102 & 0.5 & 0.65 & no \\
\hline 12 & 2 & 4 & 1 & 4 & 16987 & 883 & 0.0017 & 408 & 0.25 & 3.86 & yes \\
\hline 13 & 2 & 4 & 0.5 & 4 & 16987 & 883 & 0.0017 & 408 & 0.125 & 4.67 & yes \\
\hline 14 & 2 & 3 & 3 & 2 & 6370 & 166 & 0.0020 & 136 & 1 & 0.25 & no \\
\hline 15 & 2 & 3 & 3 & 1 & 3185 & 41 & 0.0020 & 34 & 1 & 0.062 & no \\
\hline 16 & 2 & 3 & 2 & 1 & 3185 & 41 & 0.0020 & 34 & 0.666 & 0.092 & no \\
\hline 17 & 2 & 3 & 1 & 2 & 6370 & 166 & 0.0020 & 136 & 0.333 & 0.6 & no \\
\hline 18 & 2 & 2 & 4 & 2 & 4247 & 110 & 0.0025 & 204 & 2 & 0.067 & no \\
\hline 19 & 2 & 2 & 4 & 1 & 2123 & 28 & 0.0025 & 51 & 2 & 0.017 & no \\
\hline 20 & 2 & 2 & 2 & 2 & 4247 & 110 & 0.0025 & 204 & 1 & 0.152 & no \\
\hline 21 & 2 & 2 & 2 & 1 & 2123 & 28 & 0.0025 & 51 & 1 & 0.038 & no \\
\hline 22 & 2 & 2 & 1 & 2 & 4247 & 110 & 0.0025 & 204 & 0.5 & 0.285 & no \\
\hline 23 & 2 & 2 & 1 & 1 & 2123 & 28 & 0.0025 & 51 & 0.5 & 0.071 & no \\
\hline & & & & & & & & & & \\
\hline
\end{tabular}

\subsection{Results}

Figure 3.10 shows the impact of a single drop (water-glycerol 70\%) with $D=4.2 \mathrm{~mm}$ onto a liquid layer of $h^{\prime}=h / D=0.5$ (Case 5) with non-uniform (triangular cells with different sizes) and non-adaptive (cell sizes do not change with the simulation time) computational cells. As is clearly seen, the interface of liquid and gas is not as accurately defined as in the fine mesh simulation results shown in Figure 3.11 which will be discussed later. In Fig. 3.10 the liquid volume fraction is not calculated accurately, especially during splashing (secondary atomization) and crown generation at later simulation times due to coarser mesh elements at the interface of the liquid and gas. 

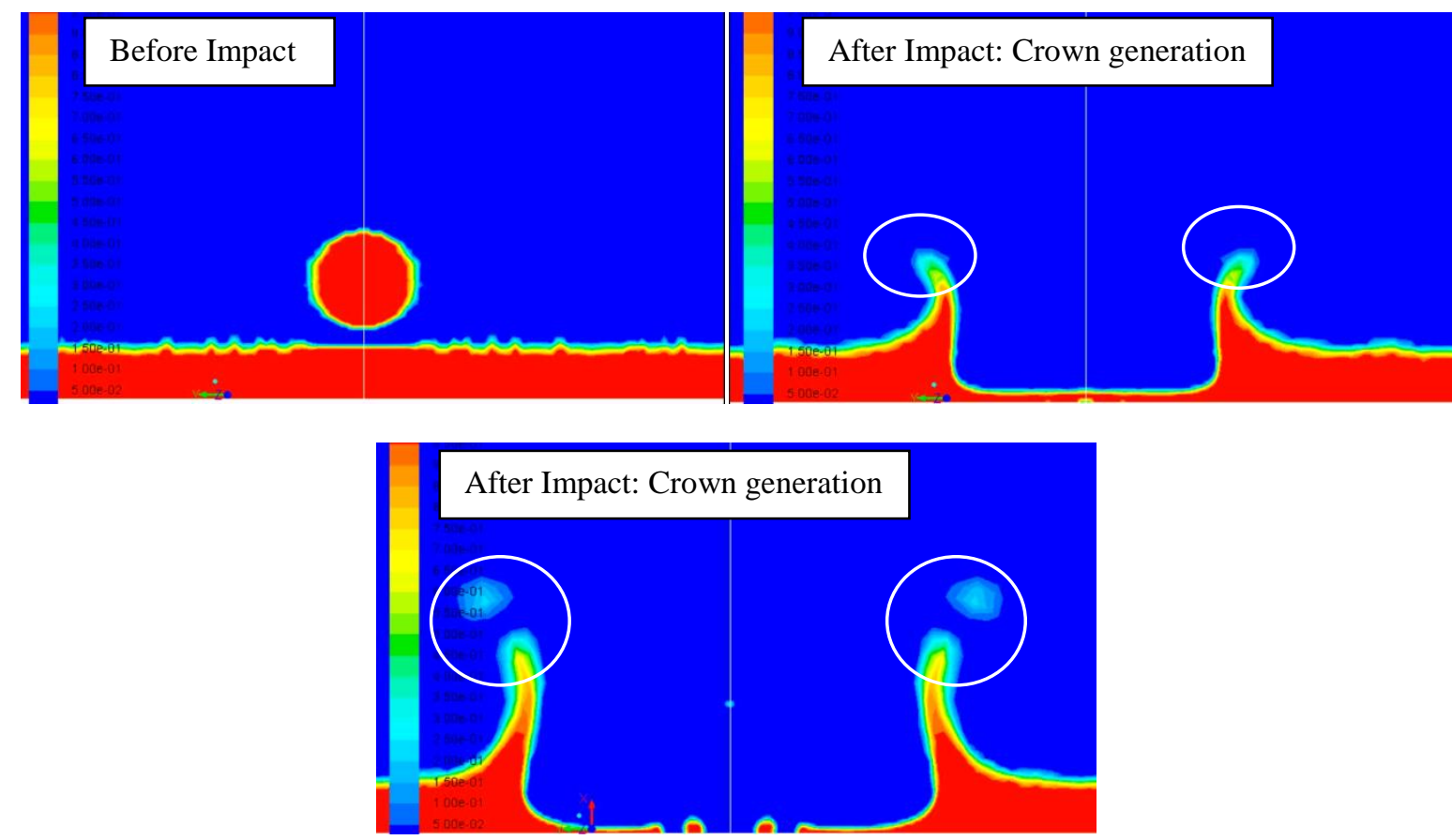

Figure 3.10 Non-uniform and non-adaptive triangular mesh results for Case 5: We $=2009, D=$ $4.2 \mathrm{~mm}$ and $h / D=0.5$. (Note: Blue refers to air and red refers to liquid)

The secondary drop atomization, crown height, $H$, upper crown diameter, $D_{U}$ and lower crown diameter, $D_{L}$ could not be obtained accurately because of the mesh size which was not small enough.

For validation purposes some simulations using adaptive mesh refinement were compared to computational and experimental studies in the literature, and they were found to be in good agreement. Figure 3.11 shows a comparison of Case 5 using a Level-4 mesh with the physical experiment of Wang and Chen (2000) and the computational study of Asadi and Passandideh-Fard (2009). The liquid interface and the ejection of drops from the crown were more accurately predicted compared to the non-uniform mesh shown in Figure 3.10. Figure 3.12 shows the good agreement of the dimensionless upper crown diameter values for Case 6 with the literature (Asadi and Passandideh-Fard, (2009); Cossali et al., (2004)). 


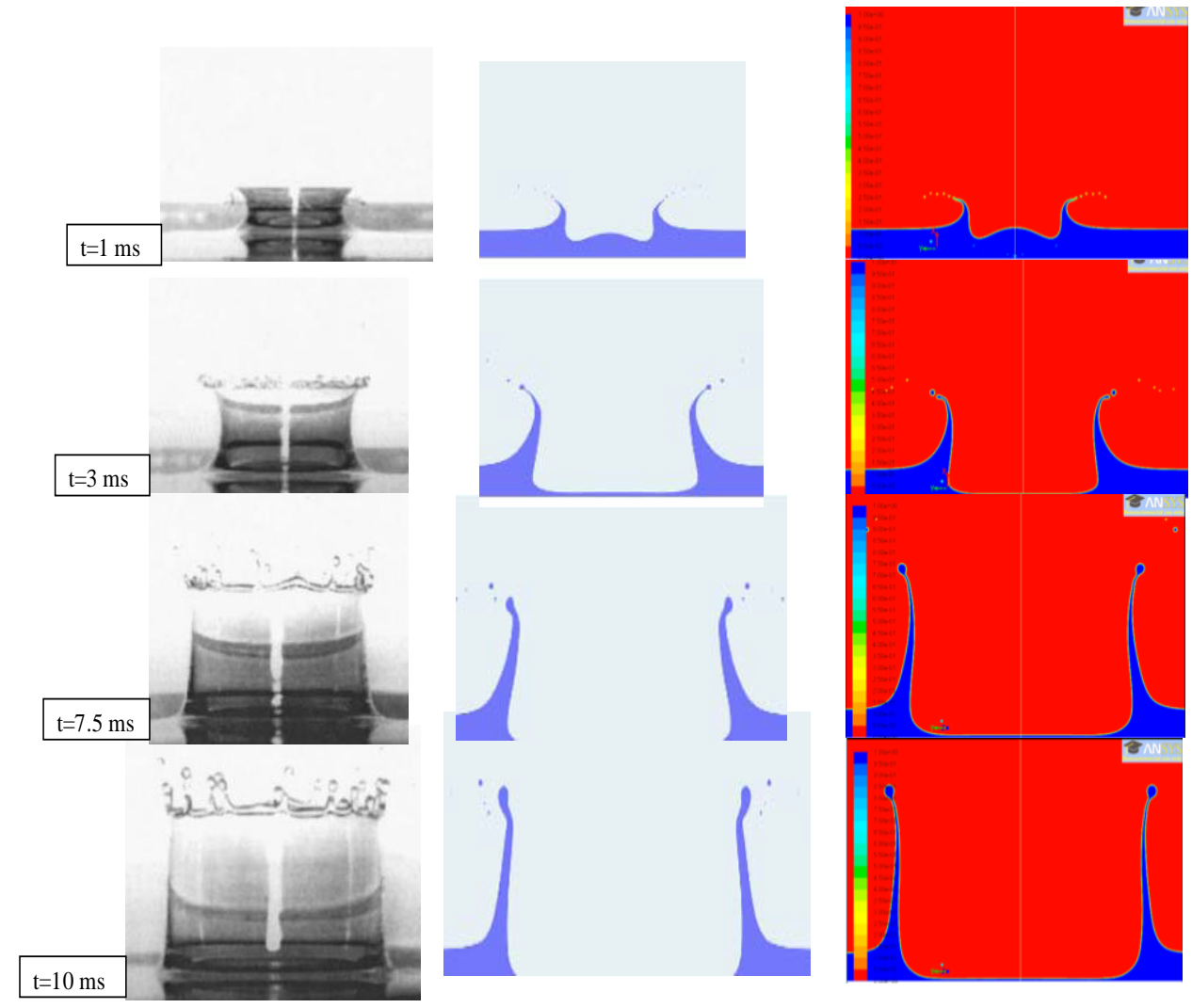

Figure 3.11 Comparison of results for Case 5 for $4.2 \mathrm{~mm}$ diameter droplet impacting onto a liquid layer with a thickness of $2.1 \mathrm{~mm}: \operatorname{Re}=1168, W e=2009, \mathrm{Fr}=631$, and $h / D=0.5$. (Experimental images from Wang and Chen (2000) in the left column, numerical results from Asadi and Passandideh-Fard (2009) in the middle column, and numerical results of the current study in the right column with blue representing water and red representing air.)

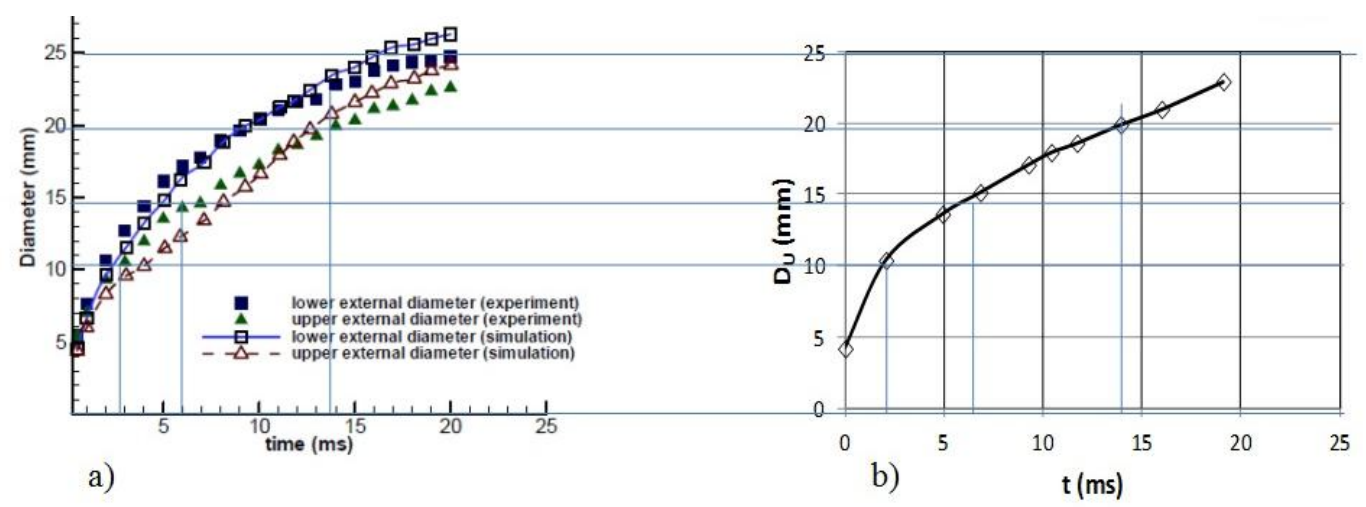

Figure 3.12 Comparison of the upper crown diameter for Case 6 with $4.2 \mathrm{~mm}$ diameter droplet impacting on a thin liquid film with a dimensionless thickness of $0.67, W e=668$ (a) Experimental results (Cossali et al., 2004) and simulation results of Asadi and Passandideh-Fard (2009). (Image taken from Asadi and Passandideh-Fard (2009).) (b) The present simulation results. (Note: Lines are extended from experimental results of Cossali et al. (2004) in order to compare with current simulation results.) 
According to the splashing criterion of Cossali et al. (1997), the simulations listed in Table 3.1 included 9 late splash cases and 14 non-splash cases. In every one, the present simulation results are in agreement with equation 3.10. Figure 3.13 shows Case 1, Case 2, and Case 3 simulation results. Case 1 and Case 3 show late splashing during crown generation, while in Case 2 the crown collapses without splashing.

Case 1 was simulated using both 2D axisymmetric and 3D models. The 2D axisymmetric model used Level-4 adaptive meshing with the smallest cells equal to $D / 80$; the 3D simulation used Level-3 non-adaptive meshing with zonal refinement, the smallest cells being equal to D/40. The simulation of Case 1 using a fully 3D simulation of one quarter of the complete domain is shown in Figure 3.14. In this simulation, the water that came from the drop and that which was initially in the water layer were separate phases similar to the $2 \mathrm{D}$ axisymmetric cases. Figure 3.14 shows the separate drop and film phases at different time steps for Case 1. These results are close to the simulations of Nikolopoulos et al. (2007). The drop has sufficient energy to displace the layer liquid and contact the surface for the dimensionless time $\tau=t /(D / U)>1$. Late splashing was observed in this case in agreement with the criterion of Cossali et al. (1997). 


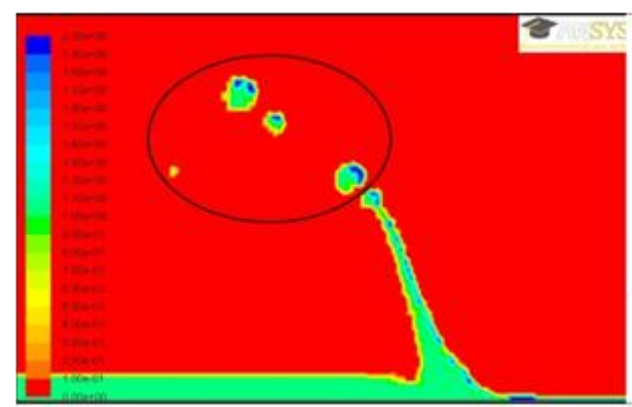

a)

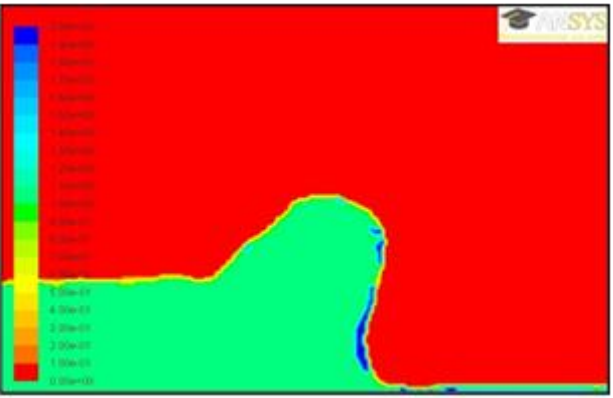

b)

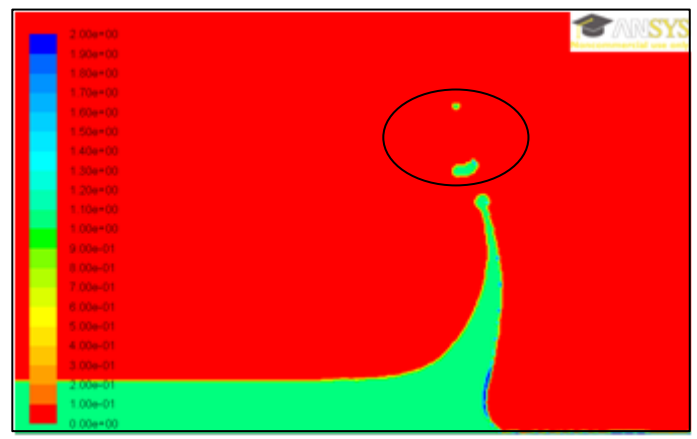

c)

Figure 3.13 2D axisymmetric simulation results: (a) late splash for Case 1 with $Y=3.5$ (b) crater collapse without late splash for Case 2 with $Y=0.398$ (c) late splash for Case 3 with $Y=4.7$. (Note: red is air, green is film liquid and blue is drop liquid. Only half of the solution domain is shown for clarity.) 


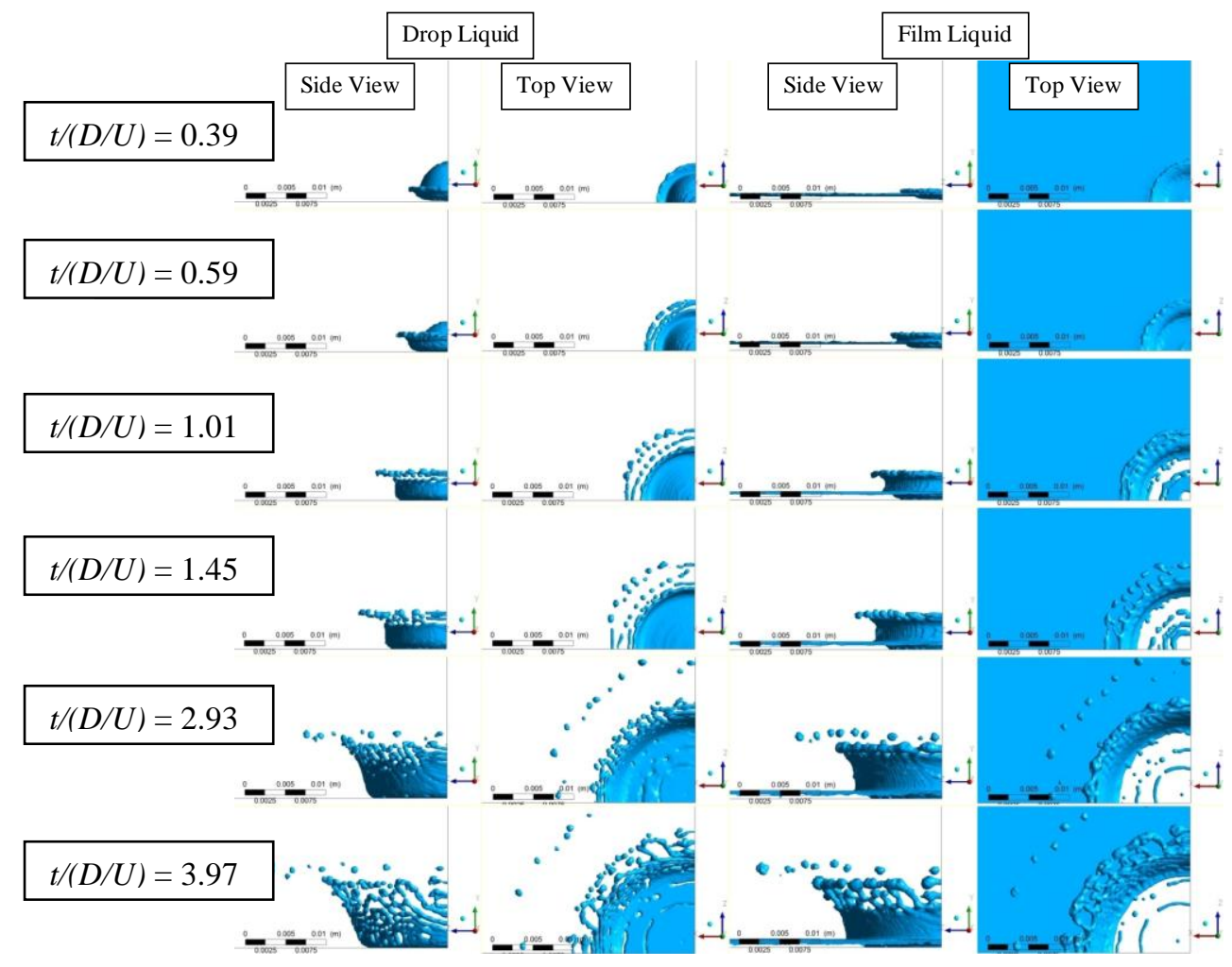

Figure 3.14 Three dimensional model liquid volume fraction results for Case 1 with respect to the dimensionless time $(t /(D / U))$. (Note: only quarter of the domain was simulated) (Hillen et al. (2012))

\subsubsection{Liquid Film and Crown Dynamics}

Figure 3.15 shows close agreement between the dimensionless centerline film thickness predicted by the 2D axisymmetric and 3D simulations for Case 1. Case 1 results for 2D axisymmetric and 3D models were also compared to the simulation results of Nikolopoulos et al. (2007). Figure 3.16 shows the dimensionless bottom crown radius $\left(R_{B}{ }^{\prime}=R_{B} / D\right)$ with respect to time in dimensionless form $(\tau=t /(D / U))$ for both 2D axisymmetric and 3D models and the Nikolopoulos et al. (2007) simulation. Results were in a good agreement with Nikolopoulos et al. (2007) for both models. 


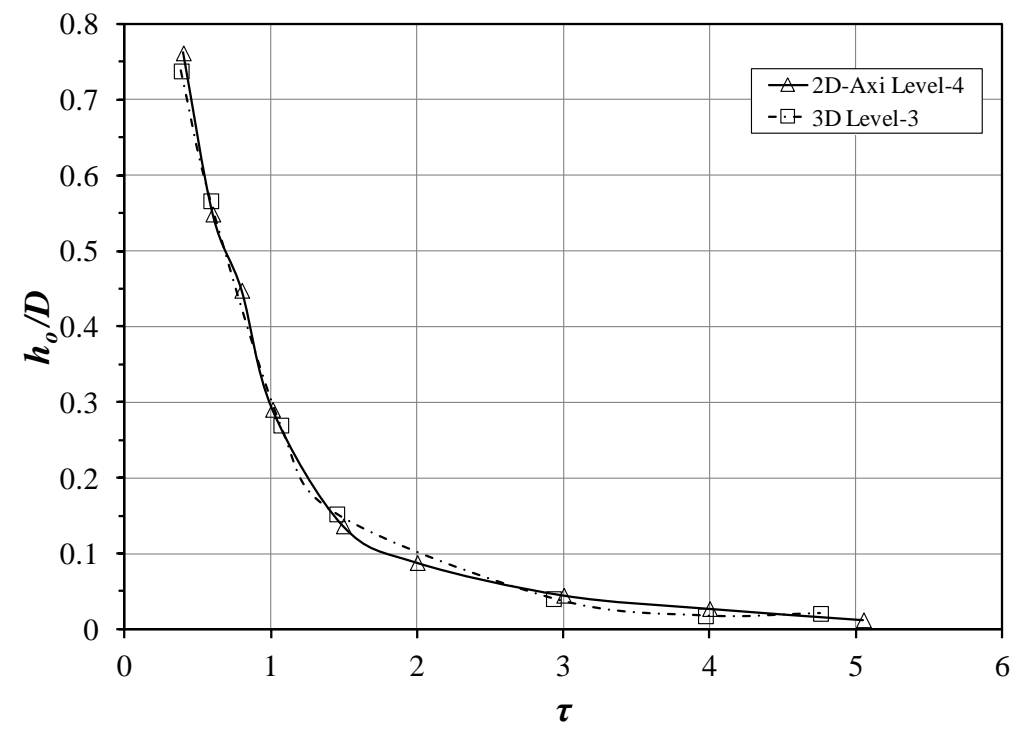

Figure 3.15 Case 1 comparisons of the 2D axisymmetric Level-4 and 3D Level-3 results of dimensionless centerline film thickness $\left(h_{0} / D\right)$ versus dimensionless time $(\tau)$ (Hillen et al. (2012))

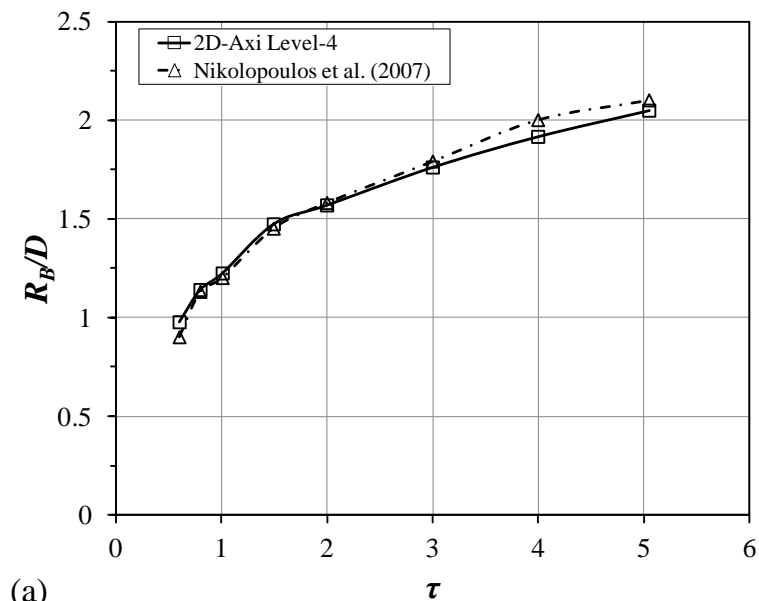

(a)

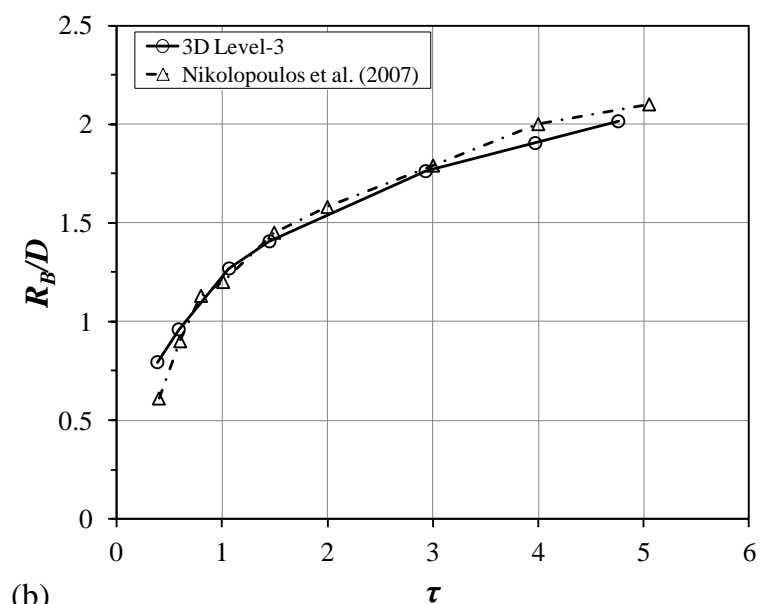

(b)

Figure 3.16 Case 1 comparisons of dimensionless bottom crown radius $\left(R_{B} / D\right)$ versus dimensionless time $(\tau)$ : (a) 2D axisymmetric Level-4 and (b) 3D Level-3 results with computational results of Nikolopoulos et al. (2007). (Hillen et al. (2012))

Figure 3.17 shows the percentage of the solid surface beneath the cavity covered by the drop and layer liquid as functions of dimensionless time for Case 1, Case 2, and Case 3. This figure illustrates that drop liquid reaches the surface in a shorter time and covers a higher area ratio in Case 1 compared to Case 2, or Case 3. This can be explained by the higher Reynolds 
number $(R e)$ and Weber number (We), along with the smaller dimensionless liquid film thickness, $h / D$ when comparing Case 1 and Cases 2-3. Moreover, it is obvious from the comparison of Case 1 and Case 3 that the thinner dimensionless liquid film thickness, $h / D$, has the most effect on the drop liquid percentage contacting the solid surface. Figures 3.18, 3.19 and 3.20 show drop and film liquid thickness at the centerline at the initial stage of the liquid crown generation for Cases 1, 2 and 3, respectively. Since the inertial energy (We and $R e$ ) is greater for Case 1 and Case 3 compared to Case 2, the initial film liquid gets very thin (less than $100 \mu \mathrm{m}$ ) for Case 1 and Case 3 during the liquid crown generation (Fig. 3.18 and Fig. 3.20). However, film liquid thickness remains thicker (more than $300 \mu \mathrm{m}$ ) for Case 2 (Fig. 3.19) because of the lower kinetic energy of the drop and the thicker layer. 


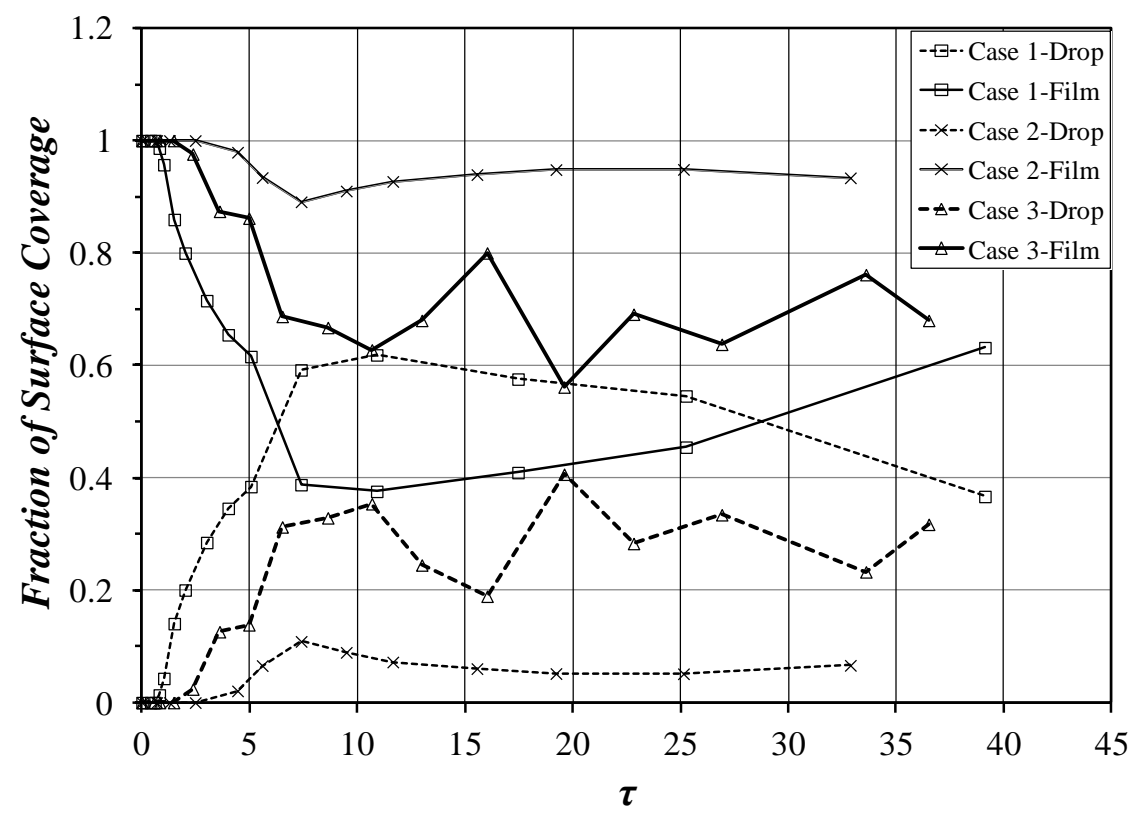

Figure 3.17 Comparison of Case 1 with $h / D=0.116$, Case 2 with $h / D=0.614$, and Case 3 with $h / D=0.5$ based on liquid (drop and film) on surface. (Note: dashed lines refer to drop liquid while solid lines refer to film liquid.)

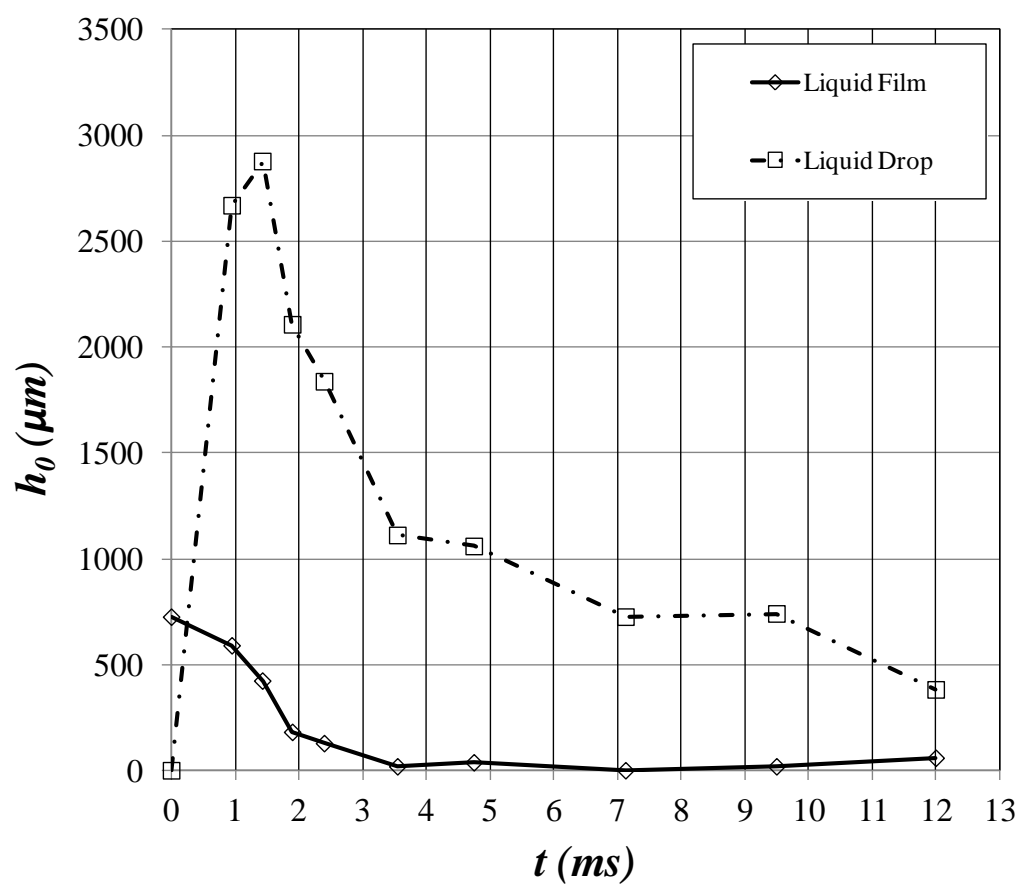

Figure 3.18 Case 1 centerline drop and film liquid thickness $\left(h_{0}\right)$ during the initial stage. 


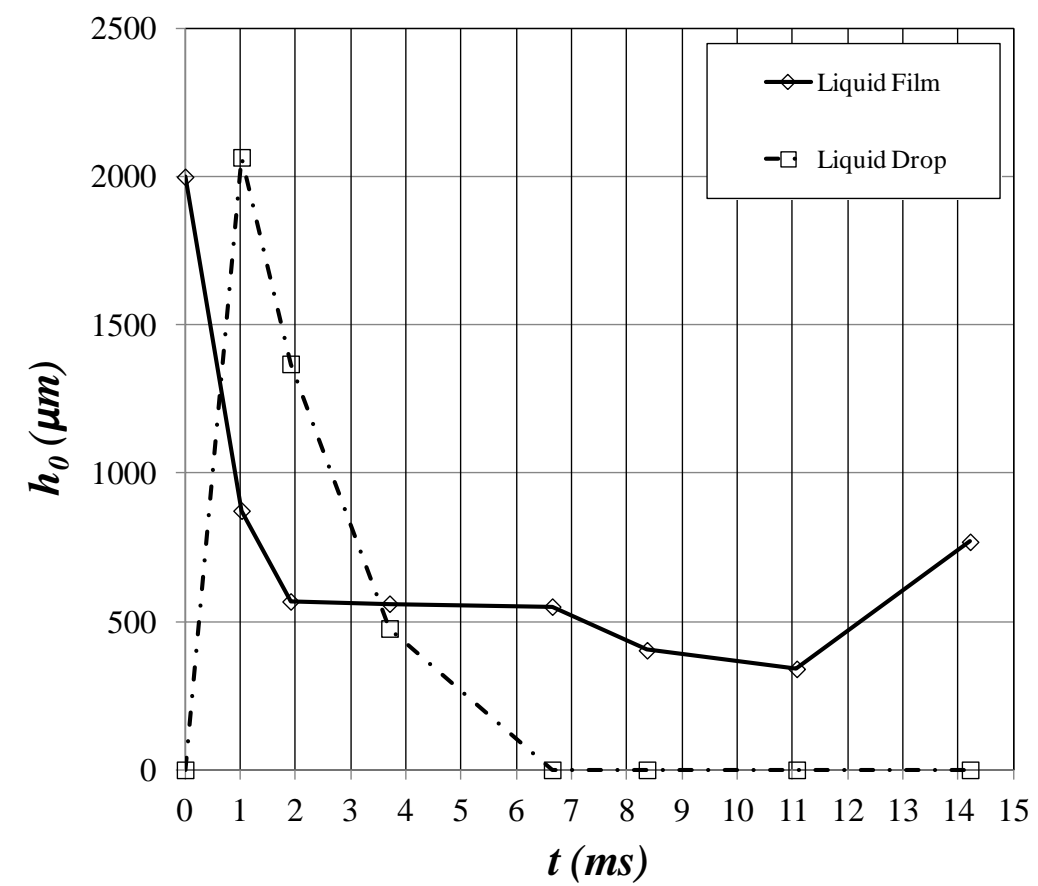

Figure 3.19 Case 2 centerline drop and film liquid thickness $\left(h_{0}\right)$ during the initial stage.

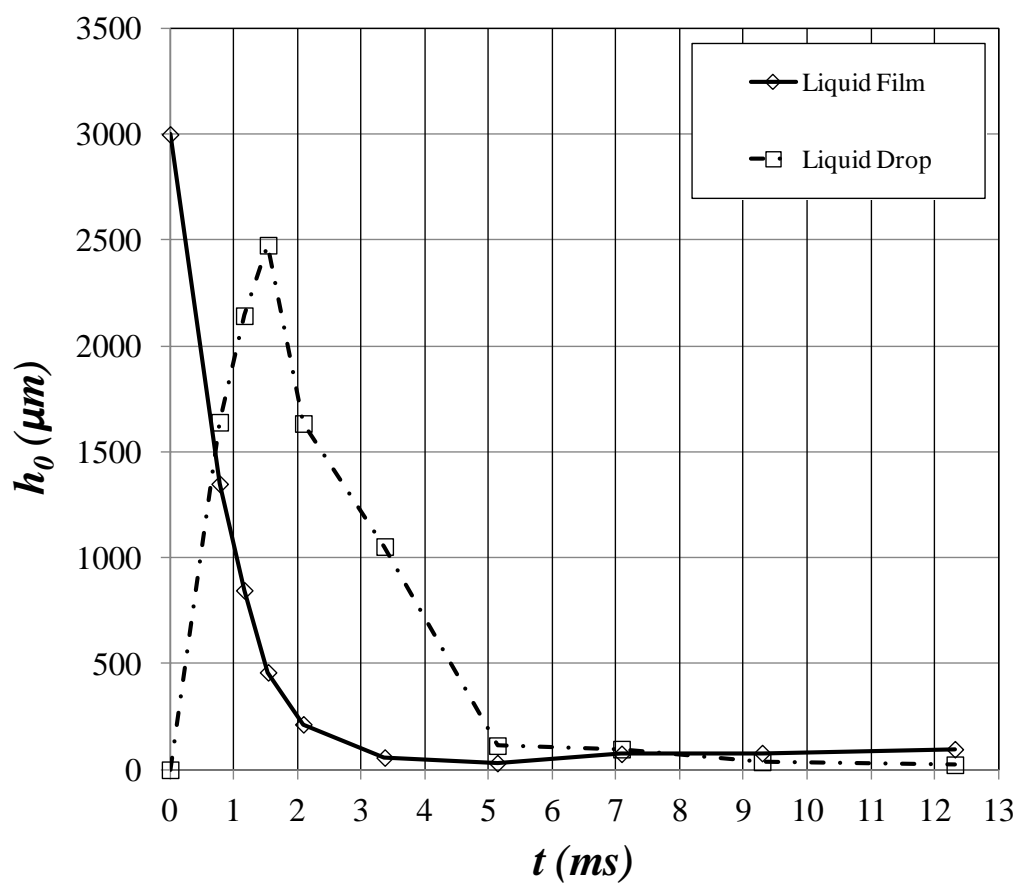

Figure 3.20 Case 3 centerline drop and film liquid thickness $\left(h_{0}\right)$ during the initial stage. 


\subsubsection{Sub-Cavity Liquid Volume}

The liquid volume beneath the cavity, called the sub-cavity liquid volume, was also calculated. This volume is very important since the liquid quantity in the sub-cavity is directly related to the heat transfer in actual spray cooling in which multiple drops interact with the liquid film and the heated surface (Kuhlman et al., 2007).

The percentages of the average thickness of the drop liquid and layer liquid, calculated based on the thickness of the sub-cavity liquid along the cavity radius throughout the cavity lifetime are shown in Figures 3.21, 3.22 and 3.23 for Cases 1-3, respectively. The behavior in the sub-cavity region is very different for Case 1 compared to the other cases. The sub-cavity liquid is almost fully occupied by the drop liquid throughout the cavity lifetime ( 100\% drop liquid) as seen in Figure 3.21. The very high percentage of drop liquid for Case 1 can be explained due to the very thin initial liquid layer $\left(h^{\prime}=h / D=0.116\right)$. For Case 2 , the percentage of drop liquid increases from $0 \%$ to around $60 \%$ from $0 \mathrm{~mm}$ to $0.5 \mathrm{~mm}$ radius. The percentages of each liquid becomes almost constant from $0.5 \mathrm{~mm}$ to radius $3 \mathrm{~mm}$ right before the sub-cavity liquid is refilled by the capillary wave (60\% drop liquid and $40 \%$ layer liquid in sub-cavity) (Figure 3.22). Case 3 shows behavior similar to Case 1 except more fluctuations occur from the centerline $(R=$ 0 ) to the maximum cavity radius $(R \sim 12 \mathrm{~mm}$ ) (Figure 3.23). Table 3.2 shows the average of the percentage $(\%)$ of the drop liquid and the layer liquid in the whole cavity region for the entire cavity lifetime. It is clear from Table 3.2 that the sub-cavity liquid in Case 1 consists of liquid dominantly from the drop $(92 \%)$. The sub-cavity liquid consisted of drop liquid with the percentages of 53\% and $48 \%$ for Case 2 and Case 3, respectively (Table 3.2). These results show that to increase the transient heat conduction between colder spray drops and the heated subcavity surface a very thin liquid layer over the heated surface during spray impact is desirable since a very high percentage of drop can reach the surface. 


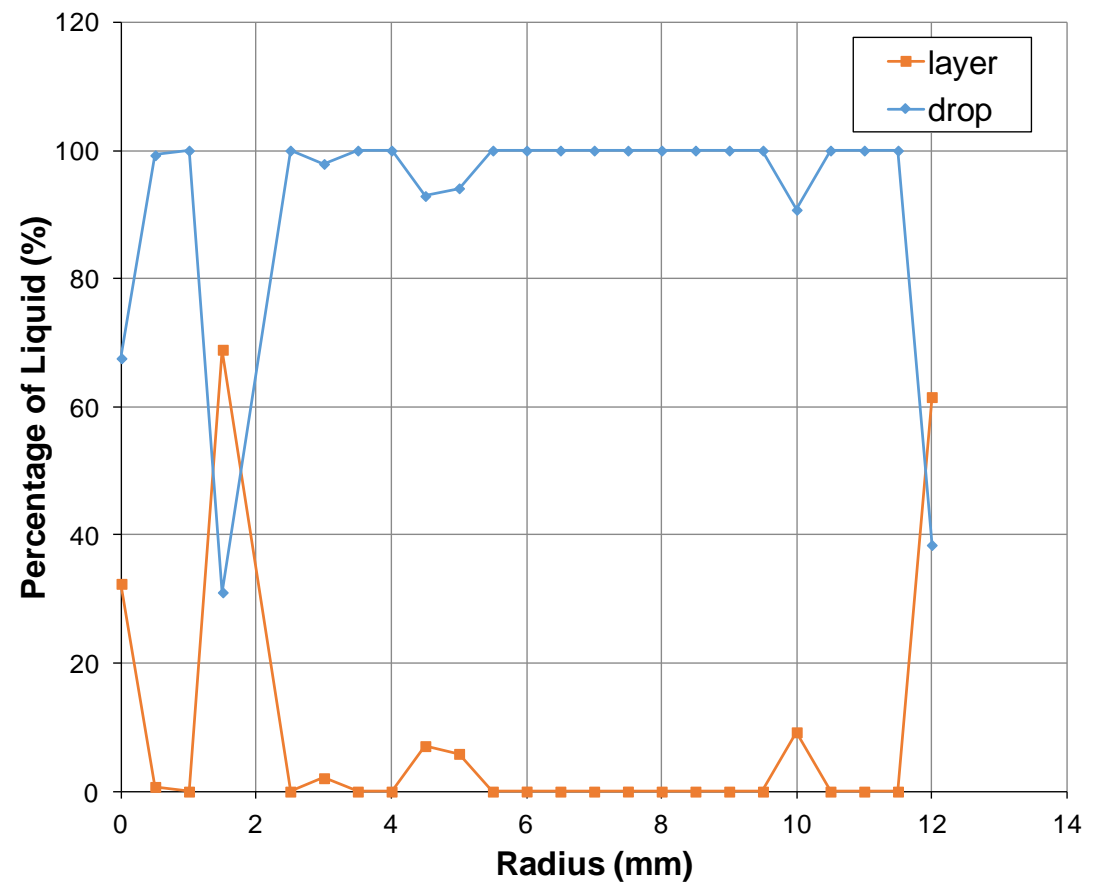

Figure 3.21 Drop liquid and layer liquid percentages (\%) below the cavity along the cavity radius $(\mathrm{mm})$ for Case 1.

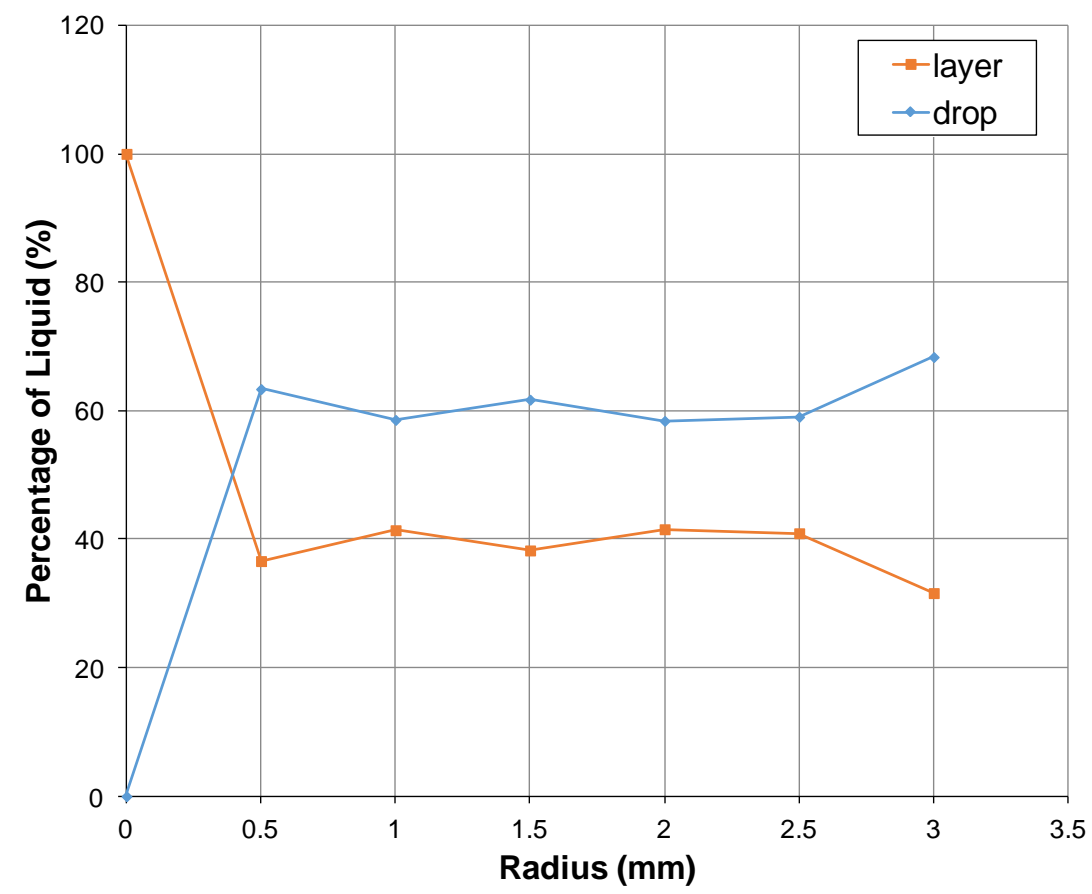

Figure 3.22 Drop liquid and layer liquid percentages (\%) below the cavity along the cavity radius (mm) for Case 2. 


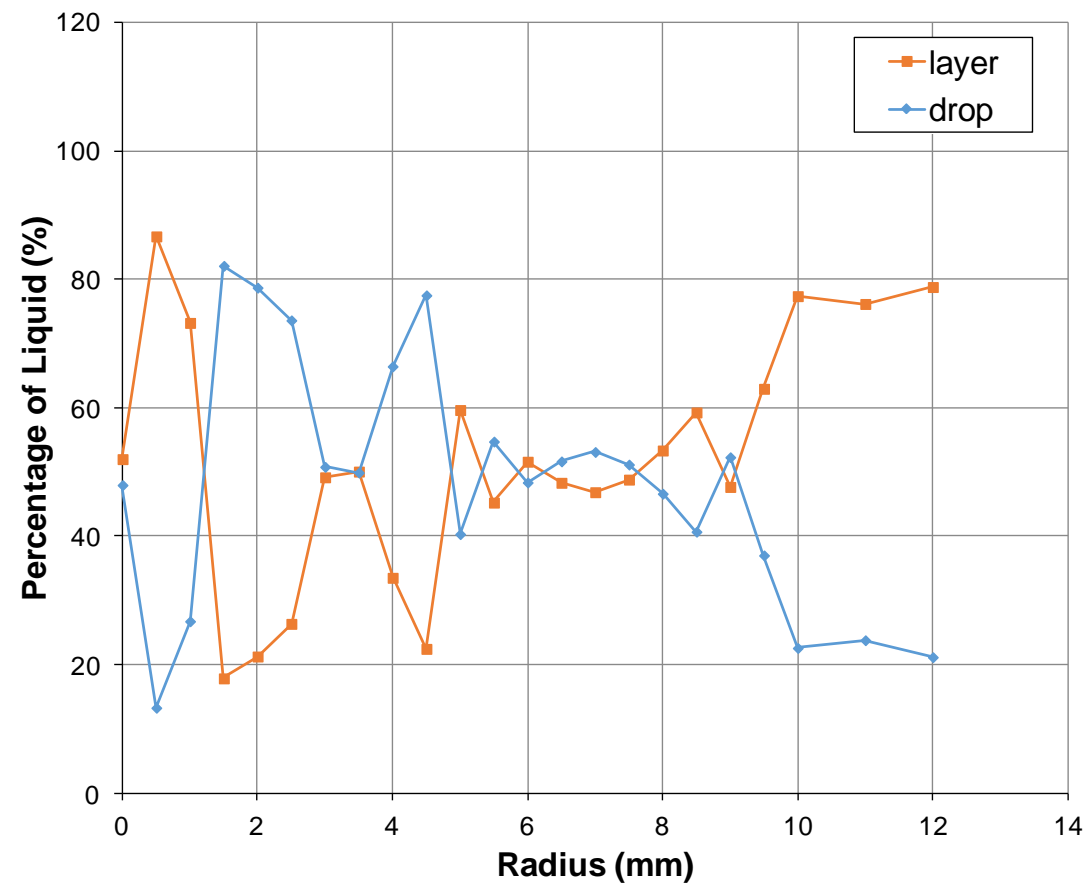

Figure 3.23 Drop liquid and layer liquid percentage (\%) below the cavity along the cavity radius (mm) for Case 3.

Table 3.2 The average of the percentages (\%) of drop liquid and layer liquid in the whole subcavity for the entire cavity lifetime.

\begin{tabular}{|c|c|c|c|}
\hline Case & $\boldsymbol{h}^{\prime}=\boldsymbol{h} / \boldsymbol{D}$ & Drop Liquid (\%) & Film Liquid (\%) \\
\hline 1 & 0.116 & 92 & 8 \\
\hline 2 & 0.614 & 53 & 47 \\
\hline 3 & 0.5 & 48 & 52 \\
\hline
\end{tabular}

\subsubsection{Comparisons with WVU Experiments}

\subsubsection{Liquid Film and Crown Dynamics}

This section is primarily based on Hillen, Kuhlman, Dinc and Gray (2012). Three cases (Cases 1, 2, and 3) were compared with the experiments performed by Hillen et al. (2012) at West Virginia University (WVU). Figure 3.24 shows the comparisons with the high-speed video images obtained from experiments by Hillen et al. (2012) for Case 1. There was a close agreement with experiments during the initial crown propagation. However, the crown had already collapsed at $28.58 \mathrm{~ms}$ in the simulation while it still existed in the experiment. This difference was attributed 
to the 2D axisymmetric nature of the simulations because secondary splashing drops are actually rings in axisymmetric simulations whereas they are clearly three dimensional in the experiments. Figure 3.25 shows the comparisons for Case 2. There is very good agreement in Case 2 during and after crown propagation because there is no secondary splashing to cause deviations from axisymmetry. Figure 3.26 shows the comparisons for Case 3. As in Case 1, at initial times the liquid interface and crown propagation were accurate but there were differences at the secondary stage in which crown collapsed earlier in CFD compared to experiment (Fig. 3.26).

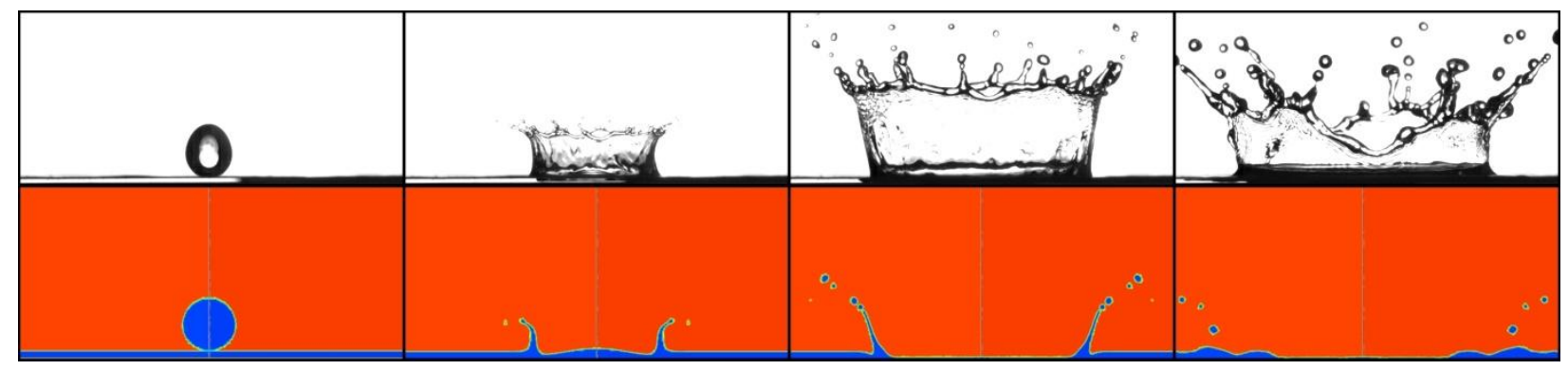

Figure 3.24 Case 1 comparisons of the high-speed video images (top) and the CFD simulations (bottom) for times $t \approx 0,3.08,12.9$, and $28.6 \mathrm{~ms}$. (Hillen et al. (2012)) (Red refers to the air phase and blue refers to liquid phase (drop and layer).)

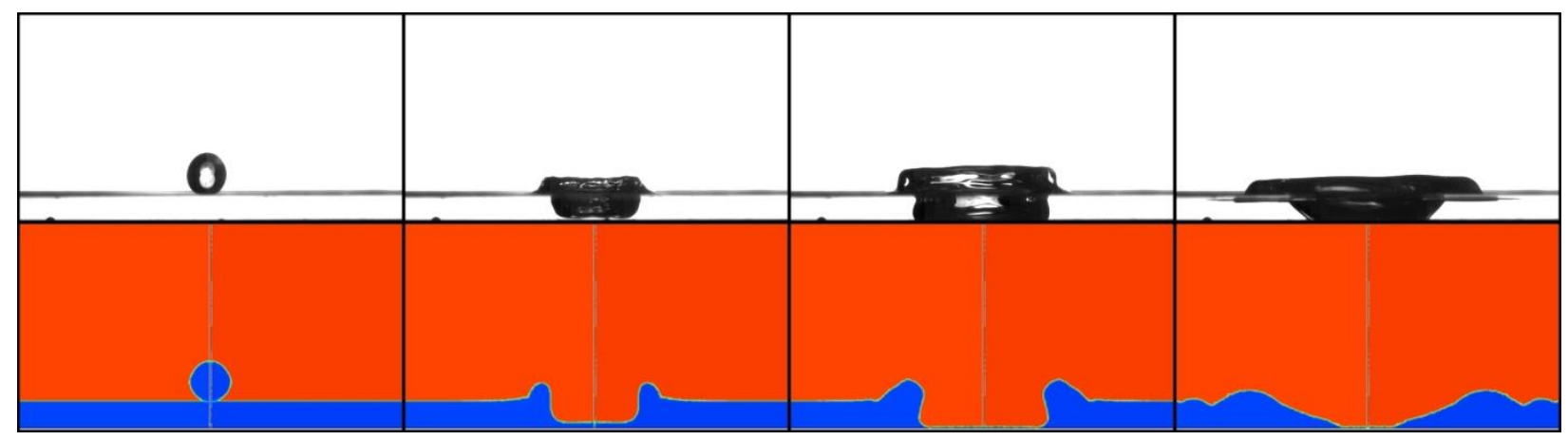

Figure 3.25 Case 2 comparisons of the high-speed video images (top) and the CFD simulations (bottom) for times $t \approx 0,3.67,8.92$, and $17.8 \mathrm{~ms}$. (Hillen et al. (2012)) (Red refers to the air phase and blue refers to liquid phase (drop and layer).) 


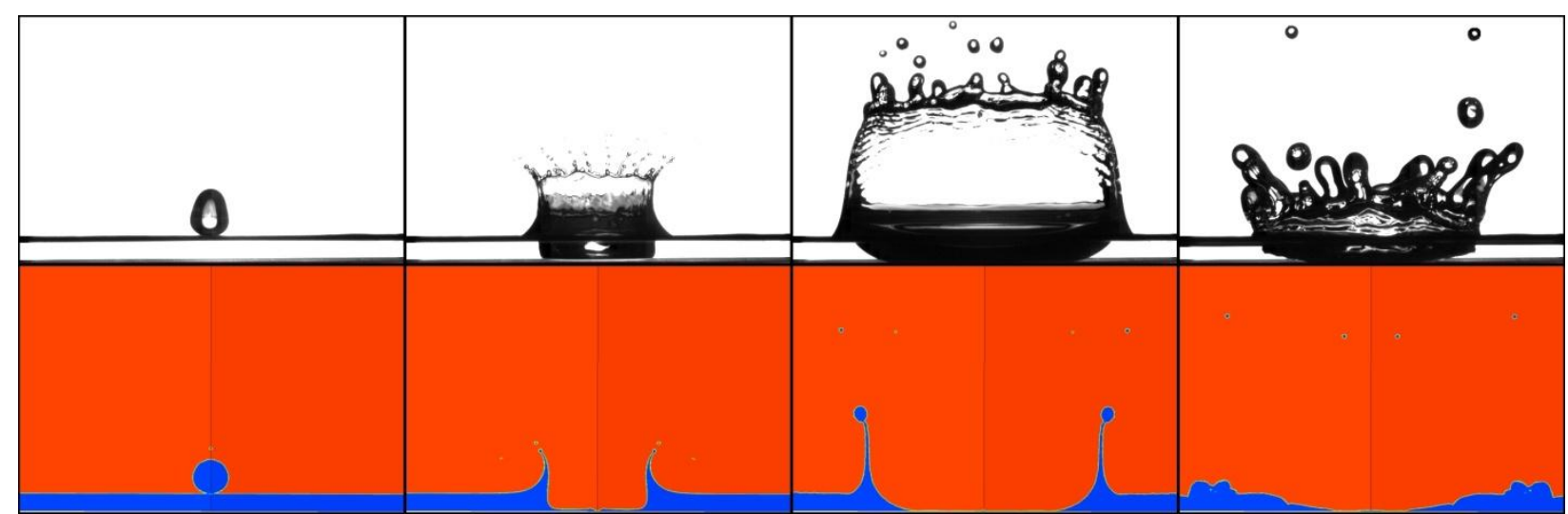

Figure 3.26 Case 3 comparisons of the high-speed video images (top) and the CFD simulations (bottom) for times $t \approx 0,4.42,33.2$, and $69.1 \mathrm{~ms}$. (Hillen et al. (2012)) (Red refers to the air phase and blue refers to liquid phase (drop and layer).)

Figures 3.27, 28 and 29 show the centerline film thickness comparisons for Cases 1, 2 and 3, respectively. The CFD results are in good agreement with the experiments. However, there are some differences at later times for Cases 1 and 3 (Figures 3.27 and 3.29, respectively). These differences in Cases 1 and 3 could be attributed to the axisymmetric geometry of the simulations as mentioned above.

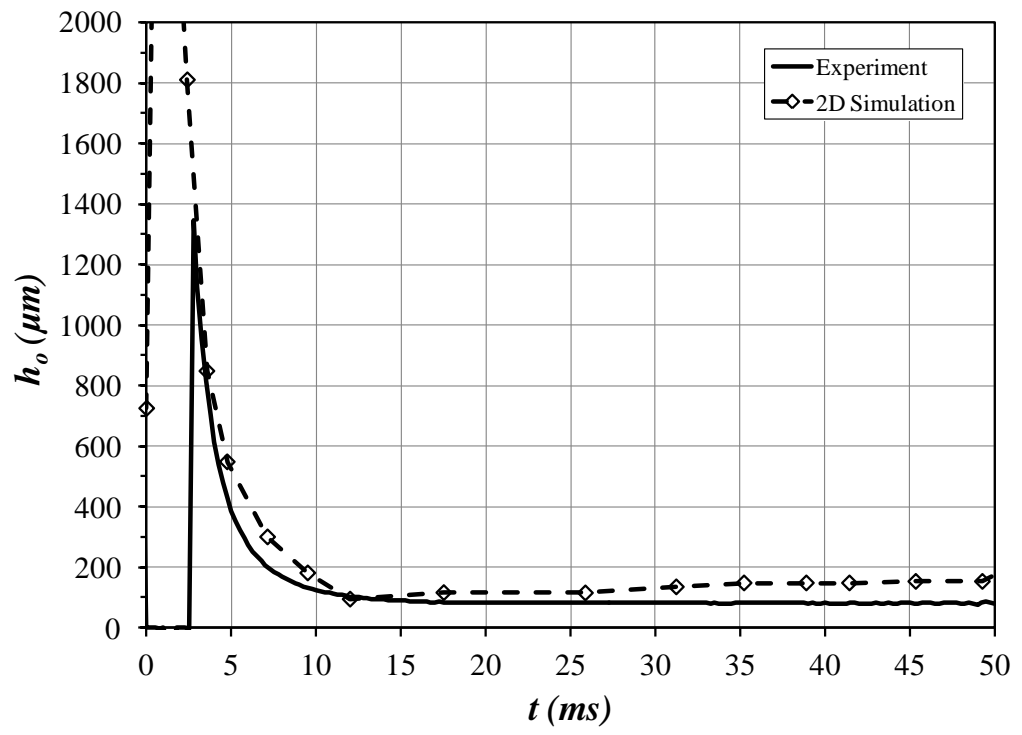

Figure 3.27 Case 1 centerline film thickness comparisons between the average experimental results and simulation results. (Hillen et al. (2012)) 


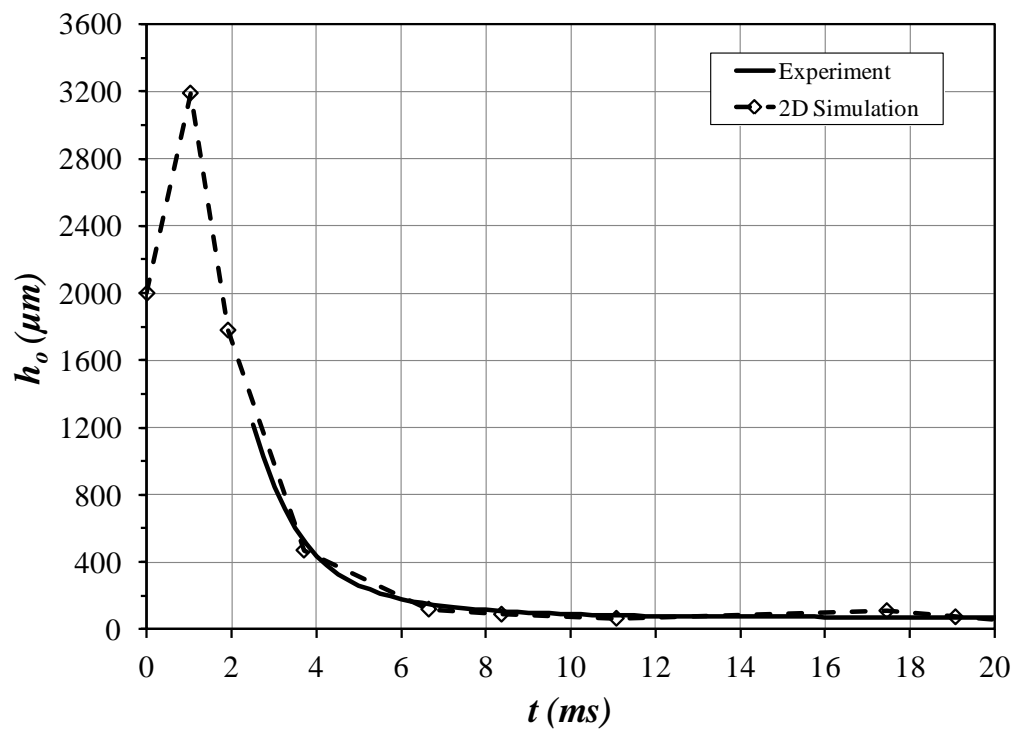

Figure 3.28 Case 2 centerline film thickness comparisons between the average experimental results and simulation results. (Hillen et al. (2012))

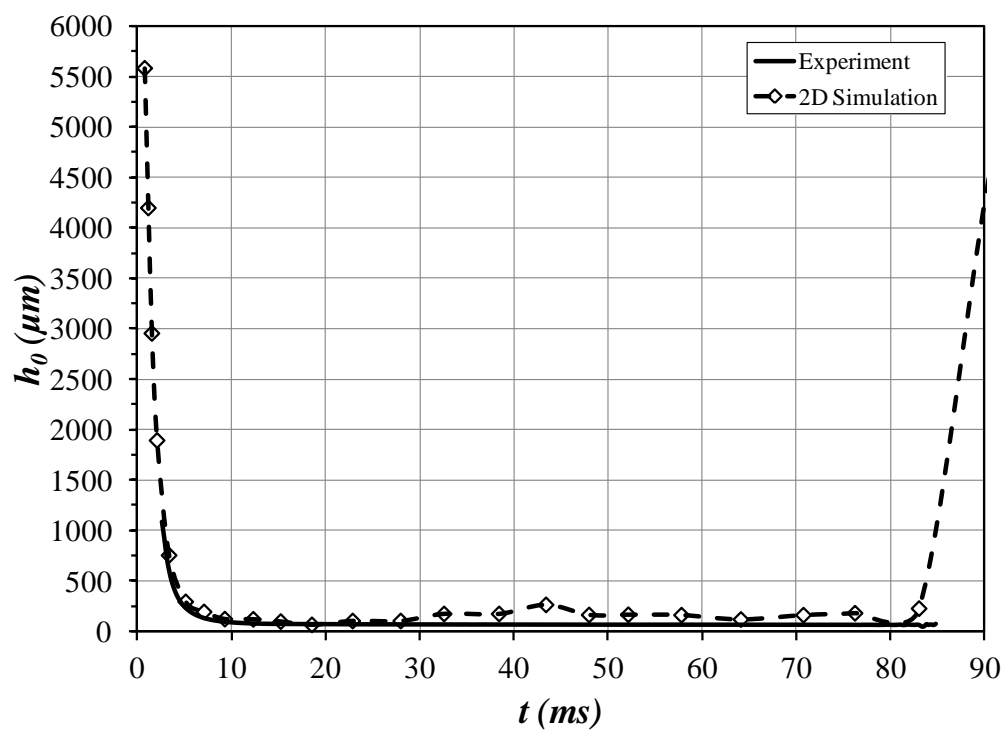

Figure 3.29 Case 3 centerline film thickness comparisons between the average experimental results and simulation results. (Hillen et al. (2012))

The dimensionless outer crown radius $\left(R_{B}{ }^{\prime}=R_{B} / D\right)$ with respect to the dimensionless time $(\tau=t /(D / U))$ is compared with the experiments for Cases 1, 2, and 3 (including the 3D simulation results for Case 1 only) in Figures 3.30, 3.31 and 3.32, respectively. For all cases, the propagation of the outer radius was similar but the simulations over predicted the experiments in 
Cases 2 and 3. The 3D and 2D axisymmetric simulation results were in good agreement for Case 1 (Figure 3.30).

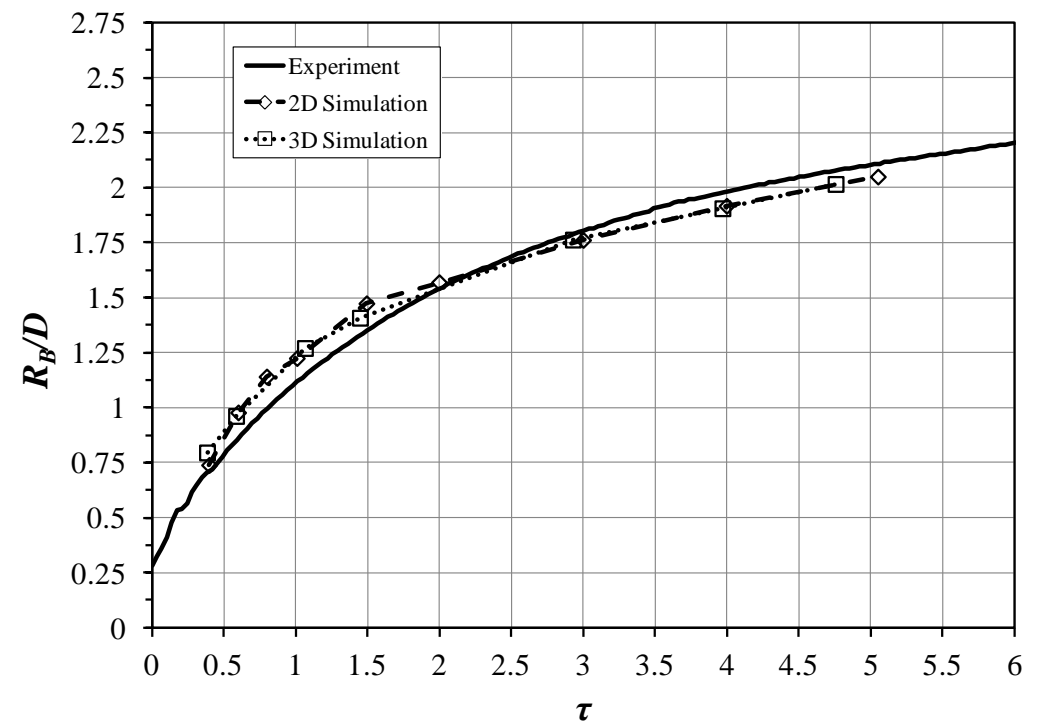

Figure 3.30 Comparison of the dimensionless bottom crown diameter experimental and simulation results with respect to the dimensionless time for Case 1. (Hillen et al. (2012))

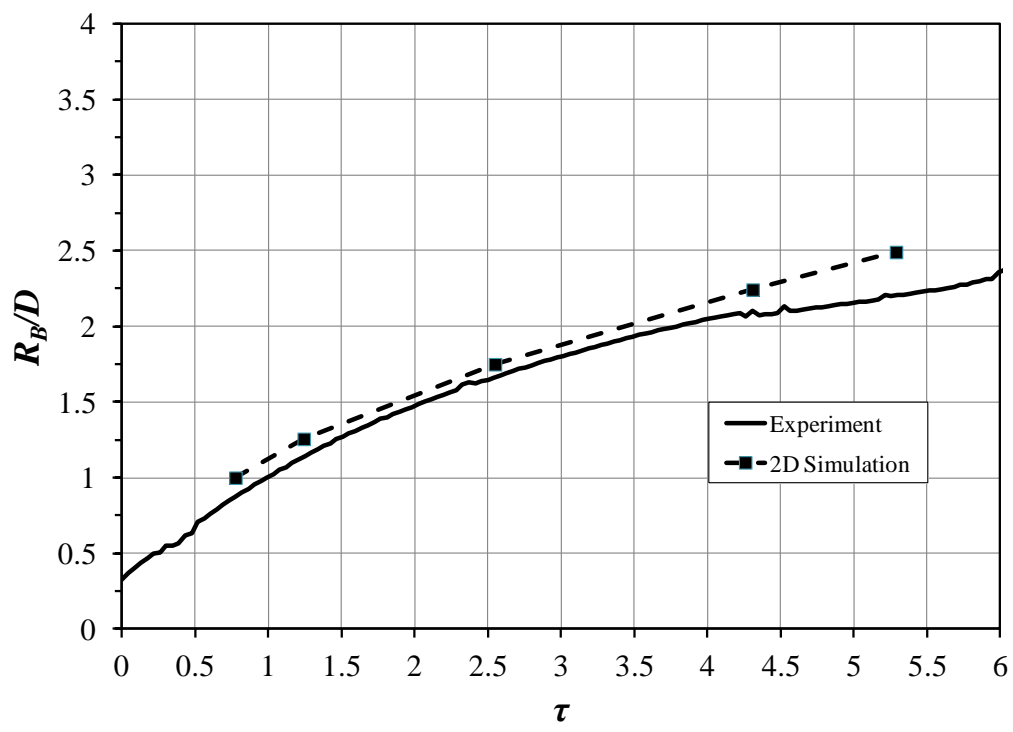

Figure 3.31 Comparison of the dimensionless bottom crown diameter experimental and simulation results with respect to the dimensionless time for Case 2. (Hillen et al. (2012)) 


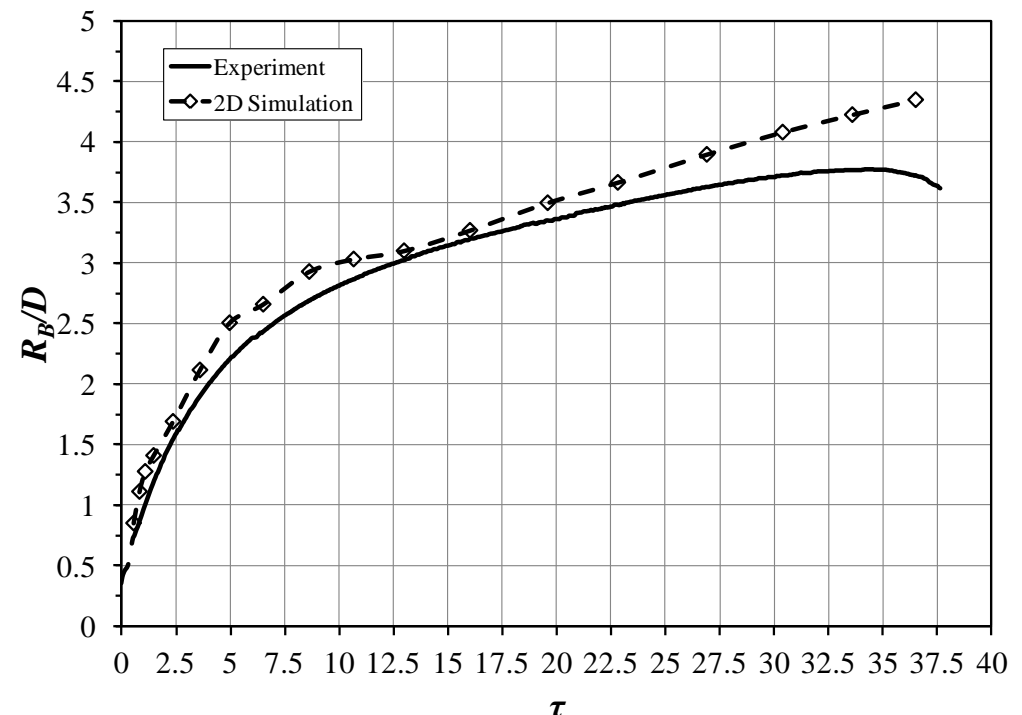

Figure 3.32 Comparison of the dimensionless bottom crown diameter experimental and simulation results with respect to the dimensionless time for Case 3. (Hillen et al. (2012))

\subsubsection{Sub-Cavity Liquid Volume}

This section is primarily based on Kuhlman, Hillen, Dinc and Gray (2014). The sub-cavity liquid volume variation with respect to both radius and time was plotted and compared with experiments for Cases 1, 2 and 3 in Figures 3.33, 3.34 and 3.35, respectively. The CFD results match with the experiments during initial cavity formation but show an earlier onset of cavity retraction and cavity collapse (Kuhlman et al., 2014). For Case 1, the maximum cavity radius is $12 \mathrm{~mm}$ for CFD, and it is around $15 \mathrm{~mm}$ for experiments. The cavity lifetime is around $70 \mathrm{~ms}$ for CFD, while it is $60 \mathrm{~ms}$ for experiments. The maximum cavity radius for Case 2 is $5 \mathrm{~mm}$, and the cavity lifetime is $25 \mathrm{~ms}$ for both experiments and CFD. For Case 3, the maximum cavity radius is $12 \mathrm{~mm}$ and the lifetime is around $80 \mathrm{~ms}$ for CFD. The Case 3 cavity radius is $10 \mathrm{~mm}$ and the lifetime is around $80 \mathrm{~ms}$ in the experiments. The discrepancy between CFD and experiments can be attributed to the axisymmetric modeling in CFD compared to the three dimensionality of the real flow. For Cases 1 and 3 secondary drops are observed. Since the axisymmetric model calculates these secondary drops as liquid rings, the amount of volume that remains in the subcavity liquid and the lifetime and radius of the cavity could be different from experiments. Thus, full 3D simulations, especially for the higher Weber and Reynolds number cases, should be more 
accurate for cavity characteristics such as radius, lifetime and sub-cavity liquid volume. In conclusion, 2D axisymmetric simulations can be used for cases with Weber and Reynolds numbers for which no late splashing formation occurs.
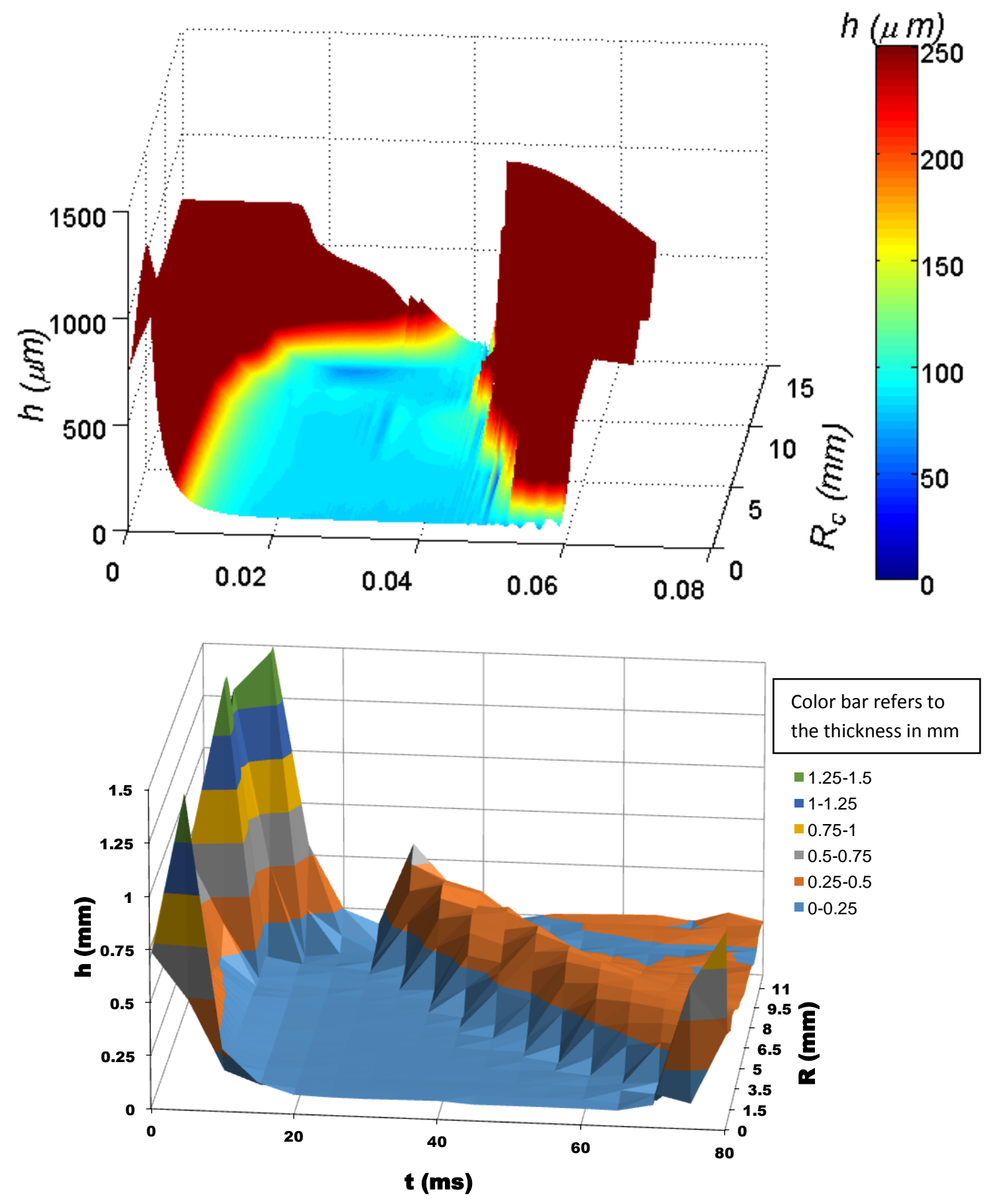

Figure 3.33 Comparison of film thickness variation with time and radius for experiments (top) and CFD (bottom) for Case 1 (Kuhlman et al., 2014). 

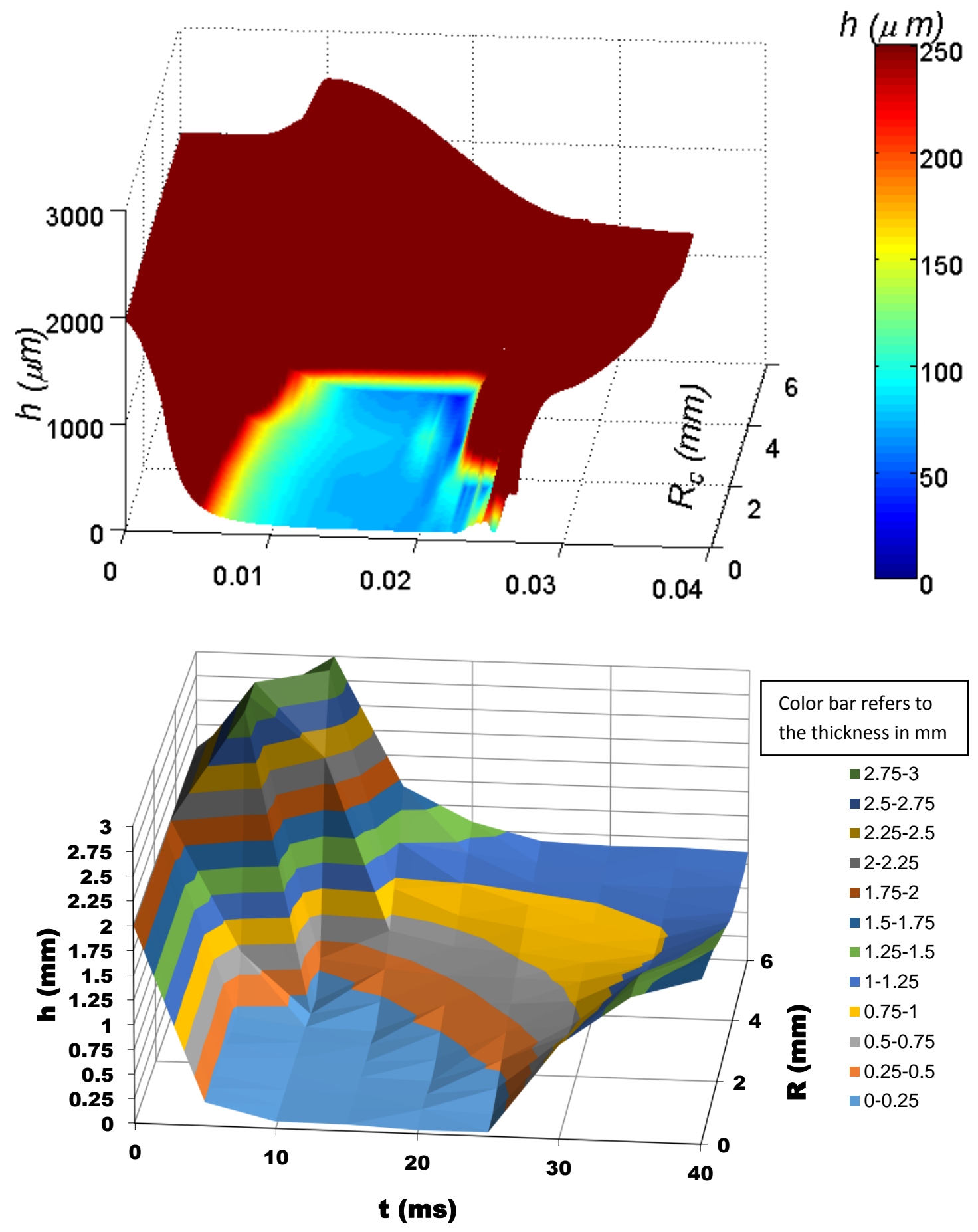

Figure 3.34 Comparison of film thickness variation with time and radius for experiments (top) and CFD (bottom) for Case 2 (Kuhlman et al., 2014). 

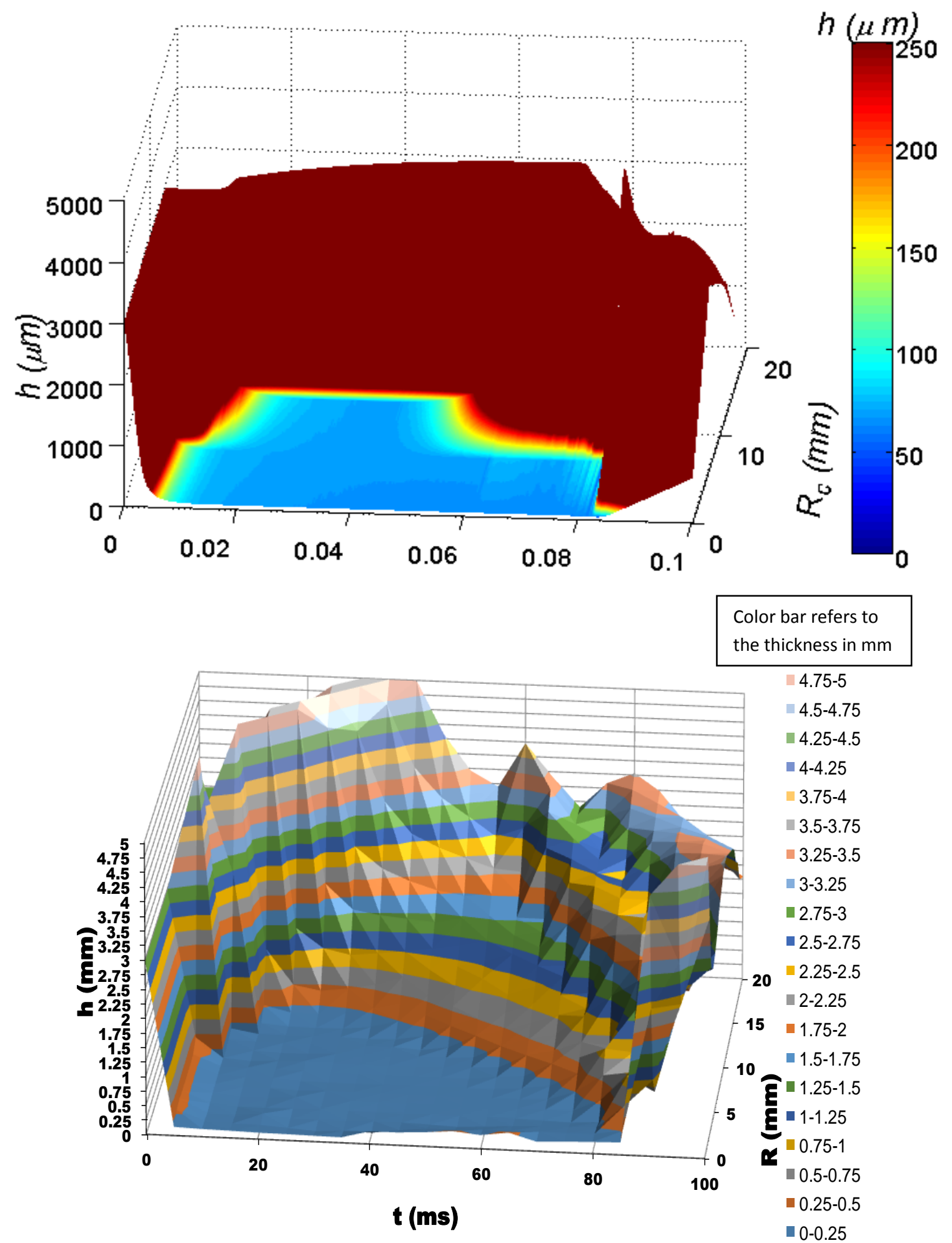

Figure 3.35 Comparison of film thickness variation with time and radius for experiments (top) and CFD (bottom) for Case 3 (Kuhlman et al., 2014). 


\subsection{Effects of Varying Gravity on Single Drop Impact Characteristics}

This section is primarily based on Dinc and Gray $(2012,2013)$. Future spacecraft, satellites, and landers may incorporate electronic packages that will require advanced spray cooling systems that can operate in a wide range of gravity fields. This is the motivation for studying the effects of a range of gravity $(g)$ values. Also, since the initial cavity formation phase is dominated by inertial and viscous effects, gravitational effects have not been previously studied to analyze the effects on the cavity retraction phase. The computational simulations reported here were obtained using the commercial Computational Fluid Dynamics (CFD) code ANSYS Fluent 14 and 14.5 running on a desktop quad core workstation. The Navier-Stokes and continuity equations were solved in 2D axisymmetric coordinates using the finite volume method for unsteady, incompressible, isothermal and laminar flow. The Volume of Fluid (VOF) multiphase model was implemented with three phases (drop liquid, film liquid, and air) to track the interface and distinguish the drop liquid from the liquid in the pre-existing film.

Since the aim of this study was the investigation of gravity for a single water drop impingement onto a liquid film, only the magnitude of $g$ was varied. For all of these cases the liquid was water, $D=4.48 \mathrm{~mm}, h=3.75 \mathrm{~mm}$, and $U=1.5 \mathrm{~m} / \mathrm{s}$ with relatively low $R e=6690$, relatively low $W e=137, h / D=0.837, \theta=0^{\circ}$ where $\theta$ is the contact angle between the liquid and surface. Therefore, $W e, R e$, and $h / D$ were the same for all simulations performed, whereas $F r$ varied. In all cases, the general sequence of events is that the drop impact creates a growing crater surrounded by an elevated crown. The crater contains a very thin liquid film with thickness equal to about $2 \%$ of the drop diameter. The crater reaches a maximum radius, begins to refill, and the inrushing liquid creates an incipient Worthington jet (except in the negative Earth gravity case). No splashing was predicted for any of the cases which make the axisymmetric simulations more accurate, as mentioned in Section 3.4.

Simulations were performed for eight upward facing liquid films with $g$ ranging from that of the Sun $\left(275 \mathrm{~m} / \mathrm{s}^{2}\right)$ to that of the $500 \mathrm{~m}$ diameter asteroid 1999RQ-36 $\left(0.038 \mathrm{~m} / \mathrm{s}^{2}\right)$. Solar gravity is an upper bound for applications in this stellar system and dramatizes the effects of very high $g$ values. Asteroid 1999RQ-36 is of interest because it approaches Earth every six years and is a likely target for a sample return mission (Spacedaily.com, 1999). Case 9 was for zero 
gravity, and Case 10 was for an upward moving drop hitting a downward facing film in Earth gravity.

Table 3.3 displays the values of $g$ and the dimensionless parameters for these simulations. It is seen that $F r$ was the only dimensionless number which varied. In all these cases, the contact angle was set to $0^{\circ}$, which had no effect on the results because for these cases the wall is always wet.

Table 3.3 Parameters used in gravity simulations.

\begin{tabular}{|c|c|c|c|c|c|c|}
\hline Case & Name & $\begin{array}{c}\text { Gravity } \\
\left(\mathbf{m} / \mathbf{s}^{\mathbf{2}}\right)\end{array}$ & $\boldsymbol{R e}$ & $\boldsymbol{W e}$ & $\boldsymbol{F r}$ & $\boldsymbol{h} \mathbf{}$ \\
\hline 1 & Sun & 275 & 6690 & 139 & 1.83 & 0.837 \\
\hline 2 & Jupiter & 24.8 & 6690 & 139 & 20 & 0.837 \\
\hline 3 & Earth & 9.81 & 6690 & 139 & 51 & 0.837 \\
\hline 4 & Mars & 3.7 & 6690 & 139 & 136 & 0.837 \\
\hline 5 & Moon & 1.68 & 6690 & 139 & 310 & 0.837 \\
\hline 6 & $\begin{array}{c}\text { dwarf planet } \\
\text { Pluto }\end{array}$ & 0.61 & 6690 & 139 & 823 & 0.837 \\
\hline 7 & $\begin{array}{c}\text { asteroid } \\
\text { Vesta }\end{array}$ & 0.252 & 6690 & 139 & 1990 & 0.837 \\
\hline 9 & $\begin{array}{c}\text { asteroid } \\
1999 \text { RQ-36 }\end{array}$ & 0.038 & 6690 & 139 & 13200 & 0.837 \\
\hline 10 & $\begin{array}{c}\text { zero gravity } \\
\text { Earth } \\
\text { gravity }\end{array}$ & -9.81 & 6690 & 139 & -51 & 0.837 \\
\hline
\end{tabular}

Figure 3.36 shows that the dimensionless time $(t U / D)$ at which liquid started to refill the crater increased as $\mathrm{Fr}$ increased, showing that gravity as well as surface tension caused the craters to refill. The role of gravity in the impact process is a new finding. Previous researchers, who considered only cases in Earth gravity, attributed the refilling process to surface tension alone. It is curious that the dimensionless times at which liquid started to refill the crater approximately follow a straight line until Pluto on this semi-log plot. The dimensional times were 9, 26, 34, 42, 45, 49, 49, $51 \mathrm{~ms}$ for Cases 1-8, respectively. Figure 3.37 shows that the dimensionless times at which the Worthington jet reached the initial undisturbed film thickness 
also increased with $F r$. In this plot the approximate linear trend continues until Vesta. The pace of events increased as Fr increased (Fig. 3.36 and 3.37).

Figure 3.38 shows the centerline liquid film thickness, $h_{0}$, as a function of time for Solar, Jupiter and Earth gravity. Figure 3.39 shows similar results for Earth, Mars and Vesta gravity in order to see the effects of lower gravity compared to Earth. The initial decrease in centerline thickness and the minimum thickness are only slightly affected by gravity. In contrast, the maximum height of the Worthington jet is greatly increased by increasing gravity, while the time of maximum height is greatly reduced. In general, the time scales of the flow are faster when the gravity is higher. The trends of faster, more extreme deformations with increasing gravity are confirmed by these results. Of course, in the case of zero gravity (Case 9), the refilling of the crater was accomplished through surface tension alone. The zero gravity simulation was discontinued before the formation of a Worthington jet could occur. The faster rate of evolution produced by higher $g$ is illustrated by the well-defined Worthington jet at $13 \mathrm{~ms}$ in Solar gravity (Fig. 3.40) whereas the jet has barely begun to form at $45 \mathrm{~ms}$ in Mars gravity (Fig. 3.41). It should be re-emphasized that splashing was not observed in Cases 1-9.

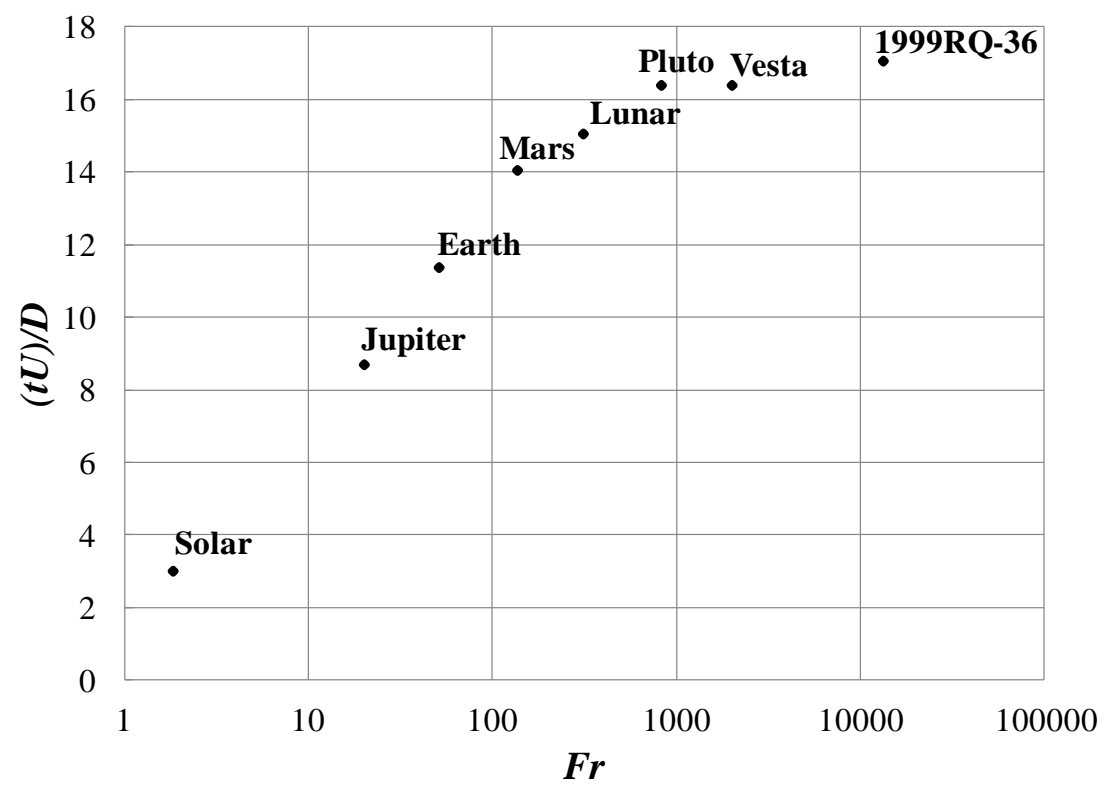

Figure 3.36 The effect of $\mathrm{Fr}$ on the time at which the crater started to refill. 


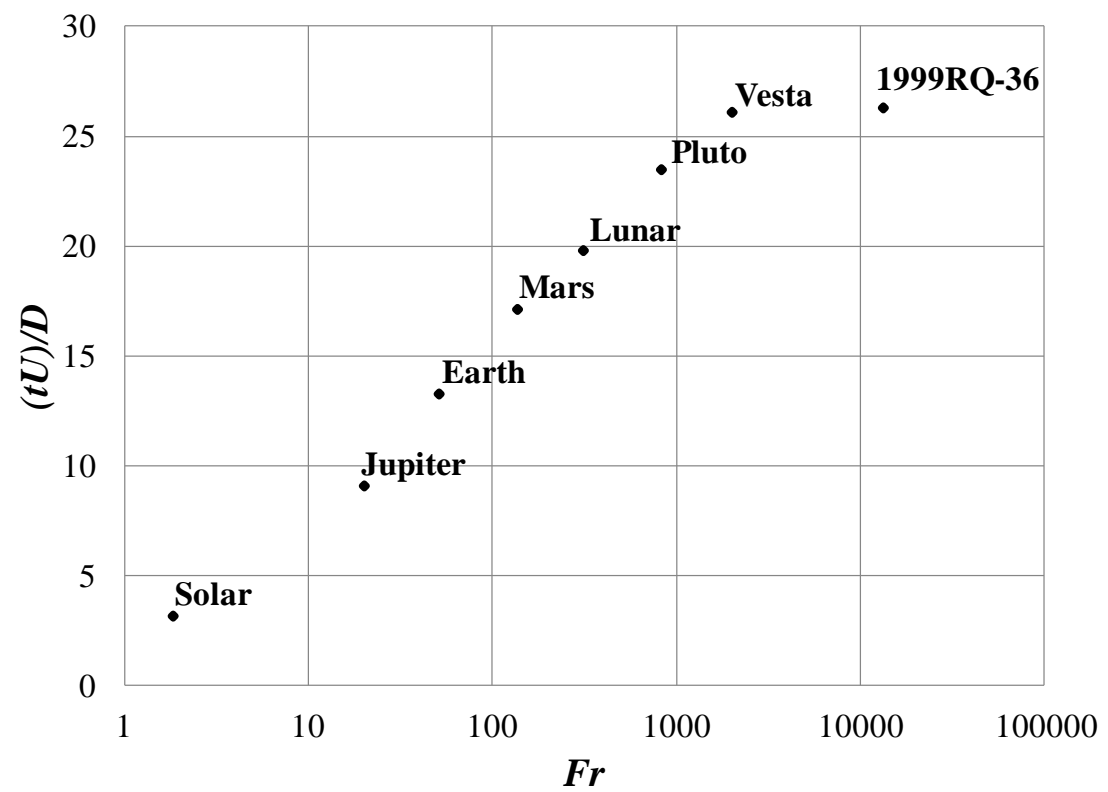

Figure 3.37 The effect of $\mathrm{Fr}$ on the time at which the Worthington jet reached the undisturbed film thickness.

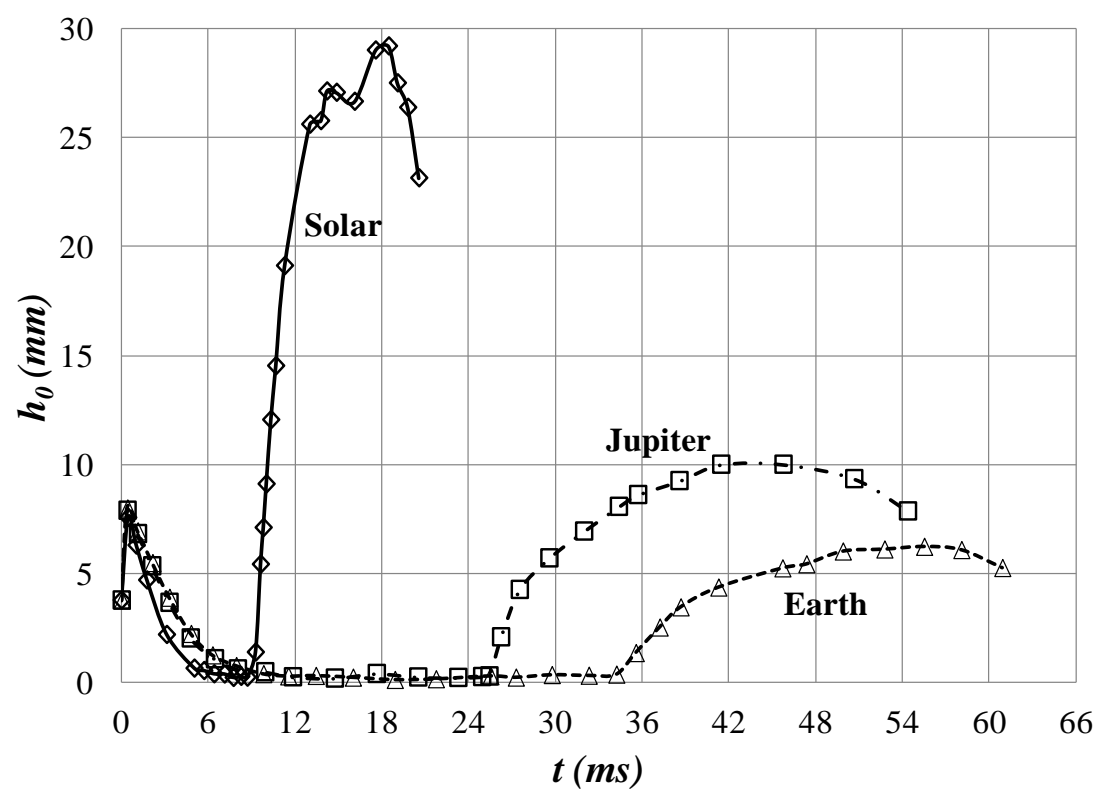

Figure 3.38 Centerline liquid film thickness, $h_{0}$, as a function of time for Solar, Jupiter and Earth gravity. 


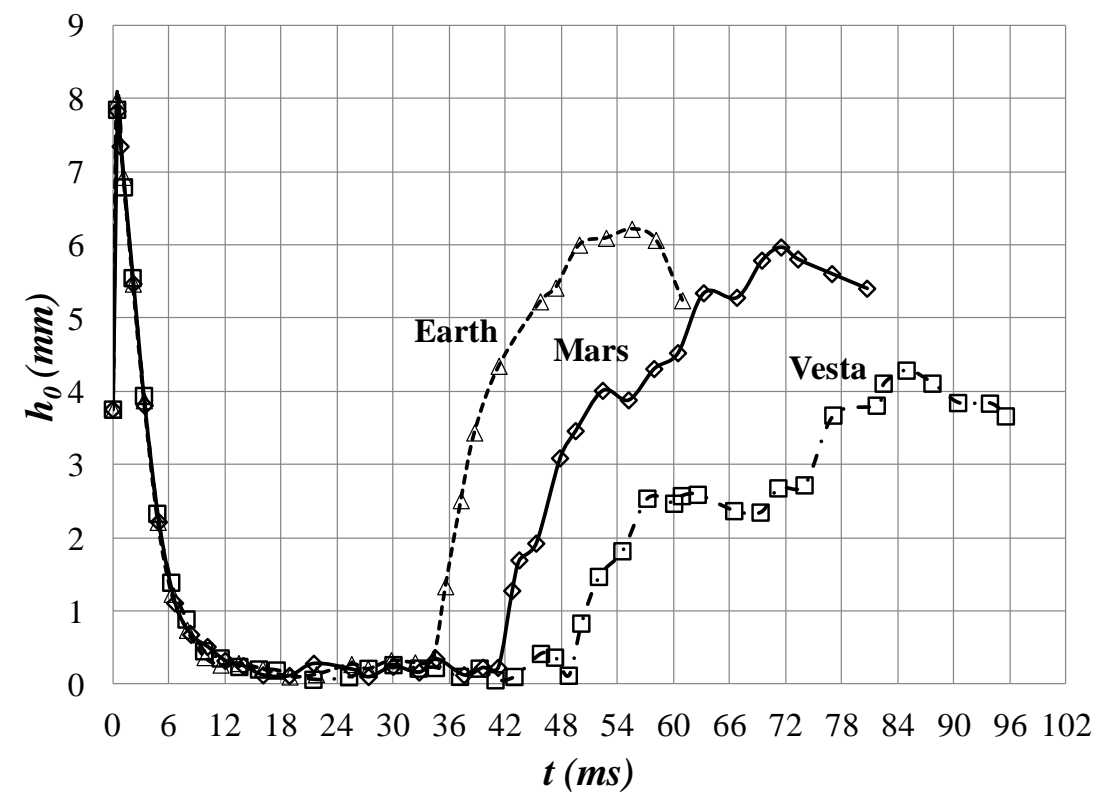

Figure 3.39 Centerline liquid film thickness, $h_{0}$, as a function of time for Earth, Mars and Vesta gravity.

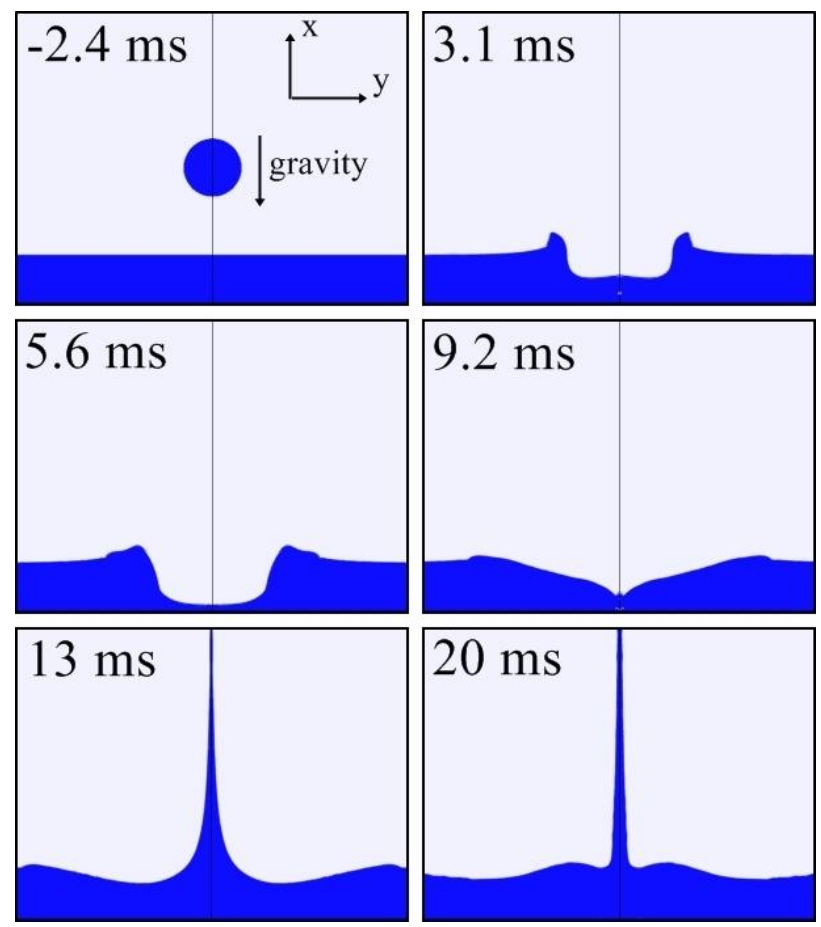

Figure 3.40 Case 1: single drop impact onto a wet surface in Solar gravity. (Note: blue is water and white is air. "ms" refers to milliseconds. Each image is cropped in order to zoom in on the impact and crater region.) 

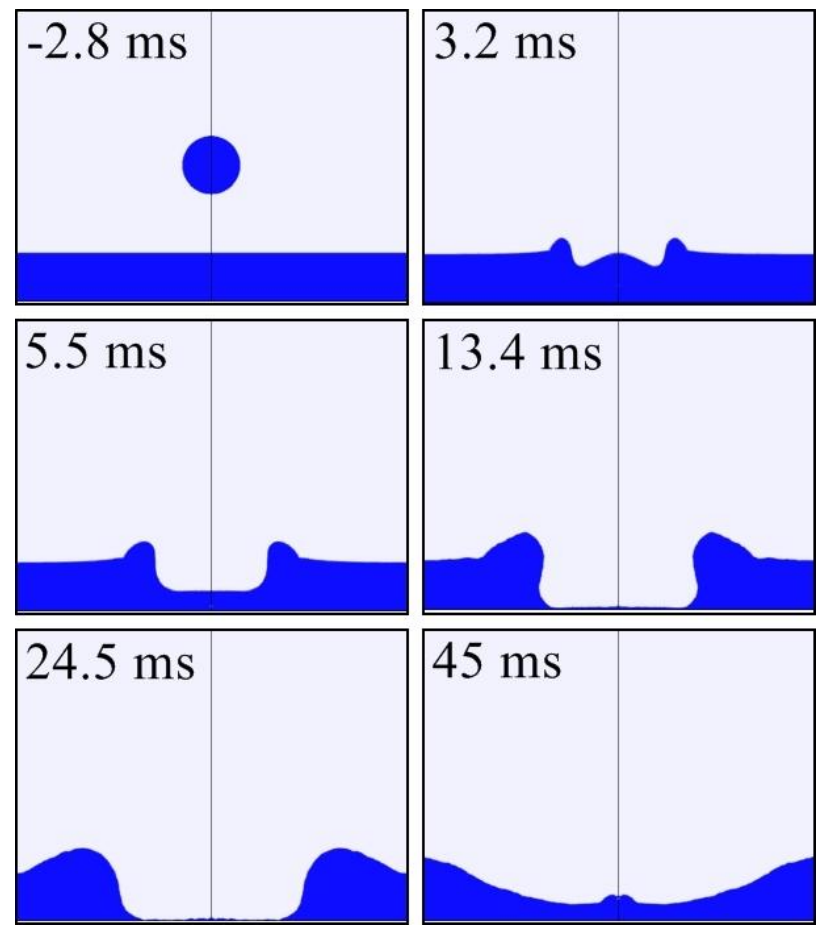

Figure 3.41 Case 4: single drop impact onto a wet surface in Mars gravity. (Note: blue is water and white is air. "ms" refers to milliseconds.)

The "negative gravity" flow of an upward moving drop hitting a downward facing liquid film in Earth surface gravity (Case 10, Fr = -51) is shown in Fig. 3.42. There was still no splashing, but the impact excited a Rayleigh-Taylor instability in which the crown of the impact crater grew large as it fell away from the surface. The crater continued to spread until most of the liquid fell from the surface (Fig. 3.43). 


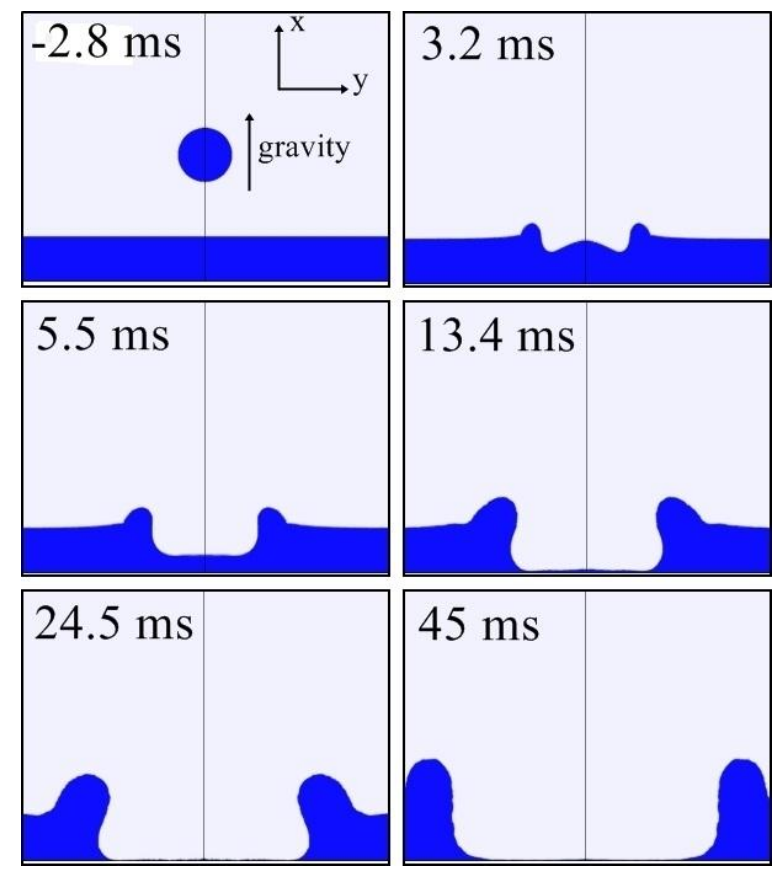

Figure 3.42 Case 10: single drop impact onto a wet surface in "negative Earth gravity". The ceiling is at the bottom of this figure. (Note: blue is water and white is air. "ms" refers to milliseconds.)

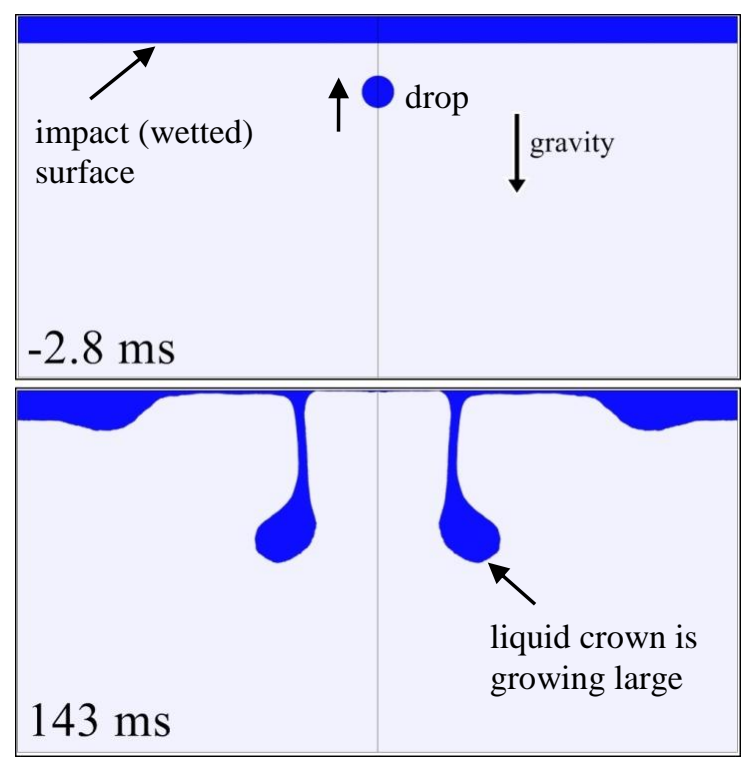

Figure 3.43 Case 10: liquid crown falling off the surface for upward moving drop hitting the downward facing water film in Earth gravity ("Negative Earth Gravity"). The ceiling is at the top of this figure. (Note: original domain size is shown. Blue is water and white is air. "ms" refers to milliseconds.) 


\subsection{Effects of Drop Shape on Single Drop Impact Characteristics}

In this section, based on Dinc and Gray $(2012,2013)$, the effects of drop shape are presented in detail. High speed videos obtained in the experimental phase of this research project demonstrated that falling drops in the 4-mm size range are seldom truly spherical (Hillen et al, 2012). Instead they quiver about a spherical mean. In order to determine if deviations from sphericity would have a significant effect on the drop impact flow, computations were performed for cylindrical "drops" which represent a much more extreme deviation from a spherical shape than is ever observed in the laboratory. Simulations were performed for single cylinders of water with a diameter $D=4.48 \mathrm{~mm}$ (the same as for the previously described spherical drops) and altitudes $L$ of 4.48, 2.99 and $2.24 \mathrm{~mm}$ impinging onto static water layers with thickness $h=3.75$ $\mathrm{mm}$ in Earth gravity. These altitudes were chosen to create cylinders of equal height, equal volume and mass, and half height, respectively, compared to the Case 3 reference sphere. Table 3.4 shows the parameters for these cases, where $D$ is the length scale used to calculate $R e, W e$, and $F r$. A contact angle of $0^{\circ}$ was specified in all these cases, but did not affect the results.

Table 3.4 Parameters used to study drop shape.

\begin{tabular}{|c|c|c|c|c|c|}
\hline Shape & $\boldsymbol{R e}$ & $\boldsymbol{W e}$ & $\boldsymbol{F r}$ & $\boldsymbol{h} / \boldsymbol{D}$ & $\boldsymbol{L} / \boldsymbol{D}$ \\
\hline $\begin{array}{c}\text { Case 3 } \\
\text { sphere }\end{array}$ & 6690 & 139 & 51 & 0.837 & NA \\
\hline $\begin{array}{c}\text { Equal } \\
\text { altitude } \\
\text { cylinder }\end{array}$ & 6690 & 139 & 51 & 0.837 & 1 \\
\hline $\begin{array}{c}\text { Equal } \\
\text { volume } \\
\text { cylinder }\end{array}$ & 6690 & 139 & 51 & 0.837 & 0.667 \\
\hline $\begin{array}{c}\text { Half } \\
\text { altitude } \\
\text { cylinder }\end{array}$ & 6690 & 139 & 51 & 0.837 & 0.5 \\
\hline
\end{tabular}


Results for the Case 3 sphere and the cylindrical drops are presented in Figs. 3.44-3.48 and in Tables 3.5 and 3.6. Figure 3.44 shows that the liquid interface is nearly identical for the Case 3 spherical drop and the equal volume cylinder, which has altitude $L=2 D / 3$. The only noticeable difference between the two is that the small air bubble trapped by the flat underside of the cylinder at $3.2 \mathrm{~ms}$ is larger than the bubble trapped by the sphere. The more prominent bubble formation under the cylinder may be attributed to the greater difficulty for the air to flow out from beneath the flat bottom of the cylinder. Figure 3.45 shows that there is very little quantitative difference in the time history of the layer thickness on the impact axis, thus confirming the visual impression given by Figure 3.44. Further confirmation is provided by Table 3.5 which shows the dimensionless time at which the impact crater started to refill, and Table 3.6 which shows the dimensionless time at which the Worthington jet reached the initial undisturbed free surface thickness. For both times, the difference between the sphere and the equal volume cylinder is less than $0.5 \%$. These comparisons suggest that the much less severe deviations from perfect sphericity which actually occur in the laboratory have insignificant effects on the flows, at least at these values of dimensionless numbers.

The equal altitude and half altitude cylinder impacts are shown in Figs. 3.46 and 3.47, respectively. As the volume of the cylindrical drop increases, the crater spreads to a greater radius, the crown reaches a greater height, and the Worthington jet becomes more prominent. At $45 \mathrm{~ms}$, the Worthington jet is thinnest for the equal altitude cylinder and fattest for the half altitude cylinder while it is very similar to the spherical drop for the equal volume cylinder. The trapped air forms a ring bubble for the half altitude cylinder as seen in Figs. 3.47 and 3.48 (b) while a single "spherical" bubble is formed under the equal altitude cylinder as shown by Figs. 3.46 and 3.48 (a). In the case of the half altitude cylinder, a secondary drop is also observed on the centerline at $14.2 \mathrm{~ms}$. Tables 3.5 and 3.6 show that the dimensionless time at which the crater begins to refill and the dimensionless time the Worthington jet reaches the elevation of the undisturbed free surface both increase with increasing cylinder volume. Because the differences in shape between a sphere and a cylinder are far more extreme than the differences observed among actual drops in the lab, it can be concluded that it is not necessary to simulate realistic nonspherical drops. Simulations of spherical drops are sufficiently accurate. 

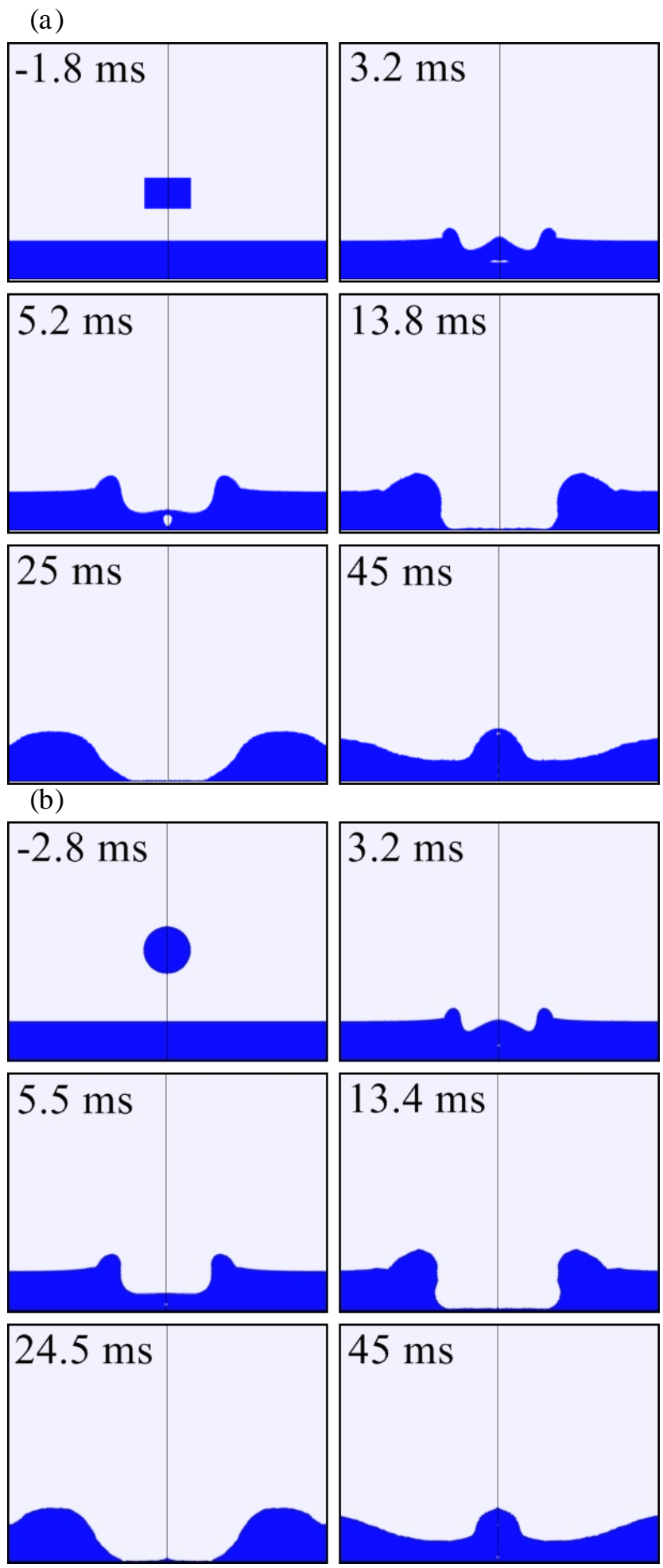

Figure 3.44 Single drop impact onto a wet surface in Earth gravity for: (a) Cylindrical drop with $D=4.48 \mathrm{~mm}, L=2.987 \mathrm{~mm}$ (equal volume cylinder) (b) Case 3 spherical drop with $D=4.48$ $\mathrm{mm}$. 


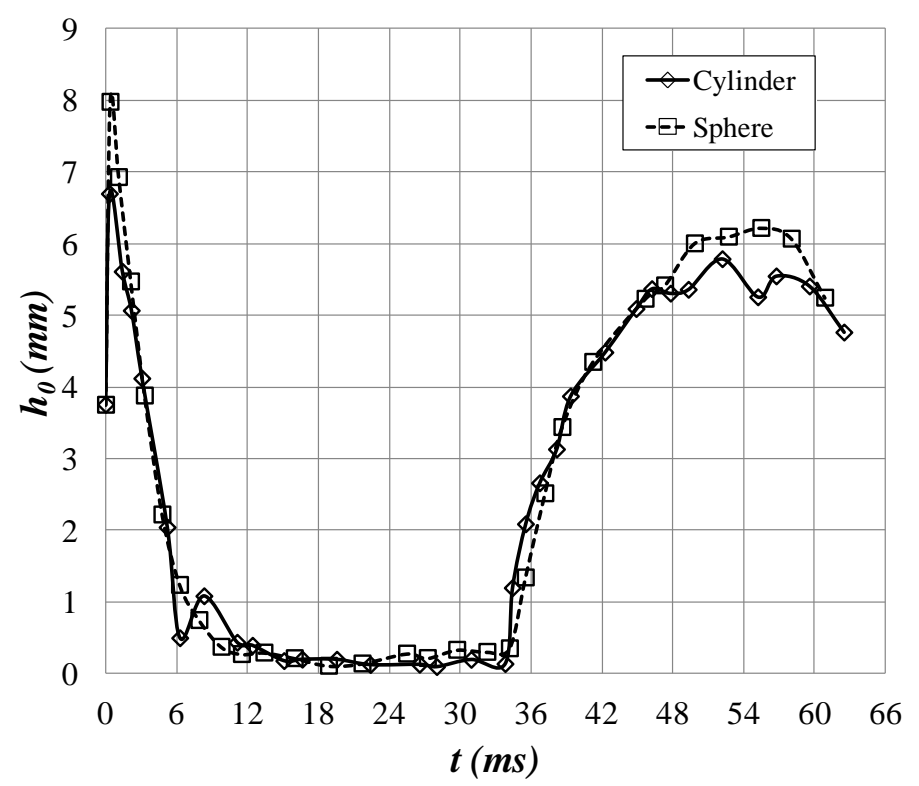

Figure 3.45 Variation of the centerline liquid layer thickness, $h_{0}$, with time for the Case 3 sphere and the cylindrical drop having the same volume.
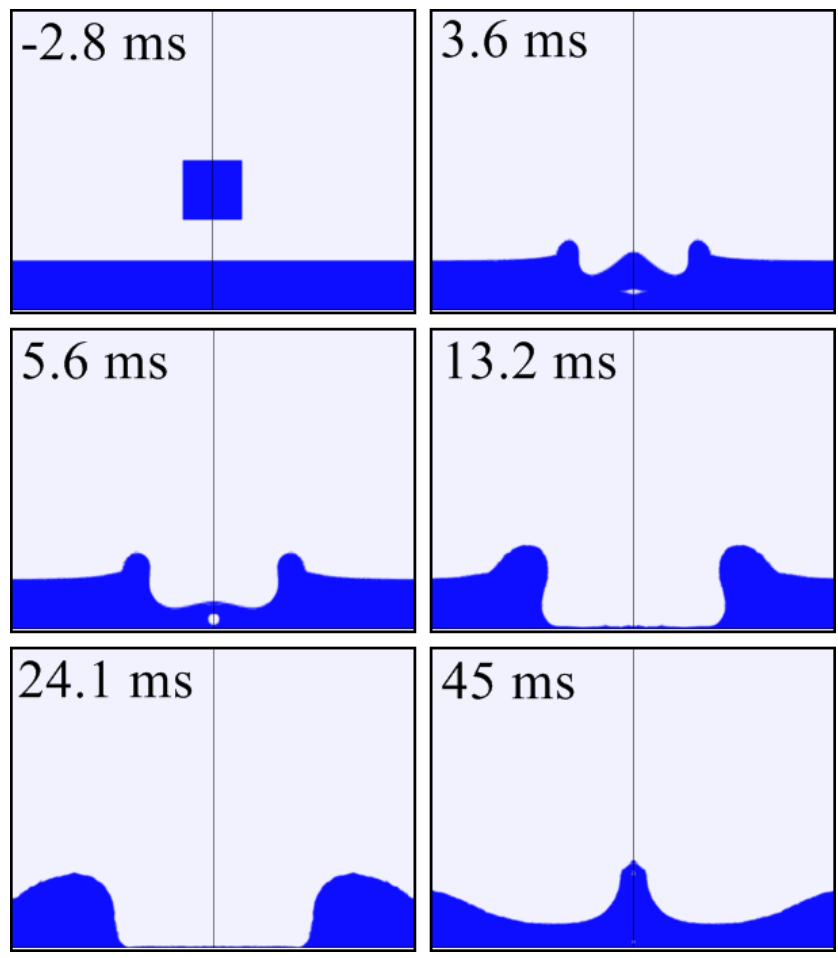

Figure 3.46 Equal altitude cylindrical drop impact onto a wet surface in Earth gravity with $D=$ $4.48 \mathrm{~mm}, L=4.48 \mathrm{~mm}$ (150\% volume of the Case 3 spherical drop). 

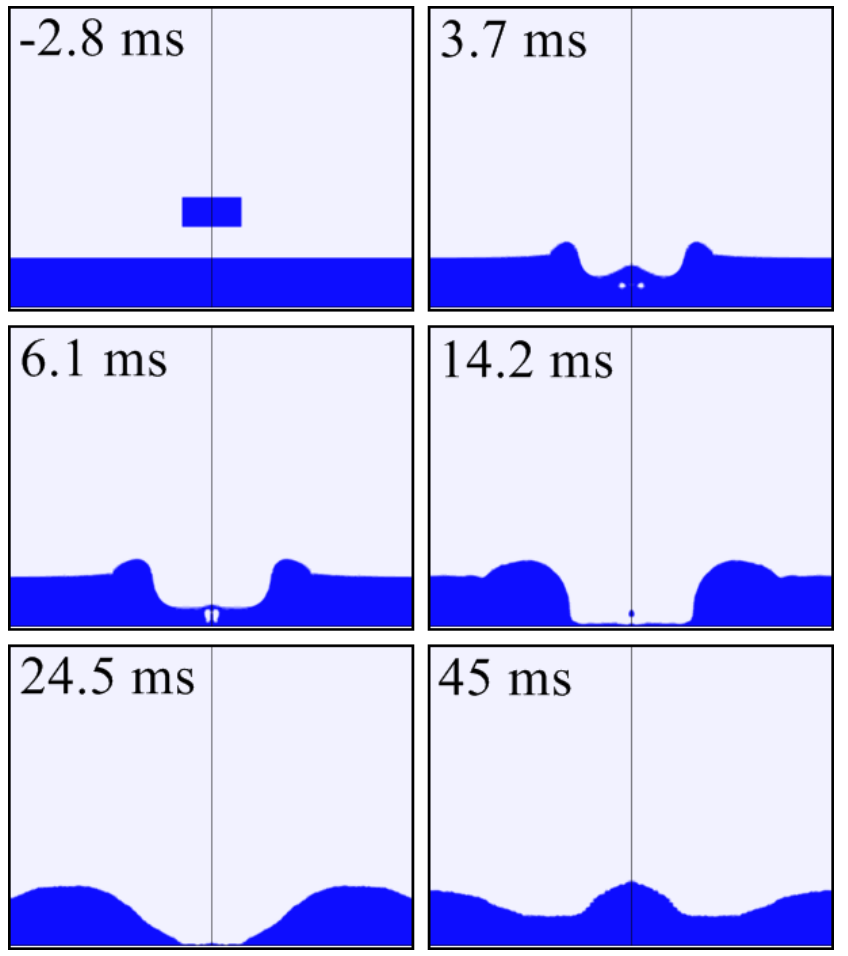

Figure 3.47 Half altitude cylindrical drop impact onto a wet surface in Earth gravity with $D=$ $4.48 \mathrm{~mm}, L=2.24 \mathrm{~mm}$ ( $75 \%$ volume of the Case 3 spherical drop).
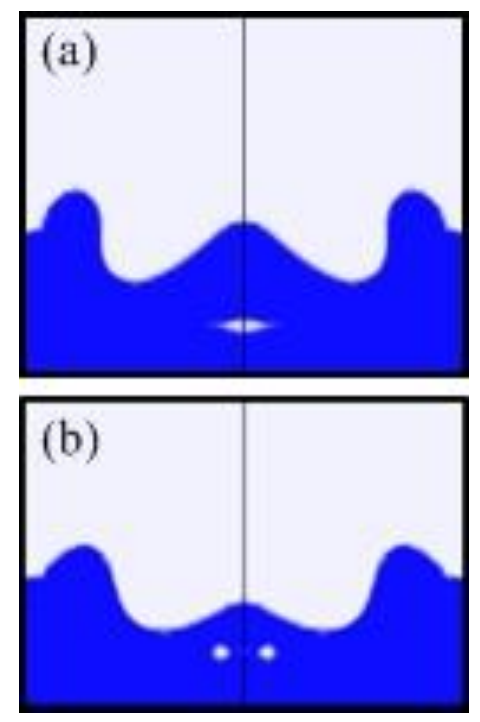

Figure 3.48 Comparison of bubble for (a) Equal altitude cylinder at $t=3.6 \mathrm{~ms}$, (b) Half altitude cylinder at $t=3.7 \mathrm{~ms}$. 
Table 3.5 The effect of drop shape on the time at which the crater started to refill.

\begin{tabular}{|c|c|c|c|}
\hline Shape & $\begin{array}{c}\text { Drop Volume Ratio } \\
\left(V_{\text {cylinder }} / V_{\text {sphere }}\right)\end{array}$ & $\boldsymbol{L} / \boldsymbol{D}$ & $(\boldsymbol{t} \mathrm{U}) / \mathrm{D}$ \\
\hline Case 3 sphere & $\mathrm{NA}$ & $\mathrm{NA}$ & 11.40 \\
\hline Equal altitude cylinder & 1.5 & 1 & 12.77 \\
\hline Equal volume cylinder & 1 & 0.667 & 11.45 \\
\hline Half altitude cylinder & 0.75 & 0.5 & 9.76 \\
\hline
\end{tabular}

Table 3.6 The effect of drop shape on the time at which the Worthington jet reached the undisturbed layer thickness.

\begin{tabular}{|c|c|c|c|}
\hline Shape & $\begin{array}{c}\text { Drop Volume Ratio } \\
\left(\boldsymbol{V}_{\text {cylinder }} / \boldsymbol{V}_{\text {sphere }}\right)\end{array}$ & $\boldsymbol{L} / \boldsymbol{D}$ & $(\boldsymbol{t U}) / \boldsymbol{D}$ \\
\hline Case 3 sphere & NA & NA & 13.29 \\
\hline Equal altitude cylinder & 1.5 & 1 & 13.61 \\
\hline Equal volume cylinder & 1 & 0.666 & 13.27 \\
\hline Half altitude cylinder & 0.75 & 0.5 & 11.83 \\
\hline
\end{tabular}




\section{CHAPTER 4: SPRAY IMPACT SIMULATIONS USING THE DISCRETE PHASE MODEL (DPM)}

In this chapter simulations of spray impingements on unheated surfaces will be discussed. The studies which are presented in this chapter are primarily based on Dinc, Gray, Hillen, Taylor and Kuhlman (2013). The methods used to perform these spray simulations will be explained in detail. These simulations were performed using the Discrete Phase Model (DPM) in ANSYS Fluent 14 on a quad-core desktop computer. The DPM is a Lagrangian-Eulerian based multiphase model used for the calculation of dispersed phase flows. In the DPM, a large number of particle trajectories are calculated in the Lagrangian formulation while the continuous phase is calculated by standard Eulerian conservation equations. Spray simulations have been performed for both full $3 \mathrm{D}$ and $2 \mathrm{D}$ axisymmetric computational domains to investigate the effects of nozzle-to-surface distance and spray half angle on spray impact at isothermal conditions. The 2D axisymmetric and 3D results have been compared for the primary spray characteristics (drop diameter, velocity, number of total drops etc.) and the wall film characteristics (liquid film mass, liquid film height, average liquid film velocity magnitude, etc.). The 2D axisymmetric simulations are far less time consuming, yet they capture many essential details of the spray and wall film characteristics for the single full-cone and hollow cone nozzles. Further 2D axisymmetric simulations have been performed to analyze spray characteristics of a full cone nozzle based on the experiments performed at WVU. Unfortunately, the centerline depth predictions of the 2D axisymmetric model for the full cone spray are unrealistic at later simulation times as discussed in Section 4.5. In this chapter and in the other chapters, the variables and Case numbers are defined separately. Thus, the symbols shown in this chapter are often not the same as the symbols shown in the other chapters.

\subsection{Numerical Modeling and Governing Equations}

The Discrete Phase Model (DPM) is a Lagrangian-Eulerian based modeling method. In order to obtain more accurate results from the DPM model, the volume fraction of the discrete phase (the second phase in ANSYS Fluent) should be less than $10 \%$ of the continuous phase (which ANSYS Fluent considers the first phase). The particle phase is defined as a dense phase if its volume fraction is more than $10 \%$, otherwise the particle phase is defined as a dilute phase. 
However, using the calculation method given by Crowe et al. (1998), the type of flow can be determined more precisely. In this method, the spray flow regime is calculated using the ratio of the momentum response time of a particle to the time between particle collisions (Crowe et al., 1998). The momentum response time, $\tau_{V}$, is a measure of the time required for a droplet to respond to an abrupt change in velocity. The momentum response time, $\tau_{V}$, is defined as

$$
\tau_{V}=\frac{\rho_{d} D^{2}}{18 \mu}
$$

where $\rho_{d}$ is the particle density (mass of a particle/volume of the particle), $D$ is the particle diameter, $\mu$ is the air viscosity.

The time between particle collisions, $\tau_{c}$, is the average time between particle-particle collisions and it is defined as

$$
\tau_{c}=\frac{1}{n \pi D^{2} V_{r}}
$$

where $n$ is the number density of particles, $V_{r}$ is the relative velocity between particles.

The ratio of the momentum response time to the time between particle collisions is then

$$
\frac{\tau_{v}}{\tau_{c}}=\frac{n \pi \rho_{d} D^{4} V_{r}}{18 \mu}
$$

Noting that the product of the number density $(n)$ and the mass $(m)$ of an individual particle is equal to the bulk density of the particles $\left(\overline{\rho_{d}}=\right.$ the mass of the dispersed phase per unit volume of mixture) allows equation 4.3 to written as

$$
\frac{\tau_{v}}{\tau_{c}}=\frac{\overline{\rho_{d}} D V_{r}}{3 \mu}
$$

The bulk density of particles is also equal to the product of the particle volume fraction $\left(\alpha_{d}\right)$ and the particle density $\left(\rho_{d}\right)$. Thus, the above ratio becomes 


$$
\frac{\tau_{v}}{\tau_{c}}=\frac{\alpha_{d} \rho_{d} D V_{r}}{3 \mu}
$$

It is assumed that the flow regime is dense if

$$
\frac{\tau_{v}}{\tau_{c}}>1
$$

and that the flow regime will be dilute if

$$
\frac{\tau_{v}}{\tau_{c}}<1
$$

Figure 4.1 shows an example calculation of the regime for a water spray with 100 micron diameter drops at room temperature with the surrounding gas being air at room temperature. In this plot, the particle volume fraction (the volume fraction of liquid drops) was calculated with respect to the particle relative velocity (the difference between liquid drop and air velocity). The region above the curve is dense flow and the region below the curve is dilute (discrete) flow.

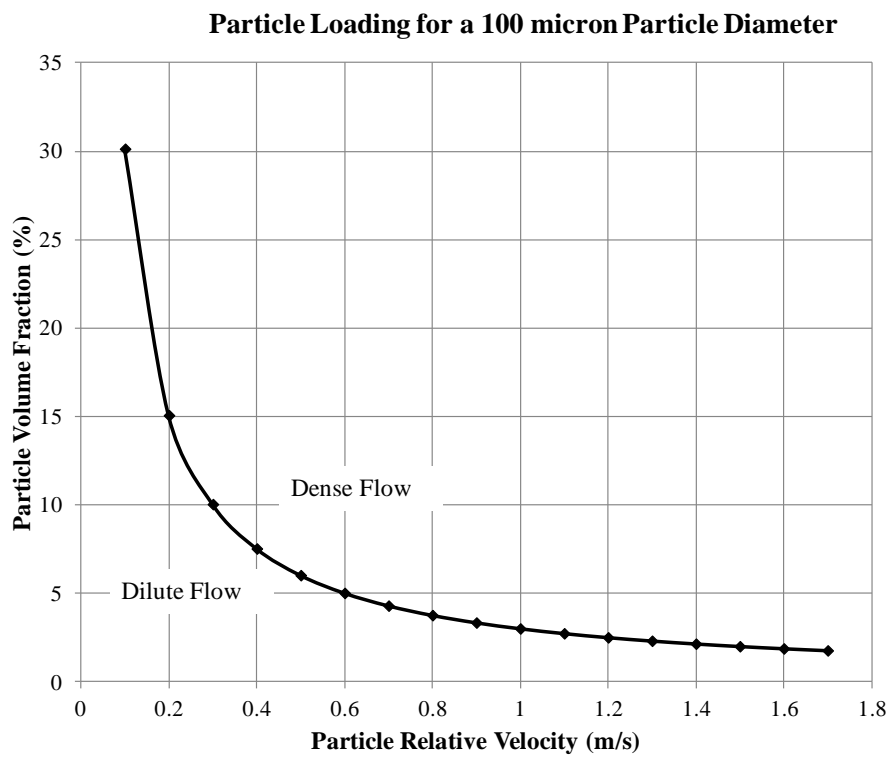

Figure 4.1 A flow regime map for a spray with 100 micron droplets. 
Figure 4.2 shows a full cone spray image obtained from a high speed camera (Kreitzer and Kuhlman, 2010). The relative particle velocity between drops in a spray is small and drops are separated with a long distance from each other especially closer to the impact surface (Fig. 4.2).

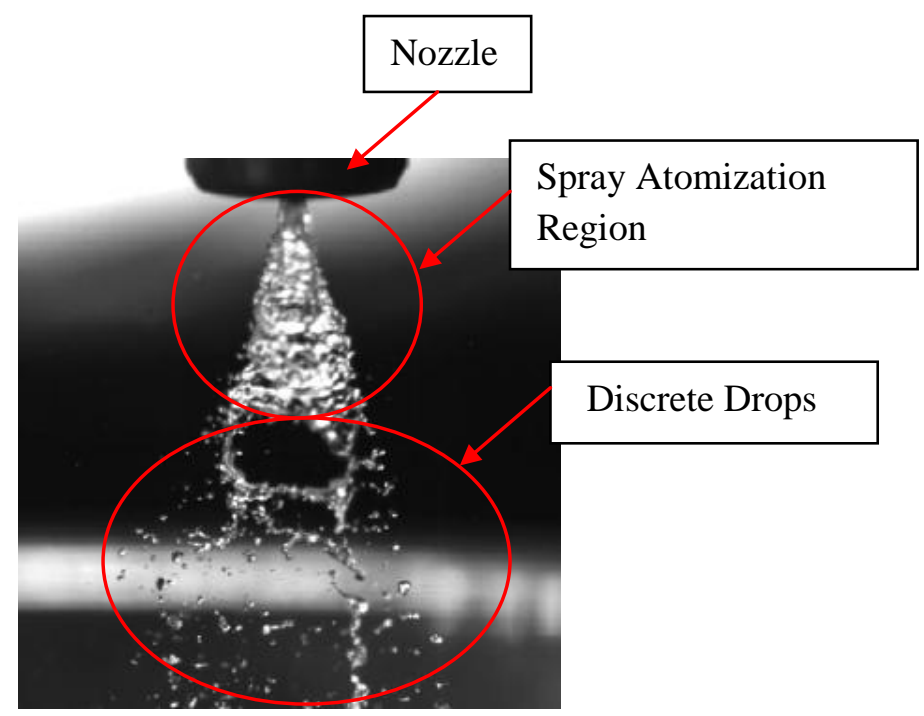

Figure 4.2 A high speed video image that shows a full cone spray formation for a nozzle (Kreitzer and Kuhlman, 2010).

The DPM includes two different phases: the continuum phase and the discrete phase. The continuum phase (e.g. air) is phase 1, and it has a high volume fraction in a spray compared to the second phase. Phase 2 is the discrete phase, and it contains a number of particles (e.g. spray drops). Particle-particle interactions and the effects of the particle volume fraction on the continuous phase were not considered in the DPM. The DPM also includes a submodel for the calculation of the spray-wall interactions called the DPM wall film model. Considering the high velocity of sprays after being injected from the nozzle, a k-epsilon turbulence model was implemented for the surrounding gas which was air at atmospheric pressure and room temperature $\left(25^{\circ} \mathrm{C}\right)$. The spray liquid was water at room temperature.

Turbulent air flow calculations were simulated numerically by solving the incompressible continuity and Navier-Stokes Equations given as

$$
\nabla \cdot(\underline{U})=S_{m}
$$




$$
\rho_{a}\left(\frac{\partial(\underline{U})}{\partial t}+\underline{U} \cdot \nabla \underline{U}\right)=-\nabla P+\mu_{a} \nabla^{2} \underline{U}+\underline{F}_{B}+\rho_{a} \underline{g}
$$

Here $S_{m}$ is the source term which includes the mass added to air from spray drops by evaporation phase change, $\underline{U}$ is the mean velocity vector, $\rho_{a}$ is the density of air, $\mu_{a}$ is the dynamic viscosity of air, $\underline{g}$ is the gravity vector, $P$ is the pressure, $\underline{F}_{B}$ is the body force.

In the Standard k-epsilon model in ANSYS Fluent, turbulence length and time scales are determined by solving the two separate transport equations for turbulence kinetic energy $(k)$ and turbulence dissipation rate $(\varepsilon)$. The Realizable k-epsilon turbulence model was developed by Shih et al. (1995). The Realizable k-epsilon model was developed to eliminate the poor performance of the Standard k-epsilon model in the calculation of the spreading rate of an axisymmetric jet (ANSYS Fluent Theory Guide, 2013). It has the same transport equation for the turbulent kinetic energy, $k$, but it has a different transport equation for the turbulent dissipation rate, $\varepsilon$ compared to the Standard k-epsilon model. The Realizable k-epsilon model also accounts for the variable turbulent viscosity coefficient, $C_{\mu}$, which is constant in the Standard k-epsilon model. The turbulent viscosity in the k-epsilon models can be calculated as

$$
\mu_{t}=\rho C_{\mu} \frac{k^{2}}{\varepsilon}
$$

where $C_{\mu}$ is a model constant in the Standard k-epsilon model but is a variable coefficient depending on the mean strain and rotation rates in the Realizable k-epsilon model (ANSYS Fluent Theory Guide, 2013). This is the main difference between the Standard and the Realizable k-epsilon models. Both the Standard and the Realizable k-epsilon models were analyzed for sprays. However, it was decided to use the Realizable k-epsilon turbulence model for the rest of the spray simulations.

\subsubsection{Calculation of the Particle Motion}

Particle motion in the DPM is calculated by using the particle equation of motion based on Newton's Second Law of motion (ANSYS Fluent Theory Guide, 2013). This equation is solved for each parcel which represents a number of particles. 


$$
m_{p} \frac{d U_{p}}{d t}=\underline{F}_{D}+\underline{F}_{\text {virtual force }}+\underline{F}_{\text {gravity }}
$$

where $m_{p}$ is the particle mass

$U_{p}$ is the particle velocity

$\underline{F}_{D}$ is the drag force

$\underline{F}_{\text {virtual force }}$ is the force required to accelerate the fluid surrounding the particle.

$\underline{F}_{\text {gravity }}$ is the gravity force.

The virtual force is important when $\rho_{a}>\rho_{p}$. In sprays this is not true so the virtual force is neglected.

The drag force acting on each particle is calculated as

$$
\underline{F_{D}}=\frac{1}{2} C_{d} \rho_{a} \underline{U_{r}^{2}} A_{p}
$$

where $C_{d}$ is the drag coefficient, $\rho_{a}$ is the air density, $A_{p}$ is the particle cross sectional area and $\underline{U_{r}}$ is the relative velocity between drop and air which is defined as

$$
\underline{U_{r}}=\underline{U_{a}}-\underline{U_{p}}
$$

where $\underline{U_{a}}$ is the air velocity and $\underline{U_{p}}$ is the particle velocity as mentioned above.

The gravity force, $F_{\text {gravity }}$, is calculated using

$$
\underline{F}_{\text {gravity }}=m_{p} \underline{g}
$$

Finally, equation 4.10 can be reduced to (King et al., 1987)

$$
m_{p} \frac{d U_{p}}{d t}=\frac{1}{2} C_{d} \rho_{a}\left(\underline{U_{a}}-\underline{U_{p}}\right)^{2} A+m_{p} \underline{g}
$$

The drag coefficient, $C_{d}$, was assumed to be 0.44 for a spherical particle and fully turbulent flow where $R e_{r}>10^{3}$ (Hinds, 1999). Relative Reynolds Number is calculated using 


$$
\operatorname{Re}_{r}=\frac{\rho_{a} D\left|\underline{U_{p}}-\underline{U_{a}}\right|}{\mu_{a}}
$$

where $\mu_{a}$ is the dynamic viscosity of air, $D$ is the drop diameter, $\left|\underline{U_{p}}-\underline{U_{a}}\right|$ is $\left|\underline{U_{r}}\right|$ (relative velocity).

\subsubsection{The DPM Wall Film Submodel}

The wall film submodel in the DPM is also based on the Lagrangian approach. The DPM parcels (a parcel consists of a number of spray droplets) are used to model the wall-film. In this submodel, film parcels are assumed to be in direct contact with the wall surface. The momentum equation is solved for each parcel on the wall film. The parcel motion is solved by integrating the force balance equation on the parcel with the integration routine which is used for particle tracking. The film height, velocity, and temperature are computed by solving the conservation equations for mass, momentum, and energy for individual parcels within the wall film. The basic assumptions in the wall film submodel in ANSYS Fluent (ANSYS Fluent Theory Guide, 2013) are

- The film thickness is less than 500 microns

- Film particles are in direct contact with the wall and there is heat conduction from the wall to the film

- The film temperature is limited to the boiling temperature of the liquid

- The model is based on is based on the work of Stanton and Rutland (1996) and O'Rourke and Bracco, (1979).

In this model, there are four regimes that can be defined based on the impact energy and wall temperature: stick, rebound, spread, splash. The impact energy, $E$, is defined as

$$
E^{2}=\frac{\rho_{p} \underline{U}_{r}^{2} D}{\sigma}\left[\frac{1}{\min \left(\frac{h_{0}}{D}, 1\right)+\frac{\delta_{b l}}{D}}\right]
$$

where $\rho_{p}$ is the particle (drop) liquid density

$\sigma$ is the liquid surface tension 
$D$ is the drop diameter

$h_{0}$ is the film thickness

$\underline{U_{r}}$ is the relative particle velocity with respect to the wall $\left(\underline{U_{r}}=\underline{U_{p}}-\underline{U_{w}}\right)$ where $\underline{U_{p}}$ is the particle velocity and $U_{w}$ is the wall velocity which is 0 in the present application)

$\delta_{b l}$ is the boundary layer thickness calculated using

$$
\delta_{b l}=\frac{D}{\sqrt{R e}}
$$

where

$$
R e=\frac{\rho_{a} D\left|\underline{U_{p}}-\underline{U_{w}}\right|}{\mu}
$$

If the wall temperature is less than the liquid boiling temperature, particles may stick to the surface, spread on the surface or splash based on the dimensionless impact energy E. Figure 4.3 summarizes these regimes. If the wall temperature is less than the boiling temperature and the dimensionless impact energy is less than 16, particles will stick to the surface. If $E$ is between 16 and 57.7 the particles will spread. If $E$ is more than 57.7, the particles will splash. If the wall temperature is more than the liquid boiling temperature, particles will either rebound or splash. However, the simulations reported in this chapter are isothermal. Therefore, particles (droplets) will splash, spread on the wall or stick to the wall based on the magnitude of $E$.

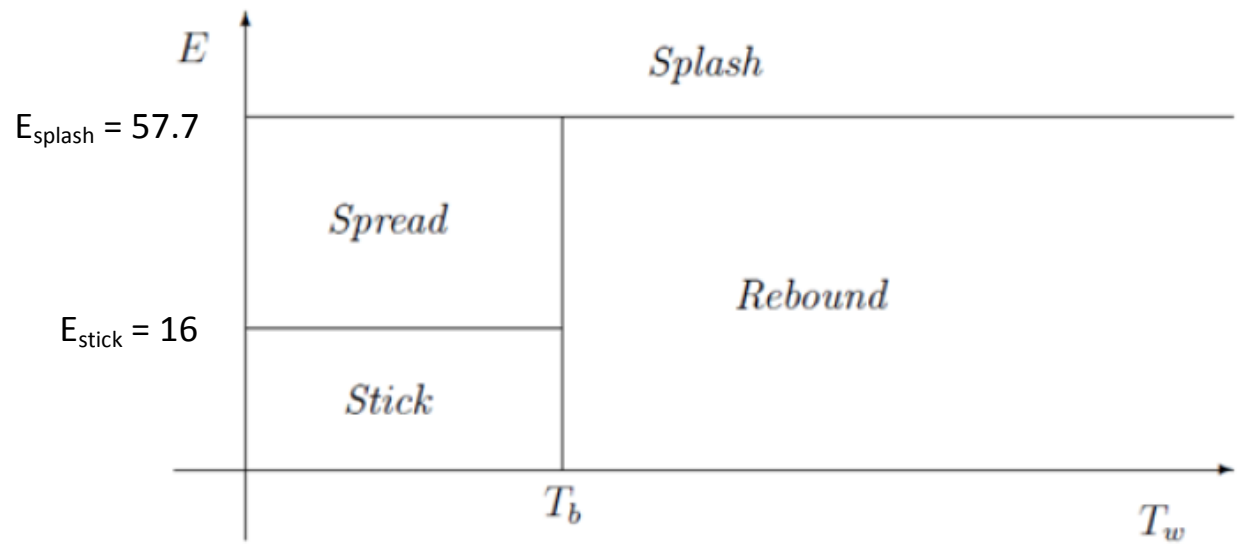

Figure 4.3 Interaction mechanisms during impact with a boundary in the DPM wall film submodel in ANSYS Fluent (ANSYS Fluent Theory Guide, 2013). 


\subsection{Preliminary Simulations of Sprays Impacting Plane Surfaces Using the Discrete Phase Model (DPM)}

The Discrete Phase Model (DPM) was used to model sprays in a full three dimensional (3D) cylindrical domain shown in Fig. 4.4. The 3D computational domain was drawn in the Design Modeler (DM) section of the ANSYS Workbench. After the solid and fluid zones were designed in the DM, meshing and assignment of boundary conditions were achieved in the Meshing module. The top surface of the cylindrical domain was defined as a constant pressure outlet while wall boundary conditions were applied at the side and bottom (spray impact) surfaces. The spray was generated using the pressure-swirl atomizer model which is based on the Linearized Instability Sheet Atomization (LISA) model of Schmidt et al. (1999). The Kelvin-Helmholtz and Rayleigh-Taylor (KHRT) model was used as the secondary droplet break-up model. This model combines two different criteria based on the effects of Kelvin-Helmholtz waves caused by the aerodynamic forces and Rayleigh-Taylor instabilities caused by the acceleration of the drops ejected from the liquid sheet (ANSYS Fluent Theory Guide, 2013).

Two different fully 3D spray cases and two exactly identical 2D axisymmetric cases simulating a pressure swirl nozzle at isothermal conditions were executed in order to determine if the 2D axisymmetric model was sufficiently accurate. The pressure swirl nozzle used in these simulations generates a hollow cone spray. Only full cone, non-swirl nozzles were used in the experimental phase of this research project. Subsequently, different spray parameters were investigated using the 2D axisymmetric full cone nozzle which represents the FullJet 1/8-G full cone spray nozzle that was used in the related experiments. The parameters of the hollow cone 3D cases are shown in Table 4.1. The only parameters that were varied for these two cases are the distance from nozzle tip to the impact surface $(h)$ and the spray half angle $(\theta)$. In this table, $P$ is the gage pressure of nozzle ( $\mathrm{Pa})$ and $M$ is the total spray mass flow rate $(\mathrm{kg} / \mathrm{s})$. 

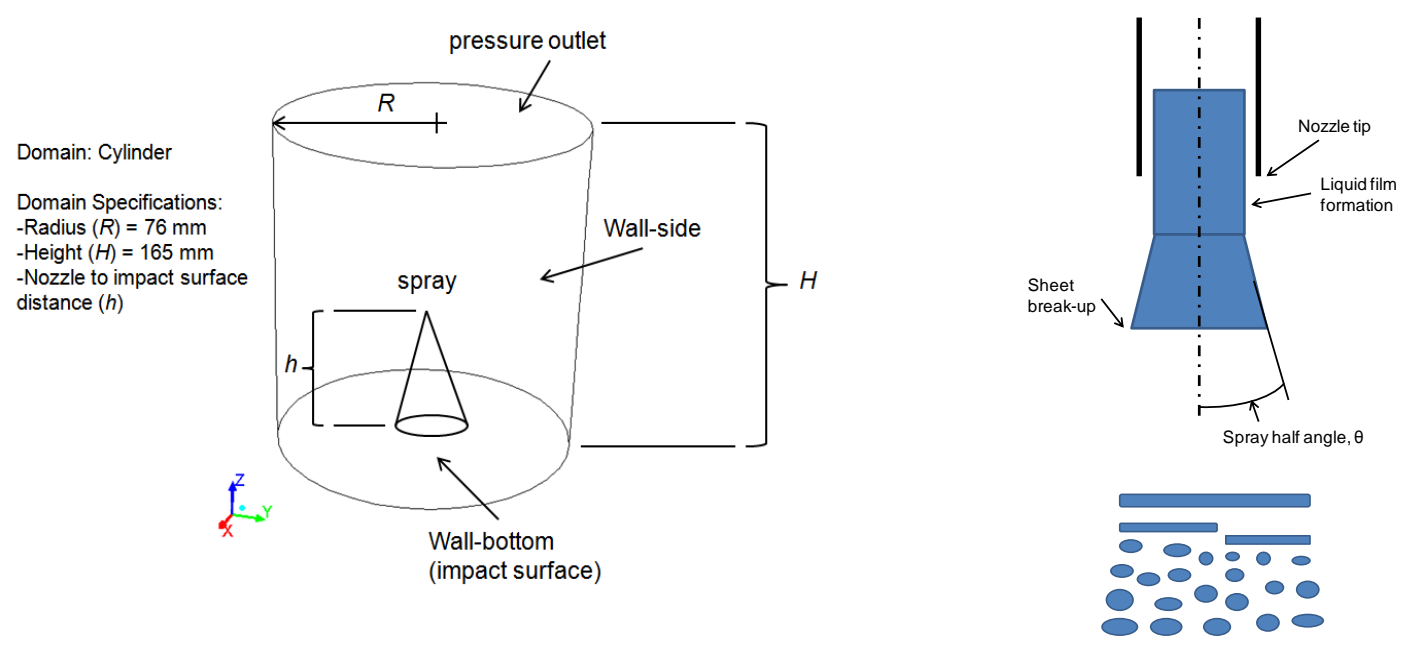

Figure 4.4 3D computational domain, domain specifications, boundary conditions and spray half angle $(\theta)$.

Table 4.1 Parameters of the hollow cone spray simulations.

\begin{tabular}{|c|c|c|c|c|c|}
\hline Case & Liquid & $\boldsymbol{P}(\mathbf{P a})$ & $\boldsymbol{M}(\mathbf{k g} / \mathbf{s})$ & $\boldsymbol{h}(\mathbf{m m})$ & $\boldsymbol{\theta}$ \\
\hline 1 & Water & $10^{6}$ & 0.003 & 40 & 10 \\
\hline 2 & Water & $10^{6}$ & 0.003 & 35 & 18 \\
\hline
\end{tabular}

\subsubsection{Results of the Preliminary Full 3D Hollow Cone Spray Simulations}

Results for the two cases specified in Table 4.1 are presented in Table 4.2 and Figs. 4.5-4.13. Simulations were run for $7.5 \mathrm{~ms}$ because these full 3D computations required approximately 1 month of run time using the available desktop workstation with Quad core processors and 24 GB memory. Table 4.2 shows the spray characteristics at $5 \mathrm{~ms}$ for the two cases considered. Figures 4.5 and 4.6 show the spray formation, spray impact, splashing drops, spray velocity distribution and spray drop accumulation on the surface for Cases 1 and 2, respectively. The drop velocity is similar in the two cases, decreasing dramatically as the impact surface is approached. The maximum spray velocity before the initial spray impact is $29.45 \mathrm{~m} / \mathrm{s}$ in the negative $\mathrm{z}$ direction for the Case 1 and $28.65 \mathrm{~m} / \mathrm{s}$ in the negative $\mathrm{z}$ direction for the Case 2. The splashed drops have a positive $z$ velocity for both Case $1(0-3.5 \mathrm{~m} / \mathrm{s})$ and Case $2(0-5 \mathrm{~m} / \mathrm{s})$. This indicates that the splashed drops move in upward direction with an angle based on the incoming drop's impingement angle onto the surface. These results are consistent with the actual experimental 
results (Kreitzer and Kuhlman, 2010). Figures 4.7 and 4.8 display the spatial distribution of drop diameters at $5 \mathrm{~ms}$. Figure 4.9 shows the time variation of the Sauter mean drop diameter $\left(d_{32}\right)$ and the arithmetic mean drop diameter $\left(d_{10}\right)$. Even though the Sauter mean drop diameters are very close, the maximum drop diameters are around $208 \mu \mathrm{m}$ for Case 1 and $334 \mu \mathrm{m}$ for Case 2. The Sauter mean drop diameters become smaller with time for both cases after the initial spray impact at around $t=1.5 \mathrm{~ms}$, and each case follows the same curve which is shown in Fig. 4.9. This is an expected result which is caused by the splashing mechanisms. Because of the initial impact of the spray drops on the surface, the number of drops increases and they become smaller.

Table 4.2 General spray characteristics at $t=5 \mathrm{~ms}$ for the hollow cone spray cases.

\begin{tabular}{|c|c|c|c|c|c|}
\hline Cases & $\begin{array}{c}\text { Simulation } \\
\text { Time }(\mathbf{m s})\end{array}$ & $\begin{array}{c}\text { Total } \\
\text { number of } \\
\text { spray drops }\end{array}$ & $\begin{array}{c}\text { Total spray } \\
\text { mass, } \boldsymbol{m} \\
(\mathbf{m g})\end{array}$ & $\begin{array}{c}\text { Total film } \\
\text { mass, } \boldsymbol{m}_{\boldsymbol{s}} \\
(\mathbf{m g})\end{array}$ & $\begin{array}{c}\text { Sauter drop } \\
\text { diameter, } \\
\boldsymbol{d}_{\mathbf{3 2}}(\boldsymbol{\mu} \mathbf{m})\end{array}$ \\
\hline 1 & 5 & $3.69 \times 10^{6}$ & 15 & 3.72 & 34.9 \\
\hline 2 & 5 & $3.62 \times 10^{6}$ & 15 & 3.50 & 34.7 \\
\hline
\end{tabular}




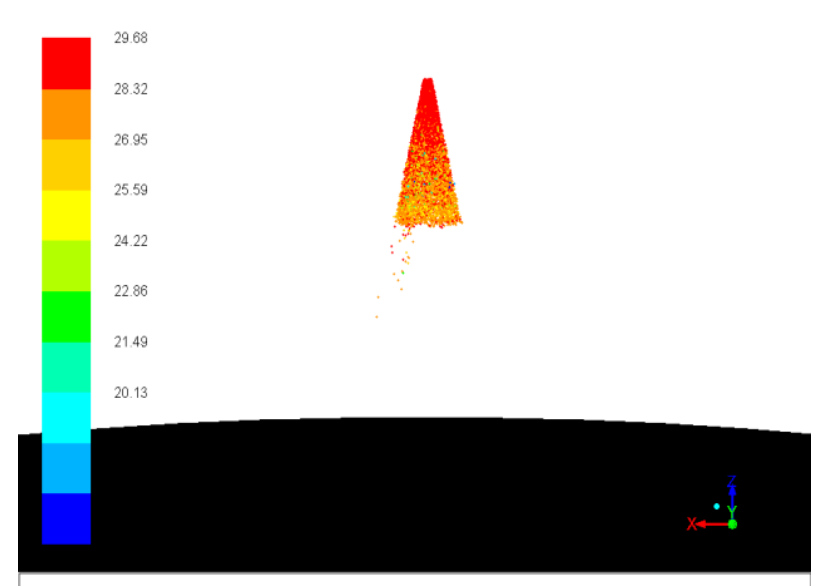

$$
t=0.5 \mathrm{~ms}
$$

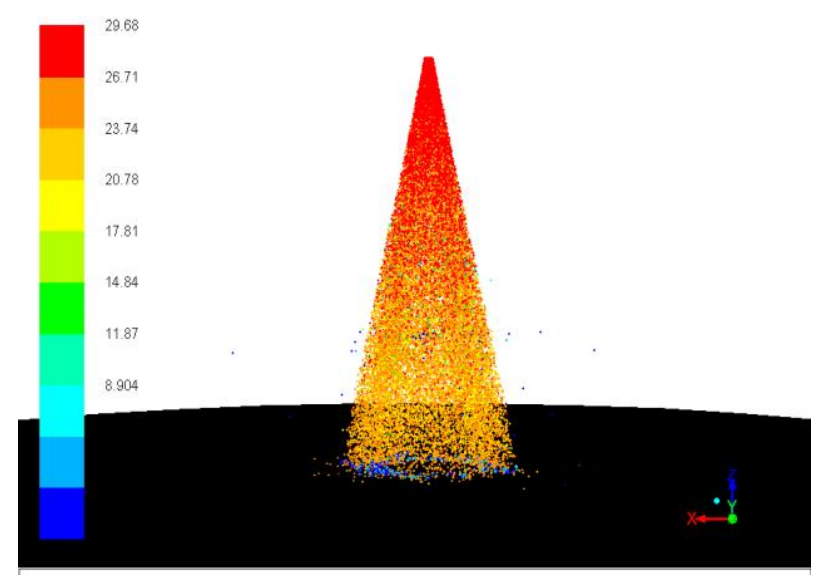

$$
t=1.5 \mathrm{~ms}
$$

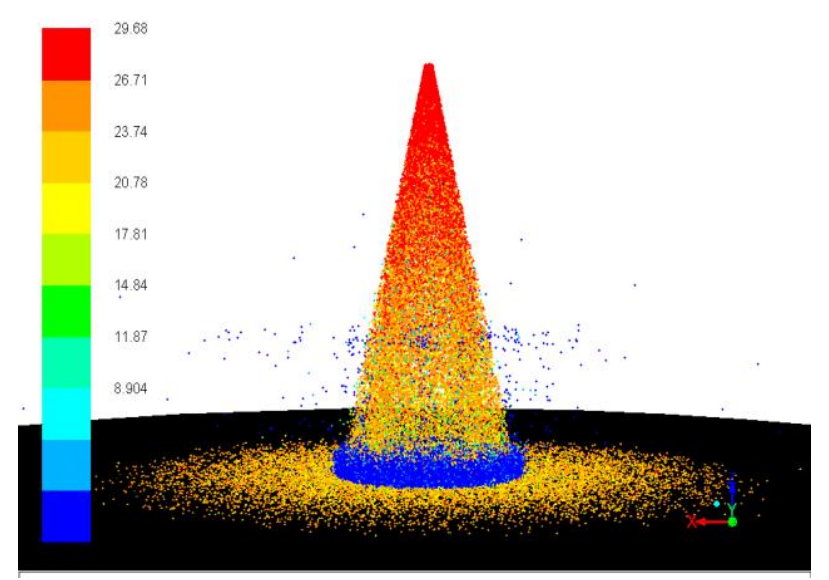

$t=2.5 \mathrm{~ms}$

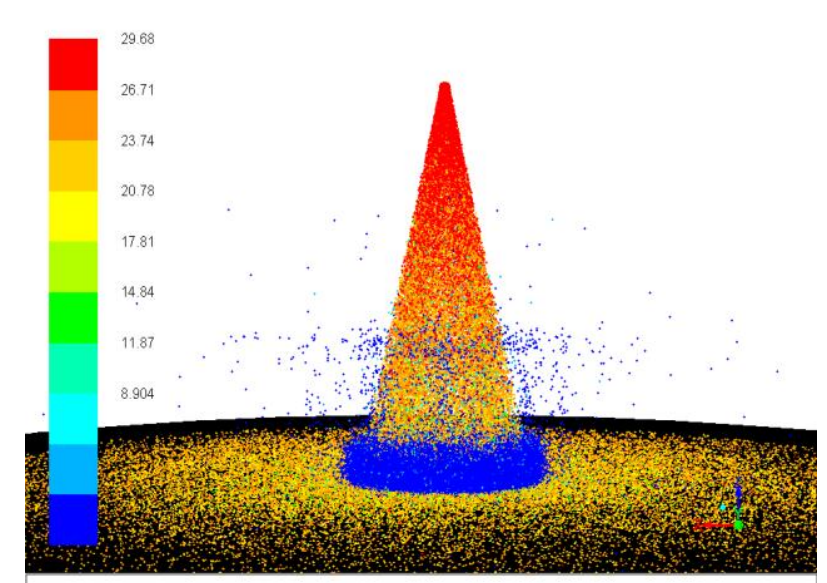

$$
t=3.5 \mathrm{~ms}
$$

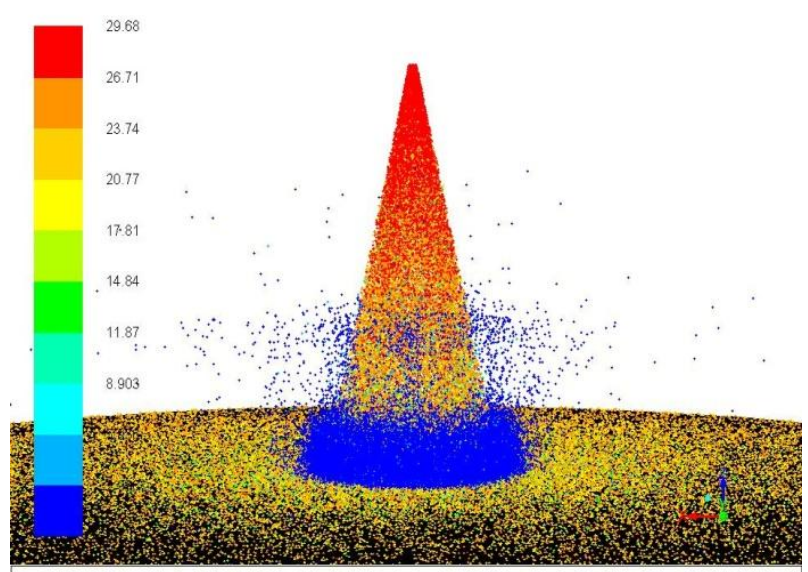

$$
t=4.5 \mathrm{~ms}
$$

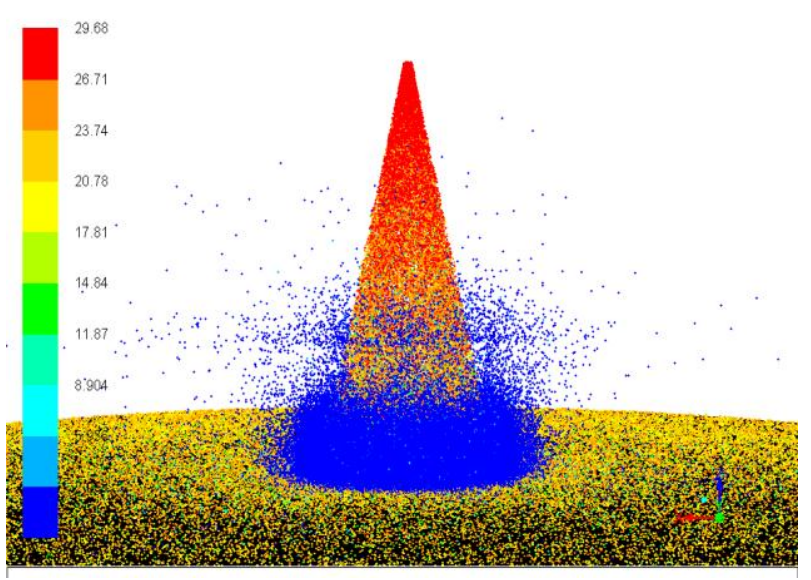

$$
t=6 \mathrm{~ms}
$$

Figure 4.5 Spray injection, spray impact and liquid film formation on the surface for Case 1 ( $h=$ $40 \mathrm{~mm}, \theta=10^{\circ}$ ). Particles are colored by the velocity magnitude-dark blue refers to lower velocity range $(0-3 \mathrm{~m} / \mathrm{s})$ and red refers to higher velocity range $(26-29.7 \mathrm{~m} / \mathrm{s})$. 


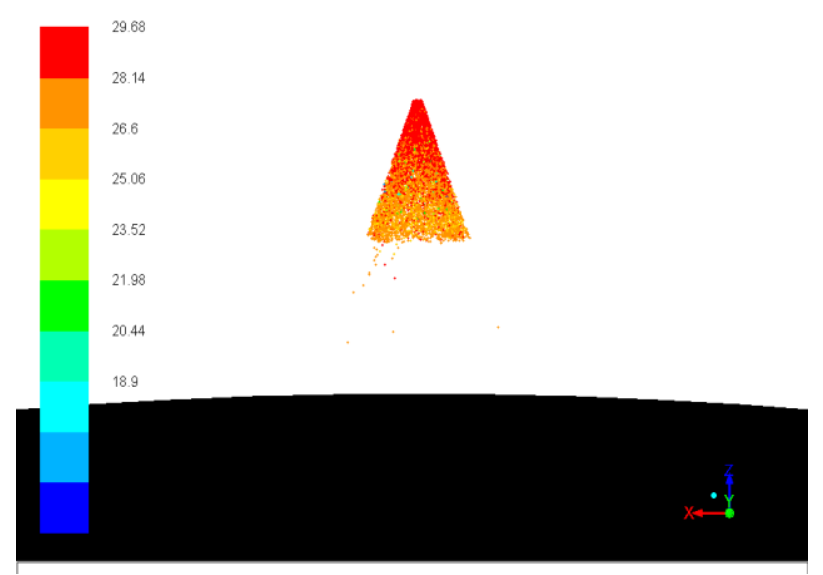

$$
t=0.5 \mathrm{~ms}
$$

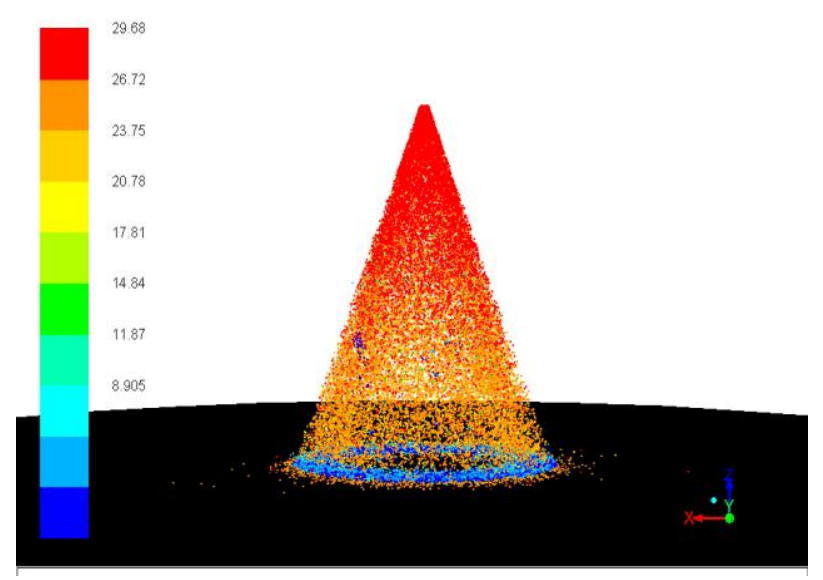

$t=1.5 \mathrm{~ms}$

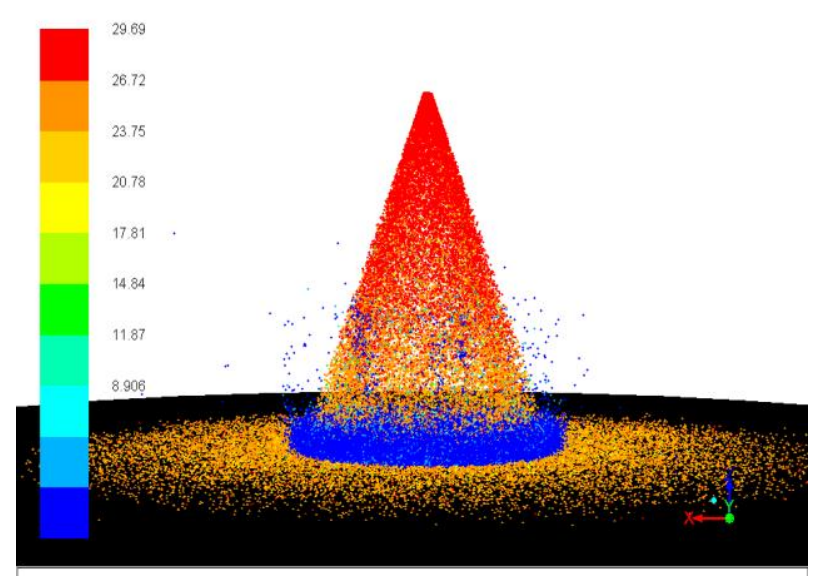

$t=2.5 \mathrm{~ms}$

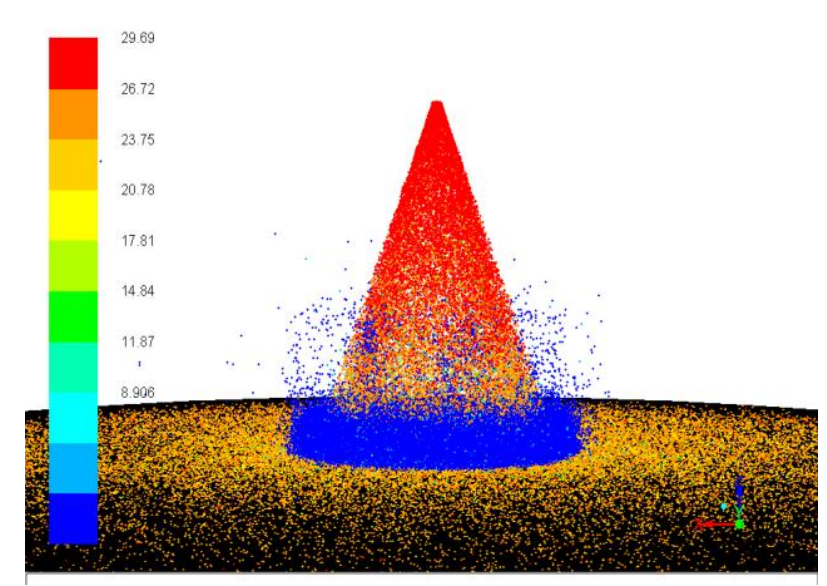

$t=3.5 \mathrm{~ms}$

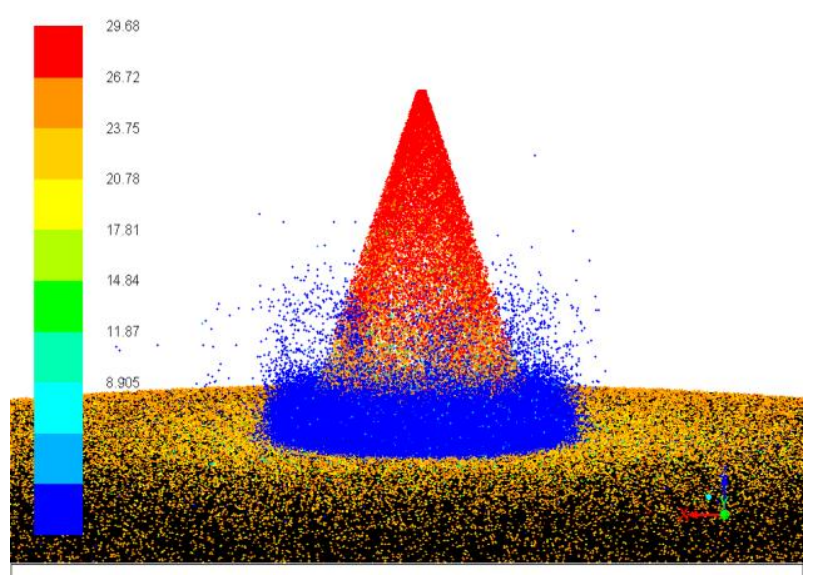

$t=4.5 \mathrm{~ms}$

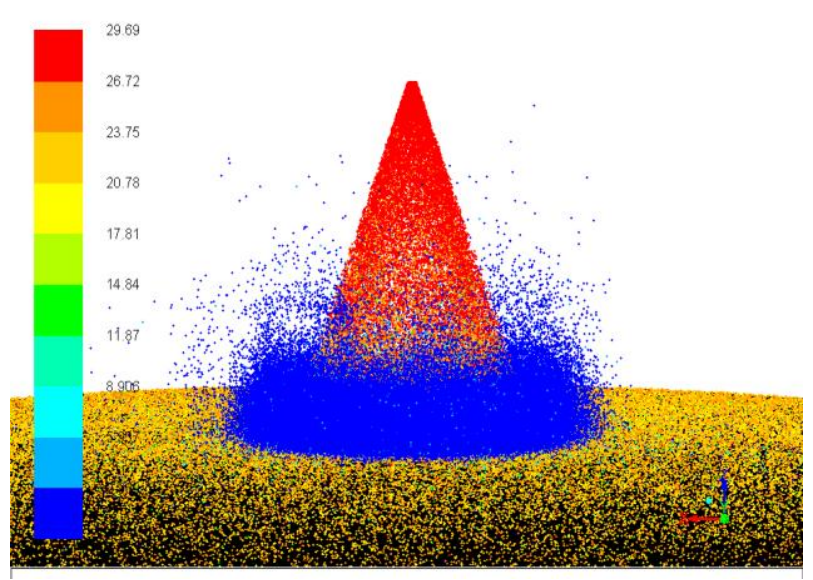

$t=6 \mathrm{~ms}$

Figure 4.6 Spray injection, spray impact and liquid film formation on the surface for Case 2 ( $h=$ $\left.35 \mathrm{~mm}, \theta=18^{\circ}\right)$. 


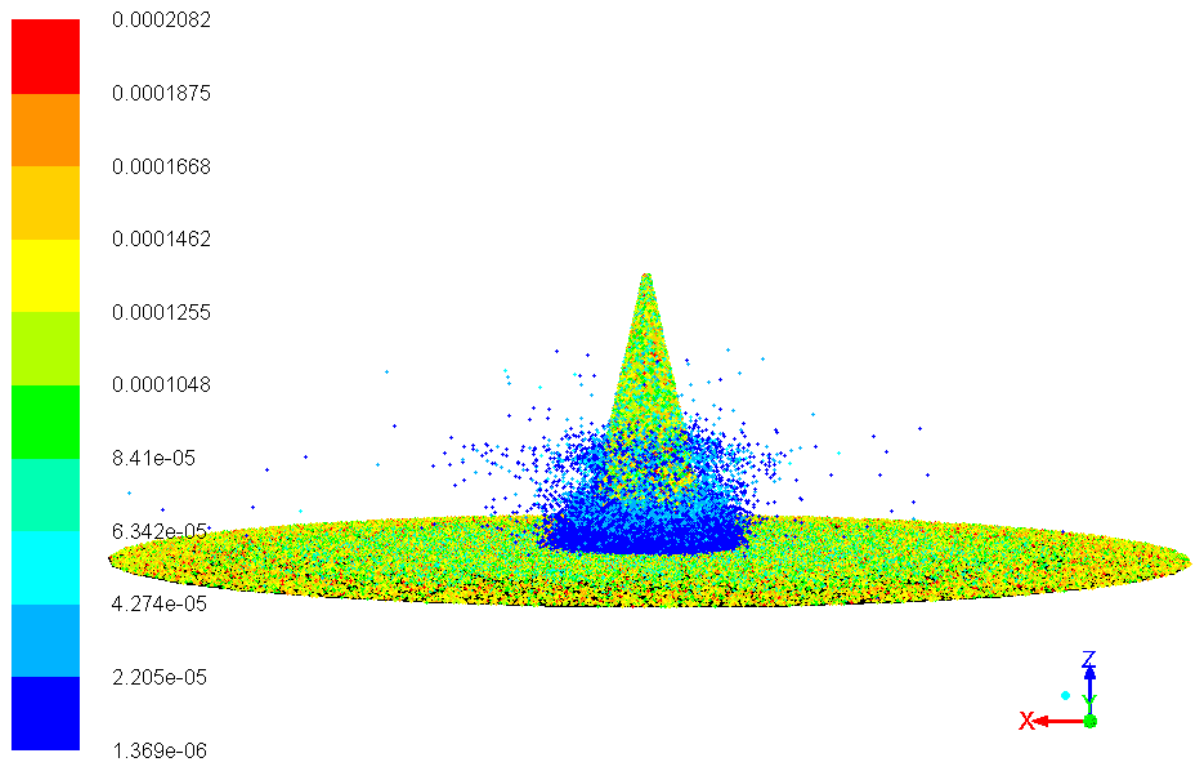

Figure 4.7 Spray drop diameter distribution $(\mathrm{m})$ for Case $1\left(h=40 \mathrm{~mm}, \theta=10^{\circ}\right)$ at $5 \mathrm{~ms}$. (Note that red refers to $188-208 \mu \mathrm{m}$, yellow refers to 126-146 $\mu \mathrm{m}$, green refers to 84-105 $\mu \mathrm{m}$, and dark blue refers to less than $22 \mu \mathrm{m}$.)

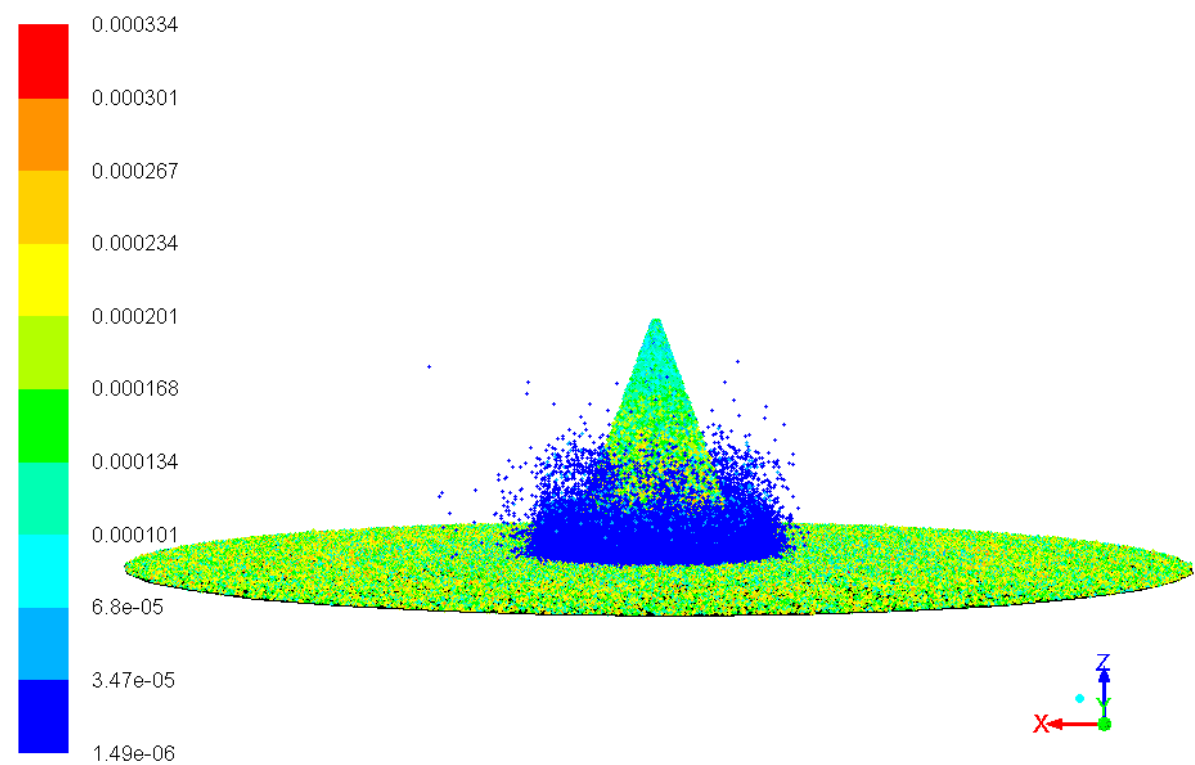

Figure 4.8 Spray drop diameter distribution $(\mathrm{m})$ for Case $2\left(h=35 \mathrm{~mm}, \theta=18^{\circ}\right)$ at $5 \mathrm{~ms}$. (Note that red refers to 301-334 $\mu \mathrm{m}$, yellow refers to 201-234 $\mu \mathrm{m}$, green refers to 134-168 $\mu \mathrm{m}$, and dark blue refers to less $35 \mu \mathrm{m}$.) 

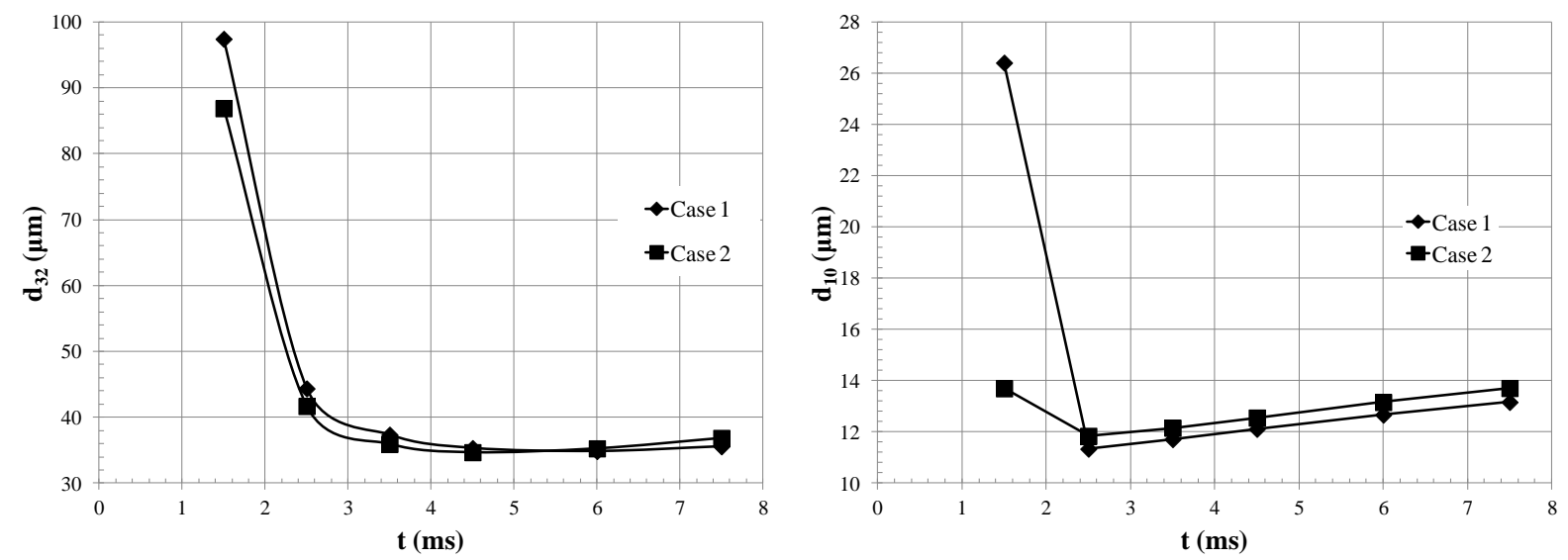

Figure 4.9 The Sauter mean drop diameter $\left(d_{32}\right)$ on the left and the arithmetic mean drop diameter $\left(d_{10}\right)$ on the right with respect to time $(\mathrm{ms})$ for Case $1\left(h=40 \mathrm{~mm}, \theta=10^{\circ}\right)$ and Case 2 $\left(h=35 \mathrm{~mm}, \theta=18^{\circ}\right)$. (Note that initial impact time is close to $1.5 \mathrm{~ms}$ and Case 2 drops hit the wall earlier than Case 1)

The variation of the total liquid film mass $\left(m_{s}\right)$ that has accumulated on the impact surface is shown in Fig. 4.10. Spray drops start to accumulate on the surface after around $1.5 \mathrm{~ms}$ which is the time when the first spray drops impinge onto the surface. The accumulated liquid film mass on the impact surface for Case 2 is more than Case 1 between the initial impact time (1.5 ms) and $4.2 \mathrm{~ms}$ (Fig. 4.10). However, the mass of spray liquid film for Case 1 becomes more than Case 2 between $4.2 \mathrm{~ms}$ and $7.5 \mathrm{~ms}$. The thickness of the liquid film that has accumulated on the impact surface at $t=5 \mathrm{~ms}$ is shown in Figs. 4.11 and 4.12. In the Case 1, the maximum thickness of $2.27 \mu \mathrm{m}$ is at the center of the spray, and the film thickness declines monotonically with increasing radius. The Case 2 film is more complex with a dimple near the centerline and an off center maximum of $1.62 \mu \mathrm{m}$. 


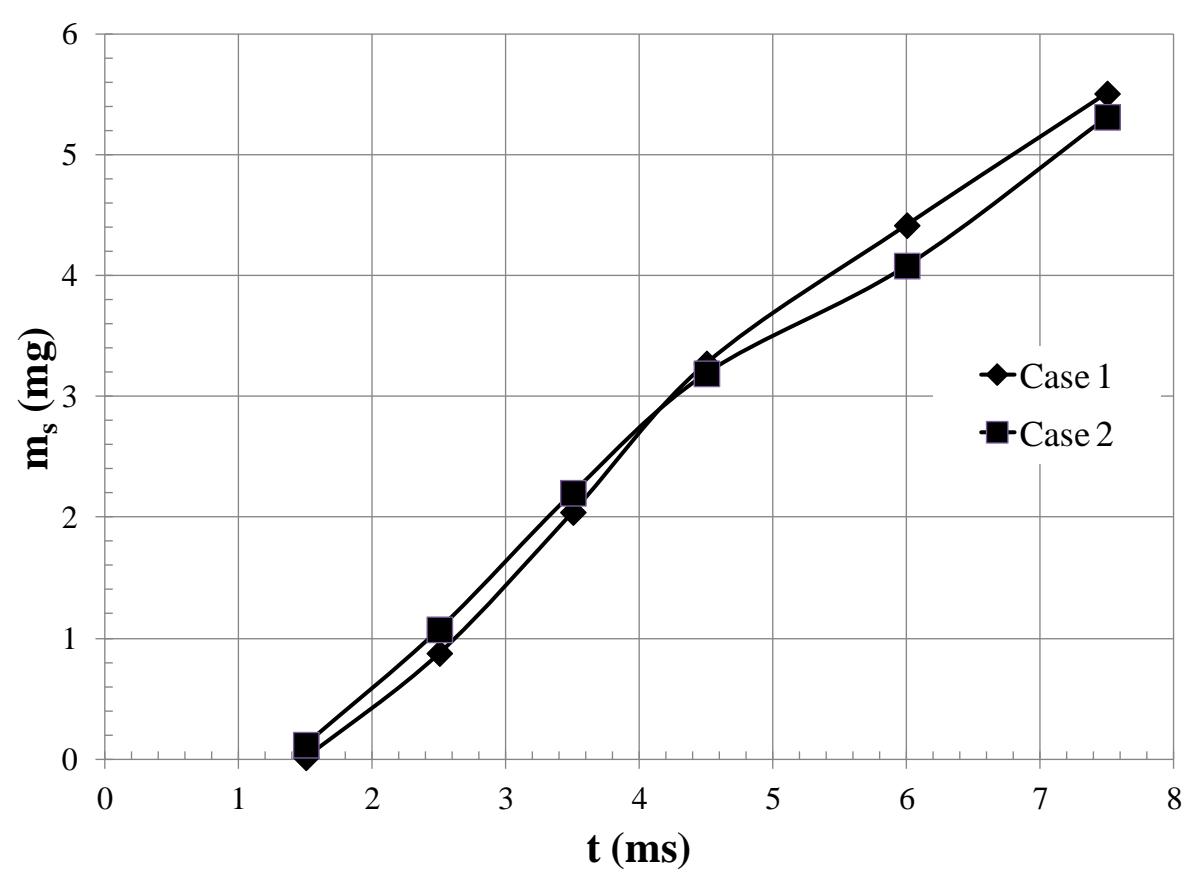

Figure 4.10 Variation of the total liquid film mass, $m_{s}(\mathrm{mg})$, on the surface with respect to time (ms) for Case $1\left(h=40 \mathrm{~mm}, \theta=10^{\circ}\right)$ and Case $2\left(h=35 \mathrm{~mm}, \theta=18^{\circ}\right)$. (Note that $t=0 \mathrm{~ms}$ is the time when spray is injected, $t \sim 1.5 \mathrm{~ms}$ is the time when spray drops start to impinge and accumulate on the surface, and the simulation end time is $7.5 \mathrm{~ms}$.)

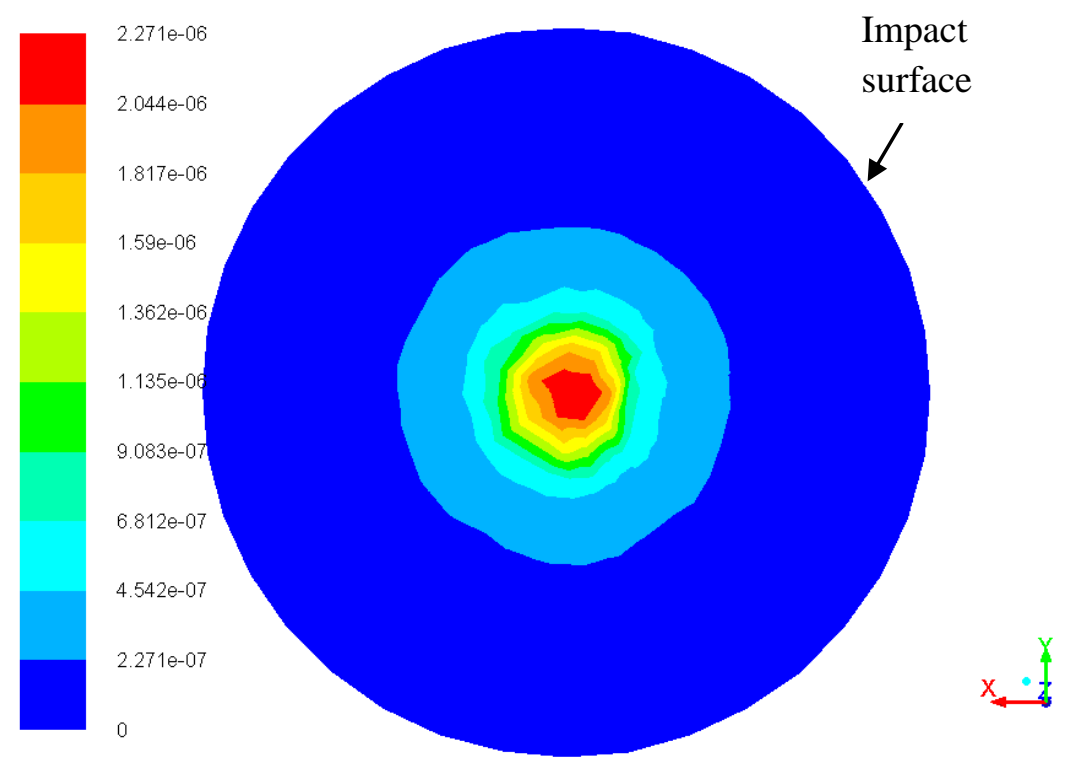

Figure 4.11 Contours of the liquid film height at the impact surface for Case 1 at $5 \mathrm{~ms}$. (Note that red refers to 2.04-2.27 $\mu \mathrm{m}$, yellow refers to 1.36-1.59 $\mu \mathrm{m}$, green refers to 0.91-1.13 $\mu \mathrm{m}$, and dark blue refers to less than $0.27 \mu \mathrm{m}$.) 


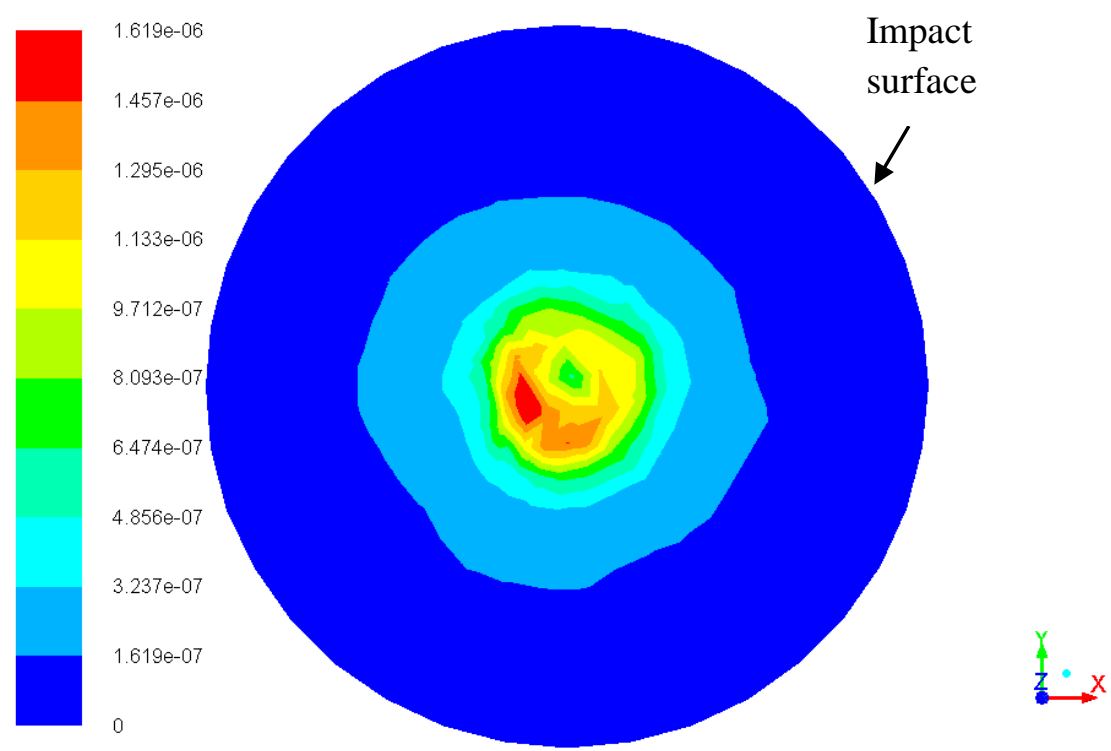

Figure 4.12 Contours of the liquid film height at the impact surface for Case 2 at $5 \mathrm{~ms}$. (Note that red refers to 1.46-1.62 $\mu \mathrm{m}$, yellow refers to 0.97-1.13 $\mu \mathrm{m}$, green refers to 0.65-0.81 $\mu \mathrm{m}$, and dark blue refers to less than $0.16 \mu \mathrm{m}$.)

Issa (2009) defined the spray impact efficiency $(\eta)$ as the ratio of the mass flow rate of the liquid leaving the domain $\left(M_{s}\right)$ to the mass flow rate of the droplets leaving the nozzle exit $(M)$ which is $\eta=M_{s} / M$.

Figure 4.13 shows the spray impact efficiency $(\eta)$ of Cases 1 and 2 . After the initial spray drops impinge onto the surface, the spray impact efficiency increases for both Cases. However, the efficiency is slightly higher for Case 2 until $4.2 \mathrm{~ms}$. The efficiency of Case 1 remains almost constant after $4.5 \mathrm{~ms}$ whereas the efficiency of the Case 2 starts to decrease after $4.5 \mathrm{~ms}$ until 7.5 ms (Fig. 4.13). A higher number of splashed spray drops for Case 2 at $5 \mathrm{~ms}$ can be clearly seen in Fig. 4.7 and Fig. 4.8. The decrease of the efficiency for the Case 2 and the constant efficiency for the Case 1 after $4.5 \mathrm{~ms}$ may be due to the higher number of splashed drops since splashed drops are not included in the spray liquid film that has accumulated on the surface. However, this should be studied for more simulation times, and also for different spray cases.

The spray liquid mass in the surface film is an important variable for spray cooling. The heat transfer between the liquid film and the heated surface should increase with the film mass. 


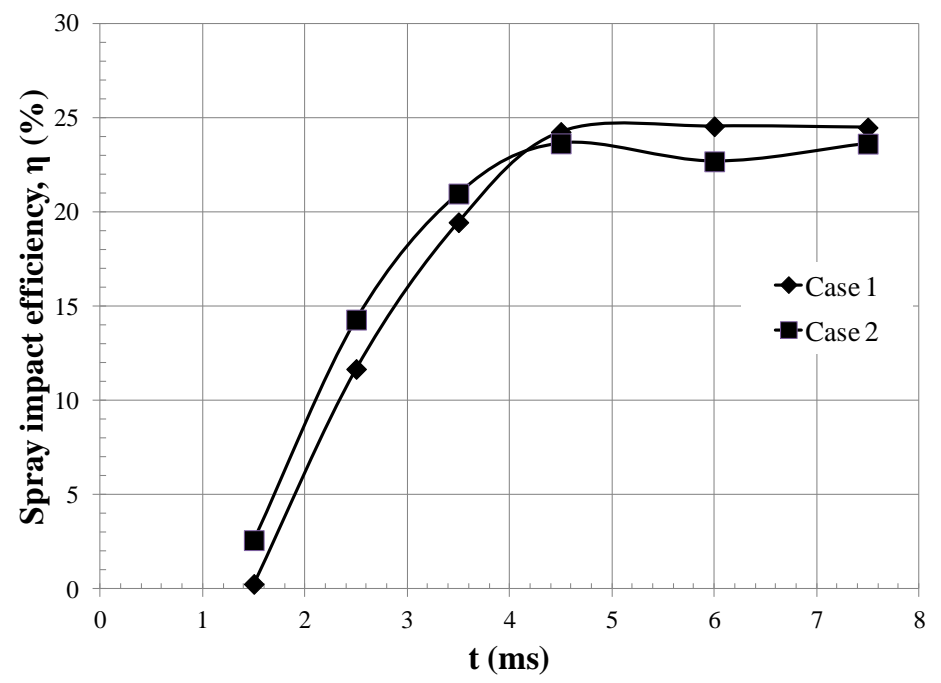

Figure 4.13 Variation of the spray impact efficiency, $\eta(\%)=M_{s} / M$, with respect to time (ms). (Note that $t=0 \mathrm{~ms}$ is the time when spray is injected, and $t \sim 1.5 \mathrm{~ms}$ is the time when spray drops start to impinge and accumulate on the surface.)

\subsubsection{Comparisons of Full 3D and 2D Axisymmetric Spray Simulations}

Full 3D spray simulations were computationally very expensive. It took about a month of computation on a Quad core desktop workstation to get a fully converged solution for a single full 3D spray simulation for a very limited spraying time (for comparison purposes 3D simulations mentioned in Section 4.2.1 were run until $10.5 \mathrm{~ms}$ ). Therefore, it would be impractical to perform full 3D simulations to analyze the effects of different parameters on sprays. For this reason, the two full 3D spray cases (Case 1 and Case 2) described in the previous section were compared to two identical 2D axisymmetric spray simulations.

For Case 1, the average drop diameters $d_{32}$ and $d_{10}$ are very close as shown in Figures 4.14.a and 4.14.b, respectively. The accumulated liquid film mass on the impact surface of Case 1 for full 3D and 2D axisymmetric sprays are also very close until $t=6 \mathrm{~ms}$ (Fig. 4.15.a). After $t$ $=6 \mathrm{~ms}$, the spray liquid film mass is higher for the 2D axisymmetric spray. This can be explained due to the number of total splashing drops. The full 3D spray generates more splashing drops with time after spray impact on the surface. Since the liquid film mass on the surface does not include these splashing drops, more liquid film mass is obtained from the $2 \mathrm{D}$ axisymmetric spray. Figure 4.15.b shows the comparison of the spray impact efficiency for full the 3D and the 
2D axisymmetric Case 1 sprays. Similar to the liquid film mass comparison shown in Figure 4.15.a, the spray impact efficiency is very close at the initial time but after $t=6 \mathrm{~ms}$ the $2 \mathrm{D}$ axisymmetric simulation results in a higher spray impact efficiency because more liquid accumulates on the impact surface, as a result of the reduced drop splashing.
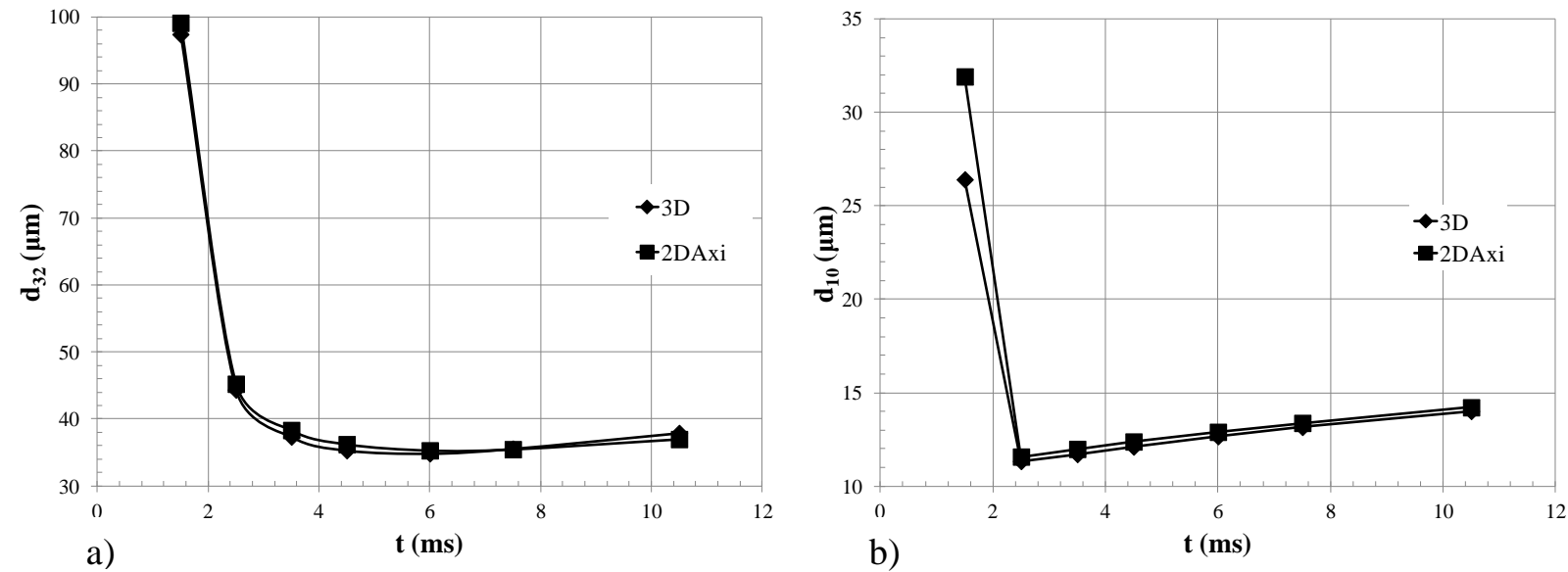

Figure 4.14 Comparison of the average drop diameters (a) $d_{32}$ (b) $d_{10}$ for Case 1 ( $h=40 \mathrm{~mm}, \theta=$ $\left.10^{\circ}\right)$

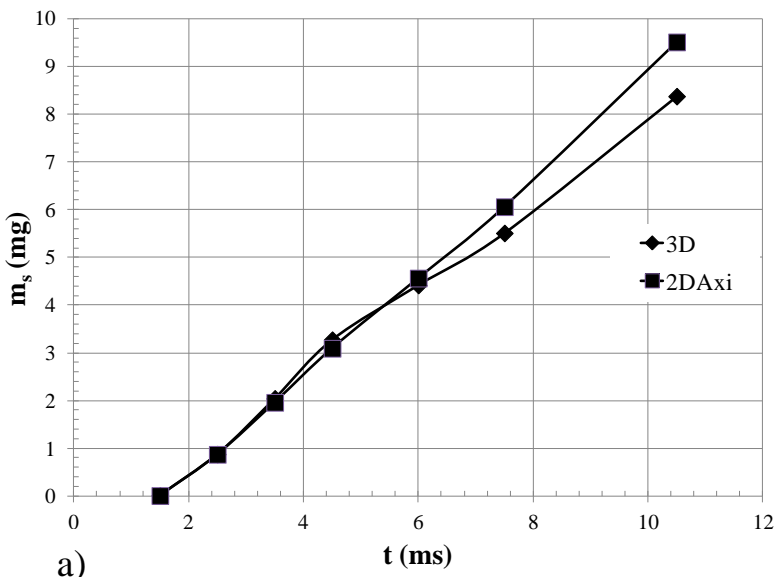

a)

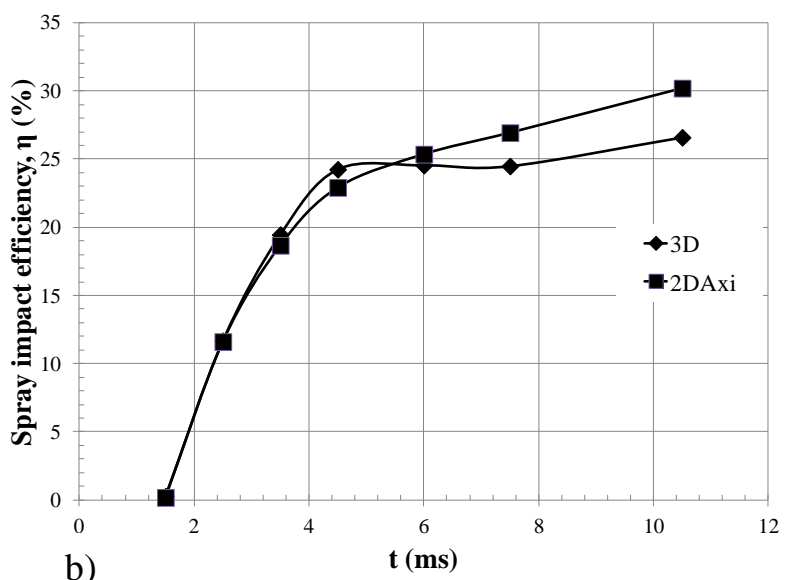

b)

Figure 4.15 Comparison of Case $1\left(h=40 \mathrm{~mm}, \theta=10^{\circ}\right)$ (a) the liquid film mass $\left(m_{s}\right)$ and (b) the spray impact efficiency, $\eta(\%)$.

Figures 4.16 and 4.17 show the drop velocity distributions for Case 1 for full 3D and 2D axisymmetric hollow cone spray simulations. The initial impact is at $t=1.5 \mathrm{~ms}$ for both $3 \mathrm{D}$ and 2D axisymmetric sprays. The liquid film formation on the impact surface is also very similar. However, there are some differences in the number of splashing drops. The comparisons of the 
full 3D and 2D axisymmetric simulations for Case 2 are not included here but were similar to those of Case 1.

It is observed from the full 3D hollow cone spray simulations that the velocity distribution of spray drops for Case 1 and Case 2 (Fig. 4.5 and 4.6), and the distribution of drop diameters (Fig. 4.7 for Case 1 and Fig. 4.8 for Case 2) are symmetric about the centerline. It is concluded based on the comparisons between Case 1 and Case 2 simulations that these sprays can be adequately simulated using a 2D axisymmetric model instead of a full 3D model since the spray characteristics such as the average drop diameters, average drop velocity distribution, spray liquid film characteristics and spray impact efficiency do not significantly change. In addition, a single 2D axisymmetric simulation can take 2-3 days to get a fully converged solution for $10.5 \mathrm{~ms}$ compared to a full 3D spray simulation which can take a month for similar conditions. Therefore, a 2D axisymmetric model will be used for the spray and spray cooling simulations using a full cone nozzle based on experiments performed at WVU. 


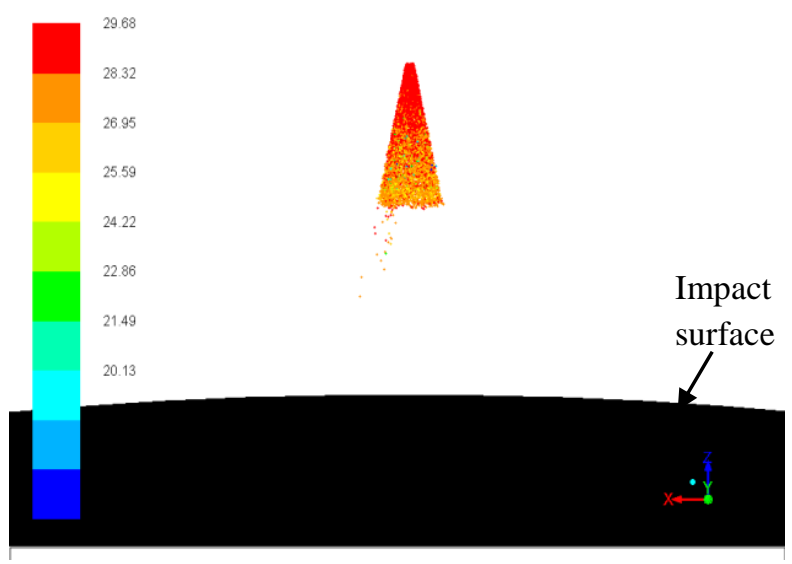

$t=0.5 \mathrm{~ms}$

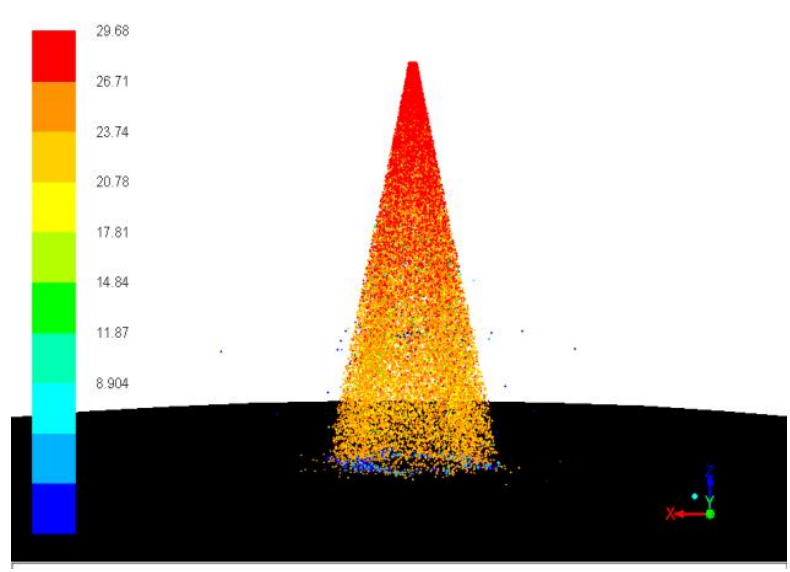

$t=1.5 \mathrm{~ms}$

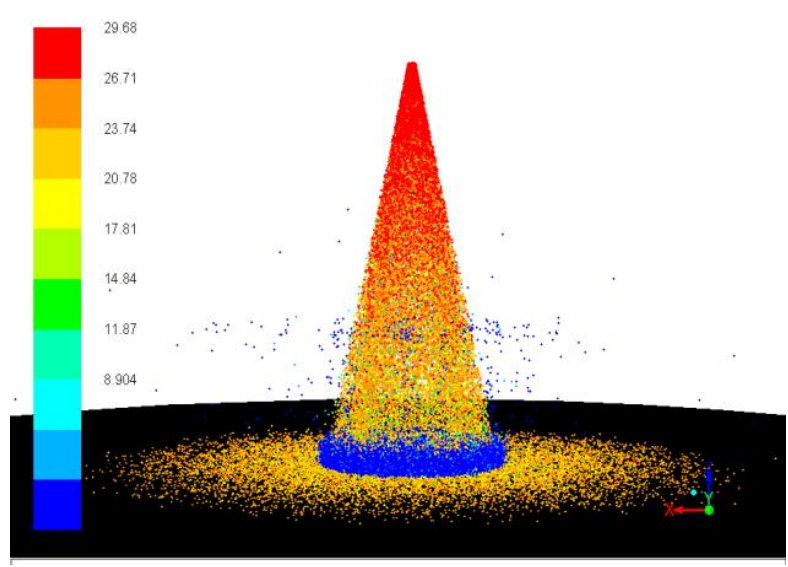

$t=2.5 \mathrm{~ms}$
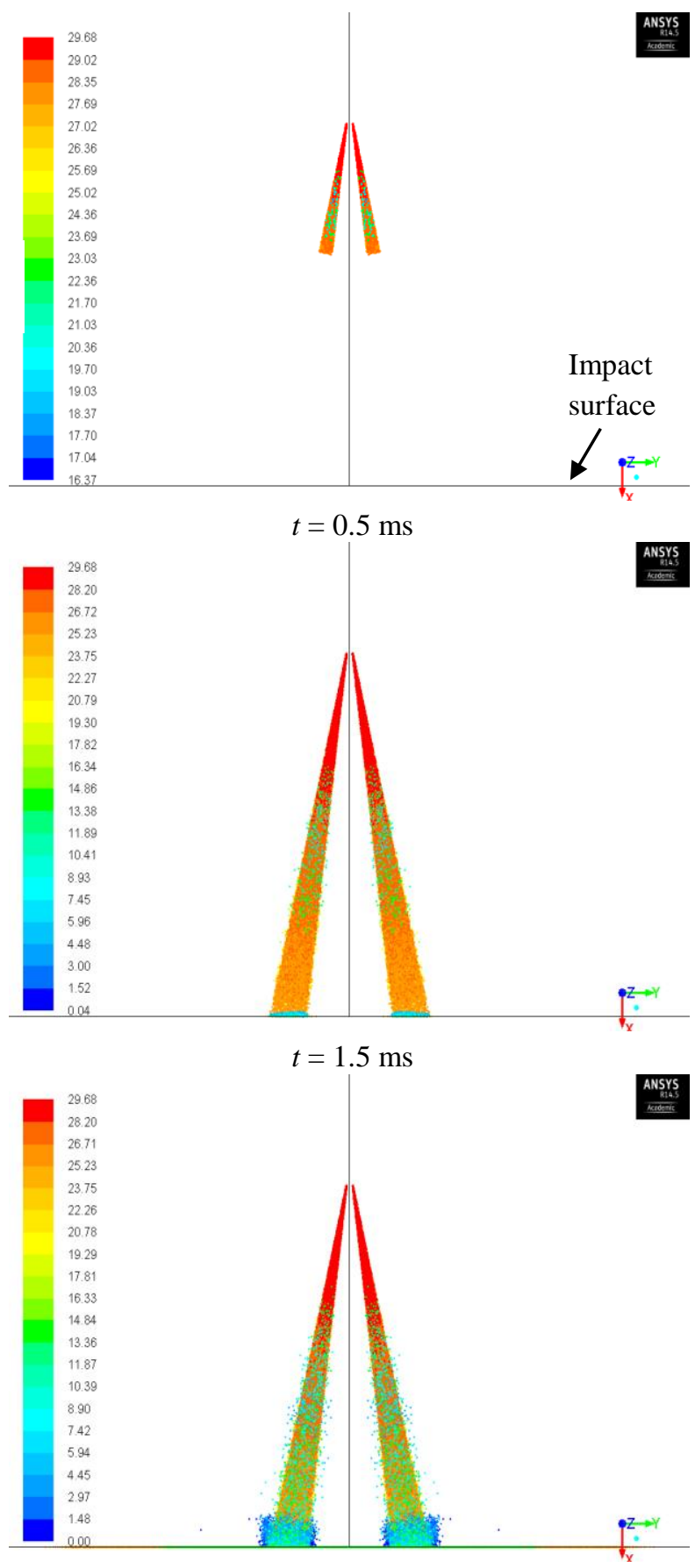

$t=2.5 \mathrm{~ms}$

Figure 4.16 Comparison of full 3D (on the left column, perspective view) and 2D axisymmetric (on the right column, vertical plane view) spray droplet velocity distribution for Case 1 ( $h=40$ $\mathrm{mm}, \theta=10^{\circ}$ ) from $t=0-2.5 \mathrm{~ms}$. (Particles are colored by the velocity magnitude-dark blue refers to lower velocity range and red refers to higher velocity range $(26-29.7 \mathrm{~m} / \mathrm{s}$.) 


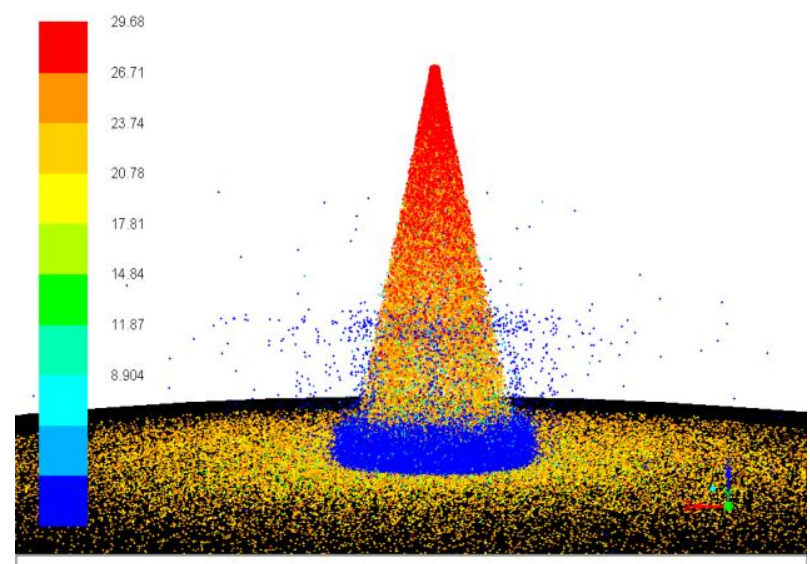

$t=3.5 \mathrm{~ms}$

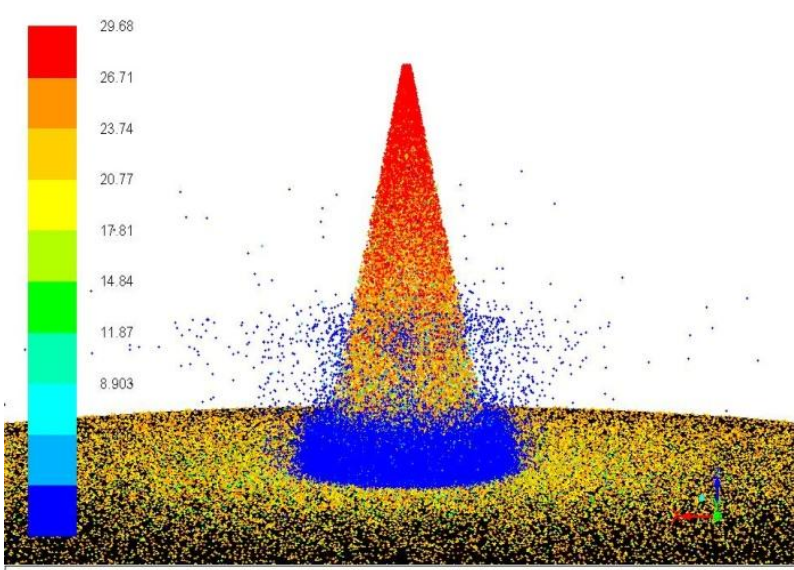

$t=4.5 \mathrm{~ms}$

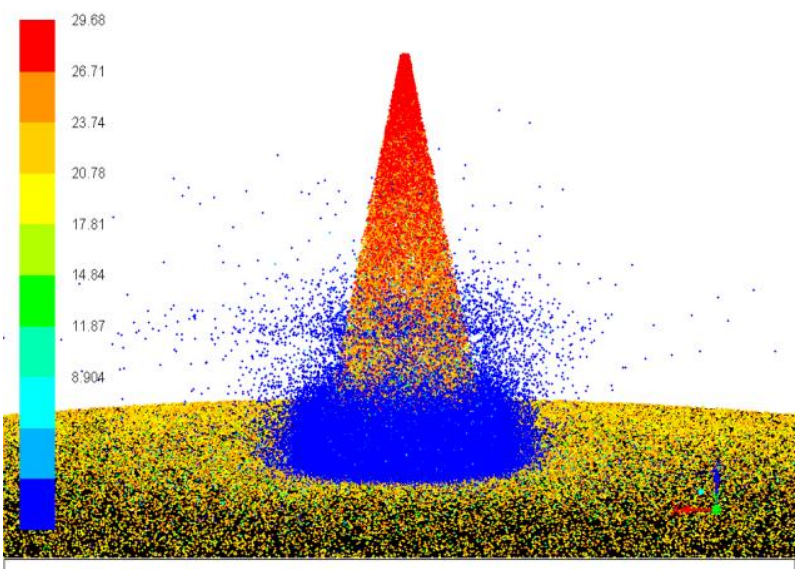

$t=6 \mathrm{~ms}$
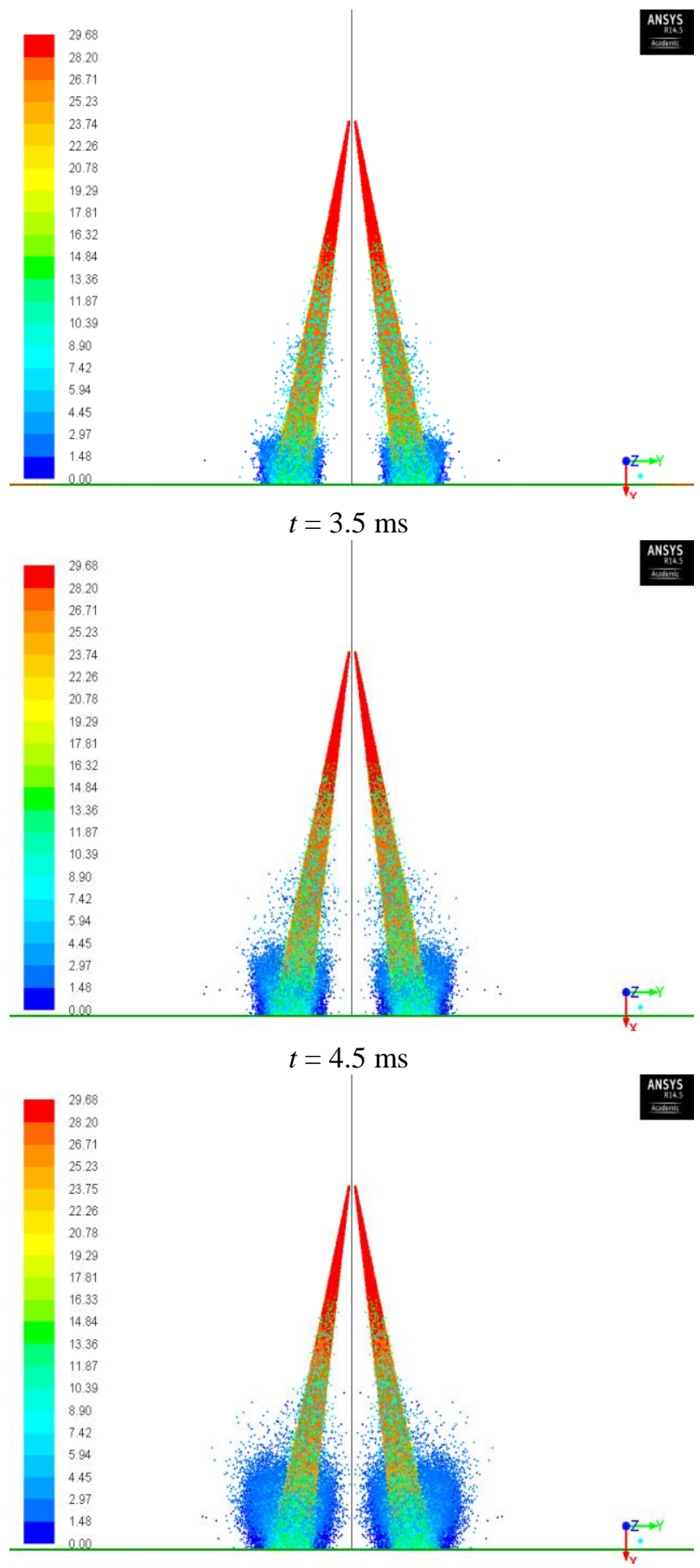

$t=6 \mathrm{~ms}$

Figure 4.17 Comparison of full 3D (on the left column, perspective view) and 2D axisymmetric (on the right column, vertical plane view) spray droplet velocity distribution for Case 1 ( $h=40$ $\mathrm{mm}, \theta=10^{\circ}$ ) from $t=3.5-6 \mathrm{~ms}$. (Particles are colored by the velocity magnitude-dark blue refers to lower velocity range and red refers to higher velocity range $(26-29.7 \mathrm{~m} / \mathrm{s})$.) 


\subsection{Simulations of Full Cone 2D Axisymmetric Sprays Using the Discrete Phase Model (DPM)}

Results and discussions mentioned in this section are mostly from Dinc, Gray, Hillen, Taylor, Kuhlman (2013). Given the good agreement of the 2D axisymmetric and full 3D simulations of Case 1 and Case 2, the detailed parametric simulations were performed with a 2D axisymmetric formulation. The parameters of the full cone nozzle were assigned based on the Spraying Systems $1 / 8 \mathrm{G}$ full cone nozzle which was being used in the experimental part of this project. The optimum size of computational cells in the spray region was obtained based on parametric studies using 2D axisymmetric full cone sprays. The smallest computational cells were defined at the spray nozzle and the spray impact regions using zonal mesh refinement with the size 0.25 $\mathrm{mm}$ as shown in Fig. 4.18. The boundary conditions of the 2D axisymmetric spray model are shown in Fig. 4.18. The boundary condition at the maximum radius was a constant pressure outlet rather than a solid wall as in the previous simulations. The computational domain was 50 $\mathrm{mm}$ high and had a radius of $50 \mathrm{~mm}$. Effects of varying nozzle-to-surface distance $(h)$, spray half angle $(\theta)$, spray mass flow rate $(M)$, gravity $(g)$, liquid surface tension $(\sigma)$, liquid density $(\rho)$ and liquid viscosity $(\mu)$ were studied as shown in Table 4.3. Table 4.4 shows the properties of the spray liquids used in these calculations. HypV is a hypothetical liquid which has the same physical properties as water at room temperature except for viscosity. HypST is also a hypothetical liquid having the same physical properties as water except for surface tension, and similarly HypD is another hypothetical liquid having the same physical properties as water except for density. 


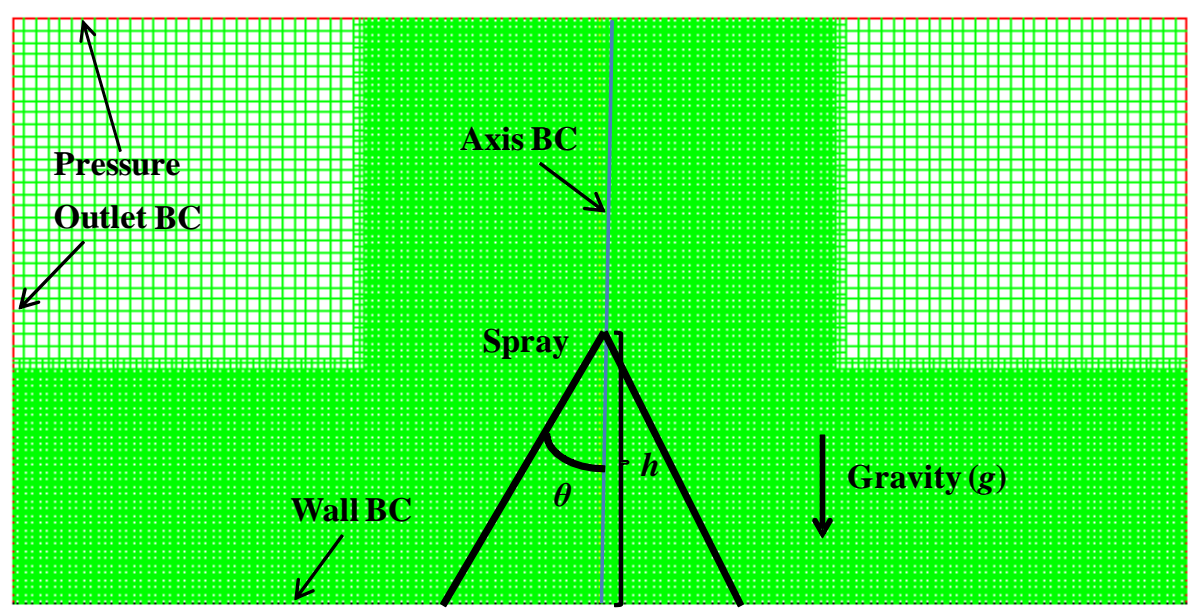

Figure 4.18 Boundary conditions and computational cells for the $2 \mathrm{D}$ axisymmetric full cone spray cases.

Table 4.3 Parameters of the 2D axisymmetric full cone spray cases.

\begin{tabular}{|c|c|c|c|c|c|}
\hline Case & $\begin{array}{c}\text { Gravity } \\
\left(\mathbf{m} / \mathbf{s}^{\mathbf{2}}\right)\end{array}$ & $\begin{array}{c}\text { Spray } \\
\text { Liquid }\end{array}$ & $\begin{array}{c}\text { Mass Flow } \\
\text { Rate, } \boldsymbol{M} \\
(\mathbf{k g} / \mathbf{s})\end{array}$ & $\begin{array}{c}\text { Nozzle-to- } \\
\text { Surface, } \boldsymbol{h} \\
(\mathbf{m m})\end{array}$ & $\begin{array}{c}\text { Spray Half } \\
\text { Angle, } \boldsymbol{\theta} \\
\left({ }^{\circ}\right)\end{array}$ \\
\hline A & -9.81 & Water & 0.01207 & 25.4 & 26.5 \\
\hline B & -9.81 & Water & 0.01207 & 31.75 & 26.5 \\
\hline C & -9.81 & Water & 0.01207 & 38.1 & 26.5 \\
\hline D & -9.81 & Water & 0.01207 & 38.1 & 20.5 \\
\hline E & -275 & Water & 0.01207 & 38.1 & 26.5 \\
\hline F & +9.81 & Water & 0.01207 & 38.1 & 26.5 \\
\hline G & -9.81 & Water & 0.01514 & 38.1 & 26.5 \\
\hline H & -9.81 & Water & 0.00400 & 38.1 & 26.5 \\
\hline I & -9.81 & HypV & 0.01207 & 38.1 & 26.5 \\
\hline J & -9.81 & HypST & 0.01207 & 38.1 & 26.5 \\
\hline K & -9.81 & HypD & 0.01207 & 38.1 & 26.5 \\
\hline
\end{tabular}


Table 4.4 Properties of spray liquids.

\begin{tabular}{|c|c|c|c|c|}
\hline Cases & $\begin{array}{c}\text { Spray } \\
\text { Liquid }\end{array}$ & $\begin{array}{c}\text { Density } \\
\left(\mathbf{k g} / \mathbf{m}^{\mathbf{3}}\right)\end{array}$ & $\begin{array}{c}\text { Dynamic } \\
\text { Viscosity } \\
(\mathbf{k g} / \mathbf{m s})\end{array}$ & $\begin{array}{c}\text { Surface } \\
\text { Tension } \\
\mathbf{( N / m )}\end{array}$ \\
\hline A-H & Water & 998 & 0.001 & 0.072 \\
\hline I & HypV & 998 & 0.005 & 0.072 \\
\hline J & HypST & 998 & 0.001 & 0.010 \\
\hline K & HypD & 3000 & 0.001 & 0.072 \\
\hline
\end{tabular}

\subsubsection{Effects of Varying Nozzle-to-Surface Distance (h)}

Comparisons of Cases A, B and C reveal the effects of nozzle-to-surface distance $(h)$. Case A is the spray which is injected closest to the impact surface $(h=25.4 \mathrm{~mm})$, Case $\mathrm{C}$ is injected farthest $(h=38.1 \mathrm{~mm})$ and Case B has the intermediate injection distance $(h=31.75 \mathrm{~mm})$. The spray half angle $\theta=26.5^{\circ}$ is constant for Cases A, B and C.

Figure 4.19 shows the average drop diameters $d_{32}$ (Fig. 4.19.a) and $d_{10}$ (Fig. 4.19.b) for Cases A, B and C. Since the initial impact time is different for all three cases, the mean diameters are different at the beginning (0-2 ms). However, after the sprays impact the surface, the average drop diameters become very similar. Figure 4.20.a shows the spray liquid film mass accumulation $\left(m_{s}\right)$ on the impact surface and Figure 4.20.b shows the spray impact efficiency $(\eta)$ for Cases A, B and C. The liquid film mass and spray impact efficiency show similar trends for all cases. Case A has a higher spray impact efficiency than Cases B and C. For instance, the Case A spray impact efficiency is $15 \%$ higher than Case $\mathrm{C}$ at $t=10 \mathrm{~ms}$. These differences in the spray impact efficiency occur because there is less mass in the spray for the closer nozzle. The drop velocity and diameter distributions for Case A (Fig. 4.21.a) and Case C (Fig. 4.21.b) are shown in Figure 4.21. In Figure 4.21, the left half shows velocity and the right half shows the diameter distribution. These full cone nozzle sprays show behavior which is similar to the hollow cone nozzle spray simulations in Section 4.2. The drop velocity decreases as the spray approaches the impact surface and the splashing drops are slower and smaller compared to the initial spray drops (Fig. 4.21). 


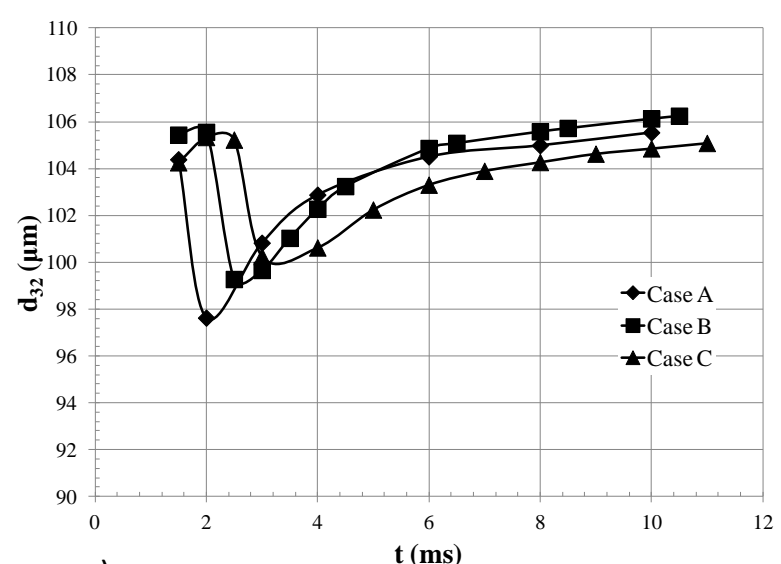

a)

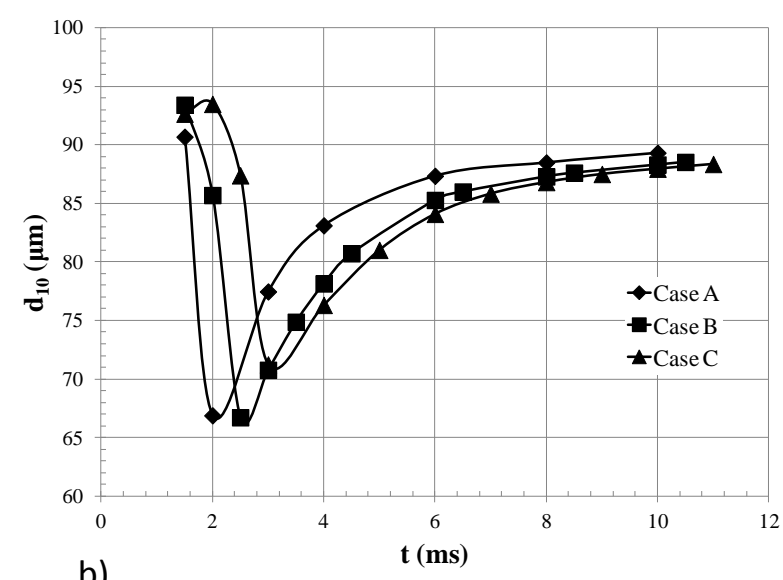

b)

Figure 4.19 (a) The Sauter mean drop diameter $\left(d_{32}\right)$ (b) the arithmetic mean drop diameter $\left(d_{10}\right)$ for Case A $(h=25.4 \mathrm{~mm})$, Case B $(h=31.75 \mathrm{~mm})$ and Case C $(h=38.1 \mathrm{~mm})$ with $\theta=26.5^{\circ}$.

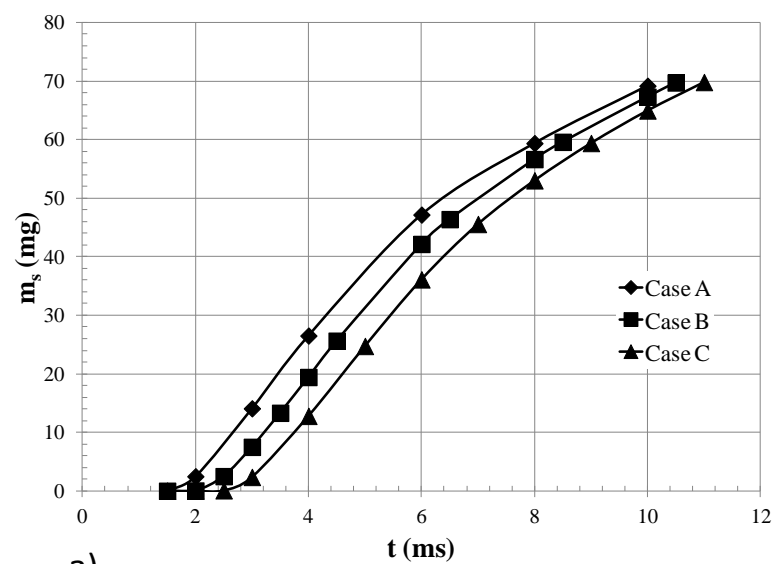

a)

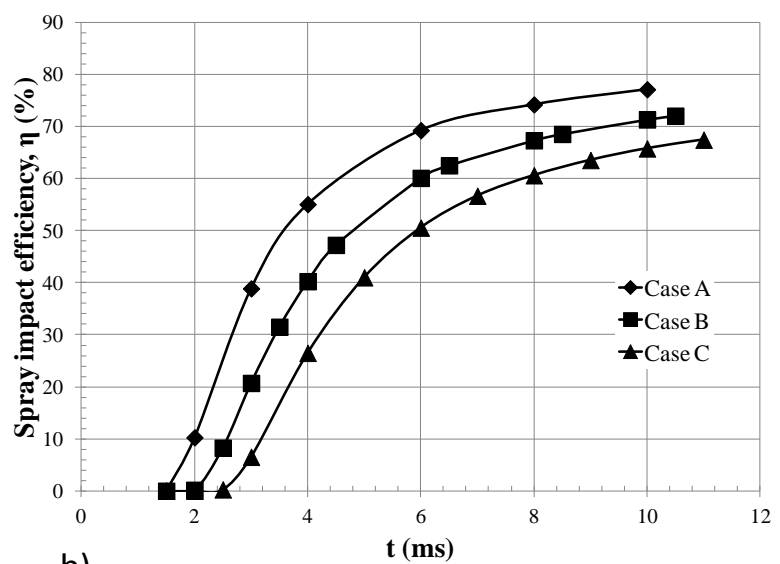

b)

Figure 4.20 (a) The liquid film mass accumulation $\left(m_{s}\right)$ (b) the spray impact efficiency $(\eta)$ for Case A $(h=25.4 \mathrm{~mm})$, Case B $(h=31.75 \mathrm{~mm})$ and Case $\mathrm{C}(h=38.1 \mathrm{~mm})$ with $\theta=26.5^{\circ}$. 


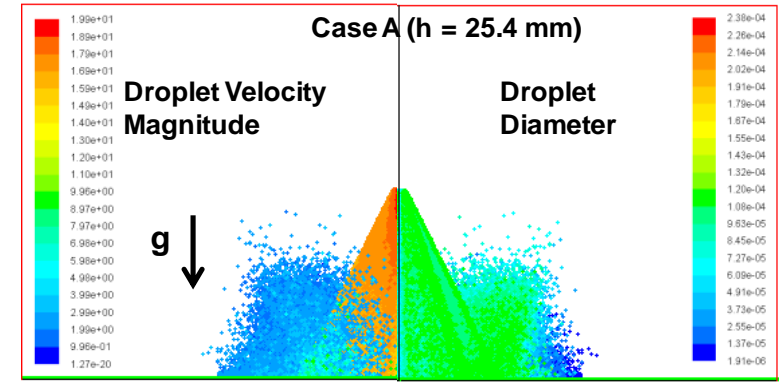

a)

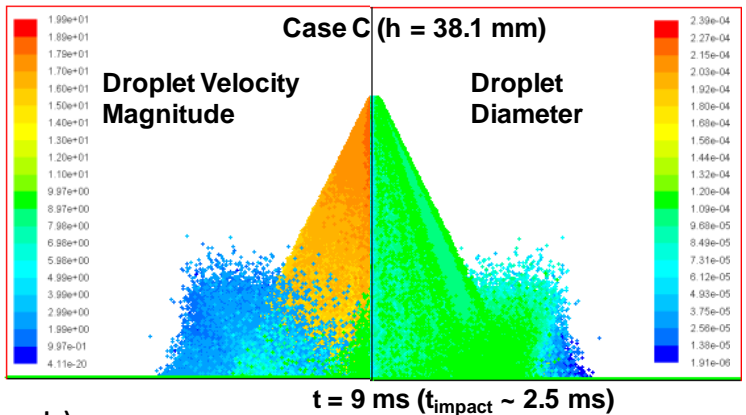

b)

Figure 4.21 Spray drop velocity and diameter distributions for (a) Case A ( $h=25.4 \mathrm{~mm})$ and (b) Case $\mathrm{C}(h=38.1 \mathrm{~mm})$ with same spray half angle, $\theta=26.5^{\circ}$. (Note: the results are shown at the equal effective time which is $t_{\text {eff }}=t-t_{\text {impact }}=6.5 \mathrm{~ms}$ for both cases. $g$ refers to gravity. Left part of images: Red refers to faster (also fastest) droplets $(18-20 \mathrm{~m} / \mathrm{s})$ while dark blue refers to slower (also slowest) droplets $(0-2 \mathrm{~m} / \mathrm{s})$. Right part of images: Green refers to bigger droplets $(0.11$ $0.155 \mathrm{~mm}$ ) while dark blue refers to smaller droplets $(0.002-0.026 \mathrm{~mm})$.)

\subsubsection{Effects of Varying Spray Half Angle ( $\theta)$}

In order to compare the effects of spray half angle $(\theta)$, Case D was simulated. Case D has the same nozzle-to-surface distance as Case $\mathrm{C}(h=38.1 \mathrm{~mm})$ but it has a smaller spray half angle, $\theta$ $=20.5^{\circ}$. Figure 4.22 shows the average drop diameters $d_{32}$ (Fig. 4.22.a) and $d_{10}$ (Fig. 4.22.b) for Cases C and D. The average drop diameters are not as similar as in Cases A, B and C. The narrower spray has significantly larger average drops. This result agrees with the manufacturer's literature (Schick, undated). Figure 4.23.a shows the liquid film mass accumulation $\left(m_{s}\right)$ on the surface and Figure 4.23.b shows the spray impact efficiency $(\eta)$ for Cases C and D. The liquid film mass and the spray impact efficiency show similar trends for both cases, but Case D has more liquid film mass and a higher impact efficiency at later times. The drop velocity and diameter distributions for Case C (Fig. 4.24.a) and Case D (Fig. 4.24.b) are shown in Figure 4.24. The left half of each figure shows the velocity and the right half shows the diameter distribution. Similar to the other spray cases, the drop velocity decreases as the spray approaches the impact surface and the splashed drops are slower and smaller compared to the initial spray drops (Fig. 4.24). 


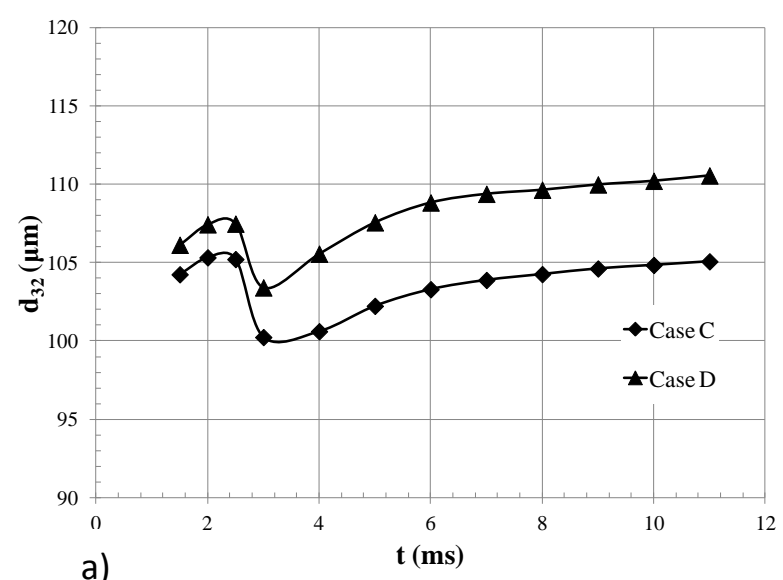

a)

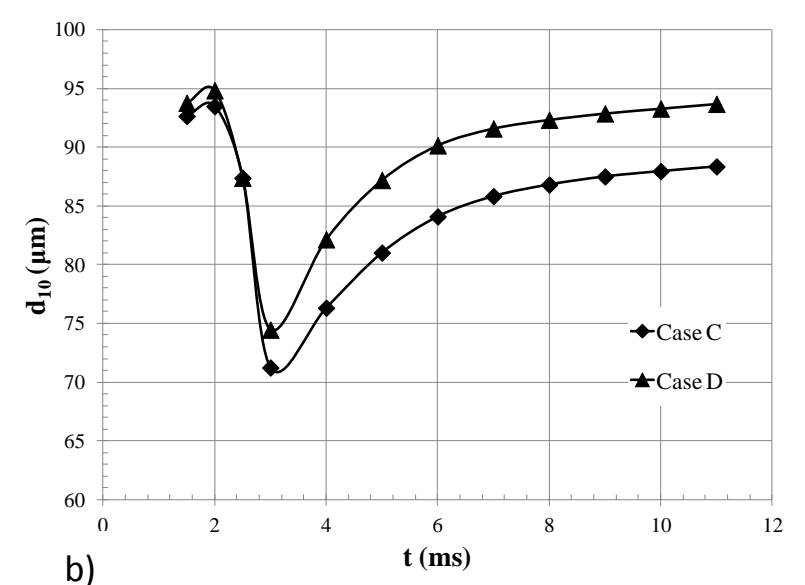

b)

Figure 4.22 (a) The Sauter mean drop diameter $\left(d_{32}\right)$ (b) the arithmetic mean drop diameter $\left(d_{10}\right)$ for Case $\mathrm{C}\left(\theta=26.5^{\circ}\right)$ and Case $\mathrm{D}\left(\theta=20.5^{\circ}\right)$ with $h=38.1 \mathrm{~mm}$.

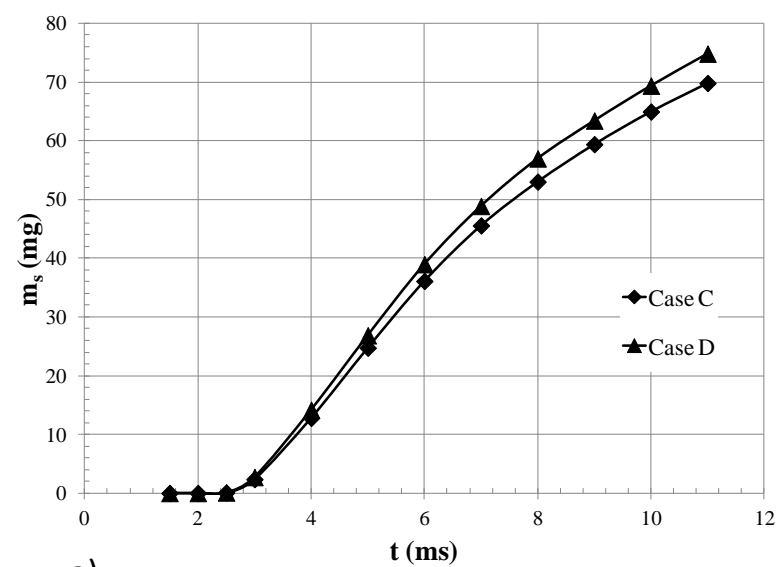

a)

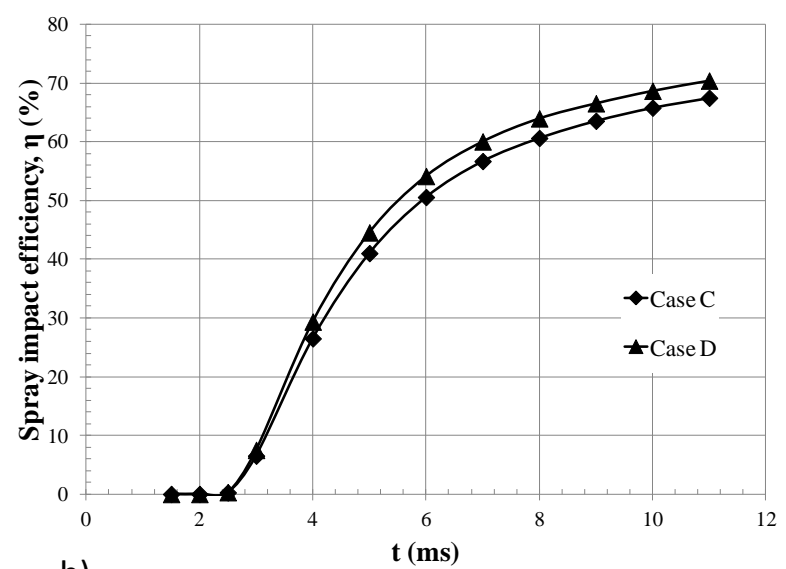

b)

Figure 4.23 (a) The liquid film mass accumulation $\left(m_{s}\right)$ (b) the spray impact efficiency $(\eta)$ for Case $\mathrm{C}\left(\theta=26.5^{\circ}\right)$ and Case $\mathrm{D}\left(\theta=20.5^{\circ}\right)$ with $h=38.1 \mathrm{~mm}$. 


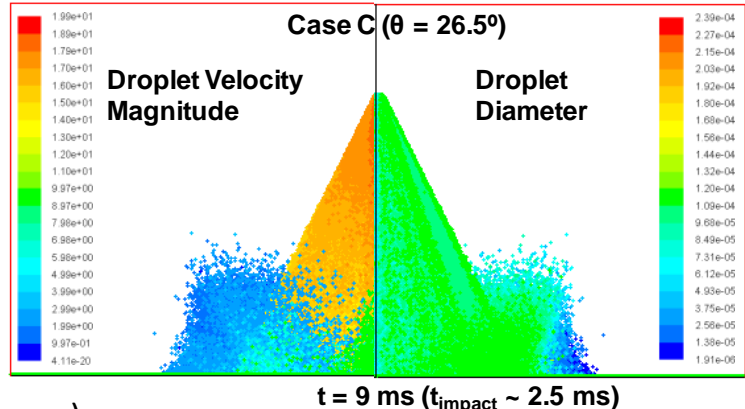

a)

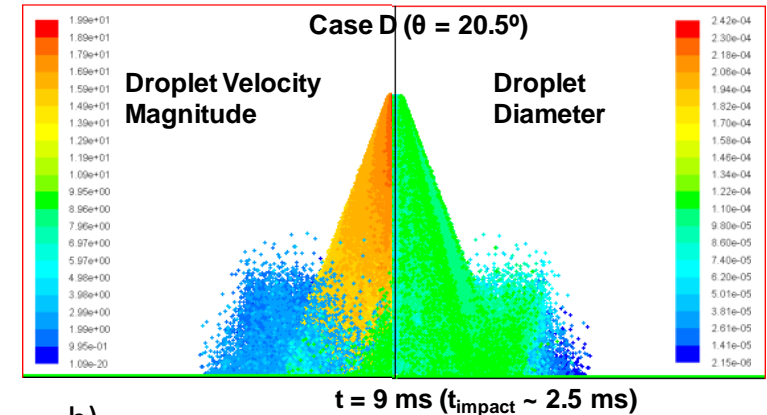

b)

Figure 4.24 The droplet velocity and diameter distributions for (a) Case C $\left(\theta=26.5^{\circ}\right)$ (b) Case D $\left(\theta=20.5^{\circ}\right)$ with same nozzle-to-surface distance, $h=38.1 \mathrm{~mm}$. (Note: the results are shown at the equal effective time which is $t_{\text {eff }}=t-t_{\text {impact }}=6.5 \mathrm{~ms}$ for both cases. Left part of images: Red refers to faster (also fastest) droplets $(18-20 \mathrm{~m} / \mathrm{s})$ while dark blue refers to slower (also slowest) droplets $(0-2 \mathrm{~m} / \mathrm{s})$ Right part of images: Green refers to bigger droplets $(0.11-0.155 \mathrm{~mm})$ while dark blue refers to smaller droplets (0.002-0.026 mm).)

\subsubsection{Effects of Varying Gravity $(g)$}

In order to compare the effects of gravity $(g)$ on sprays, Cases $\mathrm{E}$ and $\mathrm{F}$ were simulated. Cases $\mathrm{E}$ and $\mathrm{F}$ have the same spray parameters as Case $\mathrm{C}$ except gravity. Case $\mathrm{C}$ has Earth gravity $(g=-$ $\left.9.81 \mathrm{~m} / \mathrm{s}^{2}\right)$, Case E has Solar gravity $\left(g=-275 \mathrm{~m} / \mathrm{s}^{2}\right)$ and Case F has Earth gravity in the opposite direction, away from the impact surface $\left(g=+9.81 \mathrm{~m} / \mathrm{s}^{2}\right)$. Figure 4.25 shows the average drop diameters $d_{32}$ (Fig. 4.25.a) and $d_{10}$ (Fig. 4.25.b) for Cases C, E and F. The average drop diameters are similar but smaller drops occur in Case E (Solar gravity). Figure 4.26.a shows the liquid film mass accumulation $\left(m_{s}\right)$ on the surface and Figure 4.26.b shows the spray impact efficiency $(\eta)$ for Cases $\mathrm{C}, \mathrm{E}$ and $\mathrm{F}$. The liquid film mass and the spray impact efficiency show very similar trends for these cases, but Case $\mathrm{E}$ has slightly higher film mass and impact efficiency. For this spray, the effects of varying $g$ are quite small. 


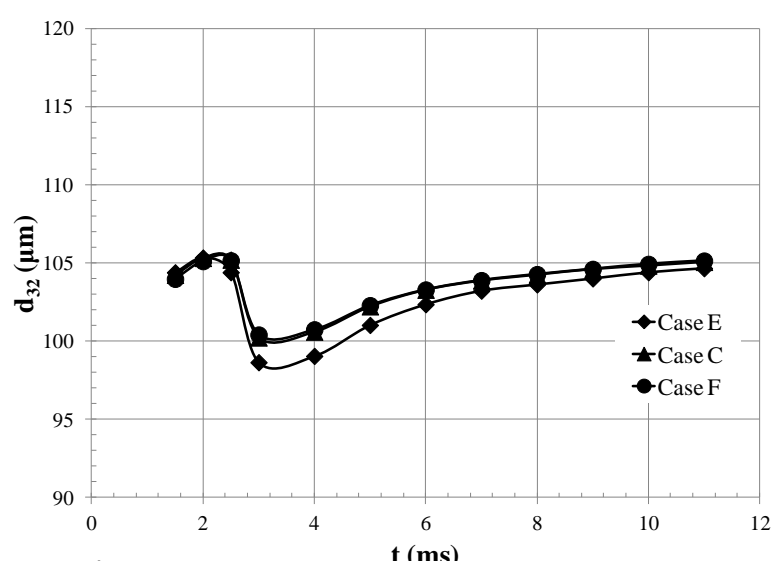

a)

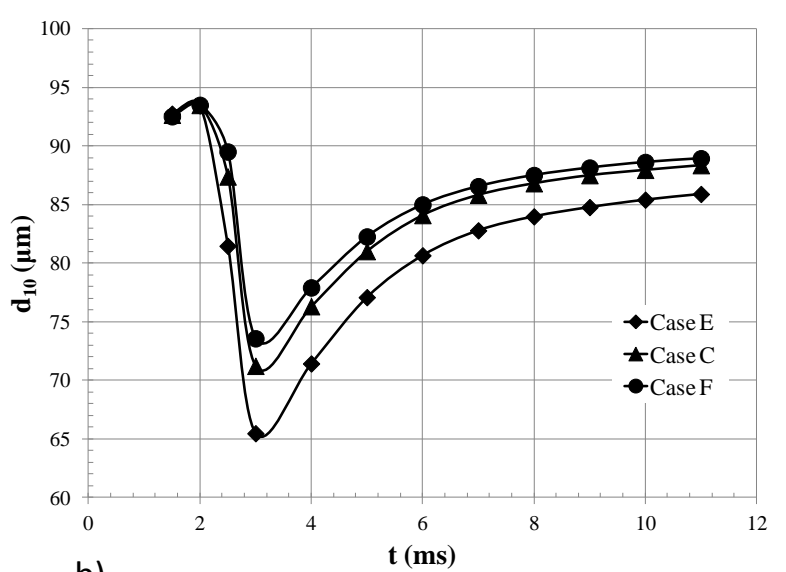

b)

Figure 4.25 (a) The Sauter mean drop diameter $\left(d_{32}\right)$ (b) the arithmetic mean drop diameter $\left(d_{10}\right)$ for Case C $\left(g=-9.81 \mathrm{~m} / \mathrm{s}^{2}\right)$, Case E $\left(g=-275 \mathrm{~m} / \mathrm{s}^{2}\right)$ and Case $\mathrm{F}\left(g=+9.81 \mathrm{~m} / \mathrm{s}^{2}\right)$. (Note that negative sign means gravity acting toward the surface.)

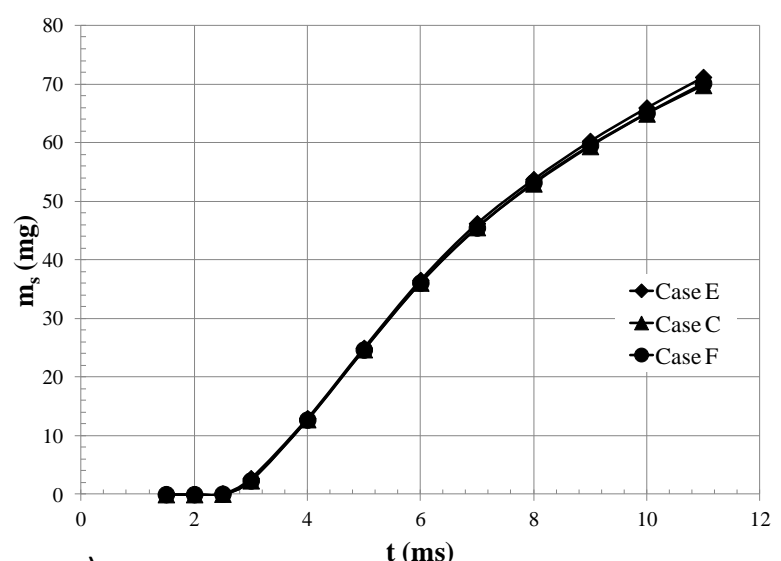

a)

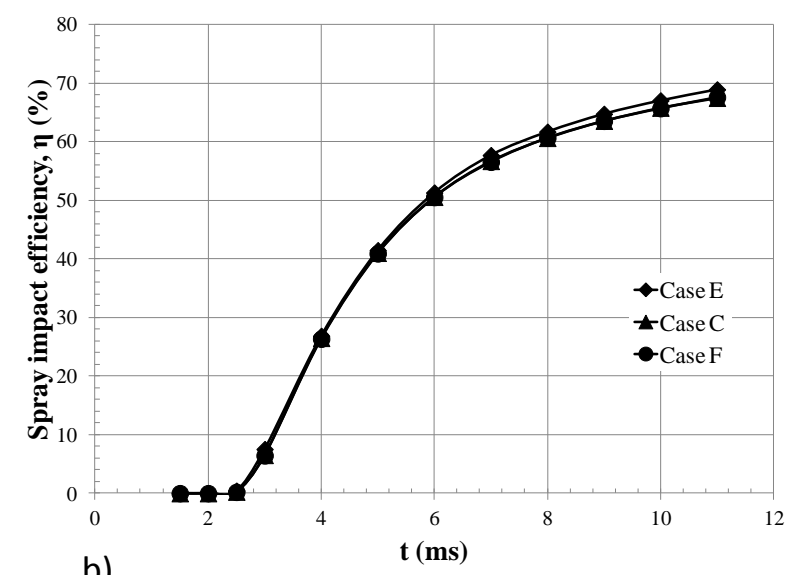

b)

Figure 4.26 (a) The liquid film mass accumulation $\left(m_{s}\right)$ (b) the spray impact efficiency $(\eta)$ for Case C $\left(g=-9.81 \mathrm{~m} / \mathrm{s}^{2}\right)$, Case E $\left(g=-275 \mathrm{~m} / \mathrm{s}^{2}\right)$ and Case F $\left(g=+9.81 \mathrm{~m} / \mathrm{s}^{2}\right)$.

\subsubsection{Effects of Varying Spray Mass Flow Rate (M)}

The effects of varying mass flow rate $(M)$ on sprays have been studied for Cases $\mathrm{C}, \mathrm{G}$ and $\mathrm{H}$. Cases $\mathrm{G}$ and $\mathrm{H}$ have the same spray parameters as Case $\mathrm{C}$ except the spray mass flow rate. Case $\mathrm{C}$ has an intermediate spray mass flow rate, $M=0.01207 \mathrm{~kg} / \mathrm{s}$, Case $\mathrm{G}$ has $M=0.01514 \mathrm{~kg} / \mathrm{s}$ (the highest mass flow rate) and Case $\mathrm{H}$ has $M=0.004 \mathrm{~kg} / \mathrm{s}$ (the lowest mass flow rate). Figure 4.27 shows the average drop diameters $d_{32}$ (Fig. 4.27.a) and $d_{10}$ (Fig. 4.27.b) for Cases C, G and H. The spray with the highest mass flow rate (Case G) has the biggest drops whereas the spray with 
the lowest mass flow rate (Case $\mathrm{H}$ ) has the smallest drops (Fig. 4.27.a and Fig. 4.27.b). This result agrees with the manufacturer's literature (Schick, undated). Figure 4.28.a shows the liquid film mass accumulation $\left(m_{s}\right)$ on the surface and Figure 4.28.b shows the spray impact efficiency $(\eta)$ for Cases $\mathrm{C}, \mathrm{G}$ and $\mathrm{H}$. The lowest mass flow rate (Case $\mathrm{H}$ ) has the least liquid film accumulation on the surface and the highest mass flow rate (Case $G$ ) has the most liquid film accumulation on the surface (Fig. 4.28.a). However, the spray impact efficiency shows similar trends for all mass flow rate cases, but the lowest mass flow rate case (Case $\mathrm{H}$ ) has a lower impact efficiency than other cases (Fig. 4.28.b).
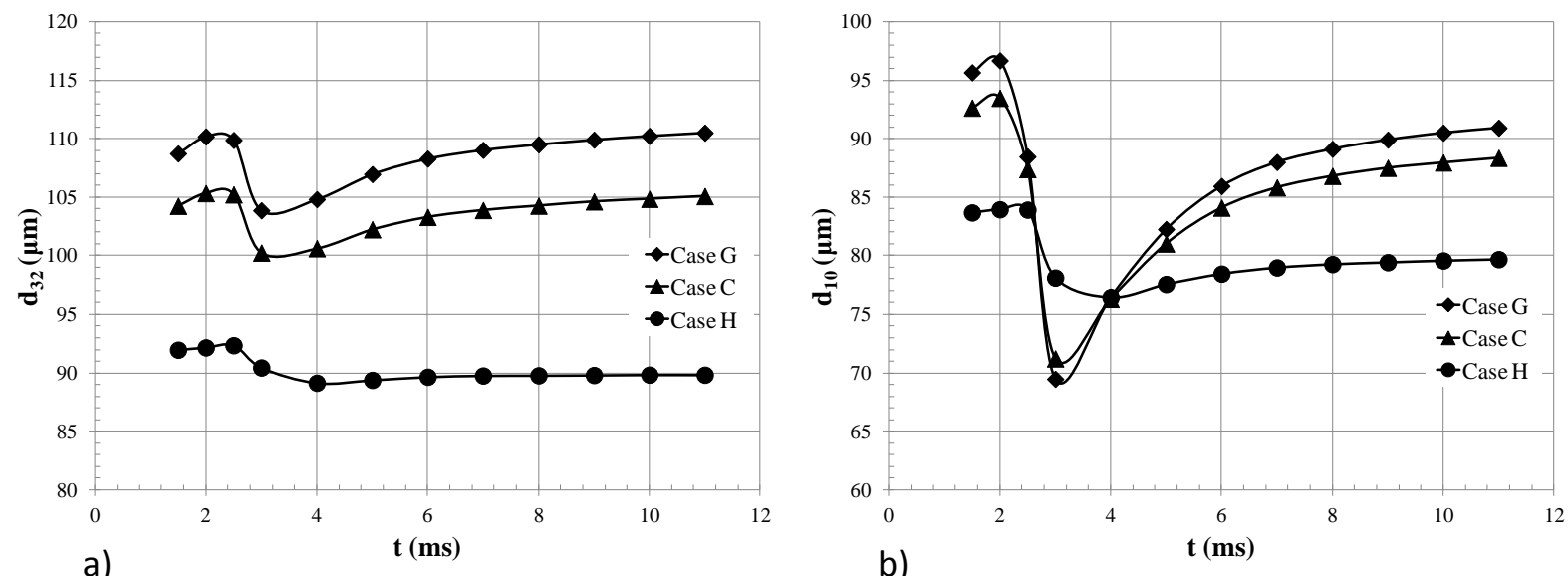

Figure 4.27 (a) The Sauter mean drop diameter $\left(d_{32}\right)$ (b) the arithmetic mean drop diameter $\left(d_{10}\right)$ for Case C $(M=0.01207 \mathrm{~kg} / \mathrm{s})$, Case $\mathrm{G}(M=0.01514 \mathrm{~kg} / \mathrm{s})$ and Case $\mathrm{H}(M=0.004 \mathrm{~kg} / \mathrm{s})$.

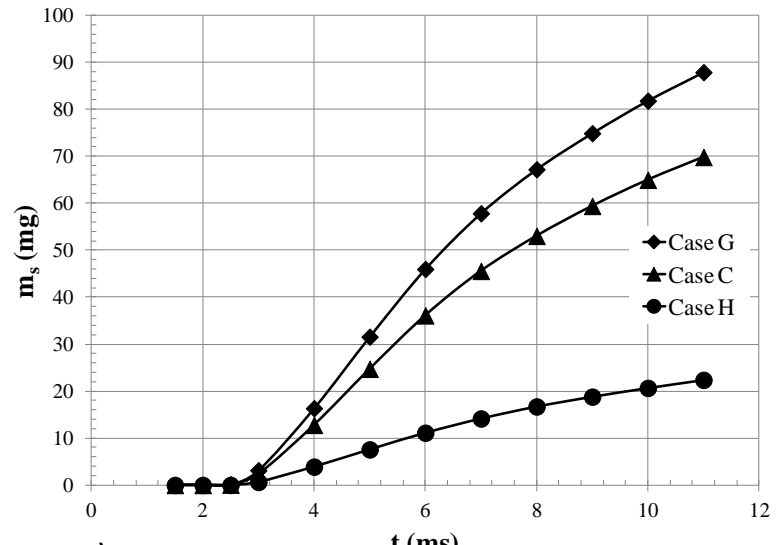

a)

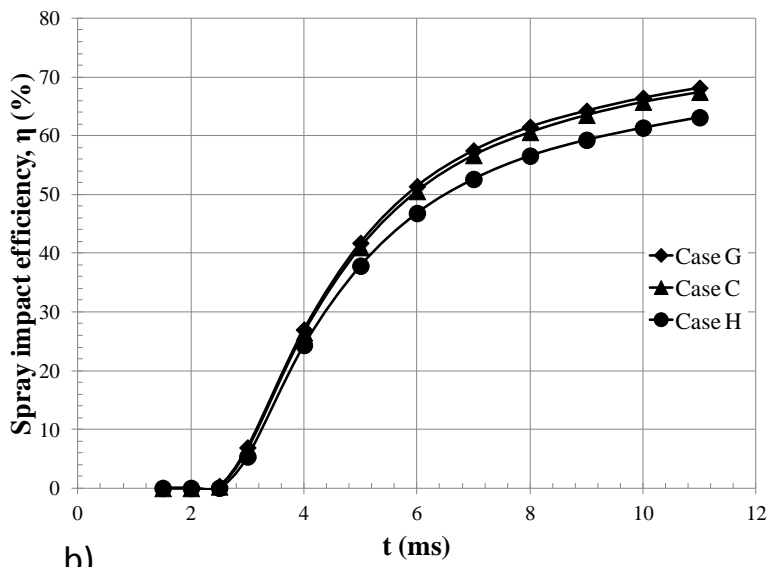

b)

Figure 4.28 (a) The liquid film mass accumulation $\left(m_{s}\right)$ (b) the spray impact efficiency $(\eta)$ for Case C $(M=0.01207 \mathrm{~kg} / \mathrm{s})$, Case $\mathrm{G}(M=0.01514 \mathrm{~kg} / \mathrm{s})$ and Case H $(M=0.004 \mathrm{~kg} / \mathrm{s})$. 


\subsubsection{Effects of Varying Spray Liquid Viscosity $(\mu)$}

The effects of varying liquid viscosity $(\mu)$ on sprays have been studied for Cases C and I. Case I has the same spray parameters as Case C except the liquid viscosity. Case I uses a hypothetical liquid (HypV) with a viscosity 5 times larger than water at room temperature. Figure 4.29 shows the average drop diameters $d_{32}$ (Fig. 4.29.a) and $d_{10}$ (Fig. 4.29.b) for Cases $\mathrm{C}$ and I. The more viscous spray (Case I) has significantly bigger drops compared to the less viscous spray (Case C). This result agrees with the manufacturer's literature (Schick, undated). Figure 4.30.a shows the liquid film mass accumulation $\left(m_{s}\right)$ on the surface and Figure 4.30.b shows the spray impact efficiency $(\eta)$ for Cases $\mathrm{C}$ and I. The liquid film accumulation on the surface and the spray impact efficiency are nearly independent of viscosity.

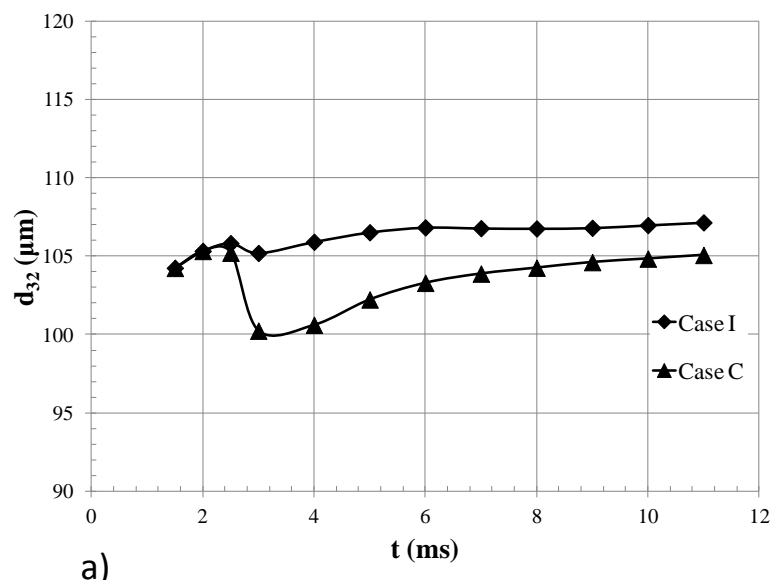

a)

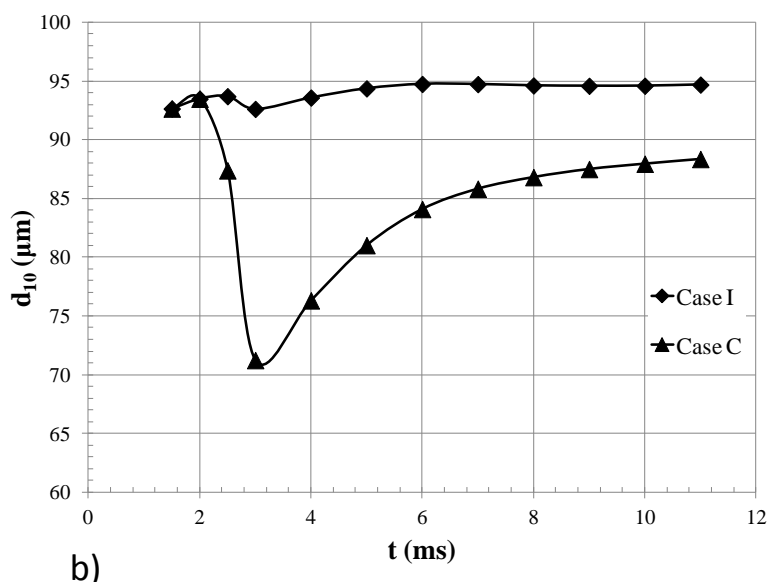

b)

Figure 4.29 (a) The Sauter mean drop diameter $\left(d_{32}\right)$ (b) the arithmetic mean drop diameter $\left(d_{10}\right)$ for Case C (water: $\mu=0.001 \mathrm{~kg} / \mathrm{m} . \mathrm{s}$ ) and Case I (HypV: $\mu=0.005 \mathrm{~kg} / \mathrm{m} . \mathrm{s}$ ). 


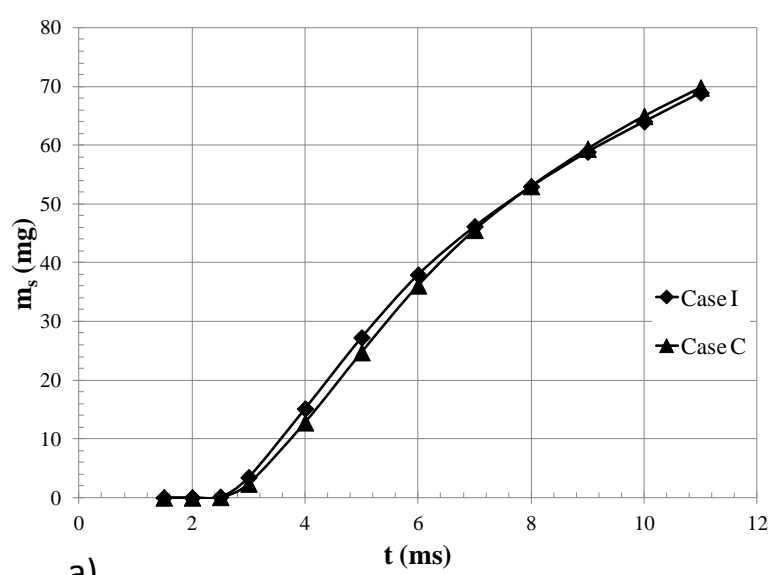

a)

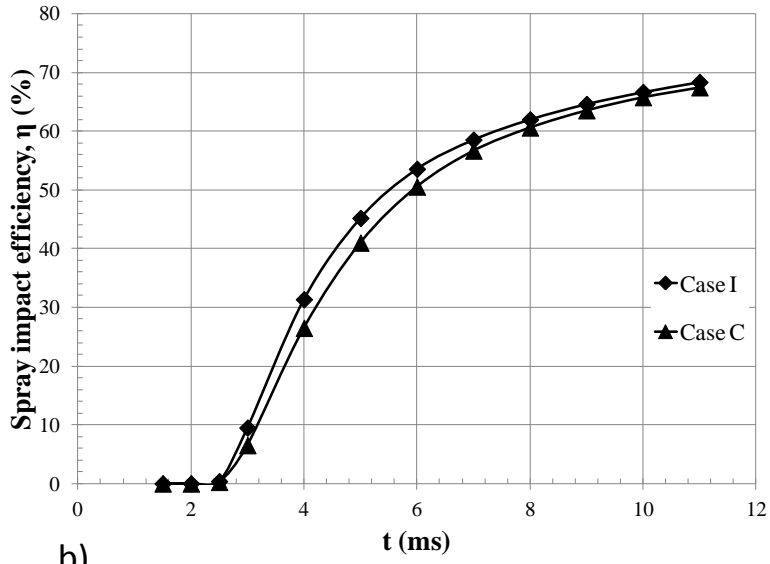

b)

Figure 4.30 (a) The liquid film mass accumulation $\left(m_{s}\right)$ (b) the spray impact efficiency $(\eta)$ for Case C (water: $\mu=0.001 \mathrm{~kg} / \mathrm{m} . \mathrm{s}$ ) and Case I (HypV: $\mu=0.005 \mathrm{~kg} / \mathrm{m} . \mathrm{s}$ ).

\subsubsection{Effects of Varying Spray Liquid Surface Tension $(\sigma)$}

The effects of varying liquid surface tension $(\sigma)$ on sprays have been studied for Cases C and J. Case $\mathbf{J}$ has the same spray parameters as Case $\mathrm{C}$ (water) except the liquid surface tension. Case $\mathrm{J}$ uses a hypothetical liquid (HypST) with surface tension equal to $0.01 \mathrm{~N} / \mathrm{m}$ which is less than water at room temperature $(\sigma=0.072 \mathrm{~N} / \mathrm{m})$. Figure 4.31 shows the average drop diameters $d_{32}$ (Fig. 4.31.a) and $d_{10}$ (Fig. 4.31.b) for Cases C and J. A lower surface tension (Case J) results in much smaller drops. This result agrees with the manufacturer's literature (Schick, undated). Figure 4.32.a shows the liquid film mass accumulation $\left(m_{s}\right)$ on the surface and Figure 4.32.b shows the spray impact efficiency $(\eta)$ for Cases $\mathrm{C}$ and $\mathrm{J}$. Initially, there is more liquid film mass accumulation on the surface for the high surface tension liquid (water, Case C) but eventually the liquid film mass becomes comparable for both cases. However, the higher surface tension liquid (water, Case C) has larger impact efficiency. 


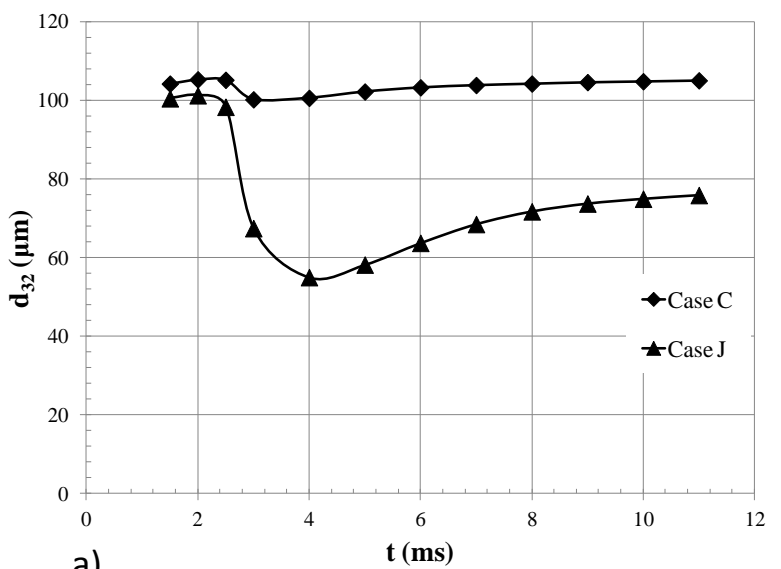

a)

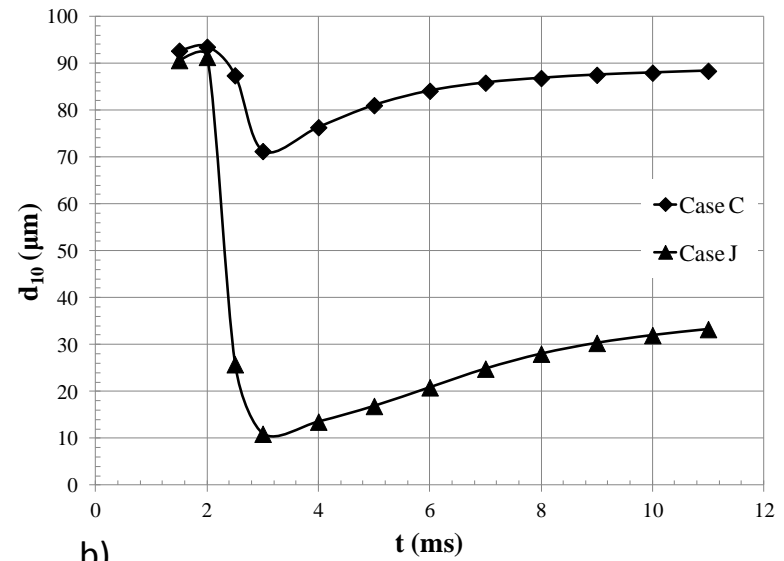

b)

Figure 4.31 (a) The Sauter mean drop diameter $\left(d_{32}\right)$ (b) the arithmetic mean drop diameter $\left(d_{10}\right)$ for Case C (water: $\sigma=0.072 \mathrm{~N} / \mathrm{m}$ ) and Case J (HypST: $\sigma=0.01 \mathrm{~N} / \mathrm{m}$ ).

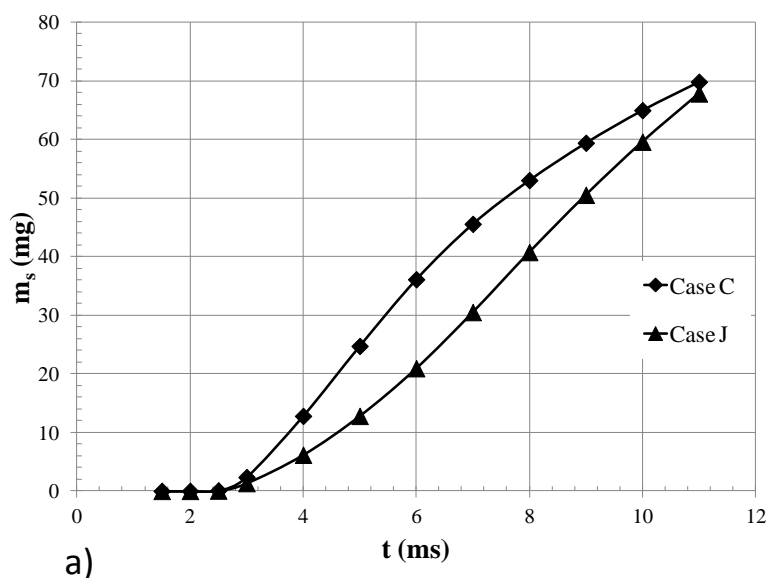

a)

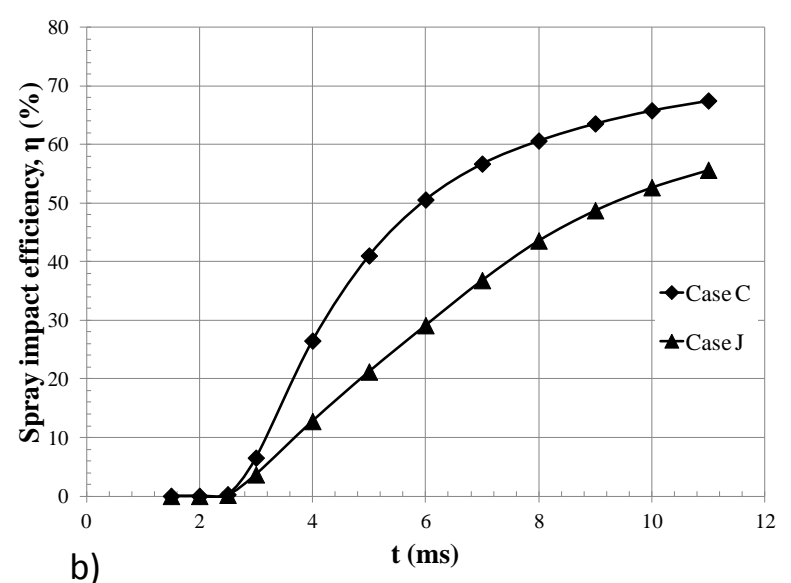

b)

Figure 4.32 (a) The liquid film mass accumulation $\left(m_{s}\right)$ (b) the spray impact efficiency $(\eta)$ for Case C (water: $\sigma=0.072 \mathrm{~N} / \mathrm{m}$ ) and Case J (HypST: $\sigma=0.01 \mathrm{~N} / \mathrm{m}$ ).

\subsubsection{Effects of Varying Spray Liquid Density ( $\rho)$}

The effects of varying liquid density $(\rho)$ on sprays have been studied for Cases C and K. Case K has the same spray parameters as Case $\mathrm{C}$ (water) except the liquid density. HypD is a hypothetical liquid with density equal to $3000 \mathrm{~kg} / \mathrm{m}^{3}$, which is around 3 times that of water at room temperature $\left(\rho=998 \mathrm{~kg} / \mathrm{m}^{3}\right)$. Figure 4.33 shows the average drop diameters $d_{32}$ (Fig. 4.33.a) and $d_{10}$ (Fig. 4.33.b) for Cases $\mathrm{C}$ and K. The more dense liquid (Case K) results in much smaller drops compared to the less dense water (Case C). Figure 4.34.a shows the liquid film mass accumulation $\left(m_{s}\right)$ on the surface and Figure 4.34.b shows the spray impact efficiency $(\eta)$ 
for Cases $\mathrm{C}$ and $\mathrm{K}$. There is more liquid film mass accumulation on the surface and higher impact efficiency for the less dense liquid (water, Case C). These results suggest that there is much more splashing with the more dense liquid.

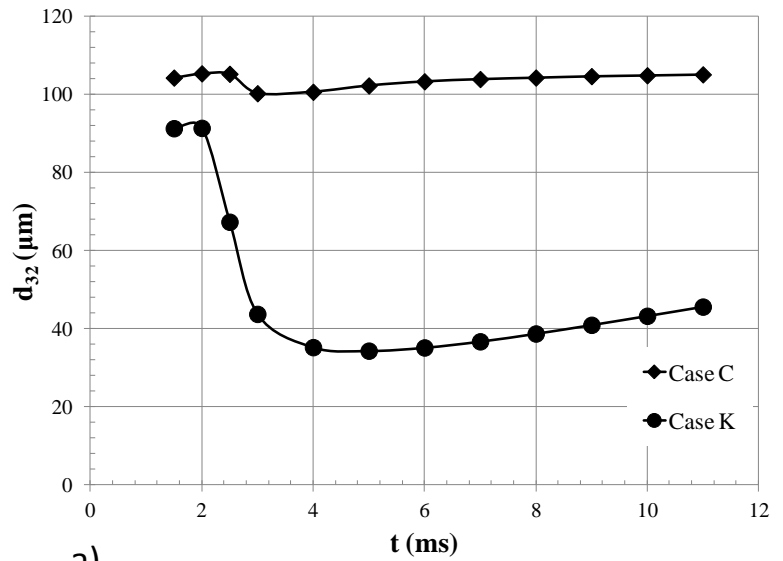

a)

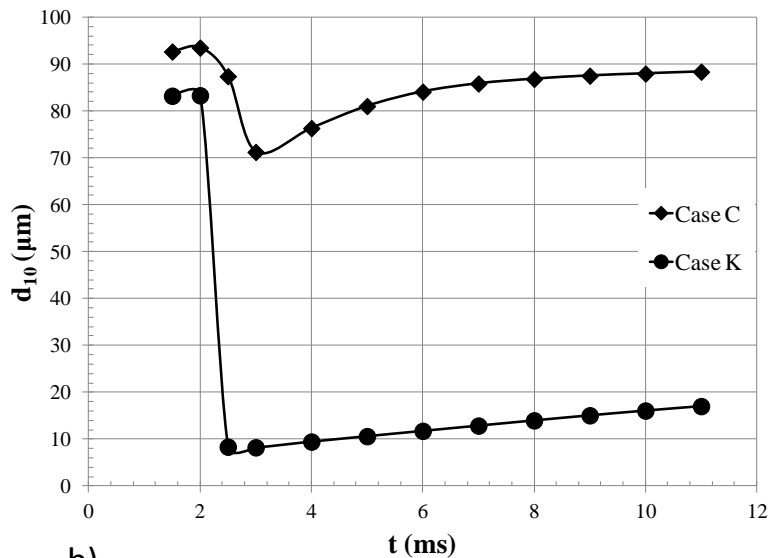

b)

Figure 4.33 (a) The Sauter mean drop diameter $\left(d_{32}\right)$ (b) the arithmetic mean drop diameter $\left(d_{10}\right)$ for Case C (water: $\rho=998 \mathrm{~kg} / \mathrm{m}^{3}$ ) and Case K (HypD: $\rho=3000 \mathrm{~kg} / \mathrm{m}^{3}$ ).

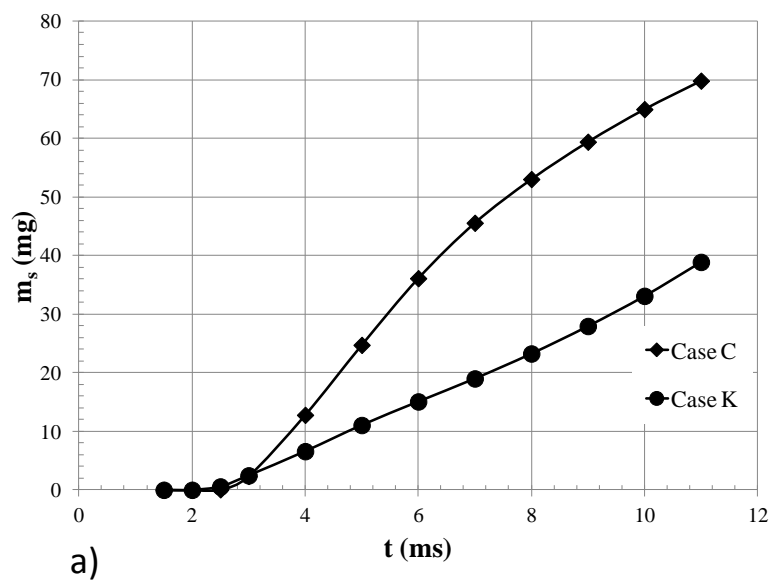

a)

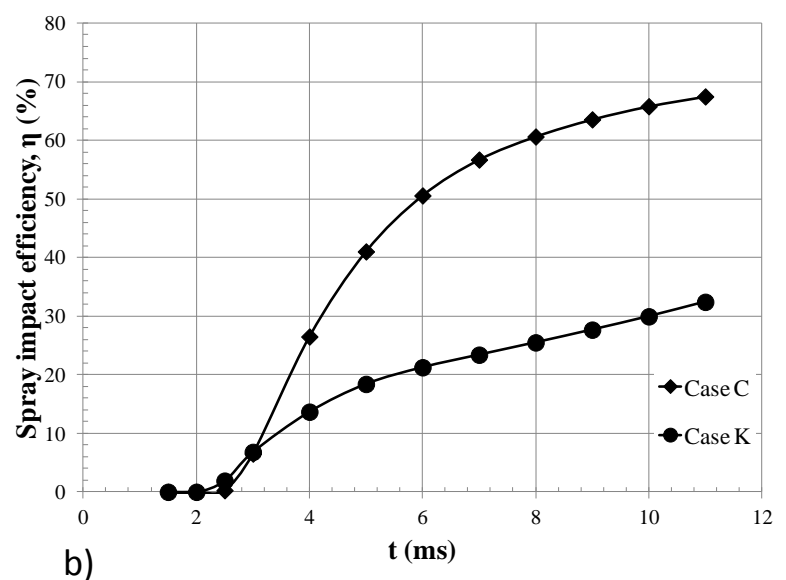

b)

Figure 4.34 (a) The liquid film mass accumulation $\left(m_{s}\right)$ (b) the spray impact efficiency $(\eta)$ for Case C (water: $\rho=998 \mathrm{~kg} / \mathrm{m}^{3}$ ) and Case K (HypD: $\rho=3000 \mathrm{~kg} / \mathrm{m}^{3}$ ). 


\subsection{Summary of the $2 D$ axisymmetric Full Cone Spray Simulations}

Figure 4.35 shows the effects of all the basic parameters presenteed previously on the total number of spray drops. For the conditions studied, these effects can be briefly summarized as

- Increasing the nozzle-to-surface $(h)$ distance increases the total number of drops (Fig. 4.35.a).

- Increasing the spray half angle $(\theta)$ increases the total number of drops (Fig. 4.35.b).

- Increasing gravity $(g)$ by a factor of 28 slightly increases the total number of drops (Fig. 4.35.c).

- Reversing the direction of Earth gravity has a negligible effect (Fig. 4.35.c).

- Increasing the mass flow rate $(M)$ increases the total number of drops (Fig. 4.35.d).

- Increasing the liquid viscosity decreases the total number of drops (Fig. 4.35.e).

- Increasing the surface tension greatly decreases the total number of drops (Fig. 4.35.f).

- Increasing the liquid density greatly increases the total number of drops (Fig. 4.35.f). 


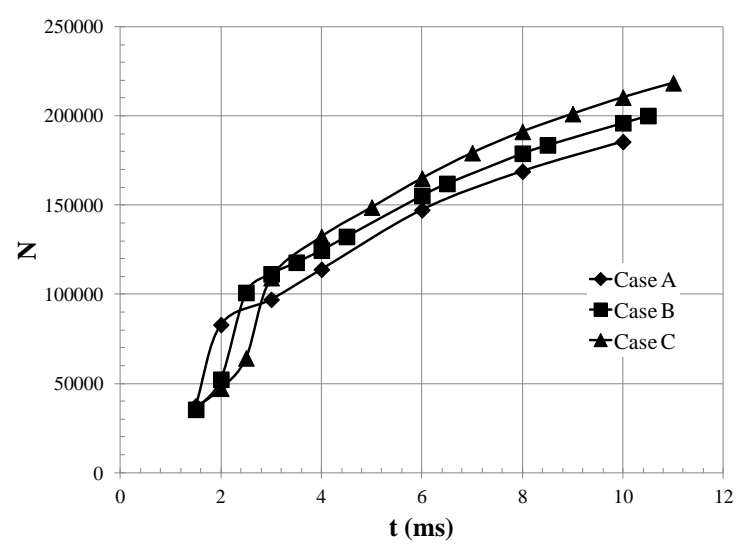

a) Effects of $h$ : Case A $(h=25.4 \mathrm{~mm})$, Case B $(h=31.75 \mathrm{~mm}$, Case C $(h=38.1 \mathrm{~mm})$

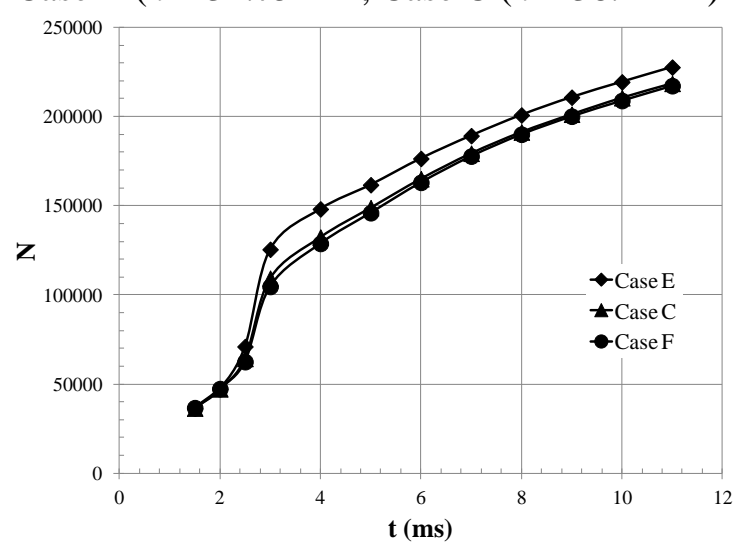

c) Effects of $\boldsymbol{g}$ : Case C $\left(g=-9.81 \mathrm{~m} / \mathrm{s}^{2}\right)$, Case $\mathrm{E}\left(g=-275 \mathrm{~m} / \mathrm{s}^{2}\right)$, Case F $\left(g=+9.81 \mathrm{~m} / \mathrm{s}^{2}\right)$

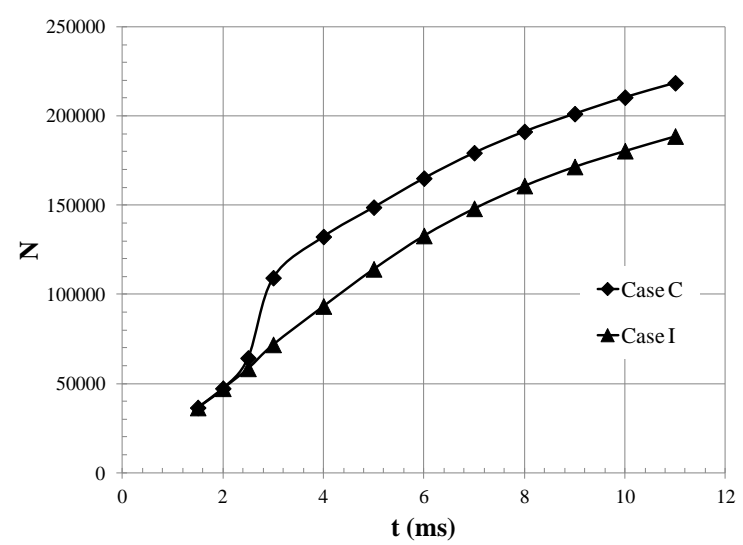

e) Effects of $\mu$ : Case $\mathrm{C}(\mu=0.001 \mathrm{~kg} / \mathrm{m} . \mathrm{s})$,

Case I $(\mu=0.005 \mathrm{~kg} / \mathrm{m} . \mathrm{s})$

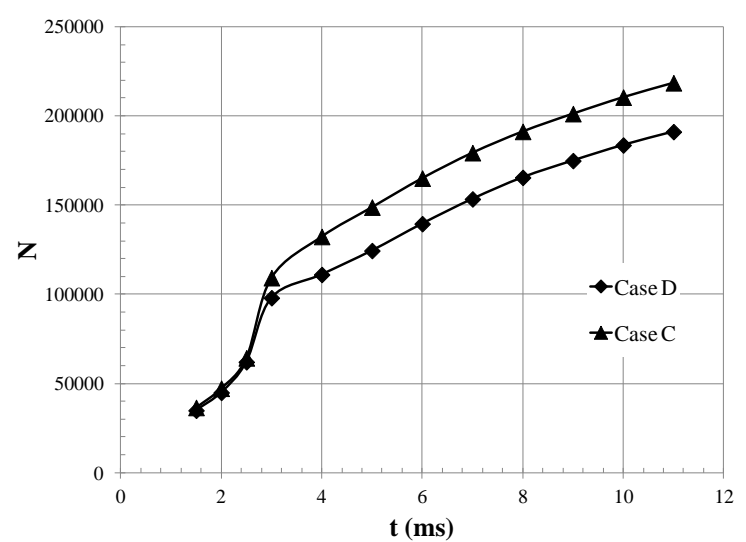

b) Effects of $\boldsymbol{\theta}$ : Case C $\left(\theta=26.5^{\circ}\right)$, Case $\mathrm{D}(\theta=$ $\left.20.5^{\circ}\right)$

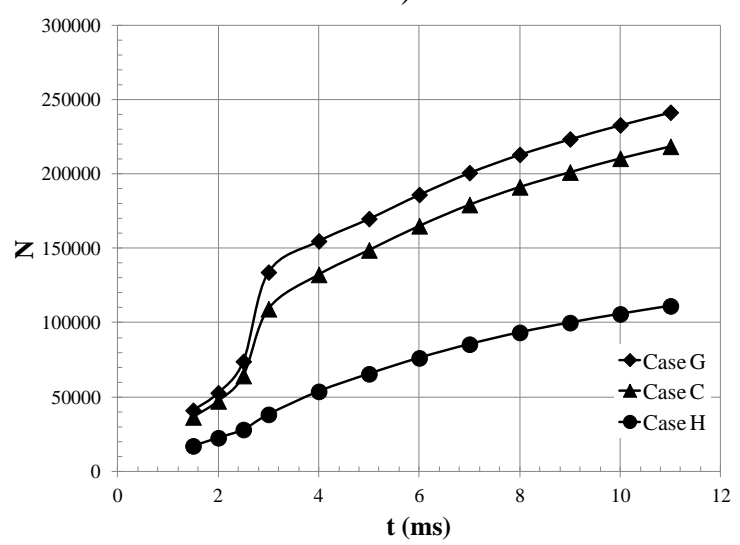

d) Effects of $\boldsymbol{M}$ : Case C $(M=0.01207 \mathrm{~kg} / \mathrm{s})$, Case $\mathrm{G}(M=0.01514 \mathrm{~kg} / \mathrm{s})$, Case H $(M=0.0040$ $\mathrm{kg} / \mathrm{s}$ )

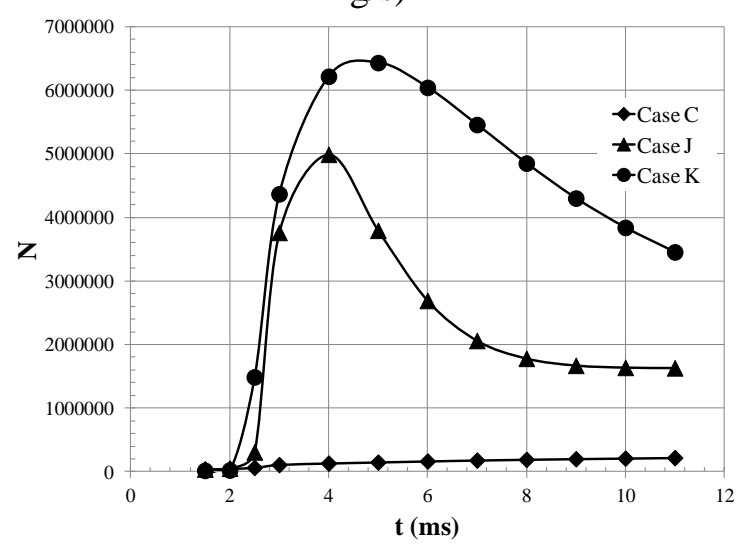

f) Effects of $\boldsymbol{\rho}$ and $\boldsymbol{\sigma}$ : Case C $\left(\rho=998 \mathrm{~kg} / \mathrm{m}^{3}, \sigma=\right.$ $0.072 \mathrm{~N} / \mathrm{m})$, Case $\mathrm{J}\left(\rho=998 \mathrm{~kg} / \mathrm{m}^{3}, \sigma=0.01 \mathrm{~N} / \mathrm{m}\right)$, Case K $\left(\rho=3000 \mathrm{~kg} / \mathrm{m}^{3}, \sigma=0.072 \mathrm{~N} / \mathrm{m}\right)$

Figure 4.35 Effects of basic spray parameters on total number of drops $(N)$. 


\subsection{Limitations of the 2D Axisymmetric Model for the Calculations of Spray-Wall Interactions}

Spray distribution and spray-wall interactions were successfully calculated using the Discrete Phase Model (DPM) and the wall film sub-model within the DPM in ANSYS Fluent as described in previous sections. However, further analysis showed that the film characteristics were not accurate compared to the experiments and that there was a serious issue of particle accumulation near the axis which resulted in an unrealistic film thickness (even more than domain height of $50 \mathrm{~mm}$ ) at that region.

This issue of unrealistic film particle accumulation near the axis could not be solved even though several test cases with different cells sizes and model parameters were tried. Therefore another approach for modeling the spray-wall interactions in ANSYS Fluent was needed. After a detailed search, it was decided that the Eulerian Wall Film (EWF) model could be used for the calculations of spray-wall interactions with the DPM being used for the calculations of spray formation and spray particle motion. This approach is the subject of the next chapter. 


\section{CHAPTER 5: SPRAY IMPACT AND COOLING SIMULATIONS WITHOUT PHASE CHANGE USING THE DISCRETE PHASE MODEL (DPM) AND THE EULERIAN WALL FILM (EWF) MODEL}

This chapter is primarily based on the Project Final Report by Kuhlman, Gray, Dinc, Hillen, Medam, and Taylor (2014) and publication by Dinc, Gray, Kuhlman, Hillen, Medam and Taylor (2014). Calculations reported in the previous chapter were successfully performed using the DPM model and its submodels, in particular the DPM wall film submodel. The predicted spray characteristics such as the drop diameter and velocity distributions were comparable to values found in the literature. However, the DPM wall film submodel in 2D axisymmetric simulations predicted film thicknesses that were unrealistic, especially at later times. In particular there was an unrealistic accumulation of water at the center of the computational domain. Efforts to remove this discrepancy using the DPM wall film submodel were unsuccessful. Therefore efforts were redirected toward using the ANSYS Fluent Eulerian Wall Film (EWF) model. In this chapter, spray and spray cooling simulations using the DPM and EWF models together are described. Simulations performed in this chapter do not include phase change. Phase change will be discussed in Chapter 6. In this chapter and in the other chapters, the variables and Case numbers are defined separately. Thus, the symbols shown in this chapter are often not the same as the symbols shown in the other chapters. The Cases mentioned in this chapter are different from the Cases mentioned in other chapters even though same Case number might be used.

\subsection{Numerical Modeling and Governing Equations}

Table 5.1 shows the outline of the cases presented in Chapter 5 for the purpose of obtaining an accurate spray-wall interaction model with the optimum computational domain and mesh size and shape. Spray-wall film interactions were calculated using two different models, the wall film submodel of the DPM (Case 1) and the Eulerian Wall Film (EWF) model (Case 2) using the 3D Discrete Phase Model (DPM) 90 cylindrical domain for a 40 psi full cone spray. The optimum computational domain size was investigated using several domain sizes $\left(90^{\circ}, 45^{\circ}, 30^{\circ}\right)$ for the 3D DPM and Eulerian Wall Film (EWF) with cylindrical domain. However, only $90^{\circ}$ (Case 2) and $30^{\circ}$ (Case 3 ) cylindrical domain cases are presented in this dissertation. The computational cell size and shape were investigated (non-uniform triangular cells (Case 3), using uniform quad 
cells (Case 4)) using the 3D DPM and Eulerian Wall Film (EWF) model. Case 5 is the finalized model for the spray cooling simulations which includes optimum computational domain size, shape and mesh size and cell shape. The details about these cases and the results and discussions about each are explained in this chapter.

Table 5.1 Outline of the Cases Simulated and Discussed in Chapter-5.

\begin{tabular}{|c|c|c|c|c|}
\hline Case & Spray Model & $\begin{array}{c}\text { Spray-Wall } \\
\text { Interaction Model }\end{array}$ & $\begin{array}{c}\text { Computational } \\
\text { Domain }\end{array}$ & $\begin{array}{c}\text { Computational } \\
\text { Cell }\end{array}$ \\
\hline 1 & $\begin{array}{c}\text { Discrete Phase Model } \\
\text { (DPM) }\end{array}$ & $\begin{array}{l}\text { Wall-film submodel } \\
\text { of the DPM }\end{array}$ & $\begin{array}{c}\text { 3D } 90^{\circ} \text { Cylindrical } \\
\text { Domain }\end{array}$ & $\begin{array}{l}\text { Non-uniform } 0.5 \\
\text { mm triangular } \\
\text { prisms }\end{array}$ \\
\hline 2 & $\begin{array}{c}\text { Discrete Phase Model } \\
\text { (DPM) }\end{array}$ & $\begin{array}{l}\text { Eulerian Wall Film } \\
\text { (EWF) model }\end{array}$ & $\begin{array}{c}\text { 3D } 90^{\circ} \text { Cylindrical } \\
\text { Domain }\end{array}$ & $\begin{array}{c}\text { Non-uniform } 0.5 \\
\text { mm triangular } \\
\text { prisms }\end{array}$ \\
\hline 3 & $\begin{array}{c}\text { Discrete Phase Model } \\
\text { (DPM) }\end{array}$ & $\begin{array}{l}\text { Eulerian Wall Film } \\
\text { (EWF) model }\end{array}$ & $\begin{array}{c}\text { 3D } 30^{\circ} \text { Cylindrical } \\
\text { Domain }\end{array}$ & $\begin{array}{c}\text { Non-uniform } 0.5 \\
\text { mm triangular } \\
\text { prisms }\end{array}$ \\
\hline 4 & $\begin{array}{c}\text { Discrete Phase Model } \\
\text { (DPM) }\end{array}$ & $\begin{array}{l}\text { Eulerian Wall Film } \\
\text { (EWF) model }\end{array}$ & $\begin{array}{c}\text { 3D } 90^{\circ} \text { Rectangular } \\
\text { Domain }\end{array}$ & $\begin{array}{l}\text { Uniform } 0.5 \mathrm{~mm} \\
\text { cubes }\end{array}$ \\
\hline 5 & $\begin{array}{c}\text { Discrete Phase Model } \\
\text { (DPM) }\end{array}$ & $\begin{array}{l}\text { Eulerian Wall Film } \\
\text { (EWF) model }\end{array}$ & $\begin{array}{c}\text { 3D } 90^{\circ} \text { Rectangular } \\
\text { Smaller Domain } \\
\text { (Final) }\end{array}$ & $\begin{array}{l}\text { Uniform } 0.5 \mathrm{~mm} \\
\text { cubes }\end{array}$ \\
\hline
\end{tabular}

\subsubsection{The Eulerian Wall Film (EWF) Model}

The Eulerian Wall Film (EWF) model in ANSYS Fluent can be used to calculate the wall film characteristics such as the liquid film velocity, thickness, and temperature. In the EWF model, the Eulerian conservation equations for mass, momentum, and energy are integrated over the film thickness resulting in 2D "depth-averaged" equations to be solved, even though this model is only applicable for 3D domains. The EWF model assumes a parabolic velocity profile with no slip at the wall and a maximum at the free surface. A temperature profile across the wall film consisting of two linear segments whose endpoints are the wall temperature, the mean film temperature, and the free surface temperature is also assumed.

Conservation of mass in the film is solved using (ANSYS Fluent Theory Guide, 2013) 


$$
\frac{\partial h}{\partial t}+\nabla_{s} \cdot\left(h \underline{V_{l}}\right)=\frac{\dot{m}_{s}}{\rho_{l}}
$$

where $h$ is the film height, $\underline{V}_{l}$ is the depth averaged film velocity, $\nabla_{\mathrm{s}}$ is the surface gradient operator, $\rho_{l}$ is the liquid density, $\dot{m}_{s}$ is the mass source per unit wall area due to droplet impact and phase change (if phase change is considered in the model).

Conservation of film momentum is solved using

$$
\frac{\partial\left(h \underline{V_{l}}\right)}{\partial t}+\nabla_{s} \cdot\left(h \underline{h V_{l} V_{l}}\right)=-\frac{h \nabla_{s}\left(P_{L}\right)}{\rho_{l}}+\left(\underline{g_{\tau}}\right) h+\left(\frac{3}{2 \rho_{l}}\right)\left(\underline{\tau_{f s}}\right)-\left(\frac{3 v_{l}}{h}\right)\left(\underline{V_{l}}\right)+\frac{\dot{q}_{i m p}}{\overline{\rho_{l}}}
$$

where

$P_{L}$ is the pressure acting on the film

$$
\begin{aligned}
& P_{L}=P_{g a s}+P_{h} \\
& P_{h}=-\rho_{l} h
\end{aligned}
$$

$\underline{g_{\tau}}$ is the acceleration due to gravity in the direction parallel to the film

$\tau_{f s}$ is the viscous shear force acting at the interface of film and air

$v_{l}$ is the film liquid kinematic viscosity

$\dot{q}_{i m p}$ is the source term due to drops impingement on the surface.

$$
\dot{\dot{q}}_{i m p}=\dot{m}_{s}\left(\underline{V_{p}}-\underline{V_{l}}\right)
$$

where $\dot{m}_{s}$ is the mass flow rate of the particle stream (droplet) impinging on the surface and $V_{p}$ is the velocity of the droplets impinging on the surface.

The first term on the right hand side represents the effects of gas-flow pressure and the gravity component normal to the surface. The second term on the right hand side includes the effect of gravity in the direction parallel to the film. The third term on the right hand side is the viscous shear force at the gas-film interface divided by the liquid density. The fourth term on the right hand side is the viscous force in the film divided by the liquid density. The fifth term on the right hand side includes the effect of the source term (e.g. droplet impact and splashing.). 
Conservation of energy in the film is solved using

$$
\frac{\partial\left(h T_{l}\right)}{\partial t}+\nabla_{s} \cdot\left(\underline{V_{l}} h T_{l}\right)=\frac{1}{\rho_{l} c_{p}}\left[2 k_{l}\left(\frac{T_{s}-T_{w}}{h}-\frac{2 T_{l}}{h}\right)+\dot{q}+\dot{m}_{v a p} h_{f g} T_{s}\right]
$$

where $T_{l}$ is the average liquid film temperature

$T_{s}$ is the temperature at the film-gas interface

$c_{p}$ is the specific heat of the liquid

$k_{l}$ is the thermal conductivity of the liquid

$T_{w}$ is the wall temperature

$\dot{q}$ is the source term coming from the spray (DPM)

$\dot{m}_{v a p}$ is the mass vaporization or condensation rate if the phase change in the film is included $h_{f g}$ is the latent heat.

\subsubsection{The Discrete Phase Model (DPM) for the Droplet Heat and Mass Transfer Calculations and the Governing Equations}

The DPM, which was described in Chapter 4, is used to calculate the spray. In the DPM, there are different heat transfer modes available in order to model energy and mass transfer between the particles (e.g. inert particles, reacting particles, droplets) and the surrounding gas (air) (ANSYS Fluent Theory Guide, 2013). Three different laws were considered in spray cooling simulations (inert heating: Law 1, droplet vaporization: Law 2, and droplet boiling: Law 3) to account for the heat and mass transfer between the spray drops and surrounding gas. In the following paragraphs these heat and mass transfer modes (laws) are explained with the relevant physical equations and assumptions.

\subsubsection{Inert Heating: Law 1}

This heating and cooling mechanism in ANSYS Fluent is implemented when the droplet temperature $\left(T_{p}\right)$ is less than the vaporization temperature ( $\left.T_{\text {vap }}\right)$ (ANSYS Fluent Theory Guide, 2013). During this stage, a heat balance is applied to calculate the droplet temperature due to convective heat transfer until it reaches to the vaporization temperature, at which time a different law is used (ANSYS Fluent Theory Guide, 2013). The heat balance equation is 


$$
m_{p} c_{p} \frac{d T_{p}}{d t}=H A_{p}\left(T_{a}-T_{p}\right)
$$

where $m_{p}$ is the mass of droplet

$c_{p}$ is the heat capacity of droplet

$T_{p}$ is the temperature of droplet

$H$ is the heat transfer coefficient of droplet

$A_{p}$ is the surface area of droplet

$T_{a}$ is the temperature of air (continuous phase).

The heat transfer coefficient $(H)$ is calculated using the Ranz and Marshall correlation (Ranz and Marshall, 1952) which is

$$
H=k_{a}\left(2+0.6 \sqrt{R e_{d}} \sqrt[3]{P r}\right) / d_{p}
$$

where $k_{a}$ is the thermal conductivity of air (continuous phase)

$R e_{d}$ is the Reynolds number based on the droplet diameter and the relative velocity between droplet and air

$\operatorname{Pr}$ is the Prandtl number of air

$d_{p}$ is the droplet diameter.

There is a coupling between the droplet phase and the continuous phase (air). Thus, the heat lost or gained by the droplet appears as a source or sink term in the continuous phase energy equation (ANSYS Fluent Theory Guide, 2013).

\subsubsection{Droplet Vaporization: Law 2}

The vaporization from the droplet is calculated when the droplet temperature $\left(T_{p}\right)$ reaches the vaporization temperature $\left(T_{\text {vap }}\right)$. This law continues until the droplet temperature reaches the boiling temperature (ANSYS Fluent Theory Guide, 2013). However, in the present spray cooling simulations since the air and droplet (spray) temperatures are either the same or close to each other so there will not be a significant effect of Law 2 (droplet vaporization). Heat transfer to the 
droplet at this stage is calculated by including the latent heat term in the heat transfer balance equation

$$
m_{p} c_{p} \frac{d T_{p}}{d t}=H A_{p}\left(T_{a}-T_{p}\right)-\frac{d m_{p}}{d t} h_{f g}
$$

where $h_{f g}$ is the latent heat of evaporation and $d m_{p} / d t$ is the rate of evaporation $(\mathrm{kg} / \mathrm{s})$. The phase change simulations will be discussed in Chapter 6.

\subsubsection{Droplet Boiling: Law 3}

This mechanism starts when the droplet temperature reaches the boiling temperature of the droplet liquid $\left(T_{b p}\right)$. The droplet diameter is calculated using the boiling rate equation which is (ANSYS Fluent Theory Guide, 2013).

$$
\frac{d\left(d_{p}\right)}{d t}=\frac{4 k_{a}}{\rho_{p} c_{p a} d_{p}}\left(1+0.23 \sqrt{R e_{d}}\right) \ln \left[1+\frac{c_{p a}\left(T_{a}-T_{p}\right)}{h_{f g}}\right]
$$

where $k_{a}$ is the thermal conductivity of air

$\rho_{p}$ is the droplet density

$c_{p a}$ is the specific heat of air.

The evaporated liquid couples to the gas phase as a species defined using the Species Transport Model. For instance water vapor is generated from liquid water in spray cooling simulations. Since no boiling is discussed in this chapter, this model will be used in the simulations mentioned in Chapter 6. 


\subsection{Initial Comparisons of the DPM Wall Film Model (Case 1) and the EWF Model (Case}

2)

In order to compare the DPM wall film submodel with the EWF model the same sprays were simulated in 3D geometries using both methods. In Case 1, the DPM model was used to calculate the trajectories of the spray droplets, the two-way coupling of the spray droplets with the surrounding air, and the surface layer characteristics using the DPM wall film submodel as explained in Chapter 4. Alternatively, in Case 2 the EWF model was used to calculate the surface film with source terms at the free surface for mass, momentum, and energy calculated using the spray characteristics computed by the DPM.

Simulations were performed using two different workstations with run times of around 7 days per case. The boundary conditions and computational domain used to compare the DPM wall film and EWF models are shown in Figure 5.1. The domain is a $90^{\circ}$ quarter cylinder containing 473,796 triangular prism computational cells with an average $0.5 \mathrm{~mm}$ size. The mesh size was determined based on a parametric analysis in which different size computational cells were used. A quarter domain was used in order to reduce the total number of computational cells compared to the full $360^{\circ}$ domain since this flow can be assumed to be approximately rotationally symmetric. Including the region below the impact surface in the domain was not necessary because it did not affect the film characteristics. This region was removed from the domain in subsequent simulations. The nozzle was assumed to be $38.1 \mathrm{~mm}$ above the impact surface so it was not included in the domain. The spray characteristics for the FullJet 1/8-G full cone spray nozzle manufactured by Spraying Systems Co. which had been measured in the laboratory by Hillen et al. (2013) using the PDA at a distance of $10 \mathrm{~mm}$ from the nozzle tip were specified as an internal boundary condition at a distance of $1.9 \mathrm{~mm}$ below the top of the domain or $28.1 \mathrm{~mm}$ above the impact surface. Additional model details are specified in Table 5.2. The characteristics of the spray were analyzed using ANSYS Fluent software version 14.5. 


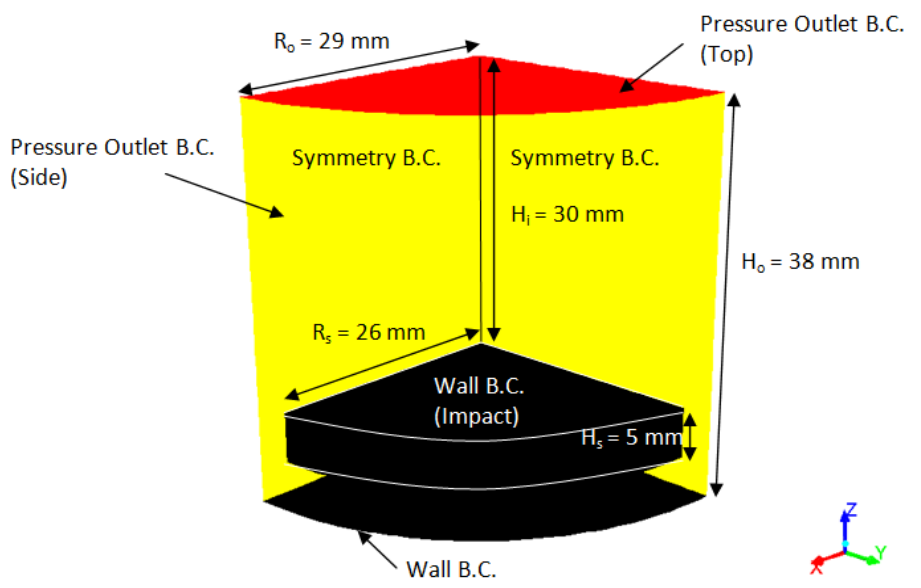

Figure 5.1 Computational domain and boundary conditions for the EWF model and DPM wall film submodel comparisons. (Note that surface below the impact surface is also assigned a wall boundary condition)

Table 5.2 Model Characteristics for Case 1 and Case 2.

\begin{tabular}{|l|l|}
\hline Model parameters & Value \\
\hline Nozzle type & FullJet 1/8-G (Full Cone) \\
\hline Spray cone half angle & $28^{\circ}$ \\
\hline Nozzle to surface distance & $38.1 \mathrm{~mm}$ \\
\hline Spray radius at surface & $20.3 \mathrm{~mm}$ \\
\hline Nozzle pressure & 40 psig \\
\hline Spray liquid & Water (boiling point 373 K) \\
\hline Drop liquid temperature & $300 \mathrm{~K}$ \\
\hline Air temperature & $300 \mathrm{~K}$ \\
\hline Surface temperature & $300 \mathrm{~K}$ \\
\hline Energy equation included? & no \\
\hline Spray model & DPM \\
\hline Film models used & Case 1. DPM wall film, Case 2. EWF \\
\hline Domain geometry & Quarter-cylinder \\
\hline Dimensionality & $3 \mathrm{D}$ \\
\hline Mesh type & \\
\hline
\end{tabular}


The following figures compare the characteristics of the liquid films predicted by the DPM wall film and EWF models. Figure 5.2 shows the flooded contours of the liquid film velocity magnitude at different simulation times for the DPM wall film model in the left column and for the EWF model in the right column. The patterns are very different, as are the magnitudes. In part c, the DPM wall film model predicts large areas where the velocity exceeds $5 \mathrm{~m} / \mathrm{s}$, but the EWF model predicts a maximum velocity of $1.14 \mathrm{~m} / \mathrm{s}$. Figure 5.3 shows the flooded contours of the liquid film thickness. The DPM wall film model gives an inexplicably unsymmetrical thickness distribution compared to the EWF model. In part c, the DPM wall film model predicts a maximum film thickness which is around 420 microns beneath the incoming spray. In contrast, the EWF predicts a fairly uniform thickness below the spray, increasing to a maximum thickness of around 205 microns outside the spray impact region. This hydraulic jump-like formation beyond the spray impact region is observed in the laboratory. It is concluded based on these comparisons that the EWF model is substantially more reliable than the DPM wall film for this problem. 


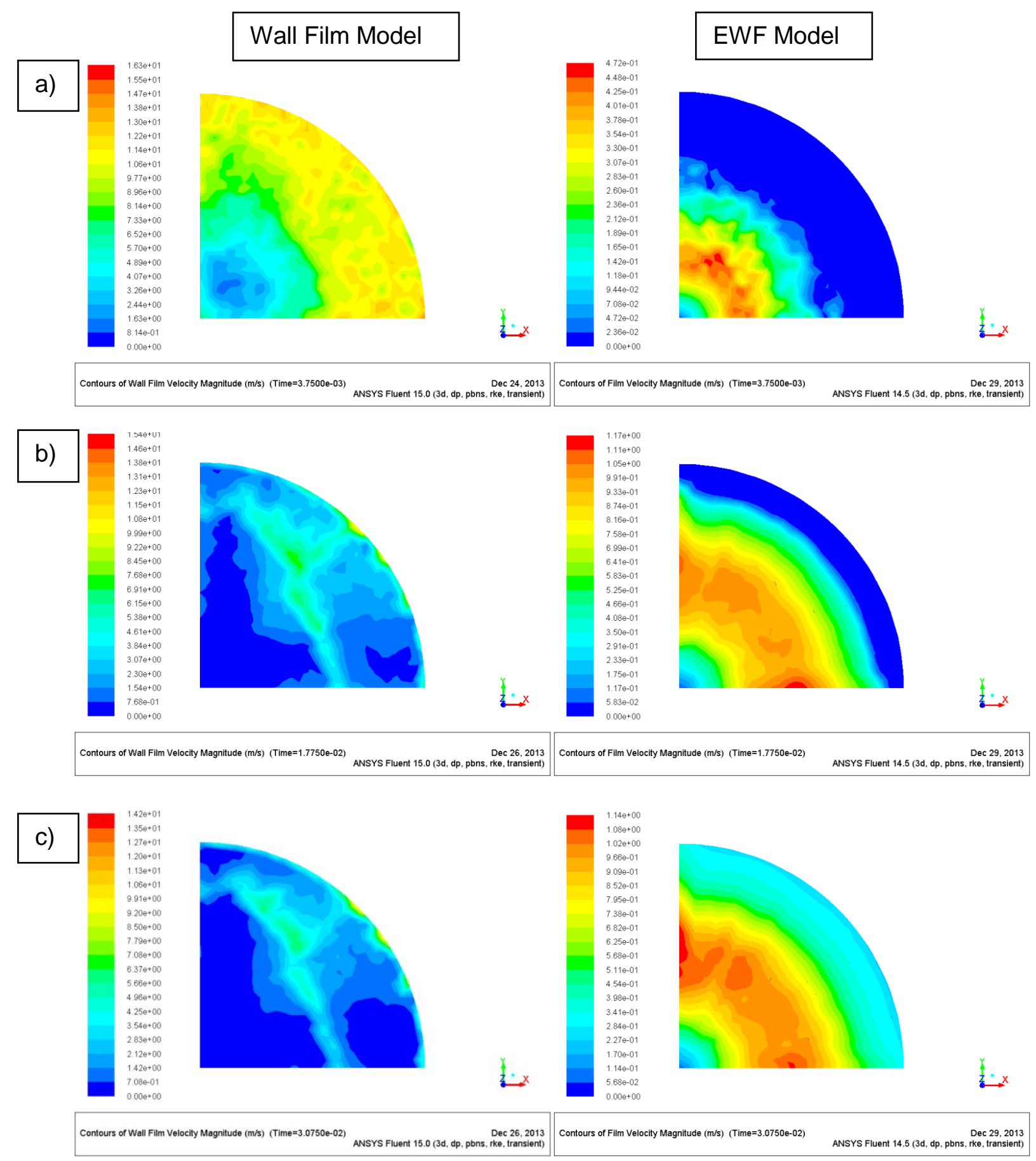

Figure 5.2 Comparisons of the liquid film velocity magnitude for the DPM wall film model (Case 1) in the left column and the EWF model (Case 2) in the right column at a) $3.75 \mathrm{~ms}$, b) $17.75 \mathrm{~ms}, \mathrm{c}) 30.75 \mathrm{~ms}$ simulation times. (Red refers to the maximum velocity and blue refers to the minimum velocity, but the color scales differ between the two models and between times for the same model. Note that the initial spray impact time on the surface is $1.75 \mathrm{~ms}$. All images show the top view of the impact surface.) 

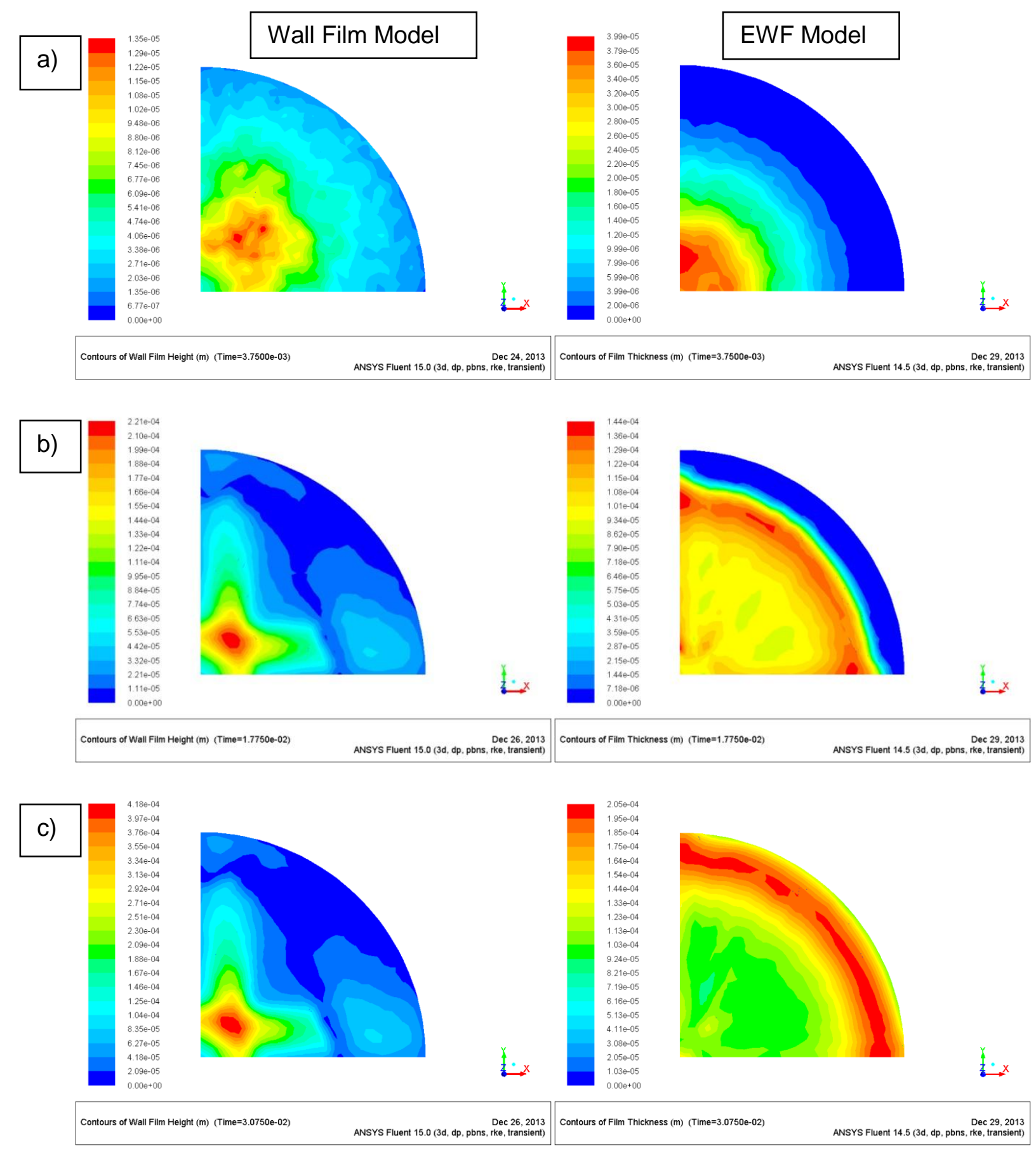

Figure 5.3 Comparisons of the liquid film thickness for the DPM wall film model (Case 1) in the left column and the EWF model (Case 2) in the right column at a) $3.75 \mathrm{~ms}, \mathrm{~b}) 17.75 \mathrm{~ms}$, c) 30.75 ms simulation times. (Red refers to the maximum thickness and blue refers to the minimum thickness, but the color scales differ between the two models and between times for the same model. Note that the initial spray impact time on the surface is $1.75 \mathrm{~ms}$. All images show the top view of the impact surface.) 


\subsection{Preliminary Spray Cooling Simulations with $30^{\circ}$ Cylindrical Domain (Case 3)}

For preliminary spray cooling simulations using the DPM for the spray and the EWF model for the wall film, a $30^{\circ}$ cylindrical $3 \mathrm{D}$ model was created. The purpose of using the $30^{\circ}$ domain was to reduce the computation time by using fewer cells. After comparing results for cylindrical domains covering $90^{\circ}, 45^{\circ}$, and $30^{\circ}$, it was concluded that the $30^{\circ}$ domain was satisfactory. One workstation was used to perform these simulations which had a run time of about 5 days per case. The computational domain was meshed with 97,740 non-uniform triangular prisms with a typical size of $0.5 \mathrm{~mm}$.

The boundary conditions and computational domain are shown in Figure 5.4. Note that the region below the impact surface shown in Figure 5.1 has been removed. The nozzle was assumed to be $38.1 \mathrm{~mm}$ above the impact surface so it was not included in the domain. The spray characteristics for the FullJet 1/8-G full cone spray nozzle at a distance of $10 \mathrm{~mm}$ from the nozzle tip were specified as an internal boundary condition at a distance of $1.9 \mathrm{~mm}$ below the top of the domain or $28.1 \mathrm{~mm}$ above the impact surface. Additional model details are specified in Table 5.3. The results were analyzed using ANSYS Fluent 14.5 and CFD-Post software.

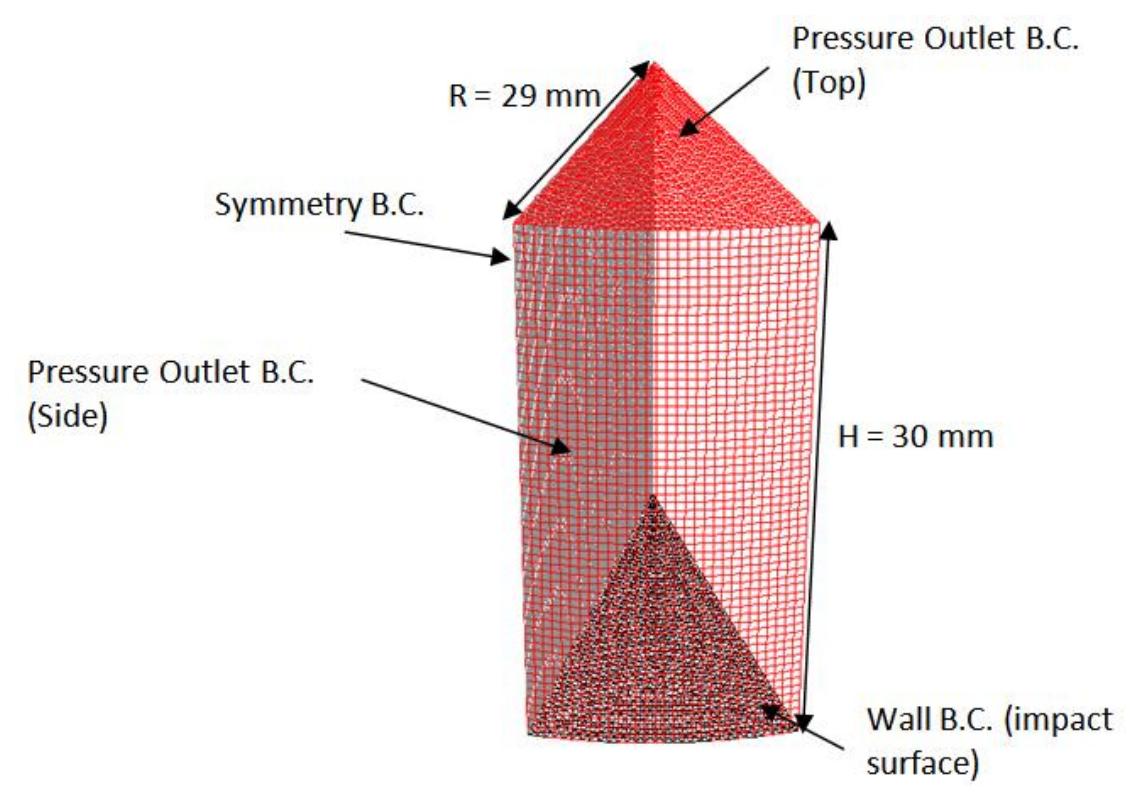

Figure 5.4 Computational domain and boundary conditions for the preliminary spray cooling simulations using the DPM and EWF models, Case 3. The viewpoint is above the impact surface looking toward the axis of symmetry. 
Table 5.3 Model Characteristics for Case 3.

\begin{tabular}{|c|c|}
\hline Model parameters & Value \\
\hline Nozzle type & FullJet 1/8-G (Full Cone) \\
\hline Spray cone half angle & $28^{\circ}$ \\
\hline Nozzle to surface distance & $38.1 \mathrm{~mm}$ \\
\hline Spray radius at surface & $20.3 \mathrm{~mm}$ \\
\hline Nozzle pressure & 40 psig \\
\hline Spray liquid & Water (boiling point $373 \mathrm{~K}$ ) \\
\hline Drop liquid temperature & $300 \mathrm{~K}$ \\
\hline Air temperature & $300 \mathrm{~K}$ \\
\hline Surface temperature & $393 \mathrm{~K}$ \\
\hline Initial film temperature & $372 \mathrm{~K}$ \\
\hline Initial film thickness & 1 micron \\
\hline Energy equation included? & Yes \\
\hline Wall thermal boundary condition & Constant temperature \\
\hline Spray model & DPM \\
\hline Film models used & EWF \\
\hline Domain geometry & $30^{\circ}$ cylindrical \\
\hline Dimensionality & $3 \mathrm{D}$ \\
\hline Mesh type & Irregular triangular prisms \\
\hline
\end{tabular}

Figure 5.5 shows the drop velocity magnitude distribution at $11.75 \mathrm{~ms}(10 \mathrm{~ms}$ after the drops first contact the surface). Drop Reynolds numbers range between 0.1 and 230, and drop Weber numbers range between 0 and 412 at this time based on the drop velocities and diameters. Figure 5.6 shows the flooded contours of the liquid film thickness distribution on the surface at $11.75 \mathrm{~ms}$ and at $51.75 \mathrm{~ms}$. At $11.75 \mathrm{~ms}$ the film created by the spray does not yet cover the entire surface. The simulations were stopped at $51.75 \mathrm{~ms}$ because after this time the results did not 
significantly vary with time (but they are not fully steady). The deviations from symmetry beyond the impact region in Figure $5.6 \mathrm{~b}$ could not be explained.

Figure 5.7 shows the variation of the film thickness along the symmetry surface parallel to the $\mathrm{x}$-axis at various times. The film thickness is fairly uniform in the spray impact region, except for a lower value on the axis. This has been attributed to the use of non-uniform triangular prism elements near the axis.

Figures 5.8 and 5.9 show the flooded contours of the liquid film depth-averaged velocity magnitude and the mid depth temperature of the liquid film, respectively. Figure 5.10 shows the temperature variation along the symmetry surface parallel to the $\mathrm{x}$-axis. The initial 1 micron thick film is cooled down in a short amount of time due to the impact of the cold spray drops $(300 \mathrm{~K})$ as shown in Figures 5.9 and 5.10. After a few milliseconds, the temperature shows a local maximum at the axis, a minimum at about $5 \mathrm{~mm}$, and a gradual increase as radius increases. Figure 5.11 shows the flooded contours of the heat flux on the surface. As the liquid film becomes thinner close to the spray axis, the surface heat flux increases and reaches its maximum value where the film is thinnest. The heat flux decreases in the radial direction, but at a decreasing rate.

These Case 3 results were promising but suggested that the computational domain and cells needed to be refined in order to obtain more accurate and uniform spray cooling results. 


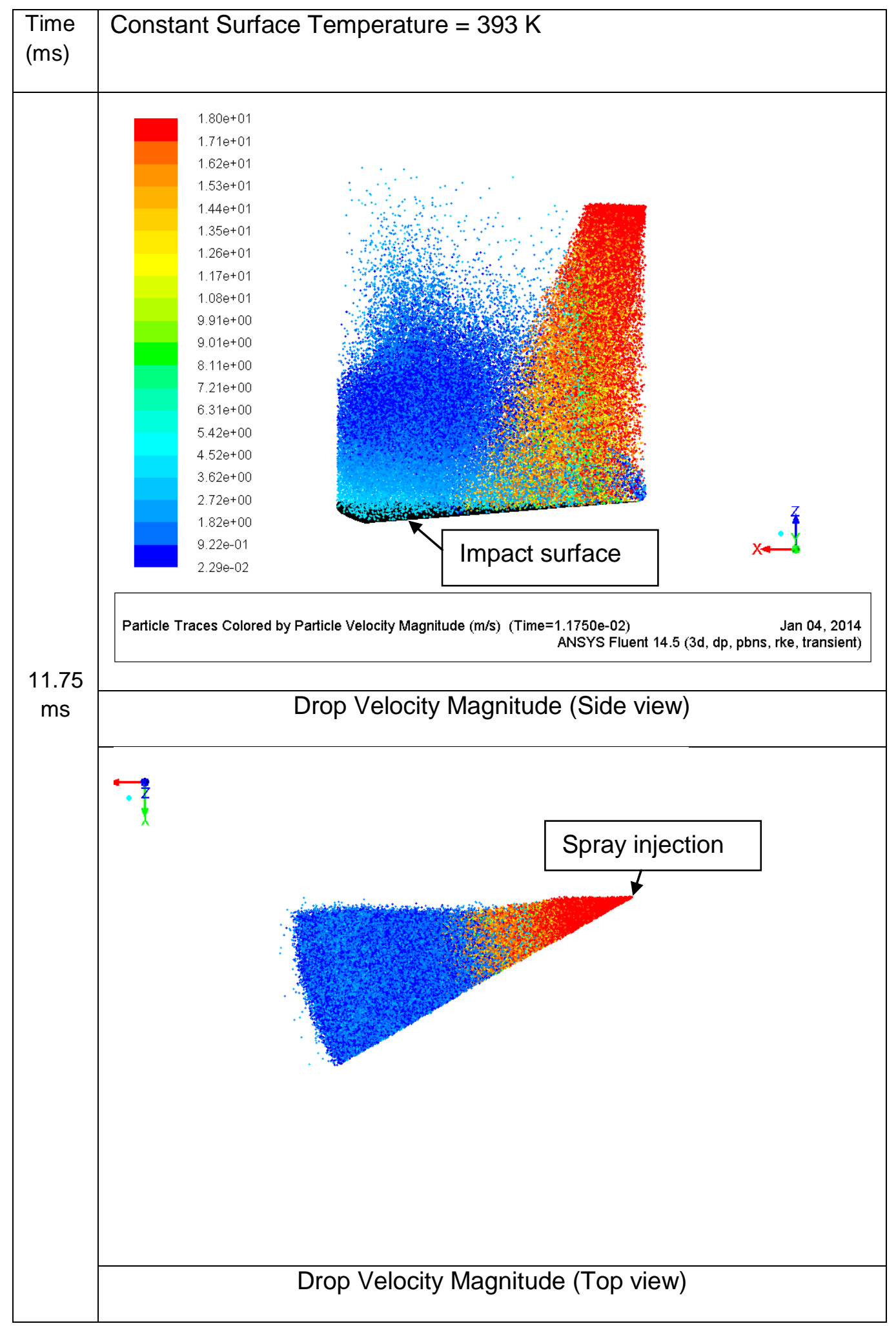

Figure 5.5 Case 3 drop velocity magnitude at $11.75 \mathrm{~ms}$. (Red refers to the maximum velocity and blue refers to the minimum velocity. Initial spray impact time is $1.75 \mathrm{~ms}$.) 

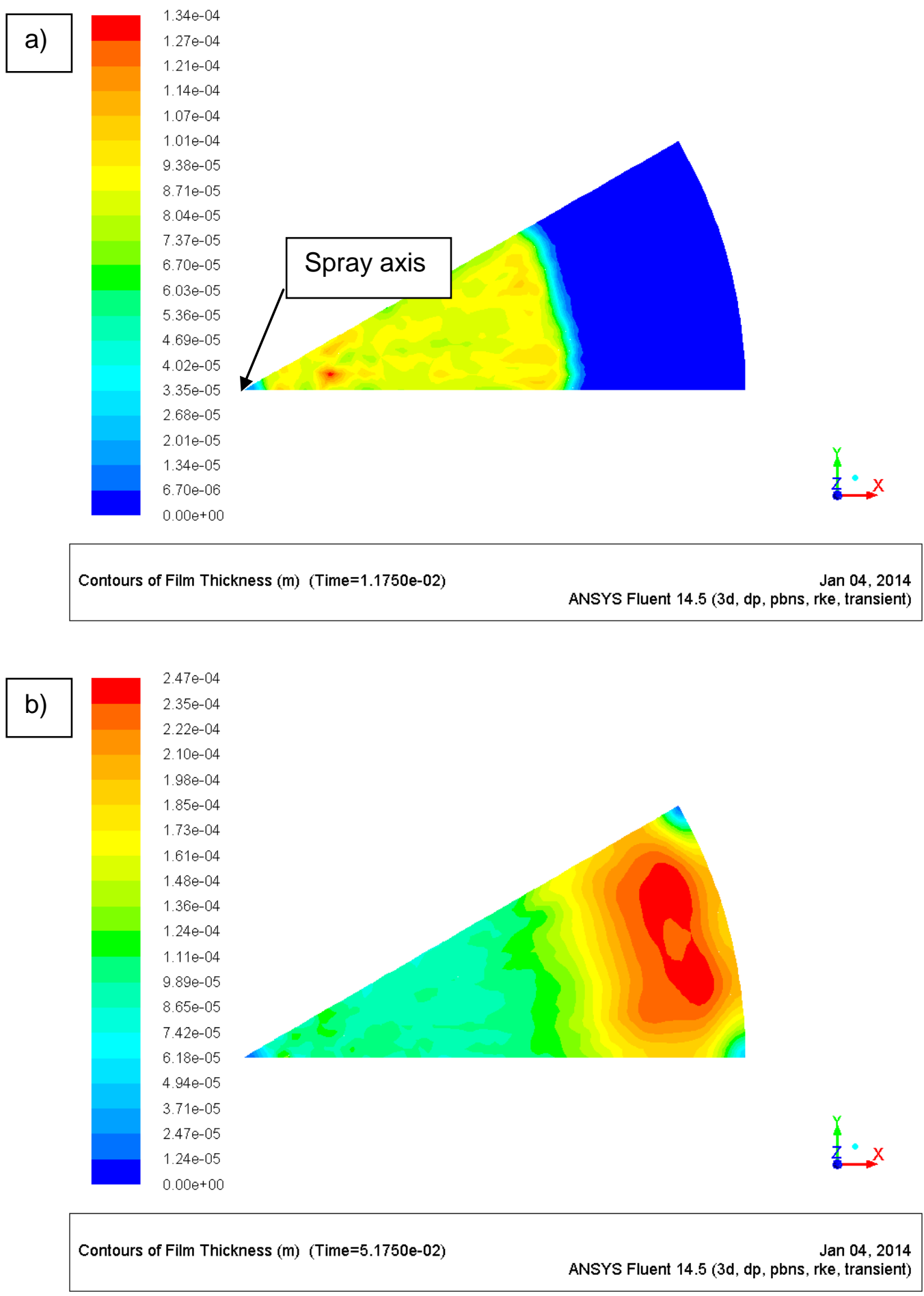

Figure 5.6 Case 3 flooded contours of the liquid film thickness at a) $11.75 \mathrm{~ms}$, b) $51.75 \mathrm{~ms}$. The color scale is different in a) and b). (All images show the top view of the impact surface.) 


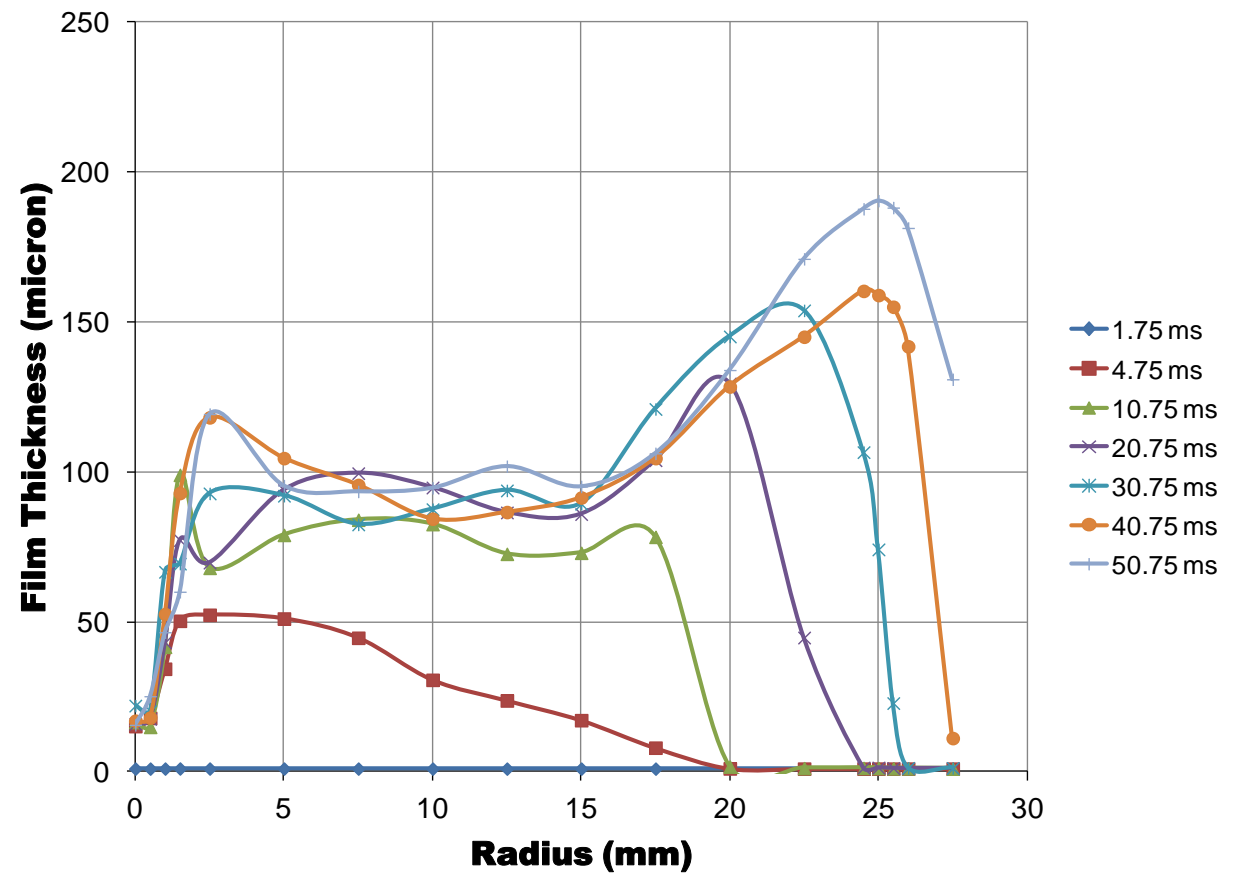

Figure 5.7 Case 3 film thickness variation with time at the symmetry surface parallel to the $x$ axis. 

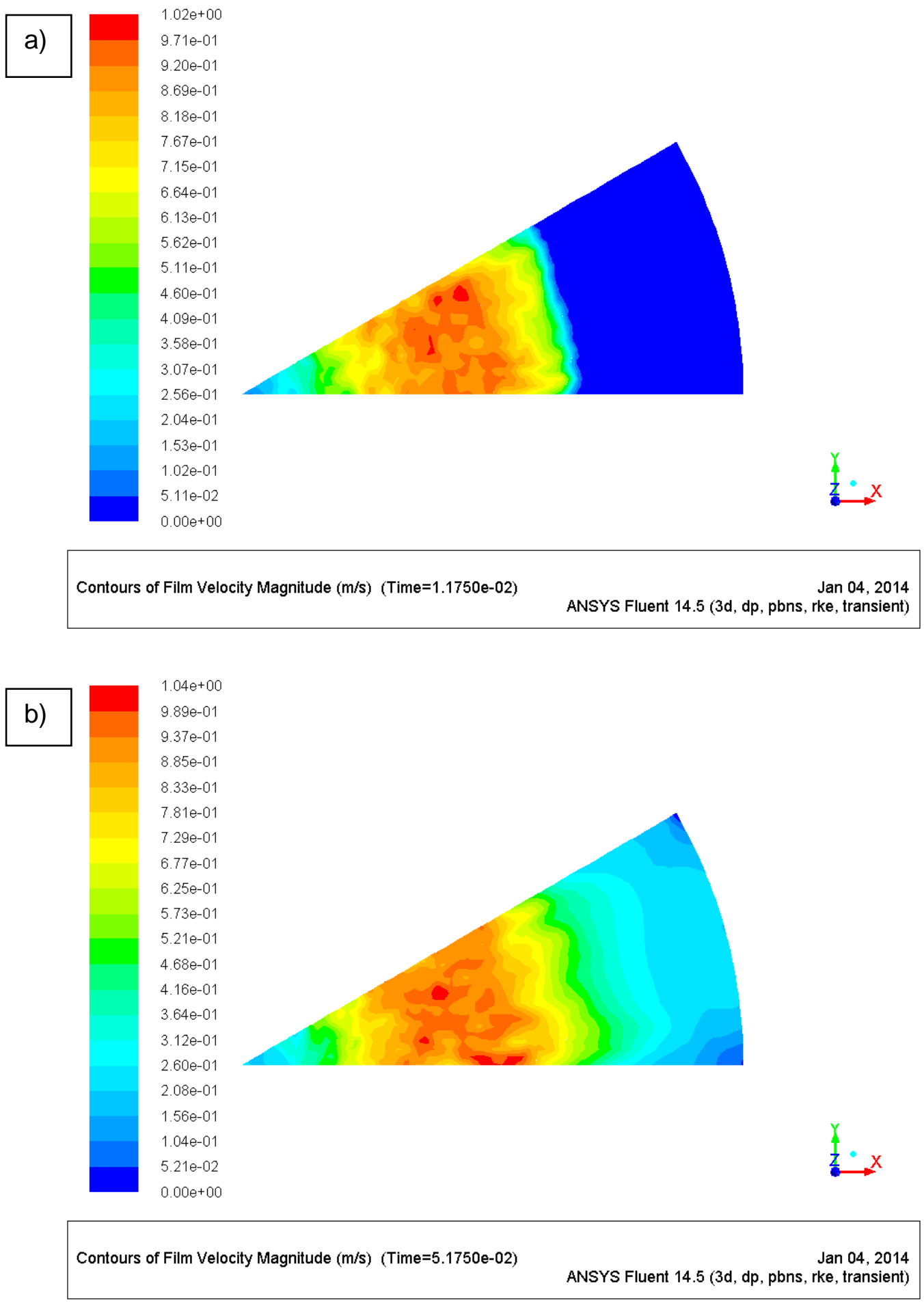

Figure 5.8 Case 3 flooded contours of the liquid film velocity magnitude at a) $11.75 \mathrm{~ms}$, b) 51.75 ms. The color scales are nearly identical in parts a) and b). (All images show the top view of the impact surface.) 

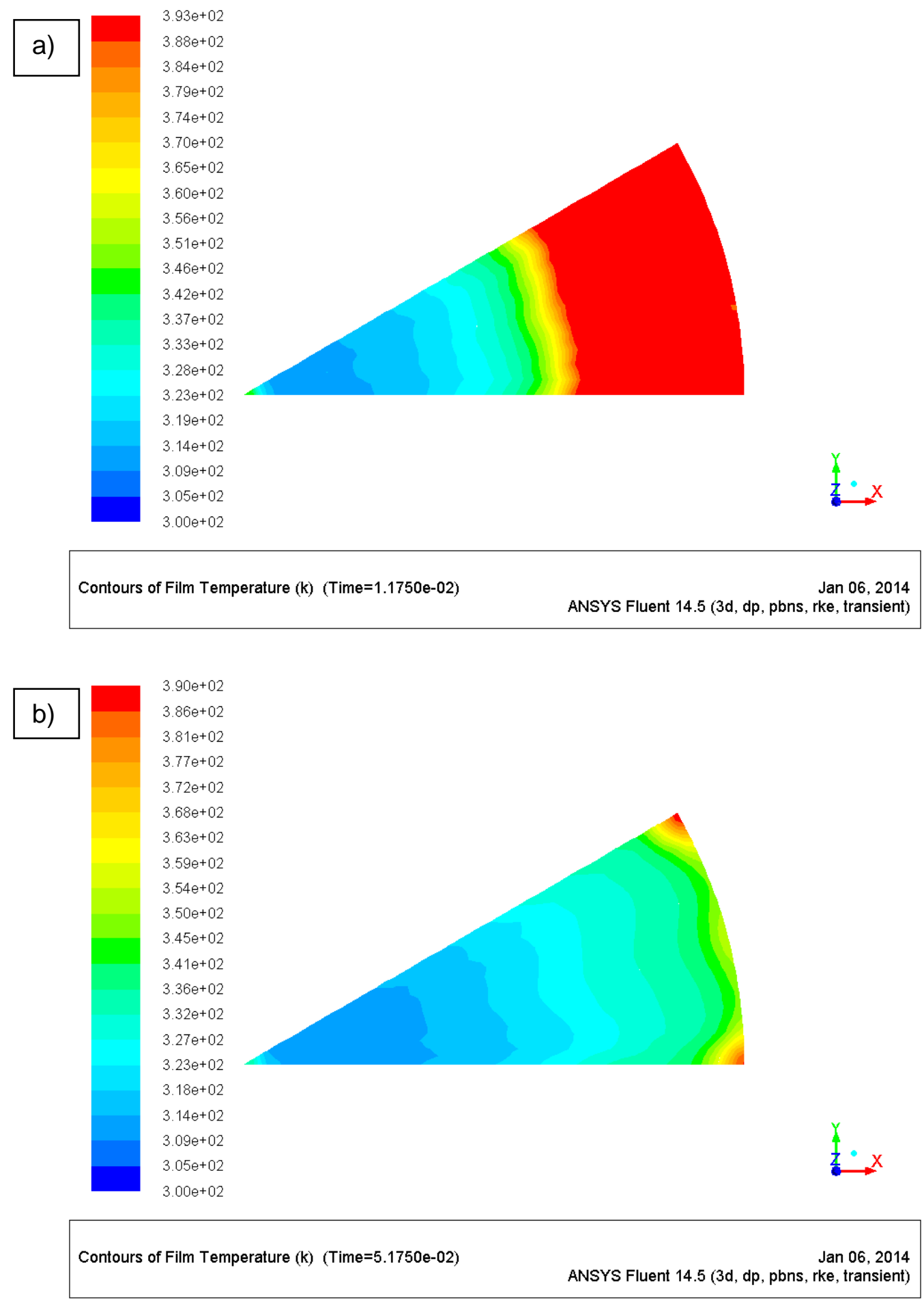

Figure 5.9 Case 3 flooded contours of the mid depth film temperature at a) $11.75 \mathrm{~ms}$, b) 51.75 ms. The color scales differ slightly in a) and b). (All images show the top view of the impact surface.) 


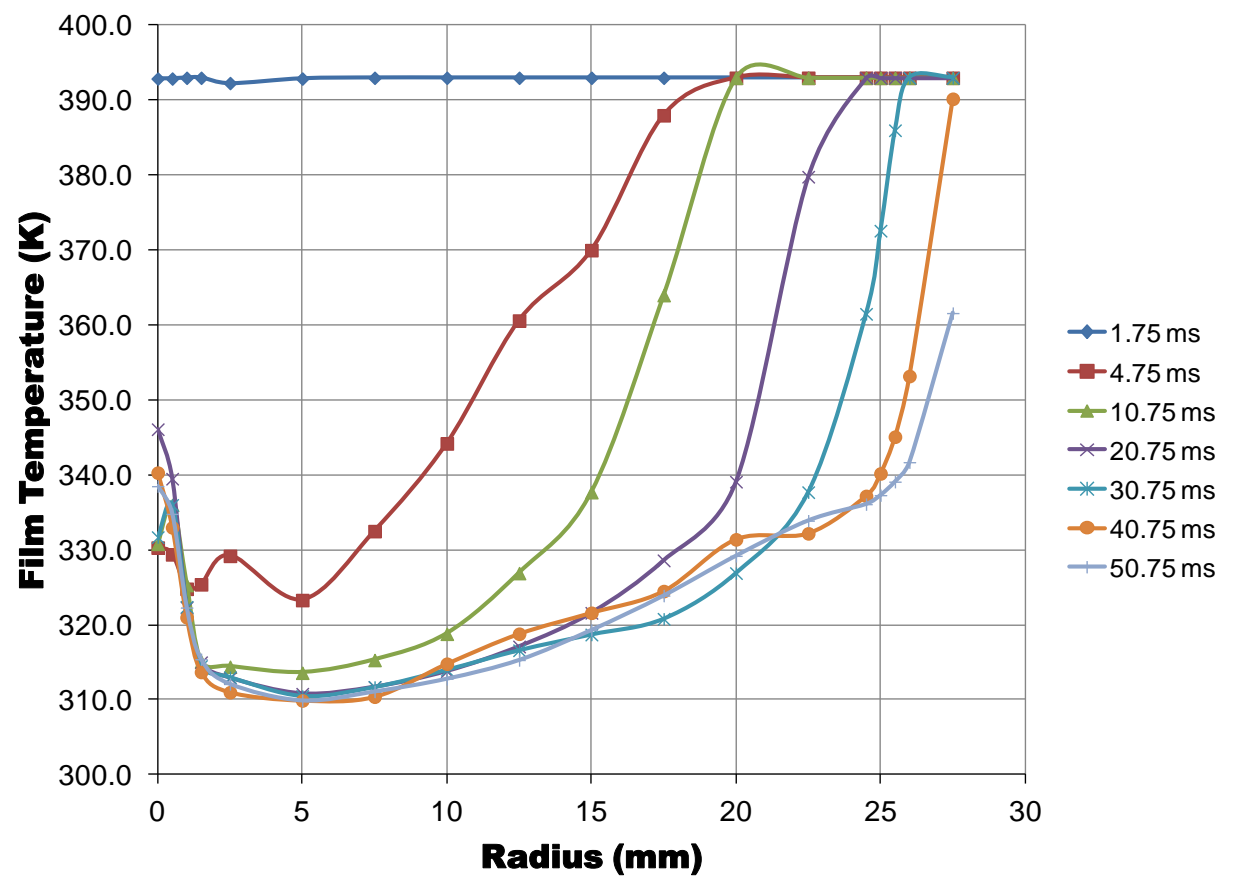

Figure 5.10 Case 3 mid depth film temperature variation with time along the symmetry surface parallel to the $x$-axis. 

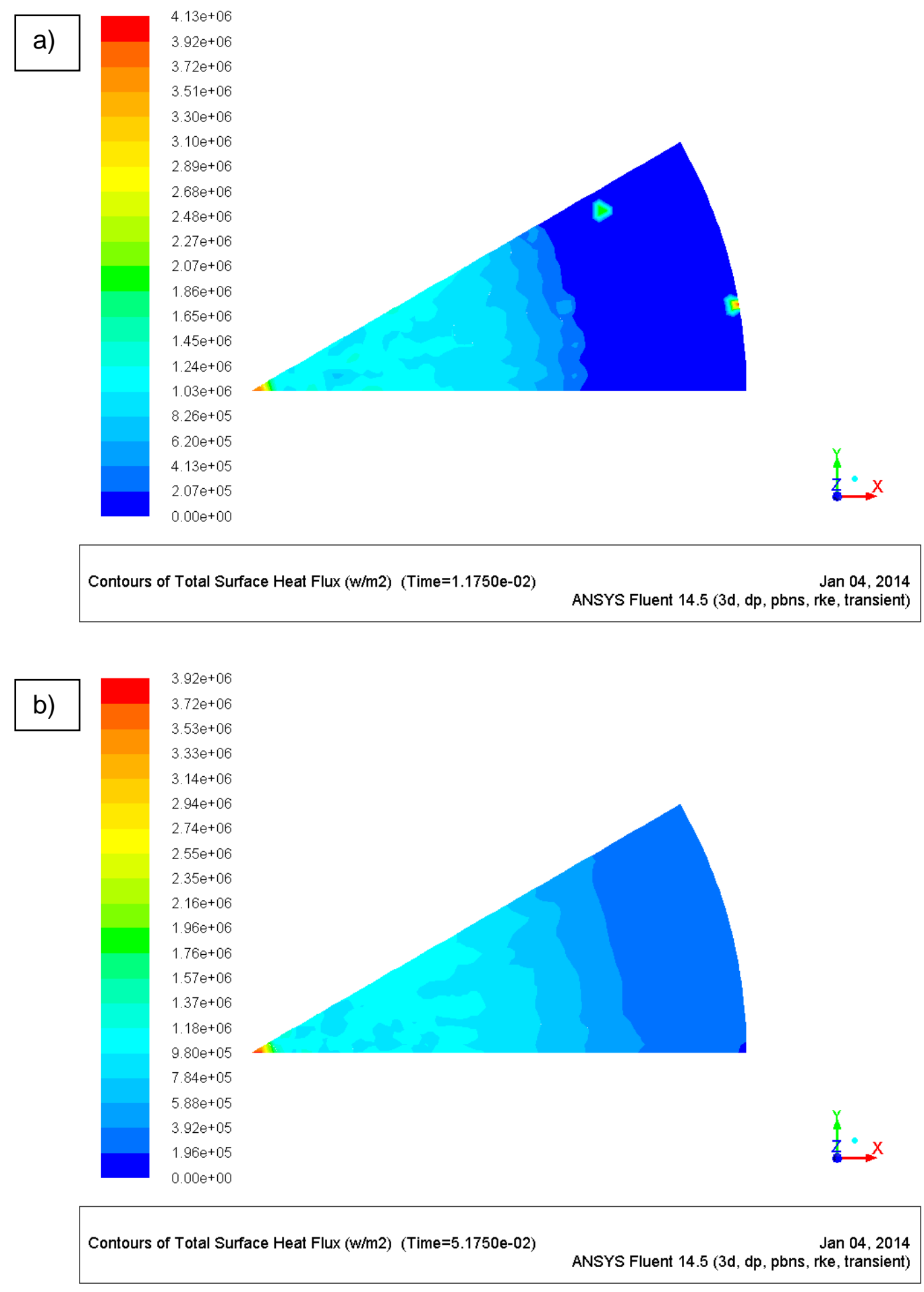

Figure 5.11 Case 3 flooded contours of the surface heat flux at a) $11.75 \mathrm{~ms}$, b) $51.75 \mathrm{~ms}$. The color scales differ in a) and b). (All images show the top view of the impact surface.) 


\subsection{Refined Spray Cooling Simulations with $90^{\circ}$ Rectangular Domain (Case 4)}

Spray cooling simulations using the DPM and EWF with a $30^{\circ}$ cylindrical wedge 3D domain gridded with non-uniform triangular prism elements showed promising results as described in the previous section. However, there were some questionable results such as non-uniform film characteristics and heat flux values on the impact surface (e.g. the highest heat flux occurred close the axis). Therefore, new simulations were performed using a quarter spray $\left(90^{\circ}\right) 3 \mathrm{D}$ rectangular domain with a uniform grid of 162,240 cubic cells with $0.5 \mathrm{~mm}$ sides as shown in Figure 5.12. These calculations were run on two workstations with about 6 day run times.

The nozzle was assumed to be $38.1 \mathrm{~mm}$ above the impact surface so it was not included in the domain. The spray characteristics for the FullJet $1 / 8-\mathrm{G}$ full cone spray nozzle which had been measured in the laboratory using the PDA at a distance of $10 \mathrm{~mm}$ from the nozzle tip were specified as an internal boundary condition at a distance of $1.9 \mathrm{~mm}$ below the top of the domain or $28.1 \mathrm{~mm}$ above the impact surface. Additional model details are specified in Table 5.4. The results were analyzed using ANSYS Fluent 14.5 and CFD-Post.

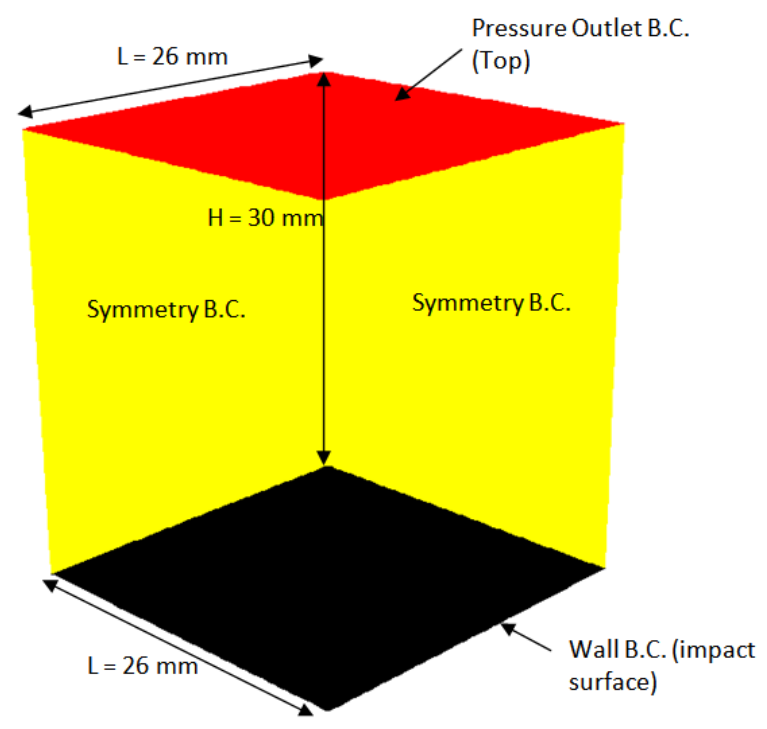

Figure 5.12 Case 4 computational domain and boundary conditions for the spray and spray cooling simulations using the DPM and EWF models. The viewpoint is above the impact surface looking toward the axis of symmetry. (Note that the missing two boundary conditions on the outer vertical surfaces are pressure outlet.) 
Table 5.4 Model Characteristics for Case 4.

\begin{tabular}{|c|c|}
\hline Model parameters & Value \\
\hline Nozzle type & FullJet 1/8-G (Full Cone) \\
\hline Spray cone half angle & $28^{\circ}$ \\
\hline Nozzle to surface distance & $38.1 \mathrm{~mm}$ \\
\hline Spray radius at surface & $20.3 \mathrm{~mm}$ \\
\hline Nozzle pressure & $40 \mathrm{psig}$ \\
\hline Spray liquid & Water (boiling point $373 \mathrm{~K}$ ) \\
\hline Drop liquid temperature & $300 \mathrm{~K}$ \\
\hline Air temperature & $300 \mathrm{~K}$ \\
\hline Surface temperature & $393 \mathrm{~K}$ \\
\hline Initial film temperature & $372 \mathrm{~K}$ \\
\hline Initial film thickness & 1 micron \\
\hline Energy equation included? & Yes \\
\hline Wall thermal boundary condition & Constant temperature \\
\hline Spray model & DPM \\
\hline Film models used & EWF model \\
\hline Domain geometry & $90^{\circ}$ rectangular domain \\
\hline Dimensionality & $3 \mathrm{D}$ \\
\hline Mesh type & Uniform 0.5 -mm cubes \\
\hline
\end{tabular}

Figures 5.13, 5.14, 5.15, 5.16 show flooded contours of liquid film velocity magnitude, liquid film thickness, mid depth film temperature, and heat flux on the impact surface, respectively, at the two different simulation times of $5.975 \mathrm{~ms}$ and $51.725 \mathrm{~ms}$. The radius of the spray at the surface is $20.3 \mathrm{~mm}$.

Figure 5.13 shows that at $51.725 \mathrm{~ms}$ the magnitude of the average liquid film velocity increases with radius to a maximum of about $1.15 \mathrm{~m} / \mathrm{s}$ at a radius of about $13 \mathrm{~mm}$ and then 
decreases smoothly. The pattern shows the expected rotational symmetry except near the symmetry boundaries.

Figure 5.14 shows that at $5.975 \mathrm{~ms}$ the liquid film is thickest ( 83 microns) near the axis and decreases in the radial direction. But at $51.725 \mathrm{~ms}$, the film thickness is about 100 microns on the axis, drops to about 80 microns under most of the spray, then increases to a maximum of about 250 microns at a radius of $26 \mathrm{~mm}$, and finally decreases to the initial thickness of 1 micron at the radius where the spray liquid has not yet reached. This general pattern is consistent with visual observations in the laboratory experiments. The film beyond about $30 \mathrm{~mm}$ remains unaffected by the spray at this time. The thickness shows rotational symmetry over most of the domain, but there are noticeable deviations near the symmetry boundaries.

The liquid film mid depth temperature is depicted in Figure 5.15. At $5.975 \mathrm{~ms}$, the temperature around the axis has dropped to about $320 \mathrm{~K}$. The temperature rises to about $380 \mathrm{~K}$ at the edge of the spray and to $393 \mathrm{~K}$ beyond about $22 \mathrm{~mm}$. Although the boiling point (373 K) has been exceeded, the film is not evaporated because the Species Transport Model (STM) has not been included yet to account for phase change. The pattern of temperature is essentially rotationally symmetric. At $51.725 \mathrm{~ms}$, the temperature around the axis has fallen to about $315 \mathrm{~K}$. It increases with radius to about $337 \mathrm{~K}$ at about $22 \mathrm{~mm}$. From $22 \mathrm{~mm}$ to about $26 \mathrm{~mm}$ there are waves in the pattern. From 26 to about $30 \mathrm{~mm}$ the temperature increases to about $365 \mathrm{~K}$. Beyond about $30 \mathrm{~mm}$ the unaffected film is at $393 \mathrm{~K}$. The pattern again shows deviations from rotational symmetry along the symmetry planes.

The surface heat flux is depicted in Figure 5.16. At both times, the overall pattern is largely rotationally symmetric with the highest values under the spray. At $5.975 \mathrm{~ms}$, the heat flux near the axis reaches a maximum of about $1.49 \mathrm{MW} / \mathrm{m}^{2}\left(149 \mathrm{~W} / \mathrm{cm}^{2}\right)$. At $51.725 \mathrm{~ms}$, the maximum heat flux has fallen to $1.18 \mathrm{MW} / \mathrm{m}^{2}\left(118 \mathrm{~W} / \mathrm{cm}^{2}\right)$ and decreases to about $0.49 \mathrm{MW} / \mathrm{m}^{2}$ $\left(49 \mathrm{~W} / \mathrm{cm}^{2}\right)$ at the edge of the spray. Beyond about $26 \mathrm{~mm}$ some waves appear in the heat flux.

Although some anomalies remain, using a quarter spray domain with a rectangular grid has provided the most believable simulations achieved so far and was used in further spray cooling simulations as described below. 

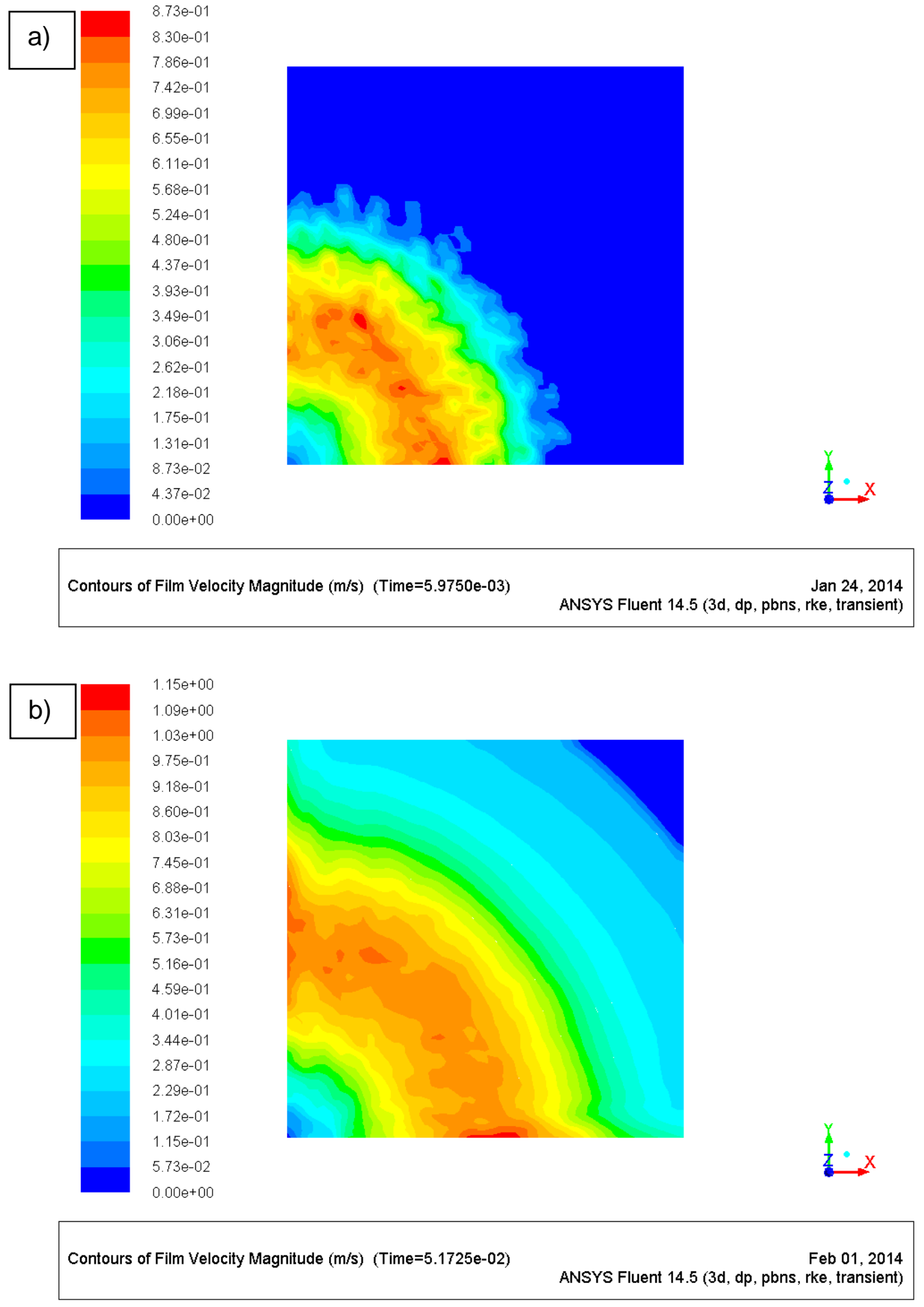

Figure 5.13 Case 4 flooded contours of the liquid film velocity magnitude at a) $5.975 \mathrm{~ms}$, b) $51.725 \mathrm{~ms}$. The color scale differs in a) and b). (All images show the top view of the impact surface.) 

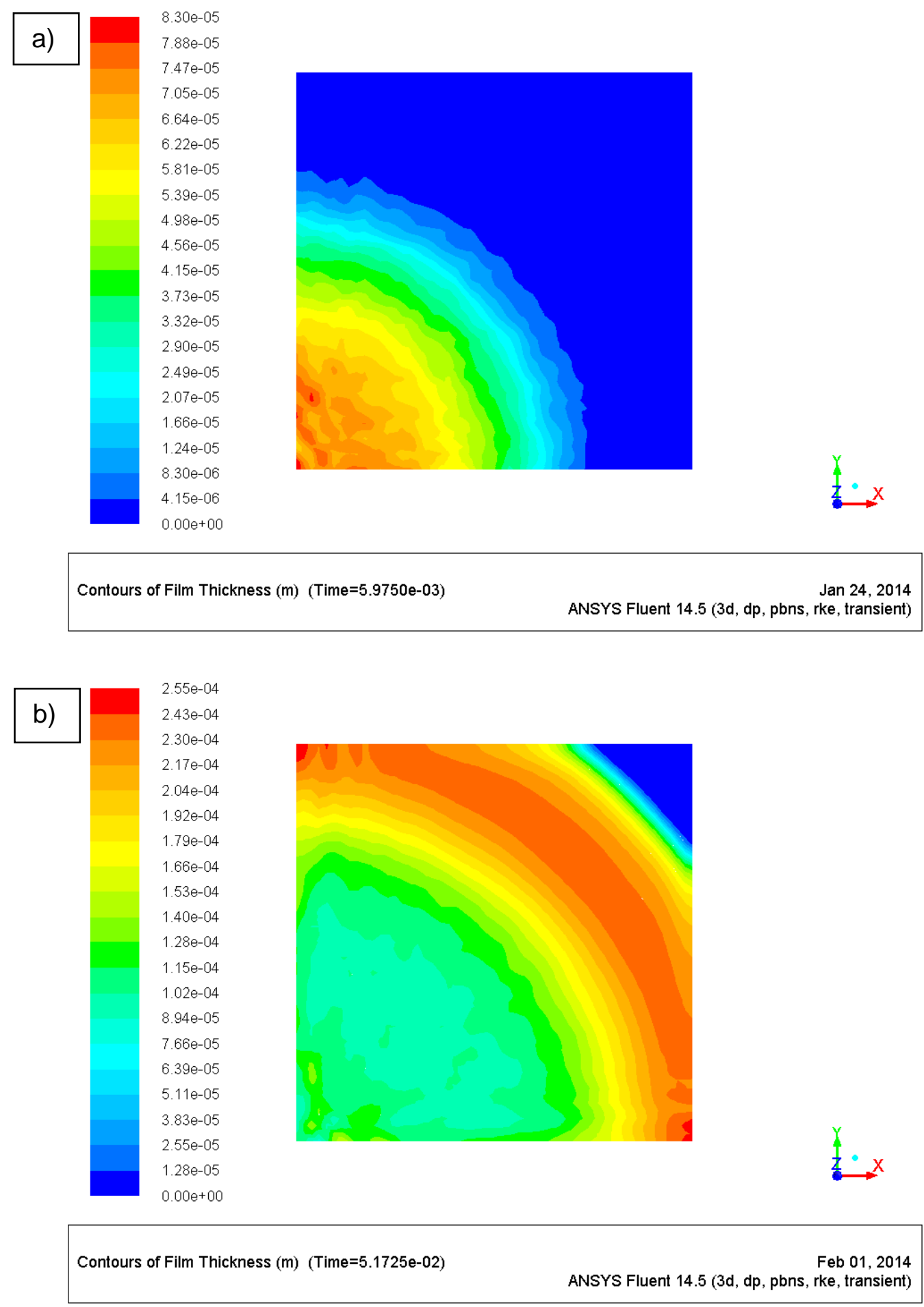

Figure 5.14 Case 4 flooded contours of the liquid film thickness at a) $5.975 \mathrm{~ms}$, b) $51.725 \mathrm{~ms}$. The color scale differs in a) and b). (All images show the top view of the impact surface.) 

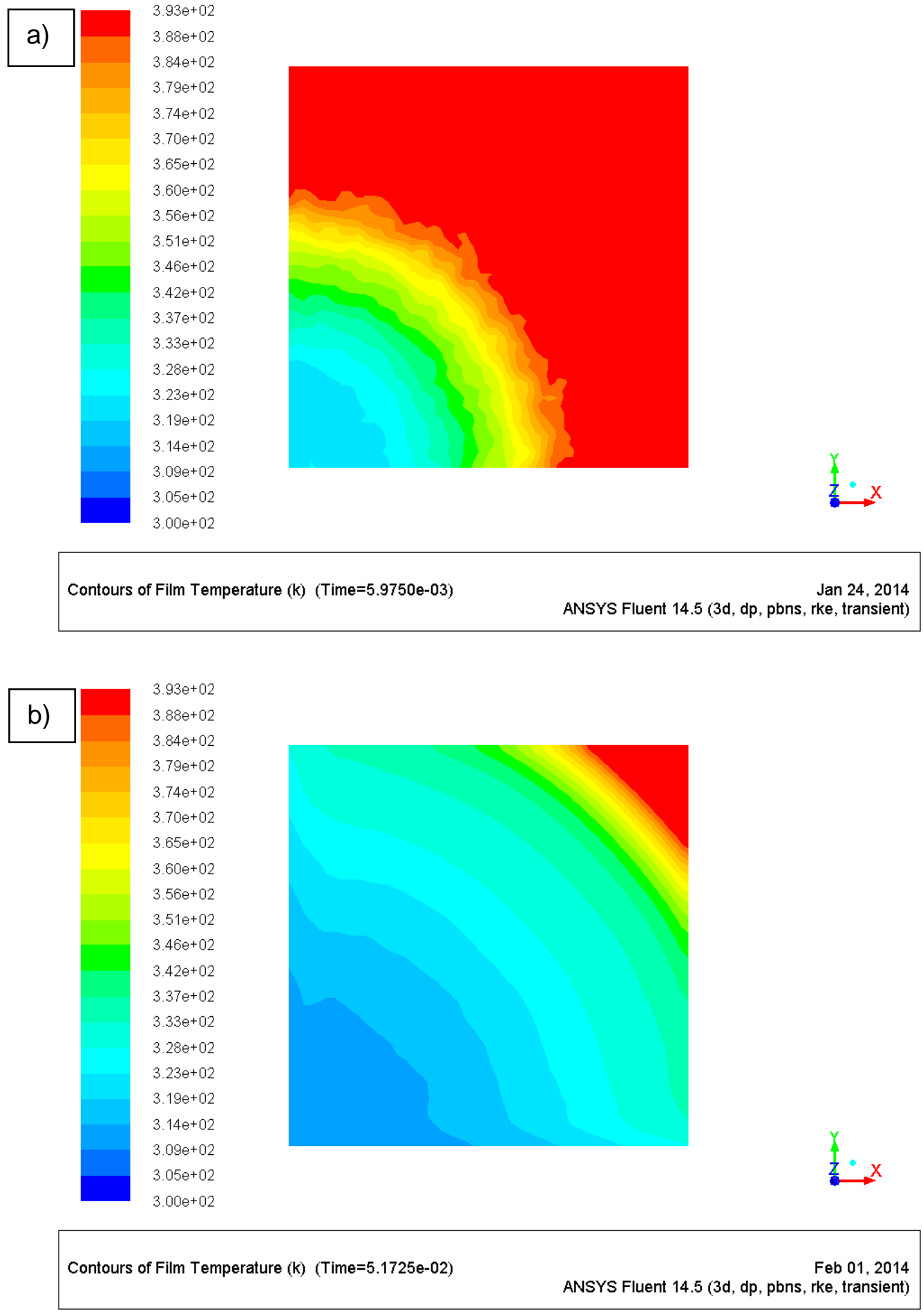

Figure 5.15 Case 4 flooded contours of the mid depth film temperature at a) $5.975 \mathrm{~ms}$, b) 51.725 $\mathrm{ms}$. The color scale is the same in a) and b). (All images show the top view of the impact surface.) 

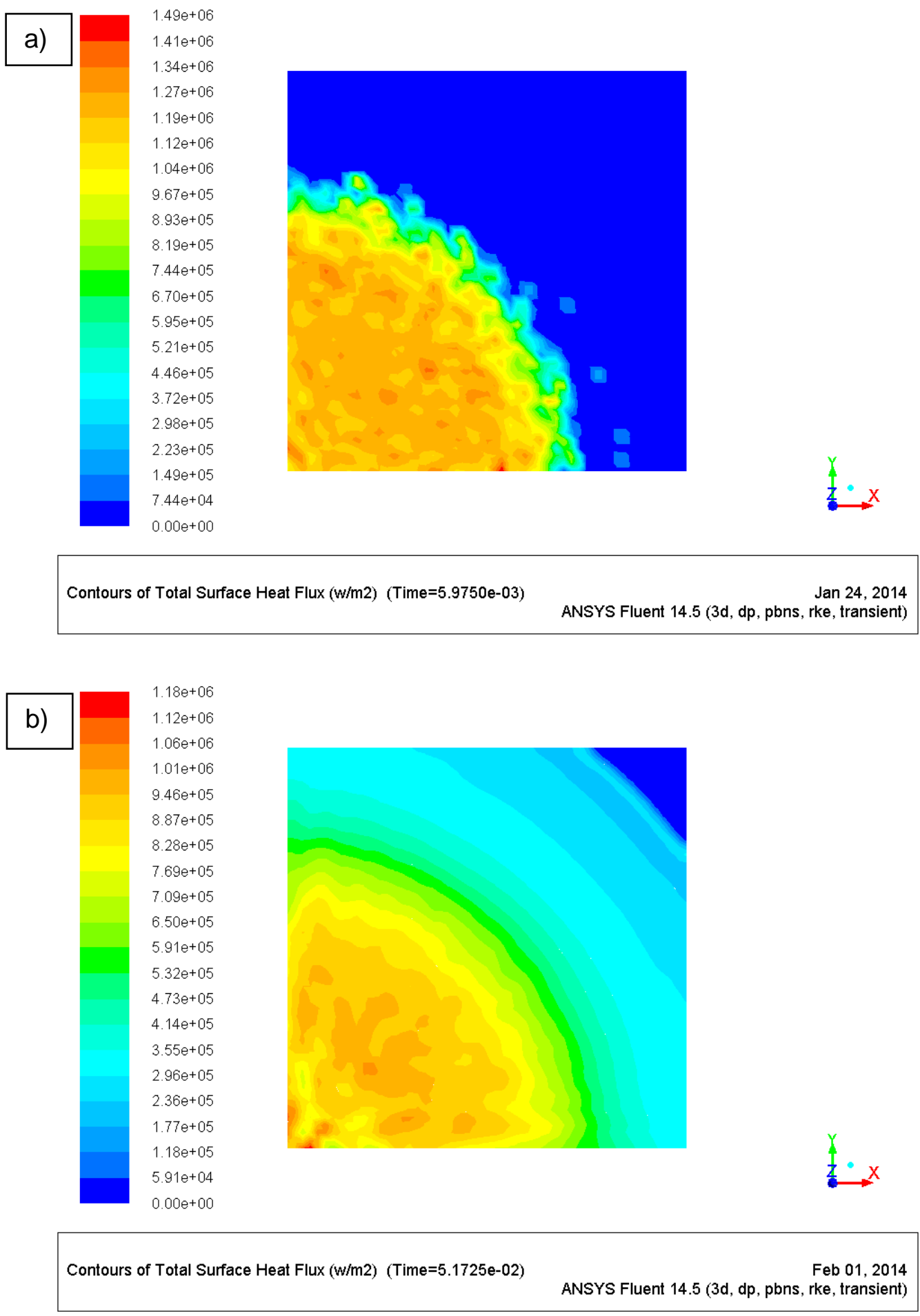

Figure 5.16 Case 4 flooded contours of the surface heat flux at a) $5.975 \mathrm{~ms}$, b) $51.725 \mathrm{~ms}$. The color scale differs in a) and b). (All images show the top view of the impact surface.) 


\subsection{Comparison of Film Thickness in Experiments and Simulations Using Turbulent Viscosity (Case 5)}

Although the simulations described in Section 5.4 (Case 4) showed good overall agreement with experiments, the thickness of the film under the spray was much less than was observed in experiments. This discrepancy will be documented below after the necessary corrections have been described. It was hypothesized that the reason for this discrepancy was that the EWF equations had been solved using molecular values for the viscosity and thermal conductivity, in effect assuming that the flow in the film was laminar. Visual observations clearly show that the film flow is chaotic due to the millions of drop impingements per second. These drop impacts should influence the mean flow by greatly increasing the mixing of momentum and energy similar to what occurs in turbulent flow. ANSYS Fluent incorporates submodels for the k-epsilon and other popular turbulence models, but these models are not appropriate for spray films because they have been derived and tuned for cases of true $3 \mathrm{D}$, shear-driven turbulence in which the enhanced mixing arises from instabilities of the basic flow. This mechanism seems unlikely in spray films.

The boundary layer on a flat plate is the most closely related flow for which a criterion assuring laminar flow is known, namely that the Reynolds number based on the free stream velocity and the distance from the leading edge should be less than 300,000 (Schlichting, 1979). Based on the simulation of Section 5.4, the maximum velocity for the assumed parabolic profile is $1.5 \times 1.2 \mathrm{~m} / \mathrm{s}=1.8 \mathrm{~m} / \mathrm{s}$. Using the spray radius at the surface of $20.3 \mathrm{~mm}$ and the kinematic viscosity of $373 \mathrm{~K}$ water $\left(2.9 \times 10^{-7} \mathrm{~m}^{2} / \mathrm{s}\right)$ gives $R e=126,000$, well below the threshold for transition. This supports the conclusion reached by direct visual observation that the mixing in

the spray film does not arise from internal instabilities but is imposed by the random impact of the spray droplets. Therefore the usual turbulence models cannot be expected to apply. As there is no existing theory for the mean flow in a spray film, it was decided to use the simplest possible model for the enhanced mixing: the eddy viscosity model of Boussinesq.

The viscous force term in the momentum equation for an incompressible Newtonian fluid is

$$
\mu \nabla^{2} \mathbf{v}
$$

where $\mu$ is the molecular dynamic viscosity and $\mathbf{v}$ is the velocity vector. 
When the momentum equation is written using the Reynolds decomposition and averaged, the mean flow momentum equation contains additional Reynolds stresses which Boussinesq suggested can be modeled as pseudo-viscous stresses using an eddy or turbulent viscosity $\mu_{t}$ so that the following term appears in the mean momentum equation

$$
\left(\mu+\mu_{t}\right) \nabla^{2} \overline{\mathbf{v}}=\mu_{e} \nabla^{2} \overline{\mathbf{v}}
$$

where $\overline{\mathbf{v}}$ is the mean (time-averaged) velocity vector and $\mu_{e}$ is the effective dynamic viscosity. In reality, it is well known that $\mu_{t}$ is a complex function of position in the flow, but for the present application it will be taken as a constant in full knowledge that this is an exceedingly crude approximation.

In a similar way, the energy equation for an incompressible fluid contains the following term for heat conduction

$$
k \nabla T
$$

where $k$ is the thermal conductivity and $T$ is the temperature.

Using the Reynolds decomposition and averaging gives the mean energy equation in which additional terms expressing the transport of heat by turbulent eddies appear. As in the case of the momentum equation, these terms can be modeled as pseudo-conduction terms so that the following term appears

$$
\left(k+k_{t}\right) \nabla \bar{T}=k_{e} \nabla \bar{T}
$$

where $k_{t}$ is the turbulent conductivity, $k_{e}$ is the effective conductivity, and $\bar{T}$ is the mean temperature. It is known that $k_{t}$ varies in a way that is similar to $\mu_{t}$. Therefore in the following simulations $k_{t}$ will also be treated as a constant recognizing this to be a crude approximation.

In laminar flow heat transfer, the Prandtl number $\mathrm{Pr}$ is an important dimensionless parameter. It can be written as 


$$
\operatorname{Pr}=\frac{c_{p} \mu}{k}
$$

where $c_{p}$ is the specific heat at constant pressure, a fluid property.

By analogy, a turbulent Prandtl number $\operatorname{Pr}_{t}$ is defined as

$$
\operatorname{Pr}_{t}=\frac{c_{p} \mu_{t}}{k_{t}}
$$

Based on the Reynolds analogy, it is customary to set $P r_{t}=1$ for turbulent convection in water. This assumption (equivalent to setting $k_{t}=c_{p} \mu_{t}$ ) has led to satisfactory results in many cases. An effective Prandtl $\mathrm{Pr}_{e}$ can be defined as

$$
\operatorname{Pr}_{e}=\frac{c_{p} \mu_{e}}{k_{e}}=\frac{c_{p}\left(\mu+\mu_{t}\right)}{\left(k+k_{t}\right)}=\frac{c_{p}\left(\mu+\mu_{t}\right)}{\left(k+c_{p} \mu_{t}\right)}
$$

After comparing results obtained using several values for the turbulent viscosity, it was decided that setting $\mu_{t}=4 \mu$ gave the best agreement with the experimental observations for film thickness, as will be demonstrated below. This choice results in an effective Prandtl number $\operatorname{Pr}_{e}$ $=1.09$ based on the properties of water at $373 \mathrm{~K}$.

Spray simulations using the constant eddy viscosity assumption were performed using the DPM and EWF models (Case 5). A quarter spray $\left(90^{\circ}\right)$ 3D rectangular domain was gridded uniformly with $0.5 \mathrm{~mm}$ cubes as in Section 5.4 (Case 4). After trying several domain sizes, the horizontal and vertical dimensions of the domain were decreased to $22 \mathrm{~mm}$ and $19 \mathrm{~mm}$, respectively, reducing the number of cells to 73,568 , which is $45 \%$ of the Case 4 domain. Figure 5.17 shows the computational domain and boundary conditions. The High Performance Computing (HPC) cluster at WVU was able to run these simulations in parallel mode with a run time of 1 to 2 days per case.

The nozzle was assumed to be $38.1 \mathrm{~mm}$ above the impact surface so it was not included in the domain. Spray was injected $18 \mathrm{~mm}$ above the impact surface or $20.1 \mathrm{~mm}$ below the nozzle. There was no available PDPA data at $20.1 \mathrm{~mm}$ below the nozzle so different initial drop 
parameters (e.g. drop velocity and diameter) were tested to achieve the best agreement with the PDA data measured at $1.6 \mathrm{~mm}$ above an impact surface.

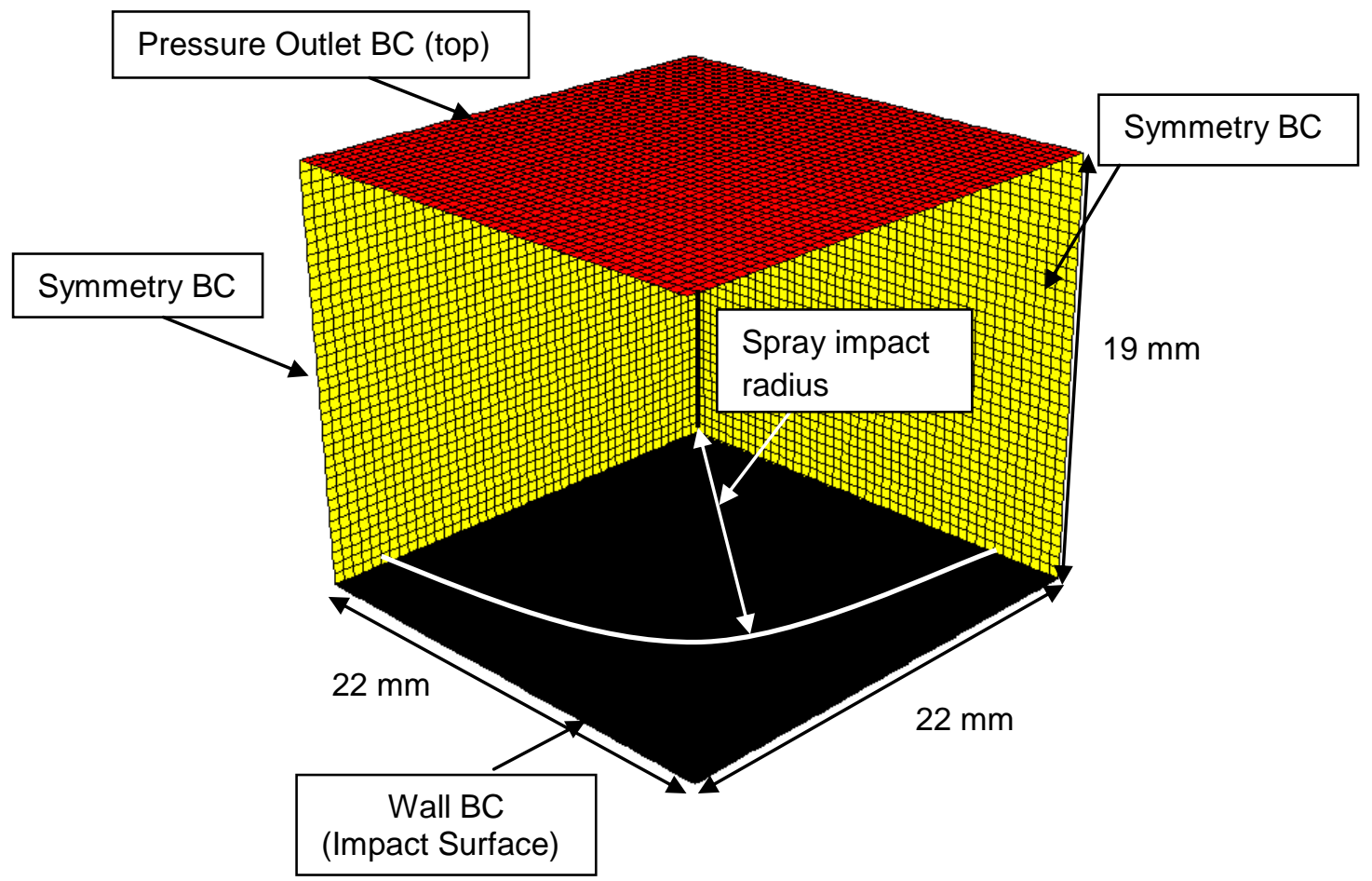

Figure 5.17 Case 5 updated computational domain and boundary conditions for the spray and spray cooling simulations using the DPM and EWF models. The viewpoint is above the impact surface looking toward the axis of symmetry. (Note that not all of the boundary conditions $(B C)$ are shown. Pressure outlet BCs at side surfaces are not included in this view.)

Figure 5.18 shows the liquid film thickness measured in the laboratory (Taylor et al., 2014a, 2014b) for the 40 psig spray compared to isothermal simulations using the domain shown in Figure 5.17 with $\mu_{e}=\mu$ (laminar) and $\mu_{e}=\mu+\mu_{t}=5 \mu$ (turbulent). Using the molecular viscosity gives a layer thickness that is smaller than the observations. For the eddy viscosity model (with $\mu_{e}=5 \mu$ ) the agreement is excellent out to $10 \mathrm{~mm}$ but becomes poor beyond $15 \mathrm{~mm}$. Due to time constraints, it was not possible to obtain better agreement by adjusting the eddy viscosity coefficient. It seems likely that better agreement could be obtained by reducing the coefficient as the radius increases beyond $10 \mathrm{~mm}$. A physical argument for this reduction can be made on the basis that the drop impacts become less forceful and more oblique as the radius increases. Nevertheless, such an adjustment was not attempted in the work presented here. 


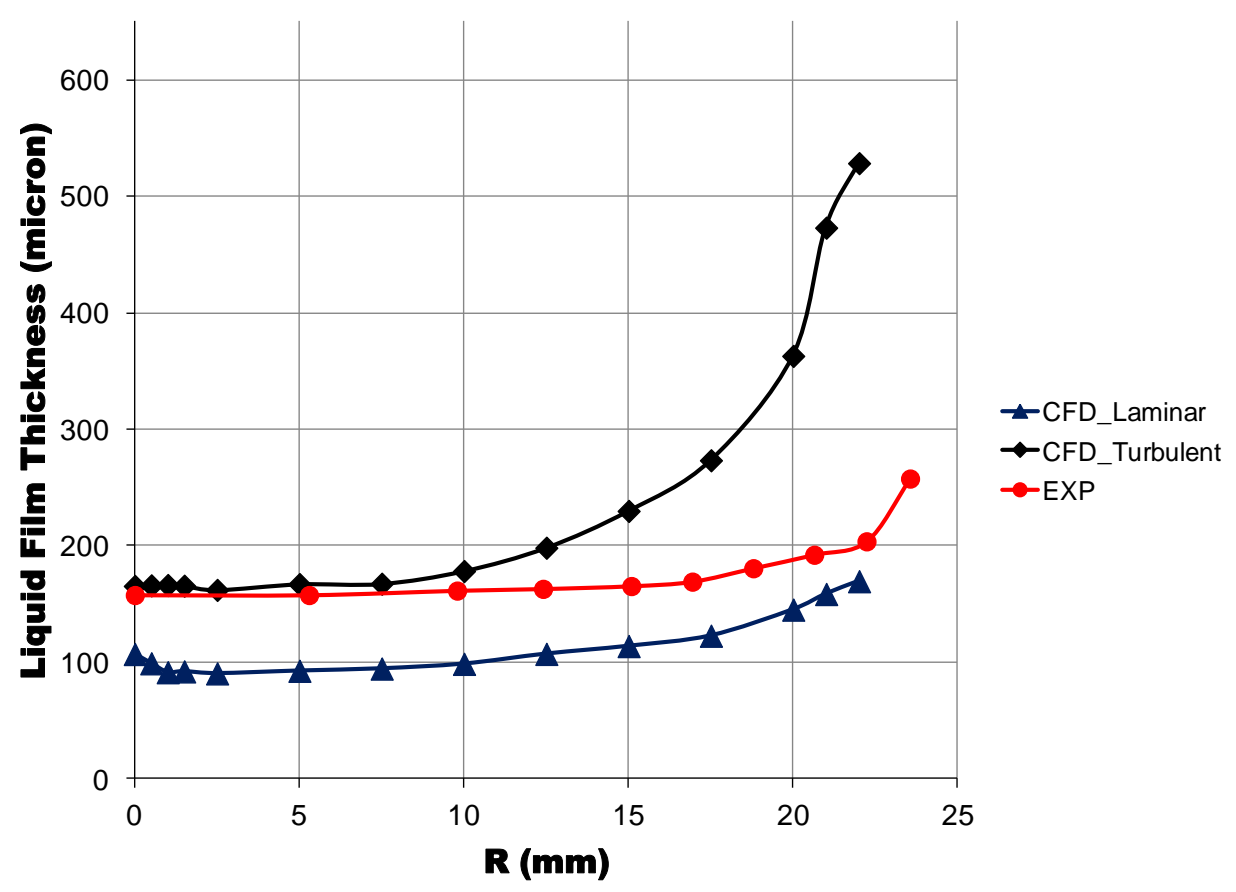

Figure 5.18 Computed liquid film thickness at $70 \mathrm{~ms}$ for laminar (CFD_Laminar) and constant turbulent viscosity $\left(\mu_{e}=5 \mu\right)$ (CFD_Turbulent) assumptions compared with experimental data (EXP) for 40 psi water spray case.

Table 5.5 shows additional details of the case presented in this section. A water spray at $300 \mathrm{~K}$ continuously impinges on the surface that is at a constant temperature of $372 \mathrm{~K}$. The surface temperature has been fixed $1 \mathrm{~K}$ below the boiling temperature since phase change in the film (e.g. bubble formation in the film, etc.) was not yet included in the model. The initial film temperature of $323 \mathrm{~K}$ is below the surface temperature $(372 \mathrm{~K})$ but is above the spray liquid temperature $(300 \mathrm{~K})$ in order to study the interaction between the hot surface and the cooler spray. The initial film thickness and temperature have been chosen to be close to the expected results in order to decrease the computational time required to approach steady state. 
Table 5.5 Model Characteristics for Case 5.

\begin{tabular}{|c|c|}
\hline Model parameters & Value \\
\hline Nozzle type & FullJet 1/8-G (Full Cone) \\
\hline Spray cone half angle & $28^{\circ}$ \\
\hline Nozzle to surface distance & $38.1 \mathrm{~mm}$ \\
\hline Spray radius at surface & $20.3 \mathrm{~mm}$ \\
\hline Nozzle pressure & 40 psig \\
\hline Spray liquid & Water (boiling point $373 \mathrm{~K}$ ) \\
\hline Drop liquid temperature & $300 \mathrm{~K}$ \\
\hline Air temperature & $300 \mathrm{~K}$ \\
\hline Surface temperature & $372 \mathrm{~K}$ \\
\hline Initial film temperature & $323 \mathrm{~K}$ \\
\hline Initial film thickness & 150 micron \\
\hline Energy equation included? & Yes \\
\hline Wall thermal boundary condition & Constant temperature \\
\hline Spray model & DPM \\
\hline Film models used & $\begin{array}{l}\text { EWF model with constant turbulent viscosity and } \\
\text { conductivity }\end{array}$ \\
\hline Domain geometry & $90^{\circ}$ rectangular domain \\
\hline Dimensionality & $3 \mathrm{D}$ \\
\hline Mesh type & Uniform $0.5 \mathrm{~mm}$ cubes \\
\hline
\end{tabular}

Figures 5.19, 5.20, 5.21 and 5.22 show flooded contours of liquid film depth-averaged velocity magnitude, thickness, mid depth liquid film temperature and surface heat flux, respectively.

Figure 5.19 shows the depth-averaged velocity magnitude of the spray film at $50 \mathrm{~ms}$ and $100 \mathrm{~ms}$. At both times, the velocity increases from 0 on the spray axis to a maximum of about 
$0.62 \mathrm{~m} / \mathrm{s}$ at a radius of about $12 \mathrm{~mm}$ and declines to about $0.3 \mathrm{~m} / \mathrm{s}$ at the edge of the spray at 20.3 $\mathrm{mm}$. Beyond the spray area, there is a wavy pattern with a maximum velocity of about $0.4 \mathrm{~m} / \mathrm{s}$ and a minimum of about $0.03 \mathrm{~m} / \mathrm{s}$. The overall pattern exhibits approximate rotational symmetry at both times, but there are noticeable deviations near the symmetry planes.

Figure 5.20 shows that the film thickness under the spray changes very little from $50 \mathrm{~ms}$ to $100 \mathrm{~ms}$. At both times the thickness is nearly constant at about 150 microns from the axis out to $14 \mathrm{~mm}$. It increases to about 500 microns at the edge of the spray $(20.3 \mathrm{~mm})$ and to a maximum of about 900 microns at $50 \mathrm{~ms}$ and about 1000 microns at $100 \mathrm{~ms}$ outside of the spray. The thickness has a wavy pattern outside of the spray at both times. At $50 \mathrm{~ms}$, the thickness decreases to less than 150 microns at about $27 \mathrm{~mm}$. At both times, the thickness is largely rotationally symmetric.

Figure 5.21 shows that there is little change in the mid depth film temperature between 50 $\mathrm{ms}$ and $100 \mathrm{~ms}$. The temperature increases from about $320 \mathrm{~K}$ on the axis to about $330 \mathrm{~K}$ at the edge of the spray. There is some waviness at radii larger than about $14 \mathrm{~mm}$. The temperature continues to increase to about $369 \mathrm{~K}$ at the largest radius, although the high temperature area is much smaller and less regular at $100 \mathrm{~ms}$. At both times, the temperature is largely rotationally symmetric.

Figure 5.22 shows little change in the surface heat flux at $50 \mathrm{~ms}$ and $100 \mathrm{~ms}$. The patterns are largely rotationally symmetric, but in both cases, the area of highest heat flux (about $1.91 \mathrm{MW} / \mathrm{m}^{2}=191 \mathrm{~W} / \mathrm{cm}^{2}$ ) has an irregular outline. It is also notable that the heat flux on the axis is only about $1.81 \mathrm{MW} / \mathrm{m}^{2}\left(181 \mathrm{~W} / \mathrm{cm}^{2}\right)$. The heat flux is about $0.76 \mathrm{MW} / \mathrm{m}^{2}\left(76 \mathrm{~W} / \mathrm{cm}^{2}\right)$ at the edge of the spray. At larger radii the heat flux varies from about $0.48 \mathrm{MW} / \mathrm{m}^{2}\left(48 \mathrm{~W} / \mathrm{cm}^{2}\right)$ to as low as $0.1 \mathrm{MW} / \mathrm{m}^{2}\left(10 \mathrm{~W} / \mathrm{cm}^{2}\right)$ in a wavy pattern. The importance of the spray in achieving high heat fluxes is quite obvious from these results. At both times, the heat flux is approximately rotationally symmetric. 


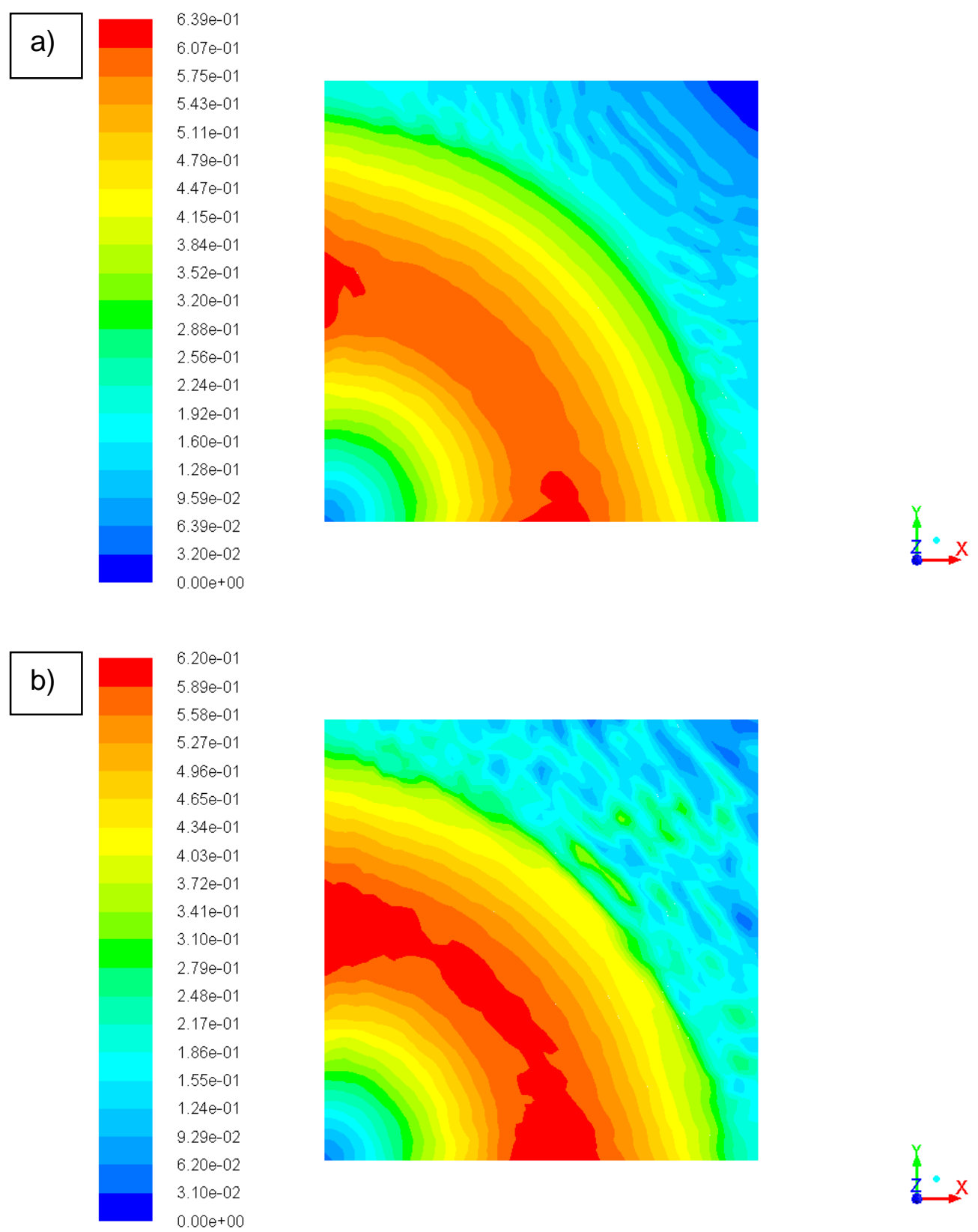

Figure 5.19 Case 5 flooded contours of the liquid velocity magnitude at a) $50 \mathrm{~ms}$, b) $100 \mathrm{~ms}$. The color scale is not the same in a) and b). Red refers to the maximum velocity, blue refers to the minimum velocity. (All images show the top view of the impact surface.) 

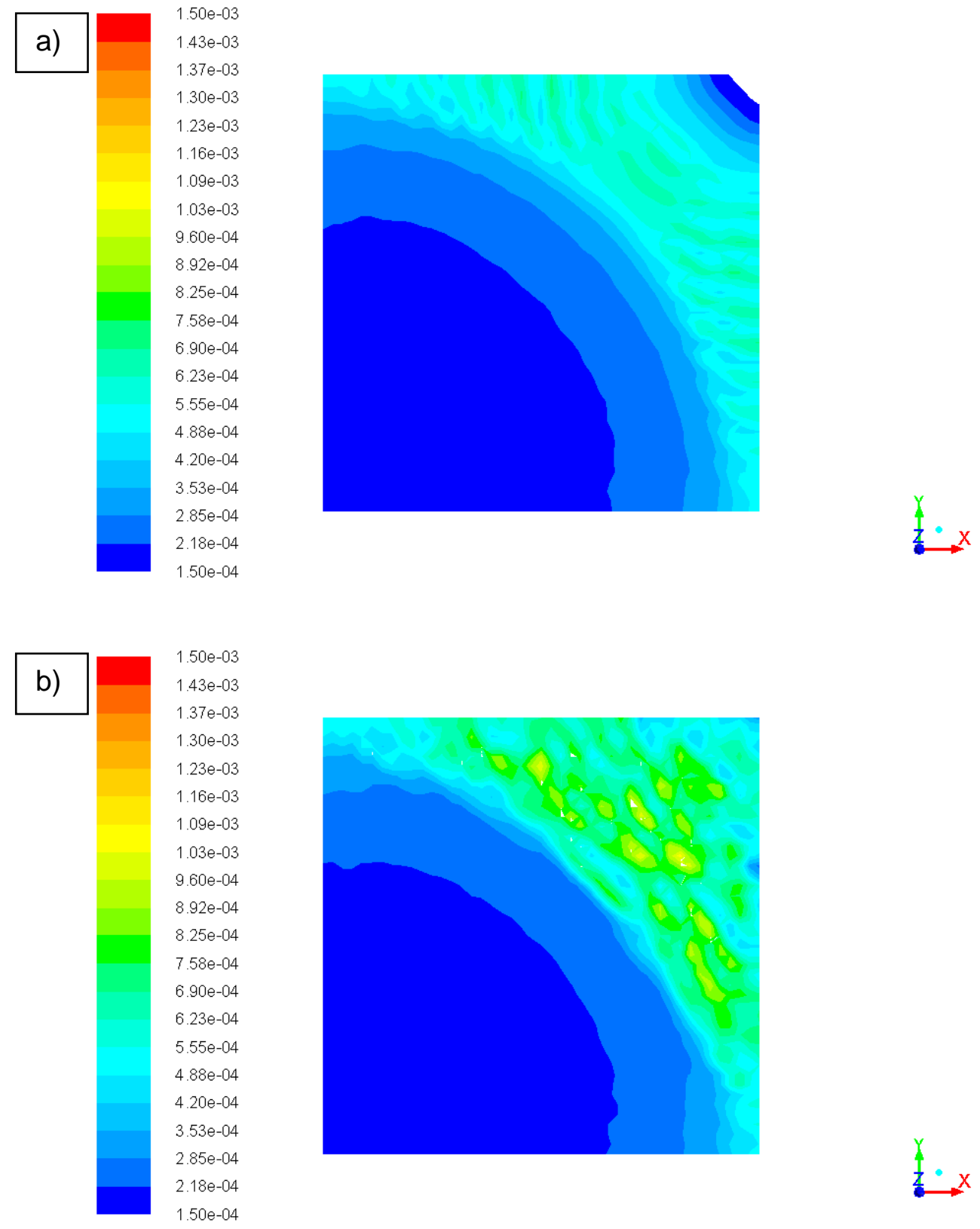

Figure 5.20 Case 5 flooded contours of the liquid film thickness at a) $50 \mathrm{~ms}$, b) $100 \mathrm{~ms}$. The color scale is the same for both times. Blue refers to 150 micron, red refers to 1500 micron (1.5 $\mathrm{mm})$. (All images show the top view of the impact surface.) 


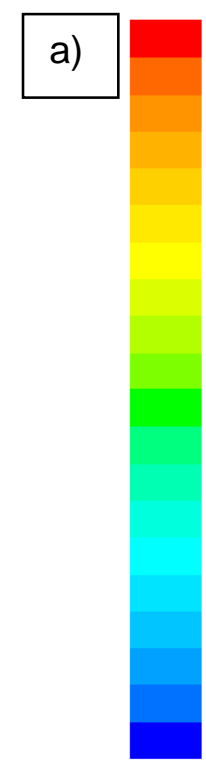

$3.70 e^{+02}$

$3.66 \mathrm{e}+02$

$3.63 e+02$

$3.59 \mathrm{e}+02$

$3.56 \mathrm{e}+02$

$3.52 e+02$

$3.49 \mathrm{e}+02$

$3.45 e+02$

$3.42 e+02$

$3.38 \mathrm{e}+02$

$3.35 e+02$

$3.31 e+02$

$3.28 \mathrm{e}+02$

$3.24 e+02$

$3.21 \mathrm{e}+02$

$3.17 e+02$

$3.14 e+02$

$3.10 e+02$

$3.07 e+02$

$3.03 e+02$

$3.00 e+02$

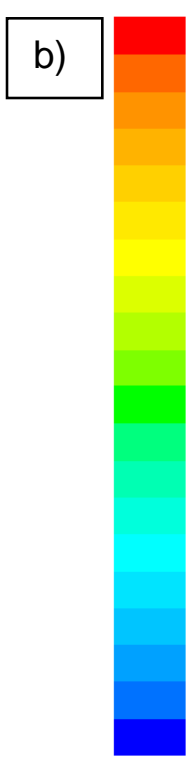

$3.69 \mathrm{e}+02$

$3.66 \mathrm{e}+02$

$3.62 e+02$

$3.59 \mathrm{e}+02$

$3.55 \mathrm{e}+02$

$3.52 e+02$

$3.48 \mathrm{e}+02$

$3.45 e+02$

$3.41 e+02$

$3.38 \mathrm{e}+02$

$3.34 \mathrm{e}+02$

$3.31 e+02$

$3.27 e+02$

$3.24 \mathrm{e}+02$

$3.20 e+02$

$3.17 e+02$

$3.13 e+02$

$3.10 \mathrm{e}+02$

$3.06 \mathrm{e}+02$

$3.03 e+02$

$2.99 e+02$
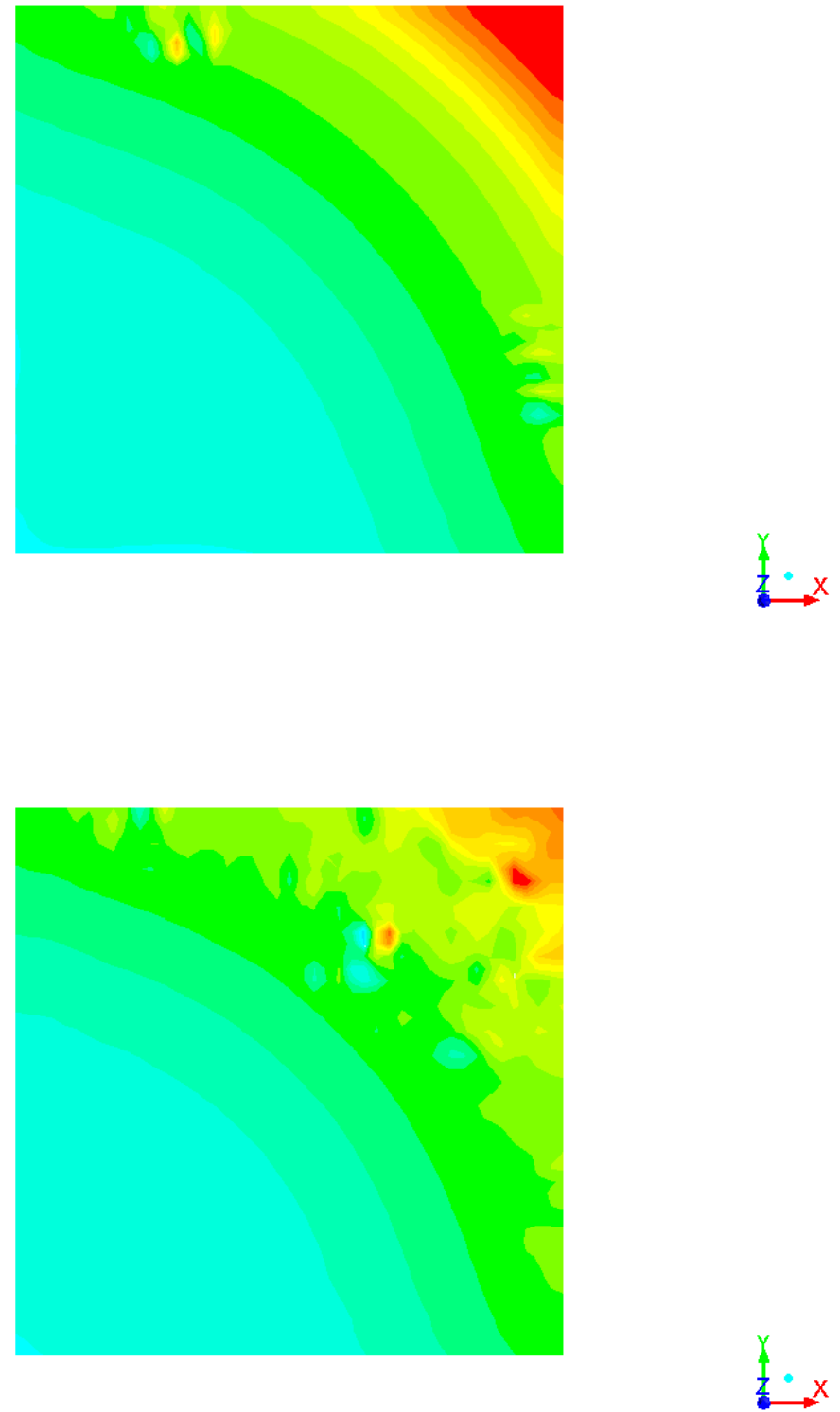

Figure 5.21 Case 5 flooded contours of the mid depth liquid film temperature at a) $50 \mathrm{~ms}$, b) 100 ms. The color scale is almost the same in a) and b). (All images show the top view of the impact surface.) 

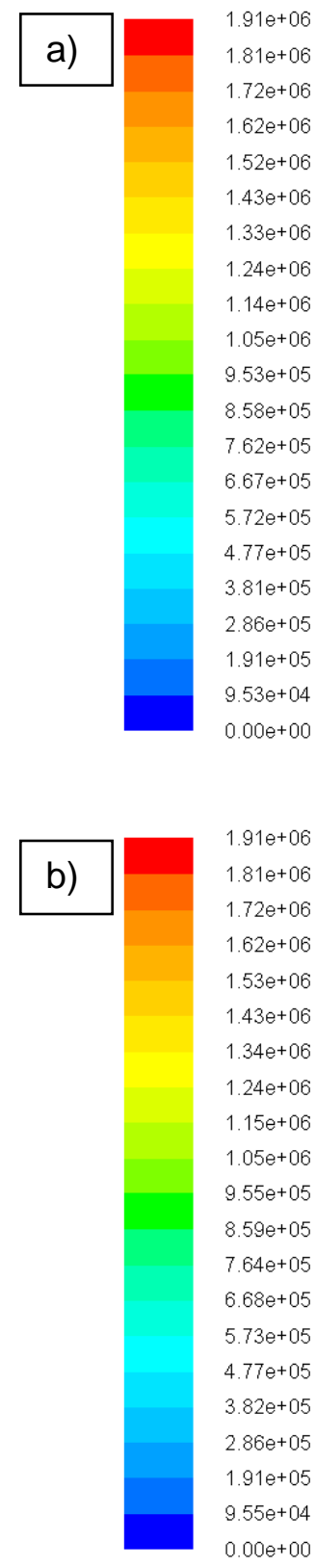
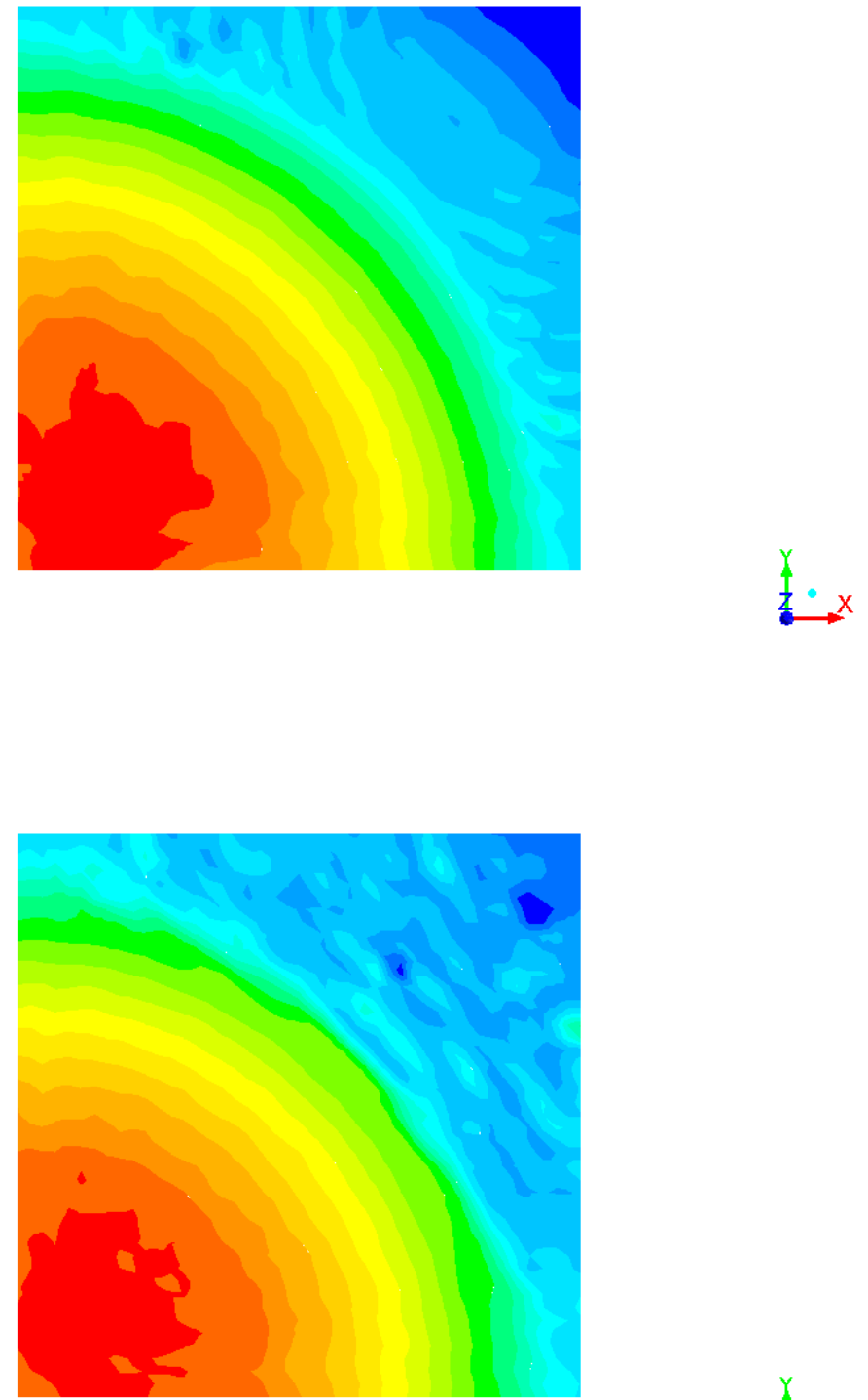

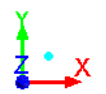

Figure 5.22 Case 5 flooded contours of the surface heat flux at a) $50 \mathrm{~ms}$, b) $100 \mathrm{~ms}$. The color scale differs in a) and b). The color scale is almost the same in a) and b). All images show the top view of the impact surface. Blue refers to $0 \mathrm{~W} / \mathrm{m}^{2}$ and red refers to $1.9 \mathrm{MW} / \mathrm{m}^{2}$.

Figure 5.23 shows the film thickness along the $x$-axis at various times. At $t=0$, the film has a uniform thickness of 150 microns. At $1.5 \mathrm{~ms}$, the layer depth is reduced within about 5 $\mathrm{mm}$ of the spray axis due to the downward momentum of the spray, with a minimum on the axis 
of about 70 microns. The film remains at its initial thickness at larger radii where the spray has yet to impact the film. At $6 \mathrm{~ms}$, the central depth has recovered to about 120 microns and the layer is deeper than its initial depth beyond about $5 \mathrm{~mm}$. At $10 \mathrm{~ms}$, the film thickness increases from about 150 microns on the axis to about 190 microns at $17.5 \mathrm{~mm}$. It then drops below 100 microns. At $30 \mathrm{~ms}$ the depth profile is approaching steady state, and the $50 \mathrm{~ms}$ and $100 \mathrm{~ms}$ profiles are nearly identical. The $100 \mathrm{~ms}$ depth profile is about 180 microns at the spray axis, dips to a minimum of about 160 microns at $2.5 \mathrm{~mm}$, recovers to about 180 microns at $10 \mathrm{~mm}$, and then rises more steeply to about 500 microns at $22 \mathrm{~mm}$. These computed liquid film thickness values are consistent with the experimental results shown in Figure 5.23 (Taylor et al., 2014a, 2014b).

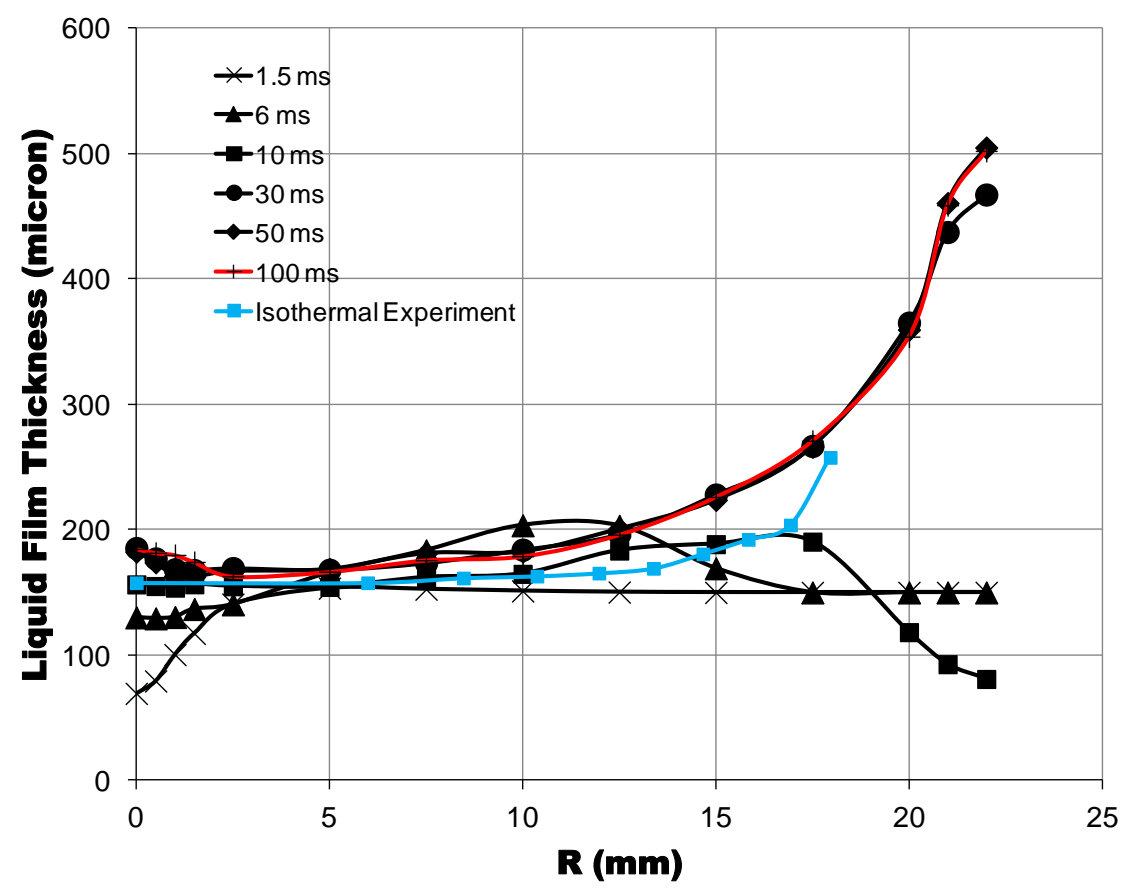

Figure 5.23 Case 5 film thickness along the $x$-axis at various times for $300 \mathrm{~K}$ spray impacting on liquid film with 150 micron initial height and $323 \mathrm{~K}$ initial film temperature. (Impact surface temperature is $372 \mathrm{~K}$. "Isothermal Experiment" shows the experimental film thickness measurements for the same spray case using 40 psi full cone nozzle without heat transfer) 
Figure 5.24 shows the film mid-point temperature along the $x$-axis at various times. The surface temperature is $372 \mathrm{~K}$ and the droplet temperature is $300 \mathrm{~K}$ at all times. At $t=0$, the film is isothermal at $323 \mathrm{~K}$. At $1.5 \mathrm{~ms}$, the decrease of the layer thickness due to the spray momentum outweighs the effect of the cooler temperature of the droplets so that the temperature rises within about $2.5 \mathrm{~mm}$ of the spray axis. The maximum temperature is about $337 \mathrm{~K}$ on the axis. Heat conduction raises the temperature of the rest of the film to about $327 \mathrm{~K}$. At $6 \mathrm{~ms}$, the entire film has been affected by the spray. The axis temperature has dropped to about $329 \mathrm{~K}$. The temperature declines to a minimum of about $323 \mathrm{~K}$ at $7 \mathrm{~mm}$ and then increases to about 337 $\mathrm{K}$ at $22 \mathrm{~mm}$. At $10 \mathrm{~ms}$, the axis temperature has dropped to $326 \mathrm{~K}$ and the temperature increases with increasing radius to about $366 \mathrm{~K}$ at $22 \mathrm{~mm}$. The entire profile is cooled significantly at 30 $\mathrm{ms}$. The temperature on the axis is about $320 \mathrm{~K}$ and it the rises to about $341 \mathrm{~K}$ at $22 \mathrm{~mm}$. The $50 \mathrm{~ms}$ and $100 \mathrm{~ms}$ temperature profiles are nearly identical, indicating the approach to steady state. The temperature rises from about $319 \mathrm{~K}$ on the axis to about $332 \mathrm{~K}$ at $22 \mathrm{~mm}$.

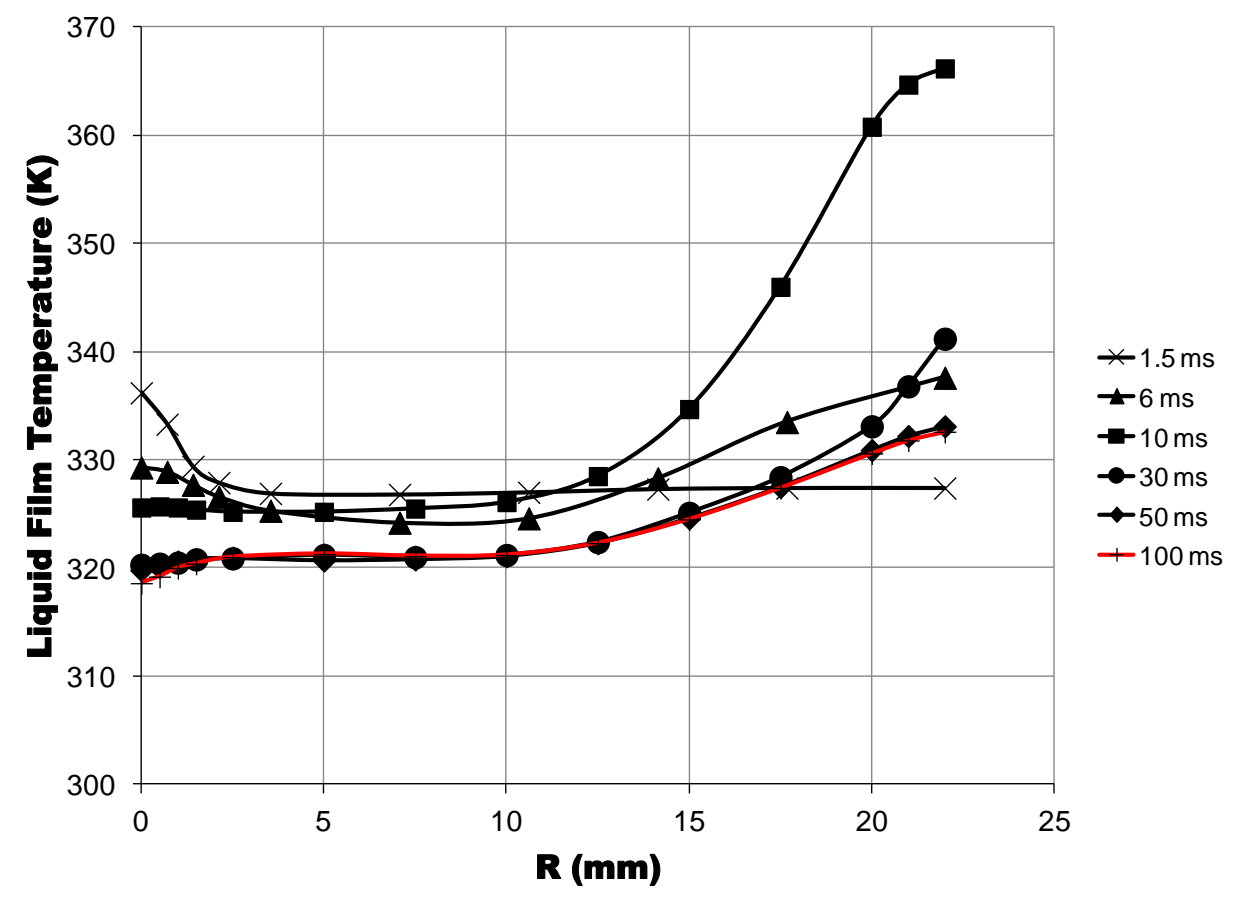

Figure 5.24 Case 5 mid depth liquid film temperature along the $\mathrm{x}$-axis for various times for 300 $\mathrm{K}$ spray impacting on liquid film with 150 micron initial height and $323 \mathrm{~K}$ initial temperature. (Impact surface temperature is $372 \mathrm{~K}$.) 
Figure 5.25 shows the heat flux averaged over the entire $22 \mathrm{~mm}$ x $22 \mathrm{~mm}$ surface as a function of time. The surface heat flux decreases from $1.9 \mathrm{MW} / \mathrm{m}^{2}\left(190 \mathrm{~W} / \mathrm{cm}^{2}\right)$ to $1 \mathrm{MW} / \mathrm{m}^{2}$ $\left(100 \mathrm{~W} / \mathrm{cm}^{2}\right)$ in around $20 \mathrm{~ms}$, and after that time it does not vary significantly.

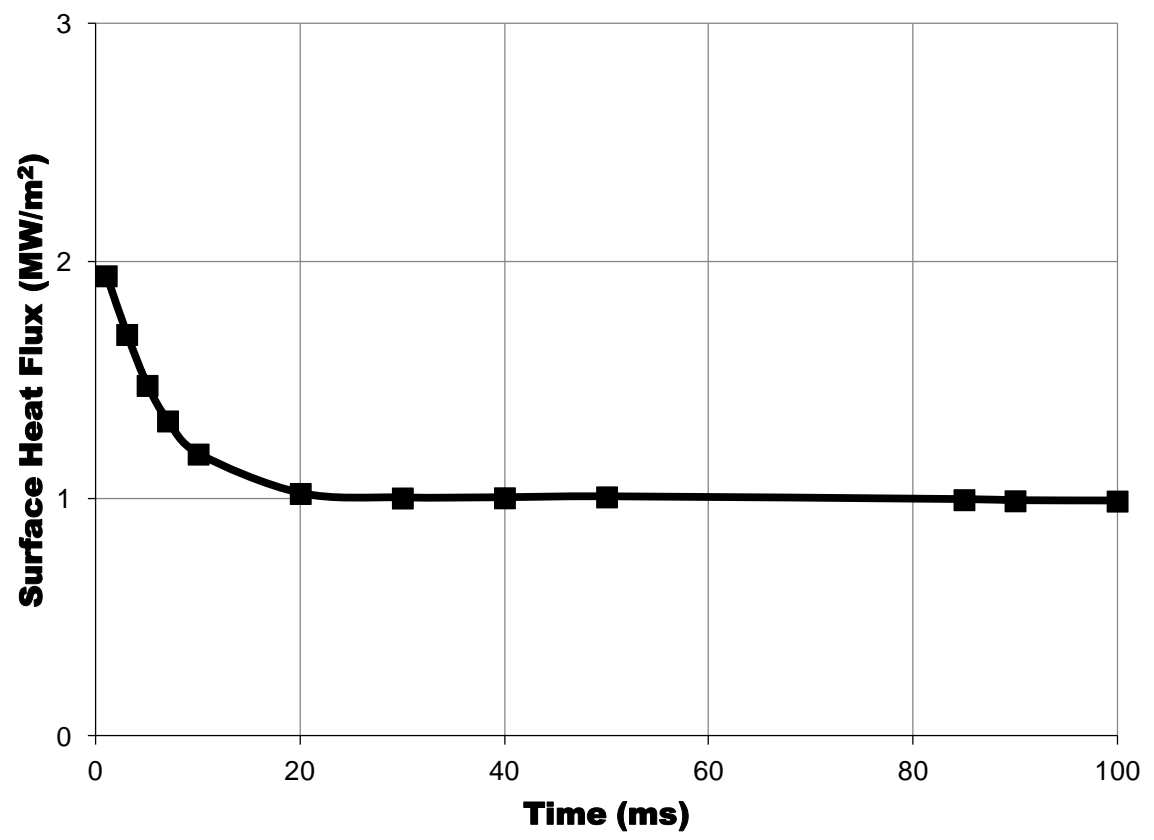

Figure 5.25 Case 5 average heat flux variation on the surface (22 $\mathrm{mm} \times 22 \mathrm{~mm})$.

As a summary of Chapter 5, the spray cooling simulations using the 3D DPM and EWF models with constant turbulent viscosity set equal to 4 times the laminar viscosity, and constant turbulent conductivity give believable results when boiling does not occur. 


\section{CHAPTER 6: SPRAY IMPACT AND COOLING SIMULATIONS WITH PHASE CHANGE USING THE DISCRETE PHASE MODEL (DPM), THE EULERIAN WALL FILM MODEL (EWF) AND THE SPECIES TRANSPORT MODEL (STM)}

In this chapter, simulations of spray cooling with phase change using the Discrete Phase Model (DPM), the Eulerian Wall Film (EWF) model and the Species Transport Model (STM) in ANSYS Fluent 15 are described. The same computational domain, initial and boundary conditions are used in this Chapter as in Case 5 of Chapter 5.

\subsection{Limitations of the EWF for Heat Transfer Calculations in Fluent 14.5}

A problem was noticed with ANSYS Fluent 14.5 in a simple test case which used the EWF and the energy equation without specifically including a liquid boiling model. A $1 \mathrm{~mm}$ thick static water layer was heated from below by a surface at a fixed temperature $372 \mathrm{~K}$. The initial water temperature was $371 \mathrm{~K}$ while the air above the film was at $300 \mathrm{~K}$. There was conduction heat transfer between the film and wall and convection heat transfer between the film and the air. The maximum temperature in the film reached close to $393 \mathrm{~K}$, greater than the wall temperature (372 $\mathrm{K})$. Of course this is physically impossible. This phenomenon had not been observed during the spray cooling simulations in Chapter 5, perhaps due to the colder spray drops causing the liquid film temperature to always become less than the wall temperature. This issue could not be solved in ANSYS Fluent 14.5.

When ANSYS Fluent 15 was used to perform the same test case the temperature inside the film never exceeded the wall temperature. However, attempts to include surface tension in version 15 of ANSYS Fluent when using the EWF failed because of an undiscovered reason which caused the simulation to stop as soon as the simulation was started.

In order to determine if accurate simulations of spray cooling could be obtained in ANSYS Fluent 15 without surface tension, the 40 psi full cone spray case (Case 5 of Chapter 5) was simulated using ANSYS Fluent 15 without the surface tension in the EWF. The results were compared to the same spray case with the surface tension in the EWF model using Fluent 14.5. Figure 6.1 shows the comparison of the flooded contours of film thickness at 90 ms simulation time. The film thickness variation at the spray impact region $(0<R<20.3 \mathrm{~mm})$ is very similar with and without surface tension. But, there are differences outside of the impact region $(R>$ $20.3 \mathrm{~mm}$ ). Figure 6.2 shows the comparison of the flooded contours of film velocity magnitude 
at $90 \mathrm{~ms}$ simulation time. The film velocity magnitude is also very similar with and without surface tension included in the EWF model. It was concluded that surface tension did not have any significant effects on the key characteristics such as film thickness and film velocity magnitude in the spray impact region $(0<R<20.3 \mathrm{~mm})$, at least under these conditions.

In addition, the comparisons of a $40 \mathrm{psi}$ spray heat transfer case (Case 5) showed that the film temperature distribution within the impact area did not change significantly between ANSYS Fluent 14.5 with surface tension and ANSYS Fluent 15 without surface tension. Therefore, it was decided that ANSYS Fluent 15 without surface tension could be used for spray cooling simulations using the EWF, and the previous spray and spray cooling simulations presented in Chapter 5 using ANSYS Fluent 14.5 are still considered valid. But the simulations in Section 6.2 which include phase change in the film have performed using ANSYS Fluent 15 with no surface tension effects included in the EWF. 


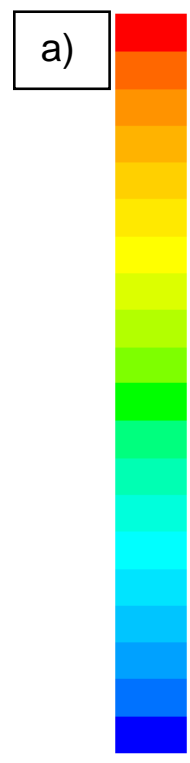

$7.16 \mathrm{e}-04$

$6.88 \mathrm{e}-04$

$6.59 \mathrm{e}-04$

$6.31 \mathrm{e}-04$

$6.03 e-04$

$5.75 \mathrm{e}-04$

$5.46 \mathrm{e}-04$

$5.18 \mathrm{e}-04$

$4.90 \mathrm{e}-04$

4. $61 \mathrm{e}-04$

4. $33 \mathrm{e}-04$

4.05e-04

$3.76 \mathrm{e}-04$

$3.48 \mathrm{e}-04$

$3.20 \mathrm{e}-04$

$2.92 \mathrm{e}-04$

2.63e-04

$2.35 \mathrm{e}-04$

$2.07 e-04$

$1.78 \mathrm{e}-04$

$1.50 \mathrm{e}-04$
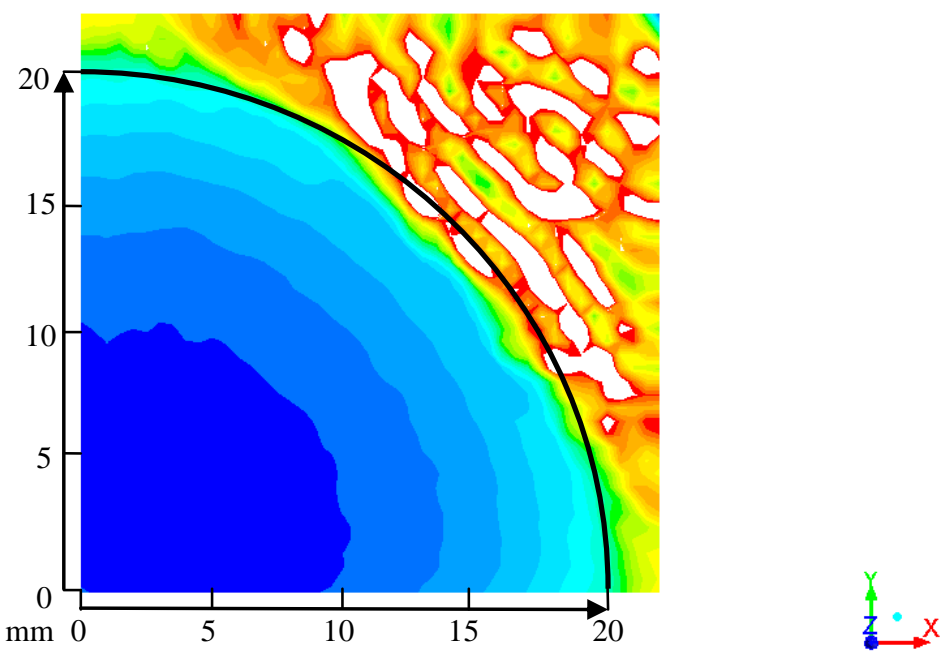

Contours of Film Thickness $(\mathrm{m})($ Time $=9.0000 \mathrm{e}-02)$

Jan 23, 2015

ANSYS Fluent 14.5 (3d, dp, pbns, rke, transient)

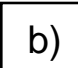

b)

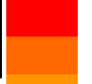

$7.16 \mathrm{e}-04$

$6.88 \mathrm{e}-04$

$6.59 \mathrm{e}-04$

$6.31 e-04$

$6.03 e-04$

$5.75 e-04$

$5.46 \mathrm{e}-04$

$5.18 \mathrm{e}-04$

$4.90 e-04$

4. $61 \mathrm{e}-04$

4. $33 \mathrm{e}-04$

$4.05 e-04$

$3.76 \mathrm{e}-04$

$3.48 \mathrm{e}-04$

3.20e-04

2.92e-04

$2.63 \mathrm{e}-04$

$2.35 \mathrm{e}-04$

$2.07 e-04$

$1.78 \mathrm{e}-04$

$1.50 \mathrm{e}-04$
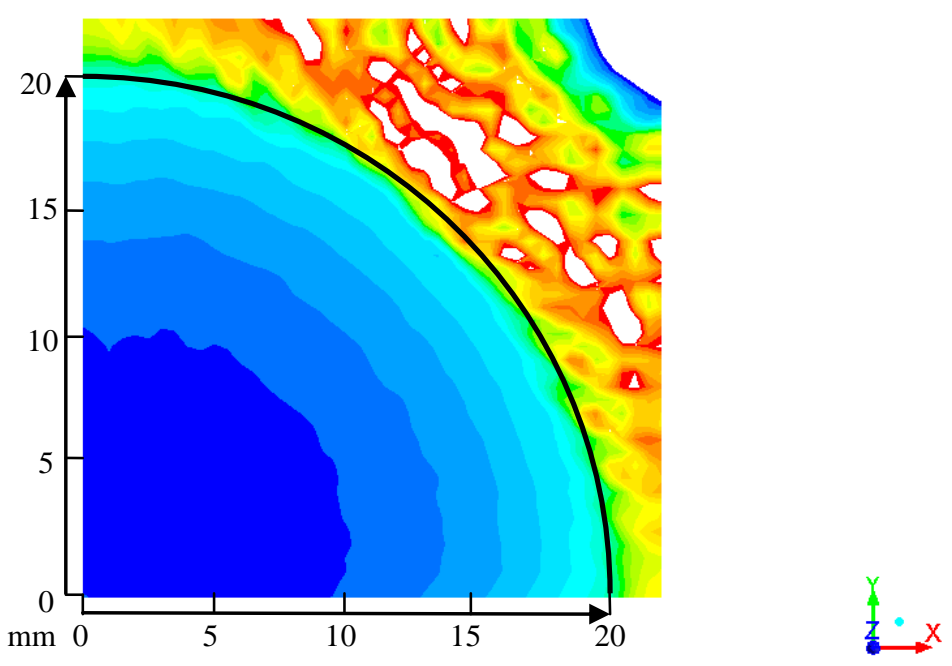

Contours of Film Thickness $(\mathrm{m})($ Time $=9.0000 \mathrm{e}-02)$

Dec 13, 2014 ANSYS Fluent 14.5 (3d, dp, pbns, rke, transient)

Figure 6.1 Flooded contours of film thickness at $90 \mathrm{~ms}$ simulation time for a $40 \mathrm{psi}$ full cone spray a) The ANSYS Fluent 15 EWF model without surface tension b) The ANSYS Fluent 14.5 EWF model with surface tension. (The color scales are the same in (a) and (b). All images show the top view of the impact surface.) 


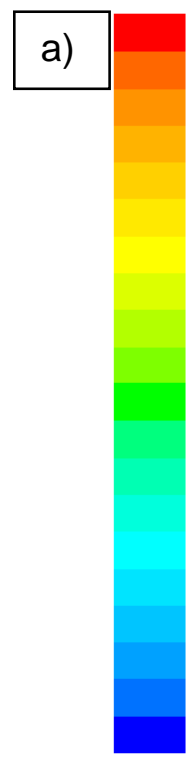

5.41e-01

$5.09 \mathrm{e}-01$

4.78e-01

4.46e-01

4. $14 \mathrm{e}-01$

3.82e-01

3.50e-01

3. $18 \mathrm{e}-01$

2.87e-01

2.55e-01

2.23e-01

$1.91 \mathrm{e}-01$

$1.59 \mathrm{e}-01$

$1.27 e-01$

$9.55 \mathrm{e}-02$

6.37e-02

$3.18 \mathrm{e}-02$

$0.00 e+00$
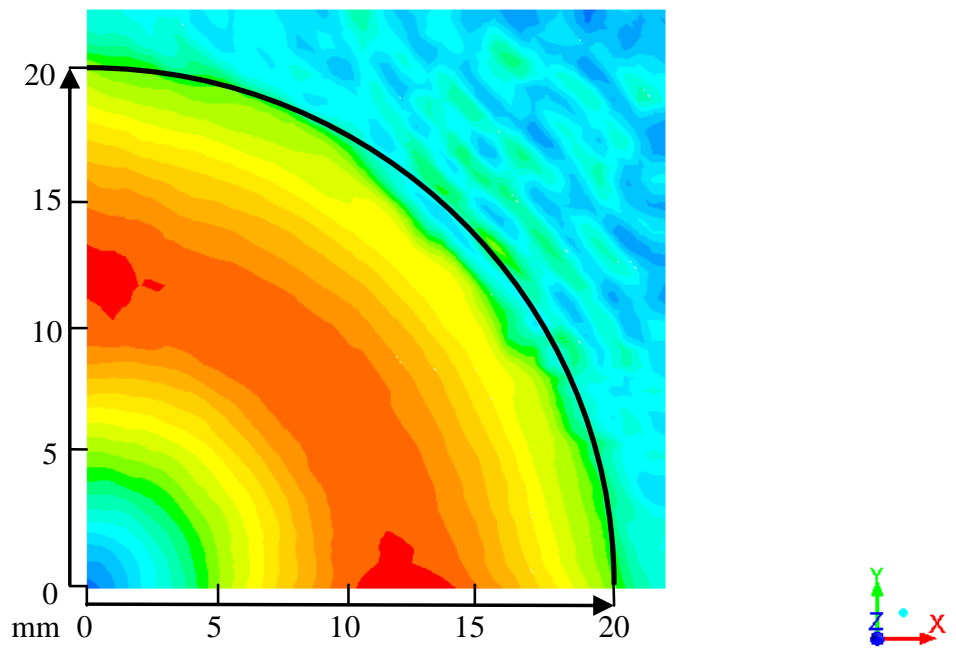

Contours of Film Velocity Magnitude $(\mathrm{m} / \mathrm{s}) \quad($ Time $=9.0000 \mathrm{e}-02)$

Jan 23, 2015

ANSYS Fluent 14.5 (3d, dp, pbns, rke, transient)

\section{b)}

$6.53 \mathrm{e}-01$

$6.20 \mathrm{e}-01$

$5.88 \mathrm{e}-01$

$5.55 \mathrm{e}-01$

$5.22 \mathrm{e}-01$

$4.90 e-01$

$4.57 e-01$

$4.24 \mathrm{e}-01$

3.92e-01

3.59e-01

3. $26 \mathrm{e}-01$

2.94e-01

2.61e-01

2.29e-01

$1.96 \mathrm{e}-01$

1.63e-01

$1.31 e-01$

9. $79 \mathrm{e}-02$

$6.53 \mathrm{e}-02$

3. $27 \mathrm{e}-02$

$0.00 e+00$
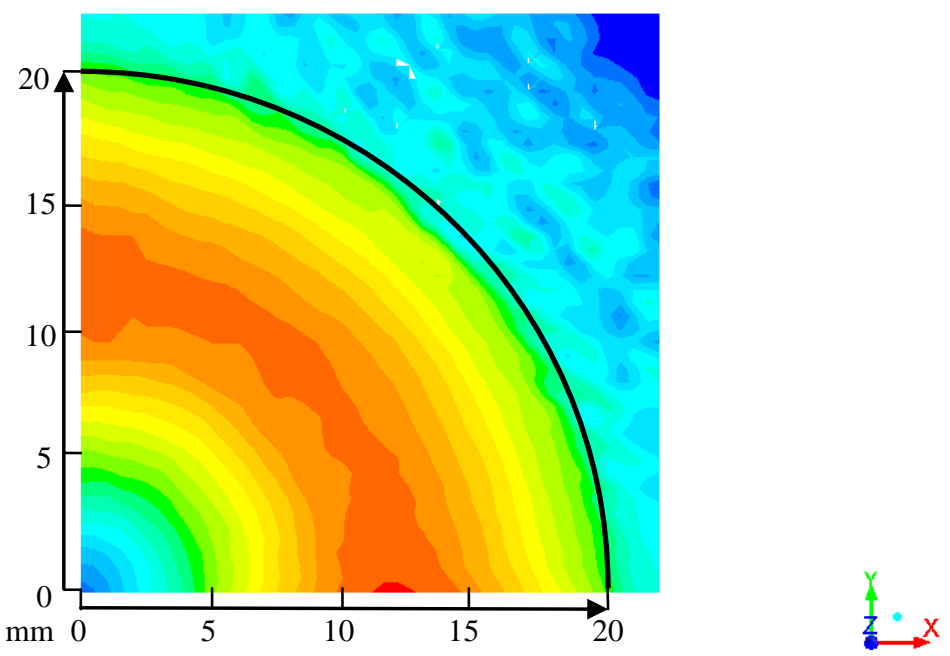

Contours of Film Velocity Magnitude $(\mathrm{m} / \mathrm{s})($ Time $=9.0000 \mathrm{e}-02)$

Dec 13, 2014 ANSYS Fluent 14.5 (3d, dp, pbns, rke, transient)

Figure 6.2 Flooded contours of film velocity magnitude at $90 \mathrm{~ms}$ simulation time for 40 psi full cone spray a) The ANSYS Fluent 15 EWF model without surface tension b) The ANSYS Fluent 14.5 EWF model with surface tension. (The color scales are very close in (a) and (b). All images show the top view of the impact surface.) 


\subsection{The Species Transport Model (STM) and the Conservation Equations for Species}

The Species Transport Model (STM) is used to account for the phase change effects in the DPM spray drops and in the EWF film. Spray cooling with phase change simulations are performed using the DPM-EWF-STM model which will be described in this chapter.

The correct coupling of the DPM, EWF and STM was the most challenging part for spray cooling simulations with phase change. In order to use the STM accurately, the species materials and their properties must be defined properly (e.g. water vapor, air, etc). The exact same name must be defined for the DPM spray liquid material and also for the EWF liquid film material otherwise the simulation either does not start or does not converge. The phase change material which is defined as a species material (e.g. water vapor) must be also the same for both the DPM and the EWF model. Several test simulations were performed in order to obtain an optimum model with correct material and model setup which would give converged and accurate results.

The Species Transport Model (STM) in ANSYS Fluent solves the governing conservation equations for each species in the computational domain (e.g. water vapor). The local mass fraction of each species $\left(Y_{i}\right)$ is calculated by solving a convection-diffusion equation (equation 6.1) (ANSYS Fluent Theory Guide, 2013).

$$
\frac{\partial\left(\rho Y_{i}\right)}{\partial t}+\nabla \cdot\left(\rho \underline{V} Y_{i}\right)=-\nabla \cdot \underline{J_{i}}+R_{i}+S_{i}
$$

where $J_{i}$ is the diffusion flux.

$R_{i}$ is the net rate of production of species, $i$, by chemical reaction

$S_{i}$ is the rate of creation by addition from the dispersed phase and other sources.

The diffusion flux, $\underline{J_{i}}$, for laminar flows is calculated based on Fick's Law (ANSYS Fluent Theory Guide, 2013) including mass transport induced by thermal gradients (the Soret effect)

$$
\underline{J_{i}}=-\rho D_{i, m} \nabla Y_{i}-D_{T, i} \frac{\nabla T}{T}
$$

where $D_{i, m}$ is the mass diffusion coefficient for species, $\mathrm{i}$ 
$D_{T, i}$ is the thermal (Soret) diffusion coefficient.

6.3. Spray Cooling Simulations with Phase Change using the Discrete Phase Model, the Eulerian Wall Film Model with Turbulent Viscosity $(\mu)$ and Thermal Conductivity $(k)$ and the Species Transport Model

In Chapter 5, spray cooling simulations using the DPM and EWF without phase change were reported. In this section, spray cooling simulations with phase change computed using the Species Transport Model (STM) with the DPM and EWF in ANSYS Fluent 15 are described. The STM enables the inclusion of phase change effects for the spray drops and the liquid film. The coupling between the DPM, EWF and STM was achieved after several test simulations were performed. The computational domain, initial conditions, boundary conditions and mesh are the same as Case 5 described in Chapter 5. A typical run time for one case was about 3 days on the High Performance Computing (HPC) cluster using 6 cores and 48 GB memory, and about 8 days on a workstation using 4 cores and 24 GB memory.

\subsubsection{Spray Cooling Simulations with Phase Change: Case I}

Table 6.1 lists the model parameters for 40 psi full cone water spray cooling simulations with phase change, Cases I, II and III. In these cases, a constant surface temperature boundary condition was implemented with $375 \mathrm{~K}$ temperature, $2 \mathrm{~K}$ more than the water boiling temperature. The initial film thickness was defined as 150 microns and the initial film temperature was $350 \mathrm{~K}$. The spray temperature and the surrounding gas (air) temperature were both $300 \mathrm{~K}$. The superheat and subcooling temperatures can be defined as

$$
\begin{aligned}
& T_{\text {superheat }}=T_{\text {surface }}-T_{\text {boiling }} \\
& T_{\text {subcooling }}=T_{\text {boiling }}-T_{\text {spray }}
\end{aligned}
$$

where $T_{\text {surface }}$ is the surface temperature

$T_{\text {boiling }}$ is the spray liquid boiling temperature

$T_{\text {spray }}$ is the spray temperature. 
Table 6.1 Model Characteristics for Cases I, II, III.

\begin{tabular}{|c|c|c|c|}
\hline Model parameters & Case I & Case II & Case III \\
\hline Nozzle type & FullJet 1/8-G (Full Cone) & FullJet 1/8-G (Full Cone) & FullJet 1/8-G (Full Cone) \\
\hline Spray cone half angle & $28^{\circ}$ & $28^{\circ}$ & $28^{\circ}$ \\
\hline $\begin{array}{l}\text { Nozzle to surface } \\
\text { distance }\end{array}$ & $38.1 \mathrm{~mm}$ & $38.1 \mathrm{~mm}$ & $38.1 \mathrm{~mm}$ \\
\hline Spray radius at surface & $20.3 \mathrm{~mm}$ & $20.3 \mathrm{~mm}$ & $20.3 \mathrm{~mm}$ \\
\hline Nozzle pressure & 40 psig & 40 psig & 40 psig \\
\hline Spray liquid & $\begin{array}{l}\text { Water } \\
\text { (boiling point } 373 \mathrm{~K} \text { ) }\end{array}$ & $\begin{array}{l}\text { Water } \\
\text { (boiling point } 373 \mathrm{~K} \text { ) }\end{array}$ & $\begin{array}{l}\text { Water } \\
\text { (boiling point } 373 \mathrm{~K} \text { ) }\end{array}$ \\
\hline Drop liquid temperature & $300 \mathrm{~K}$ & $350 \mathrm{~K}$ & $300 \mathrm{~K}$ \\
\hline Subcooling & $73 \mathrm{~K}$ & $23 \mathrm{~K}$ & $73 \mathrm{~K}$ \\
\hline Air temperature & $300 \mathrm{~K}$ & $300 \mathrm{~K}$ & $350 \mathrm{~K}$ \\
\hline Surface temperature & $375 \mathrm{~K}$ & $375 \mathrm{~K}$ & $375 \mathrm{~K}$ \\
\hline Superheat & $2 \mathrm{~K}$ & $2 \mathrm{~K}$ & $2 \mathrm{~K}$ \\
\hline Initial film temperature & $350 \mathrm{~K}$ & $350 \mathrm{~K}$ & $350 \mathrm{~K}$ \\
\hline Initial film thickness & 150 micron & 150 micron & 150 micron \\
\hline $\begin{array}{l}\text { Energy equation } \\
\text { included? }\end{array}$ & Yes & Yes & Yes \\
\hline $\begin{array}{l}\text { Wall thermal boundary } \\
\text { condition }\end{array}$ & Constant temperature & Constant temperature & Constant temperature \\
\hline Spray model & DPM & DPM & DPM \\
\hline Film models used & $\begin{array}{l}\text { EWF model with } \\
\text { Turbulent } \mu \text { and } k\end{array}$ & $\begin{array}{l}\text { EWF model with } \\
\text { Turbulent } \mu \text { and } k\end{array}$ & $\begin{array}{l}\text { EWF model with } \\
\text { Turbulent } \mu \text { and } k\end{array}$ \\
\hline Phase change model & $\begin{array}{l}\text { Species Transport Model } \\
\text { (STM) }\end{array}$ & $\begin{array}{l}\text { Species Transport Model } \\
\text { (STM) }\end{array}$ & $\begin{array}{l}\text { Species Transport Model } \\
\text { (STM) }\end{array}$ \\
\hline Domain geometry & $90^{\circ}$ rectangular domain & $90^{\circ}$ rectangular domain & $90^{\circ}$ rectangular domain \\
\hline Dimensionality & $3 \mathrm{D}$ & $3 \mathrm{D}$ & $3 \mathrm{D}$ \\
\hline Mesh type & Uniform $0.5 \mathrm{~mm}$ cubes & Uniform $0.5 \mathrm{~mm}$ cubes & Uniform $0.5 \mathrm{~mm}$ cubes \\
\hline
\end{tabular}


The superheat is $2 \mathrm{~K}$ and the subcooling is $73 \mathrm{~K}$ for Case $\mathrm{I}$.

Figure 6.3 shows the 40 psi water spray injection at $80 \mathrm{~ms}$ with the corresponding film thickness variation on the impact surface. In Figure 6.3.a, spray droplets are colored by the droplet velocity magnitude. Drops velocity decreases as they approach the impact surface. In addition, drop velocity decreases along the radius. These results are consistent with the experimental measurements performed at WVU (Hillen et al., 2013). Figure 6.3.b presents the flooded contours of the film thickness at the same simulation time. Liquid film moves along the impact surface in the positive radial direction due to the momentum of the impinging drops. Momentum of drops causes very thin film accumulation at the impact area $(0<R<20.3 \mathrm{~mm})$ with the average thickness about 150 micron, and the thickness increases after the maximum impact radius $(R=20.3 \mathrm{~mm})$.

Figure 6.4 shows the depth-averaged velocity magnitude of the spray film at $50 \mathrm{~ms}$ (Fig. 6.4.a.) and $85 \mathrm{~ms}$ (Fig. 6.4.b.). At both times, the velocity increases from 0 on the spray axis to a maximum of about $0.624 \mathrm{~m} / \mathrm{s}$ at a radius of about $12 \mathrm{~mm}$ and declines to about $0.3 \mathrm{~m} / \mathrm{s}$ at the edge of the spray at $20.3 \mathrm{~mm}$. Beyond the spray area, there is a maximum velocity of about 0.2 $\mathrm{m} / \mathrm{s}$ and a minimum of about $0.03 \mathrm{~m} / \mathrm{s}$. Similar to the previous spray and spray cooling cases, the overall pattern exhibits approximate circular symmetry at both times, but there are noticeable deviations near the symmetry planes.

Figure 6.5 shows that the film thickness under the spray changes very little from $50 \mathrm{~ms}$ to $85 \mathrm{~ms}$. At both times the thickness is nearly constant at about 150 microns from the axis out to $10 \mathrm{~mm}$. It increases to about 500 microns at the edge of the spray (20.3 microns) and to a maximum of about 600 microns at $50 \mathrm{~ms}$ and about 778 microns at $85 \mathrm{~ms}$, outside of the spray. At $50 \mathrm{~ms}$, the thickness decreases to less than 150 microns at about $27 \mathrm{~mm}$ while it decreases to around 500 microns at about $27 \mathrm{~mm}$ at $85 \mathrm{~ms}$. At both times, the thickness is largely rotationally symmetric. 


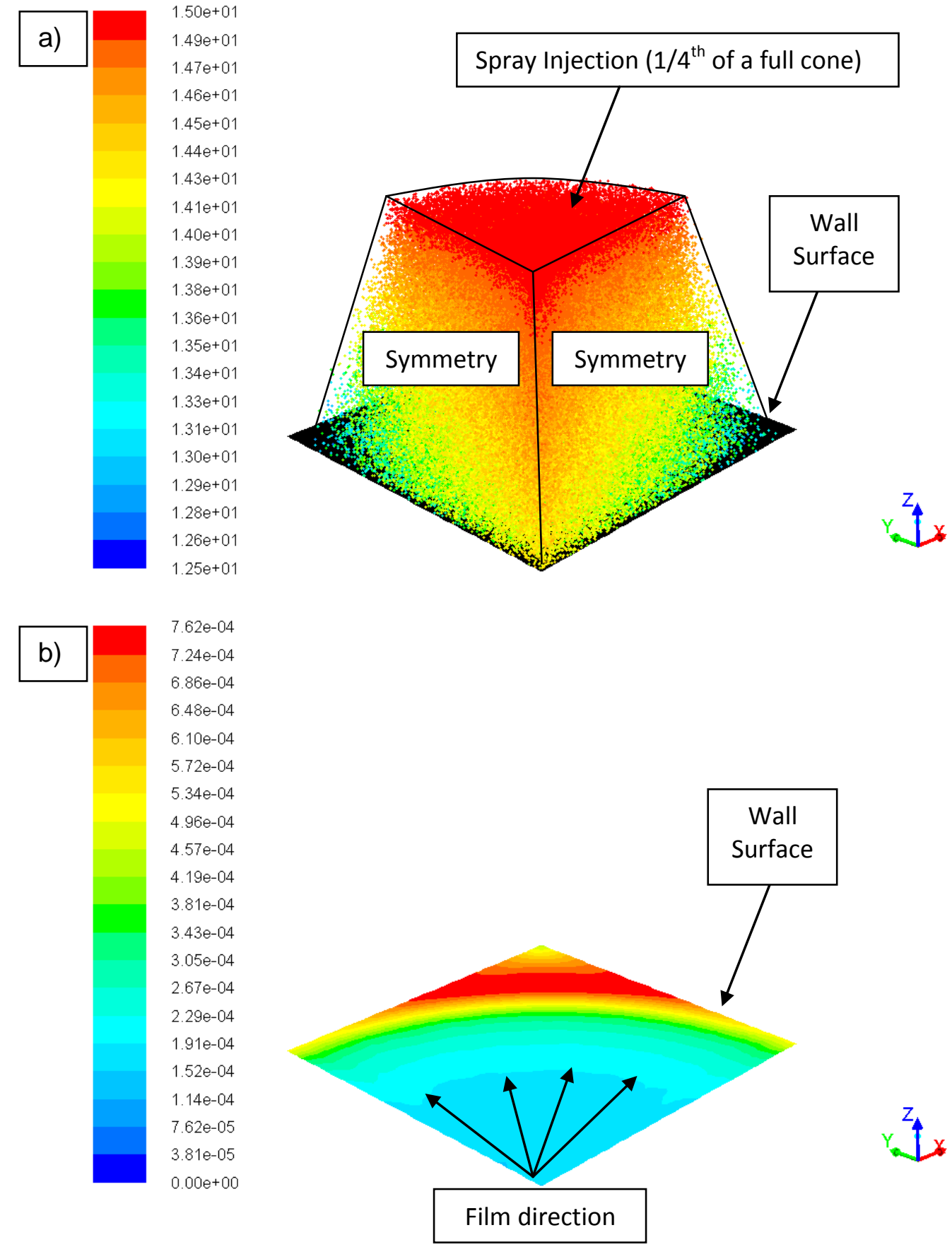

Figure 6.3 Case I: a) 40 psi full cone water spray drops at $80 \mathrm{~ms}$ colored by droplet velocity magnitude b) Perspective view of flooded contours of the corresponding film thickness at $80 \mathrm{~ms}$ simulation time. (a) Red refers to the maximum droplet velocity range: $14.9-15 \mathrm{~m} / \mathrm{s}$; blue refers to the minimum droplet velocity range: $12.5-12.61 \mathrm{~m} / \mathrm{s}$. b) red refers to the maximum film thickness: 724 -762 $\mu \mathrm{m}$; blue refers to the minimum film thickness: 0-38 $\mu \mathrm{m}$.) 

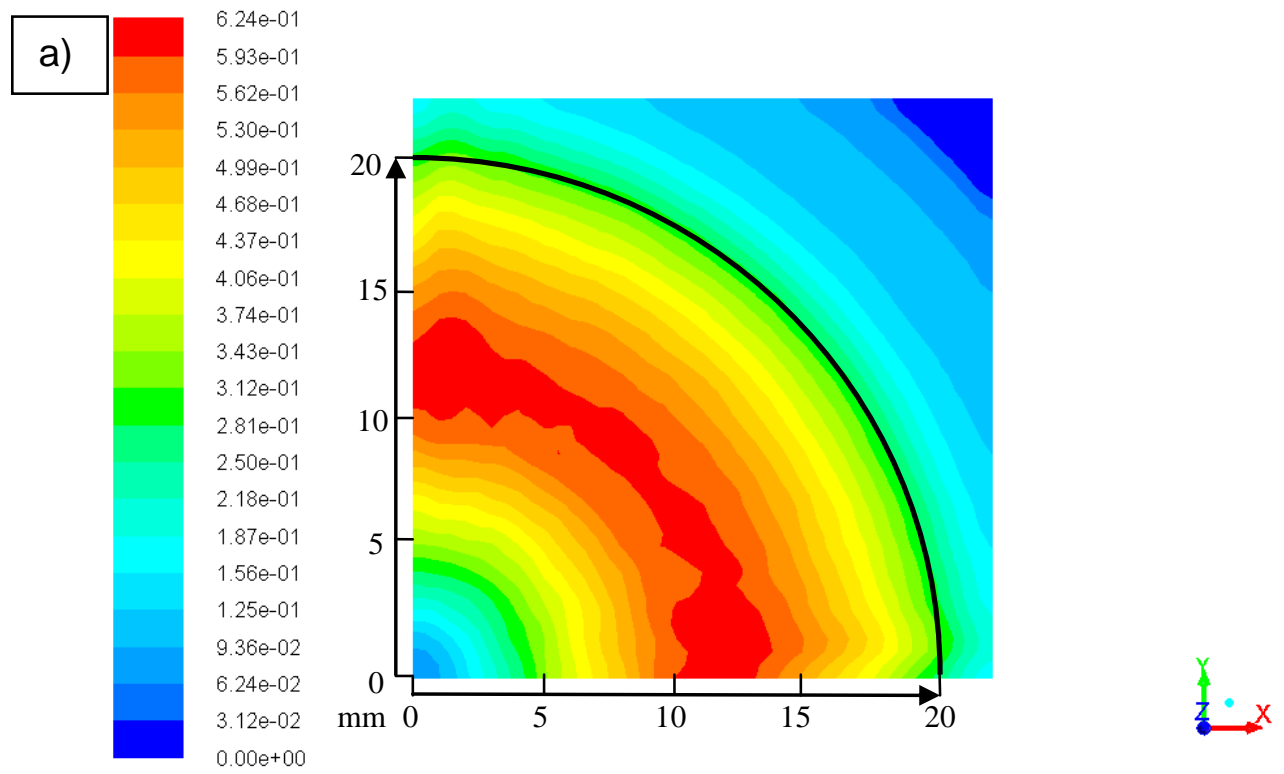

Contours of Film Velocity Magnitude $(\mathrm{m} / \mathrm{s}) \quad($ Time $=5.0000 \mathrm{e}-02)$

Jan 28,2015 ANSYS Fluent 15.0 (3d, dp, pbns, spe, rke, transient)
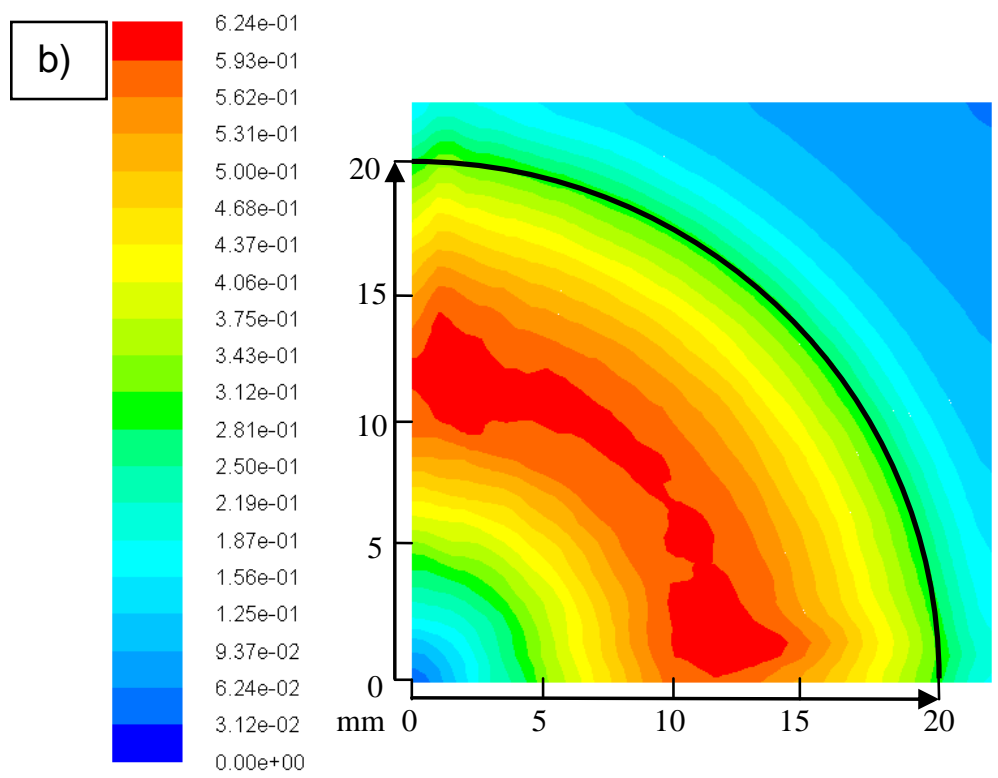

$3.12 \mathrm{e}-02$ $\mathrm{mm} 0$

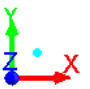

Contours of Film Velocity Magnitude $(\mathrm{m} / \mathrm{s}) \quad($ Time $=8.5000 \mathrm{e}-02)$

Jan 28, 2015

ANSYS Fluent 15.0 (3d, dp, pbns, spe, rke, transient)

Figure 6.4 Flooded contours of film velocity magnitude for 40 psi full cone water spray, Case I a) $50 \mathrm{~ms}$ simulation time b) $85 \mathrm{~ms}$ simulation time. (The color scale is the same for both times. Red refers to the maximum velocity range: $0.593-0.624 \mathrm{~m} / \mathrm{s}$; blue refers to the minimum velocity range: $0-0.031 \mathrm{~m} / \mathrm{s}$. The black arc indicates the edge of the spray. All images show the top view of the impact surface.) 

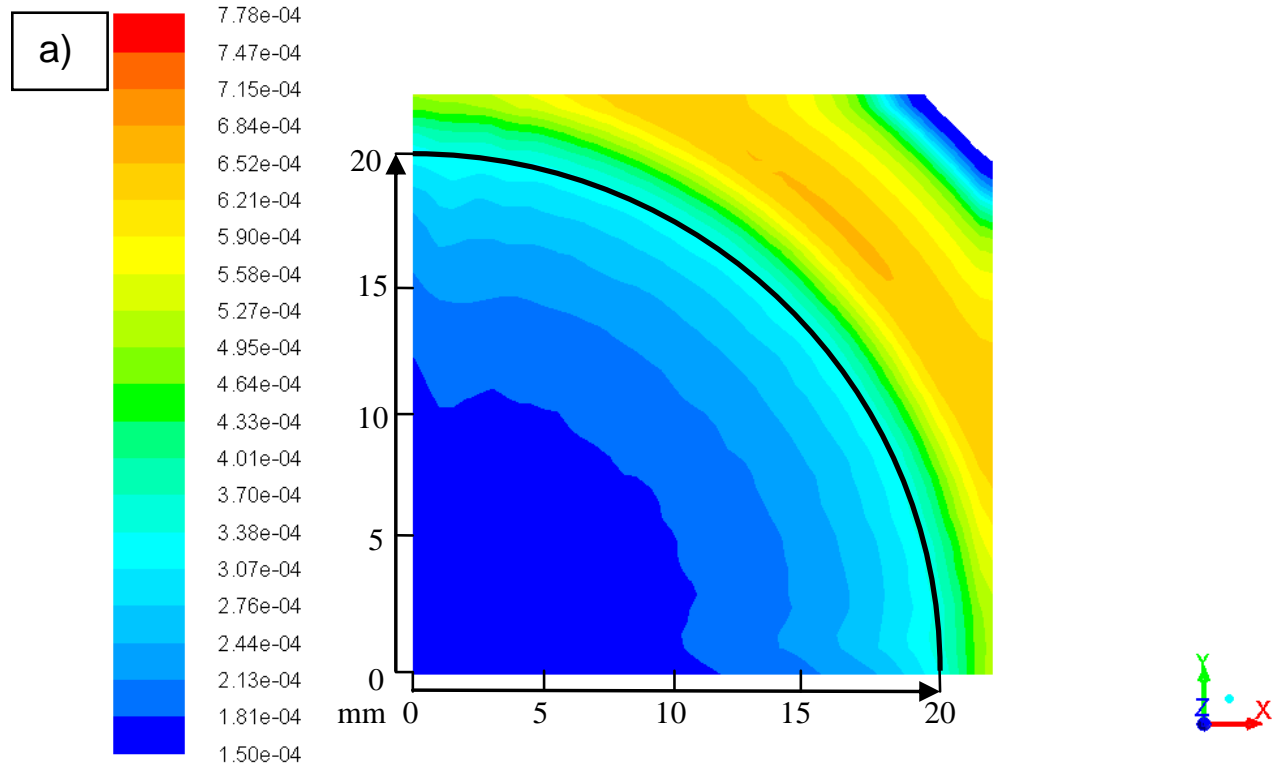

Contours of Film Thickness $(\mathrm{m})($ Time $=5.0000 \mathrm{e}-02)$

Jan 28,2015 ANSYS Fluent 15.0 (3d, dp, pbns, spe, rke, transient)

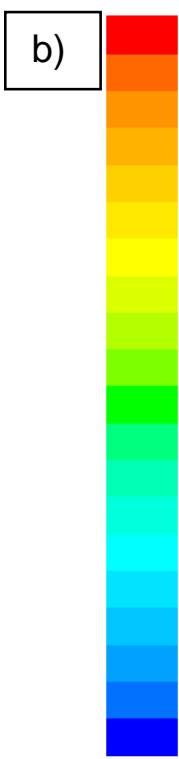

$7.78 \mathrm{e}-04$

$7.47 e-04$

$7.15 \mathrm{e}-04$

$6.84 \mathrm{e}-04$

$6.53 \mathrm{e}-04$

$6.21 \mathrm{e}-04$

$5.90 e-04$

$5.58 \mathrm{e}-04$

$5.27 e-04$

$4.96 \mathrm{e}-04$

4. $64 \mathrm{e}-04$

4.33e-04

$4.01 \mathrm{e}-04$

3.70e-04

$3.38 \mathrm{e}-04$

3.07e-04

$2.76 \mathrm{e}-04$

$2.44 \mathrm{e}-04$

$2.13 \mathrm{e}-04$

$1.81 \mathrm{e}-04$

$1.50 \mathrm{e}-04$
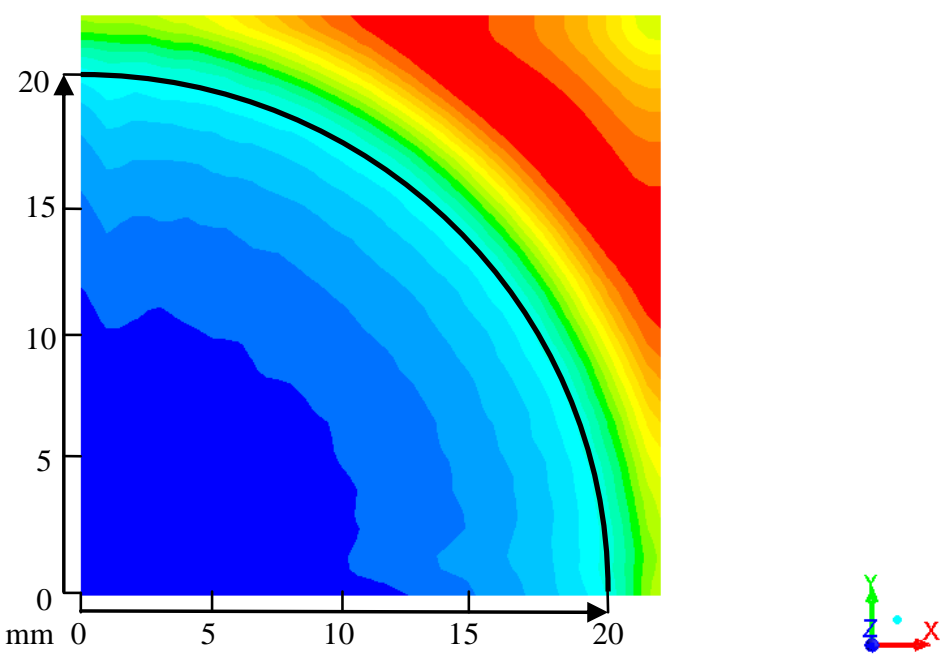

Figure 6.5 Flooded contours of film thickness for 40 psi full cone water spray, Case I a) $50 \mathrm{~ms}$ simulation time b) $85 \mathrm{~ms}$ simulation time. (The color scale is the same for both times. Blue refers to 150 microns, red refers to 778 microns. The black arc indicates the edge of the spray. All images show the top view of the impact surface.) 
Figure 6.6 shows that there is little change in the mid depth film temperature between 50 $\mathrm{ms}$ and $85 \mathrm{~ms}$. The temperature increases from about $320 \mathrm{~K}$ on the axis to about $330 \mathrm{~K}$ at the edge of the spray. The temperature continues to increase to about $366 \mathrm{~K}$ at the largest radius. At both times, the temperature distribution is largely rotationally symmetric.

Figure 6.7 shows little change in surface heat flux between $50 \mathrm{~ms}$ and $85 \mathrm{~ms}$. The patterns are largely rotationally symmetric, but in both cases, the area of highest heat flux (about 2.07 MW/m $\left.\left./ 207 \mathrm{~W} / \mathrm{cm}^{2}\right)\right)$ has an irregular outline and is within the spray impact area. The heat flux is about $0.73 \mathrm{MW} / \mathrm{m}^{2}\left(73 \mathrm{~W} / \mathrm{cm}^{2}\right)$ at the edge of the spray. At larger radii the heat flux varies from about $0.7 \mathrm{MW} / \mathrm{m}^{2}\left(70 \mathrm{~W} / \mathrm{cm}^{2}\right)$ to as low as $0.2 \mathrm{MW} / \mathrm{m}^{2}\left(20 \mathrm{~W} / \mathrm{cm}^{2}\right)$.

Figure 6.8 shows water vapor mass fraction in the film at $50 \mathrm{~ms}$ and $85 \mathrm{~ms}$, respectively. Water vapor mass fraction is very small (between 0.006 and 0.0116 ) from the axis to $R=5 \mathrm{~mm}$ but it gradually increases along the radius up to 0.05 at around $R=20.3 \mathrm{~mm}$. After $R=20.3 \mathrm{~mm}$, vapor mass fraction continues to increase with the maximum value 0.116 at both $50 \mathrm{~ms}$ and 85 ms. At both times, the vapor mass fraction is rotationally symmetric. The small value of the vapor mass fraction in the film could be due to the small value of superheat $(2 \mathrm{~K})$ and to the colder spray drops $(300 \mathrm{~K})$ impacting on the film. 


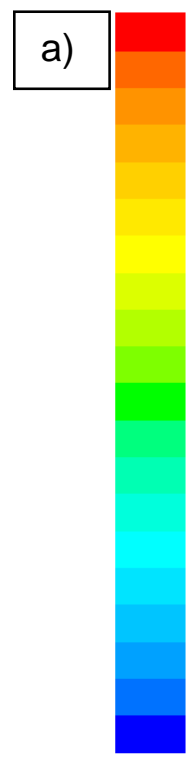

$3.66 \mathrm{e}+02$

$3.63 e+02$

$3.59 e+02$

$3.56 \mathrm{e}+02$

$3.53 e+02$

$3.50 e+02$

$3.46 \mathrm{e}+02$

$3.43 e+02$

$3.40 \mathrm{e}+02$

$3.36 \mathrm{e}+02$

$3.33 e+02$

$3.30 e+02$

$3.26 \mathrm{e}+02$

$3.23 e+02$

$3.20 \mathrm{e}+02$

$3.17 e+02$

$3.13 e+02$

$3.10 \mathrm{e}+02$

$3.07 e+02$

$3.03 e+02$

$3.00 e+02$
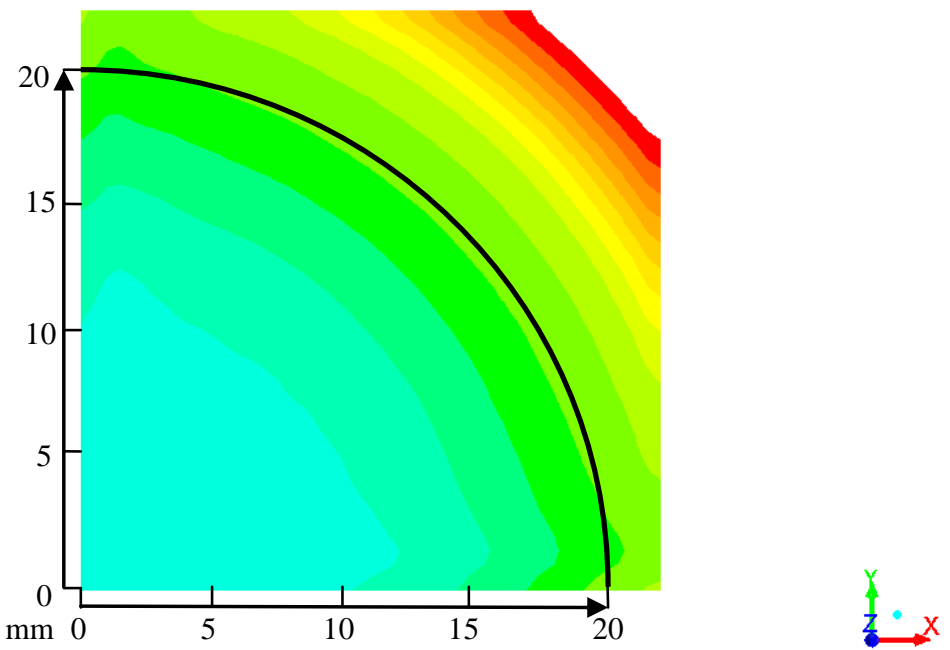

Contours of Film Temperature (k) $($ Time $=5.0000 \mathrm{e}-02)$

Jan 28,2015

ANSYS Fluent 15.0 (3d, dp, pbns, spe, rke, transient)

\section{b)}

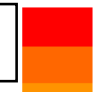

$3.66 e+02$

$3.63 \mathrm{e}+02$

$3.60 \mathrm{e}+02$

$3.56 \mathrm{e}+02$

$3.53 \mathrm{e}+02$

$3.50 e+02$

$3.46 \mathrm{e}+02$

$3.43 e+02$

$3.40 e+02$

$3.36 \mathrm{e}+02$

$3.33 \mathrm{e}+02$

$3.30 e+02$

$3.26 \mathrm{e}+02$

$3.23 e+02$

$3.20 \mathrm{e}+02$

$3.17 e+02$

$3.13 e+02$

$3.10 e+02$

$3.07 e+02$

$3.03 e+02$

$3.00 \mathrm{e}+02$
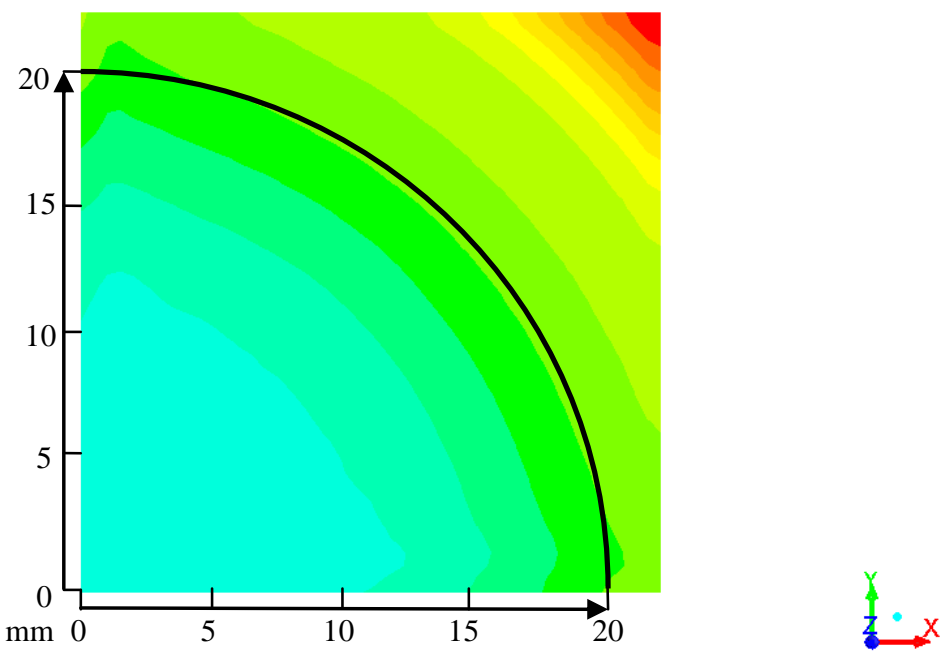

Contours of Film Temperature $(\mathrm{k})($ Time $=8.5000 \mathrm{e}-02)$

Jan 28, 2015

ANSYS Fluent 15.0 (3d, dp, pbns, spe, rke, transient)

Figure 6.6 Flooded contours of mid depth film temperature for 40 psi full cone water spray, Case I a) $50 \mathrm{~ms}$ simulation time b) $85 \mathrm{~ms}$ simulation time. (The color scale is almost the same in a) and $b$ ). Blue refers to $300 \mathrm{~K}$, red refers to $366 \mathrm{~K}$. The black arc indicates the edge of the spray. All images show the top view of the impact surface.) 


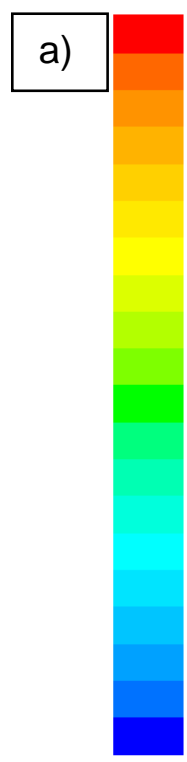

$2.07 e+06$

$1.97 e+06$

$1.86 e+06$

$1.76 \mathrm{e}+06$

$1.66 \mathrm{e}+06$

$1.55 \mathrm{e}+06$

$1.45 \mathrm{e}+06$

$1.35 e+06$

$1.24 e+06$

$1.14 e+06$

$1.04 e+06$

$9.32 e+05$

$8.28 \mathrm{e}+05$

$7.25 e+05$

$6.21 \mathrm{e}+05$

$5.18 \mathrm{e}+05$

$4.14 e+05$

$3.11 e+05$

$2.07 e+05$

$1.04 \mathrm{e}+05$

$0.00 e+00$
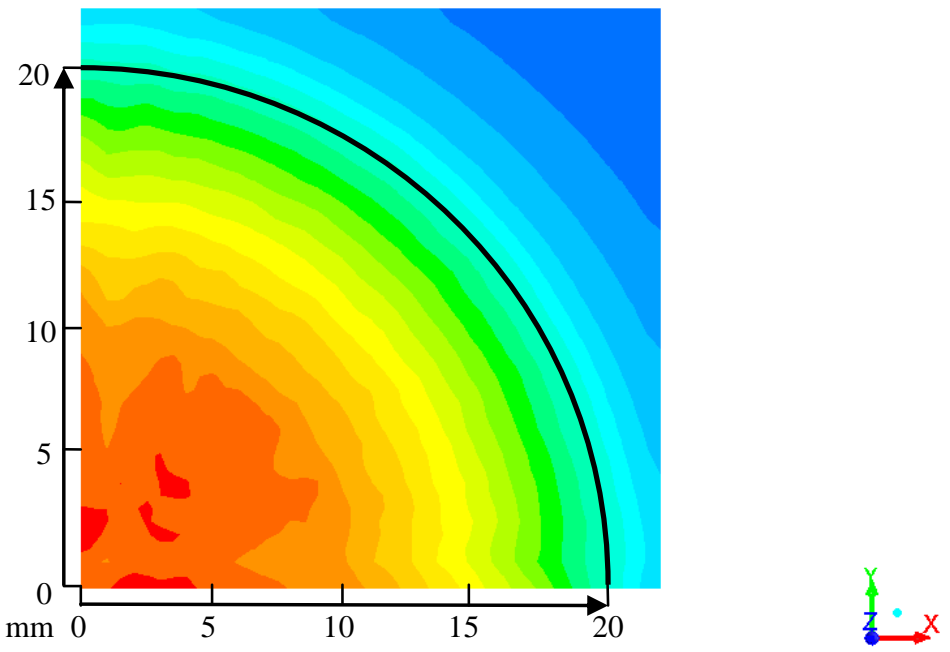

Contours of Total Surface Heat Flux (w/m2) $($ Time $=5.0000 \mathrm{e}-02)$

Jan 28,2015

ANSYS Fluent 15.0 (3d, dp, pbns, spe, rke, transient)

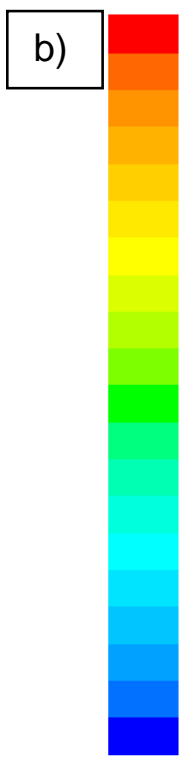

$2.07 e+06$

$1.97 e+06$

$1.87 e+06$

$1.76 \mathrm{e}+06$

$1.66 \mathrm{e}+06$

$1.55 e+06$

$1.45 e+06$

$1.35 e+06$

$1.24 e+06$

$1.14 e+06$

$1.04 e+06$

$9.33 e+05$

$8.29 \mathrm{e}+05$

$7.26 e+05$

$6.22 \mathrm{e}+05$

$5.18 \mathrm{e}+05$

$4.15 e+05$

$3.11 e+05$

$2.07 e+05$

$1.04 e+05$

$0.00 e+00$
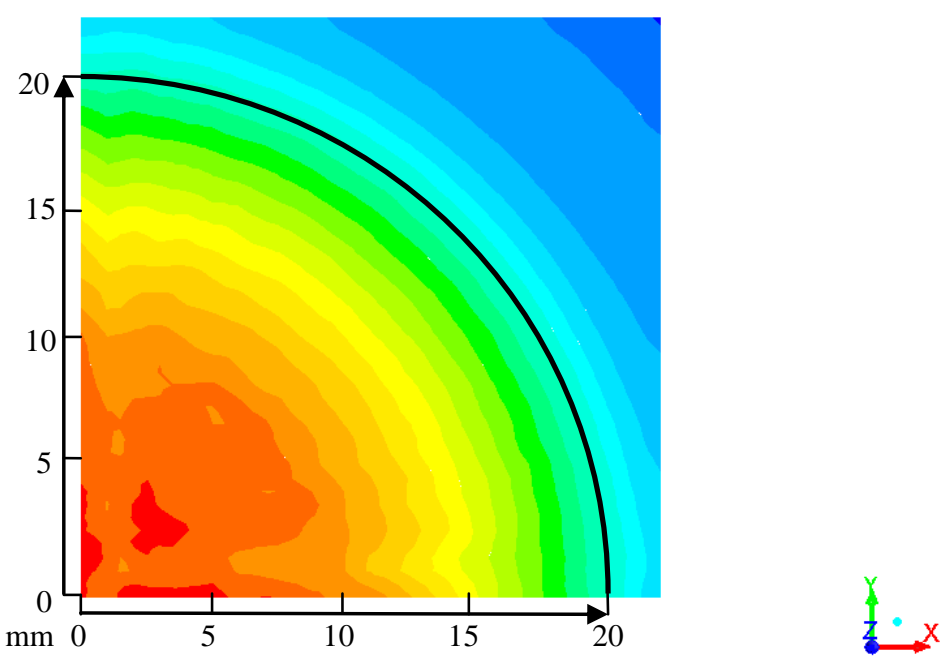

Contours of Total Surface Heat Flux (w/m2) $($ Time $=8.5000 \mathrm{e}-02)$

Jan 28,2015 ANSYS Fluent 15.0 (3d, dp, pbns, spe, rke, transient)

Figure 6.7 Flooded contours of surface heat flux for 40 psi full cone water spray, Case I a) $50 \mathrm{~ms}$ simulation time b) $85 \mathrm{~ms}$ simulation time. (The color scale is almost the same in a) and b). Blue refers to $0 \mathrm{~W} / \mathrm{m}^{2}$ and red refers to $2.07 \mathrm{MW} / \mathrm{m}^{2}$. The black arc indicates the edge of the spray. All images show the top view of the impact surface.) 


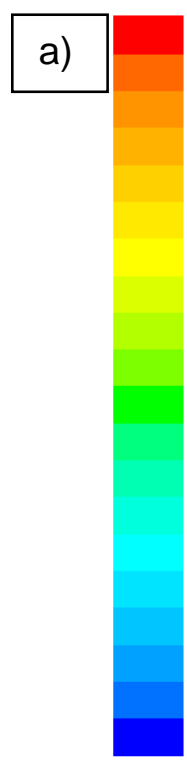

$1.16 \mathrm{e}-01$

1.10e-01

$1.04 \mathrm{e}-01$

$9.86 \mathrm{e}-02$

$9.28 \mathrm{e}-02$

$8.70 \mathrm{e}-02$

$8.12 \mathrm{e}-02$

$7.54 \mathrm{e}-02$

$6.96 \mathrm{e}-02$

6.38e-02

5.80e-02

$5.22 \mathrm{e}-02$

$4.64 \mathrm{e}-02$

4.06e-02

$3.48 \mathrm{e}-02$

2.90e-02

2.32e-02

$1.74 \mathrm{e}-02$

$1.16 \mathrm{e}-02$

$5.80 \mathrm{e}-03$

$0.00 e+00$
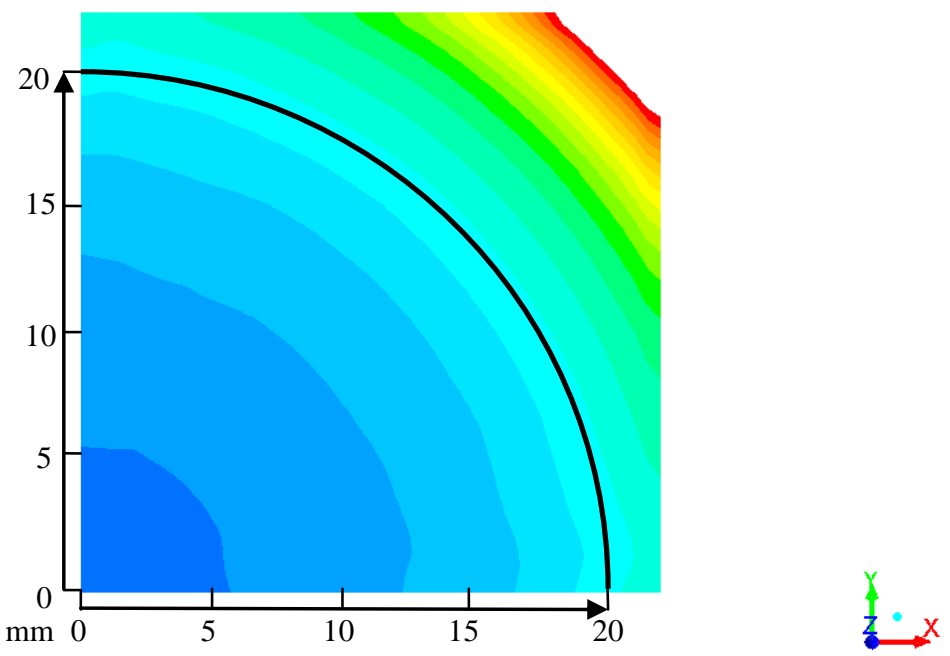

Contours of Mass fraction of h2o (Time $=5.0000 \mathrm{e}-02$ )

Jan 28, 2015

ANSYS Fluent 15.0 (3d, dp, pbns, spe, rke, transient)
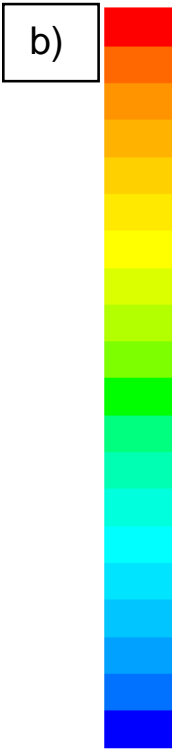

$1.16 \mathrm{e}-01$

$1.11 \mathrm{e}-01$

$1.05 \mathrm{e}-01$

$9.89 \mathrm{e}-02$

$9.31 \mathrm{e}-02$

$8.73 \mathrm{e}-02$

$8.15 \mathrm{e}-02$

$7.57 e-02$

$6.98 \mathrm{e}-02$

6.40e-02

$5.82 \mathrm{e}-02$

$5.24 \mathrm{e}-02$

$4.66 \mathrm{e}-02$

4. $07 e-02$

$3.49 \mathrm{e}-02$

2.91e-02

2.33e-02

$1.75 \mathrm{e}-02$

$1.16 \mathrm{e}-02$

5.82e-03

$0.00 e+00$
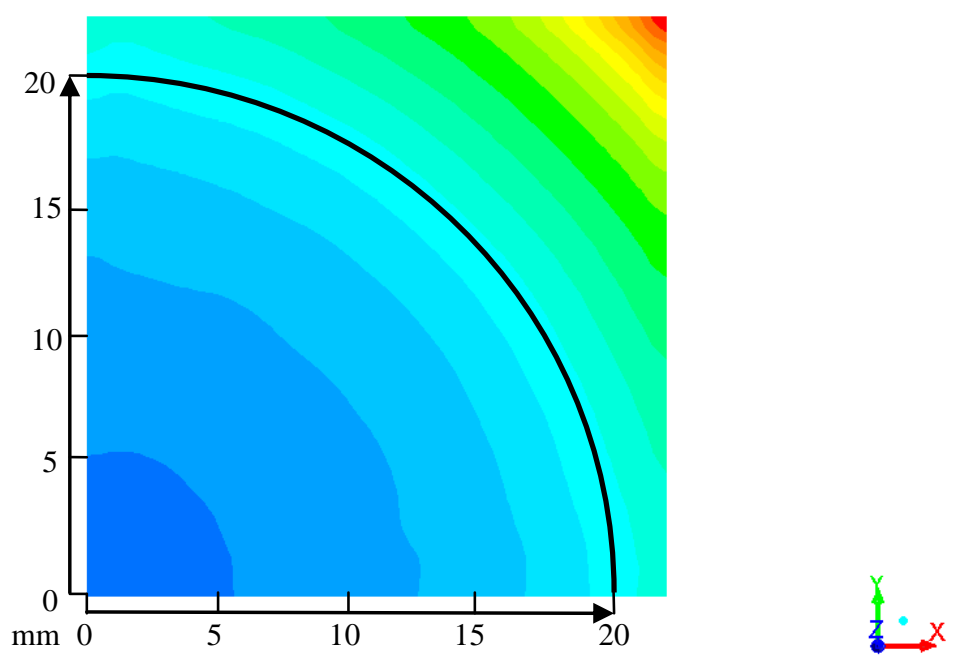

Figure 6.8 Flooded contours of water vapor mass fraction for 40 psi full cone water spray, Case I a) $50 \mathrm{~ms}$ simulation time b) $85 \mathrm{~ms}$ simulation time. (The color scale is almost the same in a) and b). Blue refers to 0 and red refers to 0.116. The black arc indicates the edge of the spray. All images show the top view of the impact surface.) 


\subsubsection{Spray Cooling Simulations with Phase Change: Case II}

In this case, a constant surface temperature is specified to be $375 \mathrm{~K}, 2 \mathrm{~K}$ more than the water boiling temperature. The initial film thickness is 150 microns and the initial film temperature is $350 \mathrm{~K}$. The spray temperature is $350 \mathrm{~K}$ and the surrounding gas (air) temperature is $300 \mathrm{~K}$. The superheat temperature is $2 \mathrm{~K}$ and subcooling temperature is $23 \mathrm{~K}$ for Case II.

Figure 6.9 shows the depth-averaged velocity magnitude of the spray film at $50 \mathrm{~ms}$ (Fig. 6.9.a.) and $85 \mathrm{~ms}$ (Fig. 6.9.b.). Similar to the Case I, at both times, the velocity increases from 0 on the spray axis to a maximum of about $0.625 \mathrm{~m} / \mathrm{s}$ at a radius of about $10-12 \mathrm{~mm}$ and declines to about $0.3 \mathrm{~m} / \mathrm{s}$ at the edge of the spray at $20.3 \mathrm{~mm}$. Beyond the spray area, there is a maximum velocity of about $0.2 \mathrm{~m} / \mathrm{s}$ and a minimum of about $0.03 \mathrm{~m} / \mathrm{s}$. The overall pattern exhibits approximate rotational symmetry at both times, but there are noticeable deviations near the symmetry planes.

Figure 6.10 shows that the film thickness under the spray changes very little from $50 \mathrm{~ms}$ to $85 \mathrm{~ms}$. These results are very close to the Case I results. At both times, the thickness is largely rotationally symmetric. 


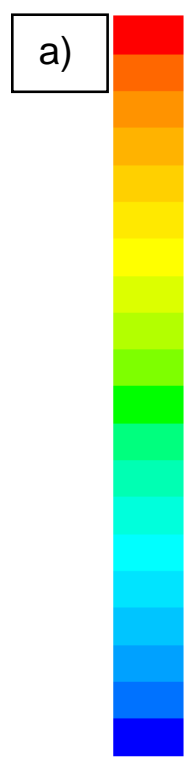

5.31e-01

5.00e-01

4.69e-01

4.38e-01

4.06e-01

3.75e-01

3.44e-01

3. $13 \mathrm{e}-01$

2.81e-01

2.50e-01

2.19e-01

$1.88 \mathrm{e}-01$

$1.56 \mathrm{e}-01$

$1.25 \mathrm{e}-01$

$9.38 \mathrm{e}-02$

6. $25 \mathrm{e}-02$

3. $13 \mathrm{e}-02$

$0.00 e+00$
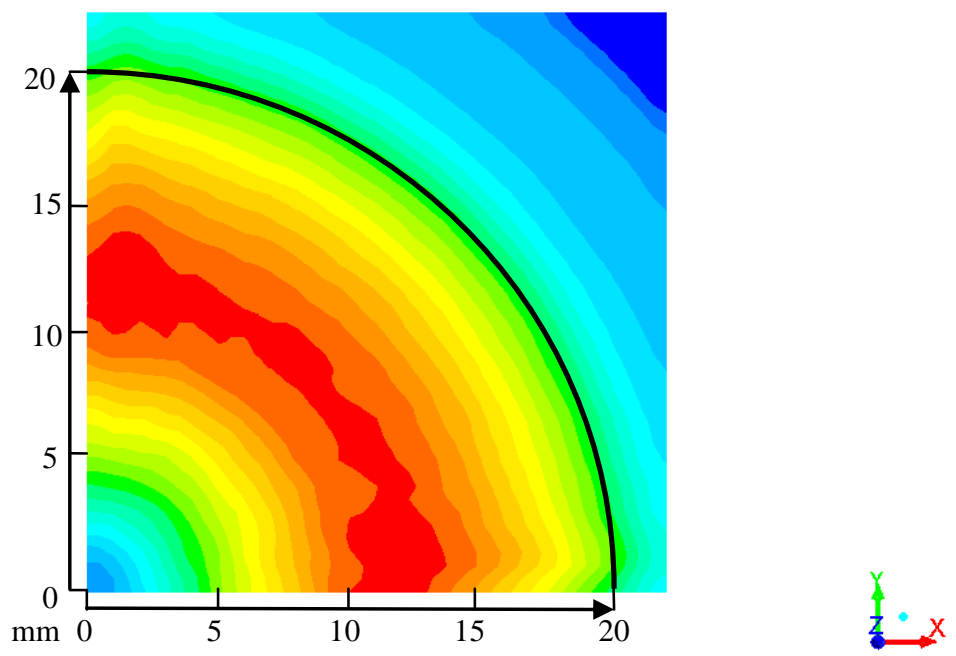

Contours of Film Velocity Magnitude $(\mathrm{m} / \mathrm{s}) \quad($ Time $=5.0000 \mathrm{e}-02)$

Jan 28, 2015

ANSYS Fluent 15.0 (3d, dp, pbns, spe, rke, transient)

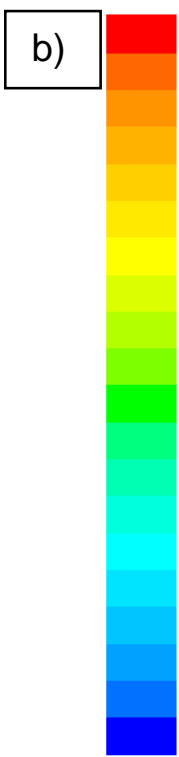

$6.25 \mathrm{e}-01$

$5.94 \mathrm{e}-01$

$5.63 e-01$

$5.31 \mathrm{e}-01$

$5.00 \mathrm{e}-01$

4.69e-01

$4.38 \mathrm{e}-01$

4. $06 \mathrm{e}-01$

3.75e-01

$3.44 \mathrm{e}-01$

3.13e-01

2.81e-01

2.50e-01

2.19e-01

$1.88 \mathrm{e}-01$

$1.56 \mathrm{e}-01$

$1.25 \mathrm{e}-01$

$9.38 \mathrm{e}-02$

$6.25 \mathrm{e}-02$

3. $13 \mathrm{e}-02$

$0.00 e+00$
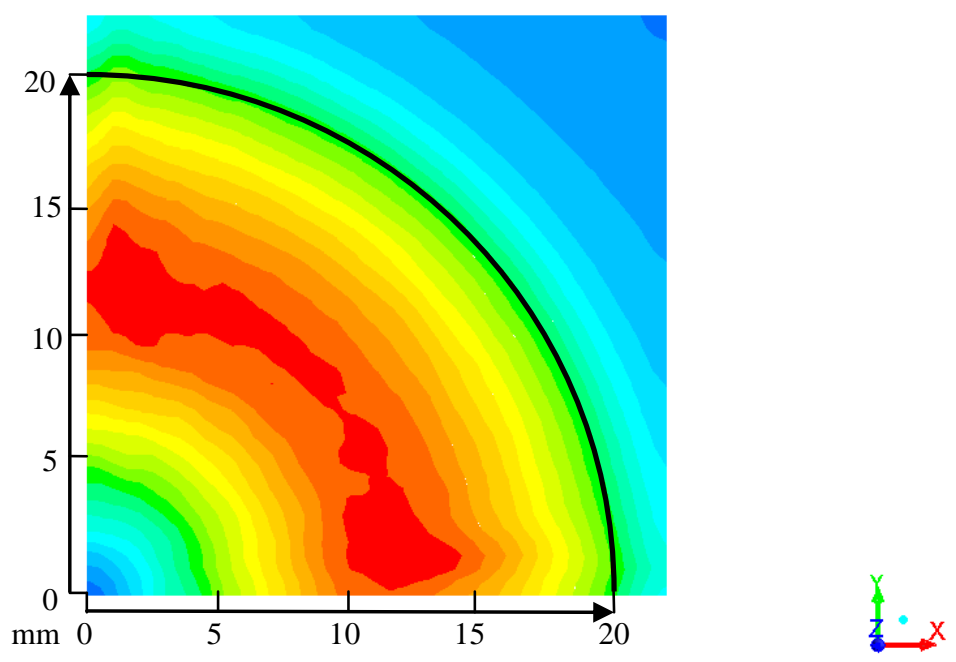

Contours of Film Velocity Magnitude $(\mathrm{m} / \mathrm{s})($ Time $=8.5000 \mathrm{e}-02)$

Jan 28, 2015 ANSYS Fluent 15.0 (3d, dp, pbns, spe, rke, transient)

Figure 6.9 Flooded contours of film velocity magnitude for 40 psi full cone water spray, Case II a) $50 \mathrm{~ms}$ simulation time b) $85 \mathrm{~ms}$ simulation time. (The color scale is the same for both times. Red refers to the maximum velocity range: $0.594-0.625 \mathrm{~m} / \mathrm{s}$; blue refers to the minimum velocity range: $0-0.031 \mathrm{~m} / \mathrm{s}$. The black arc indicates the edge of the spray. All images show the top view of the impact surface.) 

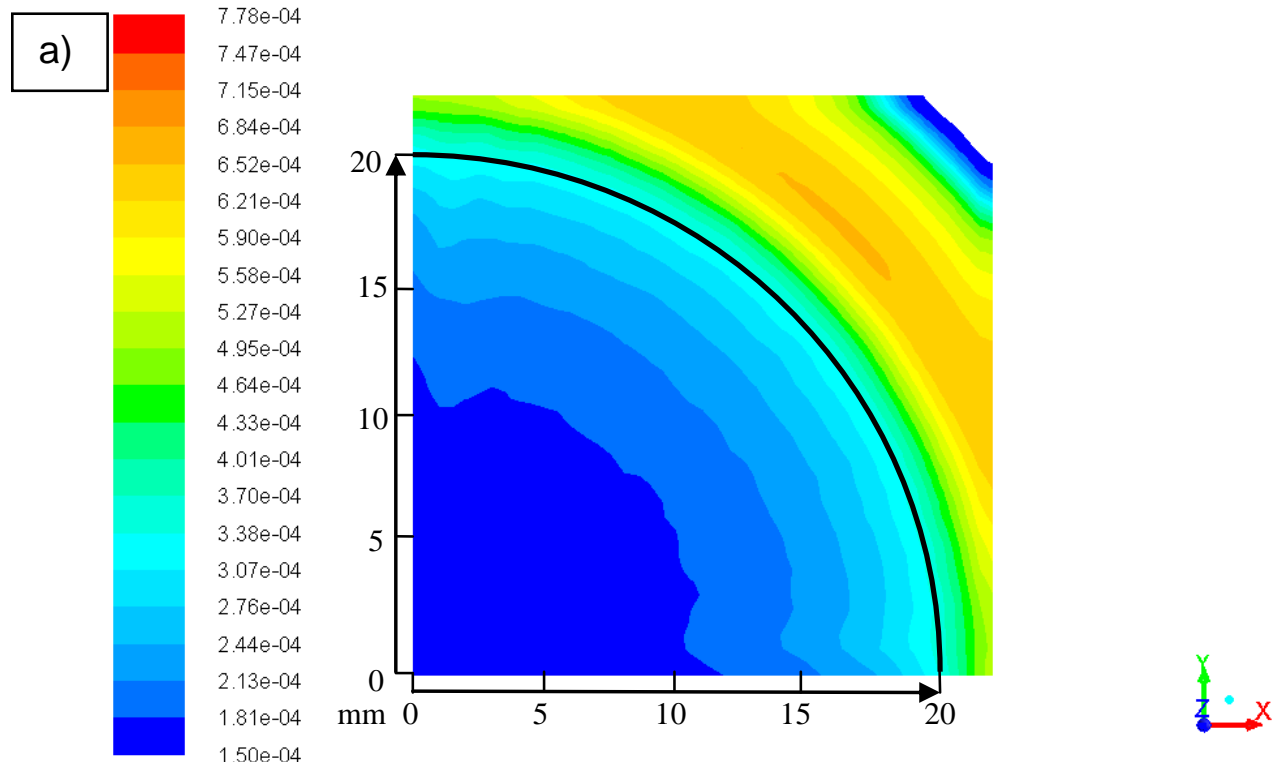

Contours of Film Thickness $(\mathrm{m})($ Time $=5.0000 \mathrm{e}-02)$

Jan 28,2015 ANSYS Fluent 15.0 (3d, dp, pbns, spe, rke, transient)

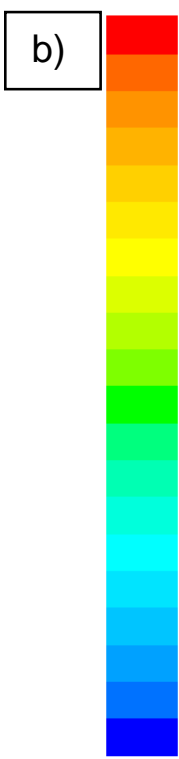

$7.78 \mathrm{e}-04$

$7.47 e-04$

$7.15 \mathrm{e}-04$

$6.84 \mathrm{e}-04$

$6.52 \mathrm{e}-04$

$6.21 \mathrm{e}-04$

$5.90 e-04$

$5.58 \mathrm{e}-04$

$5.27 e-04$

4.95e-04

4. $64 \mathrm{e}-04$

4. $33 \mathrm{e}-04$

$4.01 \mathrm{e}-04$

3.70e-04

$3.38 \mathrm{e}-04$

3.07e-04

$2.76 \mathrm{e}-04$

$2.44 \mathrm{e}-04$

$2.13 \mathrm{e}-04$

$1.81 \mathrm{e}-04$

$1.50 \mathrm{e}-04$
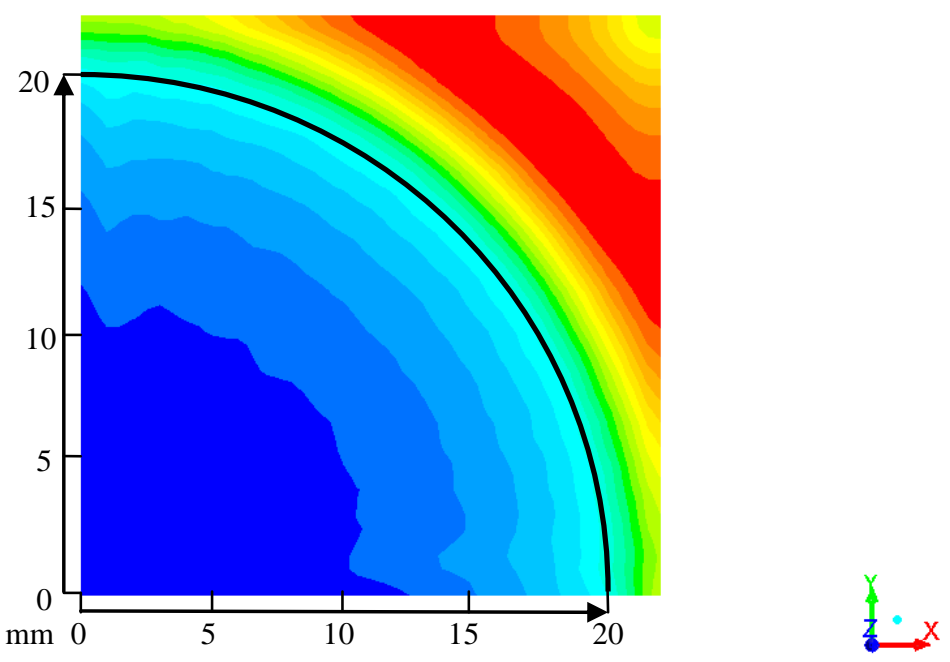

Figure 6.10 Flooded contours of film thickness for 40 psi full cone water spray, Case II a) $50 \mathrm{~ms}$ simulation time b) $85 \mathrm{~ms}$ simulation time. (The color scale is the same for both times. Blue refers to 150 microns, red refers to 778 microns. The black arc indicates the edge of the spray. All images show the top view of the impact surface.) 
Figure 6.11 shows that there is little change in the mid depth film temperature between 50 $\mathrm{ms}$ and $85 \mathrm{~ms}$. These results are completely different that Case I because of the difference of the spray liquid temperature (for Case I spray temperature is $300 \mathrm{~K}$ and for Case II spray temperature is $350 \mathrm{~K}$ ). In Case II, the temperature increases from about $354 \mathrm{~K}$ on the axis to about $358 \mathrm{~K}$ at the edge of the spray. The temperature continues to increase to about $368 \mathrm{~K}$ at the largest radius. At both times, the temperature distribution is largely rotationally symmetric.

Figure 6.12 shows a big change in surface heat flux at $50 \mathrm{~ms}$ and $85 \mathrm{~ms}$. The maximum heat flux is about $0.0083 \mathrm{MW} / \mathrm{m}^{2}\left(0.83 \mathrm{~W} / \mathrm{cm}^{2}\right)$ at $50 \mathrm{~ms}$ and it is about $0.737 \mathrm{MW} / \mathrm{m}^{2}(73.7$ $\mathrm{W} / \mathrm{cm}^{2}$ ) at $85 \mathrm{~ms}$. The high heat flux occurs within the spray impact area at both simulation times. The maximum heat flux value for Case II $\left(0.737 \mathrm{MW} / \mathrm{m}^{2}\left(73.7 \mathrm{~W} / \mathrm{cm}^{2}\right)\right)$ at $85 \mathrm{~ms}$ is very small compared to Case I $\left(2.07 \mathrm{MW} / \mathrm{m}^{2}\left(207 \mathrm{~W} / \mathrm{cm}^{2}\right)\right)$ at $85 \mathrm{~ms}$ due to colder spray drops causing a large amount of heat transfer in Case I. At both times, the heat flux is approximately rotationally symmetric and uniform within the spray impact area.

Figure 6.13 shows water vapor mass fraction in the film at $50 \mathrm{~ms}$ and $85 \mathrm{~ms}$, respectively. The water vapor mass fraction is small (between 0.03 and 0.04 ) from $0<R<3 \mathrm{~mm}$ but it gradually increases along the radius up to 0.116 at around $R=20.3 \mathrm{~mm}$ which is the radius of spray coverage on the surface. From $20.3 \mathrm{~mm}<R<27 \mathrm{~mm}$, the vapor mass fraction continues to increase to the maximum value of 0.205 at $85 \mathrm{~ms}$ at the corner of domain (about $R=27 \mathrm{~mm}$ ). At both times, the vapor mass fraction is approximately rotationally symmetric. The vapor mass fraction is higher for Case II compared to Case I. The maximum value is 0.116 for Case I and 0.205 for Case II. The minimum value within the spray impact area is 0.006 for Case I and 0.03 for Case II. This is also due to the higher temperature of the spray liquid which is $350 \mathrm{~K}$ for Case II and $300 \mathrm{~K}$ for Case I. Since, the spray temperature for Case II is closer to the phase change temperature, more vapor should be generated. 


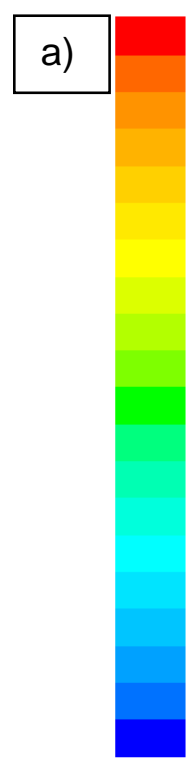

$3.68 \mathrm{e}+02$

$3.65 e+02$

$3.61 e+02$

$3.58 \mathrm{e}+02$

$3.54 \mathrm{e}+02$

$3.51 e+02$

$3.48 \mathrm{e}+02$

$3.44 \mathrm{e}+02$

$3.41 e+02$

$3.37 e+02$

$3.34 \mathrm{e}+02$

$3.31 e+02$

$3.27 e+02$

$3.24 e+02$

$3.20 \mathrm{e}+02$

$3.17 e+02$

$3.14 \mathrm{e}+02$

$3.10 e+02$

$3.07 e+02$

$3.03 e+02$

$3.00 e+02$
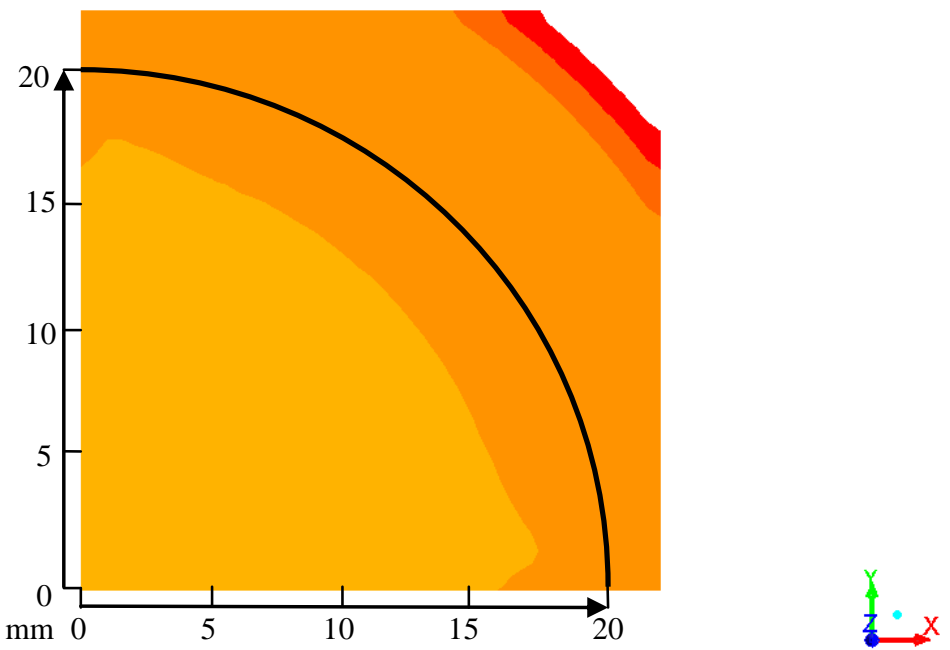

Contours of Film Temperature $(k)($ Time $=5.0000 \mathrm{e}-02)$

Jan 28,2015

ANSYS Fluent 15.0 (3d, dp, pbns, spe, rke, transient)

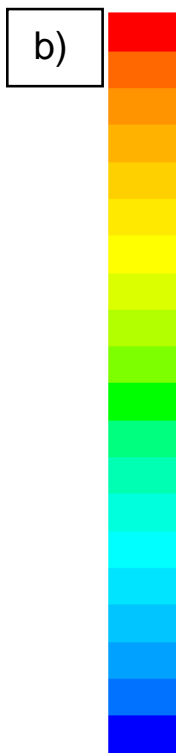

$3.68 \mathrm{e}+02$

$3.64 \mathrm{e}+02$

$3.61 e+02$

$3.58 \mathrm{e}+02$

$3.54 \mathrm{e}+02$

$3.51 e+02$

$3.47 e+02$

$3.44 \mathrm{e}+02$

$3.41 e+02$

$3.37 e+02$

$3.34 e+02$

$3.30 e+02$

$3.27 e+02$

$3.24 e+02$

$3.20 \mathrm{e}+02$

$3.17 e+02$

$3.14 e+02$

$3.10 \mathrm{e}+02$

$3.07 e+02$

$3.03 e+02$

$3.00 e+02$
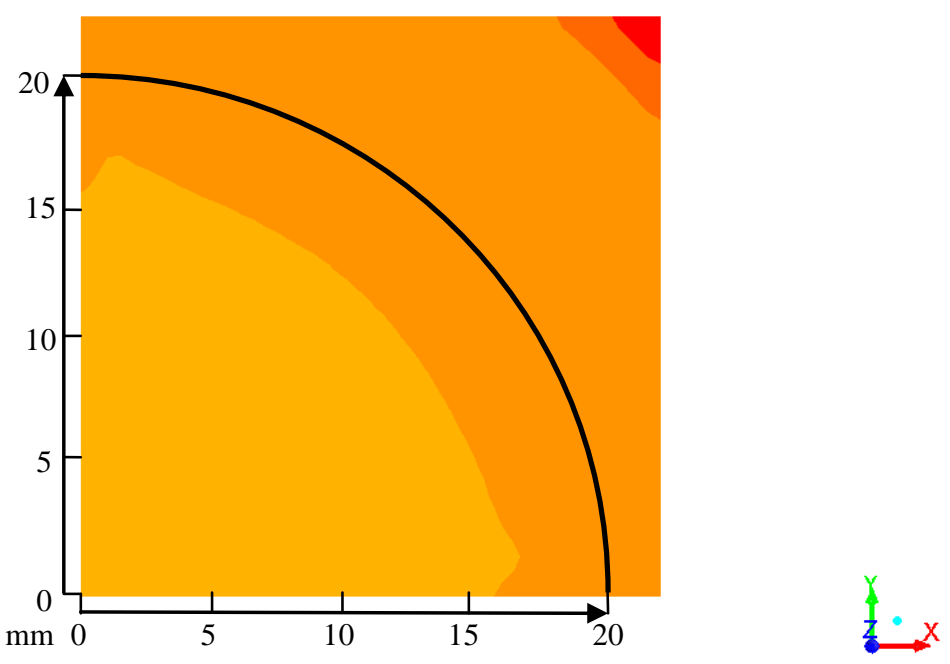

Contours of Film Temperature $(\mathrm{k})($ Time $=8.5000 \mathrm{e}-02)$

Jan 28, 2015

ANSYS Fluent 15.0 (3d, dp, pbns, spe, rke, transient)

Figure 6.11 Flooded contours of mid depth film temperature for 40 psi full cone water spray, Case II a) $50 \mathrm{~ms}$ simulation time b) $85 \mathrm{~ms}$ simulation time. (The color scale is almost the same in a) and $b$ ). Blue refers to $300 \mathrm{~K}$, red refers to $368 \mathrm{~K}$. The black arc indicates the edge of the spray. All images show the top view of the impact surface.) 

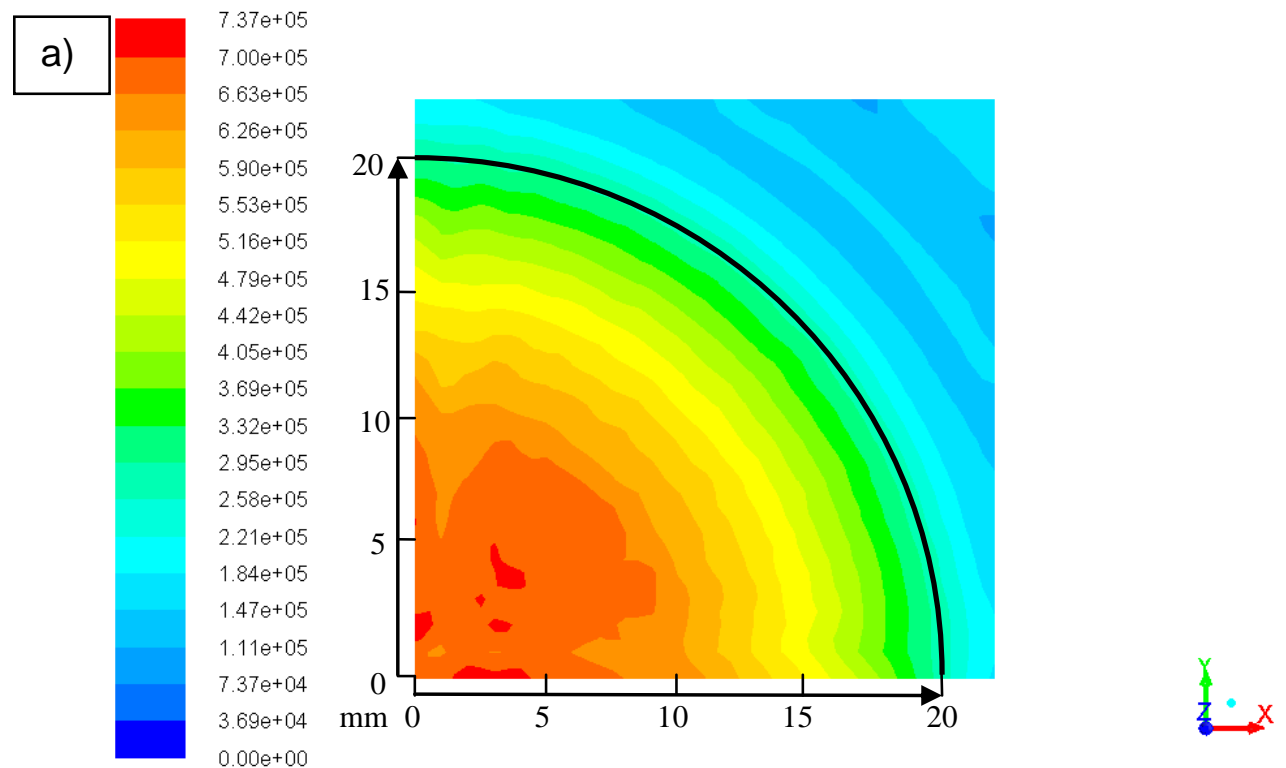

$0.00 \mathrm{e}+00$

Contours of Total Surface Heat Flux (w/m2) $($ Time $=5.0000 \mathrm{e}-02)$

Mar 15, 2015 ANSYS Fluent 15.0 (3d, dp, pbns, spe, rke, transient)

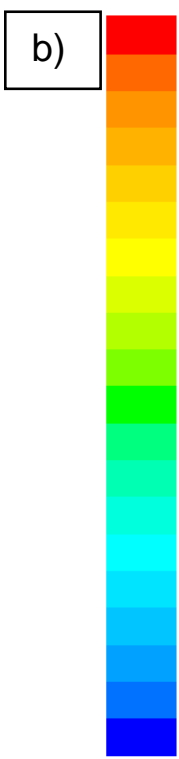

$7.37 e+05$

$7.01 \mathrm{e}+05$

$6.64 \mathrm{e}+05$

$6.27 e+05$

$5.90 e+05$

$5.53 e+05$

$5.16 \mathrm{e}+05$

$4.79 e+05$

$4.42 e+05$

$4.06 \mathrm{e}+05$

$3.69 e+05$

$3.32 e+05$

$2.95 e+05$

$2.58 \mathrm{e}+05$

$2.21 e+05$

$1.84 e+05$

$1.47 e+05$

$1.11 e+05$

$7.37 e+04$

$3.69 e+04$

$0.00 e+00$
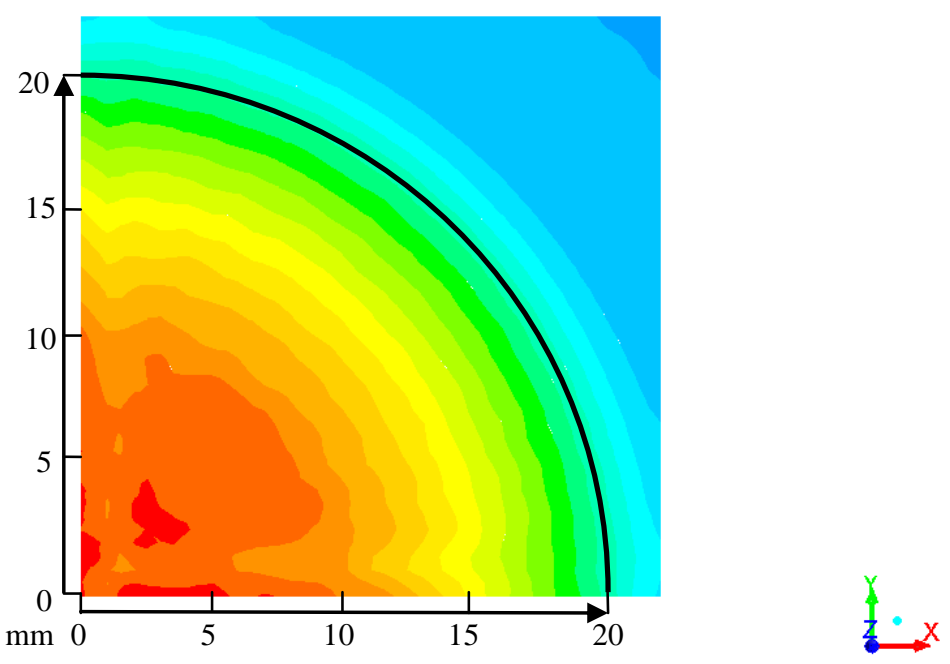

Contours of Total Surface Heat Flux (w/m2) $($ Time $=8.5000 \mathrm{e}-02)$

Jan 28, 2015 ANSYS Fluent 15.0 (3d, dp, pbns, spe, rke, transient)

Figure 6.12 Flooded contours of surface heat flux for 40 psi full cone water spray, Case II a) 50 ms simulation time b) $85 \mathrm{~ms}$ simulation time. (The color scale is different in a) and b). Blue refers to $0 \mathrm{~W} / \mathrm{m}^{2}$ and red refers to $0.0083 \mathrm{MW} / \mathrm{m}^{2}$ in a) and $0.737 \mathrm{MW} / \mathrm{m}^{2}$ in b). The black arc indicates the edge of the spray. All images show the top view of the impact surface.) 

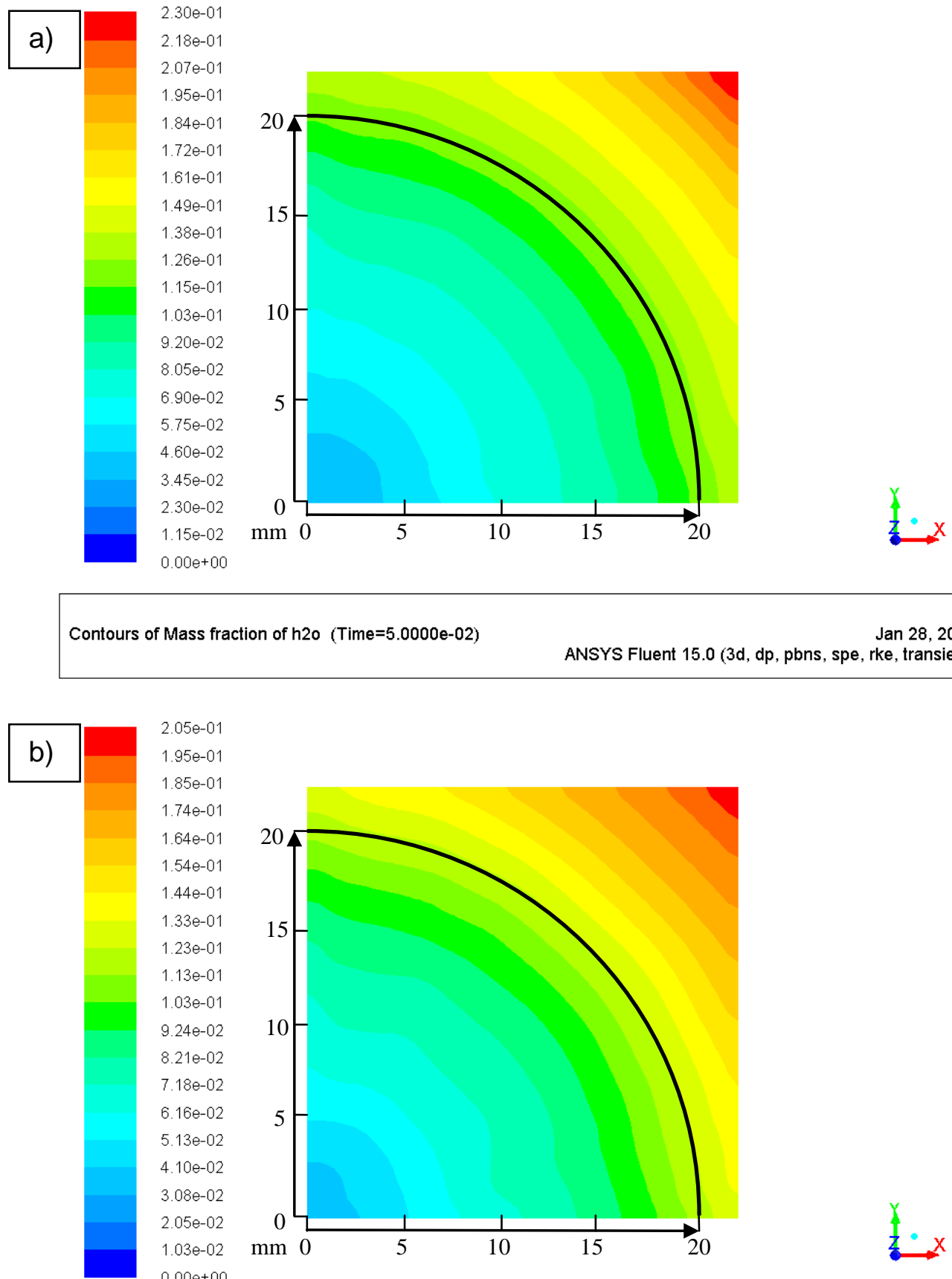

$2.05 e-01$

$1.95 \mathrm{e}-01$

$1.85 \mathrm{e}-01$

$1.74 \mathrm{e}-01$

$1.64 \mathrm{e}-01$

$1.54 \mathrm{e}-01$

$1.44 \mathrm{e}-01$

$1.33 e-01$

$1.23 e-01$

$1.13 e-01$

1.03e-01

$9.24 \mathrm{e}-02$

8.21e-02

$7.18 \mathrm{e}-02$

$6.16 \mathrm{e}-02$

$5.13 e-02$

4.10e-02

$3.08 \mathrm{e}-02$

2.05e-02

$1.03 e-02$

$0.00 e+00$
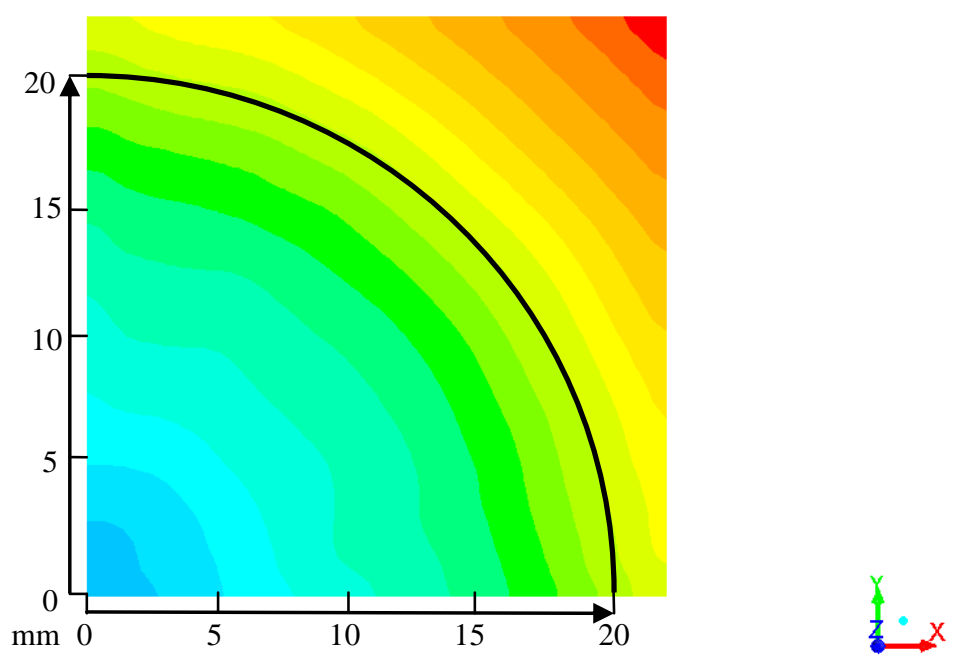

Figure 6.13 Flooded contours of water vapor mass fraction for 40 psi full cone water spray, Case II a) $50 \mathrm{~ms}$ simulation time b) $85 \mathrm{~ms}$ simulation time. (The color scale is different in a) and b). Blue refers to 0 and red refers to 0.23 in a) and 0.205 in b). The black arc indicates the edge of the spray. All images show the top view of the impact surface.) 


\subsubsection{Spray Cooling Simulations with Phase Change: Case III}

In this case, a constant surface temperature of $375 \mathrm{~K}$ is specified, $2 \mathrm{~K}$ more than the water boiling temperature. The initial film thickness is 150 micron and the initial film temperature was $350 \mathrm{~K}$. The spray temperature was $300 \mathrm{~K}$ and the surrounding gas (air) temperature was $350 \mathrm{~K}$. The superheat is $2 \mathrm{~K}$ and the subcooling is $73 \mathrm{~K}$ for Case III, the same as Case I.

Figure 6.14 shows the depth-averaged velocity magnitude of the spray film at $50 \mathrm{~ms}$ (Fig. 6.14.a.) and $85 \mathrm{~ms}$ (Fig. 6.14.b.). Similar to Cases I and II, at both times the velocity increases from 0 on the spray axis to a maximum of about $0.624 \mathrm{~m} / \mathrm{s}$ at a radius of about $10-12 \mathrm{~mm}$ and declines to about $0.3 \mathrm{~m} / \mathrm{s}$ at the edge of the spray at $20.3 \mathrm{~mm}$.

Figure 6.15 shows that the film thickness under the spray changes very little from $50 \mathrm{~ms}$ to $85 \mathrm{~ms}$. These results are very close to both Cases I and II results. At both times, the thickness is largely rotationally symmetric. 


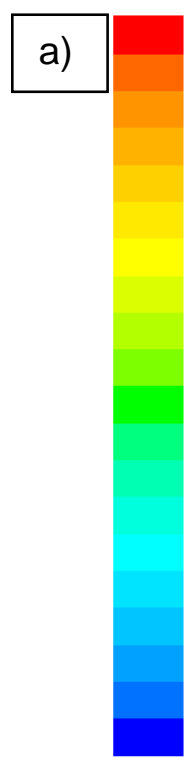

5.31e-01

5.00e-01

4.69e-01

4.38e-01

4. $06 \mathrm{e}-01$

3.75e-01

$3.44 \mathrm{e}-01$

3. $13 \mathrm{e}-01$

2.81e-01

2.50e-01

2.19e-01

$1.88 \mathrm{e}-01$

$1.56 \mathrm{e}-01$

$1.25 \mathrm{e}-01$

$9.38 \mathrm{e}-02$

6. $25 \mathrm{e}-02$

3. $13 \mathrm{e}-02$

$0.00 e+00$
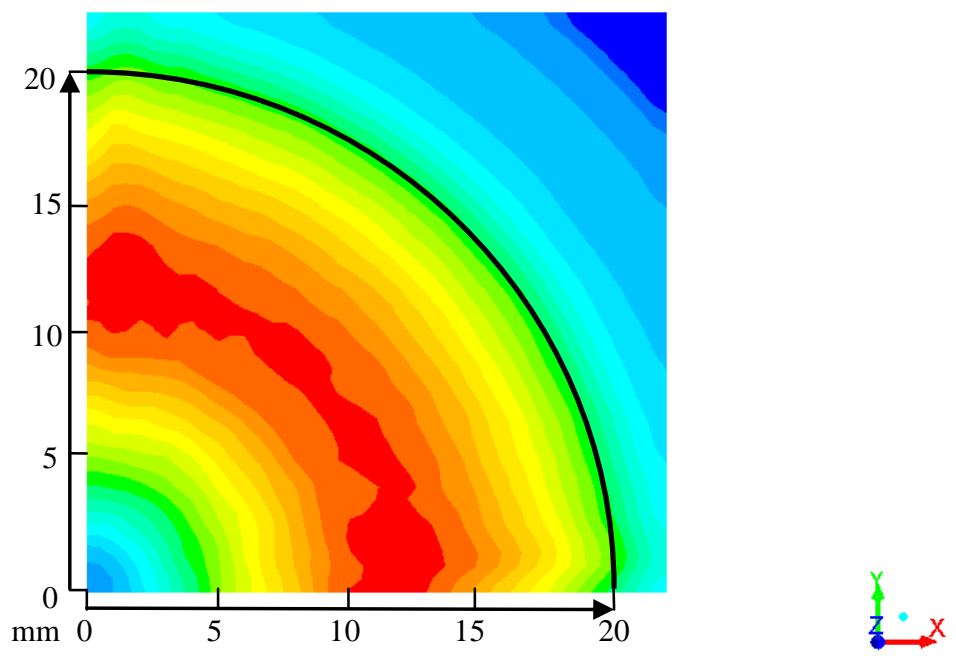

Contours of Film Velocity Magnitude $(\mathrm{m} / \mathrm{s}) \quad($ Time $=5.0000 \mathrm{e}-02)$

Jan 28, 2015

ANSYS Fluent 15.0 (3d, dp, pbns, spe, rke, transient)

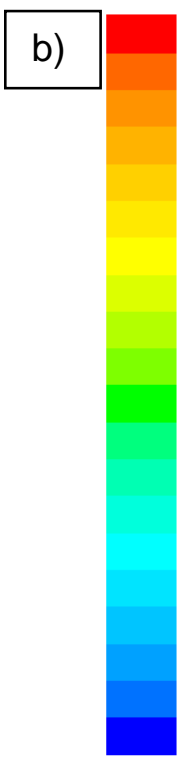

$6.25 \mathrm{e}-01$

$5.94 \mathrm{e}-01$

$5.62 \mathrm{e}-01$

$5.31 \mathrm{e}-01$

$5.00 \mathrm{e}-01$

4. $69 \mathrm{e}-01$

$4.37 e-01$

4. $06 \mathrm{e}-01$

3.75e-01

$3.44 \mathrm{e}-01$

3. $12 \mathrm{e}-01$

2.81e-01

2.50e-01

2.19e-01

$1.87 e-01$

$1.56 \mathrm{e}-01$

$1.25 \mathrm{e}-01$

9. $37 \mathrm{e}-02$

$6.25 \mathrm{e}-02$

3.12e-02

$0.00 e+00$
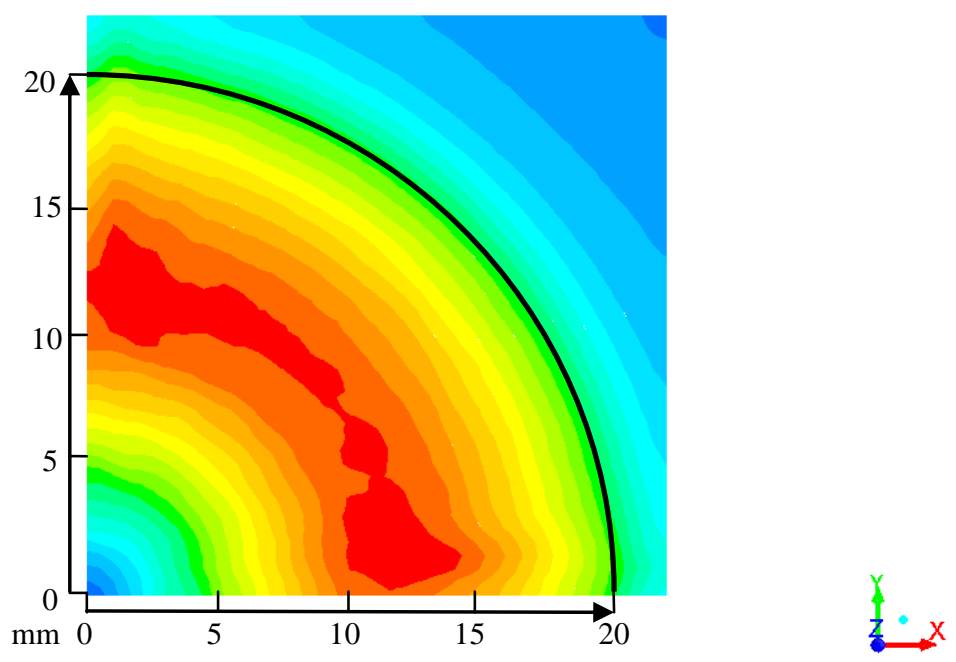

Contours of Film Velocity Magnitude $(\mathrm{m} / \mathrm{s})($ Time $=8.5000 \mathrm{e}-02)$

Jan 28, 2015 ANSYS Fluent 15.0 (3d, dp, pbns, spe, rke, transient)

Figure 6.14 Flooded contours of film velocity magnitude for 40 psi full cone water spray, Case III a) $50 \mathrm{~ms}$ simulation time b) $85 \mathrm{~ms}$ simulation time. (The color scale is the same for both times. Red refers to the maximum velocity range: $0.594-0.625 \mathrm{~m} / \mathrm{s}$; blue refers to the minimum velocity range: $0-0.032 \mathrm{~m} / \mathrm{s}$. The black arc indicates the edge of the spray. All images show the top view of the impact surface.) 

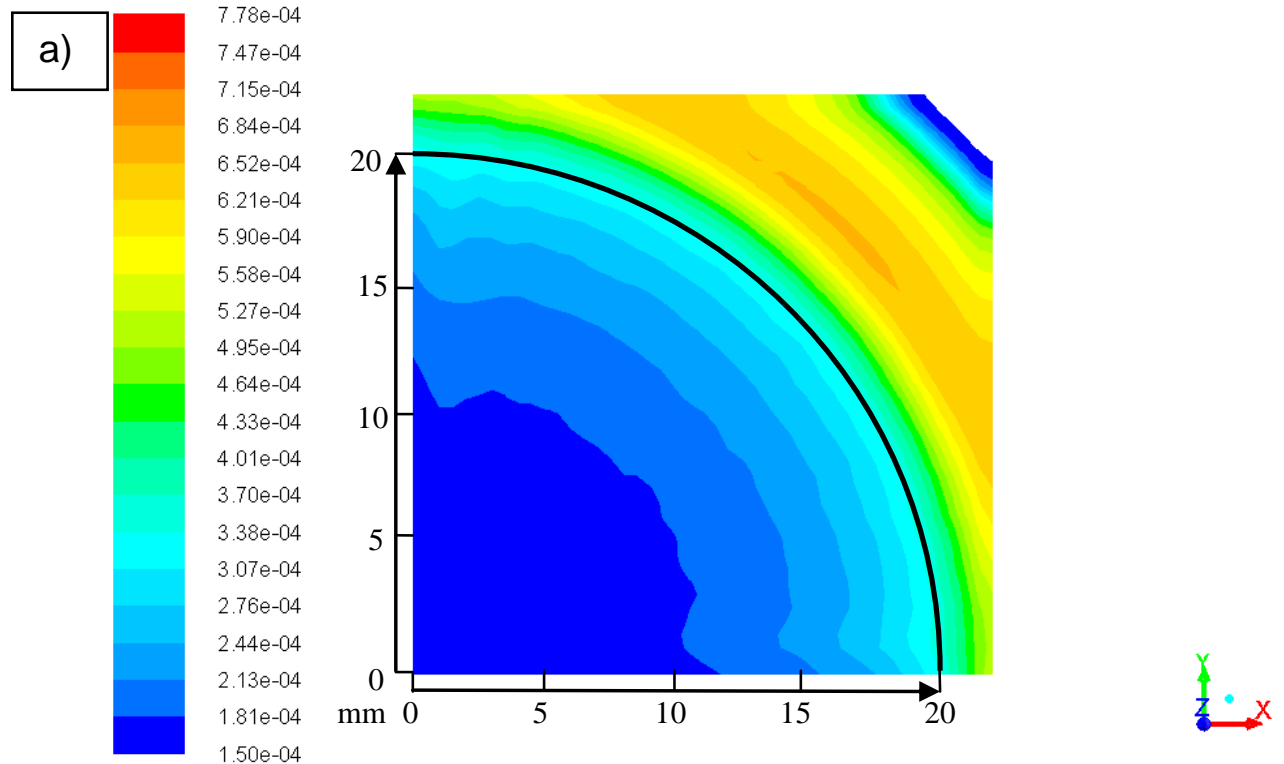

Contours of Film Thickness $(\mathrm{m})($ Time $=5.0000 \mathrm{e}-02)$

Jan 28,2015 ANSYS Fluent 15.0 (3d, dp, pbns, spe, rke, transient)

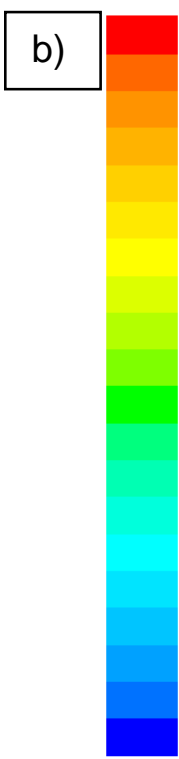

$7.78 \mathrm{e}-04$

$7.47 e-04$

$7.15 \mathrm{e}-04$

$6.84 \mathrm{e}-04$

$6.53 \mathrm{e}-04$

$6.21 \mathrm{e}-04$

$5.90 e-04$

$5.58 \mathrm{e}-04$

$5.27 e-04$

$4.96 \mathrm{e}-04$

4. $64 \mathrm{e}-04$

4.33e-04

$4.01 \mathrm{e}-04$

3.70e-04

$3.38 \mathrm{e}-04$

3.07e-04

$2.76 \mathrm{e}-04$

$2.44 \mathrm{e}-04$

$2.13 \mathrm{e}-04$

$1.81 \mathrm{e}-04$

$1.50 \mathrm{e}-04$
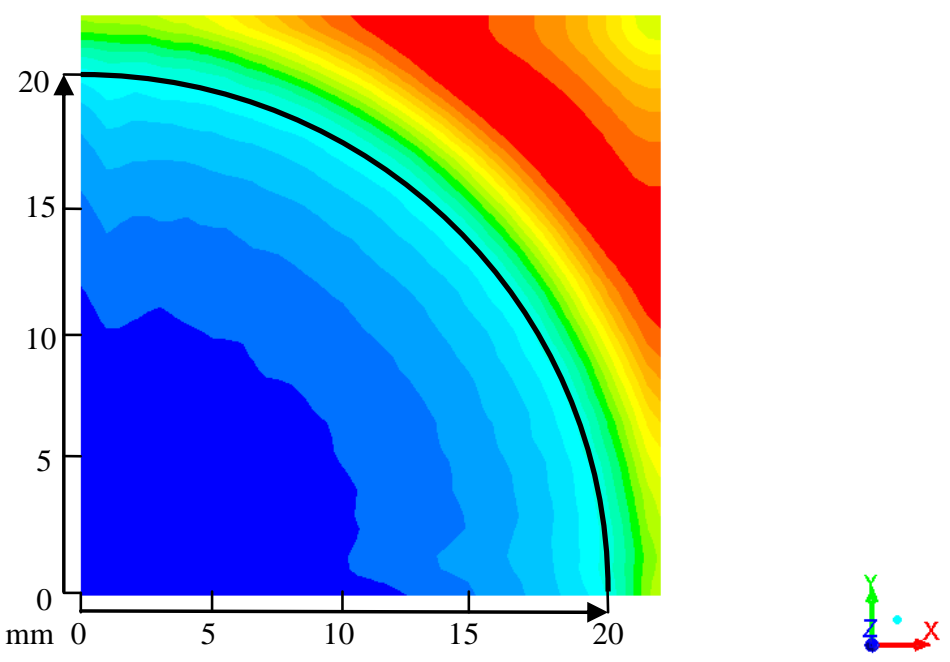

Contours of Film Thickness $(\mathrm{m})($ Time $=8.5000 \mathrm{e}-02)$

Jan 28, 2015 ANSYS Fluent 15.0 (3d, dp, pbns, spe, rke, transient)

Figure 6.15 Flooded contours of film thickness for 40 psi full cone water spray, Case III a) $50 \mathrm{~ms}$ simulation time b) $85 \mathrm{~ms}$ simulation time. (The color scale is almost the same for both times. Blue refers to 150 micron, red refers to 778 micron. The black arc indicates the edge of the spray. All images show the top view of the impact surface) 
Figure 6.16 shows that there is little change in the mid depth film temperature between 50 ms and $85 \mathrm{~ms}$. These Case III results are very close to the Case I results. Similar to Case I, the temperature increases from about $320 \mathrm{~K}$ on the axis to about $331 \mathrm{~K}$ at the edge of the spray for Case III. The temperature continues to increase to about $368 \mathrm{~K}$ at the largest radius. At both times, the temperature is largely rotationally symmetric. The results are completely different from Case II because of the difference of the spray liquid temperature (for Case III spray temperature is $300 \mathrm{~K}$ and for Case II spray temperature is $350 \mathrm{~K}$ ).

Figure 6.17 shows the surface heat flux at $50 \mathrm{~ms}$ and $85 \mathrm{~ms}$ is very similar. These results are also very close to Case I results. The maximum heat flux value is $2.08 \mathrm{MW} / \mathrm{m}^{2}\left(208 \mathrm{~W} / \mathrm{cm}^{2}\right)$ at $85 \mathrm{~ms}$ for Case III and it is $2.07 \mathrm{MW} / \mathrm{m}^{2}\left(207 \mathrm{~W} / \mathrm{cm}^{2}\right)$ for Case I.

Figure 6.18 shows water vapor mass fraction in the film at $50 \mathrm{~ms}$ and $85 \mathrm{~ms}$, respectively. The water vapor mass fraction is very small from the axis to $R=5 \mathrm{~mm}$ (between 0.006 and 0.0116 ), but it gradually increases along the radius up to 0.05 at around $R=20.3 \mathrm{~mm}$ which is very similar to the Case I results. After $R=20.3 \mathrm{~mm}$, the vapor mass fraction continues to increase to the maximum value of 0.116 at both $50 \mathrm{~ms}$ and $85 \mathrm{~ms}$. At both times, the vapor mass fraction is rotationally symmetric. 


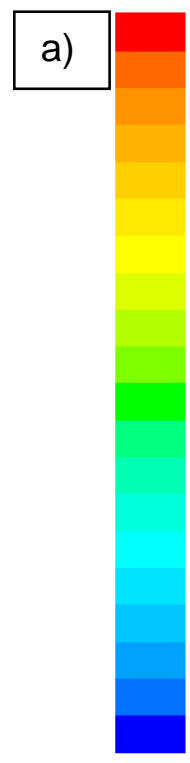

$3.68 \mathrm{e}+02$

$3.65 e+02$

$3.61 e+02$

$3.58 \mathrm{e}+02$

$3.54 \mathrm{e}+02$

$3.51 e+02$

$3.48 \mathrm{e}+02$

$3.44 \mathrm{e}+02$

$3.41 e+02$

$3.37 e+02$

$3.34 \mathrm{e}+02$

$3.31 e+02$

$3.27 e+02$

$3.24 e+02$

$3.20 \mathrm{e}+02$

$3.17 e+02$

$3.14 e+02$

$3.10 e+02$

$3.07 e+02$

$3.03 e+02$

$3.00 e+02$
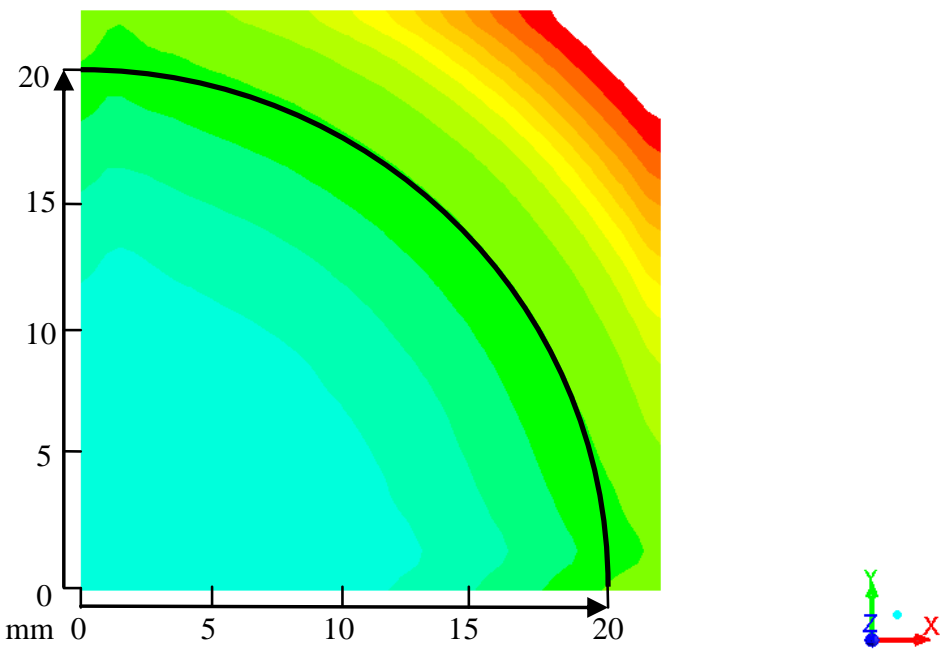

Contours of Film Temperature (k) $($ Time $=5.0000 \mathrm{e}-02)$

Jan 28,2015

ANSYS Fluent 15.0 (3d, dp, pbns, spe, rke, transient)

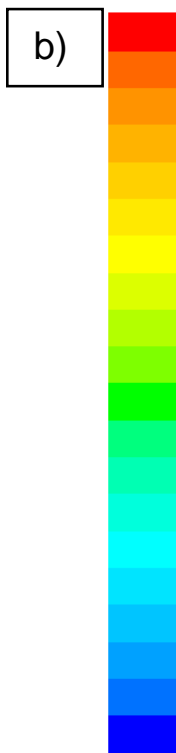

$3.68 \mathrm{e}+02$

$3.65 \mathrm{e}+02$

$3.61 \mathrm{e}+02$

$3.58 \mathrm{e}+02$

$3.54 \mathrm{e}+02$

$3.51 e+02$

$3.48 \mathrm{e}+02$

$3.44 \mathrm{e}+02$

$3.41 e+02$

$3.37 e+02$

$3.34 e+02$

$3.31 e+02$

$3.27 e+02$

$3.24 \mathrm{e}+02$

$3.20 \mathrm{e}+02$

$3.17 e+02$

$3.14 e+02$

$3.10 \mathrm{e}+02$

$3.07 e+02$

$3.03 e+02$

$3.00 e+02$
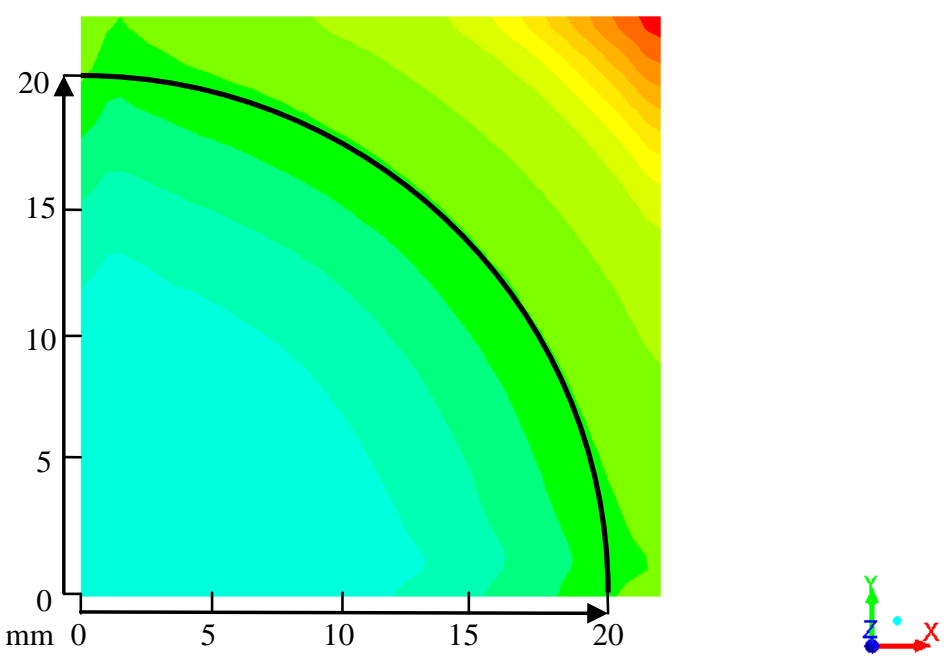

Contours of Film Temperature $(\mathrm{k})($ Time $=8.5000 \mathrm{e}-02)$

Jan 28, 2015

ANSYS Fluent 15.0 (3d, dp, pbns, spe, rke, transient)

Figure 6.16 Flooded contours of mid depth film temperature for 40 psi full cone water spray, Case III a) $50 \mathrm{~ms}$ simulation time b) $85 \mathrm{~ms}$ simulation time. (The color scale is the same in a) and $b$ ). Blue refers to $300 \mathrm{~K}$, red refers to $368 \mathrm{~K}$. The black arc indicates the edge of the spray. All images show the top view of the impact surface.) 


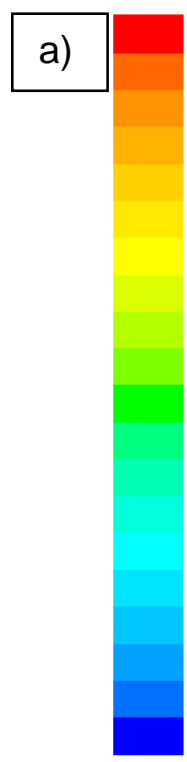

$2.07 e+06$

$1.97 e+06$

$1.86 e+06$

$1.76 \mathrm{e}+06$

$1.66 \mathrm{e}+06$

$1.55 \mathrm{e}+06$

$1.45 \mathrm{e}+06$

$1.35 e+06$

$1.24 e+06$

$1.14 e+06$

$1.04 e+06$

$9.32 e+05$

$8.28 \mathrm{e}+05$

$7.25 e+05$

$6.21 \mathrm{e}+05$

$5.18 \mathrm{e}+05$

$4.14 e+05$

$3.11 e+05$

$2.07 e+05$

$1.04 \mathrm{e}+05$

$0.00 e+00$
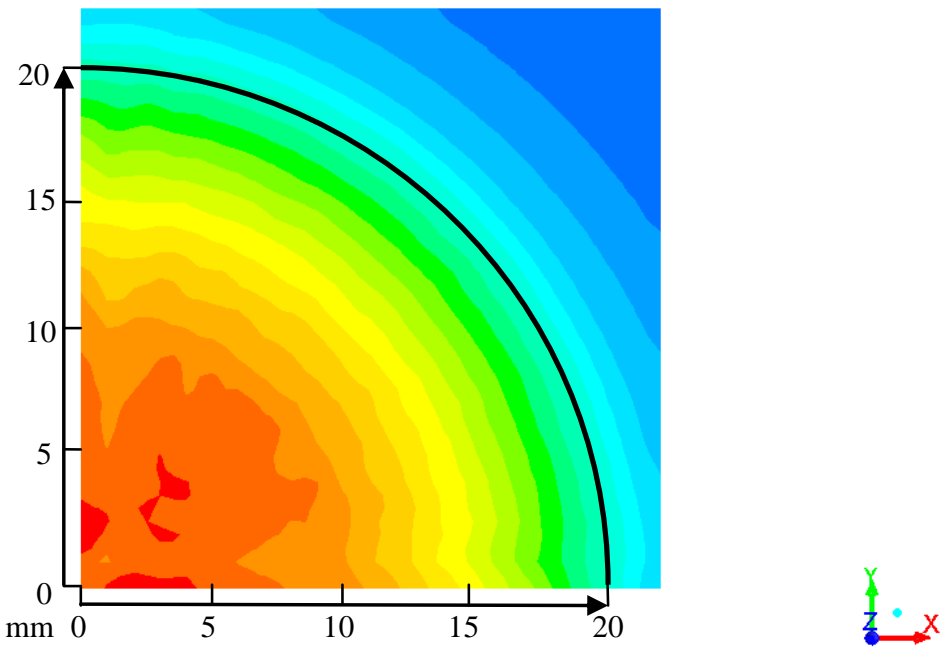

Contours of Total Surface Heat Flux (w/m2) $($ Time $=5.0000 \mathrm{e}-02)$

Jan 28,2015

ANSYS Fluent 15.0 (3d, dp, pbns, spe, rke, transient)

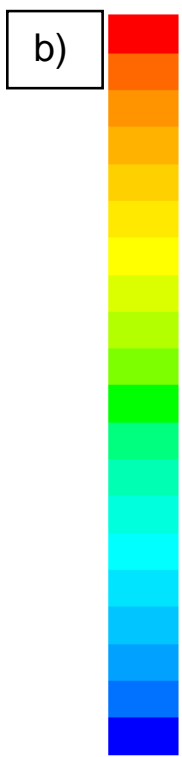

$2.08 \mathrm{e}+06$

$1.97 e+06$

$1.87 e+06$

$1.77 e+06$

$1.66 \mathrm{e}+06$

$1.56 e+06$

$1.45 e+06$

$1.35 e+06$

$1.25 \mathrm{e}+06$

$1.14 e+06$

$1.04 e+06$

$9.35 e+05$

$8.31 \mathrm{e}+05$

$7.27 e+05$

$6.23 e+05$

$5.19 e+05$

$4.16 \mathrm{e}+05$

$3.12 e+05$

$2.08 \mathrm{e}+05$

$1.04 e+05$

$0.00 e+00$
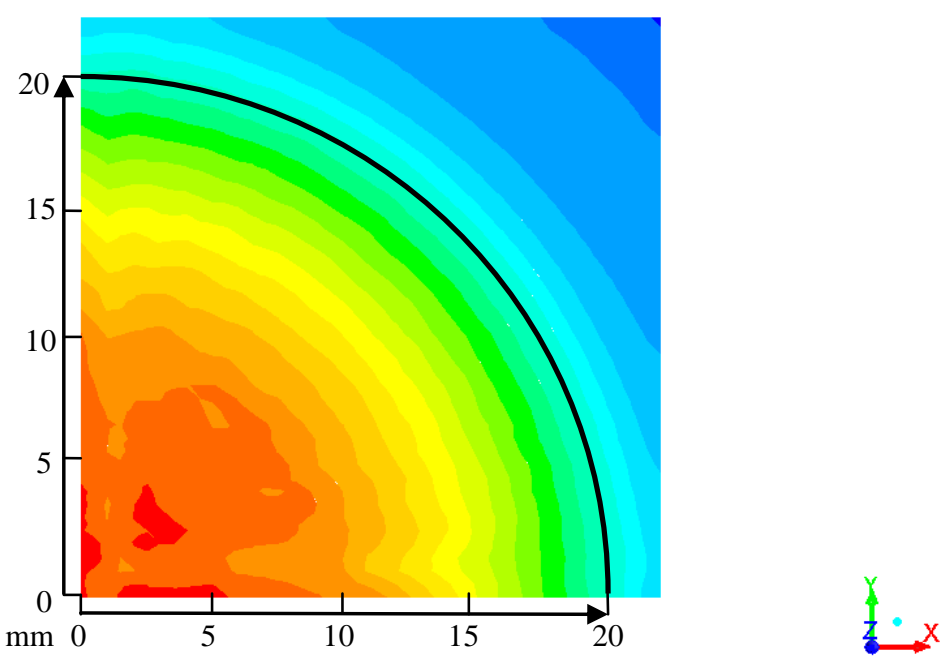

Contours of Total Surface Heat Flux (w/m2) $($ Time $=8.5000 \mathrm{e}-02)$

Jan 28, 2015 ANSYS Fluent 15.0 (3d, dp, pbns, spe, rke, transient)

Figure 6.17 Flooded contours of surface heat flux for 40 psi full cone water spray, Case III a) 50 ms simulation time b) $85 \mathrm{~ms}$ simulation time. (The color scale is different in a) and b). Blue refers to $0 \mathrm{~W} / \mathrm{m}^{2}$ and red refers to $2.07 \mathrm{MW} / \mathrm{m}^{2}$ in a) and $2.08 \mathrm{MW} / \mathrm{m}^{2}$ in b). The black arc indicates the edge of the spray. All images show the top view of the impact surface.) 


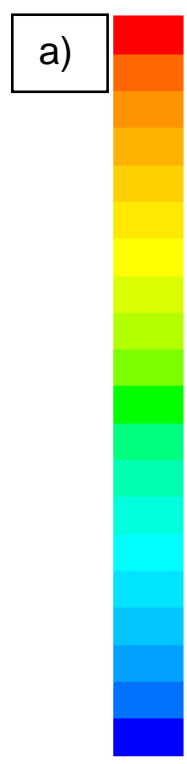

$1.16 \mathrm{e}-01$

1.10e-01

$1.04 \mathrm{e}-01$

$9.86 \mathrm{e}-02$

$9.28 \mathrm{e}-02$

$8.70 \mathrm{e}-02$

$8.12 \mathrm{e}-02$

$7.54 \mathrm{e}-02$

$6.96 \mathrm{e}-02$

6.38e-02

5.80e-02

$5.22 \mathrm{e}-02$

$4.64 \mathrm{e}-02$

4.06e-02

$3.48 \mathrm{e}-02$

2.90e-02

2.32e-02

$1.74 \mathrm{e}-02$

$1.16 \mathrm{e}-02$

$5.80 \mathrm{e}-03$

$0.00 e+00$
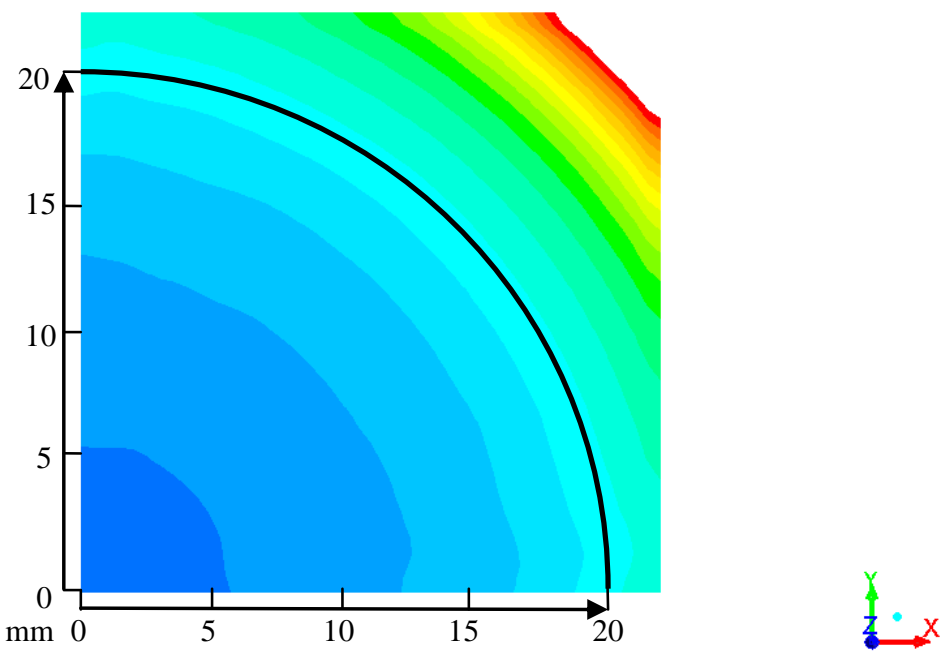

Contours of Mass fraction of h2o (Time $=5.0000 \mathrm{e}-02$ )

Jan 28, 2015

ANSYS Fluent 15.0 (3d, dp, pbns, spe, rke, transient)

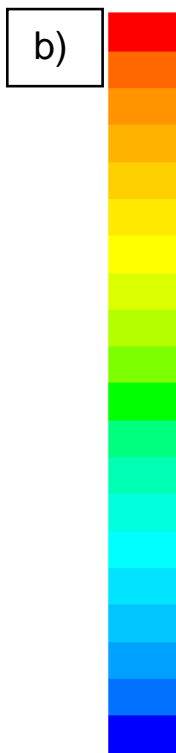

$1.16 \mathrm{e}-01$

$1.10 \mathrm{e}-01$

$1.04 \mathrm{e}-01$

$9.86 \mathrm{e}-02$

$9.28 \mathrm{e}-02$

$8.70 \mathrm{e}-02$

$8.12 \mathrm{e}-02$

$7.54 \mathrm{e}-02$

$6.96 \mathrm{e}-02$

6.38e-02

$5.80 \mathrm{e}-02$

$5.22 \mathrm{e}-02$

$4.64 \mathrm{e}-02$

4.06e-02

$3.48 \mathrm{e}-02$

2.90e-02

2.32e-02

$1.74 \mathrm{e}-02$

$1.16 \mathrm{e}-02$

$5.80 e-03$

$0.00 e+00$
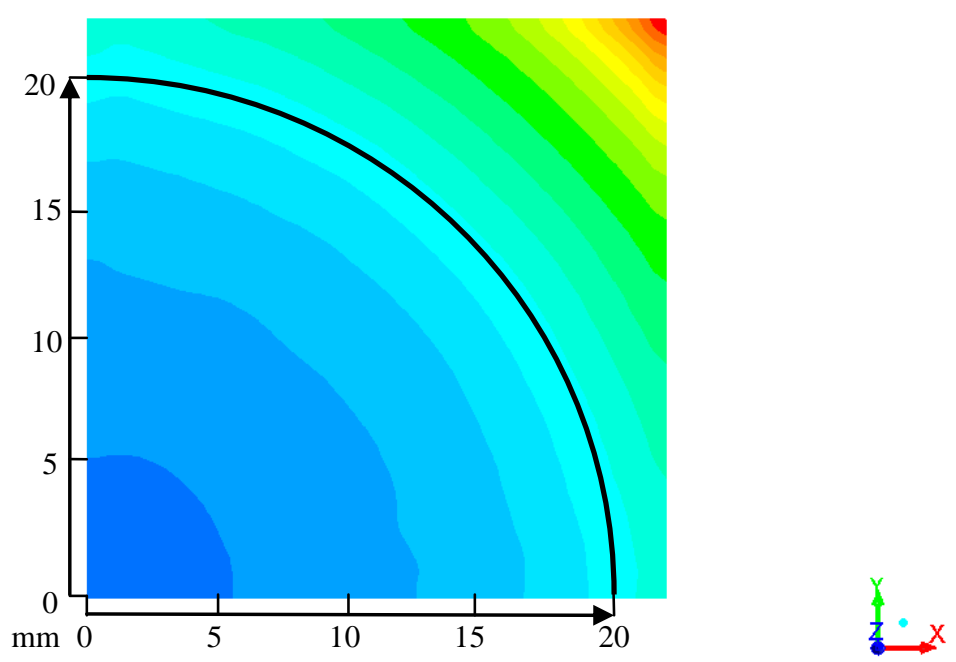

Figure 6.18 Flooded contours of water vapor mass fraction for 40 psi full cone water spray, Case III a) $50 \mathrm{~ms}$ simulation time b) $85 \mathrm{~ms}$ simulation time. (The color scale is same in a) and b). Blue refers to 0 and red refers to 0.116. The black arc indicates the edge of the spray. All images show the top view of the impact surface.) 
Figure 6.19 shows the variation of liquid film thickness (Fig. 6.19.a) and liquid film velocity magnitude (Fig. 6.19.b) along the 45 degree diagonal of the domain for Cases I, II and III at $100 \mathrm{~ms}$. These results are independent of the spray temperature (subcooling) and the air temperature at $2 \mathrm{~K}$ superheat.
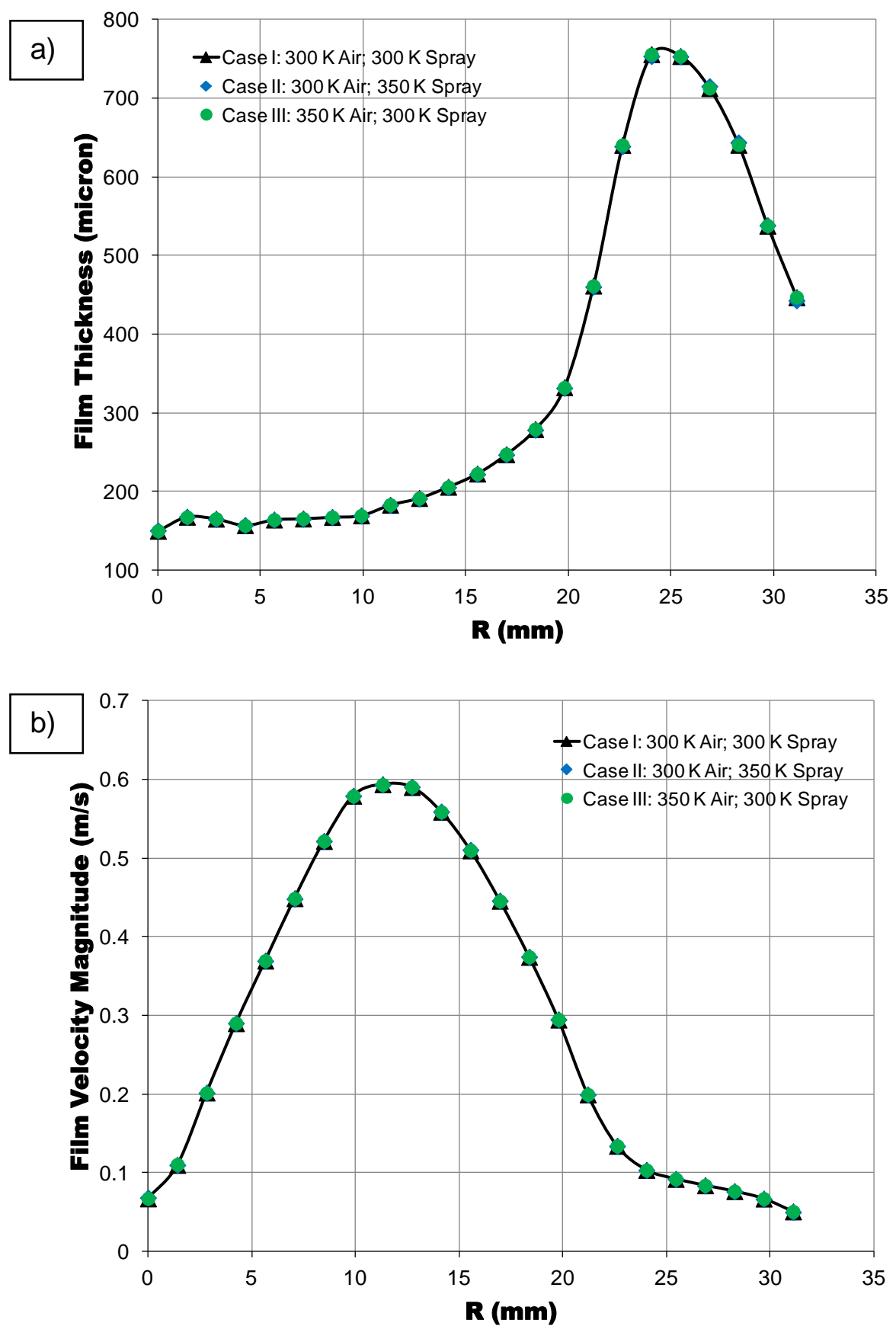

Figure 6.19 Liquid film thickness (a) and liquid film velocity magnitude vs. radius for Cases I, II and III at $100 \mathrm{~ms}$. 
Figure 6.20 shows the variation of the mid depth liquid film temperature (Fig. 6.20.a) and the vapor mass fraction (Fig. 6.20.b) along the 45 degree diagonal of the domain for Cases I, II and III at $100 \mathrm{~ms}$. The variation of liquid film temperature is significantly dependent on the spray temperature, but it is not dependent on the air temperature. Similarly, the vapor mass fraction depends on the spray temperature, but it does not depend on the air temperature. There is more vapor formation when using the warmer spray $(350 \mathrm{~K})$ compared to the colder spray at $300 \mathrm{~K}$. However, air temperature has no effect on the formation of vapor and the surface heat flux.

Table 6.4 shows the surface heat flux averaged over the entire heated surface $(22 \mathrm{~mm} \mathrm{x}$ $22 \mathrm{~mm}$ ) for the three cases at $100 \mathrm{~ms}$. Air temperature does not have any effect on the surface heat flux but there is a significant relation between the temperature of spray drops (subcooling) and surface heat flux as expected. For the colder spray (Case I: spray temperature $=300 \mathrm{~K}$ ), there is a significantly higher surface heat flux compared to the warmer spray (Case II: spray temperature $=350 \mathrm{~K}$ ). Increasing the spray temperature $50 \mathrm{~K}$ (from $300 \mathrm{~K}$ to $350 \mathrm{~K}$ ) causes a $62 \%$ decrease in the surface heat flux. 

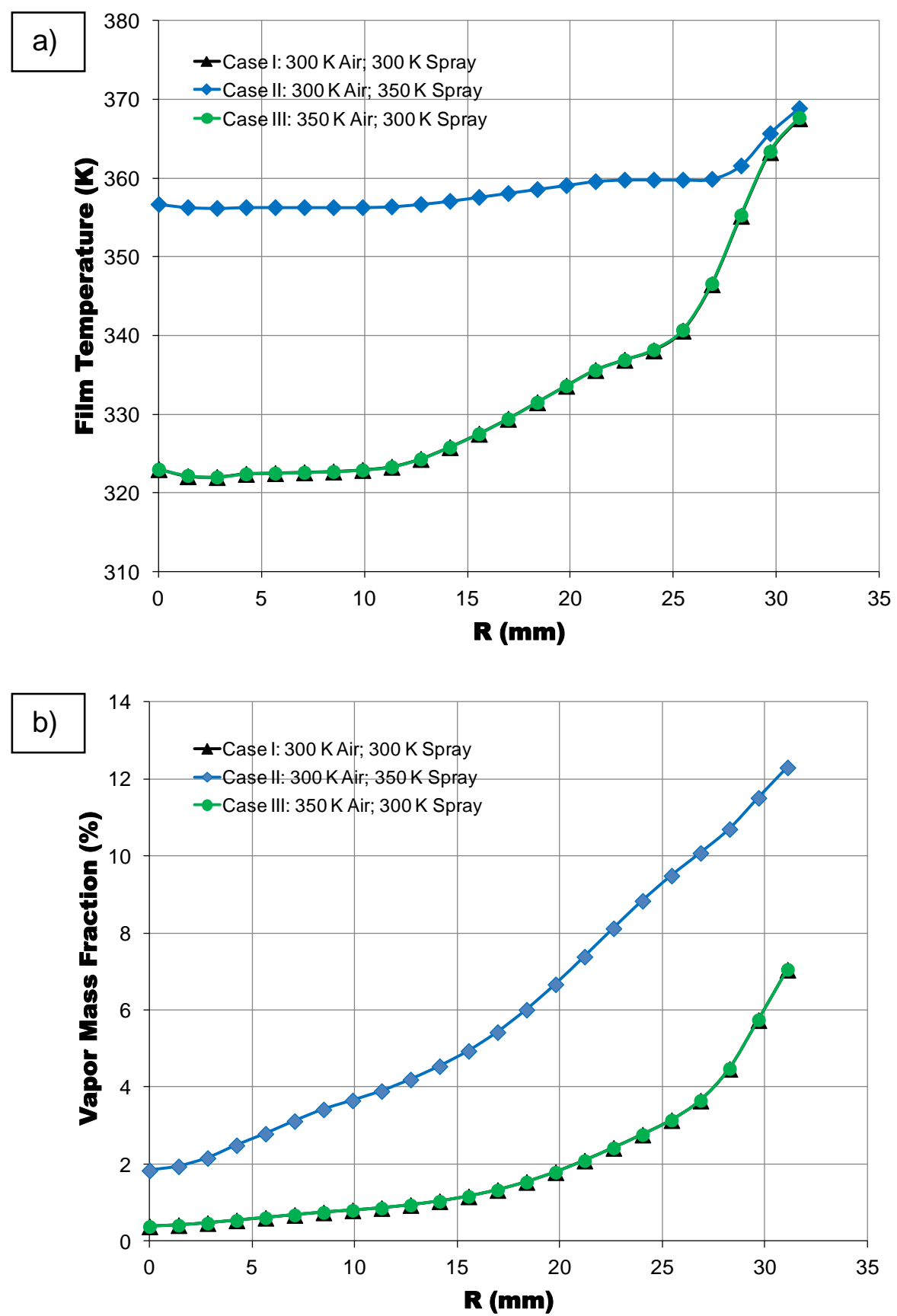

Figure 6.20 Variation of the mid depth liquid film temperature (a) and water vapor mass fraction (b) over the impact radius for Cases I, II and III at $100 \mathrm{~ms}$. 
Table 6.2 The effect of air temperature and spray temperature on the surface heat flux at $100 \mathrm{~ms}$.

\begin{tabular}{|c|c|c|c|c|c|}
\hline Case & $\begin{array}{c}\text { Air } \\
\text { Temperature } \\
(\mathbf{K})\end{array}$ & $\begin{array}{c}\text { Spray Liquid } \\
\text { Temperature } \\
(\mathbf{K})\end{array}$ & $\begin{array}{c}\text { Surface } \\
\text { Temperature } \\
(\mathbf{K})\end{array}$ & $\begin{array}{c}\text { Initial Liquid } \\
\text { Film } \\
\text { temperature } \\
(\mathbf{K})\end{array}$ & $\begin{array}{c}\text { Computed } \\
\text { Surface } \\
\text { Heat Flux } \\
\left(\mathbf{W} / \mathbf{c m}^{\mathbf{2}}\right)\end{array}$ \\
\hline I & 300 & 300 & 375 & 350 & 105.9 \\
\hline II & 300 & 350 & 375 & 350 & 39.25 \\
\hline III & 350 & 300 & 375 & 350 & 105.9 \\
\hline
\end{tabular}

As a summary of Chapter 6, spray cooling simulations with phase change were successfully performed using the 3D DPM-EWF-STM models in ANSYS Fluent 15. Thus, it is concluded that the 3D DPM-EWF-STM models in ANSYS Fluent 15 can be used for the modeling of spray/spray cooling in order to develop correlations for spray cooling. 


\section{CHAPTER 7: SPRAY IMPACT AND COOLING SIMULATIONS USING THE EULERIAN MULTIPHASE (EM) MODEL}

Although the 3D DPM-EWF-STM model used in Chapter 6 provided good results, one additional model in ANSYS Fluent was investigated to determine if it could provide more accurate and more efficient simulations. In this chapter simulations of spray impact and cooling using the Eulerian Multiphase (EM) model are described. The computational model and setup and the analysis of the results are given in detail.

\subsection{Numerical Modeling and Governing Equations}

The Eulerian Multiphase (EM) model in ANSYS Fluent can be used for the modeling of multiphase flows such as bubbly flow, droplet flow, slurry flow and fluidized bed flow (ANSYS Fluent Theory Guide, 2013). In this model, an Eulerian treatment is applied to all of the phases (e.g. liquid, gas, and vapor). The coupling between the phases is achieved through the pressure and the interphase exchange coefficients. A single pressure is shared by all phases. The conservation of mass, momentum and energy are solved separately for each phase in the EM.

The conservation of mass for phase $i$ is solved using

$$
\frac{\partial\left(\alpha_{i} \rho_{i}\right)}{\partial t}+\nabla \cdot\left(\alpha_{i} \rho_{i} \underline{U_{i}}\right)=\sum_{j=1}^{n}\left(\dot{m}_{\iota \jmath}-\dot{m}_{\jmath l}\right)+S_{i}
$$

where $\alpha_{i}$ is the volume fraction of phase $i$

$\rho_{i}$ is the density of phase $i$

$\underline{U_{i}}$ is the velocity vector of phase $i$

$m_{l j}$ is the mass transfer from phase $i$ to $j$

$\dot{m}_{\jmath l}$ is the mass transfer from phase $j$ to $i$

$S_{i}$ is the mass source term for phase $i$.

The conservation of momentum for phase $i$ is solved using

$$
\frac{\partial\left(\alpha_{i} \rho_{i} \underline{U_{i}}\right)}{\partial t}+\nabla \cdot\left(\alpha_{i} \rho_{i} \underline{U_{i} U_{i}}\right)=-\alpha_{i} \nabla P+\nabla \cdot \overline{\tau_{i}}+\alpha_{i} \rho_{i} \underline{g}+\sum_{j=1}^{n}\left(\underline{R_{i j}}+m_{l \jmath} \underline{U_{i j}}-m_{\jmath l} \underline{U_{j i}}\right)+\underline{F}
$$


where $P$ is the pressure shared by all phases

$\overline{\tau_{i}}$ is the stress tensor of phase $i$

$\underline{g}$ is the gravity vector

$R_{i j}$ is the interaction force between phases

$U_{i j}$ is the interface velocity (if $m_{l \jmath}>0$ (mass is being transferred from phase $i$ to phase $j$ ), then

$$
\left.\underline{U_{i j}}=\underline{U_{i}} \text { or else } \underline{U_{i j}}=\underline{U_{j}}\right)
$$

$\underline{F}$ is the total force vector (includes body forces).

The interaction force between phases $\left(R_{i j}\right)$ includes the effects of friction, pressure, and cohesion between phases. It is calculated using

$$
\sum_{j=1}^{n} \underline{R_{i j}}=\sum_{j=1}^{n} K_{i j}\left(\underline{U_{i}}-\underline{U_{j}}\right)
$$

where $K_{i j}$ is the interface momentum exchange coefficient. Equation 7.3 is subjected to the conditions of $R_{i j}=-R_{j i}$ and $R_{i i}=0$.

The interface exchange momentum coefficient, $K_{i j}$, for phase $i$ is calculated using

$$
K_{i j}=\frac{\rho_{i} f_{d}}{6 t_{i}} d_{i} A_{i j}
$$

where $f_{d}$ is the drag function, $t_{i}$ is the particle relaxation time, $d_{i}$ is the particle diameter, $A_{i j}$ is the interfacial area concentration. In equation 7.4, $K_{i j}=K_{j i}$.

The drag function is calculated using the Schiller and Naumann (1935) model

$$
f_{d}=\frac{c_{d} R e}{24}
$$

where $\quad c_{d}=\frac{24\left(1+0.15 R e^{0.687}\right)}{R e}$ for $R e \leq 1000$ and $c_{d}=0.44$ for $R e>1000$ 
$R e$ is the relative Reynolds number calculated based on the velocity difference between the liquid and gas phase.

$t_{i}$ is the particle relaxation time, and it is calculated as

$$
t_{i}=\frac{\rho_{i} d_{i}^{2}}{18 \mu_{j}}
$$

where $d_{i}$ is the diameter of phase $i$ (e.g. droplet diameter)

$\mu_{j}$ is the dynamic viscosity of phase $j$.

The interfacial area concentration, $A_{i}$, is used to predict mass, momentum and energy transfer through the interface between the phases. It is calculated using

$$
A_{i}=\frac{\pi d_{i}^{2}}{\frac{1}{6} \pi d_{i}^{3}}=\frac{6}{d_{i}}
$$

As a turbulence model, the Mixture k-epsilon model was used. It uses the same conservation equations of the Standard k-epsilon model but it uses volume averaged mixture fluid properties and mixture velocities (ANSYS Fluent Theory Guide, 2013).

The conservation of energy for phase $i$ is solved using

$$
\frac{\partial\left(\alpha_{i} \rho_{i} h_{i}\right)}{\partial t}+\nabla \cdot\left(\alpha_{i} \rho_{i} h_{i} \underline{\left.U_{i}\right)}=\alpha_{i} \frac{\partial P}{\partial t}+\overline{\tau_{i}}: \nabla \underline{U_{i}}-\nabla \cdot \underline{q_{i}}+S_{i}+\sum_{j=1}^{n}\left(Q_{i j}+m_{l \jmath} H_{i j}-\dot{m}_{\jmath l} H_{j i}\right)\right.
$$

where $h_{i}$ is the specific enthalpy of phase $i$

$\underline{q_{i}}$ is the heat flux

$S_{i}$ is the energy source term

$Q_{i j}$ is the heat exchange between phases $i$ and $j$

$H_{i j}$ is the interface enthalpy.

The heat transfer between the phases, $Q_{i j}$, is calculated using 


$$
Q_{i j}=h_{i j} A_{i}\left(T_{i}-T_{j}\right)
$$

where $T_{i}$ is the temperature of phase $i$

$T_{j}$ is the temperature of phase $j$

$h_{i j}$ is the interface heat transfer coefficient which is calculated using

$$
h_{i j}=\frac{k_{j} N u_{i}}{d_{i}}
$$

where $k_{j}$ is the thermal conductivity of phase $j$

$N u_{i}$ is the Nusselt number of phase $i$.

Equation 7.10 is subjected to the conditions of $Q_{i j}=-Q_{j i}$ and $Q_{i i}=0$

The Nusselt number is calculated using the Ranz-Marshall correlation (Ranz and Marshall, 1952)

$$
N u_{i}=2+0.6 R e_{i}^{1 / 2} \operatorname{Pr}_{j}^{1 / 3}
$$

where $R e_{i}$ is the Reynolds number based on diameter of phase $i\left(d_{i}\right)$ and the relative velocity between the phases $\left(\underline{U_{i}}-\underline{U_{j}}\right)$

$P r_{j}$ is the Prandtl number of phase $j$

$$
\operatorname{Pr}_{j}=\frac{c_{p j} \mu_{j}}{k_{j}}
$$

where $c_{p j}$ is the specific heat of phase $j$.

To account for boiling in the layer liquid, the Rensselaer Polytechnic Institute (RPI) boiling model is used. The RPI model is based on the wall nucleate boiling model of Kurul and Podowski (1991). In this model, the total heat flux from the wall to the liquid $\left(q_{w}\right)$ is considered to have 3 components. These 3 components of heat flux are called the convective heat flux $\left(\dot{q}_{c}\right)$, the quenching heat flux $\left(\dot{q}_{q}\right)$ and the evaporative heat flux $\left(\dot{q}_{e}\right)$. The meaning of these terms is explained below. The total wall heat flux is 


$$
\dot{q}_{w}=\dot{q}_{c}+\dot{q}_{q}+\dot{q}_{e}
$$

The heated wall area is divided into the area which is covered by nucleating bubbles $\left(A_{b}\right)$, and the area which is covered by liquid $\left(1-A_{b}\right)$. The convective heat flux is calculated using

$$
\dot{q}_{c}=h_{c}\left(T_{w}-T_{l}\right)\left(1-A_{b}\right)
$$

where $h_{c}$ is the single phase heat transfer coefficient

$T_{w}$ is the wall temperature

$T_{l}$ is the liquid temperature.

The quenching heat flux is calculated based on the energy transfer from the wall to the liquid which fills the space after bubble detachment. It is

$$
\dot{q}_{q}=\frac{2 k_{l}}{\sqrt{\pi k_{l} t}}\left(T_{w}-T_{l}\right)
$$

where $k_{l}$ is the liquid thermal conductivity

$\kappa_{l}$ is the liquid thermal diffusivity

$t$ is the periodic time.

The evaporative heat flux is calculated using

$$
\dot{q}_{e}=V_{b} n_{w} \rho_{v} h_{f g} f
$$

where $V_{b}$ is the the volume of departing bubbles

$n_{w}$ is the nucleation site density

$\rho_{v}$ is the density of vapor

$h_{f g}$ is the the latent heat of evaporation

$f$ is the frequency of bubble departure.

$$
V_{b}=\frac{\pi d_{b}^{3}}{6}
$$


where $d_{b}$ is the bubble departure diameter which is calculated in meters based on the empirical correlation of Tobulinski and Kostanchuk (1970).

$$
d_{b}=\min \left(0.0014,(0.0006)\left(e^{-\frac{\Delta T_{\text {sup }}}{45}}\right)\right)
$$

where $\Delta T_{\text {sup }}=$ superheat $=T_{w}-T_{\text {sat }}$.

The frequency of bubble departure, $f$, is calculated using the correlation of Cole (1960) which is based on inertia controlled bubble growth

$$
f=\frac{1}{T}=\sqrt{\frac{4 g\left(\rho_{l}-\rho_{v}\right)}{3 \rho_{l} d_{b}}}
$$

The nucleation site density, $n_{w}$, is calculated using

$$
n_{w}=c^{n}\left(T_{w}-T_{s a t}\right)^{n}
$$

where $c$ is the empirical constant equal to 210 (Lemmert and Chawla, (1977)) $n$ is the empirical constant equal to 1.805 (Lemmert and Chawla, (1977)).

\subsection{Meshing, Boundary and Initial Conditions}

The EM model is very demanding of computational resources because of the separate equations for each phase in the computational domain. In order to model spray cooling using the EM, a 2D axisymmetric model was used to reduce the computational cost compared to full 3D modeling.

Figure 7.1 shows the $2 \mathrm{D}$ axisymmetric computational domain. The domain size is 28.1 $\mathrm{mm} \times 28.1 \mathrm{~mm}$. A full cone spray is injected from the velocity inlet boundary condition $28.1 \mathrm{~mm}$ above the impact surface. The heated surface is treated as a wall boundary condition. The maximum radius is a pressure outlet as is the top boundary outboard of the spray inlet region. The full cone spray injection is based on the FullJet 1/8-G full cone spray nozzle used in the experiments of Taylor et al. (2014a, 2014b) for a 40 psi spray, and is identical to the 
corresponding spray conditions used in previous chapters in this dissertation. The nozzle-toimpact surface distance is $38.1 \mathrm{~mm}$ for this spray but spray is injected from $28.1 \mathrm{~mm}$ above the surface by neglecting the $10 \mathrm{~mm}$ primary atomization region. This atomization region, which requires very fine computational cells, is not of interest for these studies. In order to inject the spray from $28.1 \mathrm{~mm}$ above the surface, the experimental data was used. Based on the spray mass flow rate, the initial liquid volume fraction at the velocity inlet is $0.845 \%$ with the spray radius and velocity vector components based on the experimental data. In order to obtain a conical spray, each velocity inlet cell was defined as a separate velocity inlet boundary condition with horizontal and vertical velocity components based on the experimental velocity distribution at $28.1 \mathrm{~mm}$ above the surface (Fig. 7.2).

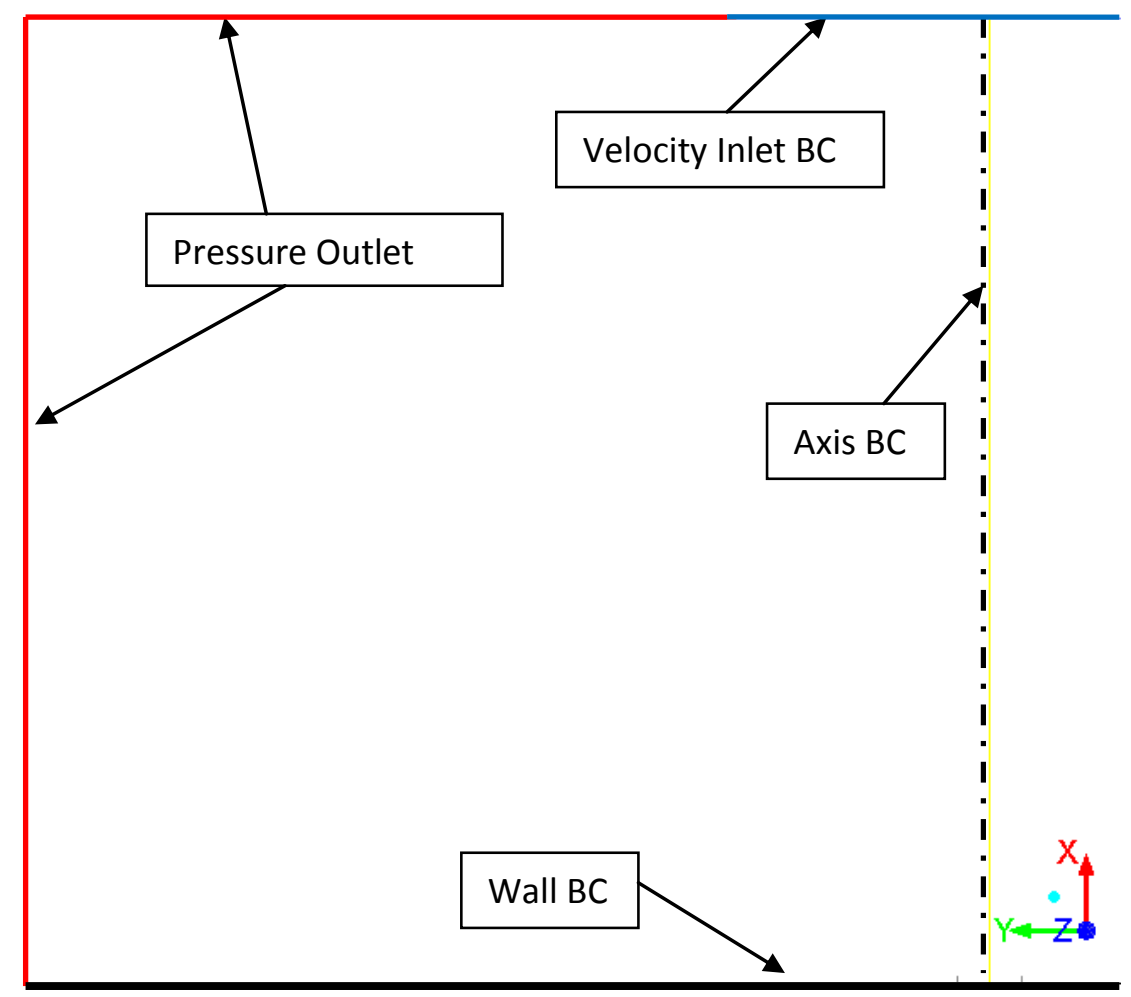

Figure 7.12D axisymmetric computational domain with the boundary conditions. 


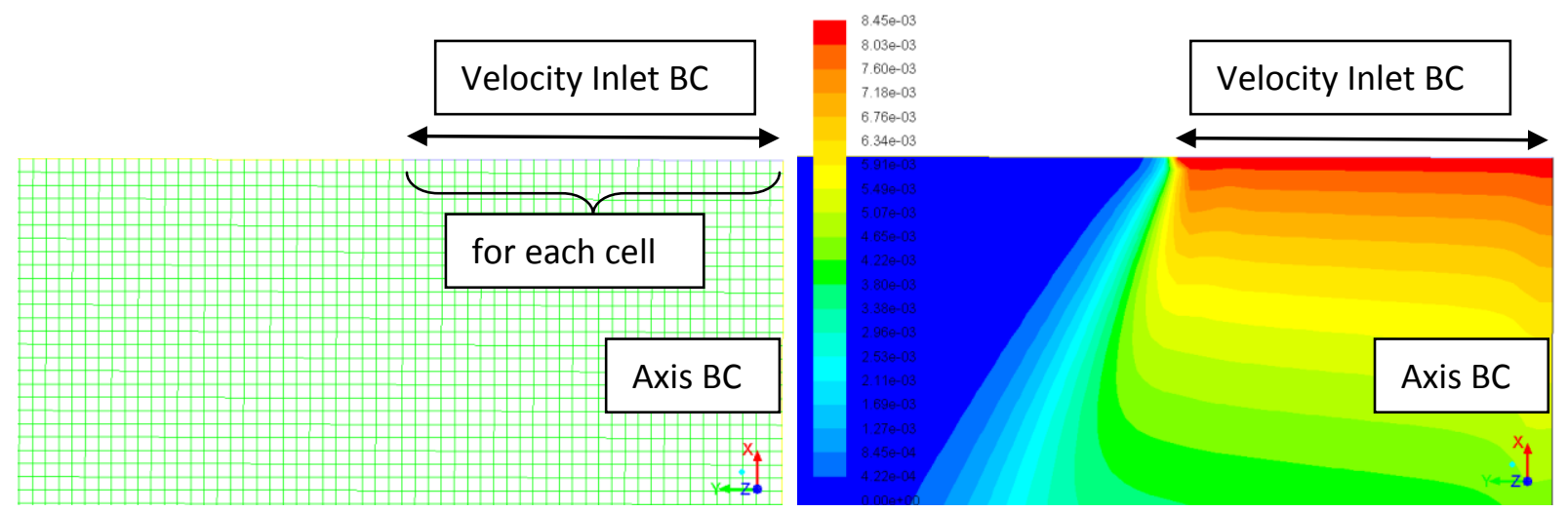

Figure 7.2 Location of the velocity inlet boundary condition cells (left) and flooded contours of liquid volume fraction (right). (Red shows the maximum liquid volume fraction $(0.845 \%)$ and blue shows zero. Note that the whole domain is not shown.).

There was a need to select an optimum computational cell size in order to obtain accurate liquid film and heat transfer characteristics for spray cooling simulations. Very fine cells are needed close to the impact surface in order to obtain grid independent film characteristics. A 4 step zonal mesh refinement was implemented near the surface with the smallest cell size being 12.5 microns and with the largest size being 200 microns as seen in Figure 7.3. 


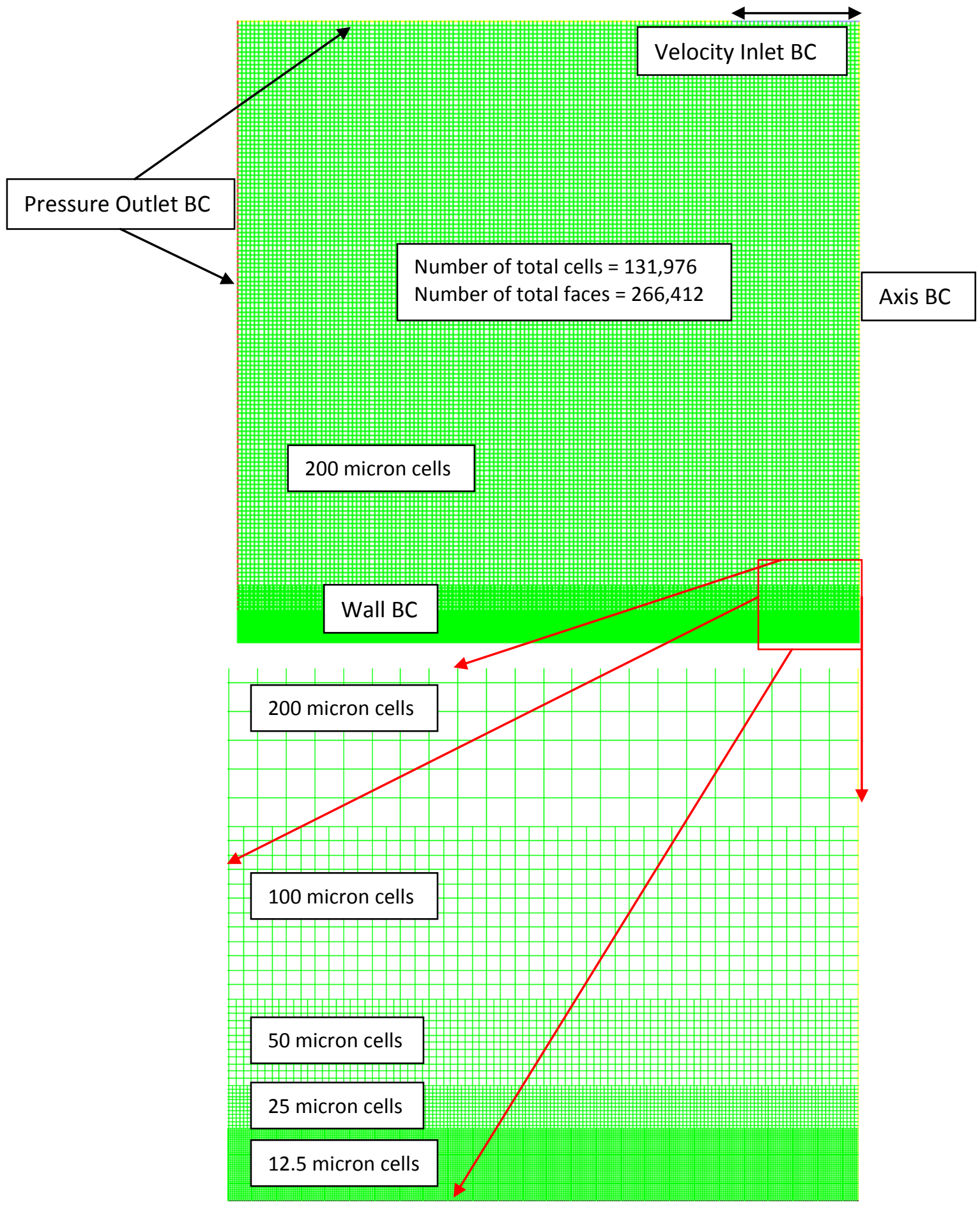

Figure 7.3 Computational cells for the whole domain (top) and for the near wall region (below). 


\subsection{Results}

First, an isothermal spray simulation for the FullJet 1/8-G full cone spray nozzle water spray was performed. Then, a spray cooling simulation for the same spray was performed for a superheated surface. The results of these simulations are described in the following sections.

\subsubsection{Isothermal Full Cone Spray Simulation}

Simulations of isothermal water sprays were performed based on the full cone spray operating at 40 psi using the HPC cluster located at WVU. Obtaining a fully converged solution from a single case took about 10-11 days using 6 cores and 48 GB memory in parallel. Table 7.1 shows the model parameters for both the isothermal and spray cooling cases.

The data analysis was done using ANSYS Fluent and CFD-Post software. The liquid film thickness over the impact surface was obtained using the liquid volume fraction values at several locations along the impact surface. Different liquid volume fraction iso-surfaces were studied in order to see which value best represented the actual gas liquid interface (Fig. 7.4). It was concluded that the liquid volume fraction range between 0.5-1 (50\%-100\%) gives the most accurate thickness since it does not include liquid volume fractions less than $50 \%$ (e.g. liquid volume fraction of impinging spray is $0.845 \%$ so it distinguishes film from spray liquid). furthermore, 0.5 values is used for the volume fraction of liquid and calculation of the film thickness. 
Table 7.1 Model parameters for Eulerian Multiphase (EM) Simulations.

\begin{tabular}{|c|c|c|}
\hline Model parameters & Isothermal Spray Case & Spray Cooling Case \\
\hline Nozzle type & FullJet 1/8-G (Full Cone) & FullJet 1/8-G (Full Cone) \\
\hline Spray cone half angle & $28^{\circ}$ & $28^{\circ}$ \\
\hline Nozzle to surface distance & $38.1 \mathrm{~mm}$ & $38.1 \mathrm{~mm}$ \\
\hline Spray radius at surface & $20.3 \mathrm{~mm}$ & $20.3 \mathrm{~mm}$ \\
\hline Nozzle pressure & 40 psig & 40 psig \\
\hline Spray liquid & Water & Water \\
\hline Liquid temperature & $300 \mathrm{~K}$ & $300 \mathrm{~K}$ \\
\hline Air temperature & $300 \mathrm{~K}$ & $300 \mathrm{~K}$ \\
\hline Surface temperature & $300 \mathrm{~K}$ & $393 \mathrm{~K}$ \\
\hline Energy equation included? & No & Yes \\
\hline Model & Eulerian Multiphase (EM) & Eulerian Multiphase (EM) \\
\hline Boiling model & No & RPI Boiling \\
\hline Dimensionality & 2D axisymmetric & 2D axisymmetric \\
\hline Mesh type & $\begin{array}{l}\text { Zonally refined mesh with } 200 \\
\text { micron maximum and } 12.5 \\
\text { micron minimum size }\end{array}$ & $\begin{array}{l}\text { Zonally refined mesh with } 200 \\
\text { micron maximum and } 12.5 \\
\text { micron minimum size }\end{array}$ \\
\hline
\end{tabular}




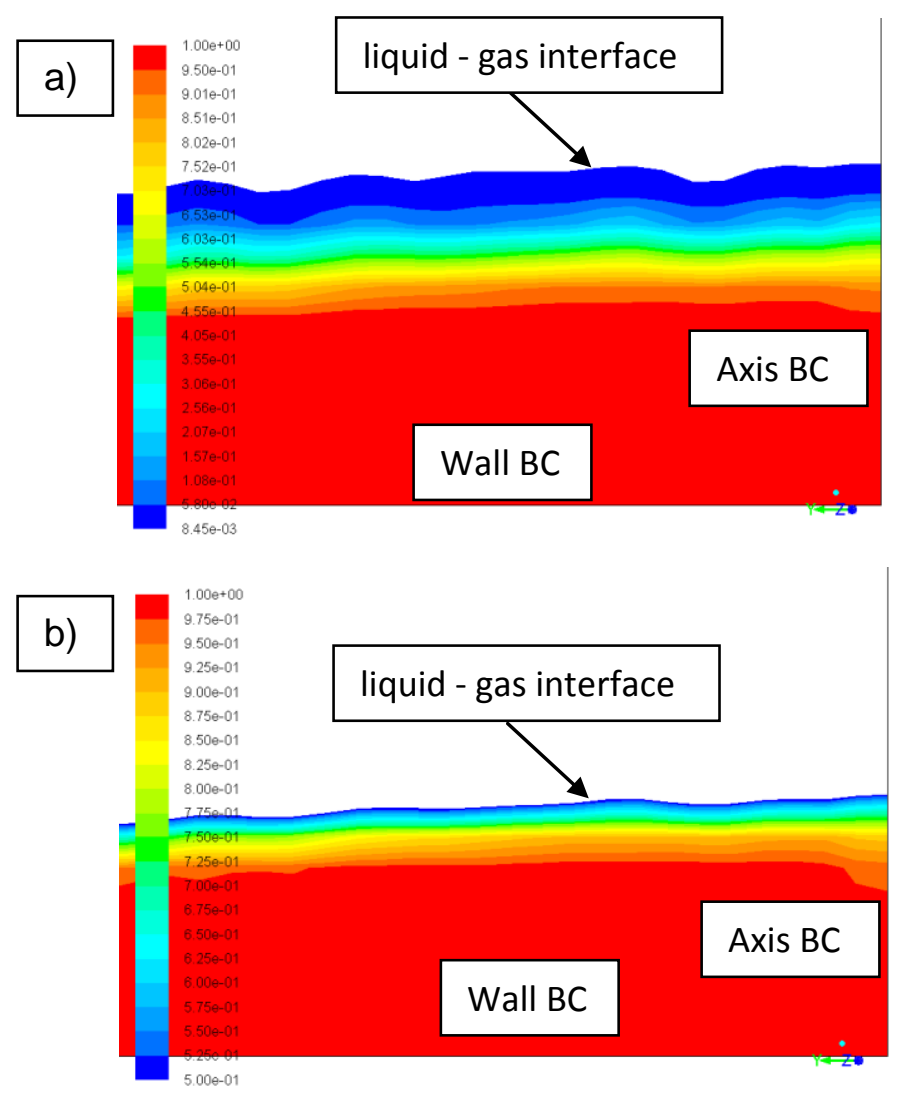

Figure 7.4 Flooded contours of liquid volume fraction at $100 \mathrm{~ms}$ simulation time near the impact surface (a) iso-surface values for 0.00845-1 (0.845\%-100\%) (Red is 1.0 and blue is 0.845.) and (b) iso-surface values for 0.5-1 (50\%-100\%) (Red is 1 and blue is 0.5. Note that the whole domain is not shown. Areas in $a$ and $b$ are the same. Horizontal distance is 320 micron and vertical distance is 200 micron.)

Figure 7.5 shows the flooded contours of liquid volume fraction near to a portion of the impact surface at different simulation times. The film characteristics in the spray impact area become almost constant after $30 \mathrm{~ms}$. 

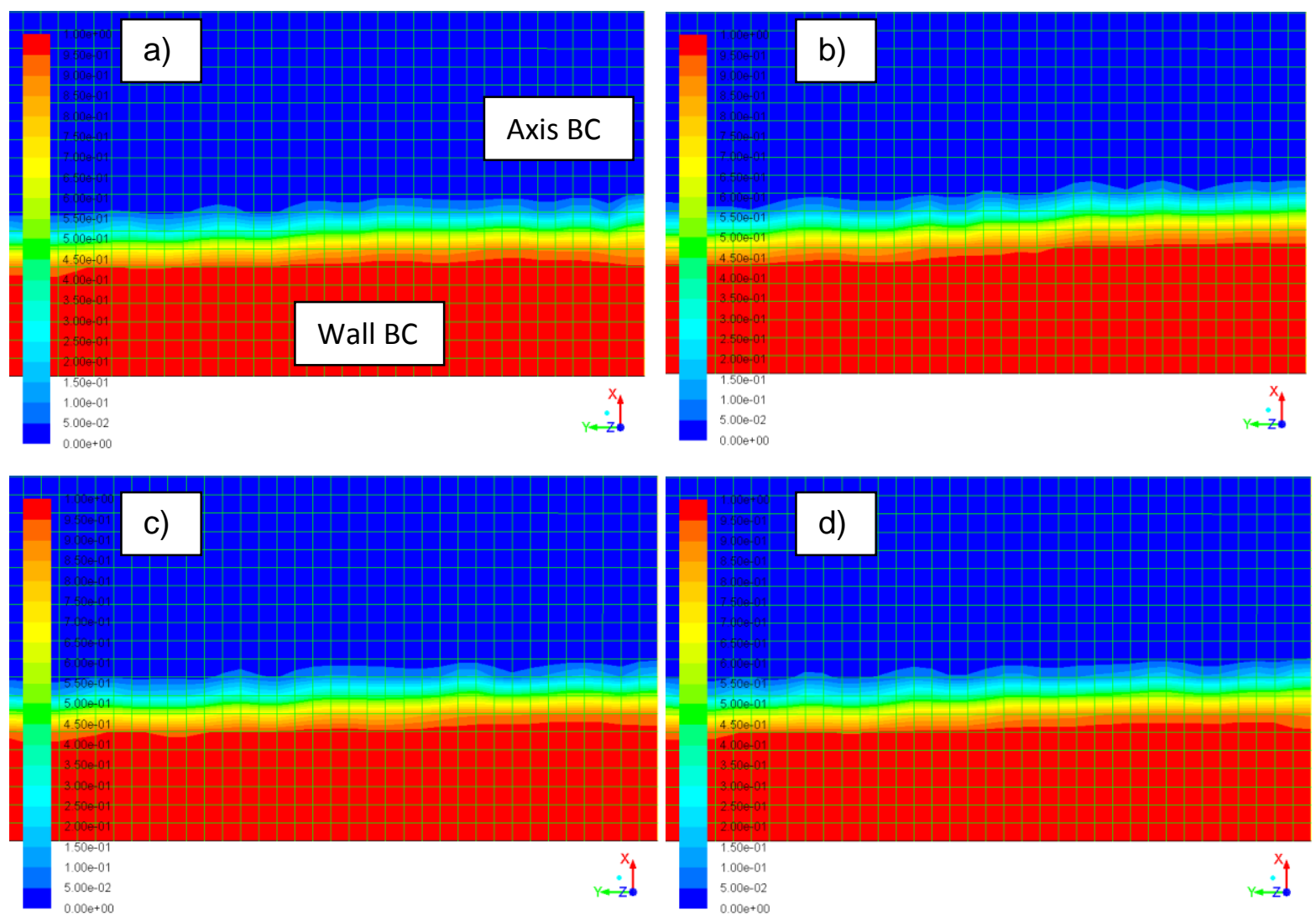

Figure 7.5 Contours of liquid volume fraction near the impact surface at (a) $30 \mathrm{~ms}$ (b) $50 \mathrm{~ms}$ (c) $70 \mathrm{~ms}$ (d) $100 \mathrm{~ms}$ simulation time. (Red is 1.0 and blue is 0 . Note that the whole domain is not shown. Areas in $a, b, c$ and $d$ are the same. Horizontal distance is 450 micron and vertical distance is 250 micron. Each cell is 12.5 micron in this figure.)

Even though the film thickness became constant after $30 \mathrm{~ms}$ within the spray impact area, the film thickness showed large temporal fluctuations outside the spray impact area. Figure 7.6 shows the arithmetic mean and Root Mean Square (RMS) values of film thickness between 30 $\mathrm{ms}$ and $110 \mathrm{~ms}$. However, the mean values in WVU experiments were obtained for a long spraying time $(0-5 \mathrm{~s})$. As seen in Fig. 7.6, the RMS increases beyond the spray coverage area $(R$ $>20 \mathrm{~mm}$ ) until the end of the domain $(R=28.1 \mathrm{~mm})$. Relative fluctuations in the film were calculated using the ratio of RMS film thickness to the mean film thickness $\left(h_{R M S} / h_{\text {mean }}\right)$. Figure 7.7 shows that the relative fluctuation is almost constant with the value of $4 \%$ within the spray impact area $(0<R<20 \mathrm{~mm})$. However, it increases gradually for $R>20 \mathrm{~mm}$ and reaches $75 \%$ due to the decreasing mean and the increasing RMS which is not consistent with the previous simulations mentioned in earlier chapters and experiments. 


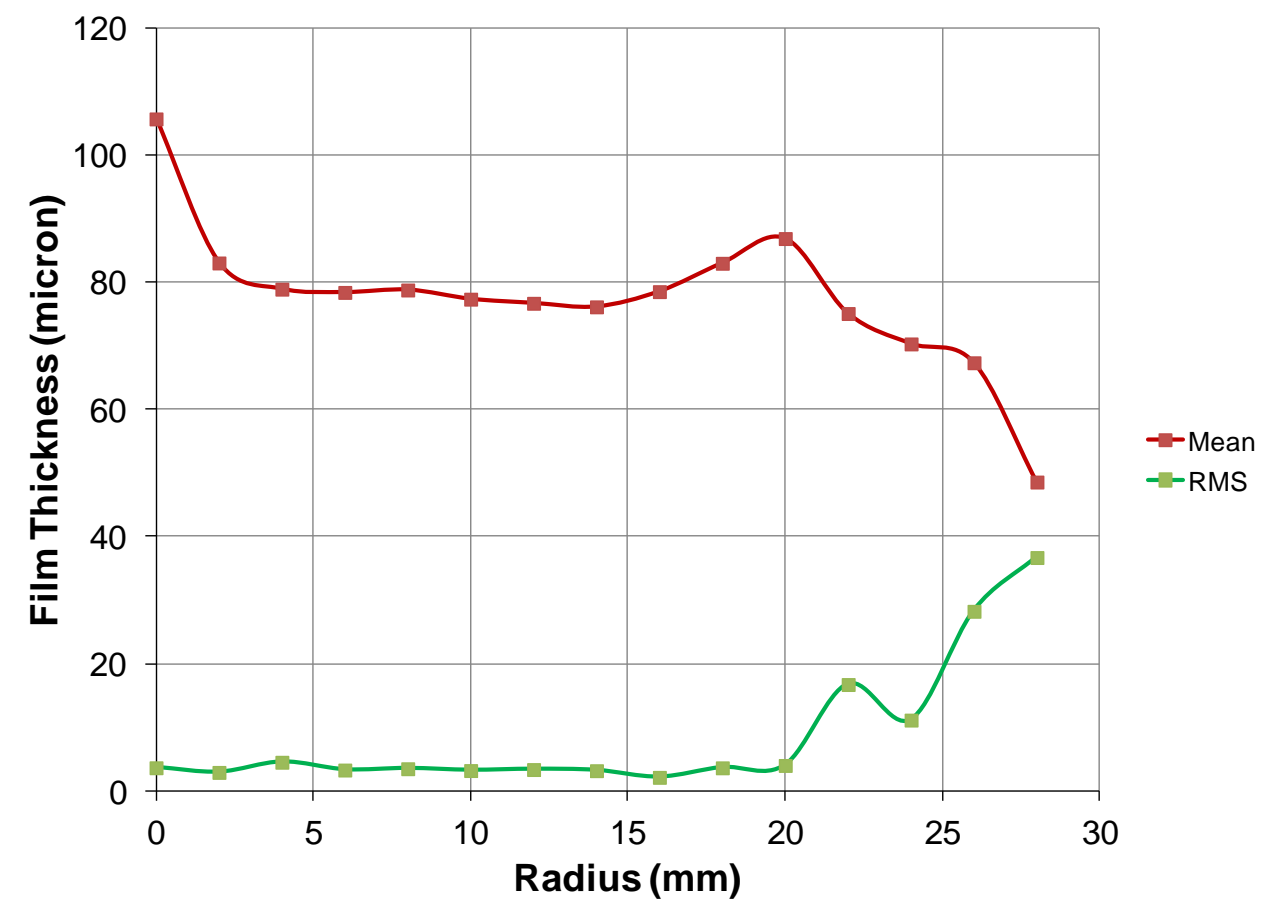

Figure 7.6 Liquid film thickness time average and Root Mean Square (RMS) thickness values vs. radius over the time interval from $30 \mathrm{~ms}-110 \mathrm{~ms}$.

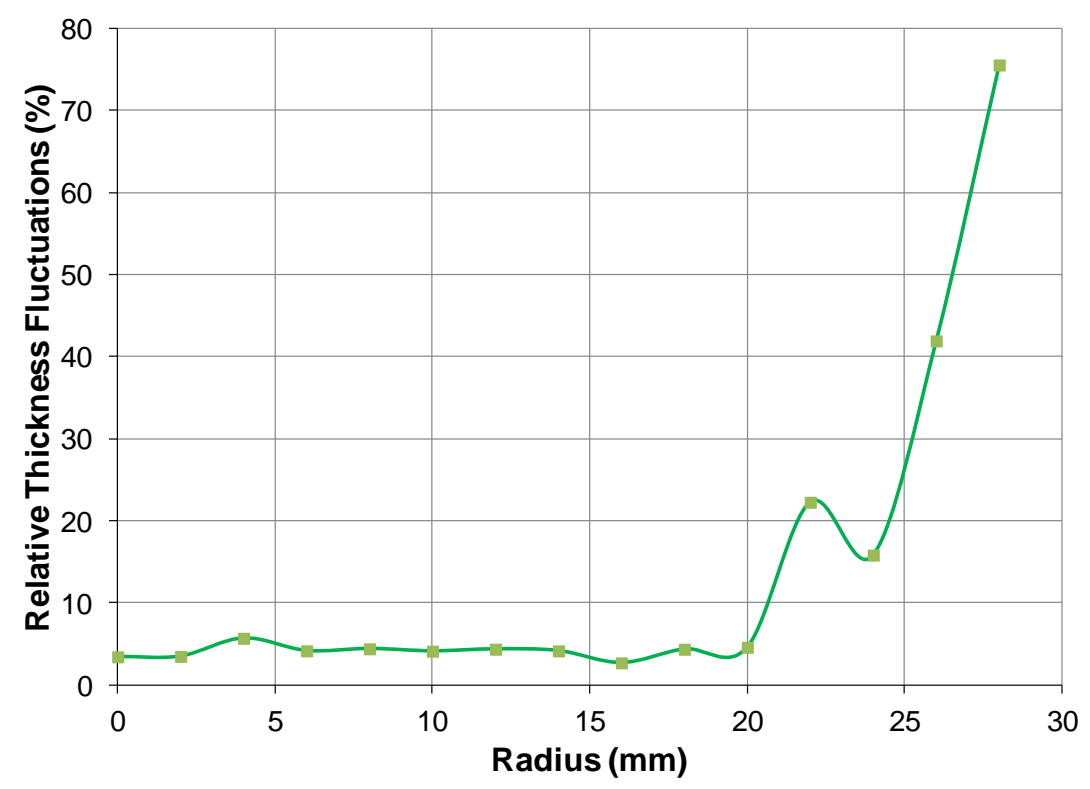

Figure 7.7 Relative thickness fluctuations in the liquid film (Relative thickness fluctuations = $\left.100\left(h_{R M S} / h_{\text {mean }}\right)\right)$.

Figure 7.8 shows examples of the film thickness variation with respect to time at different radial locations. The film is thickest at the centerline. Beneath the spray the free surface 
fluctuates in phase. Outboard of the spray at $R=26 \mathrm{~mm}$ the fluctuations are no longer in phase and the amplitude is much larger with the minimum value of 20 microns at $30 \mathrm{~ms}$ and a maximum value of 105 microns at $100 \mathrm{~ms}$. It is not known if these fluctuations would continue or damp out at later times.

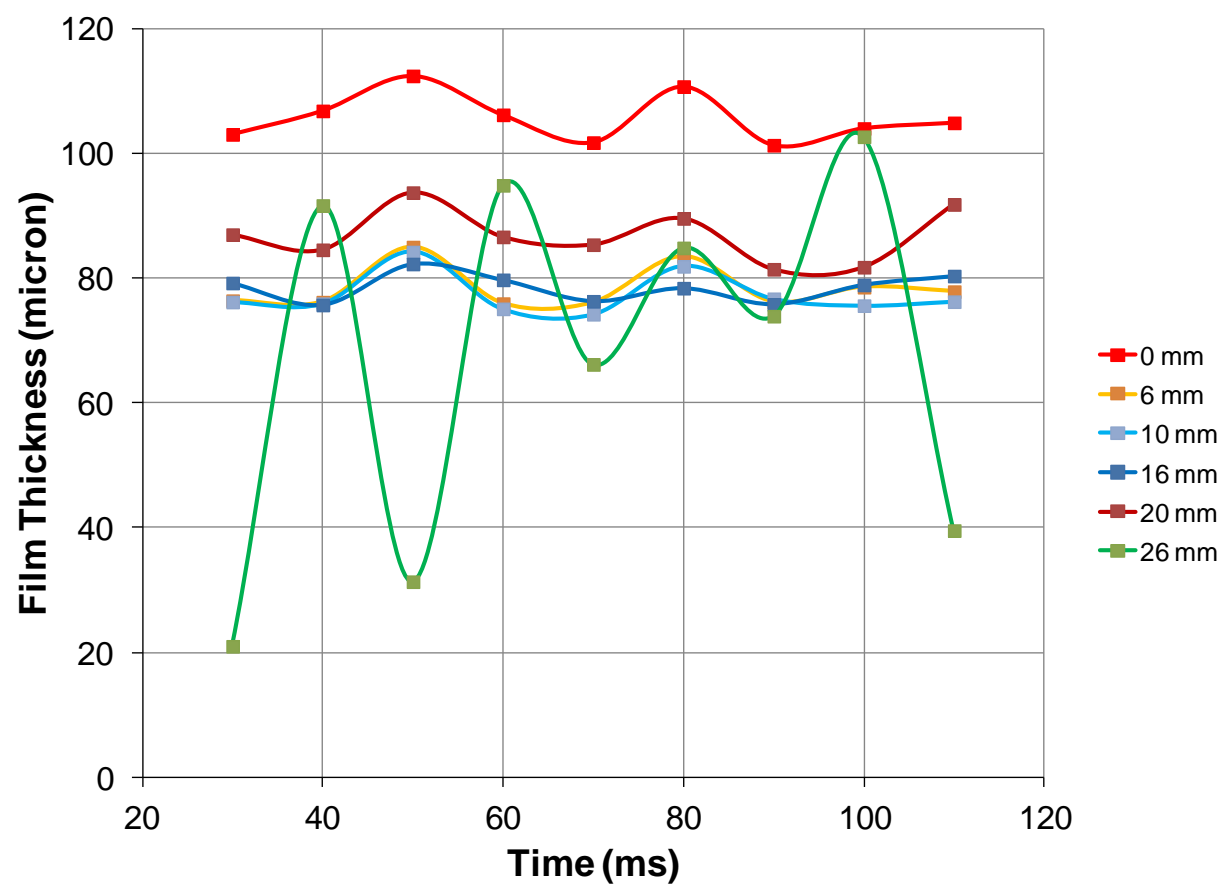

Figure 7.8 Liquid film thickness with respect to simulation time at different radial locations.

\subsubsection{Full Cone Spray Cooling Simulation}

A 40 psi full cone 2D axisymmetric spray cooling simulation was performed using the EM Model with the same 2D axisymmetric domain as in Section 7.3.1. The RPI boiling model was used to account for the phase change effects near the wall. Getting a fully converged solution from a single case took about 15 days using 6 cores and 48 GB memory in parallel. The same case was also simulated in 3D in Chapter 6 using the DPM, EWF and STM models. The cases are compared in Figs. 7.9-7.12 below.

Figure 7.9 shows that the film thickness predicted by the EM model is considerably less and decreases whereas the EWF thickness increases in general agreement with experiments. Figure 7.10 shows that the EM film velocity magnitude diverges sharply for $R>8 \mathrm{~mm}$. There does not seem to be any physical reason for the rapid increase of velocity at large radii predicted 
by the EM model. Figure 7.11 shows that the film temperature is at least $10 \mathrm{~K}$ higher in the EM simulation. Figure 7.12 shows the vapor mass fraction for both cases. The vapor mass fraction is similar from the centerline to $20 \mathrm{~mm}$. However, the vapor mass fraction increased much more rapidly beyond $20 \mathrm{~mm}$ for the EM simulation. Table 7.3 shows the calculated wall heat flux values for $3 \mathrm{D}$ and $2 \mathrm{D}$ axisymmetric cases. Even though the film characteristics were dramatically different, the wall heat flux values were reasonably close for both cases. The wall heat flux for the 2D axisymmetric EM case equals $143 \mathrm{~W} / \mathrm{cm}^{2}$, compared to $131 \mathrm{~W} / \mathrm{cm}^{2}$ for the EWF 3D simulation. Based on these comparisons, the EM simulation results presented in this chapter appear to be incorrect and should receive no further consideration. Given the extremely high computational demands of the EM model, it could not be determined if the model is inherently flawed or if one or more of the particular values of the many model constants used in these simulations are at fault.

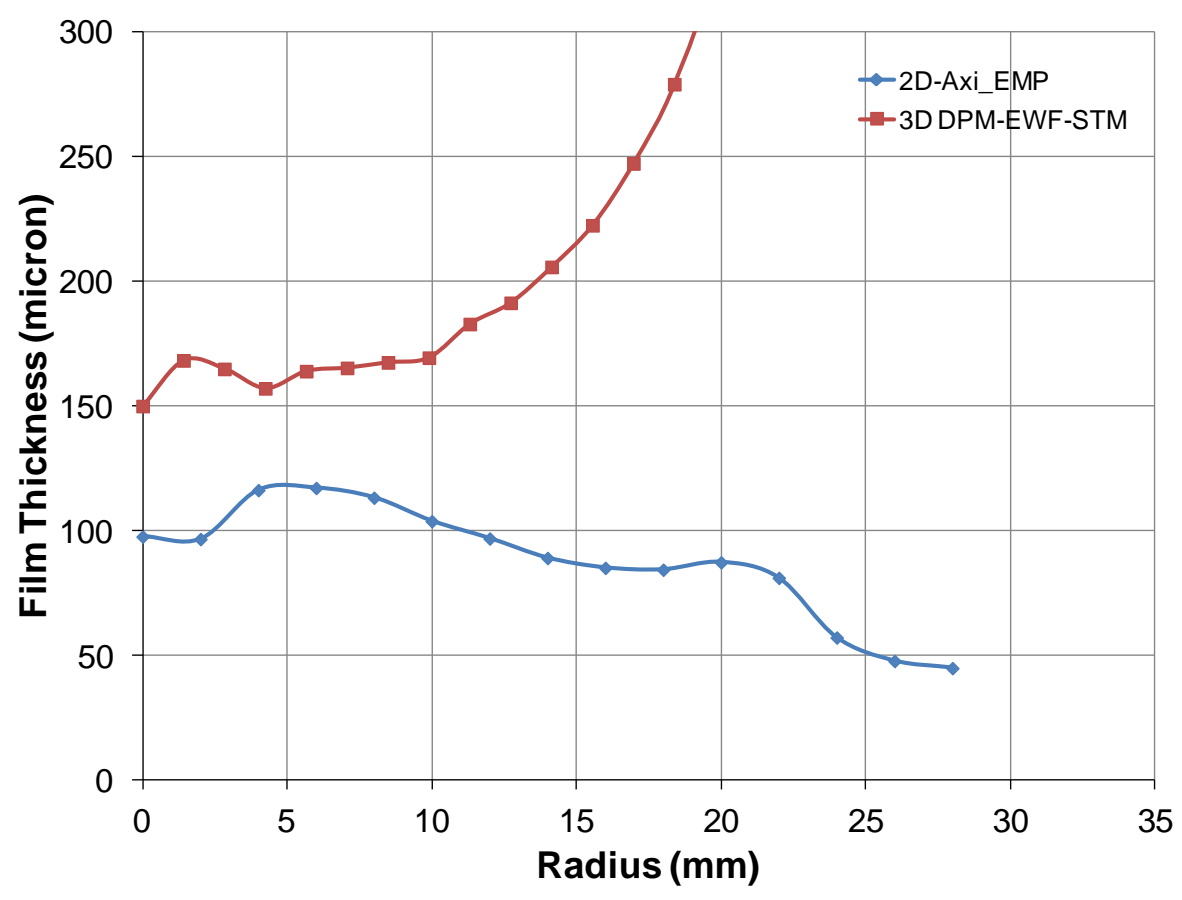

Figure 7.9 Liquid film thickness variation with radius at $100 \mathrm{~ms}$. 


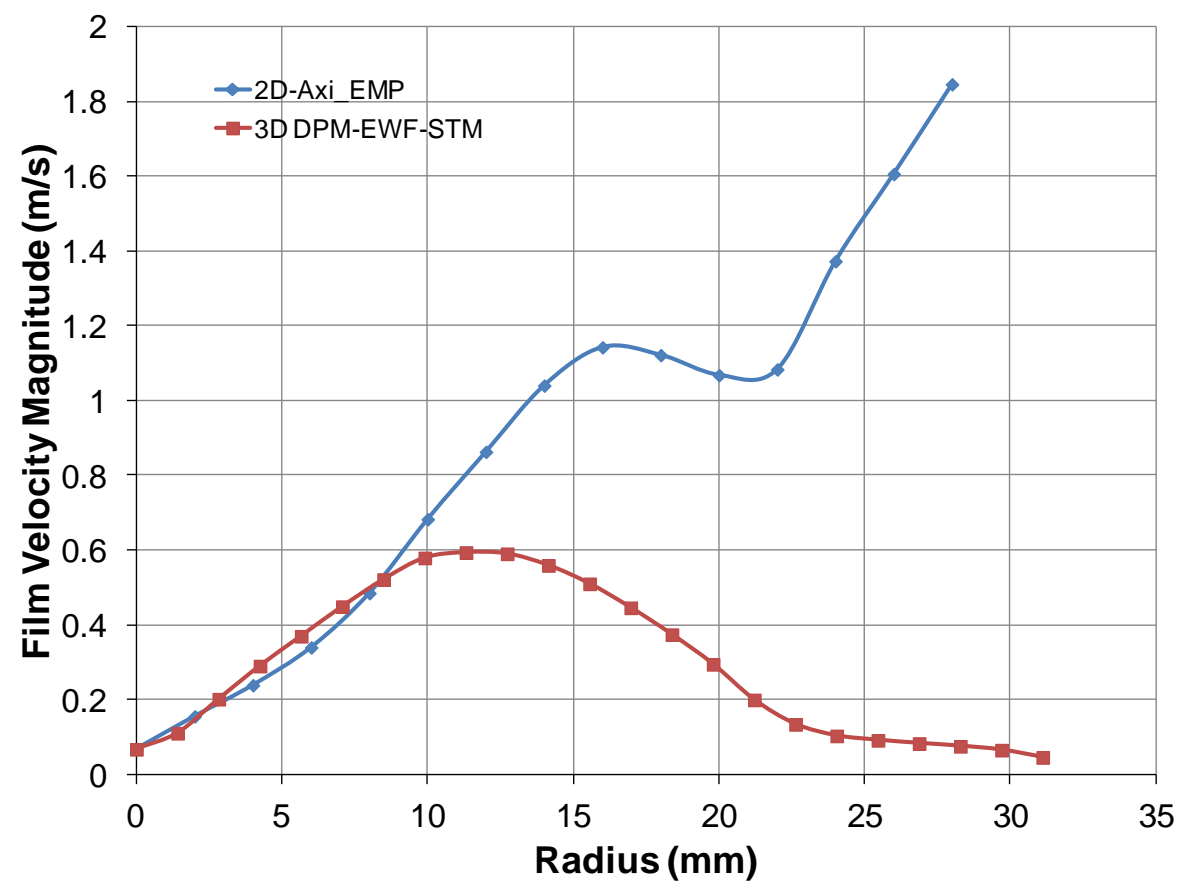

Figure 7.10 Liquid film velocity magnitude variation with radius at $100 \mathrm{~ms}$.

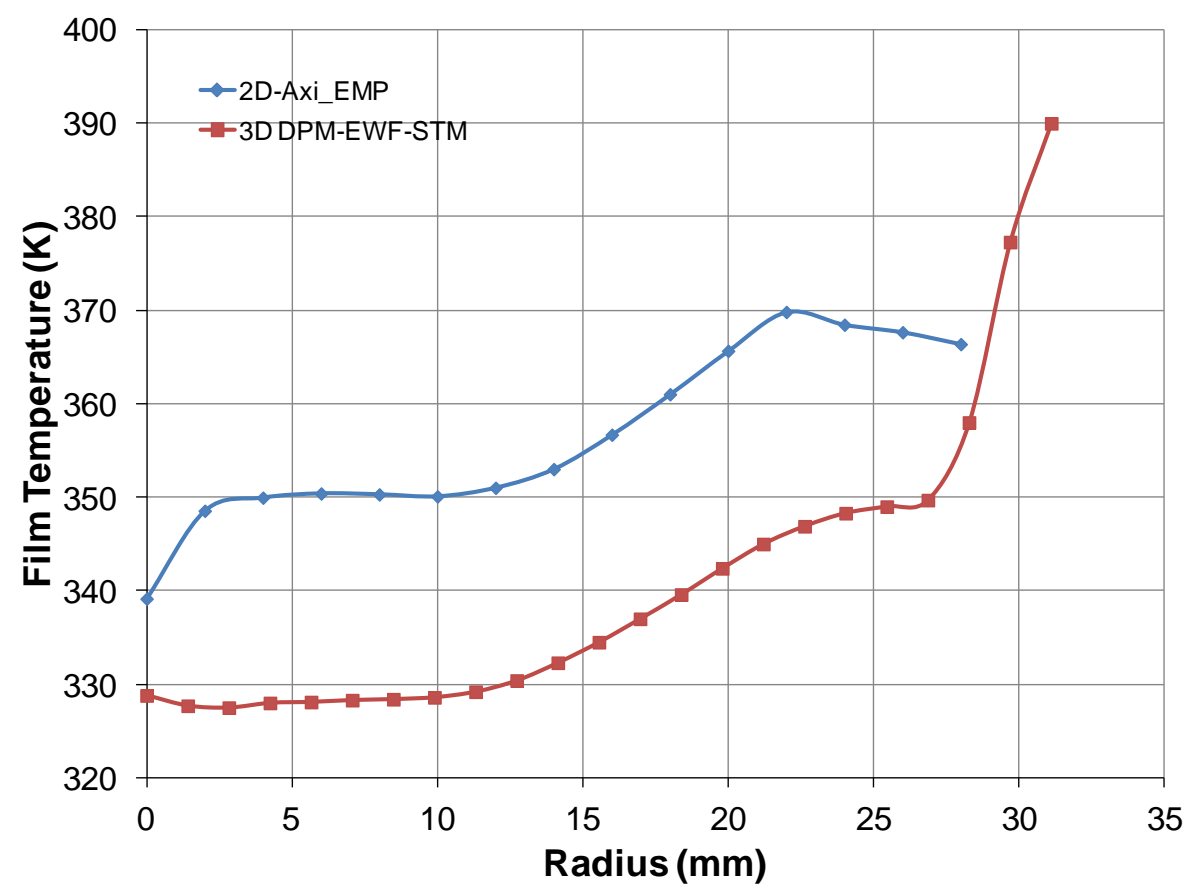

Figure 7.11 Liquid film temperature variation with radius at $100 \mathrm{~ms}$. 


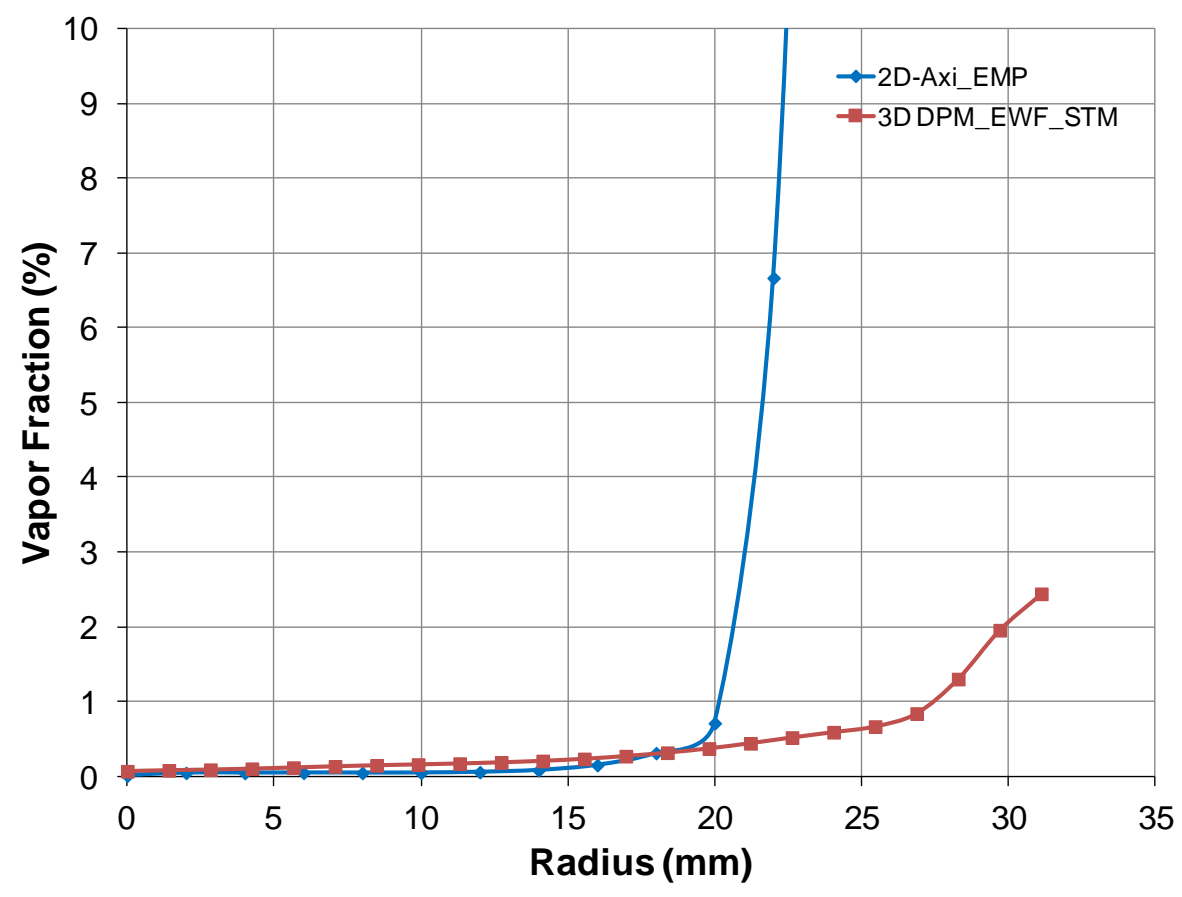

Figure 7.12 Vapor mass fraction variation with radius at $100 \mathrm{~ms}$.

Table 7.2 Computed wall heat flux values for phase change simulations.

\begin{tabular}{|c|c|c|c|c|c|}
\hline Case & $\begin{array}{c}\text { Air } \\
\text { Temperature } \\
(\mathbf{K})\end{array}$ & $\begin{array}{c}\text { Spray Liquid } \\
\text { Temperature } \\
(\mathbf{K})\end{array}$ & $\begin{array}{c}\text { Surface } \\
\text { Temperature } \\
(\mathbf{K})\end{array}$ & $\begin{array}{c}\text { Initial Liquid } \\
\text { Film } \\
\text { temperature } \\
(\mathbf{K})\end{array}$ & $\begin{array}{c}\text { Computed } \\
\text { Surface } \\
\text { Heat Flux } \\
\text { (W/.cm }\end{array}$ \\
\hline $\begin{array}{c}\text { 3D DPM- } \\
\text { EWF-STM }\end{array}$ & 300 & 300 & 393 & 300 & 131 \\
\hline $\begin{array}{c}\text { 2D- } \\
\text { axisymmetric } \\
\text { EM }\end{array}$ & 300 & 300 & 393 & 300 & 143 \\
\hline
\end{tabular}

As a summary of Chapter 7, spray cooling with phase change simulations were performed using the 2D axisymmetric Eulerian Multiphase (EM) model in ANSYS Fluent. Results were compared with the 3D DPM-EWF-STM model simulations. Comparisons showed some major discrepancies for the liquid film characteristics such as film thickness, velocity magnitude and temperature distribution along the impact radius. In addition, simulation of the 2D axisymmetric spray cooling with phase change took about 15 days which was almost three times more compared to the 3D DPM-EWF-STM model using 6 cores and 48 GB memory of the High Performance Computing (HPC) cluster at West Virginia University. The EM model can 
potentially give better results than shown in Chapter 7 when using different parameters for the models and using different drag models in ANSYS Fluent. 


\section{CHAPTER 8: CONCLUSIONS AND FUTURE WORK}

\subsection{Conclusions}

\subsubsection{Simulations of Single Drop Impact on Wetted Surfaces}

In Chapter 3, the isothermal single drop impingement on a thin liquid film has been simulated using the ANSYS Fluent 14 and 14.5 using the Volume of Fluid (VOF) multiphase model in 2D axisymmetric coordinates and 3D coordinates (for one case). Adaptive grid refinement has been implemented on the interfacial zones in order to increase the accuracy of the results while keeping the total number of computational cells relatively low. The liquid interface and crown diameter variation were in good agreement with previous experiments and numerical simulations. Full 3D Level-3 (D/40 smallest mesh size in which $D$ is the drop diameter) and 2D axisymmetric Level-4 (D/80 smallest mesh size) simulation results were in good agreement. The experiments and the CFD simulations matched closely for low $W e$ and $R e$ number range (Case 2 in Chapter 3). There were differences obtained at relatively higher $W e$ and $R e$ number range in which secondary splashing was observed (Case 1 and Case 3 in Chapter 3). These differences could be due to the axisymmetric modeling. Thus, the 3D modeling of a single drop is necessary for accurate simulations for high $W e$ and $R e$ number range cases.

Simulations of drop impacts for low $W e$ and $R e$ number have been analyzed for gravitational accelerations ranging from extremely large (Solar) to zero using the 2D axisymmetric VOF model. While the general evolution of the craters was similar, the rate at which they evolved increased along with the value of $g$. The impact of an upward moving drop on a downward facing layer caused a significantly different flow, as it excited a Rayleigh-Taylor instability which caused the liquid to fall off of the ceiling.

Laboratory observations have found that water drops in the 4-mm size range are not true spheres when they impact a liquid layer. To explore the importance of non-spherical drop shapes, three cylindrical drops were simulated, even though there is no known method to produce such shapes in the laboratory. While there were definite differences compared to the impact of a sphere, the overall similarity of the flows produced by these radically different drop shapes 
implies that the much smaller deviations from sphericity which occur in reality need not be considered in order to obtain realistic simulations.

In conclusion, single drop impact simulations can be studied using the 2D axisymmetric VOF model in ANSYS Fluent for low We and Re number range in order to develop single drop correlations for spray cooling applications.

\subsubsection{Simulations of Sprays Impacting on Dry Surfaces at Isothermal Conditions}

In Chapter 4, isothermal spray simulation results have been obtained from 3D and 2D axisymmetric models under turbulent flow conditions using the Discrete Phase Model (DPM) in ANSYS Fluent 14. The pressure-swirl atomizer model has been utilized to create swirling hollow cone spray patterns for full $3 \mathrm{D}$ and $2 \mathrm{D}$ axisymmetric domains. Comparison of these results justified the use of the 2D axisymmetric model. Full cone sprays based on the nozzle used by the WVU experimental group have been studied using the 2D axisymmetric model. The velocity and diameter distributions of the spray drops, mass of the liquid film that accumulated on the impact surface, and the spray impact efficiency have been studied by varying gravity, spray mass flow rate, nozzle-to-surface distance, spray half angle, and spray liquid properties (density, surface tension and viscosity). It can be concluded based on the cases investigated that the spray impact efficiency can be increased under these conditions:

- Smaller nozzle-to-surface distance $(h)$ based on Case C (parameters of Case C are explained in Chapter-4),

- Smaller spray half angle $(\theta)$ based on Case C,

- $\quad$ Spraying at higher gravity $(g)$ giving a lower Froude $(F r)$ number $\left(F r=V^{2} / g d\right)$ based on Case $\mathrm{C}$,

- Higher spray mass flow rate $(M)$ based on Case C,

- More viscous liquid $(\mu)$ giving a lower Reynolds $(R e)$ number $(\operatorname{Re}=\rho V d / \mu)$ based on Case C,

- Higher surface tension of liquid $(\sigma)$ giving a lower Weber $(W e)$ number $(W e=$ $\rho V^{2} d / \sigma$ ) based on Case C,

- Smaller density (less dense) of liquid ( $\rho$ ) giving a lower Reynolds $(R e)$ number $(\operatorname{Re}=\rho V d / \mu)$ based on

Case C. 


\subsubsection{Simulations of Spray Cooling without Phase Change}

In Chapter 5, simulations of partial 3D sprays and spray cooling without phase change have been performed using the DPM and EWF model using ANSYS Fluent 14.5. It has been demonstrated that the DPM is suitable for calculating droplet trajectories, but that the DPM wall film submodel gives unreasonable results compared to the Eulerian Wall Film Model (EWF). Therefore, the EWF model has been used for the calculations of the spray-wall interactions. Simulation results have been compared to the experimental measurements performed at WVU. It has been concluded that assuming laminar flow in the EWF produced thinner films when compared to laboratory data. However, this has been corrected by using a constant eddy viscosity to account for the macroscopic mixing caused by droplet impacts in the film since the other available turbulent models were not applicable for spray cooling. Based on this assumption, spray cooling simulations without phase change have been successfully performed. Film thickness results over the impact surface using the turbulent viscosity model have been close to the experimental measurements. Thus, turbulent viscosity model has been used for the further spray cooling with phase change cases which were discussed in Chapter 6.

\subsubsection{Simulations of Spray Cooling with Phase Change}

In Chapter 6, 40 psi full cone water spray cooling simulations with phase change were performed in 3D coordinates using the DPM, EWF and the Species Transport Model (STM) in ANSYS Fluent 15. Surface tension was neglected in these simulations. Film thickness results have been compared with the experiments performed at WVU using a 40 psi full cone nozzle. Different surface temperature, spray temperature and air temperature cases have been studied. It has been concluded that air temperature does not have a significant effect on the film characteristics (e.g. film thickness, film velocity magnitude, etc.) and heat transfer (surface heat flux, vapor formation, etc.). Whereas, spray temperature has significant effects on the film characteristics and heat transfer. Increasing the spray temperature $50 \mathrm{~K}$ (from $300 \mathrm{~K}$ to $350 \mathrm{~K}$ ) causes a $62 \%$ decrease on the surface heat flux. In addition, there is a high amount of vapor formation when

using the spray at $350 \mathrm{~K}$ compared to the spray at $300 \mathrm{~K}$. However, air temperature has no effect on the formation of vapor and the surface heat flux. In conclusion, 3D DPM-EWF-STM models 
in ANSYS Fluent 15 can be used for the modeling of spray/spray cooling in order to develop correlations for spray cooling applications.

In Chapter 7, 40 psi full cone water spray and spray cooling simulations have been performed in 2D axisymmetric coordinates using the Eulerian Multiphase (EM) model in ANSYS Fluent. Zonal mesh refinement has been applied close to the wall in order to increase the accuracy of the film and heat transfer calculations. The RPI boiling model has been used in order to include phase change effects in the film (bubble formation from nucleation sites over the impact surface). One spray cooling case with a constant wall temperature of $393 \mathrm{~K}$ has been simulated and results of this case have been compared to the 3D DPM-EWF-STM case. The film characteristics of the EM model were radically different compared to the 3D DPM-EWF-STM case. However, the average surface heat flux was within $8 \%$ difference between the two different spray cooling models. In conclusion, the EM model should not be used for spray cooling simulations until the reasons for its poor performance have been understood and corrected.

In conclusion, the 3D DPM-EWF-STM model is recommended for simulations of spray cooling.

\subsection{Future Work}

This study aimed to perform computational simulations of single drops and sprays to obtain models that can be used for spray cooling applications. It was found which models in ANSYS Fluent can be used for the modeling of single drops, sprays and spray cooling. It is believed that this study is unique and can be helpful for the researchers who work in the single drop and spray cooling area.

Single drops can be computationally studied using the 2D axisymmetric VOF model in ANSYS Fluent as long as the correct boundary, initial conditions and model setup is accomplished and for relatively low Reynolds $(R e)$ and Weber (We) numbers in which late (secondary drop) splashing does not occur. For high $R e$ and We number cases, it is suggested to use a full 3D VOF model to obtain more accurate splashing phenomena from single drop simulations. Single drop correlations for the Monte Carlo Spray Cooling model (Kreitzer and Kuhlman, 2010) can be derived using the VOF model. Single drop impingement on hot surfaces can be also studied using the VOF model. Single drop effects at different boiling regimes can be also studied by including phase change effects (e.g. bubble formation from nucleation sites, film 
evaporation). Gravity effects at higher $W e$ and $R e$ can be studied using a 3D VOF model in ANSYS Fluent in order to study secondary splashing.

Sprays and spray cooling can be computationally studied using the 3D DPM, EWF and STM models in ANSYS Fluent 15 with the correct coupling between the models, initial and boundary conditions and model setup. In this study, only the constant surface temperature boundary condition was studied using the 3D DPM-EWF-STM models for spray cooling with phase change. In addition, the constant surface heat flux boundary condition can be analyzed at different spray cooling conditions. Instead of constant eddy viscosity and thermal conductivity, variable eddy viscosity and conductivity $\left(\mu_{e}=\mu(r)\right.$ and $\left.k_{e}=k(r)\right)$ can give more agreement with the experiments. But, these variable viscosity and conductivity will be also dependent to spray liquid, nozzle type, nozzle-to-surface distance, etc. Some correlations for different spray cooling conditions can be derived using this model and can be implemented into the Monte Carlo Spray Cooling model. For example, the effects of nozzle pressure, nozzle flow rate, spray coolant and gravity on spray cooling can be studied. In addition, different boiling regimes in spray cooling including critical heat flux can be analyzed.

The current spray cooling simulations using the 2D axisymmetric EM model in ANSYS Fluent are not accurate compared to the 3D DPM-EWF-STM models and experimental data. The model constants and parameters can be parametrically analyzed in order to obtain more accurate results from the EM model for spray cooling simulations. Different drag and turbulent models can be also studied using the EM in order to obtain accurate spray cooling results. However, more powerful computational resources are needed to perform the EM spray cooling simulations with phase change. 


\section{REFERENCES}

Alkhedhair, A., Guan, Z., Gurgenci, H., Jahn, I., He, S., "Experimental Study on Inlet Air

Cooling by Water Spray for Natural Draft Dry Cooling Towers Enhancement", 19th Australasian Fluid Mechanics Conference, Melbourne, Australia, 8-11 December, 2014

Arienti, M., Wang, L., Corn, M., Li, X., Soteriou, M. C., Shedd, T. A., Herrmann, M., "Modeling Wall Film Formation and Breakup Using an Integrated Interface-Tracking/Discrete Phase Approach", Journal of Engineering for Gas Turbines and Power, ASME, Vol. 133, No. 0315011, 2011

Asadi, S., and Passandideh-Fard, M., "A Computational Study on Droplet Impingement onto a Thin Liquid Film", The Arabian Journal for Science and Engineering, Vol. 34, No.2B, pp. 505517,2009

ANSYS Inc., ANSYS Fluent User's Guide, Release 14.0, Canonsburg, PA, 2011

ANSYS Inc., ANSYS Fluent Theory Guide, Release 15.0, Canonsburg, PA, 2013

ANSYS Inc., ANSYS Fluent User's Guide, Release 15.0, Canonsburg, PA, 2013

Brackbill, J. U., Kothe, D. B., Zemach, C., "A Continuum Method for Modeling Surface Tension", Journal of Computational Physics, Vol. 100, pp. 335-354, 1992

Chen, R. H., Chow, L. C., Navedo, J. E., "Optimal Spray Characteristics in Water Spray Cooling", International Journal of Heat and Mass Transfer, Vol. 47, pp. 5095-5099, 2004

Chen, R. H., Tan, D. S., Lin, K. C., Chow, L. C., Griffin, A. R., Rini, D. P., "Droplet and Bubble Dynamics in Saturated FC-72 Spray Cooling on a Smooth Surface", Journal of Heat Transfer, ASME, Vol. 130, No. 101501, pp. 1-9, 2008 
Cole, R., "A Photographic Study of Pool Boiling in the Region of the Critical Heat Flux", AIChE J.. Vol. 6, pp. 533-542, 1960

Cossali, G. E., Marengo, M., Coghe, A., "The Impact of a Single drop on a Wetted Solid Surface”, Experiments in Fluids, 22, pp. 463-472, 1997

Cossali, G. E., Marengo, M., Coghe, A. and Zhdanov, S., "The Role of Time in Single Drop Splash on Thin Film”, Experiments in Fluids, 36, 2004, pp. 888, 2004

Crowe, C., Sommerfeld, M., Tsuji, Y., "Multiphase Flows with Droplets and Particles", CRC Press, 1998

Dinc, M., "The Improvements of Snow Plowing Safety and Efficiency: Computational Modeling and Experimental Verification", MS Thesis, Dept. of Mechanical Engineering, University of Nevada, Reno, NV, 2011

Dinc, M. and Gray, D. D., "Drop Impact on a Wet Surface: Computational Investigation of Gravity and Drop Shape", Advances in Fluid Mechanics, Heat and Mass Transfer, Proceedings of the $10^{\text {th }}$ World Scientific and Engineering Academy and Society (WSEAS) International Conference on Fluid Mechanics and Aerodynamics (FMA'12), Istanbul, Turkey, ISBN: 978-161804-114-2, pp. 374-379, Aug. 21-23, 2012

Dinc, M. and Gray, D. D., "Drop Impingement onto a Wetted Surface: Effects of Gravity and Shape", International Journal of Mechanics, Issue 1, Vol. 7, pp. 26-36, 2013

Dinc, M., Gray, D. D., Hillen, N. L., Taylor, J. S., Kuhlman, J. M, “Spray Simulations to Support the Development of a Monte Carlo-Based Spray Cooling Model”, Paper AIAA 2013-2975, AIAA $43^{\text {nd }}$ Fluid Dynamics Conference and Exhibit, San Diego, CA, June 24-June 27, 2013 
Dinc, M, Gray, D. D., Kuhlman, J. M., Hillen, N. L., Medam, K. T., Taylor, J. S., "Exploratory Simulations of Full-Cone Water Spray Cooling", Submitted Papers of the Proceedings of the $89^{\text {th }}$ West Virginia Academy of Science (WVAS), Vol. 86, No. 1, Shepherdstown, WV, 2014

Estes, K. A., and Mudawar, I., "Correlation of Sauter Mean Diameter and Critical Heat Flux for Spray Cooling of Small Surfaces", International Journal of Heat and Mass Transfer, Vol. 38, No. 16, pp. 2985-2996, 1995

Galvan, E. M., Ramos, J. C., Anton, R., Khodabandeh, R., "Film Thickness and Heat Transfer Measurements in a Spray Cooling System with R134a", Journal of Electronic Packaging, ASME Vol. 133, No. 011002, pp. 1-11, 2011

Gant, S. E., "CFD Modeling of Water Spray Barriers", Health and Safety Laboratory Report, HSL/2006/79, 2006

Ghani, S. A. A. Abdul., Aroussi, A., Rice, E., "Simulation of Road Vehicle Natural Environment in a Climatic Wind Tunnel", Simulation of Practice and Theory, Vol. 8, Issues 6-7, pp. 359-375, 2011

Guechi, M. R., Desevaux, P., Baucour, P., "Numerical and Experimental Study of Spray Cooling of a Heated Metal Surface", ICLASS 2012, 12th Triennial Conference on Liquid Atomization and Spray Systems, Heidelberg, Germany, September, 2012

Herrmann, M., "A Balanced Force Refined Level Set Grid Method for Two-Phase Flows on Unstructured Flow Solver Grids", Journal of Computational Physics, Vol. 227, pp. 2674-2706, 2008

Hillen, N. L., Kuhlman, J. M, Dinc, M., Gray, D. D., "Drop Impingement on Wet and Dry Surfaces", Paper AIAA 2012-2960, AIAA 42 ${ }^{\text {nd }}$ Fluid Dynamics Conference and Exhibit, New Orleans, LA, June 25-June 28, 2012 
Hillen, N. L., Taylor, J. S., Menchini, M., Morris, G., Dinc, M., Gray, D. D., Kuhlman, J. M., "Droplet Impact Time Histories for Varying Weber Number and Liquid Film Thickness for Spray Cooling Application", Paper AIAA-2013-2976, AIAA 43rd Fluid Dynamics Conference, San Diego, California, June 24-27, 2013

Hinds, W. C., "Aerosol Technology: Properties, Behavior and Measurement of Airborne Particles”, 2nd Edition, John Wiley \& Sons, Inc., 1999

Hirt, C. W., and Nichols, B. D., "Volume of Fluid (VOF) Method for the Dynamics of Free Boundaries", Journal of Computational Physics, Vol. 39, pp. 201-225, 1981

Horacek, B., Kiger, K. T., Kim, J., "Single Nozzle Spray Cooling Heat Transfer Mechanisms", International Journal of Heat and Mass Transfer, Vol. 48, pp. 1425-1438, 2005

Horacek, B., Kim, J., Kiger, K. T., "Spray Cooling Using Multiple Nozzles: Visualization and Wall Heat Transfer Measurements", Proceedings of 2004 ASME Heat Transfer/Fluids Engineering Summer Conference, Charlotte, North Carolina, USA, No. 56163, 2004

Hsieh, C. C., and Yao, S. C., "Evaporative Heat Transfer Characteristics of a Water Spray on Micro-Structured Silicon Surfaces", International Journal of Heat and Mass Transfer, Vol. 49, pp. 962-974, 2006

Issa, R. I., "Solution of Implicitly Discretized Fluid Flow Equations by Operator Splitting", Journal of Computational Physics, Vol. 62, pp. 40-65, 1986

Issa, R. J., "Numerical Investigation of the Chilling of Food Products by Air-Mist Spray", International Journal of Aerospace and Mechanical Engineering, Vol. 3:2, pp. 121-130, 2009

Issa, R. J., and Yao, S. C., "A Numerical Model for the Mist Dynamics and Heat Transfer at Various Ambient Pressures", Journal of Fluids Engineering, ASME, Vol. 127, pp. 631-639, 2005 
Kim, J. H., You, S. M., Choi, U. S., "Evaporative Spray Cooling of Plain and Microporous Coated Surfaces", International Journal of Heat and Mass Transfer, Vol. 47, pp. 3307-3315, 2004

King, W. D., Dujmovic, S., "Fluid Flow and Particle Trajectories around simple bodies: Impaction of snowflakes on car windshields", American Journal of Physics, Vol. 55, Issue 2, pp. 149, 1987

Kreitzer, P. J., "Spray Cooling Simulation Implementing Time Scale Analysis and the Monte Carlo Method," Ph.D. Dissertation, West Virginia University, Morgantown, WV, 2010

Kreitzer, P. J., and Kuhlman, J. M., "Spray Cooling Droplet Impingement Model", AIAA 10th AIAA/ASME Joint Thermophysics and Heat Transfer Conference, Chicago, IL, 2010

Kuhlman, J. M., Hillen, N. L., Dinc, M., Gray, D. D., "Liquid Volume Measurements in the Cavity Formed by Single Droplet Impacts into a Thin, Static Liquid Film", Experimental Thermal and Fluid Science, Vol. 54, pp. 179-188, 2014

Kuhlman, J. M., Gray, D. D., Dinc, M., Hillen, N. L., Medam, K. T., Taylor, J. S., "Spray Cooling Heat Transfer Mechanisms", Final Technical Report on NASA Award Number NNX10AN04A, October, 2014

Kuhlman, J. M., Kreitzer, P. J., Mehra, D., Gray, D. D., Yerkes, K. L., "Influence of the Coulomb Force on Spray Cooling", STAIF $11^{\text {th }}$ Conference on Thermophysics Applications in Microgravity, Albuquerque, NM, Feb. 11-15, 2007

Kurul, N., and Podowski, M. Z., "On the Modeling of Multidimensional Effects in Boiling Channels", In Proceedings of the 27th National Heat Transfer Conference, Minneapolis, Minnesota, USA, 1991 
Lin, L., and Ponnappan, R., "Heat Transfer Characteristics of Spray Cooling in a Closed Loop", International Journal of Heat and Mass Transfer, Vol. 46, pp. 3737-3746, 2003

Lemmert, M., Chawla, L. M., "Influence of Flow Velocity on Surface Boiling Heat Transfer Coefficient in Heat Transfer in Boiling", E. Hahne and U. Grigull, Eds., Academic Press and Hemisphere, New York, NY, USA, 1977

Medam, K. T., Dinc, M., Gray, D. D., Kuhlman, J. M., "Drop Impingement onto a Wetted Surface: Effects of Thermal Buoyancy and Gravity Level", Proceedings of the New Trends in Transport Phenomena, Ottawa, Ontario, Canada, May 1-2, 2014

Nikolopoulos, A., Theodorakakos, A., Bergeles, G., "Three-dimensional Numerical Investigation of a Droplet Impinging Normally onto a Wall Film", Journal of Computational Physics, Vol. 225, pp. 322-341, 2007

Olsen, J. E., and Cloete, S., "Coupled DPM and VOF Model for Analyses of Gas Stirred Ladles at Higher Gas Rates", Seventh International Conference on CFD in the Minerals and Process Industries CSIRO, Melbourne, Australia, 9-11 December 2009

O'Rourke, P. J., and Bracco, F. V., "Two Scaling Transformations for the Numerical Computation of Multidimensional Unsteady Laminar Flames", Journal of Computational Physics, vol. 33 (2), pp. $185-203,1979$

Pais, M. R., Chow, L. C., Mahefkey, E. T., "Surface Roughness and Its Effects on the Heat Transfer Mechanism in Spray Cooling", Journal of Heat Transfer, Vol. 114, pp. 211-219, 1992

Pautsch, A. G., and Shedd, T. A., "Adiabatic and Diabatic Measurements of the Liquid Film Thickness During Spray Cooling With FC-72", International Journal of Heat and Mass Transfer Vol. 49, pp. 2610-2618, 2006 
Peduto, D., Koch, R., Morvan, H., Dullenkopf, K., Bauer, H.-J., "Numerical Studies of Single Drop Impact onto a Plane Shallow and Deep Liquid Pool", ILASS - Europe 2011, 24th European Conference on Liquid Atomization and Spray Systems, Estoril, Portugal, September 2011

Ranz, W. E., and Marshall, W. R. Jr., "Evaporation from Drops, Part I", Chemical Engineering Progress, Vol. 48 (3), pp. 141-146, 1952

Rider, W. J., and Kothe, D. B., "Reconstructing Volume Tracking", Journal of Computational Physics, Vol.141, pp. 112-152, 1998

Rini, D. P., Chen, R. H., Chow, L. C., " Bubble Behavior and Nucleate Boiling Heat Transfer in Saturated FC-72 Spray Cooling", Journal of Heat Transfer, Vol. 124, pp. 63-72, 2002

Sarkar, M., "Multiphase Flow Modeling of Spray Cooling to Improve Heat Transfer", Dissertation, University of Arkansas, August, 2008

Sarkar, S., and Selvam, R. P., "Direct Numerical Simulation of Heat Transfer in Spray Cooling Through 3D Multiphase Flow Modeling Using Parallel Computing", Journal of Heat Transfer, ASME, Vol. 131, No. 121007, pp. 1-8, 2009

Schick, R. J., "Spray Technology Reference Guide: Understanding Drop Size", Spray Analysis and Research Services, Spraying System Co, undated

Schiller, L, and Naumann, Z., "Z. Ver. Deutch. Ing., 77, 318, 1935

Schlichting, H., "Boundary Layer Theory", 7th Edition, New York, McGraw-Hill, p. 142, 1979

Schmidt, D. P., Nouar, I., Senecal, P. K., Rutland, C. J., Martin, J. K., Reitz, R. D., "PressureSwirl Atomization in the Near Field", SAE Paper, SAE, 01-0496, 1999 
Sehmbey, M. S., Chow, L. C., Pais, M. R., Mahefkey, T., "High Heat Flux Spray Cooling of Electronics", AIP Conference Proceedings, Vol. 324, pp. 903-909, 1995

Shedd, T., Corn, M. L., Arienti, M., Soteriou, M. C., "Liquid Jet Breakup by an Impinging Air Jet", Forty-Seventh AIAA Aerospace Sciences Meeting, Paper No. AIAA-2009-0998, 2009

Shih, T.-H., Liou, W. W., Shabbir, A., Yang, Z., Zhu, J., "A New k-epsilon Eddy Viscosity Model for High Reynolds Number Turbulent Flows-Model Development and Validation", Computers Fluids, 24, 3, pp. 227-238, 1995

Silk, E. A., Golliher, E. L., R. Paneer Selvam, R. P., "Spray Cooling Heat Transfer: Technology Overview and Assessment of Future Challenges for Micro-Gravity Application", Energy Conversion and Management, Vol. 49, pp. 453-468, 2008

Silk, E., Kim, J., Kiger, K. T., "Investigation of Enhanced Surface Spray Cooling", Proceedings of the ASME IMECE, Paper 2004-61753, Anaheim, CA, USA, 2004

St-Georges, and M., Buchlin, J. M., "Detailed Single Spray Experimental Measurements and One-Dimensional Modeling", International Journal of Multiphase Flow, Vol. 20, No. 6, pp.979992, 1994

Stanton, D.W., and Rutland, C. J., "Modeling Fuel Film Formation and Wall Interaction in Diesel Engines", SAE Paper 960628, 1996

Tao, Y., Huai, X., Guo, Z., Yin, R., "Numerical Simulation of Spray Performance Based on the Euler-Lagrange Approach", Journal of Thermal Science, Vol. 18, No. 1, pp. 91-96, 2009

Taylor, J. S., Kuhlman, J. M., Gray, D. D., Dinc, M., Medam, K. T., Hillen, N. L., “Techniques to Quantify Dynamic Phenomena of Spray Droplets Impinging on a Smooth Surface", 89th Annual Meeting of the West Virginia Academy of Science, Shepherd, WV, April 12, 2014a 
Taylor, J. S., Kuhlman, J. M., Medam, K. T., Dinc, M., Gray, D. D., Hillen, N. L., "Visualization Techniques to Quantify Dynamic Phenomena of Spray Droplets Impinging on a Smooth Surface", 39th Annual AIAA Dayton-Cincinnati Aerospace Sciences Symposium (DCASS), Dayton, Ohio, March 5, 2014b

Tolubinski, V. I., Kostanchuk, D. M., "Vapor Bubbles Growth Rate and Heat Transfer Intensity at Subcooled Water Boiling", 4th International Heat Transfer Conference, Paris, France, 1970

Yang, J. L., Chow, C., Pais, M. R., "Nucleate Boiling Heat Transfer in Spray Cooling", Journal of Heat Transfer, Vol. 118, pp. 668-671, 1996

Yarin, A. L., "Drop Impact Dynamics: Splashing, Spreading, Receding, Bouncing...", Annual Review of Fluid Mechanics, Vol.38, pp. 159-192, 2006

Yiğit, C., Sözbir, N., Yao, S. C., Güven, H. R., Issa, R. J., "Experimental Measurements and Computational Modeling for the Spray Cooling of a Steel Plate Near the Leidenfrost Temperature", Journal of Thermal Science and Technology, Isı Bilimi ve Tekniği Dergisi, 31, 1, pp. 27-36, 2011

Yokoi, K., Vadillo, D., Hinch, J., Hutchings, I., "Numerical Studies of the Influence of the Dynamic Contact Angle on a Droplet Impacting on a Dry Surface", Physics of Fluids, Vol. 21, 072102, pp. 1-12, 2009

Yoshida, K., Abe. Y., Oka, T., Mori, Y., Nagashima, A., "Spray Cooling Under Reduced Gravity Conditions", Journal of Heat Transfer, Vol. 123, pp. 309-318, 2001

Yerkes, K. L., Michalek, T. E., Baysinger, K. M., Puterbaugh, R., Thomas, S. K., McQuillen, J., "Variable-Gravity Effects on a Single-Phase Partially Confined Spray Cooling System", Journal of Thermophysics Heat Transfer, Vol. 20, pp. 361-370, 2006 
Youngs, D. L., "An Interface Tracking Method for a 3D Eulerian Hydrodynamics Code", Technical Report 44/92/35, AWRE, 1984

Versteeg, H. K., and Malalasekera, W., "An Introduction to Computational Fluid Dynamics the Finite Volume Method", published by Longman Scientific \& Technical, co-published in the US by John Wiley \& Sons Inc., 1995

Wang, A.-B., and Chen, C.-C., "Splashing Impact of a Single Drop onto very Thin Liquid Films", Physics of Fluids, Vol. 12, Number 9, pp. 2155-2158, 2000

http://www.spacedaily.com/reports/Astronomers_Plan_Last_Look_at_Asteroid_1999_RQ36_Be fore_OSIRIS_REx_Launch_999.html, accessed 9/19/2012

http://www.spray.com/(Spraying Systems Co) 


\section{VITA}

Murat Dinc received his Bachelor degree from the Mechanical Engineering Department at Gazi University, Ankara in Turkey. He received his Master degree from the Department of Mechanical Engineering at University of Nevada, Reno. He was a Research Assistant and worked on the research project sponsored by the Nevada Department of Transportation (NDOT). His Master thesis was "The Improvements of Snow Plowing Safety and Efficiency: Computational Modeling and Experimental Verification". He started his $\mathrm{PhD}$ as a Graduate Research Assistant at the Department of Civil and Environmental Engineering at West Virginia University in July 2011. He has been working on the research project "Spray Cooling Heat Transfer Mechanisms" sponsored by NASA since he has started his PhD at West Virginia University. 